\title{
Verhalten reaktiver Brandschutzsysteme auf kreisförmigen Zuggliedern aus Blank- und Baustahl
}

\author{
Von der \\ Fakultät für Bauingenieurwesen und Geodäsie \\ der \\ Gottfried Wilhelm Leibniz Universität Hannover \\ zur Erlangung des akademischen Grades eines
}

Doktor der Ingenieurwissenschaften

- Dr.-Ing. -

genehmigte Dissertation

von

Dustin Häßler

geboren am 26.07.1986 in Karl-Marx-Stadt (Chemnitz) 


\section{Promotionskommission:}

Hauptberichter: $\quad$ Prof. Dr.-Ing. Peter Schaumann

Korreferent: $\quad$ Prof. Dr.-Ing. Dipl. Wirt.-Ing. (NDS) Martin Mensinger

Vorsitz: $\quad$ Prof. Dr.-Ing. Ludger Lohaus

Kommissionsmitglied: Prof. Dr.-Ing. Nabil A. Fouad

Tag der Promotion: $\quad$ 4. Mai 2016 


\section{Kurzfassung}

Im Bauwesen werden Zugglieder aus Stahl in der Regel für Aussteifungsverbände, Abhängungen und Unterspannungen von Trägern verwendet. Die dafür verbauten, meist kreisförmigen Vollprofile dienen häufig der Stabilisierung des Tragwerks. Ein Versagen der Stahlzugglieder kann zu einem Einsturz der gesamten Konstruktion führen.

Unter Brandeinwirkung verlieren ungeschützte Stahlkonstruktionen aufgrund der schnellen Erwärmung frühzeitig ihre Tragfähigkeit. Durch den Einsatz reaktiver Brandschutzsysteme auf Stahlbauteilen kann deren Erwärmung verlangsamt und dadurch der Feuerwiderstand verbessert werden. Die Optik der oftmals filigranen Stahlzugglieder bleibt dabei im Wesentlichen unverändert.

Für die Anwendung reaktiver Brandschutzsysteme auf Stahlzuggliedern mit Vollquerschnitt gibt es derzeit in den allgemeinen bauaufsichtlichen Zulassungen keine Regelungen. Die an der Bundesanstalt für Materialforschung und -prüfung (BAM) durchgeführten experimentellen und numerischen Untersuchungen [35] sowie die darauf aufbauenden Erkenntnisse dieser Dissertation hinsichtlich des Verhaltens reaktiver Brandschutzsysteme auf Stahlzuggliedern mit Vollprofil bilden die Grundlage für eine Erweiterung des Anwendungsbereiches dieser Produkte. Eine Anwendung ist grundsätzlich möglich. Aufgrund der meist für Zugglieder eingesetzten filigranen Vollquerschnitte sowie der in Bezug auf die Tragfähigkeit fehlenden Umlagerungsmöglichkeit werden besonders hohe Anforderungen an die Wirksamkeit und Zuverlässigkeit von reaktiven Brandschutzsystemen gestellt. Anhand der Ergebnisse aus den Brandversuchen sowie theoretischen Betrachtungen ist festzustellen, dass das auf kreisförmigen, zugbeanspruchten Stahlprofilen aufgebrachte reaktive Brandschutzsystem aufgrund der notwendigen dreidimensionalen Ausdehnungsrichtung der höchst möglichen Beanspruchung ausgesetzt ist. Mit Hilfe von Leistungskriterien zur Bestimmung der Tragfähigkeit, welche im Rahmen der Dissertation entwickelt wurden, lassen sich die in Brandversuchen unter axialer Zugbeanspruchung getesteten Stahlzugglieder mit reaktiver Brandschutzbeschichtung in Feuerwiderstandsklassen einordnen.

Für die Beurteilung der thermischen Schutzwirkung des reaktiven Brandschutzsystems werden die Erwärmungsgeschwindigkeit, die maximale Stahltemperatur sowie die Rissbildung und das Rissheilungsvermögen der Beschichtung herangezogen. Die Brandversuche haben gezeigt, dass die Profilgeometrie, die Höhe der Trockenschichtdicke der Beschichtung, die Höhe der aufgebrachten Zugbeanspruchung sowie die Orientierungsrichtung der Zugglieder einen wesentlichen Einfluss auf die thermische Schutzwirkung des reaktiven Brandschutzsystems ausüben. Zudem stehen diese Faktoren in gegenseitiger Wechselwirkung. Durch Abstimmung der genannten Einflussgrößen lässt sich die Wirkung der Brandschutzbeschichtung optimieren. Durch die Vielzahl an reaktiven Brandschutzsystemen und die in den Produkten variierende chemische Zusammensetzung ist deren Aufschäumverhalten 
und thermische Schutzwirkung sehr unterschiedlich und kaum vorhersagbar. Die Wirksamkeit des reaktiven Brandschutzsystems ist daher für Grenzfälle des vom Hersteller definierten Anwendungsbereichs durch Brandversuche zu überprüfen. Empfehlungen zur Durchführung dieser Versuche sind im Forschungsbericht [35] beschrieben.

Bei Verwendung von kaltverformtem Blankstahl für Stahlzugglieder mit Vollprofil sind andere Hochtemperatureigenschaften als bei dem typischerweise für Träger und Stützen eingesetzten warmgewalztem Baustahl zu berücksichtigen. Kaltverformter Blankstahl weist gegenüber herkömmlichem Baustahl einen höheren Wärmeausdehnungskoeffizient und andere temperaturabhängige Abminderungsfaktoren für die Stahlfestigkeit auf. Die in experimentellen Untersuchungen für Blankstahl ermittelten Materialeigenschaften können für die Heißbemessung von Zuggliedern mit Vollprofil aus entsprechendem Material verwendet werden. In Kombination mit Temperaturdaten aus Brandversuchen lässt sich mittels eines auf der Grundlage der Finite-Elemente-Methode (FEM) entwickelten numerischen Berechnungsmodells das Trag- und Verformungsverhalten von Zuggliedern realitätsnah berechnen. Vergleiche zur Stablängsverformung von in Brandversuchen getesteten Zuggliedern zeigen eine hohe Übereinstimmung mit den Ergebnissen aus dem im Rahmen der Dissertation neu entwickelten Bemessungsmodell.

Stichworte: Brandschutz, Stahl, Zugglied, reaktive Brandschutzsysteme, Blankstahl, Materialeigenschaften, experimentelle Untersuchungen, numerische Simulation 


\section{Abstract}

In civil engineering, steel tension members are normally used for bracings, suspensions and underbracing systems. Typically, solid steel profiles are used for such tension members, in particular circular solid section. Steel tension members often stabilize the structure; in case of failure of these members, a collapse of the entire structure may happen.

Under fire exposure, unprotected steel structures lose their load-bearing capacity at an early stage because of the rapid heating. By the use of reactive fire protection systems, which are also called intumescent coatings, the heating rate of the steel can be reduced. Thus the fire resistance of the member can be improved, while the appearance of the filigree steel tension members remains essentially unchanged.

The application of the reactive fire protection systems on steel tension members with solid section is currently not regulated in the German national approvals [59]. The research project [35] and the presented research of the thesis, both were carried out at the Federal Institute for Materials Research and Testing (BAM), aim to form the basis for an extension of the scope of application of the reactive fire protection systems applied on steel tension members with circular solid section.

The experimental as well as numerical investigations show that an application of reactive fire protection systems on steel tension members with solid section is basically possible. However, due to the relatively filigree cross-sections and the lack of stress transfer possibility within the tension member, high requirements are placed particularly on the effectiveness and reliability of the reactive fire protection system. The results of the conducted fire tests and theoretical considerations show that the necessary three-dimensional extension at mechanically loaded tension members with circular solid section result in the highest stress level for the reactive fire protection system. With the help of performance criteria to identify the load-carrying capacity, which are developed in the thesis, and fire tests under axial tensile stress the fire resistance of steel tension members with reactive fire protecion systems can be classsified.

To assess the thermal protection of the reactive fire protection system, the heating rate and maximum steel temperature of the steel as well as the foaming and cracking behaviour of the intumescent coating need to be taken into account. Based on the performed fire tests, the profile geometry and orientation of the steel tension member, the height of the dry film thickness of the intumescent coating and the amount of the applied tensile stress have significant influence on the thermal protection of the reactive fire protection system. In addition, these factors interact with each another. By adjusting these factors, the thermal protection effectiveness of the reactive fire protection system can be optimised. Because of the diversity of reactive fire protection systems in terms of their varying chemical com- 
position, the foaming behaviour and thermal protection is significantly different and hardly predictable. Therefore, the effectiveness of the reactive fire protection system has to be verified by appropriate fire tests, which must consider the limits of the scope of application. Recommendations on the implementation of these tests are described in the research report [35].

Cold formed drawn-steel, which is typically used for steel tension member with solid section, has different material properties at elevated temperatures compared to hot-rolled steel usually used for steel beams and columns. For example, cold-drawn steel has a higher thermal expansion coefficient and different temperature-dependent reduction factors for the steel strength. The mechanical material properties for cold-drawn steel obtained from small-scale material tests can be used for the fire protection design of steel tension members with solid sections manufactured accordingly. In combination with temperature data from corresponding fire tests and a numerical model based on the finite-element method (FEM), the load-bearing and deformation behaviour of steel tension members with reactive fire protection system can be realistically calculated. The longitudinal deformations of the steel tension members obtained from the experimental tests show a good agreement with that from the numerical model developed in the presented research.

Keywords: Fire protection, steel, tension member, reactive fire protection system, intumescent coating, cold-drawn steel, material properties, experimental study, numerical simulation 


\section{Danksagung}

Die vorliegende Arbeit entstand während meiner Tätigkeit als wissenschaftlicher Mitarbeiter im Fachbereich 7.3 - Brandingenieurwesen - der Bundesanstalt für Materialforschung und -prüfung (BAM). Allen Kollegen der BAM, die meine Forschungsarbeit unterstützt haben, sei hiermit gedankt.

Ein besonderer Dank gilt dem Leiter des Fachbereiches 7.3, Herrn Dr.-Ing. Sascha Hothan, der das Promotionsthema vorgeschlagen und durch vielfältige Hilfe unterstützt hat.

Für die Bereitschaft zur Übernahme der Betreuung und die Unterstützung beim Promotionsverfahren sowie die wertvollen Anregungen zur Thematik danke ich herzlich meinem Doktorvater, Herrn Univ.-Prof. Dr.-Ing. Peter Schaumann, von der Gottfried Wilhelm Leibniz Universität Hannover (LUH). Ebenso sei Herrn Univ.-Prof. Dr.-Ing. Dipl. Wirt.-Ing. (NDS) Martin Mensinger von der Technischen Universität München (TUM) für die Übernahme des Zweitgutachtens gedankt. Weiterhin danke ich Herrn Univ.-Prof. Dr.-Ing. Ludger Lohaus und Herrn Univ.-Prof. Dr.-Ing. Nabil A. Fouad von der Gottfried Wilhelm Leibniz Universität Hannover (LUH) für die Übernahme des Vorsitzes sowie die Teilnahme an der Prüfungskommission.

Die Arbeiten, die zu dieser Dissertation geführt haben, wurden überwiegend im Rahmen des Forschungsprojektes BAM-Vh 7539 "Versuchstechnische und numerische Untersuchung zur Anwendung von reaktiven Brandschutzsystemen auf Zuggliedern aus Stahl" durchgeführt und sind im Forschungsbericht [35] beschrieben. Für die finanzielle Förderung des Vorhabens sei dem Deutschen Institut für Bautechnik (DIBt) gedankt.

Abschließend möchte ich meiner Familie für die immerwährende und motivierende Unterstützung herzlichst danken.

Dustin Häßler 



\section{Inhaltsverzeichnis}

Kurzfassung III

Abstract $\quad$ V

Danksagung VII

$\begin{array}{lll}\text { Symbolverzeichnis XIII } & \text { X }\end{array}$

Abkürzungsverzeichnis $\quad$ XV

$\begin{array}{ll}\text { Definitionen } & \text { XVII }\end{array}$

1 Einführung 1

1.1 Einleitung . . . . . . . . . . . . . . . . . 1

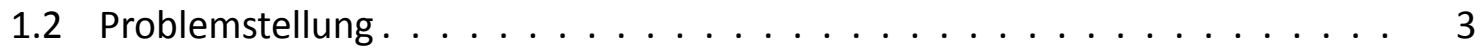

1.3 Zielstellung. ....................... 4

1.4 Methodik. . . . . . . . . . . . . . . . . . . . . 5

2 Reaktive Brandschutzsysteme im Stahlbau 7

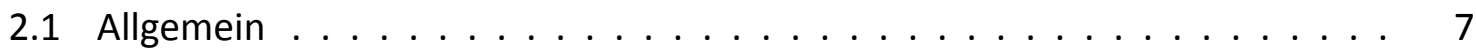

2.2 Aufbau und Funktionsweise reaktiver Brandschutzsysteme. . . . . . . . . . 7

2.3 Arten von reaktiven Brandschutzsystemen . . . . . . . . . . . . . . . . . 9

2.4 Materialeigenschaften reaktiver Brandschutzsysteme . . . . . . . . . . . . . . 10

2.5 Anwendung reaktiver Brandschutzsysteme im Stahlbau. . . . . . . . . . . . . 13

2.5.1 Anforderungen an reaktive Brandschutzsysteme . . . . . . . . . . . 13

2.5.2 Prüfung und Zulassung reaktiver Brandschutzsysteme . . . . . . . . . 13

2.5.3 Einsatz von Stahlzuggliedern im Bauwesen. . . . . . . . . . . . . . . 15

2.5.4 Anwendung reaktiver Brandschutzsysteme auf Stahlzuggliedern. . . . 16

2.6 Vorbetrachtungen . . . . . . . . . . . . . . . . . . . . . 17

2.6.1 Vergleich des Verhaltens reaktiver Brandschutzsysteme auf Biegeträgern und Zuggliedern . . . . . . . . . . . . . . . . . . . 17

2.6.2 Versagensmechanismen von Stahlzuggliedern und reaktiven Brandschutzsystemen . . . . . . . . . . . . . . . . . 20

3 Experimentelle Untersuchung von reaktiven Brandschutzsystemen auf Stahlzuggliedern 23

3.1 Herangehensweise . . . . . . . . . . . . . . . . . . 23 
3.2 Angaben zu den Versuchskörpern . . . . . . . . . . . . . . . . . . . . . . 24

3.3 Art des verwendeten reaktiven Brandschutzsystems . . . . . . . . . . . . . 24

3.4 Voruntersuchungen . . . . . . . . . . . . . . . . . . 25

3.4.1 Applikation der Thermoelemente . . . . . . . . . . . . . 26

3.4.2 Applikation des reaktiven Brandschutzsystems. . . . . . . . . . . 28

3.4.3 Messung der Schichtdicke des reaktiven Brandschutzsystems . . . . . 30

3.4.4 Orientierende Brandversuche im Einkubikmeterofen . . . . . . . . . . 32

3.4.5 Orientierende Brandversuche im Zugstabprüfofen . . . . . . . . . . 38

3.4.6 Zusammenfassung der Ergebnisse aus den Voruntersuchungen . . . . 39

3.5 Brandversuche an mechanisch belasteten und unbelasteten Versuchskörpern im Realmaßstab . . . . . . . . . . . . . . . . . . . . . . . . . . 40

3.5.1 Versuchsprogramm und Versuchskörper . . . . . . . . . . . . . . . . 40

3.5 .2 Versuchsaufbau . . . . . . . . . . . . . . . . . . . 43

3.5.3 Versuchsdurchführung . . . . . . . . . . . . . . . 45

3.5.4 Beobachtungen während der Brandversuche . . . . . . . . . . . . 47

3.6 Auswertung der Brandversuche . . . . . . . . . . . . . . . . . . . . . . 52

3.7 Einflussfaktoren auf das Verhalten des reaktiven Brandschutzsystems und der Stahlzugglieder . . . . . . . . . . . . . . . . . . . . . . 55

3.7.1 Einfluss der Rissbildung auf die Branddauer bis zum Probenbruch und auf die Stahltemperatur . . . . . . . . . . . . . . . . 55

3.7.2 Einfluss der Profilgeometrie auf die Branddauer bis zum Probenbruch und auf die Stahltemperatur . . . . . . . . . . . . . 58

3.7.3 Einfluss der Trockenschichtdicke auf die Branddauer bis zum Probenbruch und auf die Stahltemperatur . . . . . . . . . . . 62

3.7.4 Einfluss des Lastausnutzungsgrades auf die Branddauer bis zum Probenbruch und auf die Stahltemperatur . . . . . . . . . . . . . 65

3.7.5 Einfluss der axialen Zugbeanspruchung auf die Stahltemperatur. . . . 69

3.7.6 Einfluss der Bauteilorientierung auf die Stahltemperatur . . . . . . . . 71

3.7.7 Einfluss der Stablänge auf die Stahltemperatur . . . . . . . . . . . . . 74

3.7.8 Einfluss von Beschädigungen des reaktiven Brandschutzsystems . . . 76

3.7.9 Zusammenfassung der untersuchten Einflussfaktoren. . . . . . . . . . 77

3.8 Porenstruktur und Schichtenaufbau des aufgeschäumten reaktiven Brandschutzsystems . . . . . . . . . . . . . . . . . 80

3.8.1 Geometrie der Aufschäumung über den Stabquerschnitt . . . . . . . . 80

3.8.2 Untersuchung der Aufschäumung mittels CT und REM . . . . . . . . . 81

3.9 Leistungskriterien der Tragfähigkeit . . . . . . . . . . . . . . . . . . . . 85 
3.9.1 Verformungskriterium . . . . . . . . . . . . . . . 85

3.9.2 Verformungsgeschwindigkeitskriterium. . . . . . . . . . . . 88

3.9.3 Anwendung der Leistungskriterien bei Stahlzuggliedern . . . . . . . . 90

\section{Bestimmung der mechanischen Hochtemperatureigenschaften} von Blankstahl

4.1 Herstellung von Blankstahl . . . . . . . . . . . . . . . . . . 92

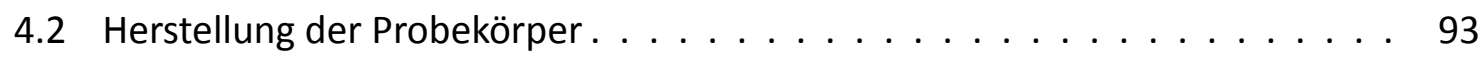

4.3 Ermittlung der chemischen Zusammensetzung. . . . . . . . . . . . . 93

4.4 Ermittlung des Elastizitätsmoduls . . . . . . . . . . . . . . . 95

4.4.1 Bestimmung des Elastizitätsmoduls mittels Resonanzverfahren . . . . 96

4.4.2 Vergleich des dynamischen E-Moduls mit Werten aus der Literatur . . 97

4.4.3 Bestimmung des Elastizitätsmoduls anhand von Kleinzugversuchen. . 98

4.4.4 Unsicherheiten bei der Bestimmung des statischen Elastizitätsmoduls 100

4.4.5 Vergleich des statischen E-Moduls mit Werten aus der Literatur . . . 103

4.4.6 Vergleich des statischen und dynamischen Elastizitätsmoduls . . . . 105

4.5 Ermittlung der Spannungs-Dehnungs-Kennlinien . . . . . . . . . . . 106

4.5.1 Kleinzugversuche bei Raumtemperatur. . . . . . . . . . . . . . . 107

4.5.2 Kleinzugversuche unter erhöhter Temperatur . . . . . . . . . . . 109

4.5.3 Vergleich der Abminderungsfaktoren der Stahlfestigkeiten . . . . . . 116

4.6 Ermittlung des Wärmeausdehnungskoeffizienten . . . . . . . . . . . 121

4.6.1 Untersuchung mittels Schubstangen-Dilatometer . . . . . . . . . . . 121

4.6.2 Untersuchung mittels Kleinzugversuchen. . . . . . . . . . . . . 122

4.6.3 Vergleich der Ergebnisse . . . . . . . . . . . . . . . . . 122

5 Numerische Analyse von Zuggliedern mit reaktiver Brandschutzbeschichtung 125

5.1 Grundlagen der Temperaturfeldberechnung . . . . . . . . . . . . 125

5.2 Untersuchung zum Erwärmungsverhalten von Stahlbauteilen . . . . . . . 126

5.2.1 Erwärmung ungeschützter Stahlprofile . . . . . . . . . . . . . 126

5.2.2 Erwärmung geschützter Stahlprofile . . . . . . . . . . . . 130

5.2.3 Vergleich zwischen Oberflächen- und Kerntemperatur . . . . . . . . 130

5.3 Entwicklung eines Bemessungsmodells für Zugglieder mit reaktiver

Brandschutzbeschichtung . . . . . . . . . . . . . . 133

5.3.1 Aufbau des Bemessungsmodells aus dem Forschungsvorhaben [35] . 133

5.3.2 Entwicklung temperaturabhängiger Spannungs-Dehnungs-Kennlinien 137 
5.3.3 Weiterentwicklungen des Bemessungsmodells . . . . . . . . . . . . 140

5.3.4 Vergleich zwischen Bemessungsmodell und Brandversuch . . . . . . . 144

5.3.5 Spannungs- und Dehnungszustand der mechanisch belasteten Stahlzugglieder bei Erreichen der Tragfähigkeit bzw. des Probenbruchs . . . . . . . . . . . . . . . . . . . . . . 146

5.4 Möglichkeiten zur Bestimmung der Feuerwiderstandsdauer für Stahlzugglieder mit reaktiver Brandschutzbeschichtung . . . . . . . . . . . . . . 149

5.4.1 Variante 1 - Brandversuch an mechanisch belasteten Zuggliedern . . 149

5.4.2 Variante 2 - Brandversuch an mechanisch unbelasteten Zuggliedern 150

5.4.3 Variante 3 - Auswertung mittels Bemessungsfestigkeit . . . . . . . . . 151

6 Zusammenfassung, Schlussfolgerung und Ausblick 155

6.1 Zusammenfassung und Schlussfolgerung . . . . . . . . . . . . . . . . . 155

6.2 Ausblick . . . . . . . . . . . . . . . . . . . . . . . 159

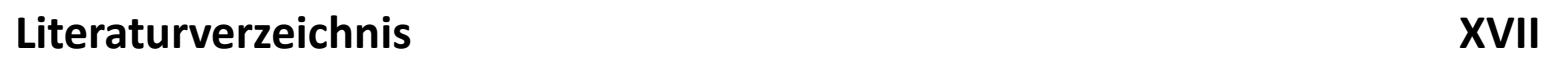

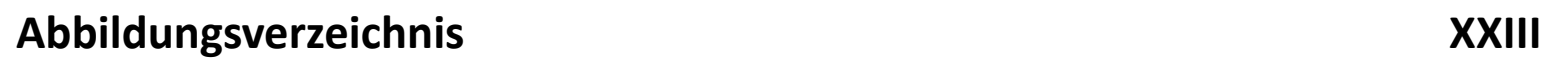

$\begin{array}{ll}\text { Tabellenverzeichnis } & \text { XXIX }\end{array}$

A Ergänzende Angaben zu den in Kapitel 3 beschriebenen Brandversuchen im Realmaßstab XXXI

B Zusätzliche Informationen zu den in Kapitel 4 durchgeführten Materialuntersuchungen

C Information zu den in Kapitel 5 verwendeten Materialmodellen LVII

D Gegenüberstellung der in Brandversuchen gemessenen und numerisch berechneten Stablängsverformungen 


\section{Symbolverzeichnis}

\section{Buchstaben}

$\begin{array}{ll}\text { a } & \text { Dicke } \\ \mathrm{A} & \text { Fläche / Bruchdehnung } \\ \mathrm{b} & \text { Breite } \\ \mathrm{c} & \text { Spezifische Wärmekapazität } \\ \mathrm{d} & \text { Durchmesser } \\ \mathrm{e} & \text { Exzentrizität } \\ \mathrm{E} & \text { Elastizitätsmodul (E-Modul) } \\ \mathrm{F} & \text { Kraft } \\ \mathrm{f} & \text { Spannung } \\ \mathrm{g} & \text { Gravitationskonstante } \\ \mathrm{I} & \text { Trägheitsmoment } \\ \mathrm{k} & \text { Proportionalitäts-/ Anpassungs-/ } \\ & \text { Abminderungsfaktor } \\ \mathrm{L} & \text { Länge } \\ \mathrm{N} & \text { Zugkraft } \\ \mathrm{r} & \text { Radius } \\ \mathrm{R} & \text { Festigkeit / Bauteilwiderstand / } \\ & \text { Feuerwiderstanddauer } \\ \mathrm{S}, \mathrm{A} & \text { Querschnittsfläche } \\ \mathrm{t} & \text { Zeit } \\ \mathrm{T} & \text { Temperatur } \\ \mathrm{u} & \text { Längsverschiebung } \\ \mathrm{U} & \text { Umfang } \\ \mathrm{w} & \text { Durchbiegung } \\ \mathrm{Z} & \text { Brucheinschnürung } \\ & \\ \end{array}$

\section{Spezielle Bezeichnungen}

$\begin{array}{ll}A_{m} / V & \text { Profilfaktor } \\ c_{p} & \text { spezifische Wärmekapazität } \\ E_{a, \theta} & \text { temperaturabhängiger E-Modul } \\ E_{d y n} & \text { dynamischer E-Modul } \\ E_{s t a t} & \text { statischer E-Modul } \\ E_{d, f i} & \text { Bemessungswert der } \\ & \text { Einwirkungen im Brandfall } \\ \dot{e} & \text { Dehngeschwindigkeit } \\ \mathrm{f}_{\mathrm{y}, \theta} & \text { effektive Fließgrenze } \\ \mathrm{f}_{\mathrm{p}, \theta} & \text { Proportionalitätsgrenze }\end{array}$

\section{$\underline{\text { Griechisch }}$}

$\begin{array}{ll}\alpha & \text { Wärmeausdehnungskoeffizient/ } \\ \gamma & \text { Winkel } \\ \varepsilon & \text { Sicherheitsbeiwert } \\ \theta, \vartheta & \text { Dehnung; Emissionsgrad } \\ \lambda & \text { Wärmeleitfähigkeit } \\ \nu & \text { Poissonzahl } \\ \rho & \text { Dichte } \\ \sigma & \text { Spannung } \\ \mu & \text { Lastausnutzungsgrad } \\ \eta & \text { Reduktionsfaktor } \\ \Delta & \text { Differenz/ Änderung }\end{array}$

\begin{tabular}{|c|c|}
\hline Indizes & \\
\hline 0 & bezieht sich auf den Kaltfall \\
\hline dyn & dynamisch \\
\hline fi & bezieht sich auf den Brandfall \\
\hline $\max$ & Maximalwert \\
\hline $\min$ & Minimalwert \\
\hline Mises & Mises-Spannung \\
\hline nom & nominal/ technisch \\
\hline stat & statisch \\
\hline tech & technisch \\
\hline wahr & wahr \\
\hline zul & zulässig \\
\hline
\end{tabular}

$\alpha_{c} \quad$ Konvektiver Wärmeübergangsbeiwert

$\theta_{0}, \vartheta_{0} \quad$ Raumtemperatur

$\theta_{\max } \quad$ maximale Strahltemperatur

$\sigma_{\mathrm{B}} \quad$ Stefan-Boltzmann-Konstante

$\sigma_{\text {nom }} \quad$ technische Spannung

$\mu_{\mathrm{fi}} \quad$ Lastausnutzungsgrad im Brandfall

$\eta_{\mathrm{fi}} \quad$ Reduktionsfaktor für den Brandfall

$\varepsilon_{\text {nom }} \quad$ technische Dehnung

$\varepsilon^{\mathrm{E}} \quad$ Gesamtdehnung 
$\mathrm{f}_{\mathrm{p} 0,01} \quad$ technische Proportionalitätsgrenze

$\mathrm{f}_{\mathrm{p} 0,2} \quad 0,2 \%$-Dehngrenze

$\mathrm{f}_{\mathrm{u}} \quad$ Zugfestigkeit

$\mathrm{f}_{\mathrm{u}, \mathrm{I}} \quad$ Zugfestigkeit (Bereich I)

$\mathrm{f}_{\mathrm{u}, \mathrm{II}} \quad$ Zugfestigkeit (Bereich II)

$\mathrm{f}_{\mathrm{b}} \quad$ Bruchspannung

$\mathrm{F}_{\text {Zug }} \quad$ Zugkraft / Zugbeanspruchung

$\mathrm{k}_{\mathrm{E}} \quad$ Abminderungsfaktor für den Elastizitätsmodul

$\mathrm{k}_{\mathrm{p} 0,01}$ Abminderungsfaktor der Proportionalitätsgrenze

$\mathrm{k}_{\mathrm{p} 0,2} \quad$ Abminderungsfaktor der 0,2\%-Dehngrenze

$\mathrm{k}_{\mathrm{u}, \mathrm{I}} \quad$ Abminderungsfaktor der Zugfestigkeit (Bereich I)

$\mathrm{k}_{\mathrm{u}, \mathrm{II}} \quad$ Abminderungsfaktor der Zugfestigkeit (Bereich II)

$\mathrm{L}_{\mathrm{b}} \quad$ beflammte Bauteillänge

$\mathrm{L}_{c} \quad$ Versuchslänge der Zugprobe

$\mathrm{L}_{\text {Grenz }}$ Verformungskriterium

R30 feuerhemmend

$\mathrm{R}_{\mathrm{a}} \quad$ mittlere Rauheit

$\mathrm{R}_{\mathrm{d}, \mathrm{fi}} \quad$ Bemessungswert des Bauteilwiderstandes im Brandfall

$\mathrm{R}_{\mathrm{z}} \quad$ mittlere Rautiefe

$t_{I} \quad$ Versuchsdauer Bereich I

$\mathrm{t}_{\mathrm{II}} \quad$ Versuchsdauer Bereich II

$t_{\text {ges }}$ gesamte Versuchsdauer

$t_{\mathrm{F}} \quad$ Feuerwiderstandsdauer

$t_{\text {Trag }} \quad$ Zeitpunkt bis zum Erreichen der Tragfähigkeit

$t_{\theta} \quad$ gesamte Versuchsdauer

$\mathrm{T}_{\mathrm{m}} \quad$ Schmelztemperatur

$\mathrm{T}_{\mathrm{R}} \quad$ Rekristallisationstemperatur

U/A Profilfaktor

$\mathrm{V}_{\text {Grenz }}$ Verformungsgeschwindigkeitskriterium
$\varepsilon^{\mathrm{EE}} \quad$ elastischer Dehnungsanteil

$\varepsilon^{\mathrm{PE}} \quad$ plastischer Dehnungsanteil

$\varepsilon_{\mathrm{b}} \quad$ Bruchdehnung

$\varepsilon_{\mathrm{k}} \quad$ Kriechdechnung

$\varepsilon_{\mathrm{p}} \quad$ Dehnung für Proportionalitätsgrenze

$\varepsilon_{\mathrm{p} 0,01} \quad$ Dehnung für techn. Proportionalitätsgrenze

$\varepsilon_{\mathrm{p} 0,2} \quad$ Dehnung an der 0,2\%-Dehngrenze

$\varepsilon_{\mathrm{t}} \quad$ Grenzdehnung für Fließgrenze

$\varepsilon_{\text {th }} \quad$ Thermische Dehnung

$\varepsilon_{\mathrm{y}} \quad$ Fließdehnung

$\varepsilon_{\mathrm{u}} \quad$ Dehnung für die Zugfestigkeit

$\varepsilon_{\mathrm{u}, \mathrm{I}} \quad$ Dehnung für die Zugfestigkeit (Bereich I)

$\varepsilon_{\mathrm{u}, \mathrm{II}} \quad$ Dehnung für die Zugfestigkeit (Bereich II) 


\section{Abkürzungsverzeichnis}

$\begin{array}{ll}\text { Abb. } & \text { Abbildung } \\ \text { abZ } & \text { Allgemeine bauaufsichtliche Zulassung } \\ \text { B } & \text { Belasteter Versuchskörper } \\ \text { BAM } & \text { Bundesanstalt für Materialforschung und -prüfung } \\ \text { BRT } & \text { Brandraumthermoelement / Brandraumtemperatur } \\ \text { CEN } & \text { European Committee for Standardization } \\ \text { CT } & \text { Computerthermographie } \\ \text { DEP } & \text { Deckenprüfstand des Fachbereiches 7.3 der BAM } \\ \text { DIBt } & \text { Deutsches Institut für Bautechnik } \\ \text { DIN } & \text { Deutsches Institut für Normung } \\ \text { EC2 } & \text { DIN EN 1992 } \\ \text { EC3 } & \text { DIN EN 1993 } \\ \text { EN } & \text { Europäische Norm } \\ \text { ETB } & \text { Europäische Technische Bewertung } \\ \text { ETK } & \text { Einheits-Temperaturzeitkurve } \\ \text { ISO } & \text { International Organization for Standardization } \\ \text { Mat } & \text { Materialmodell } \\ \text { MTE } & \text { Mantelthermoelement } \\ \text { MW } & \text { Mittelwert } \\ \text { Nr. } & \text { Nummer } \\ \text { P } & \text { Probekörper } \\ \text { PKT } & \text { Probekörpertemperatur } \\ \text { PTE } & \text { Probekörperthermoelement } \\ \text { RBS } & \text { Reaktives Brandschutzsystem } \\ \text { REM } & \text { Rasterelektronenmikroskop } \\ \text { TE } & \text { Thermoelement } \\ \text { U } & \text { Unbelasteter Versuchskörper } \\ \text { VR } & \text { Versuchsreihe } \\ \text { ZiE } & \text { Zustimmung im Einzelfall } \\ & \end{array}$





\section{Definitionen}

\section{Stahltemperatur}

Durch unterschiedliches Aufschäumen sowie Rissbildung kann die thermische Schutzwirkung des reaktiven Brandschutzsystems lokal variieren. Ferner können in Stabbereichen nahe der Brandraumwände geringere Stahltemperaturen aufgrund der dort vorhandenen Randeinflüsse und Abschattungseffekte auftreten. Temperaturunterschiede in Stablängsrichtung der Stahlzugglieder sind daher zu erwarten. Im Gegensatz dazu ist aufgrund der guten Wärmeleitfähigkeit von Stahl und den meist filigranen Querschnitten von Stahlzuggliedern davon auszugehen, dass sich im Stabquerschnitt eine einheitliche Stahltemperatur einstellt.

Für Stahlzugglieder mit mechanischer Zugbeanspruchung ergibt sich über das gesamte Bauteil eine konstante Zugbeanspruchung. Da sich bei Zuggliedern aus Stabbereichen mit geringerer Stahltemperatur keine Tragfähigkeitsreserven erschließen lassen, ist in Stablängsrichtung mit einem Probenbruch des Bauteils an der Stelle mit der maximalen Stahltemperatur zu rechnen. Bei der Auswertung der Brandversuche wird daher immer das am Stahlzugglied angebrachte Probekörperthermoelement mit dem maximal gemessenen Temperaturverlauf betrachtet. Dies gilt auch für die in den Brandversuchen getesteten Vergleichsprüfkörper ohne mechanische Zugbeanspruchung. Wird die Stahltemperatur zu einem bestimmten Zeitpunkt betrachtet, wie z.B. dem Probenbruch des Zuggliedes, kann ein einzelner Temperaturwert angegeben werden. In der Dissertation wird vereinfachend auch die Bezeichnungen "maximale Stahltemperatur" gebraucht.

\section{Vergleichsprüfkörper}

Es handelt sich um Stahlzugglieder mit reaktiver Brandschutzbeschichtung, die in den Brandversuchen ohne mechanische Zugbeanspruchung getestet werden. Zusätzlich zur horizontalen Bauteilorientierung werden einige der Vergleichsprüfkörper in vertikaler Einbaulage geprüft. Mit Ausnahme der Bauteillänge und der Anzahl der angebrachten Thermoelemente entsprechen die Vergleichsprüfkörper den unter mechanischer Zugbeanspruchung getesteten Stahlzuggliedern mit reaktiver Brandschutzbeschichtung. Mit Hilfe der Vergleichsprüfkörper lässt sich der Einfluss der Zugbeanspruchung sowie der Bauteilorientierung auf das Verhalten und die thermische Schutzwirkung des reaktiven Brandschutzsystem beurteilen.

\section{Branddauer}

Die Branddauer entspricht der Zeitdauer in Minuten vom Beginn bis zum Ende eines Brandversuches. Der Beginn des Brandversuches erfolgt mit dem Einsetzen der Beflammung. Der Brandversuch endet, wenn ein im Vorfeld definiertes Kriterium, z.B. der Probenbruch des Versuchskörpers, erreicht ist. 



\section{Einführung}

\subsection{Einleitung}

Ein Brandereignis kann in und an Bauwerken und technischen Anlagen zu jeder Zeit auftreten. Brandgefahren sind dort allgegenwärtig. Selbige lassen sich oftmals nicht sofort als potentielle Gefahren erkennen. Umso wichtiger sind vorbeugende Schutzmaßnahmen, um Brandkatastrophen zu verhindern. Maßnahmen, die einer Entstehung und Ausbreitung von Bränden entgegenwirken, werden allgemein unter dem Begriff „,vorbeugender Brandschutz“ zusammengefasst. In vielen Bereichen des täglichen Lebens lassen sich Maßnahmen zum Brandschutz finden. Meist sind diese unscheinbarer Art und für Laien kaum wahrnehmbar.

Einen wichtigen Teil des vorbeugenden Brandschutzes bildet der "bauliche Brandschutz". Brandschutzanforderungen an Gebäude sind in der Musterbauordnung und den Landesbauordnungen geregelt. Gebäude, an die Anforderungen bezüglich des Brandschutzes gestellt werden, müssen durch entsprechende bauliche Maßnahmen geschützt werden. Dabei sind unter anderem das Brandverhalten der Baustoffe sowie der Feuerwiderstand der Bauteile und Tragkonstruktionen zu berücksichtigen. Die Verwendung von Baustoffen und Bauteilen wird im Allgemeinen durch Normen und Richtlinien geregelt. Das Deutsche Institut für Bautechnik (DIBt) erstellt und veröffentlicht dazu entsprechende Listen mit den technischen Regeln für Bauprodukte und Bauarten. Bauprodukte, für die es weder normative Regelungen, Richtlinien oder Gleichwertiges gibt, werden als „nicht geregelte Bauprodukte" bezeichnet. Für die Verwendung von nicht geregelten Bauprodukten sind Verwendbarkeitsnachweise erforderlich, z.B. eine allgemeine bauaufsichtliche Zulassung (abZ), eine Europäische Technische Bewertung (ETB), ein allgemeines bauaufsichtliches Prüfzeugnis (abP) oder eine Zustimmung im Einzelfall (ZiE). Ein Beispiel für nicht geregelte Bauprodukte sind reaktive Brandschutzsysteme (RBS). Die auch als reaktive Brandschutzbeschichtung bezeichneten Systeme sind laut Bauregelliste nach Erteilung einer abZ, ETB oder ZiE an und in Bauwerken verwendbar.

Reaktive Brandschutzsysteme haben ihren Ursprung als Feuerschutzmittel zur brandschutztechnischen Ertüchtigung von hölzernen Tragwerken. Seit den 1960er Jahren werden reaktive Brandschutzsysteme zur Erhöhung des Feuerwiderstandes von Stahlbauteilen eingesetzt [23], [24]. Diese Systeme eignen sich aufgrund der geringen Beschichtungsdicken besonders, wenn aus architektonischen Gründen die Optik der Stahlkonstruktion im Wesentlichen erhalten bleiben soll oder andere Brandschutzbekleidungen nicht ausführbar sind. Das Wirkprinzip reaktiver Brandschutzsysteme besteht darin, unter Brandeinwirkung aufzuschäumen und eine thermische Schutzschicht um das Stahlbauteil auszubilden. Die Erwärmung des Stahls wird dadurch verlangsamt. Die mit zunehmender Temperatur einsetzende Festigkeitsabnahme des Stahls wird verzögert und die Tragfähigkeit des Bauteils bleibt länger erhalten. Das Verhalten reaktiver Brandschutzsysteme auf Stahlbauteilen, die 
direkt an die raumabschließenden Elemente angeschlossen sind, wird in [39] untersucht. Als raumabschließende Bauteile werden übliche Fassadenelemente wie Trapez-, Kassettenund Sandwichprofile verwendet. Informationen zu den Einsatzmöglichkeiten von reaktiven Brandschutzsystemen auf Stahlbauteilen sind auch in [37] und [38] veröffentlicht.

Vor behördlicher Zulassung für ein reaktives Brandschutzsystem muss selbiges verschiedene Tests bestehen. In Brandversuchen wird beispielsweise der Feuerwiderstand von Stahlbauteilen mit reaktiver Brandschutzbeschichtung anhand von mechanisch belasteten und unbelasteten Versuchskörpern geprüft. Auf Grundlage der Brandprüfung wird die thermische Schutzwirkung des reaktiven Brandschutzsystems nachgewiesen. Der Anwendungsbereich des reaktiven Brandschutzsystems wird von der Feuerwiderstandsdauer und zahlreichen anderen Faktoren, wie z.B. der Profilgeomtrie und der Art der mechanischen Beanspruchung des Stahlbauteils, begrenzt. In den Zulassungen für reaktive Brandschutzsysteme wird zwischen den drei verschiedenen Beanspruchungsarten Biegung, Druck und Zug unterschieden. Brandprüfungen für reaktive Brandschutzsysteme werden in der Regel an Stahlträgern und/oder -stützen durchgeführt. Für die Prüfung von Stahlzuggliedern existieren gegenwärtig keine normativen Vorgaben. Aus diesem Anlass wurden im Fachbereich 7.3 - Brandingenieurwesen der Bundesanstalt für Materialforschung und -prüfung (BAM) unter Leitung von Herrn Dr.-Ing. Sascha Hothan Untersuchungen zur Anwendung von reaktiven Brandschutzsystemen auf Zuggliedern aus Stahl durchgeführt. Der Autor der Dissertation war als Projektbearbeiter an dem Vorhaben maßgeblich beteiligt. Die vorliegende Arbeit basiert auf den Ergebnissen des Forschungsprojektes [35] und stellt eine Fortführung der Untersuchungen dar. Die Dissertation konzentriert sich auf die vergleichende Auswertung der einzelnen Brandversuche und die Auswirkung der maßgebenden Einflussfaktoren, wie z.B. Profilgeometrie, Trockenschichtdicke des Brandschutzsystems, Lastausnutzungsgrad der mechanischen Zugbeanspruchung sowie Orientierung des Bauteils. Ferner werden Kriterien für die Bestimmung der Tragfähigkeit erarbeitet, anhand der sich die in Brandversuchen unter axialer Zugbeanspruchung getesteten Stahlzugglieder mit reaktiver Brandschutzbeschichtung in Feuerwiderstandsklassen einordnen lassen. Darüber hinaus werden Materialuntersuchungen zu den mechanischen Hochtemperatureigenschaften von kaltverformten Blankstahl ausgeführt. Dieser Stahl wird in der Regel für Zugglieder mit Vollprofil verwendet. Selbiger besitzt aufgrund des Herstellungsverfahrens andere Materialeigenschaften, als der üblicherweise im Bauwesen für Stahlträger und -stützen eingesetzte warmgewalzte Baustahl. Materialkennwerte für den mechanischen Hochtemperaturbereich von gezogenem, kaltverformtem Blankstahl sind derzeit weder in den Normen noch in der Literatur benannt. Auf Grundlage der in den Brandversuchen gemessenen Stahltemperaturen an den mit reaktivem Brandschutzsystem beschichteten Zuggliedern sowie den Ergebnissen aus den Materialuntersuchungen wird ein numerisches Bemessungsmodell entwickelt. Durch das Modell wird eine Berechnung des Trag- und Verformungsverhaltens sowie die Ermittlung der Tragfähigkeit von mit reaktiven Brandschutzsystemen geschützten Stahlzuggliedern aus kaltverformten Blankstahl ermöglicht. 


\subsection{Problemstellung}

Für die Anwendung reaktiver Brandschutzsysteme auf Stahlzuggliedern existieren derzeit nur Vorgaben bei der Verwendung von offenen Profilen. Die Regelung basiert auf den in [32] und [33] beschriebenen numerischen Untersuchungen, welche die Dehnungen in der Biegezugzone eines belastet geprüften Stahlträgers mit denen eines Zuggliedes vergleicht. Sofern die auftretenden Dehnungen für das reaktive Brandschutzsystem auf dem Zugglied die des Trägers nicht überschreiten, wird davon ausgegangen, dass eine ausreichende Haftfähigkeit der Brandschutzbeschichtung anhand der Prüfung des belasteten Trägers nachgewiesen ist. Dies setzt jedoch voraus, dass der Träger und das Zugglied die gleiche Profilgeometrie und die gleiche Trockenschichtdicke der reaktiven Brandschutzbeschichtung, d.h. Dicke des intumeszierenden Anstriches nach Abb. 2.01, aufweisen. Auf Grundlage von numerischen Vergleichsuntersuchungen zwischen den vorhandenen Dehnungen eines Zuggliedes und der Biegezugzone eines Trägers ist derzeit die Anwendung von reaktiven Brandschutzsystemen auf Zuggliedern mit offenen Profilen bis zu einem Lastausnutzungsgrad im Brandfall von $\mu_{\mathrm{fi}} \leq 0,5$ möglich. Die Anwendung der genannten Regelung für Zugglieder mit offenem Profil, welche Brandprüfungen an belasteten Stahlträgern mit reaktiver Brandschutzbeschichtung vorraussetzt, beschränkt sich im Wesentlichen auf die zugbeanspruchten Bauteile von Stahlfachwerkträgern. Eine Zusammenfassung der derzeitig für Zugglieder geltenden Regelungen sowie ergänzende numerische Berechnungen sind in [37] beschrieben.

In der Baupraxis kommen für Stahlzugglieder häufig Vollprofile zum Einsatz. Nach den derzeitigen Regelungen in den Zulassungen des DIBt ist die Verwendbarkeit von reaktiven Brandschutzsystemen auf Vollprofilen gesondert nachzuweisen. Das Gleiche gilt für Zugglieder mit offenem oder geschlossenem Profil, die von den in den Zulassungen angegebenen Regelungen abweichen. Der Nachweis kann beispielsweise durch eine Zustimmung im Einzelfall (ZiE) erfolgen. Grundlage für die Zustimmung im Einzelfall bildet in der Regel eine gutachterliche Stellungnahme, welche häufig mit weiteren Brandprüfungen verbunden ist. Da eine Zustimmung im Einzelfall nur Gültigkeit für das jeweilige Projekt besitzt und stets ein zeit- und kostenaufwändiges Verfahren darstellt, gibt es seitens der Hersteller Bestrebungen, den Anwendungsbereich von Zulassungen für reaktive Brandschutzsysteme zu erweitern, um auch Vollprofile für Zugglieder einsetzen zu können. Auch seitens der deutschen Bauaufsicht, d.h. dem DIBt, wird eine Regelung dieser Anwendung mittels Zulassungen favorisiert, um ein einheitliches Sicherheitsniveau definieren zu können.

Die Prüfung von mechanisch belasteten Zuggliedern mit reaktivem Brandschutzsystem ist derzeit normativ nicht geregelt. Entsprechende Empfehlungen für die Versuchsdurchführung müssen dazu erarbeitet werden. Vorschläge für ein Versuchsprogramm sind im Forschungsbericht [35] enthalten. 
Das Festlegen von Regelungen für die Erweiterung der Zulassungen gestaltet sich schwierig, da die Anwendbarkeit reaktiver Brandschutzsysteme auf Stahlzuggliedern bisher kaum untersucht wurde. Diesbezüglich liegen fast keine veröffentlichten Prüfdaten vor. Geregelte Prüfverfahren zur Untersuchung von reaktiven Brandschutzsystemen auf Stahlzuggliedern existieren derzeit nicht. Bei den Prüfinstitutionen und bei den Herstellern von Brandschutzbeschichtungen ist nur wenig zum Verhalten reaktiver Brandschutzsysteme auf Vollprofilen und im Besonderen die bei kreisförmigen Vollprofilen vorhandene Oberflächenkrümmung und deren Auswirkung auf die Haftfähigkeit des reaktiven Brandschutzsystems bekannt. Die Versagensmechanismen des reaktiven Brandschutzsystems sowie der Einfluss von Fehlstellen im Beschichtungssystem auf die thermische Schutzwirkung sind bisher unerforscht. Die Norm zur Bemessung von Stahlbauteilen unter Brandeinwirkung [11] enthält lediglich Materialeigenschaften für warmgewalzten Baustahl und kaltverformte dünnwandige Profile. Inwieweit sich diese Angaben auf den typischerweise für Vollprofile eingesetzten gezogenen, kaltverformten Blankstahl übertragen lassen, ist unbekannt.

\subsection{Zielstellung}

Die vorliegende Arbeit hat das Ziel, neue Erkenntnisse hinsichtlich des Verhaltens reaktiver Brandschutzsysteme auf Stahlzuggliedern mit kreisförmigem Querschnitt unter Brandbeanspruchung zu erlangen. Im Fokus der Forschung stehen die überwiegend für Zugglieder verwendeten Kreisvollprofile. Das Aufschäumverhalten der reaktiven Brandschutzbeschichtung, die Ausbildung der thermischen Schutzschicht sowie die Wirkungsweise verschiedener Einflussfaktoren, wie z.B. Profilgeometrie des Zuggliedes, Trockenschichtdicke des reaktiven Brandschutzsystems, Lastausnutzungsgrad der mechanischen Zugbeanspruchung sowie Orientierung des Bauteils im Brandraum sind zu untersuchen. Im Ergebnis dessen sollen Empfehlungen für die Bestimmung der Tragfähigkeit erarbeitet werden, die eine Einordnung von Brandprüfungen an mechanisch belasteteten Stahlzuggliedern mit reaktiver Brandschutzbeschichtung in die Feuerwiderstandsklassen ermöglichen.

Darüber hinaus sind Materialuntersuchungen zu den mechanischen Hochtemperatureigenschaften von gezogenem kaltverformtem Blankstahl vorzunehmen, da derzeit weder in den Normen noch in der Literatur Kennwerte vorliegen. Auf Grundlage der in den Brandversuchen an den mit einem reaktiven Brandschutzsystem beschichteten Zuggliedern gemessenen Stahltemperaturen und den Ergebnissen aus den Materialuntersuchungen soll ein numerisches Bemessungsmodell entwickelt werden. Die Berechnung des Trag- und Verformungsverhaltens sowie die Ermittlung der Tragfähigkeit von mit reaktiven Brandschutzsystemen geschützten Stahlzuggliedern aus kaltverformten Blankstahl sollen dadurch ermöglicht werden. 
Die Untersuchungen dienen als Verfahrensgrundlage für die Durchführung und Auswertung von Brandversuchen an Stahlzuggliedern mit reaktiver Brandschutzbeschichtung. Die Erweiterung des Anwendungsbereiches reaktiver Brandschutzsysteme auf Zugglieder mit Vollprofil wird dadurch ermöglicht. Das derzeit aufwendige Verfahren mittels Zustimmungen im Einzelfall (ZiE) soll damit abgelöst werden. Die Ausführung kompletter Bauwerke in Stahlbauweise mit Brandschutzanforderungen für die tragende Konstruktion aus Trägern, Stützen und Zuggliedern könnte somit im Rahmen von Zulassungen abgedeckt werden.

\subsection{Methodik}

Um das Verhalten reaktiver Brandschutzsysteme auf Stahlzuggliedern mit kreisförmigem Querschnitt unter Brandbeanspruchung beurteilen zu können, werden allgemeine Grundlagen für den Einsatz dieser Systeme im Stahlbau erörtert. Neben der Erläuterung des Aufbaus und der Funktionsweise reaktiver Brandschutzbeschichtungen wird zu Forschungsaktivitäten zur Bestimmung der Materialeigenschaften reaktiver Brandschutzsysteme, dem Stand der Normung für diese Systeme sowie den Einsatzgebieten von Stahlzuggliedern im Bauwesen ausgeführt. Vorbetrachtungen zum Aufschäumverhalten reaktiver Brandschutzsysteme mit dem Schwerpunkt eines Vergleiches zwischen den zu erwartenden Beanspruchungen für das reaktive Brandschutzsystem an Zuggliedern mit Kreisvollprofil und der Biegezugzone eines Trägers mit I-Profil werden angestellt. Reaktive Brandschutzsysteme auf Biegeträgern und Zuggliedern werden sowohl mit als auch ohne mechanische Beanspruchung miteinander verglichen (siehe Abb. 2.06).

Im Rahmen der Vorbetrachtungen zum Verhalten reaktiver Brandschutzsysteme werden maßgebende Einflussfaktoren identifiziert. Ziel ist die Bestimmung von Anwendungsgrenzen und kritischen Zuständen für reaktive Brandschutzbeschichtungen, die es durch entsprechende Prüfungen zu ermitteln gilt. Im Rahmen des an der BAM durchgeführten Forschungsvorhabens [35] wird erstmalig das Verhalten reaktiver Brandschutzsysteme auf kreisförmigen Stahlzuggliedern untersucht. Dabei wird die Mehrzahl der Versuchskörper einer axialen Zugbeanspruchung ausgesetzt, welche über die komplette Branddauer bis zum Probenbruch aufrecht erhalten bleibt. Die innerhalb des Versuchsprogramms variierten Einflussfaktoren, wie z.B. Profilgeometrie des Zuggliedes, Trockenschichtdicke des reaktiven Brandschutzsystems, Lastausnutzungsgrad der mechanischen Zugbeanspruchung sowie Orientierung des Bauteils im Brandraum, werden hinsichtlich ihrer Wirkung auf das Aufschäumverhalten der reaktiven Brandschutzbeschichtung und der thermischen Schutzwirkung bewertet. Dafür werden Erwärmungsgeschwindigkeit, maximale Stahltemperatur des Zuggliedes sowie Haftfähigkeit, Rissbildung und Rissheilungsvermögen der reaktiven Brandschutzbeschichtung betrachtet. Durch gezielt vorgenommene lokale Beschädigungen an der Brandschutzbeschichtung wird an einigen Versuchskörpern ein Zustand simuliert, wie er unter Baustellenbedingungen vorkommen kann. Damit wird die Wirksamkeit bzw. 
Robustheit des Gesamtsystems getestet. Anhand von Videoaufzeichnungen aus den Brandversuchen und der Fotodokumentation der getesteten Zugglieder wird eine Typologie der Aufschäumformen des reaktiven Brandschutzsystems vorgenommen. Untersuchungen mittels Computertomographie (CT) und Rasterelektronenmikroskop (REM) geben Aufschluss über die Struktur des aufgeschäumten Brandschutzsystems und die Porengröße. Um eine Einordnung in die Feuerwiderstandsklassen zu ermöglichen, werden für die unter mechanischer Zugbeanspruchung getesteten Stahlzugglieder Kriterien zur Bestimmung der Tragfähigkeit erarbeitet.

Dabei sind jedoch die Materialeigenschaften des Stahls zu berücksichtigen. Die an Bauwerken eingesetzten Stahlzugglieder mit Vollprofil bestehen in der Regel aus kaltverformtem Blankstahl. Aufgrund des Herstellungsprozesses weist dieser Stahl andere Materialeigenschaften auf, als der üblicherweise im Bauwesen verwendete warmgewalzte Baustahl, welcher typischerweise für Träger- und Stützenprofile eingesetzt wird. Da in den Normen und der Fachliteratur keine speziellen Werte zu den mechanischen Hochtemperatureigenschaften für kaltverformten Blankstahl von Vollprofilen vorhanden sind, d.h. es liegen lediglich Informationen für kaltverformte dünnwandige Profile vor, werden entsprechende Materialuntersuchungen angestellt.

Zusammen mit den in den Brandversuchen gemessenen Stahltemperaturen der beschichteten Zugglieder werden die ermittelten mechanischen Hochtemperatureigenschaften als Eingangsgrößen für die Entwicklung eines numerischen Bemessungsmodells verwendet. Mit diesem Modell soll die Möglichkeit geschaffen werden, das Trag- und Verformungsverhalten sowie die Tragfähigkeit von mit reaktiven Brandschutzsystemen geschützten Stahlzuggliedern aus kaltverformtem Blankstahl zu berechnen. 


\section{Reaktive Brandschutzsysteme im Stahlbau}

\subsection{Allgemein}

Stahlkonstruktionen können unter Brandeinwirkung aufgrund der schnellen Erwärmung frühzeitig ihre Tragfähigkeit verlieren. Um dies zu vermeiden, sind entsprechende Schutzmaßnahmen zu ergreifen. Ursprünglich als Feuerschutzmittel zur brandschutztechnischen Ertüchtigung von hölzernen Tragwerken entwickelt, werden seit den 1960er Jahren reaktive Brandschutzsysteme zur Erhöhung des Feuerwiderstandes von Stahlbauteilen eingesetzt [23], [24]. Laut den Zulassungsgrundsätzen des Deutschen Instituts für Bautechnik (DIBt) [26] werden reaktive Brandschutzsysteme als Beschichtung auf Stahlbauteilen zur Erhöhung der Feuerwiderstandsdauer aufgebracht. Durch die geringen Beschichtungsdicken eignen sich diese besonders dann, wenn aus architektonischen Gründen die Optik der Stahlkonstruktion erhalten bleiben soll oder andere Brandschutzbekleidungen nicht ausführbar sind. Das Wirkprinzip des reaktiven Brandschutzsystems besteht darin unter Brandeinwirkung aufzuschäumen und eine thermische Schutzschicht um das Stahlbauteil auszubilden. Die Erwärmung des Stahls kann dadurch verlangsamt werden. Die mit zunehmender Temperatur einsetzende Festigkeitsabnahme des Stahls wird verzögert und die Tragfähigkeit des Bauteils bleibt länger erhalten.

\subsection{Aufbau und Funktionsweise reaktiver Brandschutzsysteme}

Reaktive Brandschutzsysteme (RBS) bestehen in der Regel aus einer Grundierung, welche als Korrosionsschutz und als Haftvermittler dient, einem intumeszierenden Anstrich und einem Deckanstrich (siehe Abb. 2.01). Der intumeszierende Anstrich kann ein Dämmschichtbildner, eine Ablationsbeschichtung, eine Sublimationsbeschichtung oder eine Kombination dieser Produkte sein. Das Aufbringen der Beschichtungsstoffe kann durch Streichen, Sprühen, Spritzen sowie Tauchen in einem oder mehreren Arbeitsschritten erfolgen.

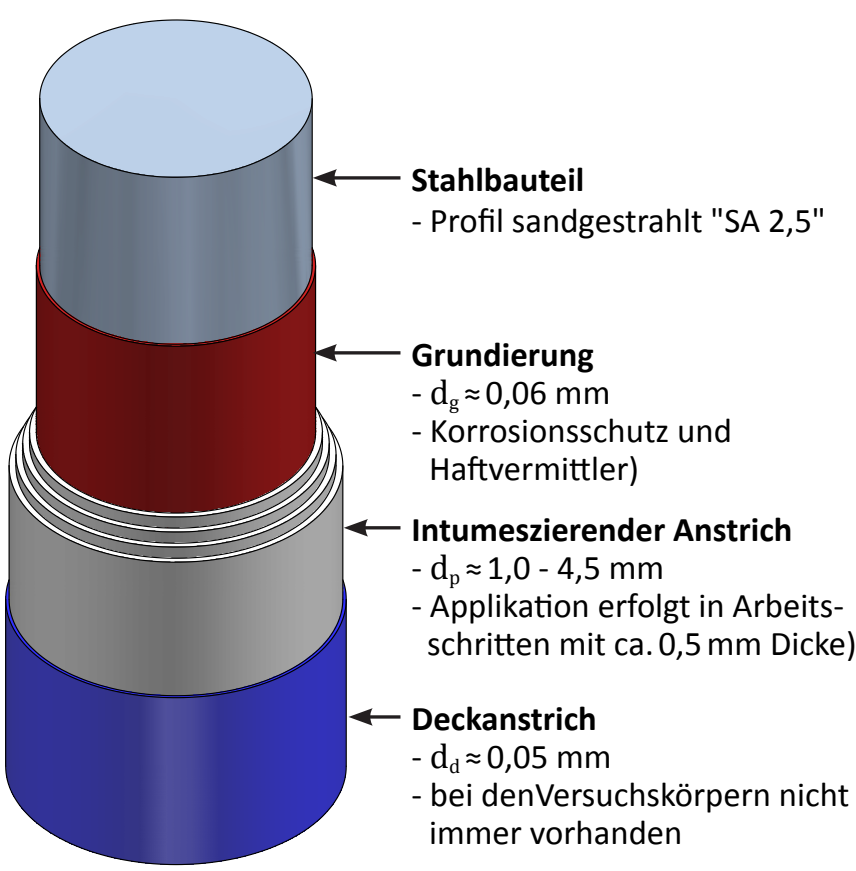

Abb. 2.01: Schematischer Aufbau des auf die Versuchskörper applizierten reaktiven Brandschutzsystems 
In der Regel bestehen reaktive Brandschutzbeschichtungen aus den nachfolgenden Komponenten: Säurequelle (z.B. Ammoniumpolyphosphat), Kohlenstoffquelle (z.B. Pentaerythrit oder Dipentaerythrit), Treibmittel (z.B. Melamin), Additiven (z.B. verschiedene Salze) und einem Bindemittel (z.B. Acrylat). Additive sind nicht zwingend für die Aufschäumung des reaktiven Brandschutzsystems erforderlich. Mit Hilfe dieser Zusatzstoffe lassen sich jedoch gezielt Verbesserungen der chemischen und physikalischen Reaktionen erreichen. Aufgabe des Bindemittels ist die Bildung einer festen und zusammenhängenden Matrix des reaktiven Brandschutzsystems sowie die Sicherstellung einer ausreichenden Haftfähigkeit der Brandschutzbeschichtung. Der Verlauf der chemischen Reaktionen des intumeszierenden Anstriches, d.h. der Aufschäumung, lässt sich vereinfacht wie folgt beschreiben: Die chemische Startreaktion des Aufschäumprozesses wird durch Temperaturbeanspruchung infolge des Brandereignisses ausgelöst. Die chemischen Substanzen in der Säurequelle reagieren miteinander und es bildet sich eine Säure. Durch Reaktion der Säure mit der Kohlenstoffquelle wird Kohlenstoff freisetzt, welcher die Grundlage für die Ausbildung der Schaumstruktur des reaktiven Brandschutzsystems ist. Parallel dazu bilden sich durch die thermische Zersetzung des Treibmittels (Gasbildner) gasförmige Produkte, welche zu einer Aufschäumung der Kohleschicht führen. Um eine gute Aufschäumung zu erreichen, muss die Schaumbildung mit dem Erweichen der Bindemittelmatrix korrelieren.
(1) Inaktive Phase

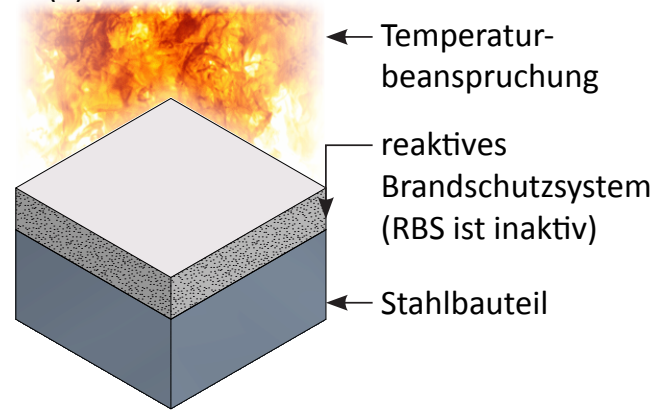

(2) Aufschäumphase

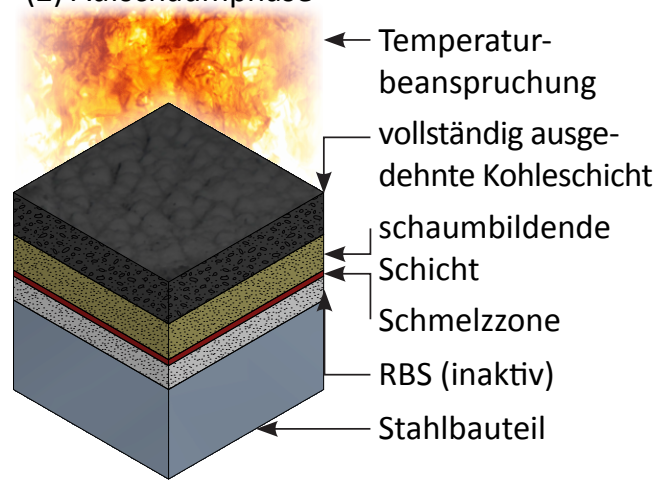

(3) Stabile Phase

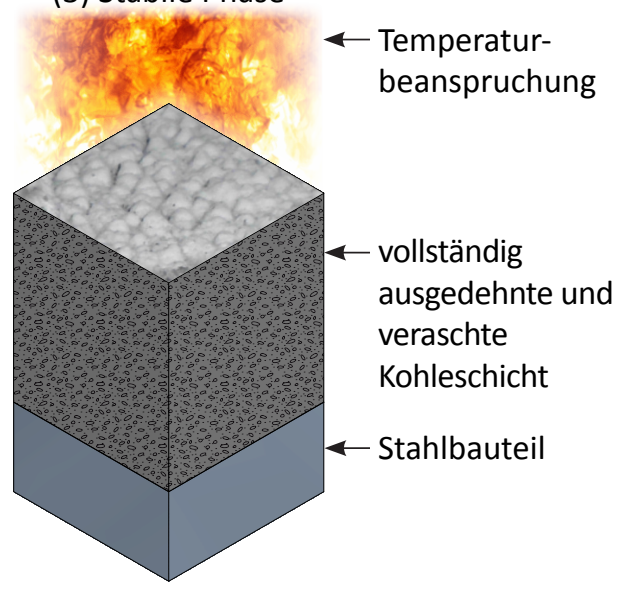

Abb. 2.02: Schematische Darstellung des Aufschäumprozesses des RBS

Die vollständig ausgedehnte und veraschte Kohleschicht ist hoch porös und hat eine geringe Wärmeleitfähigkeit. Dadurch wird der Wärmetransport zwischen den heißen Brandgasen und der sich unter dem reaktiven Brandschutzsystem befindlichen Stahloberfläche erheblich reduziert. Um einen effektiven Schutz der Kohleschicht zu gewähleisten, muss die Rezeptur des reaktiven Brandschutzsystems hinsichtlich der physikalischen und chemi- 
schen Eigenschaften gut abgestimmt sein. Weitere Ausführungen zum Aufbau und den chemischen Prozessen von reaktiven Brandschutzsystemen sind in den Literaturquellen [60] und [62] beschrieben.

Neben dem Aufschäumungsprozess des intumeszierenden Anstrichs lässt sich auch das Verhalten des reaktiven Brandschutzsystems über die Branddauer durch verschiedene Phasen beschreiben. Eine entsprechende Unterteilung wurde bereits von Chen \& Shen [25] vorgeschlagen. Die einzelnen Phasen sind in Abb. 2.02 dargestellt und werden nachfolgend näher erläutert.

\section{(1) Inaktive Phase}

Die Temperatur der Beschichtung ist geringer als die Aktivierungstemperatur, so dass es zu keinem Aufschäumen der Beschichtung kommt. Im Allgemeinen liegt die Aktivierungstemperatur bei etwa 200 bis $250^{\circ} \mathrm{C}$. Die thermischen Eigenschaften des reaktiven Brandschutzsystems bleiben bis zum Erreichen der Aktivierungsenergie unverändert.

\section{(2) Aufschäumphase}

Die Temperatur der Beschichtung übersteigt die Aktivierungstemperatur. Das reaktive Brandschutzsystem beginnt aufzuschäumen. Folglich nimmt die Dicke der Beschichtung zu. Es bildet sich eine schwarze, blasenartige Kohleschicht. Die Schwarzfärbung resultiert aus der Verbrennung von Kohlenstoff, welcher bei der Aufschäumung gebildet wird. Die wärmedämmende Wirkung der Brandschutzbeschichtung entwickelt sich, so dass die Erwärmung des Stahls wesentlich verlangsamt wird.

\section{(3) Stabile Phase:}

In der stabilen Phase erfolgt keine weitere Aufschäumung des reaktiven Brandschutzsystems. Die Dicke der Aufschäumung und folglich auch die wärmedämmenden Eigenschaften der Beschichtung bleiben unverändert. Der Aufschäumungsprozess ist beendet. Dadurch, dass kein Kohlenstoff mehr gebildet wird, verascht das Brandschutzsystem und färbt sich weiß. Infolge der Temperatureinwirkung entsteht eine stabile anorganische Isolationsschicht. Die Wirkungsweise der Brandschutzbeschichtung in Form der Aufschäumung ist nicht reversibel.

\subsection{Arten von reaktiven Brandschutzsystemen}

Reaktive Brandschutzsysteme lassen sich in 1- und 2-Komponenten-Systeme unterscheiden. Innerhalb der 1-Komponenten-Systeme wird in lösemittelhaltige und wasserbasierte Systeme unterschieden. 1-Komponenten-Systeme basieren auf Acrylatharzen und werden auch als "herkömmliche" Brandschutzbeschichtung bezeichnet. Durch eine hohe Anzahl an Additiven wird eine hohe Aufschaumhöhe und gute Wärmeisolierung bei relativ geringen 
Trockenschichtdicken erreicht. Der Schaum von 1-Komponenten-Systemen ist relativ weich und anfällig gegenüber mechanischen Beschädigungen. Der hohe Anteil an Additiven führt zu einer begrenzten Korrosions- und Chemikalienbeständigkeit, welche bei aggressiver Umwelteinwirkung einen Einsatz im Außenbereich ausschließt.

2-Komponenten-Systeme werden auf der Basis von Epoxydharzen hergestellt. Brandschutzbeschichtungen auf Epoxydharzbasis sind lösemittelfrei. Durch einen hohen Bindemittelanteil, z.B. Aminhärter, bilden diese Systeme bei der Aufschäumung ein wesentlich dichteres und widerstandsfähigeres Gefüge als 1-Komponenten-Systeme. Durch einen Faseranteil im Beschichtungsstoff kann die Widerstandsfähigkeit weiter gesteigert werden. 2-Komponenten-Systeme sind daher für den Außeneinsatz geeignet, härten zudem schneller aus und lassen sich mit wesentlich höheren Trockenschichtdicken pro Arbeitsgang applizieren. Diese Systeme besitzen allerdings wesentlich weniger Additive, wodurch die Aufschäumung in der Regel geringer ausfällt.

\subsection{Materialeigenschaften reaktiver Brandschutzsysteme}

Über das Aufschäumverhalten reaktiver Brandschutzsysteme sowie dessen Materialeigenschaften wird bereits in den Dissertationen von Raveglia [49], Sothmann [57] und Tabeling [60] ausgeführt. Der Einfluss der Morphologie, d.h. der Gestalt und Form der Aufschäumung sowie der Oberflächenbeschaffenheit aufgeschäumter Brandschutzbeschichtungen und deren Auswirkung auf die thermische Schutzwirkung wird in [42] beschrieben. Die Ergebnisse der genannten Untersuchungen werden nachfolgend kurz erläutert.

In der Dissertation von Raveglia werden numerische Simulationen zum Einfluss von Fehlstellen im reaktiven Brandschutzsystem und die damit verbundenen Auswirkungen auf die Tragfähigkeit von Stahlbauteilen durchgeführt. Bei den numerischen Berechnungen wird für das reaktive Brandschutzsystem eine spezifische Wärmekapazität von $c_{p}=1,0 \mathrm{~kJ} /(\mathrm{kg} \mathrm{m})$ sowie eine Dichte von $\rho=10 \mathrm{~kg} / \mathrm{m}^{3}$ verwendet. Die beiden Größen werden als konstant und temperaturunabhängig angenommen. Die Wärmeleitfähigkeit wird so lange variiert, bis sich zwischen numerischer Simulation und den experimentellen Untersuchungen eine Übereinstimmung zeigt. Genaue Werte für die Wärmeleitfähigkeit der Brandschutzbeschichtung sind in der Dissertation von Raveglia nicht angegeben.

Sothmann untersucht in seiner Dissertation [57] die Materialkennwerte des reaktiven Brandschutzsystems "Unitherm LSA" der Firma Sika. Dafür werden Wärmeleitfähigkeit und Gesamtwärmekapazität der Beschichtung sowie die Dichte experimentell bestimmt. Für die Ermittlung der Gesamtwärmekapazität wird die dynamische Differenzkalometrie (DSC) verwendet. Durch Verwendung der Thermogravimetrie (TG) wird die Dichte und die Wärmeleitfähigkeit des reaktiven Brandschutzsystems ermittelt. Die Untersuchungen zeigen, 
dass die thermische Schutzwirkung maßgeblich auf die geringe Wärmeleitfähigkeit des aufgeschäumten reaktiven Brandschutzsystems zurückzuführen ist. Die Erhöhung der Gesamtwärmekapazität, welche sich infolge des Aufschäumungsprozesses ergibt, ist von nachgeordneter Bedeutung. Die ermittelten temperaturabhängigen Verläufe für die Wärmeleitfähigkeit und die spezifische Wärmekapazität sind in Abb. 2.03 angegeben. In den durchgeführten numerischen Simulationen wird für die Dichte ein konstanter Wert von

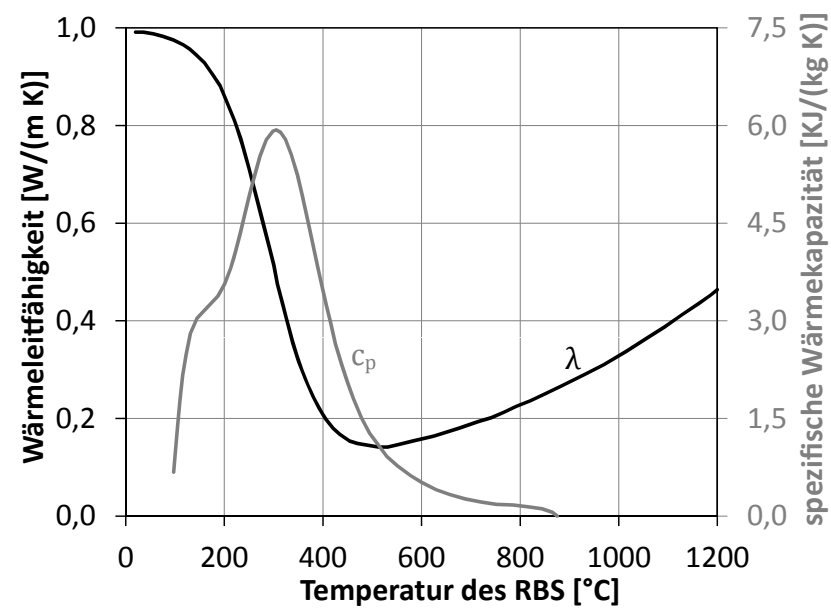

Abb. 2.03: Wärmeleitfähigkeit und Gesamtwärmekapazität aus den Untersuchungen von Sothmann [57] $\rho=1300 \mathrm{~kg} / \mathrm{m}^{3}$ angenommen .

Die Dissertation von Tabeling [60] umfasst eine ausführliche Zusammenstellung der Materialeigenschaften von reaktiven Brandschutzsystemen aus zahlreichen Veröffentlichungen. Daraus geht hervor, dass für die bisherigen Untersuchungen zur Beschreibung von reaktiven Brandschutzsystemen vereinfachte Annahmen genutzt wurden. Beispielsweise wird meist von einem linearen Temperaturgradienten für die Brandschutzbeschichtung ausgegangen. Ferner erfolgt oftmals in der numerischen Simulation eine Anpassung der thermischen Materialeigenschaften des reaktiven Brandschutzsystems, so dass die Ergebnisse mit den experimentelle Untersuchungen übereinstimmen. Belastbare Angaben hinsichtlich der Materialkennwerte liegen demnach nicht vor. Ein Schwerpunkt der Dissertation von Tabeling ist die Entwicklung eines numerischen Modells, welches in der Lage ist, den Aufschäumprozess des Brandschutzsystems sowie das Erwärmungsverhalten von beschichteten Stahlbauteilen wiederzugeben. Um das Verhalten des reaktiven Brandschutzsystems in der numerischen Simulation beschreiben zu können, werden temperaturabhängige Verläufe für den Wärmeausdehnungskoeffizient, die spezifische Wärmekapazität, die Materialdichte und die Wärmeleitfähigkeit der Beschichtung ermittelt (siehe Abb. 2.04). Entsprechende Materialuntersuchungen wurden dazu von Tabeling durchgeführt. Für die Wärmeübertragung innerhalb des reaktiven Brandschutzsystems wird die Wärmeleitung sowie die Wärmestrahlung in den Poren der aufgeschäumten Brandschutzbeschichtung betrachtet. Der Einfluss aus Konvektion auf die Wärmeübertragung wird vernachlässigt, da die Porengröße relativ klein ist und dadurch keine signifikanten Luft- und Gasbewegungen auftreten. Für die numerische Simulation wird eine äquivalente Wärmeleitfähigkeit des reaktiven Brandschutzsystems gebildet, welche aus den Anteilen des Grundmaterials, der Wärmeleitfähigkeit des Mediums innerhalb der Poren und Gaseinschlüsse sowie der Wärmestrahlung in den Poren und Gaseinschlüssen besteht. 
Neben den thermischen Eigenschaften des reaktiven Brandschutzsystems ist für die numerische Simulation des Aufschäumprozesses auch ein Wert für das Elastizitätsmodul erforderlich. Im nicht aufgeschäumten Zustand der Beschichtung werden von Tabeling Zugversuche an Kleinproben durchgeführt. In Abhängigkeit von der Art des Brandschutzsystems, d.h. epoxidharzbasierend oder lösemittelhaltig, ergeben sich unterschiedlich große Werte für den Elastizitätsmodul. Für die numerische Simulation wird von Tabeling ein temperaturunabhängiger Elastizitätsmodul von $\mathrm{E}=1,0 \mathrm{~N} / \mathrm{mm}^{2}$ verwendet. Um die Aufschäumung des Brandschutzsystems auch in den Ausrundungsbereichen eines I-förmigen Profils korrekt wiederzugeben, wird der Elastizitätsmodul in diesen Bereichen auf $E=5,0 \mathrm{~N} / \mathrm{mm}^{2}$ erhöht. Neben seiner Dissertation [60] hat Tabeling die Ergebnisse der durchgeführten Untersuchungen auch in den Veröffentlichungen [54], [55] und [61] beschrieben.

Die Untersuchungen von Morys [42] zeigen, dass nicht nur die Form der Aufschäumung, die Struktur der Poren und die Höhe des aufgeschäumten reaktiven Brandschutzsystems, sondern auch die Oberflächenbeschaffenheit die thermische Schutzwirkung der Brandschutzbeschichtung beeinflusst. Einegeschlossene und dichte Oberfläche der aufgeschäumten Beschichtung erhöht die thermische Schutzwirkung des Brandschutzsystems. Die Porenstruktur und der Schichtenaufbau des in der Dissertation verwendeten reaktiven Brandschutzsystems wird in Kapitel 3.8 untersucht.

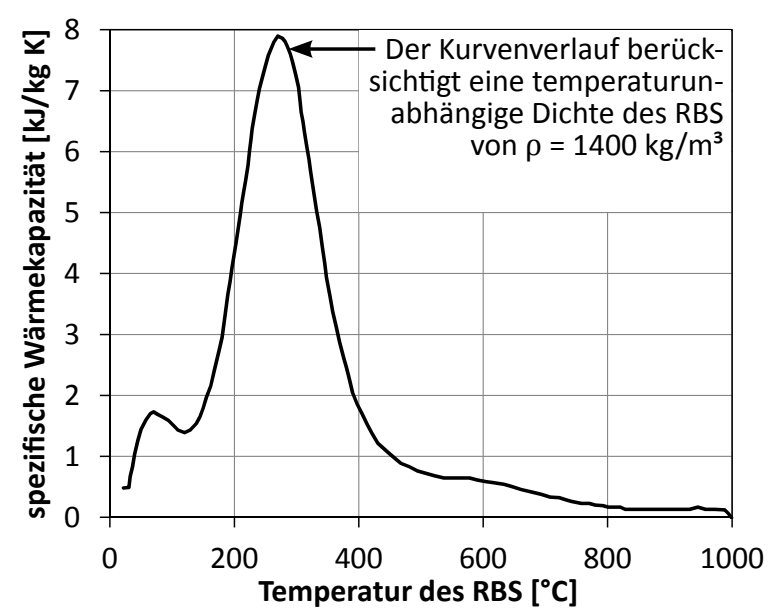

a) Verlauf der spezifischen Wärmekapazität

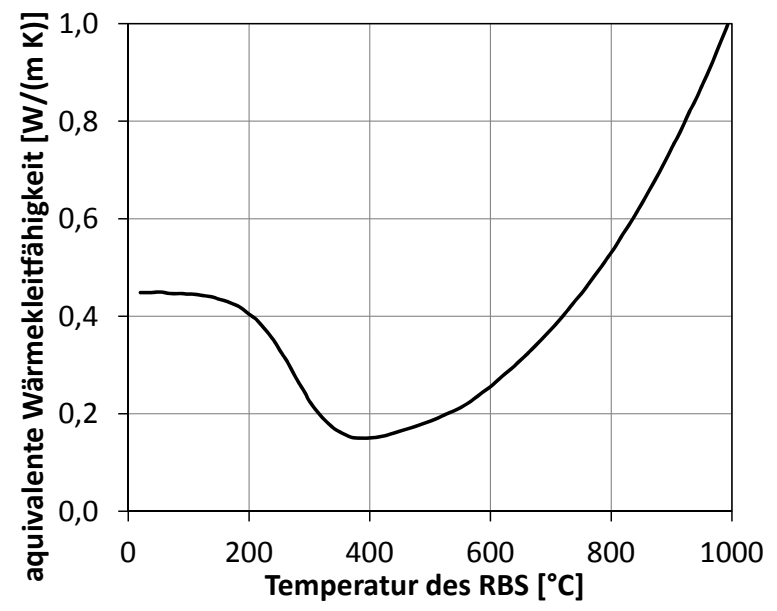

b) äquivalente Wärmeleitfähigkeit

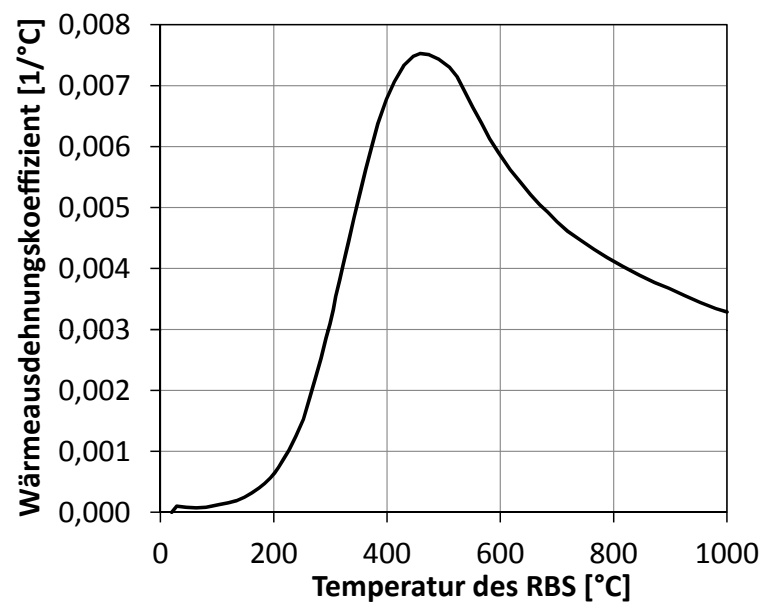

c) gemittelter Wärmesausdehnungskoeffizient

Abb. 2.04: Ergebnisse der Untersuchungen des RBS von Tabeling [60] 


\subsection{Anwendung reaktiver Brandschutzsysteme im Stahlbau}

\subsubsection{Anforderungen an reaktive Brandschutzsysteme}

In den Zulassungsgrundsätzen des DIBt [26] werden verschiedene Anforderungen an reaktive Brandschutzsysteme gestellt. Unter anderem muss eine dauerhafte Wirksamkeit bei Brandbeanspruchung, eine ausreichende Haftung am Stahlbauteil sowie ein Korrosionsschutz sichergestellt werden. Reaktive Brandschutzsysteme müssen auf Stahlbauteilen daher bestimmte thermische und mechanische Anforderungen erfüllen. Die Wärmeleitfähigkeit muss so weit verringert werden, dass sich der Stahl unter Brandbeanspruchung nur langsam erwärmt und die Festigkeit des Stahls möglichst lange erhalten bleibt. Die Bestimmung der mechanischen und thermischen Materialeigenschaften des reaktiven Brandschutzsystems gestaltet sich aufgrund des zeitlich veränderlichen Aufschäumverhaltens der Systeme schwierig. Zwischen den verschiedenen Produkten gibt es zudem teilweise große Unterschiede im Aufschäumverhalten und der Höhe der thermischen Schutzwirkung, welche eine Vereinheitlichung bestimmter Prämissen erschweren.

Als Vergleichskriterium für die thermischen Eigenschaften des reaktiven Brandschutzsystems wird häufig auf die Erwärmungsgeschwindigkeit [13] des beschichteten Stahlbauteils zurückgegriffen. Diese lässt sich relativ einfach messtechnisch erfassen. Die Erwärmungsgeschwindigkeit des Stahls ist im Wesentlichen von den im Brandraum herrschenden Brandgastemperaturen, den thermischen Eigenschaften und der Dicke des reaktiven Brandschutzsystems sowie der Profilgeometrie bzw. des Profilfaktors $\left(A_{m} / V\right)$ des verwendeten Bauteils abhängig. Für die Bestimmung der mechanischen Hochtemperatureigenschaften des Stahls ist die Erwärmungsgeschwindigkeit von Bedeutung, da dadurch das Kriechverhalten des Stahls beeinflusst wird. Ein Vergleich zur Erwärmungsgeschwindigkeit von in der BAM getesteten Träger- und Stützenprofilen mit reaktiver Brandschutzbeschichtung ist in [35] und [37] angegeben.

Die Haftfähigkeit der reaktiven Brandschutzbeschichtung wird als ausreichend angesehen, wenn diese während der gesamten Dauer eines Brandversuches am Bauteil haften bleibt [26]. Die Prüfung belasteter Stahlbauteile ist dabei zwingend erforderlich, da die Verformungen des Stahlbauteils eine besonders hohe Beanspruchung für das reaktive Brandschutzsystem darstellen.

\subsubsection{Prüfung und Zulassung reaktiver Brandschutzsysteme}

\section{Prüfung}

Die Anwendung von reaktiven Brandschutzsystemen auf Stahlträger und -stützen hat sich im Bauwesen etabliert. Um eine zuverlässige Anwendung dieser Systeme sicherzustellen, werden Brandversuche an belasteten und unbelasteten Bauteilen durchgeführt. 
Die Prüflast ist unter Zugrundelegung anerkannter Bemessungsverfahren zu wählen und über die gesamte Versuchsdauer konstant zu halten. Die Temperaturbeanspruchung erfolgt nach DIN 4102-2 [03] mit der Einheits-Temperaturzeitkurve. Die Stahltemperatur eines im Brandfall voll ausgelasteten Bauteils darf an keiner Messstelle mehr als $500{ }^{\circ} \mathrm{C}$ betragen. Bei Einhaltung dieser "kritischen Temperatur" ist der Feuerwiderstand des Bauteils ohne eine Reduzierung des Lastausnutzungsgrades sichergestellt. Durch eine Beschränkung des Lastausnutzungsgrades, d.h. Reduzierung der maximalen Beanspruchbarkeit des Bauteils, ist es auch möglich, Temperaturen von mehr als $500^{\circ} \mathrm{C}$ zuzulassen. Regelungen zum Versuchsumfang sowie der Durchführung und Auswertung der Brandversuche an Stahlträgern und -stützen sind für die Ausstellung einer abZ in den Zulassungsgrundsätzen des DIBt [26] sowie für die Erlangung einer ETB in DIN EN 13381-8 [09] beschrieben. Des Weiteren sind in DIN EN 1363-1 [07] allgemeine Anforderungen für Feuerwiderstandsprüfungen angegeben.

\section{Zulassung}

Bei reaktiven Brandschutzsystemen handelt es sich um nicht geregelte Bauprodukte, für die eine allgemeine Bauaufsichtliche Zulassung (abZ), eine europäische technische Bewertung (ETB) oder eine Zustimmung im Einzelfall (ZiE) erforderlich ist [59]. Die Zulassungen können auf nationaler oder europäischer Ebene erteilt werden. Für die Erteilung von allgemeinen bauaufsichtlichen Zulassungen ist in Deutschland das Deutsche Institut für Bautechnik (DIBt) zuständig. Vor Erteilung einer allgemeinen bauaufsichtlichen Zulassung für ein reaktives Brandschutzsystem wird dieses verschiedenartig getestet. Einen wesentlichen Bestandteil der Prüfung bilden Brandversuche, bei denen der Feuerwiderstand an mechanisch belasteten und unbelasteten Stahlbauteilen unter Verwendung der reaktiven Brandschutzbeschichtung überprüft wird. In der Regel erfolgt dies an Trägern und/oder Stützen. Für Stahlzugglieder existieren gegenwärtig keine normativen Vorgaben, die mittels Prüfung eine Zuordnung in eine Feuerwiderstandsklasse zulassen. Der Einsatz reaktiver Brandschutzsysteme bleibt derzeit auf die üblicherweise für Träger und Stützen verwendeten offenen und geschlossenen Profile, wie z.B. I-Profile und Hohlprofile, begrenzt. Ein festgelegter Bereich des Profilfaktors $A_{m} / V$ (nach ETB) bzw. U/A (nach abZ) ist dabei einzuhalten. Die Anwendung beschränkt sich auf die Baustähle der Festigkeitsklasse S235 und S355. Für andere Stahlsorten sind reaktive Brandschutzsysteme gesondert nachzuweisen. Weitere Einschränkungen ergeben sich aus der maximal möglichen Feuerwiderstandsklasse (feuerhemmend bis feuerbeständig) sowie der statischen Beanspruchung des jeweiligen Bauteils, z.B. als Biegeträger, Druck- oder Zugglied. Mittels der genannten Kriterien, die anhand von Brandprüfungen ermittelt wurden, kann aus den Zulassungen die Mindesttrockenschichtdicke des reaktiven Brandschutzsystems bestimmt werden. Für die Anwendung reaktiver Brandschutzsysteme, die von den Regelungen in den Zulassungen abweichen, ist eine Zustimmung im Einzelfall erforderlich. Selbige ist bei der jeweiligen obersten Bauaufsichtsbehörde eines Bundeslandes zu beantragen. 
In den bis Dezember 2010 erteilten Zulassungen des DIBt ist die Verwendung von reaktiven Brandschutzsystemen auf Stahlzuggliedern gesondert nachzuweisen. In allen seit Januar 2011 neu ausgestellten Zulassungen sind die Regelungen dahingehend geändert worden, dass reaktive Brandschutzsysteme unter gewissen Einschränkungen auf Stahlzugglieder aus offenen und geschlossenen Profilen verwendet werden können [59]. Stahlbauteile mit Vollquerschnitt sind nach wie vor von einer Anwendung ausgeschlossen und sind gesondert nachzuweisen. Die dafür erforderliche Vorgehensweise ist im Kapitel 1.2 beschrieben. Um eine Anwendung von reaktiven Brandschutzsystemen auf Zuggliedern mit Vollprofil mittels Zulassungen zu ermöglichen wird derzeit auf europäischer (CEN) und internationaler Ebene (ISO) daran gearbeitet, eine entsprechende Norm [18] und [19] für die Durchführung von Brandprüfungen an Stahlzuggliedern mit Vollquerschnitt zu erstellen.

\subsubsection{Einsatz von Stahlzuggliedern im Bauwesen}

Stahlzugglieder finden unter anderem in Fachwerkträgern, Aussteifungsverbänden, Abhängungen und Unterspannungen Anwendung. Die Zugglieder werden im Bauwesen in horizontaler, vertikaler oder schräger Position eingebaut. Für Fachwerkträger werden in der Regel relativ große Stahlprofile mit offenem oder geschlossenem Profil, wie zum Beispiel I-Profile und Hohlprofile, eingesetzt. Bei Aussteifungsverbänden, Abhängungen oder Unterspannungen, wie beispielsweise bei unterspannten Trägern, werden üblicherweise schlanke Vollprofile, insbesondere mit kreisförmigem Querschnitt, verwendet. Für deren Einsatz sind neben statisch-konstruktiven Gesichtspunkten vor allem architektonische und optische Aspekte ausschlaggebend. In Abb. 2.05 ist das Dachtragwerk des Carports einer Firma für Abfallwirtschaft in München dargestellt, bei dem Kreisvollprofile als Stahlzugglieder verwendet wurden.
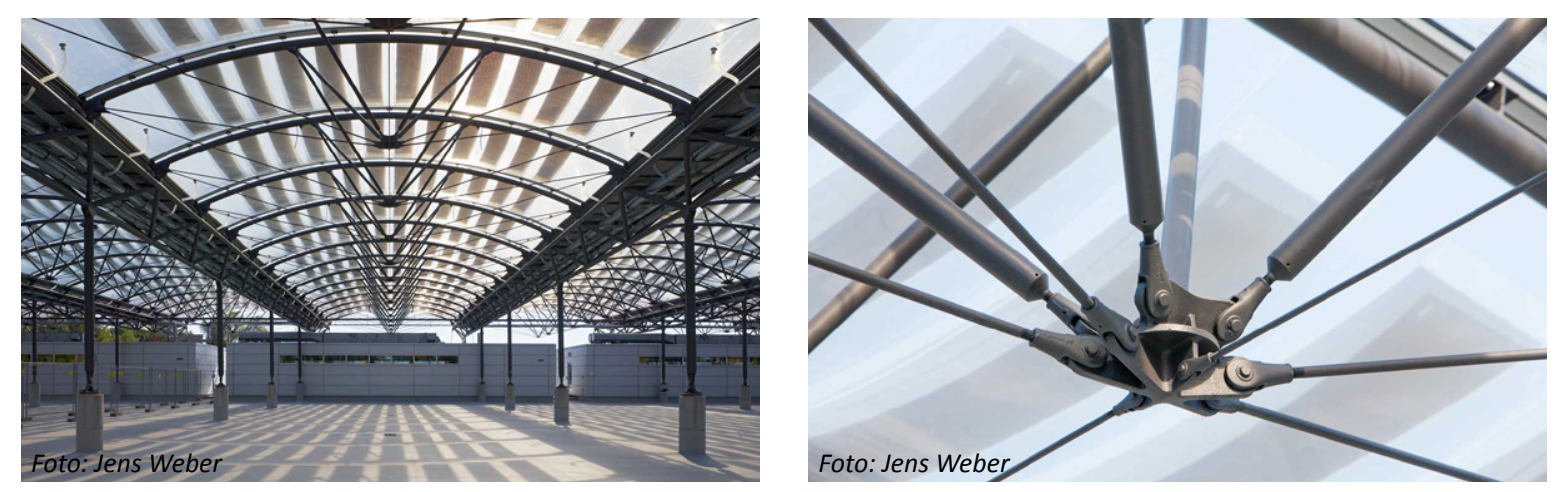

Abb. 2.05: Einsatzbereich von Zugliedern am Beispiel der Überdachung des Carports der Abfallwirtschaft München [66]

Übernehmen Zugglieder wichtige Funktionen in einem Tragwerk, beispielsweise die Aufrechterhaltung der Gesamtstabilität der Konstruktion, kann deren Versagen im Brandfall schnell zu kritischen Situationen führen. Dies gilt insbesondere dann, wenn eine Umlage- 
rung der Kräfte innerhalb der Konstruktion nicht möglich ist und das Tragwerk über keine Redundanzen verfügt. Im Brandfall sind die Ausdehnung der Bauteile bzw. der Konstruktion zu berücksichtigen und können ebenfalls Auswirkung auf die Stabilität der Gesamtkonstruktion haben. Für Stahlzugglieder sind daher entsprechende Schutzmaßnahmen unverzichtbar, um eine ausreichende Feuerwiderstandsdauer für diese Bauteile zu gewährleisten.

\subsubsection{Anwendung reaktiver Brandschutzsysteme auf Stahlzuggliedern}

\section{Zugglieder mit offenem oder geschlossenem Profil}

Ein 2010 von der BAM durchgeführtes Forschungsprojekt [33] beschäftigt sich mit der Fragestellung, ob eine Anwendung reaktiver Brandschutzsysteme auf Stahlzugglieder mit offenem oder geschlossenem Profil möglich ist und inwieweit sich bereits bestehende Brandversuche an mit reaktiven Brandschutzsystemen beschichteten Biegeträgern auf Zugglieder übertragen lassen. Die Bewertung erfolgt im Hinblick auf die Haftfähigkeit einer reaktiven Brandschutzbeschichtung durch einen numerischen Vergleich der auftretenden Dehnungen an einem Stahlzugglied und der Biegezugzone eines Stahlträgers. Träger und Zugglied müssen dabei die gleiche Querschnittsgeometrie besitzen. Eine ausführliche Beschreibung der numerischen Untersuchung ist in den Veröffentlichungen [32], [33] und [37] enthalten. Eine Fortführung dieser Untersuchungen sowie eine Bewertung der derzeitigen Zulassungsregelungen und der normativen Grundlagen ist in [36] beschrieben.

Auf Grundlage der numerischen Untersuchungen aus [32] wird in die seit 2011 ausgestellten allgemeinen bauaufsichtlichen Zulassungen (abZ) die Anwendung reaktiver Brandschutzsysteme auf Stahlzugglieder mit offenen Profilen, z.B. I-Profile, aufgenommen [59]. Der Anwendungsbereich dieser Zugglieder beschränkt sich auf Bauteile mit einem maximalen Lastausnutzungsgrad für den Brandfall von $\mu_{\mathrm{fi}} \leq 0,5$. Ferner muss das reaktive Brandschutzsystem das Prozedere zur Erlangung einer abZ erfolgreich durchlaufen [26]. Brandprüfungen müssen zudem an mechanisch belasteten Trägern mit offenem Profil erfolgen, um die Haftungseigenschaften des reaktiven Brandschutzsystems am verformten Bauteil zu überprüfen. Soll die Anwendung der Zugglieder hinsichtlich der Bauteilorientierung nicht begrenzt werden, sind Brandprüfungen mit Versuchskörpern in horizontaler und vertikaler Einbaulage durchzuführen. Die Regelung für Zugglieder aus offenen Profilen lässt sich grundsätzlich auch auf geschlossene Profile, wie z.B. Hohlprofile, übertragen. Entsprechende Brandprüfungen an Bauteilen mit geschlossenem Profil werden vorausgesetzt.

\section{Zugglieder mit Vollprofil}

Für die im Stahlbau beispielsweise in Aussteifungsverbänden, Abhängungen oder für Unterspannungen von Stahlträgern eingesetzten Vollprofile ist eine Anwendung reaktiver Brandschutzsysteme derzeit in den Zulassungen nicht geregelt. Der in Deutschland für offene und geschlossene Stahlprofile angewendete Vergleich zwischen Biegebauteil und Zugglied ist 
für Vollquerschnitte nicht möglich, da in der Regel keine Brandversuche an Biegeträgern mit Vollprofil durchgeführt werden. Die Haftfähigkeit und thermische Schutzwirkung der Brandschutzbeschichtung ist anhand von zusätzlichen Brandversuchen nachzuweisen. Regelungen über Art, Umfang und Aufbau von belasteten oder unbelasteten Brandprüfungen an Stahlzuggliedern existieren gegenwärtig weder in Deutschland noch in Europa. Ziel eines an der BAM durchgeführten Forschungsprojektes [35] ist es, Vorschläge für Zulassungsgrundsätze bzw. Leitlinien zur Beurteilung reaktiver Brandschutzsysteme Stahlzuggliedern, insbesondere mit Vollquerschnitt, zu entwickeln. Im Fokus der Untersuchungen stehen insbesondere kreisförmige Vollprofile mit relativ kleinem Stabdurchmesser. Als Ergebnis des Forschungsprojektes werden Empfehlungen für die Durchführung und Auswertung von Brandversuchen an Stahlzuggliedern mit reaktivem Brandschutzsystem erarbeitet. Die vorliegende Dissertation stellt eine Fortführung der Untersuchungen des Forschungsprojektes dar. Die Erkenntnisse aus den beiden Arbeiten bilden die Grundlage für eine mögliche Erweiterung des Anwendungsbereiches von Zulassungen für reaktive Brandschutzsysteme auf Stahlzugglieder mit Vollprofil.

\subsection{Vorbetrachtungen}

\subsubsection{Vergleich des Verhaltens reaktiver Brandschutzsysteme auf Biegeträ- gern und Zuggliedern}

Die Übertragung der Ergebnisse von reaktiven Brandschutzsystemen aus Brandprüfungen an Biegeträgern mit I-Profil auf Stahlzugglieder mit Kreisvollprofil ist problematisch, da sich aus der jeweiligen Profilart unterschiedliche Anforderungen an das Aufschäumverhalten der reaktiven Brandschutzbeschichtung ergeben. In Abb. 2.06 ist das unterschiedliche Aufschäumverhalten des reaktiven Brandschutzsystems an einem I-Profil und einem Kreisvollprofil mit und ohne mechanische Beanspruchung schematisch dargestellt.

Für den unbelasteten Träger ergibt sich aufgrund der vornehmlich geraden Oberflächen eine eindimensionale Aufschäumung des reaktiven Brandschutzsystems. Da die Brandschutzbeschichtung fast ausschließlich in Dickenrichtung, d.h. senkrecht zur Bauteiloberfläche, aufschäumt, stellt dies keine besondere Beanspruchung für das System dar. Die Rissbildung des reaktiven Brandschutzsystems in kritischen Bereichen, wie z.B. Kanten und Ausrundungsbereichen mit relativ starker Oberflächenkrümmung, bleiben beim I-Profil unberücksichtigt, da die Messung der Stahltemperaturen nach DIN EN 13381-8 [09] nur in Bereichen mit gerader Oberfläche, z.B. für den Trägerflansch jeweils in der Mitte zwischen Flanschfuß und Steg bzw. für den Trägersteg in der Mitte zwischen beiden Flanschen, erfolgt. Die eventuell auftretenden höheren Stahltemperaturen in den kritischen Querschnittsbereichen bleiben bei der Brandprüfung unberücksichtigt. Im Gegensatz dazu treten an einem unbelasteten Zugglied mit kreisförmigem Querschnitt aufgrund der gekrümm- 
ten Oberfläche und der Umfangsvergrößerung der Brandschutzbeschichtung während des Aufschäumprozesses radiale Zugspannungen auf. Das Brandschutzsystem muss folglich in zwei Richtungen aufschäumen, d.h. in Dickenrichtung der Brandschutzbeschichtung sowie in Stabumfangsrichtung. Ist die aus der Umfangsvergrößerung des reaktiven Brandschutzsystems auftretende radiale Zugspannung größer als die Zugfestigkeit der aufschäumenden Brandschutzbeschichtung, kann es zur Bildung von Längsrissen kommen, die in der Regel eine Reduzierung der thermischen Schutzwirkung zur Folge hat.

Einen weiteren Einfluss auf das Aufschäumverhalten des reaktiven Brandschutzsystems stellt die Art der Lasteinwirkung dar. Die Biegebeanspruchung bei Trägern führt im Untergurt zu Zugspannungen in Bauteillängsrichtung. Durch die daraus resultierenden Dehnungen ist eine Aufschäumung des reaktiven Brandschutzsystems in zwei Richtungen erforderlich, d.h. in Dickenrichtung der Brandschutzbeschichtung und in Stablängsrichtung.

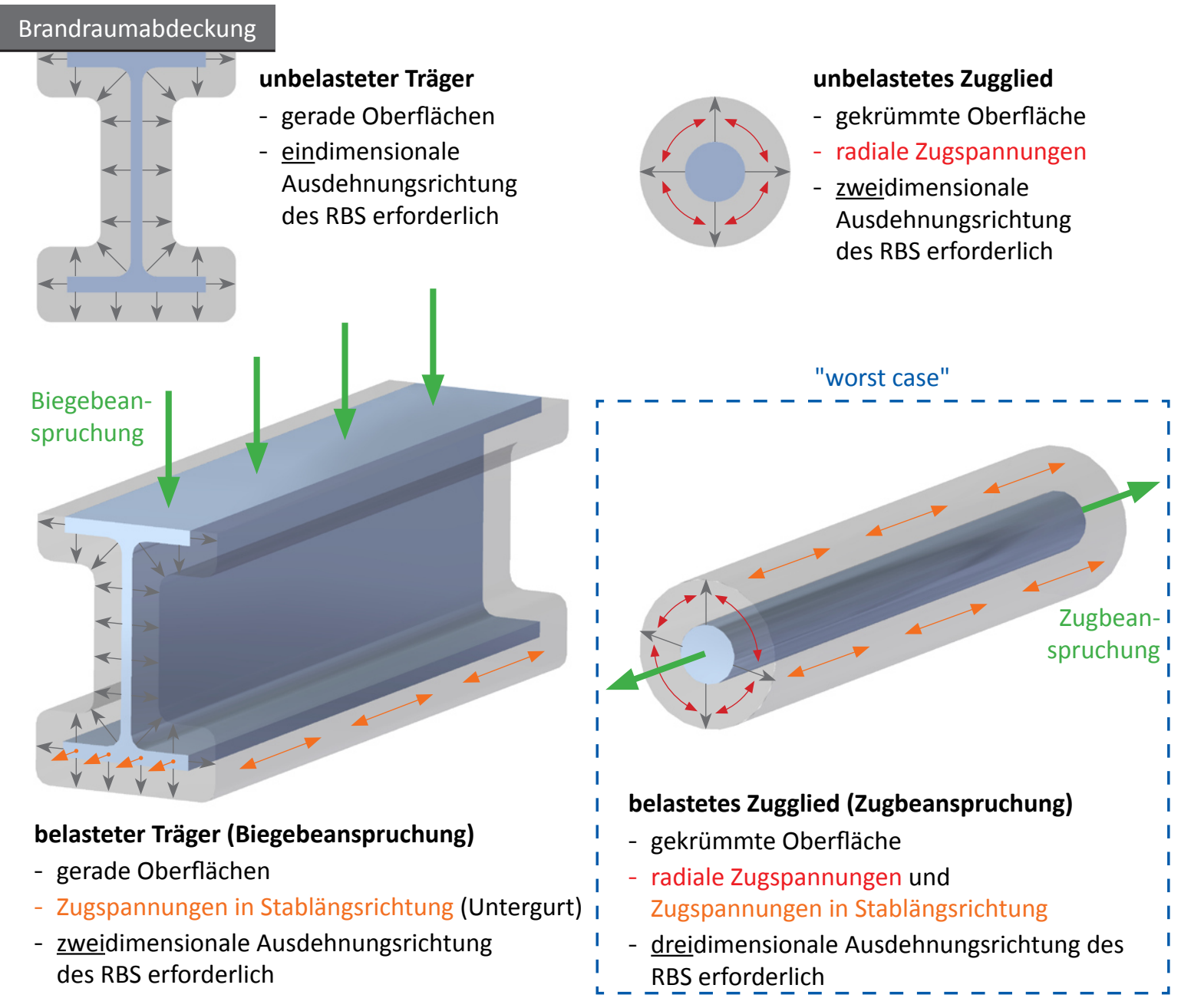

Abb. 2.06: Vergleich des Aufschäumverhaltens reaktiver Brandschutzsysteme auf Biegeträgern und Zuggliedern [34] 
Bei einem belasteten Zugglied treten ebenfalls Zugspannungen in Stablängsrichtung auf. Reaktive Brandschutzsysteme müssen bei einem Zugglied mit Kreisvollprofil zusätzlich auch die aus der gekrümmten Bauteiloberfläche resultierenden radialen Spannungen vom Brandschutzsystem aufnehmen. Für das belastete Zugglied ist eine dreidimensionale Aufschäumung erforderlich, d.h. in Dickenrichtung der Beschichtung, in Stabumfangsrichtung sowie Stablängsrichtung. Dies stellt besonders hohe Anforderungen an die Wirkungsweise des reaktiven Brandschutzsystems. Die in [32] und [33] beschriebenen numerischen Vergleichsrechnungen zwischen Trägern und Zuggliedern mit I-förmigen Querschnitt haben bereits ergeben, dass ein im Kaltfall voll ausgelastetes Zugglied für Stahltemperaturen von mehr als $300{ }^{\circ} \mathrm{C}$ höhere Dehnungen in Stablängsrichtung aufweist, als ein Biegeträger mit identischem Querschnitt und Lastausnutzungsgrad. Da die Höhe der Dehnungen die Haftfähigkeit des reaktiven Brandschutzsystems beeinflusst, ist dies ein weiterer Beleg für eine höhere Beanspruchung des reaktiven Brandschutzsystems auf Stahlzuggliedern als auf Biegeträgern. Die Stahltemperatur, ab der die Dehnungen des Zuggliedes größer sind als die des Biegeträgers, lässt sich durch eine Reduzierung des Lastausnutzungsgrades erhöhen. Allerdings sind in der Baupraxis Bauteile mit geringem Lastausnutzungsgrad aus Gründen der Wirtschaftlichkeit eher unüblich und daher kaum anzutreffen.

Zuverlässige Aussagen zur thermischen Schutzwirkung des reaktiven Brandschutzsystems sind nur mit Hilfe von Brandprüfungen an mechanisch belasteten Zuggliedern möglich, da das dabei auftretende dreidimensionale Aufschäumen den kritischsten Fall für die Brandschutzbeschichtung darstellt. Die Prüfung von unbelasteten Zuggliedern entspricht zudem nicht der tatsächlichen Situation in der Baupraxis. Eine Kombination der Ergebnisse aus Brandprüfungen an reaktiven Brandschutzsystemen, bei der entweder Stablängsspannungen oder radiale Umfangsspannungen auftreten, beispielsweise an belasteten Trägern mit I-förmigen Querschnitt und unbelasteten Zuggliedern mit Kreisvollprofil, besitzt derzeit keine wissenschaftliche Grundlage. Hinzu kommt, dass die zum Erreichen einer entsprechenden Feuerwiderstandsdauer benötigten Trockenschichtdicken des reaktiven Brandschutzsystems bei Zuggliedern mit Kreisvollprofil in der Regel wesentlich größer sind, als die üblicherweise an Biegeträgern getesteten Dicken. Ein Vergleich zwischen Träger und Zugglied ist jedoch nur sinnvoll, wenn beide Bauteile mit der gleichen Trockenschichtdicke geprüft werden. Bereits vorhandene Prüfdaten reaktiver Brandschutzsysteme auf Biegeträgern sind daher nur begrenzt anwendbar. Ein im Rahmen der Normungsarbeit [19] und [18] diskutierter Vorschlag, die Bauteiltemperaturen aus Brandversuchen an unbelastet geprüften Zuggliedern mit Kreisvollprofil mittels eines Korrekturfaktors aus Brandprüfungen an belasteten und unbelasteten Biegeträgern zu modifizieren, ist bisher nicht wissenschaftlich untersucht worden und strittig. Darüber hinaus ist ein solches Verfahren auf die maximale Trockenschichtdicke des Biegeträgers begrenzt. Gegen die Verwendung von Prüfdaten oder Korrekturfaktoren aus Brandprüfungen an Trägern spricht zudem, dass für Träger und Zugglieder unterschiedliche Stähle zum Einsatz kommen. Da sich die Materialeigenschaften zwischen warmgewalzten Baustahl und kaltverformten Blankstahl hinsichtlich des Wär- 
meausdehnungskoeffizienten und der temperaturabhängigen Abminderungsbeiwerte der Stahlfestigkeit unterscheiden, ist eine Beurteilung zu den Auswirkungen auf das reaktive Brandschutzsystem vakant. Zum Einfluss der Stahlsorte auf die Haftfähigkeit und Rissbildung des reaktiven Brandschutzsystems gibt es bisher ebenfalls keine Untersuchungen.

$\underline{\text { Fazit }}$

Ein Vergleich zwischen der Biegezugzone eines Trägers mit I-Profil und einem auf Zug belasteten Zugglied mit Kreisvollquerschnitt ist aufgrund fehlender wissenschaftlich fundierter Grundlagen nicht möglich. Zuverlässige Aussagen zur Feuerwiderstandsdauer von Stahlzuggliedern mit reaktiver Brandschutzbeschichtung können einzig durch Brandprüfungen mit mechanischer Zugbeanspruchung getroffen werden.

\subsubsection{Versagensmechanismen von Stahlzuggliedern und reaktiven Brand- schutzsystemen}

Aus instationären Warmzugversuchen zur Bestimmung der Materialeigenschaften von Stahl ist bekannt, dass der Probenbruch von zugbeanspruchten Stahlbauteilen mit kreisförmigem Vollquerschnitt in einem lokal begrenzten Bereich auftritt. Dieser Bereich weist eine ausgeprägte Brucheinschnürung, d.h. Querschnittsverjüngung der Zugprobe, auf. Im Einschnürungsbereich treten relativ große Verformungen bzw. Dehnungen in Stablängsrichtung auf. Mit zunehmender Stahltemperatur stellt sich ein immer duktileres Bauteilverhalten im Bruchbereich ein. Da bei Stahlzuggliedern die aus der Zugbeanspruchung resultierenden Spannungen über das gesamte Bauteil konstant sind und sich aus Stabbereichen mit geringerer Stahltemperatur keine Tragfähigkeitsreserven erschließen lassen, ist ein Probenbruch des Bauteils an der Stelle der maximalen Stahltemperatur wahrscheinlich.

Wie bereits in Kapitel 2.6.1 beschrieben, tritt bei Profilen mit gekrümmten Bauteiloberfläche eine stärkere Beanspruchung des reaktiven Brandschutzsystems auf. Durch lokal erhöhte Brandgastemperaturen, eine Akkumulation von Beschichtungsmaterial oder Abschattungseffekte des Versuchskörpers im Brandversuch können sowohl in Stablängsrichtung als auch über den Querschnitt eines kreisförmigen Stahlprofils unterschiedliche Aufschäumhöhen des reaktiven Brandschutzsystems auftreten. Darüber hinaus ist aus Brandversuchen an Stahlträgern und -stützen mit reaktiver Brandschutzbeschichtung bekannt, dass es durch die große Volumenzunahme beim Aufschäumen des Brandschutzsystems zu einer Rissbildung in der Schaumstruktur kommen kann. Risse in der aufgeschäumten Brandschutzbeschichtung können sowohl an mechanisch belasteten als auch an unbelasteten Stahlbauteilen auftreten. Der Verlauf der Risse, sowie deren Länge, Breite und Tiefe kann variieren. An Stellen mit Rissbildung wird die thermische Schutzwirkung der reaktiven Brandschutzbeschichtung erheblich reduziert und eine schnelle Erwärmung des Stahls ist die Folge. Bei mechanisch belasteten Zuggliedern kann durch den temperaturbedingten Festigkeitsverlust 
ein frühzeitiger Probenbruch eintreten. Bei kreisförmigen Stahlprofilen ist es wahrscheinlich, dass aufgrund des geringeren Querschnitts des reaktiven Brandschutzsystems die Rissbildung in Stabbereichen mit reduzierter Aufschäumhöhe auftritt. Bei horizontal eingebauten Zuggliedern kann eine Aufweitung eines Längsrisses auf der Staboberseite zu einem Abgleiten der aufgeschäumten Brandschutzbeschichtung führen. Für vertikal angeordnete Stahlbauteile kann ein Abgleiten des reaktiven Brandschutzsystems in Stablängsrichtung infolge des Eigengewichts der Brandschutzbeschichtung auftreten. Das Abgleiten der Brandschutzbeschichtung ist als besonders kritisch anzusehen, da sich dadurch große nahezu ungeschützte Stabbereiche ergeben, welche zu einem frühzeitigen Verlust der Tragfähigkeit des Bauteils führen. Die für Zugglieder mit Kreisvollprofil zu erwartenden Versagensmechanismen des reaktiven Brandschutzsystems sind in horizontaler und vertikaler Prüflage in Abb. 2.07 schematisch dargestellt.

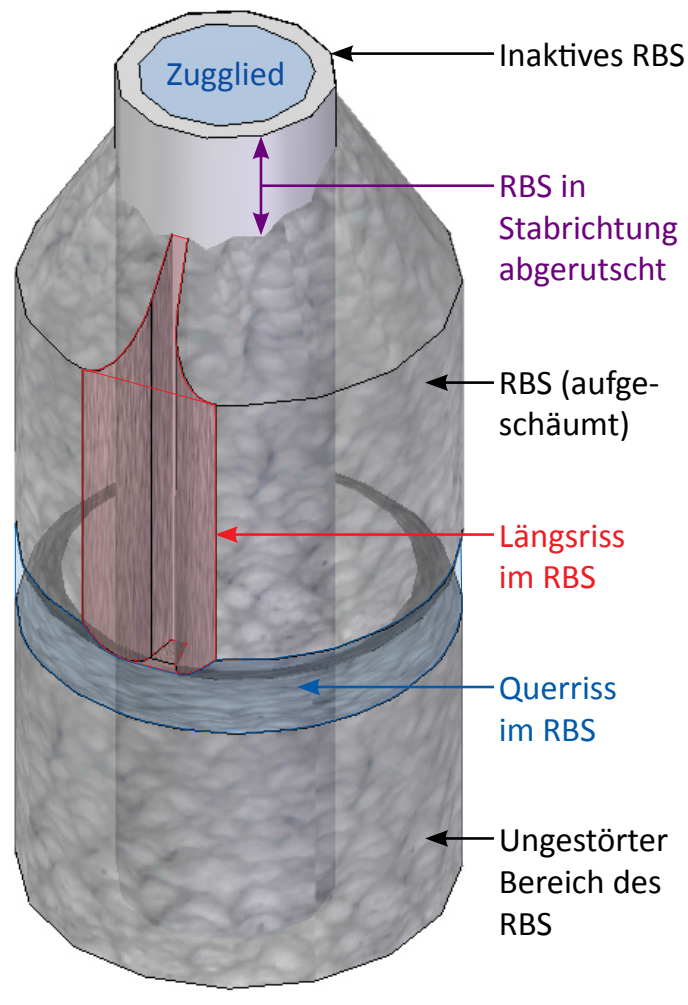

Perspektivischer Querschnitt

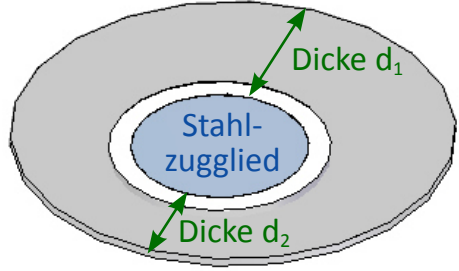

Unterschiede in der Aufschäumung des RBS

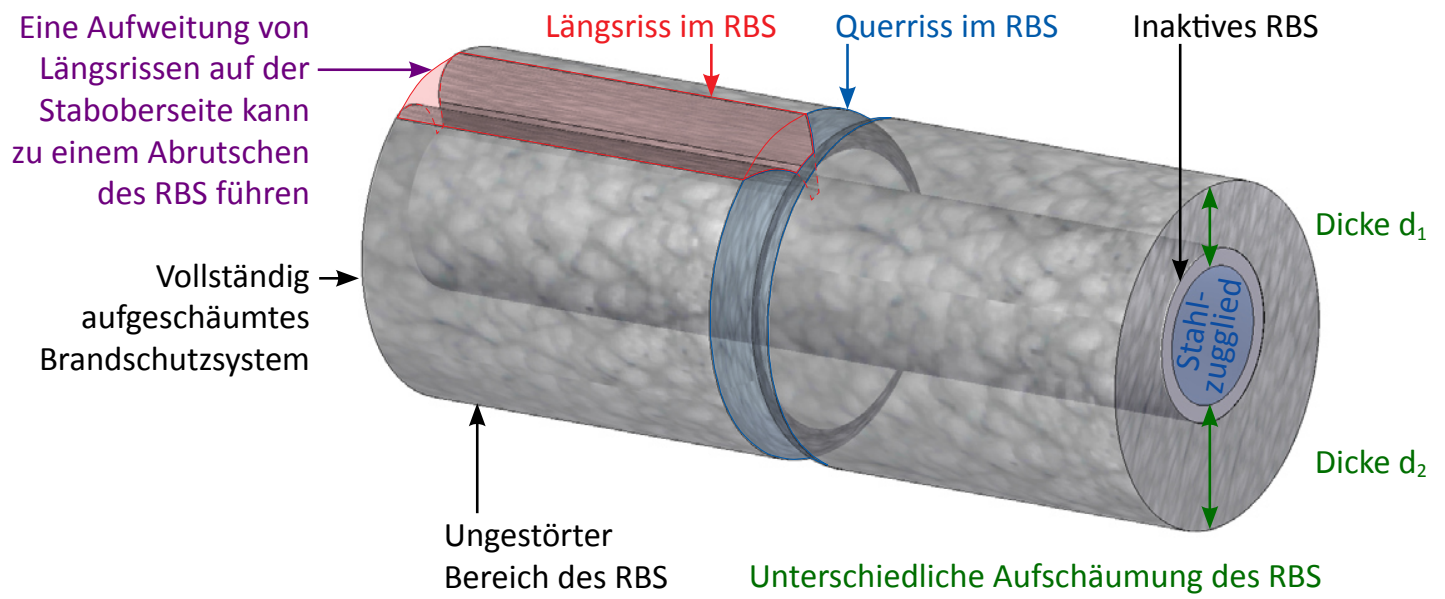

Abb. 2.07: Schematische Darstellung möglicher Versagensmechanismen des RBS auf Kreisvollprofilen (KVP) (oben: Zugglied in vertikaler Prüflage; unten: Zugglied in horizontaler Prüflage) 


\section{Fazit}

Ungleiche Aufschäumhöhen, Rissbildung und Abgleiten des reaktiven Brandschutzsystems können sowohl bei horizontal als auch vertikal eingebauten Stahlzuggliedern zu unterschiedlichen Stahltemperaturen führen und ein Versagen an dieser Stelle induzieren. 


\section{Experimentelle Untersuchung von reaktiven Brand- schutzsystemen auf Stahlzuggliedern}

\subsection{Herangehensweise}

Im Rahmen eines Forschungsprojektes [35] werden erstmalig Brandversuche an Stahlzuggliedern mit reaktiver Brandschutzbeschichtung durchgeführt. Die Prüfungen finden im Realmaßstab 1:1 statt. Die beschichteten Zugglieder werden mehrheitlich mit Zugbeanspruchung geprüft. In den Brandversuchen wird besonders das Aufschäumverhalten und die thermische Schutzwirkung des reaktiven Brandschutzsystems untersucht. Der Einfluss der Profilgeometrie des Zuggliedes, der Trockenschichtdicke des reaktiven Brandschutzsystems, des Lastausnutzungsgrades der mechanischen Zugbeanspruchung sowie die Orientierung des Bauteils im Brandraum werden ebenfalls untersucht. Der Versuchsaufbau der Brandversuche an mechanisch belasteten Zuggliedern ist schematisch in Abb. 3.01 dargestellt. Der Aufbau und die Funktionsweise des Prüfstandes, welcher nachfolgend als Zugstabprüfofen bezeichnet wird, ist in Kapitel 3.5.2 beschrieben. Anhand der Brandversuche werden Empfehlungen für die Bestimmung der Tragfähigkeit erarbeitet, um damit eine Einordnung von Brandprüfungen an mechanisch belasteteten Stahlzuggliedern mit reaktiver Brandschutzbeschichtung in die Feuerwiderstandsklassen zu ermöglichen. Da der Einsatz reaktiver Brandschutzsysteme auf Stahlzuggliedern bisher kaum untersucht wurde und nur wenig Informationen zum Verhalten dieser Systeme auf Vollprofilen, insbesondere auf Kreisvollprofilen, bekannt ist, werden im Vorfeld der Brandversuche an mechanisch belasteten Stahlzuggliedern verschiedene Voruntersuchungen hinsichtlich geeigneter Trockenschichtdicken und der Applikation der Thermoelemente durchgeführt. Des Weiteren wird die Wirkungsweise des reaktiven Brandschutzsystems an Teilen der aufgeschäumten Brandschutzbeschichtung mittels Computertomographie und Rasterelektronenmikroskop untersucht.

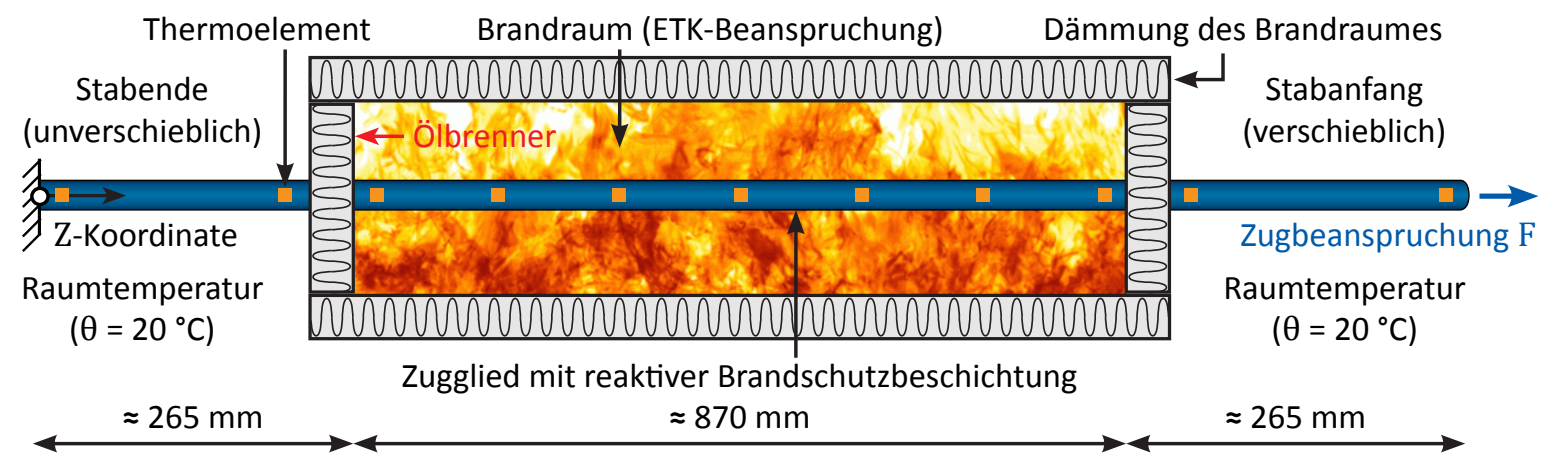

Abb. 3.01: Vereinfachte Darstellung der im Zugstabprüfofen durchgeführten Brandversuche im Realmaßstab 


\subsection{Angaben zu den Versuchskörpern}

In Kapitel 2.6.1 wurden bereits theoretische Überlegungen zum Aufschäumverhalten reaktiver Brandschutzsysteme auf Biegeträgern mit I-Profil und Stahlzuggliedern mit kreisförmigem Vollquerschnitt ausgeführt. Danach besteht für kreisförmige Profile mit Zugbeanspruchung aufgrund der vorhandenen Oberflächenkrümmung und der zu erwartenden Stablängsverformungen eine besonders hohe Beanspruchung für das reaktive Brandschutzsystem. Für die Untersuchungen werden mehrheitlich Vollprofile mit dem für Zugstabsysteme üblichen kreisförmigem Querschnitt sowie möglichst großer Oberflächenkrümmung, d.h. kleinem Stabdurchmesser, ausgewählt. Da negative Auswirkungen der mechanischen Zugbeanspruchung auf das Aufschäumungsverhalten und die thermische Schutzwirkung des reaktiven Brandschutzsystems vermutet werden, besteht das Versuchsprogramm mehrheitlich aus belasteten Brandprüfungen. Um den Einfluss größerer Stabdurchmesser zu untersuchen und gleichzeitig die maximale Zugkraft des Prüfstandes von 400 kN nicht zu überschreiten, wird ein Kreishohlprofil in das Versuchsprogramm aufgenommen. In den derzeitigen Zulassungen beschränkt sich die Anwendung reaktiver Brandschutzsysteme auf Stahl der Festigkeitsklassen S235 und S355. Für Zugglieder werden in der Regel Stähle mit hoher Festigkeit eingesetzt, wodurch der Profilquerschnitt reduziert werden kann. Für die Untersuchungen werden Stahlzugglieder der Festigkeitsklasse S355 gewählt.

Die Abmessungen und Materialeigenschaften der in den Brandprüfungen verwendeten Stahlprofile sind in Tabelle A.1 angegeben. Alle Zugglieder mit dem gleichen Profil stammen aus einer Charge und weisen identische Materialeigenschaften auf. Die Kreisvollprofile bestehen aus gezogenem, kaltverformten Blankstahl. Für die nahtlos gefertigten Kreishohlprofile wird warmgewalzter Baustahl verwendet. Um eine gute Haftfähigkeit zwischen Stahlbauteil und reaktiver Brandschutzbeschichtung zu gewährleisten, ist eine aufgeraute Oberfläche (SA 21/2) nach DIN EN ISO 8501-1 [16] erforderlich. Dies wird mittels Sandstrahlen erzeugt. Die sandgestrahlten Kreisvollprofile weisen eine mittlere Rauheit $\left(R_{a}\right)$ von 7,5 $\mu \mathrm{m}$ und eine mittlere Rautiefe $\left(R_{z}\right)$ von $44,5 \mu$ m auf.

\subsection{Art des verwendeten reaktiven Brandschutzsystems}

Für die Untersuchungen kommt als Brandschutzbeschichtung ein herkömmliches reaktives Brandschutzsystem mit einer derzeit gültigen Zulassung zum Einsatz [02], [17]. Laut der Zulassung des Produktes und den Angaben des Herstellers beschränkt sich die Anwendung auf Stützen und Träger im Innenbereich von Gebäuden. Anwendungsschwerpunkte des Systems sind "H"- oder "I"-Profile sowie rechteckige Hohlprofile bis zu einer Feuerwiderstandsklasse von R120. Bei dem verwendeten reaktiven Brandschutzsystem handelt es sich um ein wasserbasiertes 1-Komponenten-System. Das System besteht aus einer Grundierung und einem intumeszierenden Anstrich. Auf einen Decklack wird in den Brandversu- 
chen verzichtet. Die Applikation der Brandschutzbeschichtung erfolgt durch den Hersteller selbst. Die anhand von Brandversuchen mit dem verwendeten Produkt gewonnenen Erkenntnisse sind grundsätzlicher Art. Da sich reaktive Brandschutzsysteme hinsichtlich der erforderlichen Trockenschichtdicke, Höhe und Struktur der Aufschäumung, thermischen Schutzwirkung sowie Rissbildung und Rissheilungsvermögen unterscheiden ist jedoch eine produktbezogene Prüfung in Form von Brandversuchen unverzichtbar.

In der Zulassung des verwendeten reaktiven Brandschutzsystems [02], [17] sind Angaben zur erforderlichen Trockenschichtdicke nur für offene und geschlossene Profile enthalten. Entsprechende Brandversuche an Stahlträgern und -stützen wurden dafür durchgeführt. Eine Übertragung der ermittelten Trockenschichtdicken auf die Vollprofile der zu testenden Stahlzugglieder ist aufgrund der unterschiedlichen Profil- und Beanspruchungsart nicht möglich. Um geeignete Trockenschichtdicken für die zu prüfenden Profile der Stahlzugglieder zu ermitteln, werden zunächst orientierende Brandversuche im Einkubikmeterofen durchgeführt. Eine ausführliche Beschreibung dieser Untersuchungen erfolgt in Kapitel 3.4.

Für die Wirksamkeit des reaktiven Brandschutzsystems auf mechanisch belasteten Zuggliedern wird neben der Höhe der Zugbeanspruchung auch ein Einfluss des Zeitpunktes der Belastung vermutet. Generell wird bei reaktiven Brandschutzsystemen zwischen einer Werks- und einer Baustellenbeschichtung unterschieden. Bei einer Werksbeschichtung erfolgt die Belastung des Stahlbauteils erst nachdem das Brandschutzsystem appliziert und ausgehärtet ist. Beim Aufbringen der Beanspruchung auf ein Stahlbauteil mit Werksbeschichtung wird die Brandschutzbeschichtung von Anfang an mit belastet. Bei zugbeanspruchten Bauteilen könnten dadurch Mikrorisse in der Brandschutzbeschichtung entstehen, welche sich negativ auf das Aufschäumverhalten des Brandschutzsystems auswirken, z.B. durch eine erhöhte Rissbildung. Für die Baustellenbeschichtung wird das Brandschutzsystem auf das bereits voll oder teilweise mechanisch belastete Bauteil aufgebracht. Eine mechanische Beanspruchung ist nur für das Stahlbauteil vorhanden. Das ausgehärtete Brandschutzsystem bleibt nahezu spannungslos. Auf das Problem des Zeitpunktes der Applikation des reaktiven Brandschutzsystems wird bereits in [38] hingewiesen. Durch die mechanische Beanspruchung der Brandschutzbeschichtung und die daraus folgende höhere Beanspruchung sowie aus weiteren praktischen Gesichtspunkten zur Versuchsdurchführung wird für die in der BAM untersuchten Stahlzugglieder eine Werksbeschichtung gewählt.

\subsection{Voruntersuchungen}

Im Rahmen von Voruntersuchungen werden im Einkubikmeterofen und im Zugstabprüfofen orientierende Brandversuche an unbelasteten Kreisvollprofilen mit und ohne reaktiver Brandschutzbeschichtung durchgeführt. Auf Grundlage dieser Brandversuche werden geeignete Trockenschichtdicken des reaktiven Brandschutzsystems sowie die Art, Anzahl 
und Position der Thermoelemente für die Brandversuche im Realmaßstab ausgewählt. Um eine Querschnittsschwächung des Zuggliedes durch das Anbringen von Thermoelementen mittels Bohrungen zu vermeiden, wird bei der Herstellung der Versuchskörper eine neue Methode der Applikation von Thermoelementen erprobt. Ferner werden erste Erfahrungen zum Auftragen der reaktiven Brandschutzbeschichtung auf Profilen mit stark gekrümmter Oberfläche und schlanken Querschnitt gesammelt. Durch Vergleichsmessungen zwischen herkömmlichen Schichtdickenmessgeräten und einer mikroskopischen Untersuchung wird der Einfluss der gekrümmten Oberfläche des Stahlbauteils auf die Messgenauigkeit zur Bestimmung der Trockenschichtdicke untersucht.

Im Rahmen der Vorversuche werden insgesamt neun unbelastete Versuchskörper mit Kreisvollprofil untersucht. Mit Ausnahme der Versuchskörper V5 und V6, welche der Überprüfung und Kalibrierung der Schichtdickenmessung des reaktiven Brandschutzsystems dienen, werden mit allen anderen Versuchskörpern Brandversuche durchgeführt. Eine Übersicht zur Bezeichnung und Verwendung der einzelnen Versuchskörper ist in Tabelle 3.1 angegeben.

Tabelle 3.1: Art und Aufbau der Versuchskörper sowie deren Verwendungszeck

\begin{tabular}{|c|c|c|c|c|c|}
\hline $\begin{array}{l}\text { Versuchs- } \\
\text { körper }\end{array}$ & Profil & $\begin{array}{l}\text { Länge } \\
\text { [mm] }\end{array}$ & $\begin{array}{l}\text { Dicke RBS } \\
\text { [mm] }\end{array}$ & $\begin{array}{l}\text { Anzahl der Probekör- } \\
\text { perthermoelemente }\end{array}$ & Ziel der Untersuchung \\
\hline V1 & \multirow{7}{*}{ 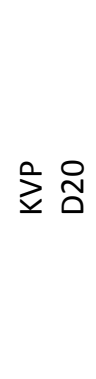 } & \multirow{5}{*}{700} & 1,0 & \multirow{5}{*}{$\begin{array}{c}\text { je } 3 \text { Stück im } \\
\text { Abstand von } 150 \mathrm{~mm}\end{array}$} & \multirow{5}{*}{$\begin{array}{l}\text { - Feuerwiderstand nach ETK } \\
\text { - Entwicklung der Stahltemperatur } \\
\text { und Haftungsvermögen des RBS }\end{array}$} \\
\hline V2 & & & 1,0 & & \\
\hline V3 & & & 2,0 & & \\
\hline V4 & & & 2,0 & & \\
\hline V7 & & & unbeschichtet & & \\
\hline V5 & & 50 & 1,0 & \multirow{2}{*}{ keine } & \multirow{2}{*}{$\begin{array}{l}\text { - Überprüfung und Kalibrierung der } \\
\text { Schichtdickenmessung des RBS }\end{array}$} \\
\hline V6 & & 30 & 2,0 & & \\
\hline U91 & \multirow{2}{*}{ 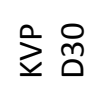 } & \multirow{2}{*}{800} & 2,5 & \multirow{2}{*}{$\begin{array}{c}\text { je } 3 \text { Stück im } \\
\text { Abstand von } 150 \mathrm{~mm}\end{array}$} & \multirow{2}{*}{$\begin{array}{l}\text { - Einfluss der Thermoelementart, } \\
\text {-anzahl und -applikation auf das RBS }\end{array}$} \\
\hline U92 & & & 2,5 & & \\
\hline
\end{tabular}

Die Versuchskörper U91 und U92 werden nicht im Einkubikmeterofen, sondern im Zugstabprüfofen getestet.

\subsubsection{Applikation der Thermoelemente}

Um die thermische Schutzwirkung des reaktiven Brandschutzsystems und die Feuerwiderstandsfähigkeit der Versuchskörper bewerten zu können, ist eine Temperaturmessung des Stahlbauteils während des Brandversuches unerlässlich. Im Allgemeinen erfolgt das Anbringen der Thermoelemente vor der Applikation der Brandschutzbeschichtung. Die Messstelle ist so auszubilden, dass der Einfluss auf die Aufschäumung und die thermische Schutzwirkung der Brandschutzbeschichtung so gering wie möglich ist. Zur Messung der Bauteiltemperatur werden üblicherweise Mantelthermoelemente verwendet. Diese werden mittels kleiner Bohrungen, welche nur wenige Millimeter breit und tief sind, am 
Versuchskörper befestigt. Da für Träger und Stützen relativ große Profile eingesetzt werden, kann die dadurch im Bereich der Temperaturmessstellen entstehende Querschnittsschwächung vernachlässigt werden. Bei den für Zugglieder verwendeten filigranen Profilen kann diese Art der Applikation von Thermoelementen zu einer Störstelle führen, die den Zeitpunkt und den Ort des Probenbruchs beeinflusst. Im Rahmen der Voruntersuchungen wird ein neues Verfahren zum Anbringen der Thermoelemente erprobt. Für die Thermoelemente werden herkömmliche, mit Keramikfasern ummantelte Thermoleitungen vom Typ K mit einem Drahtdurchmesser von $0,5 \mathrm{~mm}$, welche eine Temperaturbeständigkeit bis $1200{ }^{\circ} \mathrm{C}$ besitzen, verwendet. Bei der Auswahl der Thermoelemente ist darauf zu achten, dass das Kabel relativ kompakt ist und einen möglichst kleinen Gesamtdurchmesser aufweist. Störende Einflüsse der Kabel auf das Aufschäumen der reaktiven Brandschutzbeschichtung sollen dadurch minimiert werden. Die verwendeten Thermoelemente besitzen einen Außendurchmesser von ca. 2,2 bis 2,8 mm und werden mit Hilfe eines Punktschweißgerätes auf der Oberfläche des Stahlbauteils angeschweißt. Ein vor dem Anschweißen am Kabelende aufgebrachter Schrumpfschlauch verhindert ein Entflechten der Keramikfasern. Nach dem Anschweißen des Thermoelementes wird über eine Länge von etwa $3 \mathrm{~cm}$ ein dünner Draht in Serpentinen über die Thermoleitung gezogen und punktuell am Stahlbauteil verschweißt. Der eng anliegende Draht dient als Zugentlastung und zur Lagesicherung des Thermoelements. Um das spätere Aufschäumen des reaktiven Brandschutzsystems nicht zu behindern, wird das Thermoelementkabel nach der Zugentlastung senkrecht vom Versuchskörper weggeführt. Eine entsprechende Detailansicht der Messstelle vor und nach der Applikation des reaktiven Brandschutzsystems ist in Abb. 3.02 dargestellt. An die Versuchskörper V1 bis V4 sowie V7 werden jeweils drei Thermoelemente angeschweißt. Die Messstellen befinden sich in einer Reihe entlang der Stablängsachse. Um lokale Effekte im reaktiven Brandschutzsystem, wie z.B. Temperaturunterschiede infolge von Rissen oder unterschiedlichen Aufschäumhöhen, zu erfassen, wird der Abstand der Thermoelemente in Stablängsrichtung auf jeweils 150 mm festgelegt. Der Ausfall einer Messstelle kann bei der Auswertung des Brandversuches aufgrund der relativ kurzen Abstände der Messquerschnitte kompensiert werden. Aufgrund der guten Wärmeleitfähigkeit von Stahl und der filigranen Bauteilform von Zuggliedern ist davon auszugehen, dass sich im Querschnitt eine einheitliche Temperatur einstellt. Die Anordnung einer Messstelle pro Messquerschnitt ist daher ausreichend. Um ein repräsentatives Ergebnis der Temperaturmessung zu erhalten, ist beim Beschichten eine Konzentration des reaktiven Brandschutzsystems an der Messstelle zu vermeiden. Die Brandschutzbeschichtung ist vor dem Brandversuch von den Thermoelementen zu entfernen.

Zum Vergleich der neu entwickelten Applikationsmethode für Thermoelemente mit dem herkömmlichen Verfahren mittels Mantelthermoelementen werden im Zugstabprüfofen zwei Brandprüfungen durchgeführt. Die Versuchskörper U91 und U92 werden jeweils mit drei Messquerschnitten im Abstand von $150 \mathrm{~mm}$ ausgestattet (siehe Abb. 3.03). An zwei Messquerschnitten werden jeweils vier $3 \mathrm{~mm}$ tiefe Löcher im Winkel von jeweils $90^{\circ}$ in 
den Stab gebohrt. Darin werden ein 1,5 mm dickes Mantelthermoelement sowie drei etwa $15 \mathrm{~cm}$ lange Mantelthermoelementattrappen befestigt. Dies simuliert einen Messquerschnitt mit vier Mantelthermoelementen. Der dritte Messquerschnitt wird mit einem keramikfaserummantelten Thermoelement nach dem neu entwickelten Verfahren ausgestattet.
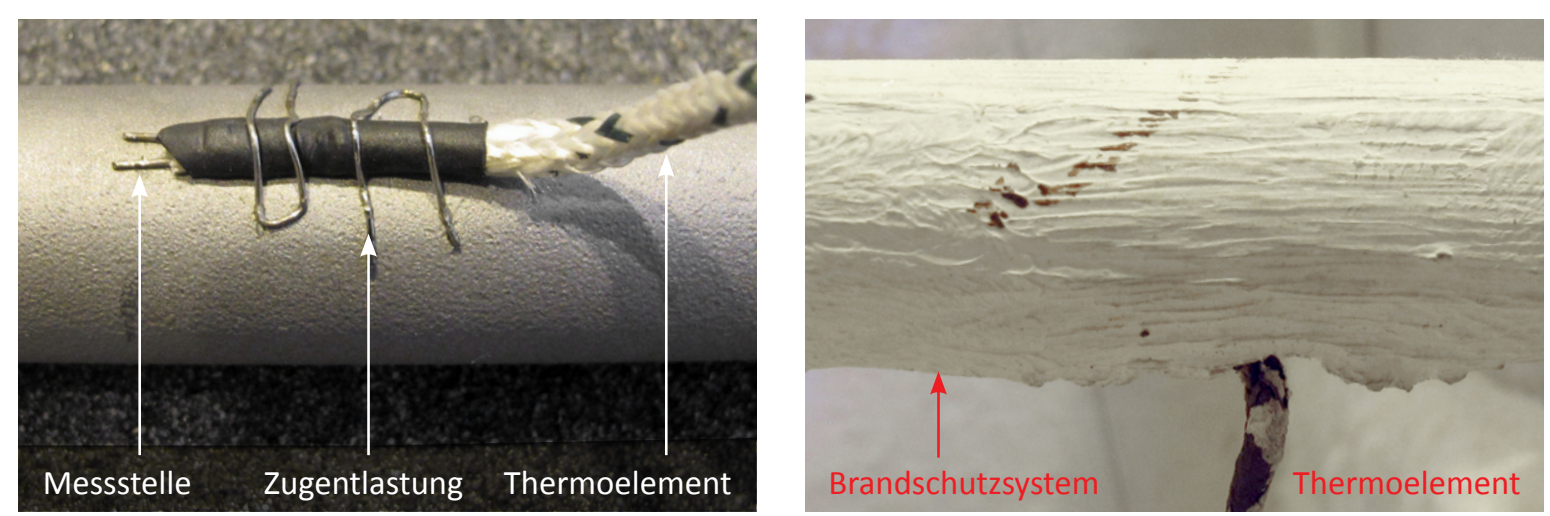

Abb. 3.02: Detailansicht der Messstelle mit angeschweißtem Thermoelement nach dem neu entwickelten Applikationsverfahren (links: vor der Applikation des RBS, rechts: kurz vor der Brandprüfung)

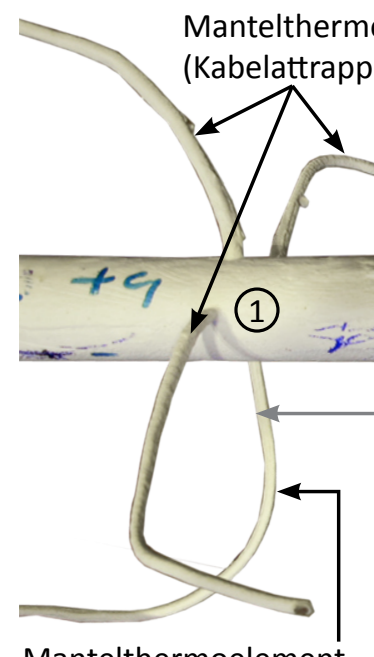

Mantelthermoelement
Kreisvollprofil KVP D30 mit drei Messquerschnitten (Nr. 1, 2 und 3)

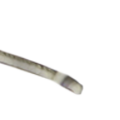

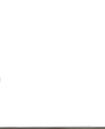
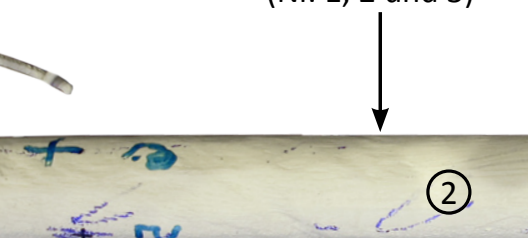

$150 \mathrm{~mm}$

Thermoelement mit Keramikfaserummantelung (RBS noch nicht entfernt)
Mantelthermoelement

$$
\text { (Kabelattrappe) }
$$

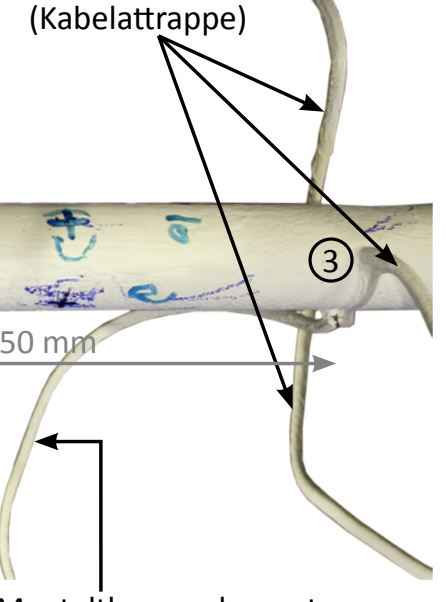

Mantelthermoelement

Abb. 3.03: Versuchskörpers U91 nach der Applikation der Thermoelemente und des RBS

\subsubsection{Applikation des reaktiven Brandschutzsystems}

Auf die sandgestrahlten und mit Thermoelementen versehenen Versuchskörper wird mittels Pinsel eine ca. $50 \mu \mathrm{m}$ dicke Grundierung aufgebracht. Die lösemittelhaltige rot-braune Grundierung gewährleistet den Korrosionsschutz für das Stahlbauteil und dient als Haftvermittler zwischen Stahl und dem reaktiven Brandschutzsystem. Die Beschichtung der Ver- 
suchskörper mit dem reaktiven Brandschutzsystem erfolgt etwa 14 Tage nach der Fertigstellung der Grundierung. Die Brandschutzbeschichtung ist eine weiße Emulsion. Selbige lässt sich gut auf die grundierten Stahlbauteile auftragen. Die Applikation des reaktiven Brandschutzsystems erfolgt in mehreren Arbeitsgängen mit jeweils etwa 0,5 mm Schichtdicke pro Arbeitsgang. Zwischen den einzelnen Arbeitsgängen werden die Versuchskörper etwa einen Tag bei Raumtemperatur getrocknet, um ein Abbinden der aufgebrachten Schicht zu gewährleisten. Wegen der einfachen und schnellen Handhabung sowie der Vermeidung von Materialverlusten bei der Applikation wird in den ersten Arbeitsgängen die reaktive Brandschutzbeschichtung mittels Streichverfahren aufgetragen. Da dadurch jedoch nur eine vergleichsweise raue Oberfläche erzielt werden kann, werden die späteren Arbeitsgänge im Airless-Spritzverfahren durchgeführt. Die durch das Spritzverfahren erzeugte Oberfläche ist wesentlich glatter als beim Streichverfahren. Beim Spritzverfahren sind der höhere Materialverbrauch sowie eventuell erforderliche Schutzmaßnahmen nachteilig. Nach dem Aufbringen der letzten Schicht werden die Versuchskörper über mehrere Wochen bei Raumtemperatur zum Durchtrocknen gelagert. Die Applikation des reaktiven Brandschutzsystems ist in Abb. 3.04 dargestellt. Die erzielte Trockenschichtdicke der Brandschutzbeschichtung ist in Tabelle 3.1 angegeben.
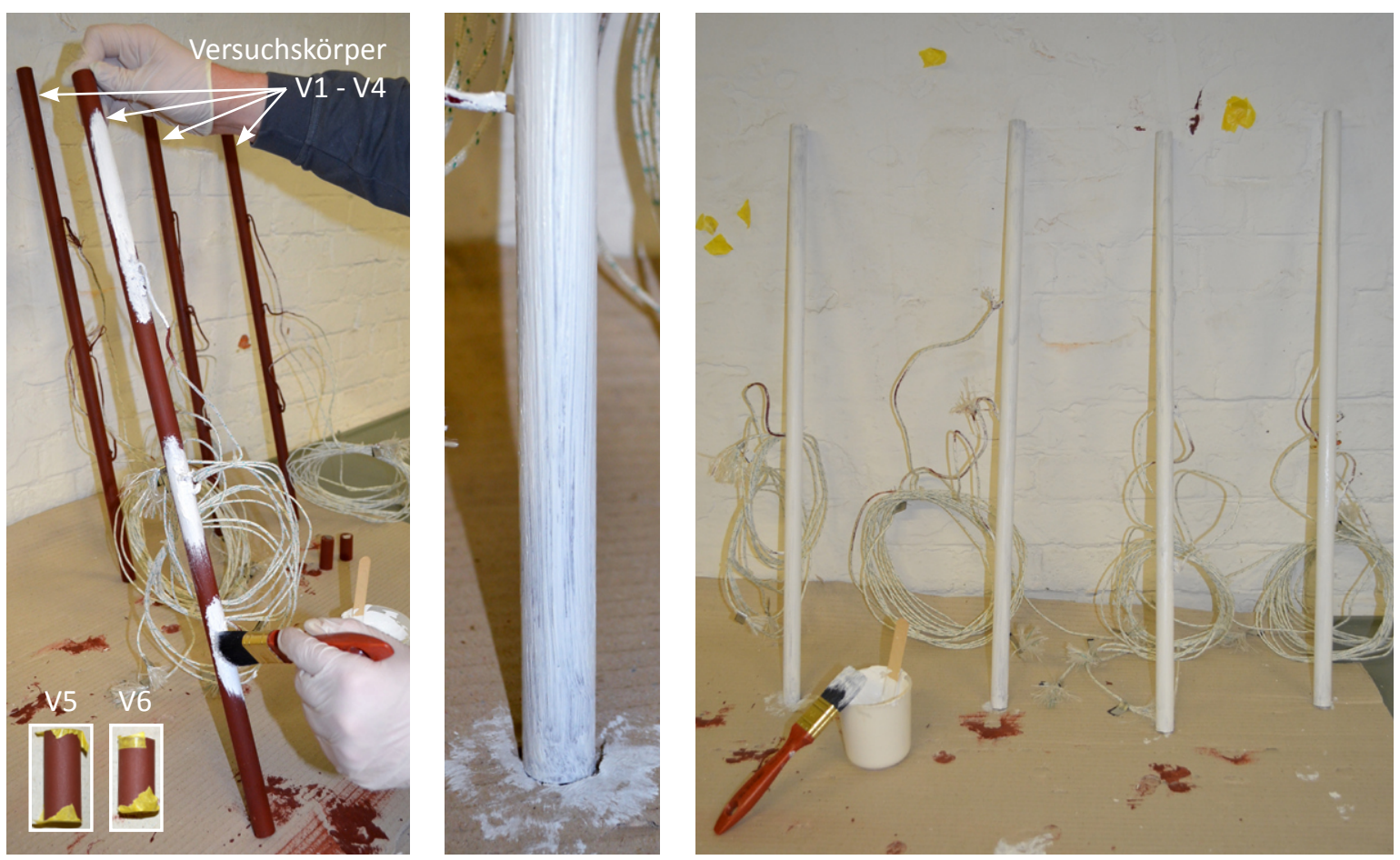

Abb. 3.04: Applikation des reaktiven Brandschutzsystems auf die grundierten Versuchskörper V1 - V4 


\subsubsection{Messung der Schichtdicke des reaktiven Brandschutzsystems}

Für Stahlbauteile mit gekrümmter Oberfläche liegen bei der BAM keine Erfahrungen über die Genauigkeit der Schichtdickenmessung des reaktiven Brandschutzsystems vor. Um den geometrischen Einfluss beurteilen zu können, werden Vergleiche zwischen herkömmlichen Messgeräten und einer mikroskopischen Untersuchung vorgenommen. Die Untersuchungen erfolgen mit zwei Messgeräten der Firma Fischer (DELTASCOPE ${ }^{\circledR}$ MP30E und DUALSCOPE ${ }^{\circledR}$ FMP 100) sowie verschiedener Messsonden (FGB2, F2OH und FKB10). An den Versuchskörpern V5 und V6 werden zusätzlich Vergleichsmessungen mittels Mikroskop

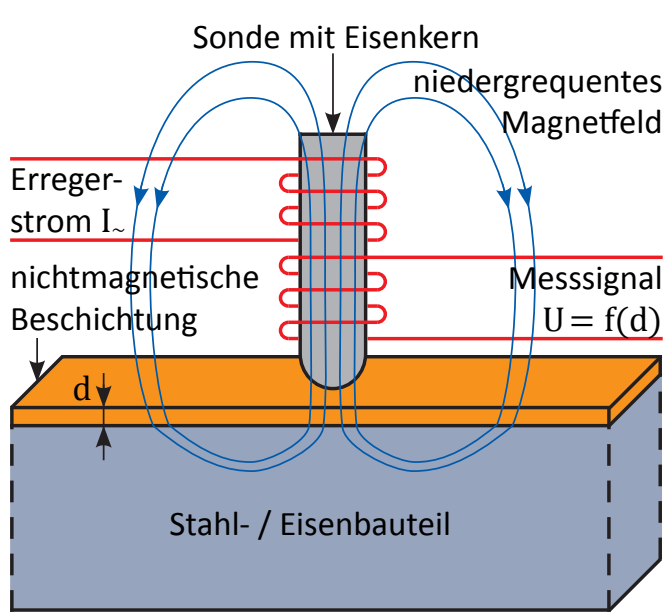

Abb. 3.05: Funktionsprinzip des magnetinduktiven Verfahrens durchgeführt.

Die Messgeräte zur Bestimmung der Schichtdicke basieren in der Regel auf einem magnetinduktiven Verfahren oder der Wirbelstrommethode. Das magnetinduktive Verfahren wird beim Messgerät DELTASCOPE ${ }^{\circledR}$ MP30E verwendet. Beim DUALSCOPE ${ }^{\circledR}$ FMP 100 sind Messungen mit Hilfe des magnetinduktiven Verfahrens und dem Wirbelstromverfahren möglich. Für die Bestimmung der Trockenschichtdicke reaktiver Brandschutzsysteme auf Stahlbauteilen kommt in der Regel das magnetinduktive Verfahren zum Einsatz. Die physikalische Grundlage bzw. die Funktionsweise dieser Messmethode ist in Abb. 3.05 dargestellt. Durch einen Erregerstrom wird ein niederfrequentes Magnetfeld erzeugt. Die Stärke des Feldes hängt vom Abstand zwischen Messsonde und Substrat (Stahl) ab. Durch eine Messspule wird die Stärke des Magnetfeldes erfasst und in ein Messsignal umgewandelt. Mit Hilfe einer entsprechenden Sondenkennlinie wird aus dem Messsignal die Schichtdicke berechnet. Das Hauptanwendungsgebiet dieser Methode ist die Schichtdickenbestimmung von Farb-, Emaille-, Lack- oder Kunststoff-Schichten auf Stahl oder Eisen.

Die Grundierungsdicke wird nach einer Trocknungszeit von 14 Tagen gemessen. An den Versuchskörpern V1 bis V4 werden je vier Messreihen im Abstand von ca. $150 \mathrm{~mm}$ mit jeweils vier Messpunkten in einem Winkel von $90^{\circ}$ um den Stabquerschnitt angeordnet. Um exakte Messergebnisse zu erzielen, werden die Messsonden möglichst senkrecht auf die Oberfläche des Versuchskörpers aufgesetzt. Für die Versuchskörper V5 und V6 wird die Grundierungsdicke jeweils in halber Bauteilhöhe anhand von acht gleichmäßig in Umfangsrichtung verteilten Messstellen bestimmt. Im Mittel beträgt die Grundierung an den Versuchskörpern etwa 50 bis $70 \mu \mathrm{m}$. Dies entspricht den Vorgaben des Herstellers der reaktiven Brandschutzbeschichtung. Die Abweichungen zwischen den verwendeten Messgeräten betragen 
etwa $10 \mu \mathrm{m}$ und sind für den praktischen Gebrauch vernachlässigbar. Die Messtoleranzen sind unter anderem auf die händisch erfolgten Messungen, das Messgerät selbst und das angewandte Verfahren zurückzuführen. Laut den Angaben des Herstellers des Messgerätes können sich bei relativ kleinen Stabdurchmessern von ca. 20 mm erhöhte Messabweichungen von mehr als $10 \%$ ergeben.

Nach dem Beschichten der Versuchskörper und einer Trocknungszeit der reaktiven Brandschutzbeschichtung von etwa vier Wochen erfolgt die Messung der Gesamtschichtdicke des Beschichtungssystems. Die Messpunkte werden analog zur Messung der Grundierungsdicke gewählt. Aufgrund des größeren Mess-

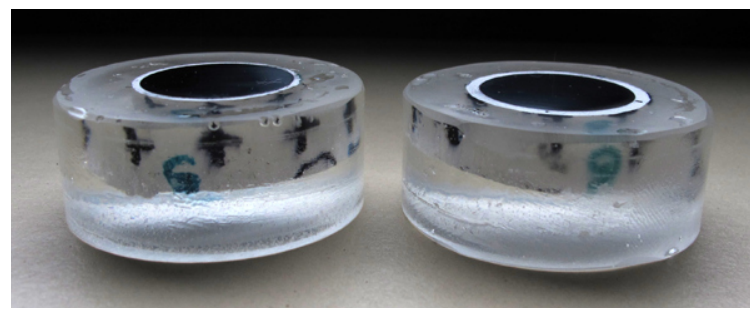

Abb. 3.06: Detail der Versuchskörper V5 und V6 für die mikroskopische Untersuchung bereiches wird bei beiden Messgeräten in erster Linie die Zweipunktmesssonde FKB10 verwendet. Für die Versuchskörper V5 und V6 erfolgt zusätzlich eine optische Untersuchung mittels Mikroskop (siehe Abb. 3.06). Die beiden Proben werden dafür in drei Teile zerschnitten. Aus dem mittleren Teilstück wird anschließend ein Querschliff hergestellt. Die Schichtdicke des reaktiven Brandschutzsystems, auch als Trockenschichtdicke (DFT - dry film thickness) bezeichnet, ergibt sich aus der nach dem Trocknen der Beschichtung gemessenen Gesamtschichtdicke abzüglich der Dicke der Grundierung. Die Abweichungen der gemessenen Mittelwerte sind zwischen den verwendeten Messgeräten relativ klein. Für die Versuchskörper mit einer Trockenschichtdicke des reaktiven Brandschutzsystems von ca. $1 \mathrm{~mm}$ beträgt die größte Schichtdickendifferenz ca. 0,14 mm. Bei den Proben mit einer Trockenschichtdicke von etwa $2 \mathrm{~mm}$ vergrößert sich diese Abweichung auf 0,28 mm. Die Ergebnisse der an den Versuchskörpern V5 und V6 durchgeführten mikroskopischen Untersuchung sind nahezu identisch mit den gemessenen Mittelwerten der Schichtdickenmessgeräte. Bei den untersuchten Kreisvollprofilen ist nur ein geringer Einfluss der gekrümmten Oberfläche auf die Bestimmung der Schichtdicke der reaktiven Brandschutzbeschichtung zu verzeichnen. Auf kreisförmigen Profilen mit kleinem Durchmesser können die üblicherweise zur Schichtdickenmessung eingesetzten Messgeräte uneingeschränkt angewendet werden.

Durch die mikroskopische Untersuchung lassen sich Fehlstellen, wie z.B. Luftblasen und kleinere Unregelmäßigkeiten der Beschichtungsdicke über den Querschnitt feststellen (siehe Abb. 3.07). Die Trockenschichtdicken des reaktiven Brandschutzsystems unterliegen auch bei sorgfältiger Herstellung stets Schwankungen, wie selbige auch bei offenen und geschlossenen Bauteilen auftreten. In den Brandversuchen hat sich jedoch gezeigt, dass kleinere Schwankungen der Trockenschichtdicke einen vernachlässigbaren Einfluss auf die Höhe der aufgeschäumten Brandschutzbeschichtung ausüben. 

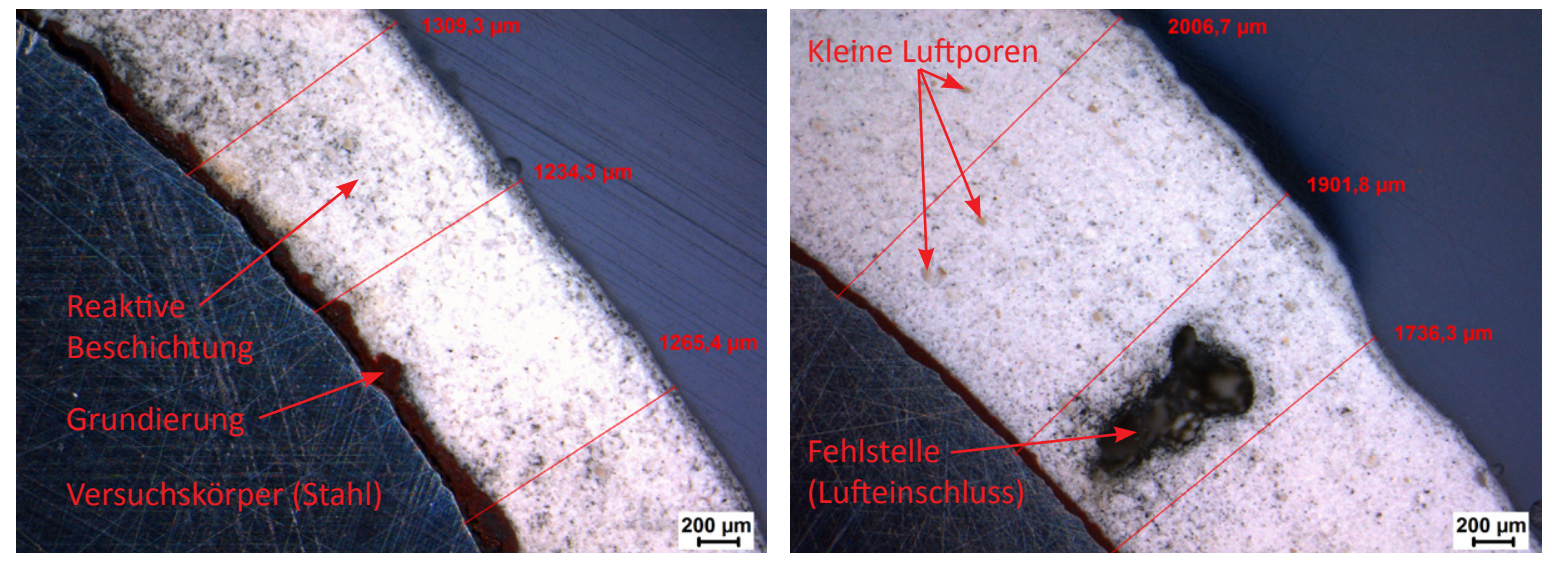

Abb. 3.07: Querschliff des Versuchskörpers V5 (links) und V6 (rechts)

\subsubsection{Orientierende Brandversuche im Einkubikmeterofen}

Die zur Orientierung geplanten Brandversuche (Kennzeichnung „V“) werden im Einkubikmeterofen (1 $\mathrm{m}^{3}$-Ofen) der BAM durchgeführt. Der hoch hitzebeständige Mehrzweckprüfofen ist nicht genormt. Der Brandraum hat bei Kantenlängen von $1 \mathrm{~m}$ ein Volumen von $1 \mathrm{~m}^{3}$. Der Ofen ist allseitig mit Feuerleichtsteinen bekleidet und außenseitig mit einem $5 \mathrm{~mm}$ dicken Stahlblech ummantelt. Die Beflammung des Brandraumes erfolgt durch zwei Ölbrenner (je $465 \mathrm{~kW}$ ), die auf einer Seite in eine Wand und im Ofenboden eingelassen sind. Für die Vorversuche wird lediglich der wandseitige Ölbrenner verwendet. Die Steuerung der Brandraumtemperatur erfolgt durch zwei Stabthermoelemente (BRT 1 und BRT 2), welche parallel zur Achse des horizontalen Ölbrenners ebenfalls in der gleichen Wand eingebaut sind. In den verbleibenden drei Wänden des Ofens befindet sich je eine ca. $500 \times 500 \mathrm{~mm}$ große Öffnung, die je nach Bedarf mit Feuerfeststeinen verschlossen werden kann. Zur Beobachtung der Brandversuche ist in der Öffnung seitlich vom Ölbrenner (Südseite) ein Feuerfeststein mit integriertem Beobachtungsfenster eingebaut. Die Brandversuche werden mittels Videokamera aufgezeichnet.

Am Einkubikmeterofen werden insgesamt zwei Brandprüfungen durchgeführt. Versuchskörper mit gleicher Schichtdicke des reaktiven Brandschutzsystems werden gemeinsam getestet. Bei der Prüfung der Proben V3 und V4 wird die unbeschichtete Probe V7 mit untersucht. Die Beflammung erfolgt jeweils tangential zu den Versuchskörpern entsprechend nach Einheits-Temperaturzeitkurve (ETK). Zusätzlich zu den beiden fest im Brandraum verbauten Stabthermoelementen werden vier weitere Mantelthermoelemente (MTE 1 bis MTE 4) zur Messung der Brandgastemperaturen unterhalb der Versuchskörper positioniert. Um während der Prüfung nahezu adiabatische Randbedingungen an den Stabenden des Versuchskörpers sicherzustellen, werden die Stabenden ca. $50 \mathrm{~mm}$ in einen Vermiculit-Block eingelassen. Dadurch ergibt sich für die Versuchskörper jeweils eine beflammte Stablänge von etwa $600 \mathrm{~mm}$. Die Probekörperthermoelemente (TE 1 bis TE 3) werden für die auf der 
Nordseite befindlichen Versuchskörper (V1 bzw. V3) nach unten senkrecht zum Stab weggeführt. Für die auf der Südseite angeordneten Versuchskörper (V2 bzw. V4) werden die Thermoelemente nach oben abgehängt, um den Einfluss der unterschiedlichen Position der Thermoelemente auf das Aufschäumverhalten der Brandschutzbeschichtung zu untersuchen (siehe Abb. 3.08). Eine möglichst geringe Beeinträchtigung des Aufschäumprozesses des reaktiven Brandschutzsystems wird dabei angestrebt. Eine detaillierte Zeichnung des Brandraumes mit die Position der Versuchskörper ist im Forschungsbericht [35] enthalten.

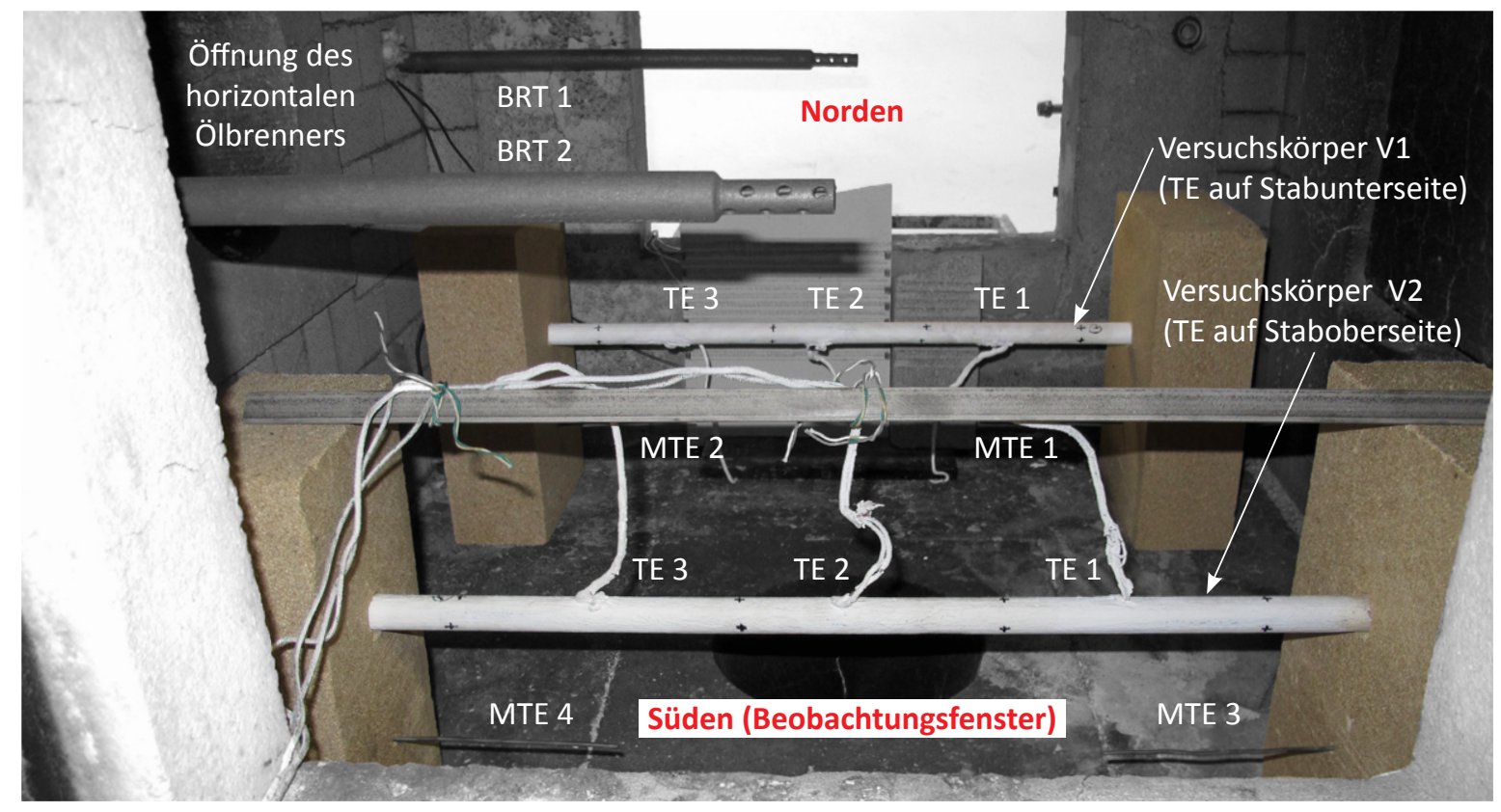

Abb. 3.08: Versuchsaufbau der Brandprüfung im Einkubikmeterofen (Versuchskörper V1 und V2; Blick aus Richtung Süden)

\section{Beobachtungen während der Brandversuche}

Im Unterschied zu den Versuchskörpern V3 und V4 ist an den Proben V1 und V2 die reaktive Brandschutzbeschichtung im Vorfeld des Brandversuches nicht von den Thermoelementkabeln entfernt worden. Aufgrund der fehlenden Wärmekapazität der Kabel setzte bereits kurz nach Versuchsstart das Aufschäumen der Beschichtung ein. Um eine Akkumulation des reaktiven Brandschutzsystems und somit eine Beeinflussung der Aufschäumung im Bereich der Messstellen zu verhindern, ist das Entfernen des an den Thermoelementkabeln anhaftenden Beschichtungsmaterials notwendig. In beiden Brandversuchen kommt es etwa drei Minuten nach Versuchsstart an den beschichteten Versuchskörpern zum Aufschäumen des reaktiven Brandschutzsystems. Zunächst bilden sich auf der Brandschutzbeschichtung Blasen. Die komplette Oberfläche färbt sich schwarz. Die Schwarzfärbung resultiert aus dem beim Aufschäumen des reaktiven Brandschutzsystems entstehenden Kohlenstoff. Nach einer Branddauer von etwa 10 Minuten erreicht die Aufschäumung des reaktiven Brandschutzsystems die augenscheinlich größte Volumenausdehnung. Durch die große 
Volumenzunahme während des Aufschäumprozesses und der damit verbundenen Vergrößerung des äußeren Umfangs der Brandschutzbeschichtung bilden sich Risse in Stablängsrichtung im reaktiven Brandschutzsystem (siehe Versuchskörper V2 und V4 in Abb. 3.09). Mit zunehmender Aufschäumhöhe der Brandschutzbeschichtung nimmt die Breite und Tiefe der Längsrisse zu. In einigen Fällen reicht die Risstiefe fast bis zur Stahloberfläche. Am Versuchskörper V3 ist zu beobachten, dass durch die Aufweitung eines Längsrisses auf der Staboberseite ein teilweises Abgleiten der reaktiven Brandschutzbeschichtung auftritt (siehe Abb. 3.09). Der durch die Thermoelemente angezeigte schnelle Temperaturanstieg an dem Versuchskörper zeigt, dass für diese Stabbereiche keine ausreichende thermische Schutzwirkung des Stahlbauteils mehr vorhanden ist.
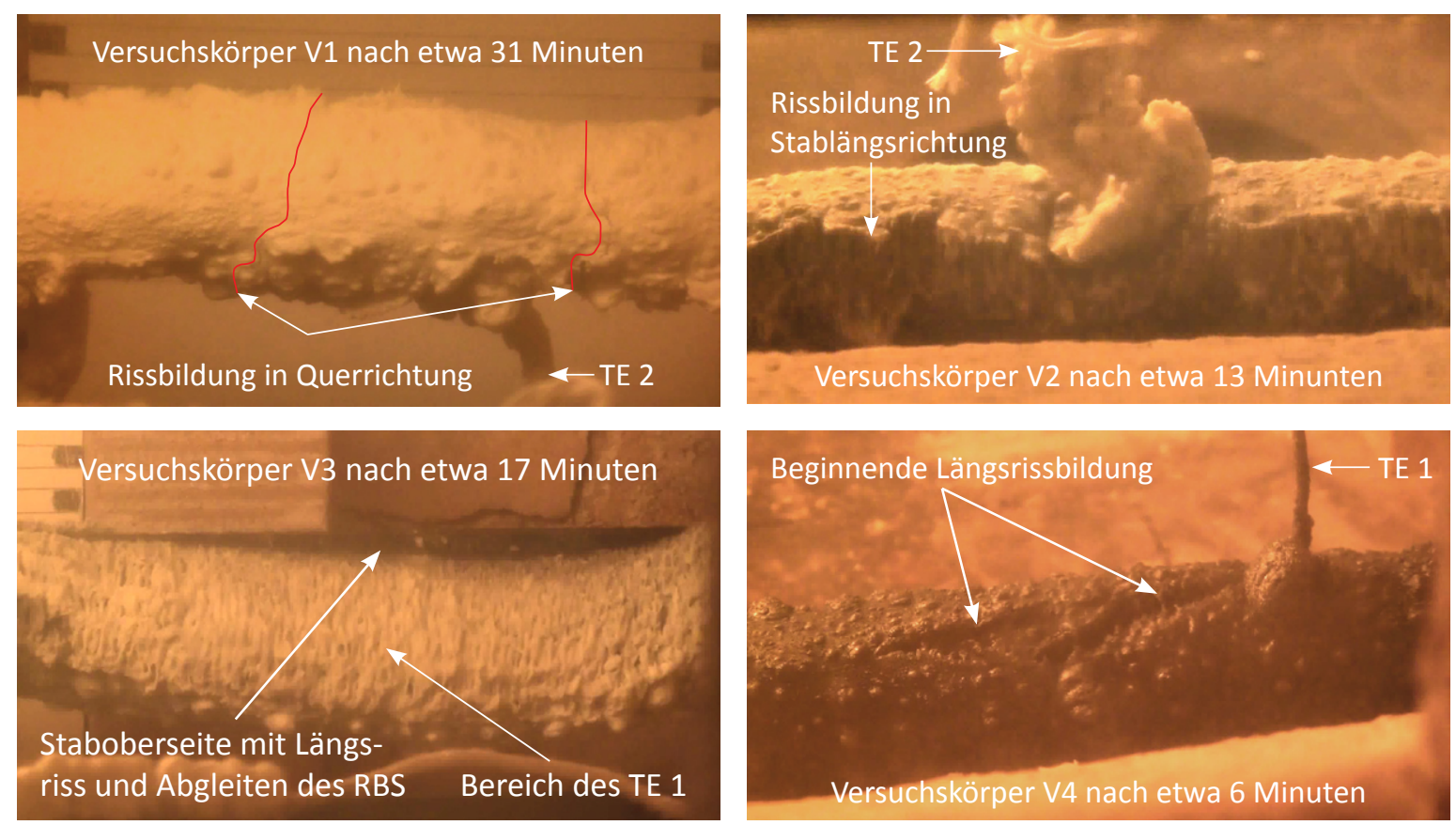

Abb. 3.09: Detailaufnahmen der Rissbildung in den Brandversuchen

Neben den Längsrissen sind auch Querrisse im reaktiven Brandschutzsystem vorhanden (siehe Versuchskörper V1 in Abb. 3.09). Diese sind in der Regel weniger als einen Millimeter breit und führen meist zu keiner Beeinträchtigung der thermischen Schutzwirkung. Die Bildung der Querrisse ist mit hoher Wahrscheinlichkeit auf die Temperaturunterschiede zwischen der brennerzu- und -abgewandten Seite des Versuchskörpers und der damit verbundenen unterschiedlichen Aufschäumung der Brandschutzbeschichtung zurückzuführen. Die während der Brandversuche festgestellten Beobachtungen zum Aufschäumungs- und Rissverhalten des reaktiven Brandschutzsystems spiegeln damit die theoretischen Vorbetrachtungen in Kapitel 2.6.2 wieder. Des Weiteren ist zu beobachten, dass sich durch teilweises Nachschäumen von noch nicht oder nur teilweise reagierten Teilen des reaktiven Brandschutzsystems entstandene Risse wieder schließen können. Dies ist jedoch auf die 
Aufschäumphase begrenzt. Etwa 15 Minuten nach dem Versuchsstart verascht die Oberfläche des Brandschutzsystems und färbt sich langsam weiß. Nach weiteren ca. 5 Minuten ist der komplette Versuchskörper weiß und hat eine krustenartige, feste Oberfläche. Damit ist der Aufschäumprozess abgeschlossen. Das am Versuchskörper V3 beobachtete Abgleiten des reaktiven Brandschutzsystems kommt zum Stillstand. Im weiteren Verlauf der Brandversuche ist augenscheinlich keine wesentliche Veränderung der aufgeschäumten Brandschutzbeschichtung mehr erkennbar. Aufgrund der hohen Stahltemperaturen von über $800^{\circ} \mathrm{C}$ werden die Brandversuche nach 60 Minuten beendet. Ein Abfallen der Brandschutzbeschichtung von den Versuchskörpern ist über die gesamte Branddauer nicht aufgetreten. Die gemessenen Brandgas- und Probekörpertemperaturen sind in Abb. 3.11 dokumentiert.

\section{Feststellungen nach dem Brandversuch}

An den Versuchskörpern V1 und V2 sind große Längsrisse auf der vom Ölbrenner abgewandten Seite vorhanden (siehe Abb. 3.10). Die aufgeschäumte reaktive Brandschutzbeschichtung beträgt im Bereich der Längsrisse nur wenige Millimeter. An lokalen Stellen reichen die Risse bis auf die Stahloberfläche. Neben Rissen in Stablängsrichtung zeigen sich im Brandschutzsystem vereinzelt auch Querrisse. Die weniger als einen Millimeter breiten Querrisse verlaufen in der Regel auf der dem Ölbrenner zugewandten Seite. Im Mittel beträgt die Höhe der aufgeschäumten Brandschutzbeschichtung an den Proben V1 und V2 etwa $17 \mathrm{~mm}$. Die Aufschäumung variiert zwischen $0 \mathrm{~mm}$ und $35 \mathrm{~mm}$. An den Versuchskörpern V3 und V4 sind in der Aufschäumung ebenfalls Risse in Längs- und Querrichtung zur Stabachse vorhanden. Am Versuchskörper V3 ist durch das Abgleiten der Brandschutzbeschichtung die halbe Stablänge nahezu ungeschützt (siehe Abb. 3.10). Die Höhe der Aufschäumung beträgt im Mittel etwa $19 \mathrm{~mm}$. Die Extremwerte betragen 0 und $49 \mathrm{~mm}$. Der unbeschichtete Vergleichsprobekörper V7 bleibt während des gesamten Brandversuches nahezu unverändert. Lediglich auf der Oberfläche bildet sich im Verlauf der Brandprüfung eine schwarze Oxidschicht. Eine ausführliche Dokumentation der Risse und Höhe der Aufschäumung des reaktiven Brandschutzsystems sind im Forschungsbericht [35] enthalten.
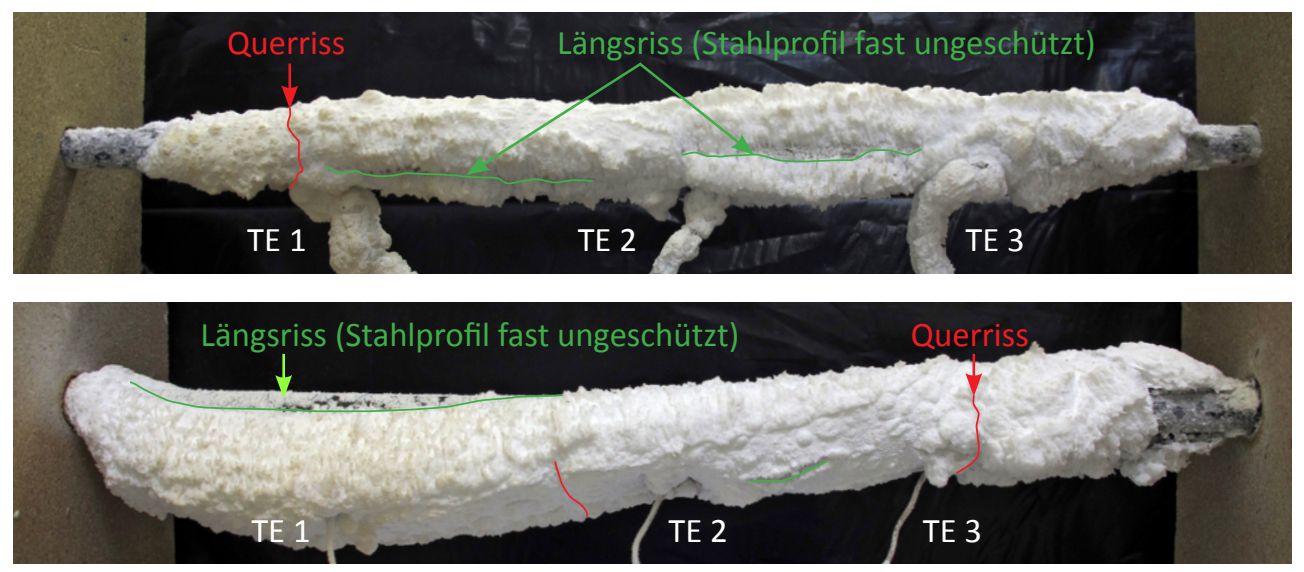

Abb. 3.10: Versuchskörper V1 (oben) und V3 (unten) nach dem Brandversuch (Blick aus Richtung Norden) 
Auswertung der Temperaturverläufe

Die Temperaturen der im Einkubikmeterofen vorhandenen Brandraumthermoelemente befinden sich innerhalb der in DIN EN 1363-1 [07] angegebenen Grenzen. An den unterhalb der Versuchskörper angeordneten Mantelthermoelementen zeigen sich aufgrund von Abschattungseffekten etwas geringere Brandgastemperaturen als an den fest eingebauten Stabthermoelementen des Einkubikmeterofens.

Da bei belasteten Stahlzuggliedern die aus der Zugbeanspruchung resultieren-

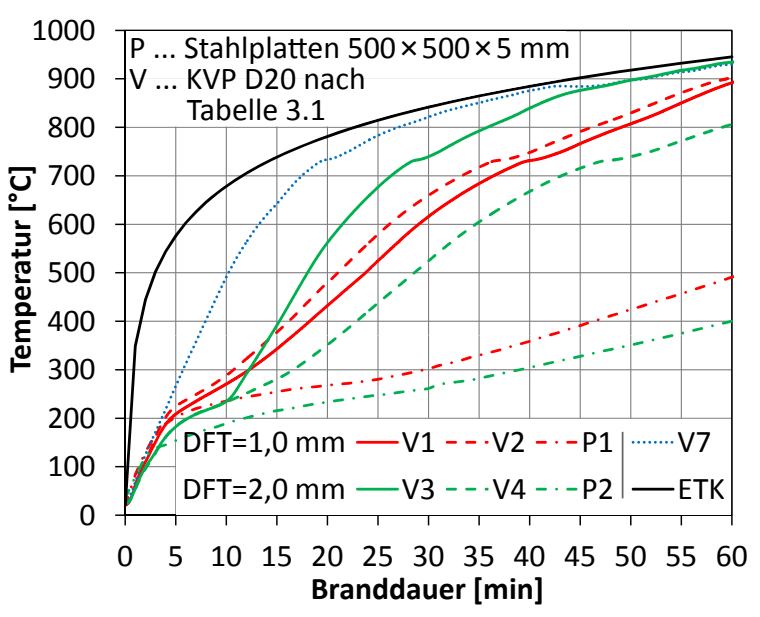

Abb. 3.11: Verlauf der gemessenen maximalen Stahltemperaturen den Spannungen über das gesamte Bauteil konstant sind und sich aus Stabbereichen mit geringerer Stahltemperatur keine Tragfähigkeitsreserven erschließen lassen, ist mit einem Probenbruch des Bauteils an der Stelle der maximalen Stahltemperatur zu rechnen. Bei der Auswertung der gemessenen Probekörpertemperaturen werden daher die maximal gemessenen Stahltemperaturen betrachtet (siehe Kapitel Definitionen). Für die im Einkubikmeterofen untersuchten Versuchskörper, welche ohne Zugbeanspruchung getestet wurden, sind die gemessenen Temperaturverläufe in Abb. 3.11 dargestellt. Mit Ausnahme des ungeschützten Stahlprofils weisen alle Versuchskörper im Bereich zwischen 5 und 15 Minuten Branddauer eine deutliche Reduzierung der Erwärmungsgeschwindigkeit auf. Dies lässt sich auf den Aufschäumungsvorgang des reaktiven Brandschutzsystems zurückführen, welcher eine Energiezufuhr in Form von Wärme benötigt. Nach Abschluss des Aufschäumprozesses steigt die Erwärmungsgeschwindigkeit der Versuchskörper wieder leicht an. Durch die ausgebildete thermische Schutzschicht des aufgeschäumten reaktiven Brandschutzsystems verläuft die Erwärmung des Stahls immer noch wesentlich langsamer als bei dem ungeschützten Stahlbauteil. Durch die Erhöhung der Trockenschichtdicke lässt sich im Allgemeinen die thermische Schutzwirkung der reaktiven Brandschutzbeschichtung weiter steigern.

Die an den Versuchskörpern V1 und V2 mit einer Trockenschichtdicke von 1,0 mm gemessenen maximalen Stahltemperaturen differieren um weniger als $50 \mathrm{~K}$. Die Temperaturdifferenz ist mit hoher Wahrscheinlichkeit auf die unterschiedliche Rissbildung im reaktiven Brandschutzsystem zurückzuführen. Strömungsbedingte Temperaturunterschiede der Brandgase im Einkubikmeterofen können ebenfalls nicht ausgeschlossen werden. Nach einer Branddauer von 30 Minuten beträgt die maximale Stahltemperatur $616^{\circ} \mathrm{C}$ (V1) bzw. $660^{\circ} \mathrm{C}$ (V2). Bei einer Trockenschichtdicke von 2,0 mm an den Versuchskörpern V3 und V4 verringert sich die maximale Stahltemperatur um etwa $100 \mathrm{~K}$. Durch das teilweise Abgleiten 
der aufgeschäumten Brandschutzbeschichtung kommt es am Versuchskörper V3 nach etwa 10 Minuten zu einer schnellen Erhöhung der Stahltemperaturen. Nach einer Branddauer von 30 Minuten beträgt die maximale Stahltemperatur $740{ }^{\circ} \mathrm{C}(\mathrm{V} 3)$ und $525^{\circ} \mathrm{C}$ (V4). Am ungeschützten Versuchskörper V7 ist eine Temperatur von $822^{\circ} \mathrm{C}$ vorhanden.

Als Ergänzung zu den im Einkubikmeterofen getesteten Kreisvollprofilen mit reaktiver Brandschutzbeschichtung sind in Abb. 3.11 die Temperaturverläufe von zwei Brandprüfungen an beschichteten Stahlplatten (P1 und P2) angegeben. Die Brandprüfungen wurden vom Hersteller des reaktiven Brandschutzsystems im Ofen nach DIN 4102-8 [04] durchgeführt. Die Trockenschichtdicke der Brandschutzbeschichtung beträgt 1,0 und 2,0 mm. Der Profilfaktor ( $A_{m} / V$-Verhältnis) der Stahlplatten und der Kreisvollprofile beträgt $200 \mathrm{~m}^{-1}$. Der Vergleich der maximalen Stahltemperaturen zeigt, dass sich die Stahlplatten nach etwa 5 Minuten wesentlich langsamer erwärmen, als die getesteten Kreisvollprofile. Ursache hierfür ist unter anderem die unterschiedliche Profilart der Versuchskörper, welche die Aufschäumung und die Rissbildung des reaktiven Brandschutzsystems beeinflusst. Die Brandschutzbeschichtung besitzt auf Profilen mit stark gekrümmter Oberfläche eine wesentlich schlechtere thermische Schutzwirkung als bei Profilen mit gerader Oberfläche. Ein weiterer Grund für die Temperaturunterschiede zwischen Kreisprofil und Platte liegt in der Beflammung der Bauteile. Die Kreisprofile werden allseitig beflammt. Im Unterschied dazu werden die Stahlplatten lediglich einseitig beflammt. Der Vergleich zeigt, dass eine Übertragung der thermischen Schutzwirkung reaktiver Brandschutzsysteme zwischen Stahlbauteilen unterschiedlicher Profilarten nicht möglich ist.

\section{Auswertung des Einflusses der Thermoelemente an den Versuchskörpern}

Das neu entwickelte Verfahren zum Anbringen der Thermoelemente auf der Staboberfläche der Versuchskörper hat sich in den Brandprüfungen bewährt. Durch die Zugentlastung besitzen die Messstellen eine hohe Robustheit, wodurch ein Abreißen der Thermoelemente verhindert wird. Darüber hinaus lässt sich das nach dem Beschichten der Versuchskörper an den Thermoelementkabeln anhaftende überschüssige Beschichtungsmaterial relativ problemlos entfernen. Die Brandversuche zeigen, dass im Bereich der Messstelle das Aufschäumen durch das Thermoelement nur unwesentlich beeinflusst wird. Ein Einfluss der Position der Thermoelemente auf das Aufschäumungs- und Rissverhalten konnte nicht eindeutig festgestellt werden. Um eine mögliche Beeinflussung der Messstellen auf die Längsrissbildung auf der Staboberseite und ein damit verbundenes Abgleiten der reaktiven Brandschutzbeschichtung zu vermeiden, werden in den Brandversuchen im Realmaßstab die Thermoelemente in der Regel auf der Stabunterseite angeordnet. Durch den gewählten Abstand der Messstellen von 150 mm können lokale Unterschiede in der thermischen Schutzwirkung des reaktiven Brandschutzsystems, welche sich z.B. durch Risse ausbilden, erfasst werden. Die Anordnung von einem Thermoelement pro Messquerschnitt ist bei den verwendeten filigranen Kreisvollquerschnitten ausreichend. Die neu entwickelte Applikationsmethode wird auch bei den Brandversuchen im Realmaßstab eingesetzt. 


\subsubsection{Orientierende Brandversuche im Zugstabprüfofen}

Im Rahmen der Voruntersuchungen werden neben den Brandprüfungen im Einkubikmeterofen zwei weitere Brandversuche im Zugstabprüfofen durchgeführt. Damit soll die neu entwickelte Applikationsmethode für Thermoelemente mit dem herkömmlichen Verfahren mittels Mantelthermoelementen verglichen werden. Wichtigstes Vergleichskriterium ist die Gestalt der Aufschäumung im Bereich der Thermoelemente und der Stabbereiche ohne Messstelle. Der Aufbau der Versuchskörper und die Applikation der Thermoelemente ist bereits in Kapitel 3.4.1 beschrieben. Trotz sorgfältiger Applikation lösen sich einige der Mantelthermoelemente bzw. der Mantelthermoelementattrappen beim Transport und während des Beschichtens vom Versuchskörper. Ursächlich sind die fehlende Zugentlastung und die höhere Steifigkeit der Kabel der Mantelthermoelemente. Zudem lassen sich im Gegensatz zu den mit Keramikfasern ummantelten Thermoelementen an den Mantelthermoelementen die an den Kabeln anhaftende überschüssige Brandschutzbeschichtung oft nicht entfernen, ohne dass Kabel abreißen.
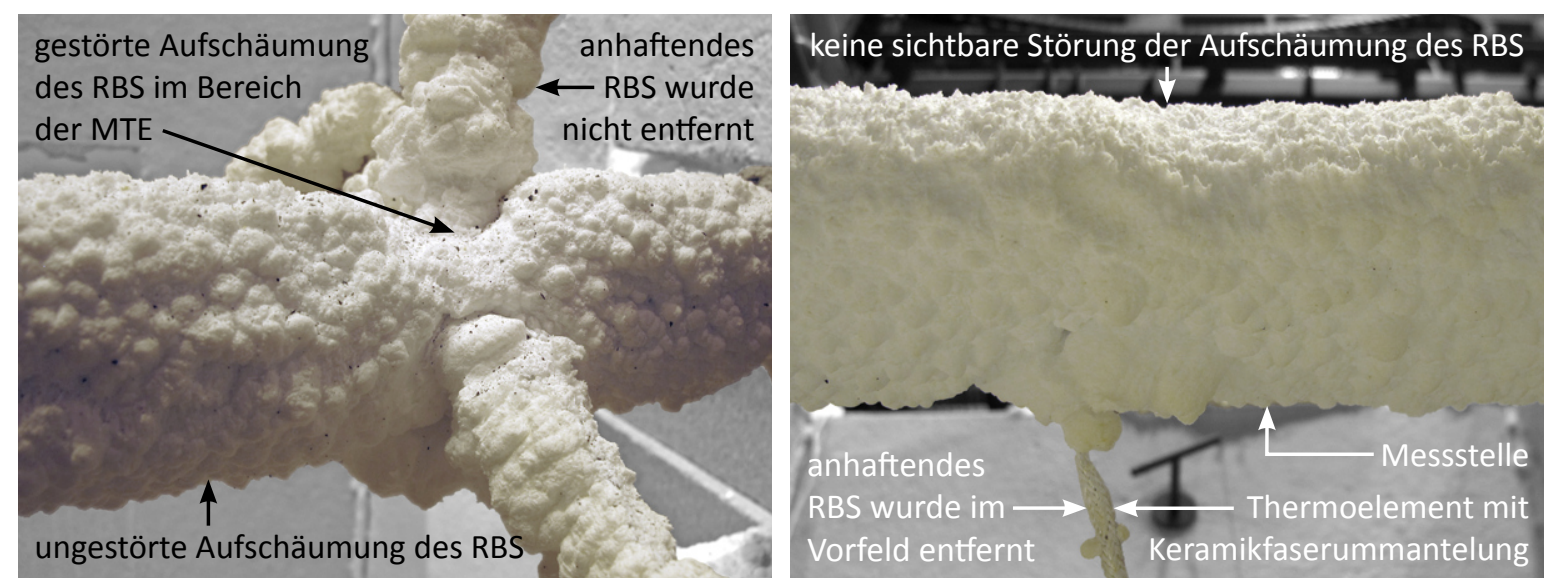

Abb. 3.12: Detail des Versuchskörpers U91 nach dem Brandversuch (links: Bereich des Messquerschnittes 1; rechts: Bereich des Messquerschnittes 2)

Die Brandversuche zeigen, dass durch das an den Kabeln der Mantelthermoelemente befindliche Beschichtungsmaterial das Aufschäumen des auf dem Zugglied aufgebrachten reaktiven Brandschutzsystems maßgeblich beeinflusst wird. Da sich in den mit Mantelthermoelementen ausgestatteten Messquerschnitten vier Messstellen inklusive der drei Kabelattrappen befinden, ist die Aufschäumung des reaktiven Brandschutzsystems im Bereich des Messquerschnittes gegenüber den Stabbereichen ohne Messstellen gestört (siehe Abb. 3.12). Inwieweit die in diesem Bereich mit Hilfe der Mantelthermoelemente gemessenen Stahltemperaturen als repräsentativ für das Zugglied angesehen werden können, ist daher fraglich. Für den Messquerschnitt, bei dem das mit Keramikfasern ummantelte Thermoelement eingesetzt und nach dem neu entwickelten Verfahren appliziert wird, ist gegenüber dem Stabbereich ohne Messstelle kaum eine Änderung der Aufschäumung fest- 
stellbar (siehe Abb. 3.12). Die Messstelle befindet sich zudem aufgrund der Zugentlastung ca. $3 \mathrm{~cm}$ von der Stelle entfernt, an der das Kabel vom Versuchskörper weggeführt wird. Darüber hinaus besitzt das neu entwickelte Verfahren zur Applikation von Thermoelementen den Vorteil, dass der Bauteilquerschnitt nicht beeinträchtigt wird. Dies ist insbesondere im Hinblick auf Bauteile mit mechanischer Zugbeanspruchung wichtig. Der Vergleich der beiden Applikationsmethoden zeigt, dass das neu entwickelte Verfahren für den Einsatz in Brandprüfungen geeignet ist und gegenüber dem herkömmlichen Verfahren mittels Mantelthermoelementen zahlreiche Vorteile aufweist.

\subsubsection{Zusammenfassung der Ergebnisse aus den Voruntersuchungen}

Die Voruntersuchungen an unbelasteten Zuggliedern zeigen, dass eine Applikation von reaktiven Brandschutzsystemen auf Stahlbauteilen mit Kreisvollprofil und kleinem Durchmesser möglich ist. Die Haftfähigkeit der Brandschutzbeschichtung mit Trockenschichtdicken von 1,0, 2,0 und 2,5 mm konnte an Kreisvollprofilen über eine Branddauer von 60 Minuten prinzipiell nachgewiesen werden. Bei der Probe (V3) trat jedoch ohne erkennbare Ursachen ein partielles Abgleiten der Beschichtung auf. Ein schneller Temperaturanstieg und höhere Stahltemperaturen waren die Folge.

Die Brandversuche haben gezeigt, dass die Erwärmungsgeschwindigkeit des Stahls durch die auf die Versuchskörper aufgebrachte Trockenschichtdicke des reaktiven Brandschutzsystems signifikant verringert werden kann. Im Allgemeinen gilt, je größer die Trockenschichtdicke der reaktiven Brandschutzbeschichtung, desto langsamer ist die Erwärmung des Stahls. Die Bildung von Rissen im aufgeschäumten Brandschutzsystem kann die thermische Schutzwirkung erheblich reduzieren und zu lokal höheren Stahltemperaturen führen. Risse können sowohl in Längs- als auch in Querrichtung auftreten. Die Bildung von Längsrissen resultiert mit hoher Wahrscheinlichkeit aus der Vergrößerung des Umfangs zwischen unaufgeschäumtem und aufgeschäumtem Zustand der Brandschutzbeschichtung. Im Gegensatz zu den Längsrissen sind Querrisse im reaktiven Brandschutzsystem weniger als einen Millimeter breit und führen meist zu keiner signifikanten Reduzierung der thermischen Schutzwirkung des Systems. Mit Hilfe von Brandprüfungen an mechanisch belasteten Zuggliedern ist zu prüfen, ob sich die Querrisse bei axialer Zugbeanspruchung des Versuchskörpers aufweiten und sich negativ auf die thermische Schutzwirkung und die Haftfähigkeit der Brandschutzbeschichtung auswirken. Bei einer ausreichenden Trockenschichtdicke des reaktiven Brandschutzsystems können Rissheilungseffekte aktiviert werden. Dabei können entstandene Risse in der Brandschutzbeschichtung durch das Nachschäumen von noch nicht vollständig reagierten Bereichen teilweise wieder geschlossen werden. Diese Fähigkeit beschränkt sich jedoch auf die Aufschäumphase des reaktiven Brandschutzsystems, d.h. auf die ersten 10 bis 20 Minuten des Brandversuches. 
Da nach einer Branddauer von 30 Minuten die Stahltemperaturen des Kreisvollprofils mit einem Durchmesser von $20 \mathrm{~mm}$ bei einer Trockenschichtdicke des reaktiven Brandschutzsystems von $1,0 \mathrm{~mm}$ bereits $600^{\circ} \mathrm{C}$ übersteigen, wird für die Brandversuche im Realmaßstab die minimale Beschichtungsdicke auf $1,5 \mathrm{~mm}$ festgelegt. Die Untersuchungen hinsichtlich der Genauigkeit der Schichtdickenmessung haben gezeigt, dass sich die Trockenschichtdicke des reaktiven Brandschutzsystems auch bei Profilen mit relativ starker Oberflächenkrümmung mit den herkömmlichen Messgeräten ausreichend genau bestimmen lässt.

Die im Rahmen der Voruntersuchungen neu entwickelte Methode zur Applikation von Thermoelementen hat sich in den Brandversuchen bewährt und bietet gegenüber dem herkömmlichen Verfahren mittels Mantelthermoelementen zahlreiche Vorteile. Wichtig ist eine Zugentlastung an der Messstelle vorzusehen und vor dem Brandversuch die reaktive Brandschutzbeschichtung von den Thermoelementkabeln zu entfernen. Ein störender Einfluss der Messstellen auf das Aufschäumverhalten des reaktiven Brandschutzsystems ist mit dem neuen Applikationsverfahren für Thermoelemente nicht festzustellen. In den Brandversuchen im Realmaßstab werden die Messstellen analog zu den im Einkubikmeterofen getesteten Versuchskörpern ausgebildet. Um eine mögliche Beeinflussung der Messstellen auf die Längsrissbildung auf der Staboberseite und ein damit erhöhtes Risiko des Abgleitens der reaktiven Brandschutzbeschichtung zu vermeiden, werden in den Brandversuchen im Realmaßstab die Thermoelemente meist auf der Stabunterseite angeordnet.

\subsection{Brandversuche an mechanisch belasteten und unbelasteten Versuchskörpern im Realmaßstab}

\subsubsection{Versuchsprogramm und Versuchskörper}

Bei der Gestaltung des Versuchsaufbaus und der Durchführung der Brandprüfungen wird sich an den Empfehlungen für Feuerwiderstandprüfungen nach DIN EN 1361-1 [07] orientiert. Mit Hilfe des Versuchsprogramms der Brandprüfungen an mechanisch belasteten und unbelasteten Stahlzuggliedern mit reaktiver Brandschutzbeschichtung soll gezielt die Wirkung der Einflussfaktoren, wie Profilgeometrie und Ausrichtung des Bauteils, der Trockenschichtdicke der Brandschutzbeschichtung sowie der Höhe der mechanischen Beanspruchung untersucht und bewertet werden. Aus Gründen der Vergleichbarkeit werden alle Brandversuche mit dem gleichen reaktiven Brandschutzsystem durchgeführt. Äußere Einflüsse auf die Feuerwiderstandsdauer der Zugglieder, wie z.B. die Größe des Brandraumes oder die Anzahl und Art der verwendeten Beflammungseinrichtung und dessen Brennmaterial werden im Rahmen der Brandversuche nicht untersucht.

Insgesamt werden Brandversuche an 42 mechanisch belasteten und an 25 unbelasteten Stahlzuggliedern mit reaktiver Brandschutzbeschichtung durchgeführt. Alle Zugglieder wei- 
sen einen kreisförmigen Querschnitt auf und werden mit dem gleichen reaktiven Brandschutzsystem beschichtet. Versuchskörper mit gleicher Trockenschichtdicke des reaktiven Brandschutzsystems sind in einer Versuchsreihe (VR) zusammengefasst. Das Versuchsprogramm für die Brandversuche im Realmaßstab ist in Tabelle 3.2 angegeben.

Tabelle 3.2: Versuchsprogramm für die Brandprüfungen im Realmaßstab

\begin{tabular}{|c|c|c|c|c|c|c|c|c|c|c|c|c|c|}
\hline \multirow{2}{*}{ 돌 } & \multirow{2}{*}{$\begin{array}{l}\text { Bezeichnung } \\
\text { der Versuchs- } \\
\text { reihe (VR) }\end{array}$} & \multicolumn{4}{|c|}{$\begin{array}{c}\text { Unbelastete } \\
\text { Versuchskörper (U) }\end{array}$} & \multicolumn{8}{|c|}{$\begin{array}{l}\text { Mechanisch belastete } \\
\text { Versuchskörper (B) }\end{array}$} \\
\hline & & \multicolumn{4}{|c|}{ Stablänge $=750 \mathrm{~mm}$} & \multicolumn{8}{|c|}{ Stablänge = $1400 \mathrm{~mm}$} \\
\hline \multirow{3}{*}{ 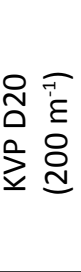 } & $\begin{array}{c}\text { VR a } \\
(\mathrm{DFT}=1,5 \mathrm{~mm})\end{array}$ & U30 & U31 & U32 & - & $\begin{array}{c}\mathrm{B} 6 \\
{[15 \%]}\end{array}$ & $\begin{array}{c}\text { B3 } \\
{[25 \%]}\end{array}$ & \begin{tabular}{|l}
$\mathrm{B} 11^{\#}$ \\
{$[35 \%]$}
\end{tabular} & $\begin{array}{c}\mathrm{B} 7 \\
{[40 \%]}\end{array}$ & $\begin{array}{c}\text { B12 } \\
{[45 \%]}\end{array}$ & $\begin{array}{c}\text { B8* } \\
{[45 \%]}\end{array}$ & $\begin{array}{c}B 2 * \\
{[45 \%]}\end{array}$ & $\begin{array}{c}\text { B4* } \\
{[45 \%]}\end{array}$ \\
\hline & $\begin{array}{c}\text { VR b } \\
(\mathrm{DFT}=2,5 \mathrm{~mm})\end{array}$ & U34 & U35 & U33 & U36 & $\begin{array}{c}\text { B1 } \\
{[15 \%]}\end{array}$ & $\begin{array}{c}\text { B5 } \\
{[25 \%]}\end{array}$ & $\begin{array}{c}\text { B9 } \\
{[35 \%]}\end{array}$ & $\begin{array}{l}\text { B13\# } \\
{[45 \%]}\end{array}$ & $\begin{array}{c}\mathrm{B} 10 \\
{[45 \%]}\end{array}$ & $\begin{array}{l}\text { B15\# } \\
{[45 \%]}\end{array}$ & $\begin{array}{c}\text { B14 } \\
{[55 \%]}\end{array}$ & $\begin{array}{r}\text { B16 } \\
{[65 \%]}\end{array}$ \\
\hline & $\begin{array}{c}\text { VR c } \\
(\mathrm{DFT}=3,5 \mathrm{~mm})\end{array}$ & U37 & U38 & U39 & U40 & $\begin{array}{c}\text { B22 } \\
{[15 \%]}\end{array}$ & $\begin{array}{c}\text { B21 } \\
{[25 \%]}\end{array}$ & $\begin{array}{c}\text { B20 } \\
{[35 \%]}\end{array}$ & $\begin{array}{l}\text { B23 }{ }^{\#} \\
{[45 \%]}\end{array}$ & $\begin{array}{c}\text { B17 } \\
{[45 \%]}\end{array}$ & $\begin{array}{l}\text { B24\# } \\
{[45 \%]}\end{array}$ & $\begin{array}{c}\text { B19 } \\
{[55 \%]}\end{array}$ & $\begin{array}{r}\text { B18 } \\
{[65 \%]}\end{array}$ \\
\hline \multirow{2}{*}{ 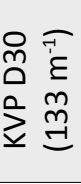 } & $\begin{array}{c}\text { VR d } \\
(\mathrm{DFT}=2,5 \mathrm{~mm})\end{array}$ & U41 & U42 & U43 & U44v & $\begin{array}{c}\text { B45 } \\
{[15 \%]}\end{array}$ & $\begin{array}{c}\text { B47 } \\
{[25 \%]}\end{array}$ & $\begin{array}{c}\text { B48 } \\
{[35 \%]}\end{array}$ & - & $\begin{array}{c}\text { B49 } \\
{[42 \%]}\end{array}$ & - & $\begin{array}{c}\text { B50 } \\
{[55 \%]}\end{array}$ & $\begin{array}{r}\text { B46 } \\
{[65 \%]}\end{array}$ \\
\hline & $\begin{array}{c}\mathrm{VRe} \mathrm{e}^{* *} \\
(\mathrm{DFT}=3,5 \mathrm{~mm})\end{array}$ & U51 & U52 ${ }^{n}$ & U53 & U54 ${ }^{\mathrm{n}}$ & $\begin{array}{l}\text { B55 } \\
{[15 \%]}\end{array}$ & $\begin{array}{c}\text { B56 } \\
{[25 \%]}\end{array}$ & $\begin{array}{c}\text { B60 } \\
{[35 \%]}\end{array}$ & - & $\begin{array}{c}\text { B58 } \\
{[45 \%]}\end{array}$ & - & $\begin{array}{c}\text { B59 } \\
{[55 \%]}\end{array}$ & $\begin{array}{r}\text { B57 } \\
{[65 \%]}\end{array}$ \\
\hline \multirow{2}{*}{ 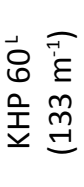 } & $\begin{array}{c}\text { VR } \mathrm{f} \\
(\mathrm{DFT}=2,5 \mathrm{~mm})\end{array}$ & U67v & U68 & U69 & - & - & $\begin{array}{l}\text { B66 } \\
{[25 \%]}\end{array}$ & - & - & $\begin{array}{c}\text { B64 } \\
{[45 \%]}\end{array}$ & - & - & $\begin{array}{r}B 65 \\
{[60 \%]}\end{array}$ \\
\hline & $\begin{array}{c}\text { VR g } \\
(\mathrm{DFT}=3,5 \mathrm{~mm})\end{array}$ & U61 & U62 & U63 & - & - & $\begin{array}{l}\text { B71 } \\
{[25 \%]}\end{array}$ & - & - & $\begin{array}{c}\text { B72 } \\
{[45 \%]}\end{array}$ & - & - & $\begin{array}{l}B 70^{\mathrm{T}} \\
{[60 \%]}\end{array}$ \\
\hline
\end{tabular}

KVP ... Kreisvollprofil;

KHP ... Kreishohlprofil

DFT ... Trockenschichtdicke des Brandschutzsystems

[15\%] ... Lastausnutzungsgrad im Brandfall $\mu_{\mathrm{fi}}$

n ... In gleicher Ebene wie das belastete Zugglied geprüft (Position 2, vertikaler Abstand = $150 \mathrm{~mm}$ )

$\checkmark$... In vertikaler Prüflage getestet (Position 3)

${ }^{\mathrm{T}}$... Thermoelemente auf der Staboberseite angeordnet

L... Länge der Versuchskörper $1240 \mathrm{~mm}$

* ... definierte Beschädigung des reaktiven Brandschutzsystems vorgenommen.

* Bauteile mit höherer Trockenschichtdicke des RBS (B8=3,0 mm;B2 =3,5 mm;B4 =4,5 mm)

** Im Rahmen der Dissertation durchgeführte Brandversuche (Dokumentation siehe Anhang A)

Die Herstellung der Versuchskörper erfolgt analog zu den Voruntersuchungen in Kapitel 3.4. Aufgrund der größeren Stablängen der mechanisch belasteten Zugglieder werden entsprechend mehr Thermoelemente angeordnet. Für die Applikation der Thermoelemente kommt das in den Voruntersuchungen neu entwickelte Verfahren zur Anwendung (siehe Kapitel 3.4.1). Mit wenigen Ausnahmen werden bei allen horizontal getesteten Versuchskörpern die Thermoelemente auf der Stabunterseite angeordnet, um eine Beeinflussung auf die Bildung von Längsrissen auf der Staboberseite und ein damit verbundenes mögliches Abgleiten der Brandschutzbeschichtung zu minimieren. Die Einbausituation der Versuchskörper im Zugstabprüfofen und die Lage der Thermoelemente ist in Abb. 3.13 dargestellt. 
Position 2 - Probe befindet sich in horizontaler Lage oberhalb des belasteten Zuggliedes mit einem vertikalen Abstand von ca. 150 mm (siehe Abb. A.06)

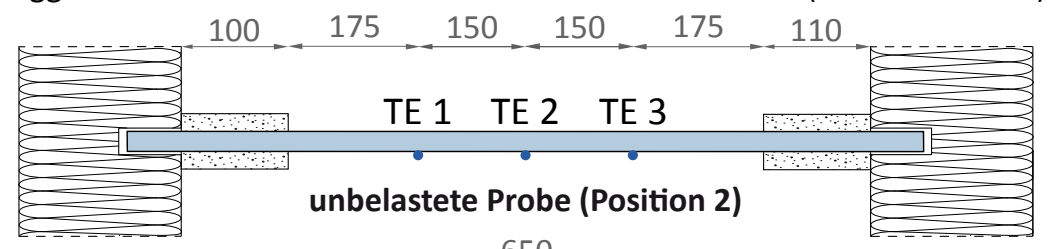

650

Mechanisch belastetes Zugglied - Probe befindet sich in horizontaler Lage auf der Höhe und parallel zu den Brennerachsen (siehe Abb. 3.14).

Ölbrenner

Hydraulikzylinder

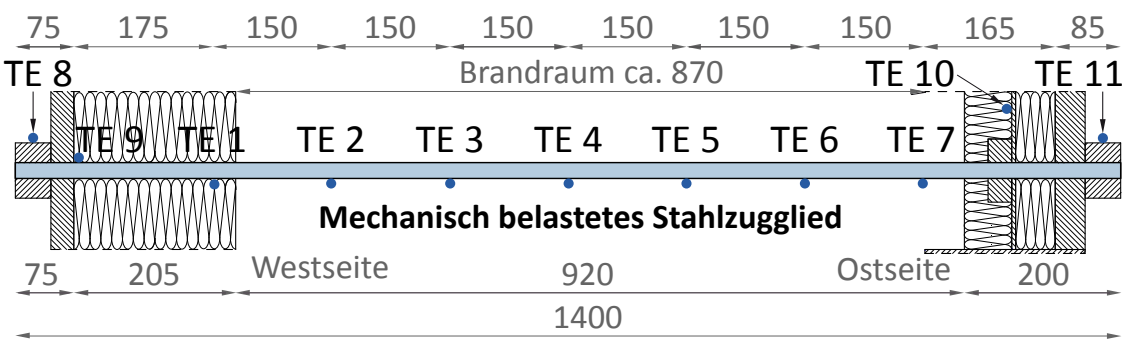

Position 1 - Probe befindet sich in horizontaler Lage unterhalb des belasteten Zuggliedes mit $250 \mathrm{~mm}$ vertikalem und horizontalem Abstand (siehe Abb. 3.14).
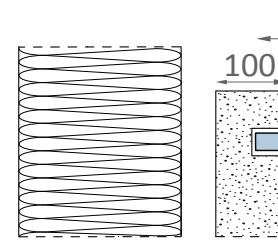

225 15 150 225

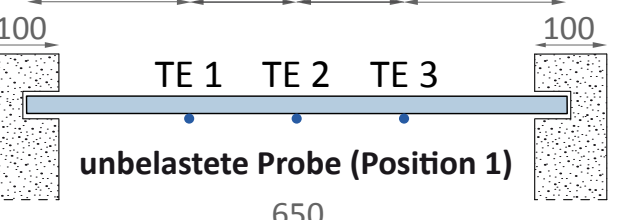

650

\section{Probekörperaufnahme}

Wärmedämmung

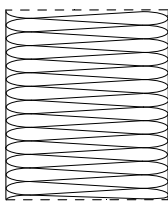

Versuchskörper
Position 3

Probe befindet sich in vertikaler Lage und wird an der Stelle des Brandraumthermoelements (BRT 7) angeordnet (siehe Abb. 3.14; BRT 7 wird versetzt).

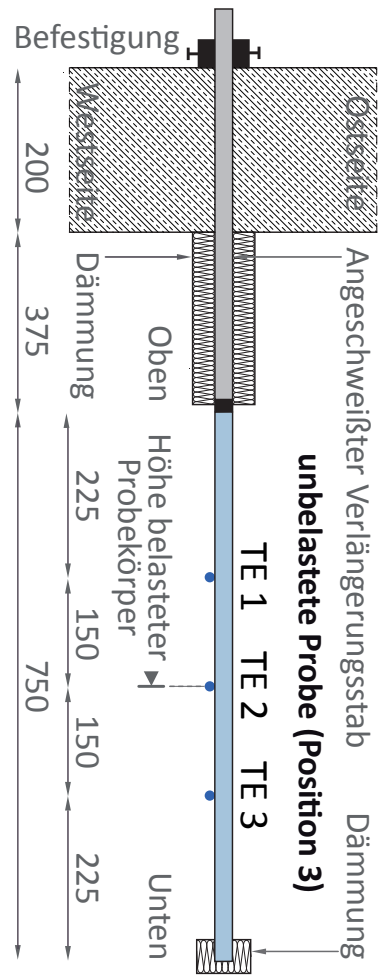

Hinweis: Unbelastete Proben (Vergleichsprüfkörper) werden in verschiedenen Positionen (1 - 3) getestet.

Abb. 3.13: Querschnitt eines Teils des Zugstabprüfofens mit Bezeichnung und Bemaßung der Thermoelemente für getesteten Versuchskörper (Blick aus Richtung Süden)

Die Brandversuche werden an Kreisvollprofilen mit einem Durchmesser von 20 und $30 \mathrm{~mm}$ (KVP D20 und KVP D30) sowie Kreishohlprofilen mit einem Durchmesser von 60,3 mm und einer Wanddicke von 8,8 mm (KHP D60) durchgeführt. Auf Grundlage der Ergebnisse aus den orientierenden Brandversuchen im Einkubikmeterofen (siehe Kapitel 3.4) wird der Einfluss der Trockenschichtdicke (DFT) an allen Profilen für Beschichtungsdicken von 2,5 und 3,5 mm untersucht. An den KVP D20 werden zusätzlich die Trockenschichtdicken 1,5, 3,0 und 4,5 mm getestet. Zur Untersuchung des Einflusses der Bauteilorientierung werden an Versuchskörpern ohne Zugbeanspruchung Brandversuche mit vertikaler und horizontaler Probenausrichtung durchgeführt. Die Beeinflussung der mechanischen Zugbeanspruchung auf das Aufschäumverhalten und die thermische Schutzwirkung des reaktiven Brandschutzsystem erfolgt anhand des Vergleichs von mechanisch belasteten Zuggliedern mit unter- 
schiedlichen Lastausnutzungsgraden im Brandfall und unbelasteten Vergleichsprüfkörpern (siehe Kapitel Definitionen). Der Profilquerschnitt und die Trockenschichtdicke der unbelasteten Proben sind mit den mechanisch belasteten Versuchskörpern identisch. Die Höhe des Lastausnutzungsgrades der Zugglieder variiert von $\mu_{\mathrm{fi}}=0,15$ (gering ausgelastet) bis 0,65 (voll ausgelastet) in einem Intervall von 0,1. Ein Lastausnutzungsgrad im Brandfall von $\mu_{\mathrm{fi}}=$ 0,65 entspricht einem im Kaltfall voll ausgelastetem Zugglied. Um die Robustheit des reaktiven Brandschutzsystems bewerten zu können, werden einige der Zugglieder mit definierten Beschädigungen des Beschichtungssystems versehen.

\subsubsection{Versuchsaufbau}

Für die Einrichtung des Versuchsstandes zur Untersuchung von Stahlprofilen mit mechanischer Zugbeanspruchung in einem sogenannten Zugstabprüfofen wird ein vorhandener Deckenprüfstand (DEP) umgebaut (siehe Abb. 3.15 und Abb. 3.14). Ein Stahlrahmen (Belastungsrahmen) sowie ein kleinerer Brandraum von etwa $0,9 \times 1,5 \times 1,5 \mathrm{~m}$ werden in den Deckenprüfstand eingebaut. Auf einer Seite des Stahlrahmes wird ein servohydraulischer Prüfzylinder zum Aufbringen der Zugbeanspruchung auf Versuchskörper montiert. Das mit einer Zugkraft zu belastende Zugglied wird mittels einer dafür gefertigten Einspannvorrichtung auf der Westseite mit dem Stahlrahmen und auf der Ostseite mit dem Hydraulikzylinder montiert. Der Prüfstand ist so konzipiert, dass sowohl Profile mit Vollquerschnitt, als auch offene und geschlossene Profile unter Brandbeanspruchung und axialer Zugbeanspruchung untersucht werden können. Auf die Versuchskörper kann durch den Hydraulikzylinder eine maximale Zugkraft von $400 \mathrm{kN}$ aufgebracht werden. Aufgrund des in sich geschlossenen Stahlrahmens entstehen für den Deckenprüfstand keine äußeren Beanspruchungen.

Im Zugstabprüfofen können Zugglieder auf einer Länge von ca. 0,9 m nach Einheits-Temperaturzeitkurve (ETK) beflammt werden. Für die Beflammung des Brandraumes werden zwei bereits auf der Westseite des Deckenprüfstandes vorhandene Heizölbrenner genutzt. Diese ermöglichen eine tangentiale Beflammung der horizontal eingebauten Versuchskörper. Die Achsen der Brenner befinden sich auf der gleichen Höhe wie das mechanisch belastete Zugglied. Die Brandgase werden durch die im DEP vorhandenen Rauchgaskanäle abgeleitet. Mittels eines hitzebeständigen Sichtfensters auf der Südseite des Brandraumes werden die Versuchskörper über die gesamte Branddauer beobachtet.

Mittels zweier herausnehmbarer Wandsegmente an der Ost- und Westseite des Brandraumes lassen sich die mechanisch belasteten Versuchskörper relativ schnell ein- und ausbauen. Die Oberseite des Brandraumes wird durch drei bewehrte Porenbetonplatten abgedeckt. Durch Bohrungen in der mittleren Abdeckplatte werden zwei Plattenthermoelementlanzen zur Messung der Brandgastemperaturen befestigt. An der Nordseite des Brandraumes befindet sich jeweils $15 \mathrm{~cm}$ vom Boden bzw. von der Decke entfernt eine Sonde zur Messung des Brandraumdruckes. Die Lage der Versuchskörper im Brandraum 
sowie die Position der Brandraumthermoelemente und Brandraumdrucksonden sind in Abb. 3.14 dargestellt. Die Positionierung der Brandraumthermoelemente erfolgt in Anlehnung an DIN EN 1361-1 [07]. Demzufolge werden für die Brandgastemperaturmessung zwei Plattenthermoelemente im Abstand von 100 mm zu den Versuchkörpern angeordnet. Aufgrund der vorhandenen Brandraumabmessungen des Zugstabprüfofen wurde in Stablängsrichtung der Zugglieder der laut Norm erforderliche Abstand zwischen Brandraumthermoelement und der inneren Begrenzung des Brandraumes von $450 \mathrm{~mm}$ auf etwa $300 \mathrm{~mm}$ verringert. Ferner wurde die nach Norm [07] geforderte wechselseitige Anordnung der Plattenthermoelemente aufgrund der Videoaufzeichnung der Brandversuche nicht umgesetzt. Bei der Durchführung der Brandversuche für die VR e werden zusätzlich zur Darstellung in Abb. 3.14 zwei weitere Plattenthermoelementlanzen im Bereich des unbelasteten Versuchskörpers hinzugefügt. Ferner wird bei einem Teil dieser Brandprüfungen der unbelastete Vergleichsprüfkörper oberhalb des mechanisch belasteten Zuggliedes angeordnet (Position 2 nach Abb. 3.13).

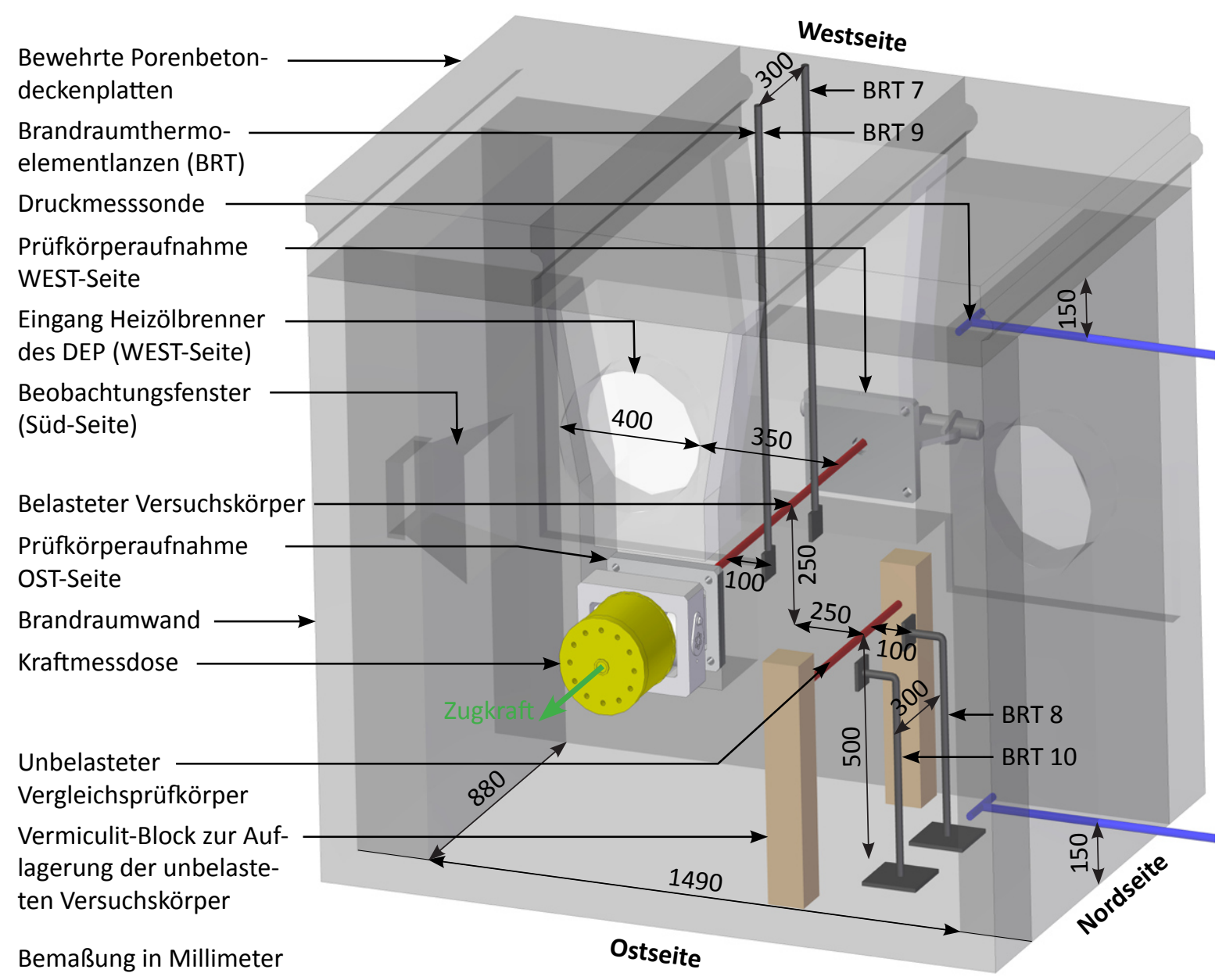

Abb. 3.14: 3D-Modell des Brandraumes des Zugstabprüfofens mit den eingebauten Versuchskörpern und den installierten Temperatur- und Druckmessinstrumenten 


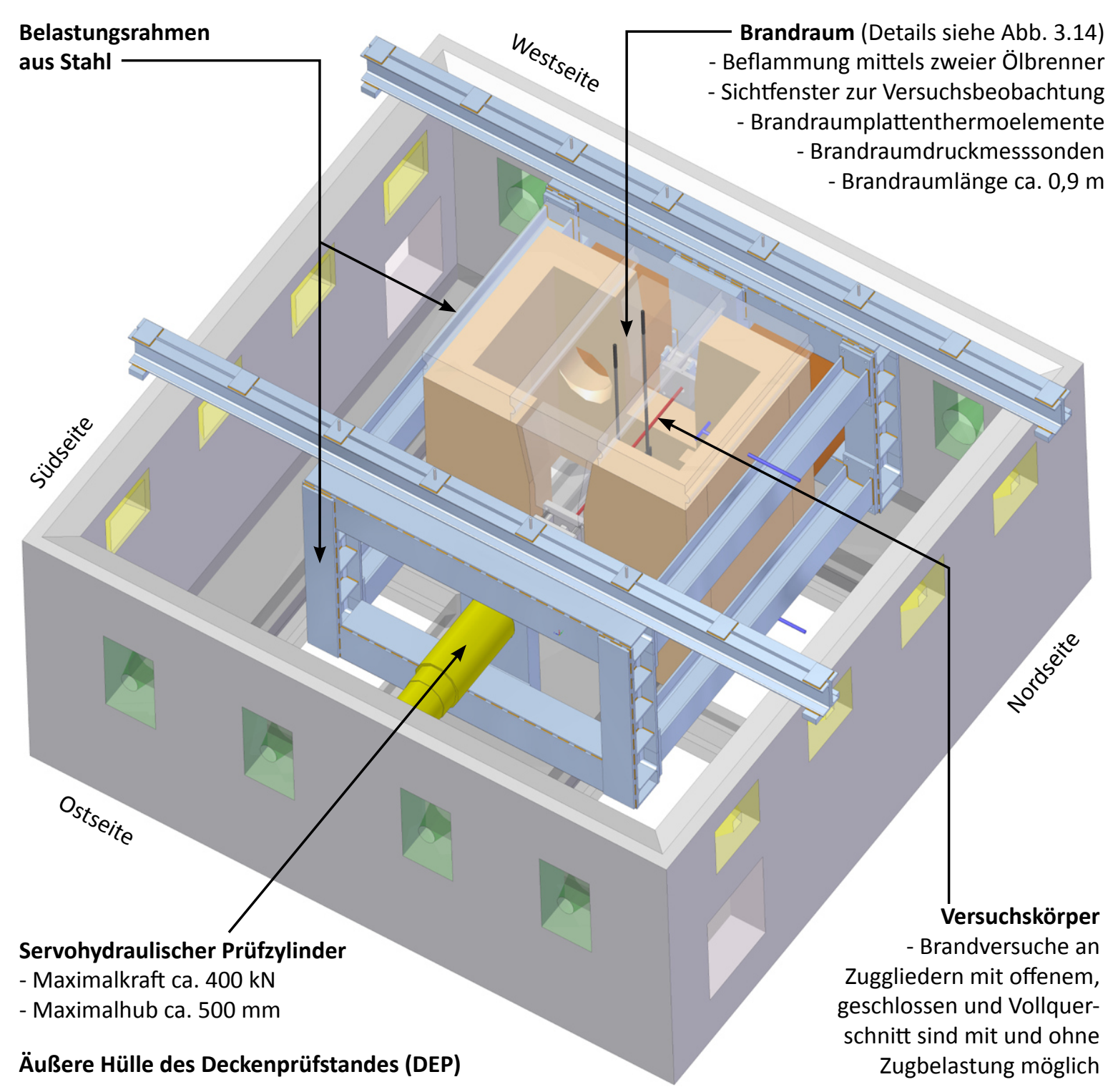

Abb. 3.15: 3D-Modell des Zugstabprüfofens für die Durchführung der Brandversuche im Realmaßstab

\subsubsection{Versuchsdurchführung}

Der unter mechanischer Zugbeanspruchung zu prüfende Versuchskörper wird zusammen mit dem unbelasteten Vergleichsprüfkörper in den Versuchsstand eingebaut. In der Regel werden die Proben so positioniert, dass sich die Thermoelemente auf der Stabunterseite befinden. Das mechanisch zu belastende Zugglied wird mittels entsprechender Einspannvorrichtungen mit dem Belastungsrahmen sowie dem Hydraulikzylinder des Zugstabprüfofens verbunden. Um auch außerhalb des Brandraumes die Stahltemperatur des Versuchskörpers messen zu können, werden nachträglich vier zusätzliche Thermoelemente an das 
mechanisch belastete Zugglied geschweißt (TE 8 bis TE 11 nach Abb. 3.13). Anschließend werden die Probekörperthermoelemente an das Messsystem angeschlossen. Bei einigen Brandversuchen wird zusätzlich ein unbelasteter Vergleichsprüfkörper in den Brandraum eingebaut. In der Regel befindet sich der Vergleichsprüfkörper parallel zum belasteten Zugglied mit einem horizontalen und vertikalen Abstand von jeweils $250 \mathrm{~mm}$ (Position 1 nach Abb. 3.13). Durch den gewählten Abstand zwischen den Versuchskörpern soll eine Beeinflussung durch Abschattungseffekte verhindert werden. Um den Einfluss der Bauteilorientierung auf das Verhalten des reaktiven Brandschutzsystems zu untersuchen, werden die unbelasteten Vergleichsprüfkörper in horizontaler und vertikaler Einbaulage getestet (Position 1 und 3 nach Abb. 3.13). Bei horizontaler Orientierung der Vergleichsprüfkörper befinden sich die Probekörperthermoelemente auf der Stabunterseite und bei vertikaler Prüfposition auf der brennerzugewandten Seite (Westseite). Vor dem Brandversuch werden eventuelle Beschädigungen oder Auffälligkeiten an den Versuchskörpern dokumentiert.

Die Abläufe des Aufschäumens der reaktiven Brandschutzbeschichtung werden per Video aufgezeichnet. Die Höhe der aufgebrachten Zugbeanspruchung sowie die Brandgas- und Probekörpertemperaturen werden ebenfalls messtechnisch erfasst. Die Berechnung der Prüflast erfolgt unter Verwendung der bei Raumtemperatur in Kleinzugversuchen nach DIN EN ISO 6892-1 [14] bestimmten Werte für die 0,2\%-Dehngrenze $\left(\mathrm{f}_{\mathrm{p} 0,2}\right)$ bzw. Streckgrenze $\left(f_{y}\right)$ (siehe Kapitel 4). Die Prüflast des Zuggliedes berechnet sich nach Gleichung (3.1). In die Berechnung fließen die bei Raumtemperatur vorhandene Festigkeit des Stahls, der Stabdurchmesser sowie der gewählte Lastausnutzungsgrad ein. Da es sich bei den durchgeführten Brandprüfungen im Realmaßstab um instationäre Warmzugversuche handelt, wird die aufgebrachte Zugbeanspruchung über die gesamte Versuchsdauer konstant gehalten.

Berechnung der Prüflast: $\quad F_{z u g}=\frac{\pi \cdot \mathrm{d}^{2}}{4} \cdot \mathrm{f}_{p 0,2} \cdot \mu_{f i}$

Brandversuche ohne unbelasteten Vergleichsprüfkörper enden nach dem Eintreten des Probenbruchs des mechanisch belasteten Zuggliedes. Bei Prüfungen mit unbelastetem Vergleichsprüfkörper muss zusätzlich bei beiden Versuchskörpern eine maximale Stahltemperatur von $750{ }^{\circ} \mathrm{C}$ erreicht werden. Dies lässt sich dadurch begründen, dass der Stahl für Temperaturen von mehr als $750^{\circ} \mathrm{C}$ nur über eine sehr geringe Festigkeit verfügt und das Zugglied dadurch seine statische Funktion innerhalb des Tragwerkes verliert. Nach Beendigung des Brandversuchs und Abkühlung des Brandraumes erfolgt eine erneute Dokumentation der Versuchskörper. Mittels eines Drahtes wird die Höhe der Aufschäumung des reaktiven Brandschutzsystems entlang der Stabachse in einem Abstand von ca. 50 mm gemessen. Die Messreihen sind jeweils im Winkel von $90^{\circ}$ um den Stabquerschnitt angeordnet. Nach dem Ausbau der Versuchkörper aus dem Zugstabprüfofen und dem Entfernen der aufgeschäumten Brandschutzbeschichtung werden die unter mechanischer Zugbeanspruchung geprüften Zugglieder hinsichtlich ihrer Längenänderung und Brucheinschnürung vermessen. 


\subsubsection{Beobachtungen während der Brandversuche}

\section{Stahlzugglieder mit KVP D20 (VR a bis c)}

Das Aufschäumen der reaktiven Brandschutzbeschichtung setzt an den Versuchskörpern mit KVP D20 nach etwa zwei Minuten ein. Auf dem Brandschutzsystem bilden sich zunächst Blasen. Die Oberfläche färbt sich dabei schwarz. Die Schwarzfärbung resultiert aus dem beim Aufschäumprozess freigesetzten Kohlenstoff. Durch die große Volumenausdehnung und Umfangszunahme der aufschäumenden Brandschutzbeschichtung bilden sich Risse in Quer- und Längsrichtung (siehe Abb. 3.17). Bei ausreichender Trockenschichtdicke kann das Rissheilungsvermögen des reaktiven Brandschutzsystems aktiviert werden. Entstandene Risse können sich durch Nachschäumen von noch nicht vollständig reagiertem Beschichtungsmaterial teilweise wieder schließen. Zu beobachten ist auch, dass sich bei fehlendem Rissheilungsvermögen einzelne Risse weiter öffnen. Folglich nimmt die thermische Schutzwirkung in diesen Bereichen ab und es kommt zu einem lokal erhöhten Temperaturanstieg des Versuchskörpers. Wie bereits bei den Untersuchungen im Einkubikmeterofen (siehe Kapitel 3.4.4), kommt es auch bei den Brandversuchen im Zugstabprüfofen bei horizontal eingebauten Versuchskörpern infolge von Längsrissen auf der Staboberseite vereinzelt zu einem Abgleiten der aufgeschäumten reaktiven Brandschutzbeschichtung (siehe Abb. 3.17). Der Stahl ist in diesem Bereich nahezu ungeschützt der Brandeinwirkung ausgesetzt. Infolge des schnellen Anstiegs der Stahltemperatur tritt an den mechanisch belasteten Zuggliedern ein frühzeitiger Probenbruch ein. Das Abgleiten der Brandschutzbeschichtung konnte ausschließlich auf den Versuchskörpern mit Zugbeanspruchung beobachtet werden. Besonders häufig kommt es an den Zuggliedern der VR a mit einer vergleichsweisen kleinen Trockenschichtdicke von 1,5 mm zum Abgleiten des reaktiven Brandschutzsystems. Mit hoher Wahrscheinlichkeit besteht hinsichtlich des Abgleitens der Brandschutzbeschichtung ein Zusammenhang zwischen Trockenschichtdicke, Rissheilungsvermögen, Profilgeometrie und Höhe der Zugbeanspruchung. Die bereits in Abb. 2.06 vorgestellten Versagensmechanismen der aufschäumenden reaktiven Brandschutzbeschichtung haben sich in den Brandversuchen im Realmaßstab bestätigt.

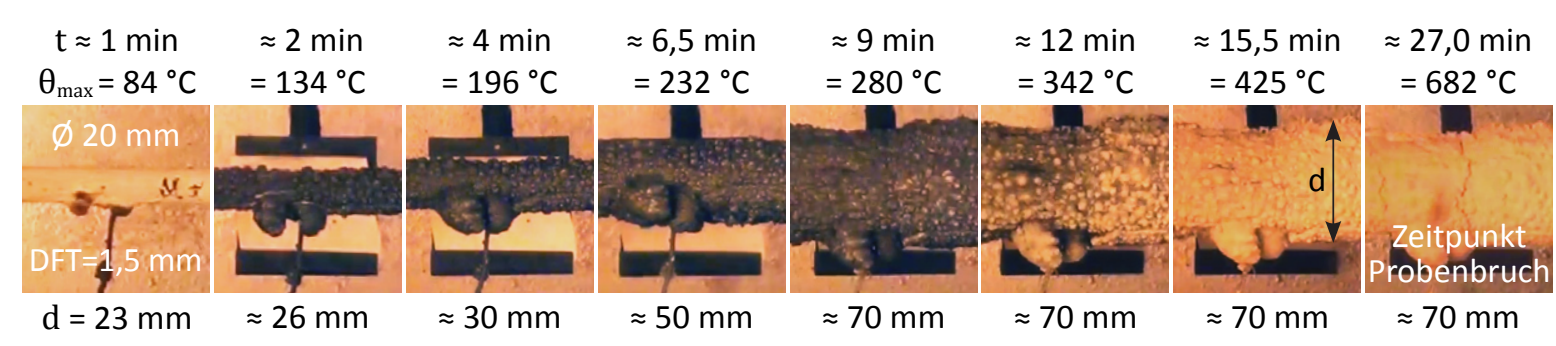

Abb. 3.16: Aufschäumungsprozess des reaktiven Brandschutzsystems am Beispiel des Versuchskörpers B3 (Branddauer $\mathrm{t}$ in min; maximale Stahltemperatur in $\theta_{\max }{ }^{\circ} \mathrm{C}$; äußere Probenabmessung $\mathrm{d}$ in $\mathrm{mm}$ )

Je nach Profilgeometrie und Trockenschichtdicke wird die größte Ausdehnung der Aufschäumung augenscheinlich nach ca. 10 bis 20 Minuten Branddauer erreicht. Danach beginnt 
die Oberfläche des aufgeschäumten Brandschutzsystems zu veraschen und färbt sich weiß. Neuer Kohlenstoff wird nicht mehr gebildet und der Aufschäumvorgang ist damit beendet. Die weiße krustenartige Oberfläche des reaktiven Brandschutzsystems verändert sich im weiteren Verlauf des Brandversuches nur noch unwesentlich. Das Aufschäumverhalten der Brandschutzbeschichtung ist am Beispiel des Versuchskörpers B3 in Abb. 3.16 dargestellt.

Der Probenbruch der unter mechanischer Zugbeanspruchung getesteten Versuchskörper deutet sich in der Regel durch mehrere Querrisse in der aufgeschäumten reaktiven Brandschutzbeschichtung an. Die Rissaufweitung resultiert aus den großen mechanischen Dehnungen, welche im Einschnürungsbereich des Zuggliedes besonders groß sind. Anhand der auftretenden Querrisse und der Position der gemessenen maximalen Stahltemperatur lässt sich die Bruchstelle der Probe relativ gut vorhersagen. Je nach Lastausnutzungsgrad, Trockenschichtdicke und Aufschäumverhalten des reaktiven Brandschutzsystems tritt der Probenbruch an den mechanisch belasteten Versuchskörpern nach etwa 21 bis 46 Minuten ein. Durch die Erschütterung beim Probenbruch kommt es häufig zu einem teilweisen Abfallen des aufgeschäumten reaktiven Brandschutzsystems vom mechanisch belasteten Zugglied.

Die im Zugstabprüfofen in vertikaler Prüfposition getesteten unbelasteten Vergleichsprüfkörper zeigen meist ein ähnliches Aufschäumungs- und Rissverhalten des reaktiven Brandschutzsystems wie die in horizontaler Einbaulage getesteten Versuchskörper. Die bereits in Abb. 2.07 der Vorbetrachtungen beschriebenen Versagensmechanismen der aufschäumenden Brandschutzbeschichtung sind in den Brandversuchen aufgetreten. Im Gegensatz zu den Versuchskörpern mit horizontaler Bauteilorientierung tritt ein teilweises Abgleiten des reaktiven Brandschutzsystems an den vertikalen Versuchskörpern nur in Stablängsrichtung auf (siehe Abb. 3.35). Ein Abfallen der aufgeschäumten Brandschutzbeschichtung ist an den vertikalen Vergleichsprüfkörpern nicht zu beobachten.

\section{Stahlzugglieder mit KVP D30 (VR d bis e)}

Die Versuchskörper mit KVP D30 zeigen in den Brandversuchen ein ähnliches Verhalten wie die beschichteten Stahlzugglieder mit KVP D20. Während des Aufschäumprozesses bilden sich ebenfalls Längs- und Querrisse im reaktiven Brandschutzsystem aus (siehe Abb. 3.17). Durch das vorhandene Rissheilungsvermögen der Brandschutzbeschichtung können die entstandenen Risse jedoch meist wieder vollständig geschlossen werden, so dass die thermische Schutzwirkung erhalten bleibt. Zudem befinden sich Längsrisse meist auf der Stabunterseite. Ein durch Längsrisse auf der Staboberseite verursachtes Abgleiten der Brandschutzbeschichtung ist bei den KVP D30 nicht aufgetreten. Generell zeigt sich an den Versuchskörpern mit KVP D30 sowohl in Stablängsrichtung als auch über den Querschnitt eine relativ gleichmäßige Aufschäumung des reaktiven Brandschutzsystems. Die Zeitpunkte des Probenbruchs der mechanisch belasteten Versuchskörper variieren je nach Lastausnutzungsgrad, Trockenschichtdicke und Aufschäumverhalten des reaktiven Brandschutzsystems zwischen 42 bis 72 Minuten. 

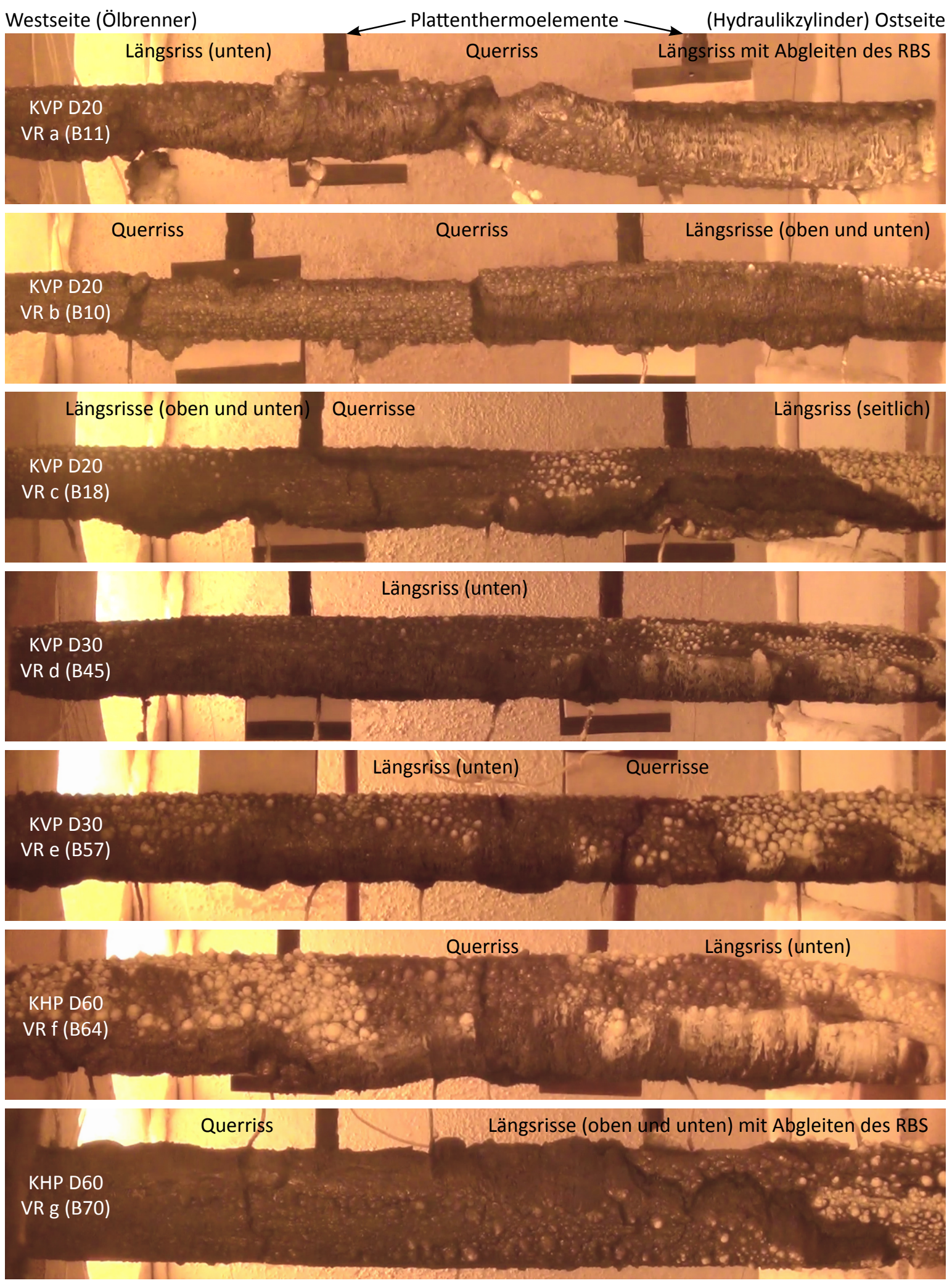

Abb. 3.17: Beispiele mechanisch belasteter Zugglieder mit aufgeschäumten RBS (nicht maßstäblich) 

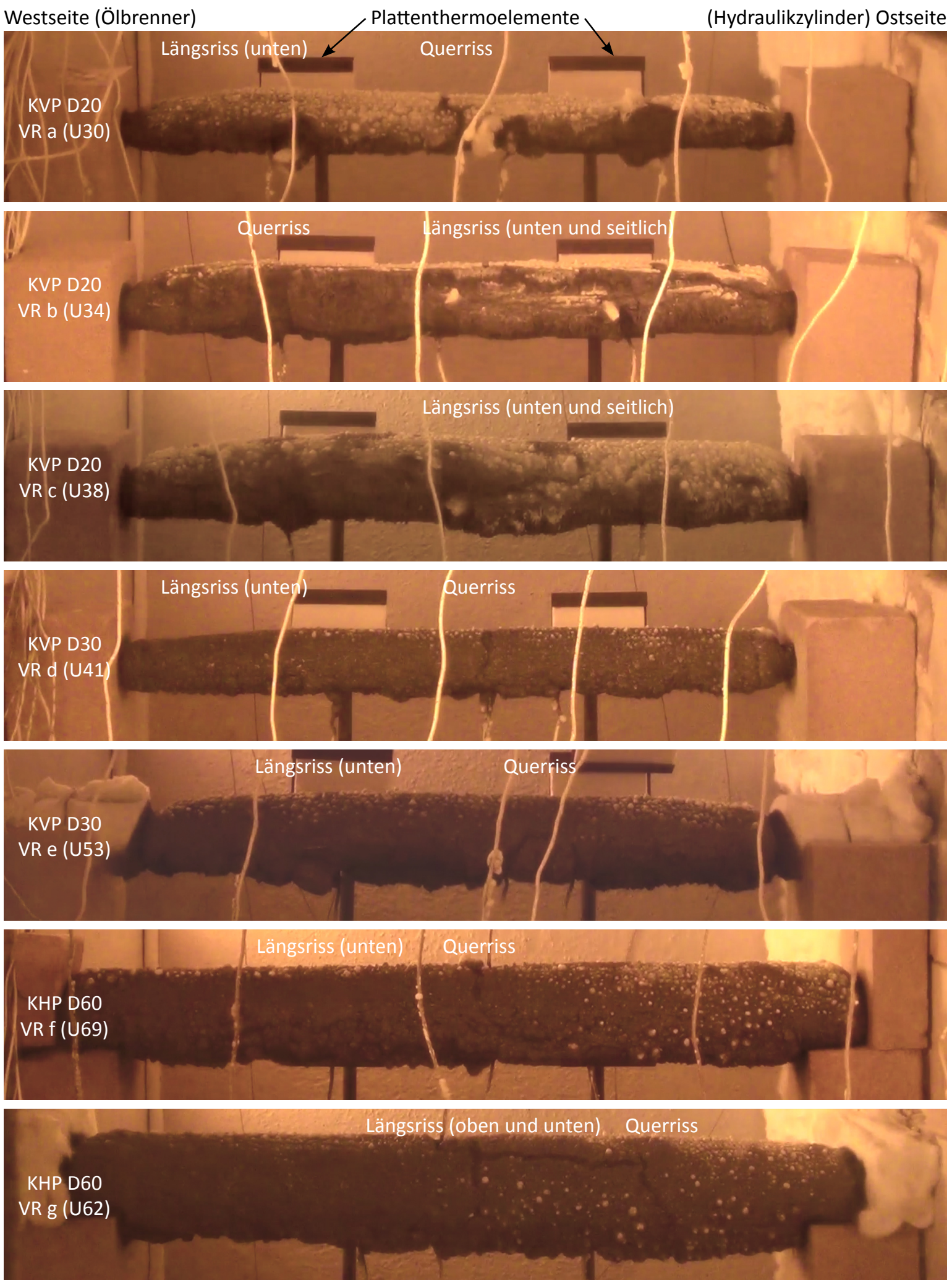

Abb. 3.18: Beispiele unbelasteter horizontaler Vergleichsprüfkörper mit aufgeschäumten RBS (nicht maßstäblich) 


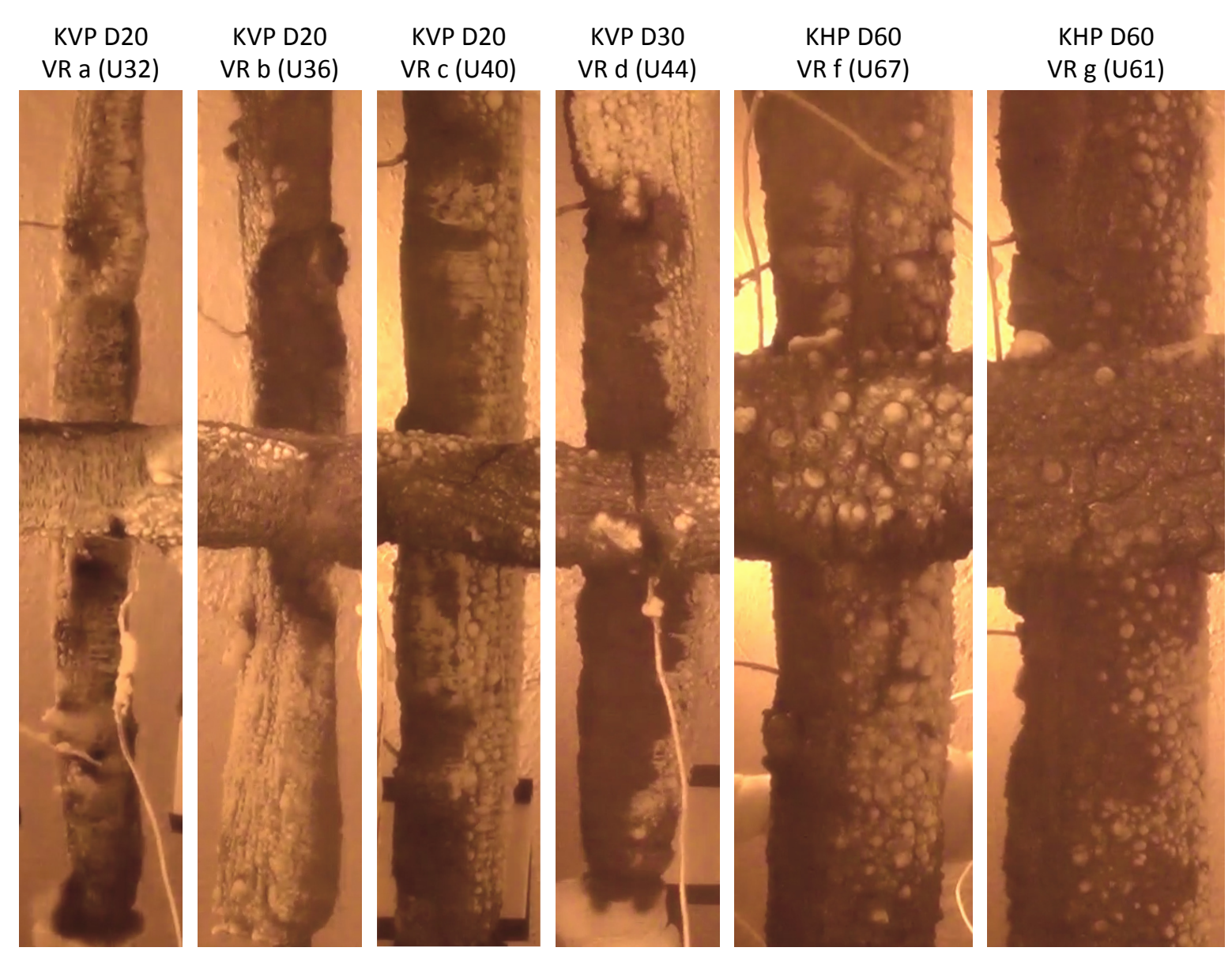

Jeweils in der Bildmitte befindet sich das horizontal angeordnete mechanisch belastete Zugglied. In der VR e wurde kein Versuchskörper vertikal getestet.

Abb. 3.19: Beispiele unbelasteter vertikaler Vergleichsprüfkörper mit aufgeschäumten RBS (nicht maßstäblich)

\section{Stahlzugglieder mit KVP D60 (VR $f$ bis $g$ )}

An den getesteten Versuchskörpern mit KHP D60 entspricht die Aufschäumung und Rissbildung des reaktiven Brandschutzsystems im Wesentlichen dem bereits an den Kreisvollprofilen beobachteten Verhalten. Der größere Stabdurchmessers des KHP D60 und die daraus resultierende geringere Oberflächenkrümmung haben meist nicht zu einer Reduzierung der Rissbildung geführt. Teilweise hat sich die Rissbildung sogar verstärkt. Längsrisse befinden sich überwiegend auf der Stabunterseite. Diese sind meist relativ breit und reichen oft bis auf die Stahloberfläche. Um einen Einfluss der Thermoelemente auf die Rissbildung an der Stabunterseite auszuschließen, werden einige der Versuchskörper so eingebaut, dass sich die Messstellen auf der Staboberseite befinden. Die Längsrisse treten dennoch auf der Stabunterseite auf (siehe Abb. 3.20). An dem mechanisch belasteten Versuchskörper B70 führt die Anordnung der Thermoelemente auf der Staboberseite zu einer Längsrissbildung auf selbiger, wodurch es zu einem teilweisen Abgleiten des reaktiven Brandschutzsystems 
kommt. Auf eine weitere Positionierung der Thermoelemente auf der Staboberseite wird daher verzichtet. An den KHP D60 ist, wie bereits bei den Kreisvollprofilen, eine deutlich stärkere Rissbildung der Brandschutzbeschichtung an den mechanisch belasteten Zuggliedern zu beobachten. Folglich zeigt sich an den unter mechanischer Zugbeanspruchung geprüften Versuchskörpern meist ein schnellerer Anstieg der Stahltemperaturen. Obwohl die KHP D60 den gleichen Profilfaktor besitzen wie die KVP D30 und die gleichen Trockenschichtdicken getestet werden, unterliegen die Kurven der maximalen Stahltemperatur der getesteten mechanisch belasteten KHP D60 einer deutlich stärkeren Streuung. Je nach Lastausnutzungsgrad, Trockenschichtdicke und Aufschäumverhalten des reaktiven Brandschutzsystems tritt der Probenbruch an den mechanische belasteten Versuchskörpern nach etwa 37 bis 90 Minunten ein.

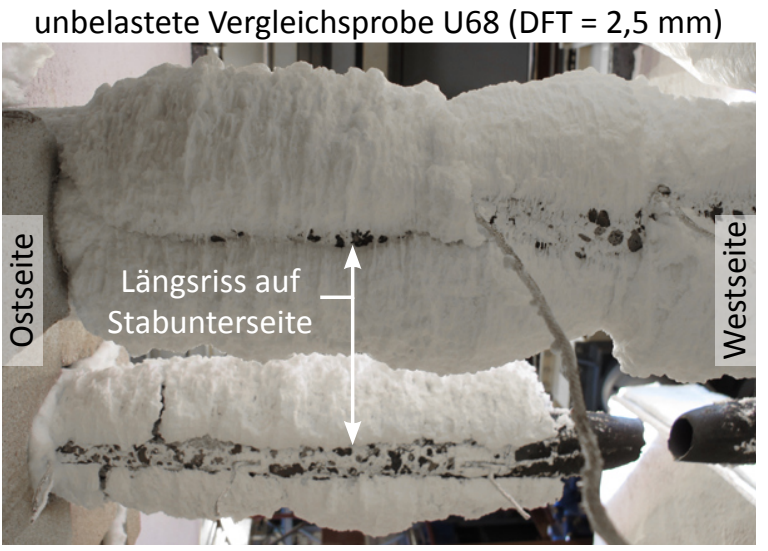

belastetes Zugglied B65 (DFT = 2,5 mm, $\mu_{\mathrm{fi}}=0,45$ )

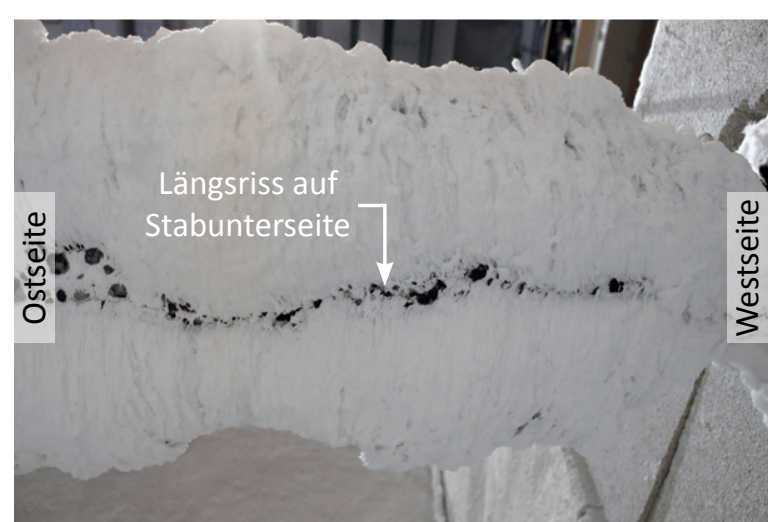

belastetes Zugglied B66 (DFT = 2,5 mm, $\mu_{\mathrm{fi}}=0,60$ )

Abb. 3.20: Längsrissbildung an Versuchskörpern (links: Thermoelemente auf der Stabunterseite; rechts Thermoelemente auf der Staboberseiteseite)

\subsection{Auswertung der Brandversuche}

Zeitdauer bis zum Probenbruch der mechanisch belasteten Zugglieder

Die Auswertung der mechanisch belasteten Zugglieder in Tabelle 3.3 zeigt, dass sich bei Reduzierung des Lastausnutzungsgrades im Brandfall, d.h. Verringerung der Zugbeanspruchung, die Zeitdauer bis zum Probenbruch erhöht. Eine Verlängerung der Branddauer bis zum Probenbruch lässt sich auch durch Erhöhung der Trockenschichtdicke des reaktiven Brandschutzsystems erreichen. Die Profilgeometrie des Zuggliedes spielt ebenfalls eine Rolle. Für die KVP D20 tritt der Probenbruch je nach Höhe des Lastausnutzungsgrades im Brandfall bei einer Trockenschichtdicke von DFT $=1,5 \mathrm{~mm}$ nach etwa 21 bis 38 Minuten, bei DFT $=2,5 \mathrm{~mm}$ nach 29 bis 44 Minuten und bei DFT = 3,5 $\mathrm{mm}$ nach 33 bis 46 Minuten ein. Für die KVP D30 vergrößert sich die Branddauer bis zum Probenbruch bei DFT = 2,5 $\mathrm{mm}$ auf ca. 42 bis 63 Minuten und bei DFT = 3,5 mm auf etwa 51 bis 73 Minuten. Die Vergrößerung des Stabdurchmessers von 20 auf $30 \mathrm{~mm}$ bewirkt eine Verlängerung der Branddauer 
um das ca. 1,5-fache. Für die KHP D60 ergibt sich eine Zeitdauer bis zum Probenbruch bei DFT $=2,5 \mathrm{~mm}$ von etwa 37 bis 55 Minuten und bei DFT = 3,5 mm von ca. 43 bis 90 Minuten.

Tabelle 3.3: Zeitpunkt bis zum Probenbruch und gemessene maximale Stahltemperaturen der im Zugstabprüfofen getesteten Versuchskörper

\begin{tabular}{|c|c|c|c|c|c|c|c|c|c|c|c|c|c|}
\hline \multirow{3}{*}{ 둥 } & \multirow{3}{*}{$\begin{array}{c}\text { Bezeichnung } \\
\text { der Versuchs- } \\
\text { reihe (VR) }\end{array}$} & \multirow{3}{*}{\multicolumn{4}{|c|}{$\begin{array}{c}\text { Unbelastete } \\
\text { Versuchskörper (U) } \\
* * *\end{array}$}} & \multicolumn{8}{|c|}{ Mechanisch belastete Versuchskörper (B) } \\
\hline & & & & & & \multicolumn{8}{|c|}{ Lastausnutzungsgrad im Brandfall $\mu_{\mathrm{fi}}$} \\
\hline & & & & & & 0,15 & 0,25 & 0,35 & & 0,45 & & 0,55 & 0,65 \\
\hline \multirow{3}{*}{ 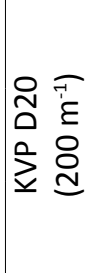 } & $\begin{array}{r}\text { VR } \\
\text { (DFT=1, }\end{array}$ & \begin{tabular}{|l|}
$26,8)$ \\
{$[555]$} \\
\end{tabular} & $\begin{array}{l}(38,3) \\
{[739]}\end{array}$ & $\begin{array}{l}(21,3)^{\mathrm{v}} \\
{[624]}\end{array}$ & - & & \begin{tabular}{|l|}
$(26,8)$ \\
{$[678]$} \\
\end{tabular} & \begin{tabular}{|c|}
$(22,6)^{\#}$ \\
{$[628]$} \\
\end{tabular} & \begin{tabular}{|l|}
$(21,3)$ \\
{$[645]^{1}$} \\
\end{tabular} & & & & $\begin{array}{l}(39,9)^{*} \\
{[648]^{3}}\end{array}$ \\
\hline & $\begin{array}{r}V R \\
\text { (DFT=2 }\end{array}$ & & & & & & & & & & & & \\
\hline & $\begin{array}{r}\mathrm{VP} \\
\text { (DFT }=3\end{array}$ & $\begin{array}{c}(42,6)^{\mathrm{v}} \\
{[627]} \\
\end{array}$ & & & & & & & & & & & \\
\hline \multirow{2}{*}{ 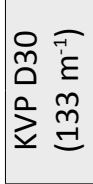 } & $\begin{array}{c}\text { VR d } \\
(\mathrm{DFT}=2,5 \mathrm{~mm})\end{array}$ & \begin{tabular}{|l|}
$(42,1)$ \\
{$[523]$} \\
\end{tabular} & $\begin{array}{l}(49,5) \\
{[648]}\end{array}$ & $\begin{array}{c}(44,9)^{\mathrm{v}} \\
{[650]}\end{array}$ & \begin{tabular}{|c|}
$(54,6)^{v}$ \\
{$[738]$}
\end{tabular} & $\begin{array}{l}(63,4) \\
{[745]} \\
\end{array}$ & $\begin{array}{l}(54,6) \\
{[692]} \\
\end{array}$ & & - & & - & & \\
\hline & $\begin{array}{c}\text { VR e } * * \\
(\mathrm{DFT}=3,5 \mathrm{~mm})\end{array}$ & \begin{tabular}{l|}
$(64,6)$ \\
{$[730]$} \\
\end{tabular} & $\begin{array}{l}(50,9)^{\mathrm{n}} \\
{[556]}\end{array}$ & $\begin{array}{l}(56,4) \\
{[576]}\end{array}$ & \begin{tabular}{|c|}
$(53,2)^{n}$ \\
{$[608]$} \\
\end{tabular} & $\begin{array}{l}(72,8) \\
{[740]}\end{array}$ & $\begin{array}{l}(64,6) \\
{[696]} \\
\end{array}$ & $\begin{array}{l}15 \\
{[6} \\
\end{array}$ & - & & - & & \\
\hline \multirow{2}{*}{ 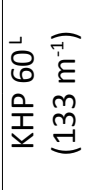 } & $\begin{array}{c}\text { VR f } \\
(\mathrm{DFT}=2,5 \mathrm{~mm})\end{array}$ & $\begin{array}{c}(55,0)^{\mathrm{v}} \\
{[696]}\end{array}$ & $\begin{array}{l}(37,1) \\
{[468]}\end{array}$ & $\begin{array}{l}(42, \\
{[57}\end{array}$ & - & - & $\begin{array}{l}(55, \\
{[72} \\
\end{array}$ & - & - & 1 & - & - & $\begin{array}{l}(37,1) \\
{[648]^{4}}\end{array}$ \\
\hline & $\begin{array}{c}\text { VR g } \\
(\mathrm{DFT}=3,5 \mathrm{~mm})\end{array}$ & \begin{tabular}{|c|}
$(89,8)^{\mathrm{v}}$ \\
{$[856]$}
\end{tabular} & $\begin{array}{l}(43,2) \\
{[406]}\end{array}$ & $\begin{array}{c}(72,1)^{\mathrm{T}} \\
{[647]}\end{array}$ & - & - & $\begin{array}{c}(89,8)^{\mathrm{T}} \\
{[736]}\end{array}$ & - & - & $\begin{array}{l}(72,1) \\
{[665]}\end{array}$ & - & - & \\
\hline
\end{tabular}

KVP ... Kreisvollprofil; KHP ... Kreishohlprofil

DFT ... Trockenschichtdicke des Brandschutzsystems

(...) ... Zeitpunkt des Probenbruchs der mechanisch belasteten Zugglieder in Minuten

[...] ... Am Versuchskörper gemessene maximale Stahltemperatur in ${ }^{\circ} \mathrm{C}$ zum Zeitpunkt des Probenbruchs

$1 ; 2 ; 3 ; 4$... veränderter Lastausnutzungsgrad im Brandfall von $\mu_{\mathrm{fi}}=0,40 ; 042 ; 0,45 ; 0,60$

n ... In gleicher Ebene wie das belastete Zugglied geprüft (Position 2, vertikaler Abstand $=150 \mathrm{~mm}$ )

v ... In vertikaler Prüflage getestet (Position 3)

${ }^{\mathrm{T}}$... Thermoelemente auf der Staboberseite angeordnet

L ... Länge der Versuchskörper $1240 \mathrm{~mm}$

\# ... definierte Beschädigung des reaktiven Brandschutzsystems vorgenommen.

* Bauteile mit höherer DFT des RBS (B8 =3,0 mm; B2 =3,5 mm; B4=4,5 mm; siehe Tabelle 3.2)

** Im Rahmen der Dissertation durchgeführte Brandversuche (Dokumentation siehe Anhang A)

*** Für die unbelasteten Versuchskörper wird die gemessene maximale Stahltemperatur für den Zeitpunkt des Probenbruchs des simultan getesteten mechanisch belasteten Zuggliedes bestimmt.

\section{Stahltemperatur zum Zeitpunkt des Probenbruchs}

Für den Zeitpunkt des Probenbruchs zeigt sich, dass im Allgemeinen eine Reduzierung des Lastausnutzungsgrades im Brandfall zu einer Erhöhung der am Versuchskörper gemessenen maximalen Stahltemperatur führt (siehe Tabelle 3.3). Abweichungen sind auf die Messtellen der Temperaturmessung, die nicht immer genau mit der Versagensstelle übereinstimmen, zurückzuführen. Mechanisch belastete Zugglieder mit gleichem Lastausnutzungsgrad im Brandfall zeigen aufgrund der übereinstimmenden Zugbeanspruchung nahezu identische Werte für die maximale Stahltemperatur zum Zeitpunkt des Probenbruchs. Dies ist 
unabhängig von der Profilgeometrie der Zugglieder und der aufgebrachten Trockenschichtdicke des reaktiven Brandschutzsystems.

Bei den unbelasteten Versuchskörpern sind die gemessenen Stahltemperaturen in der Regel geringer als an den Versuchskörpern mit Zugbeanspruchung. Dies ist auf die stärkere Rissbildung der reaktiven Brandschutzbeschichtung an den mechanisch belasteten Zuggliedern zurückzuführen. Zudem zeigen unbelastete Versuchskörper in vertikaler Prüfposition meist höhere Stahltemperaturen, als horizontal getestete Proben. Ursächlich hierfür ist ebenfalls die stärkere Rissbildung des Brandschutzsystems.

\section{Erwärmungsgeschwindigkeit}

Zahlreiche durchgeführte Brandprüfungen an mechanisch belasteten und unbelasteten Stahlbauteilen mit offenem und geschlossenem Profil haben gezeigt, dass für die Untersuchung der mechanischen Hochtemperatureigenschaften des Stahls eine Erwärmungsgeschwindigkeit zwischen 4 und 20 $\mathrm{K} / \mathrm{min}$ angenommen werden kann [37]. Die im Realmaßstab getesteten Zugglieder mit Vollprofil werden in gleicher Art und Weise ausgewertet. Aufgrund des über die Branddauer veränderlichen Zustandes des reaktiven Brandschutzsystems und der damit verbundenen unterschiedlichen thermischen Schutzwirkung erfolgt eine Unterteilung in mehrere Erwärmungsphasen. Phase 1, in der ein noch nicht oder nur kaum aufgeschäumtes reaktives Brandschutzsystem vorliegt, erstreckt sich bis zu einer maximalen Stahltemperatur von etwa $200^{\circ} \mathrm{C}$. In Phase 2 , mit einem Temperaturbereich bis etwa $700{ }^{\circ} \mathrm{C}$,

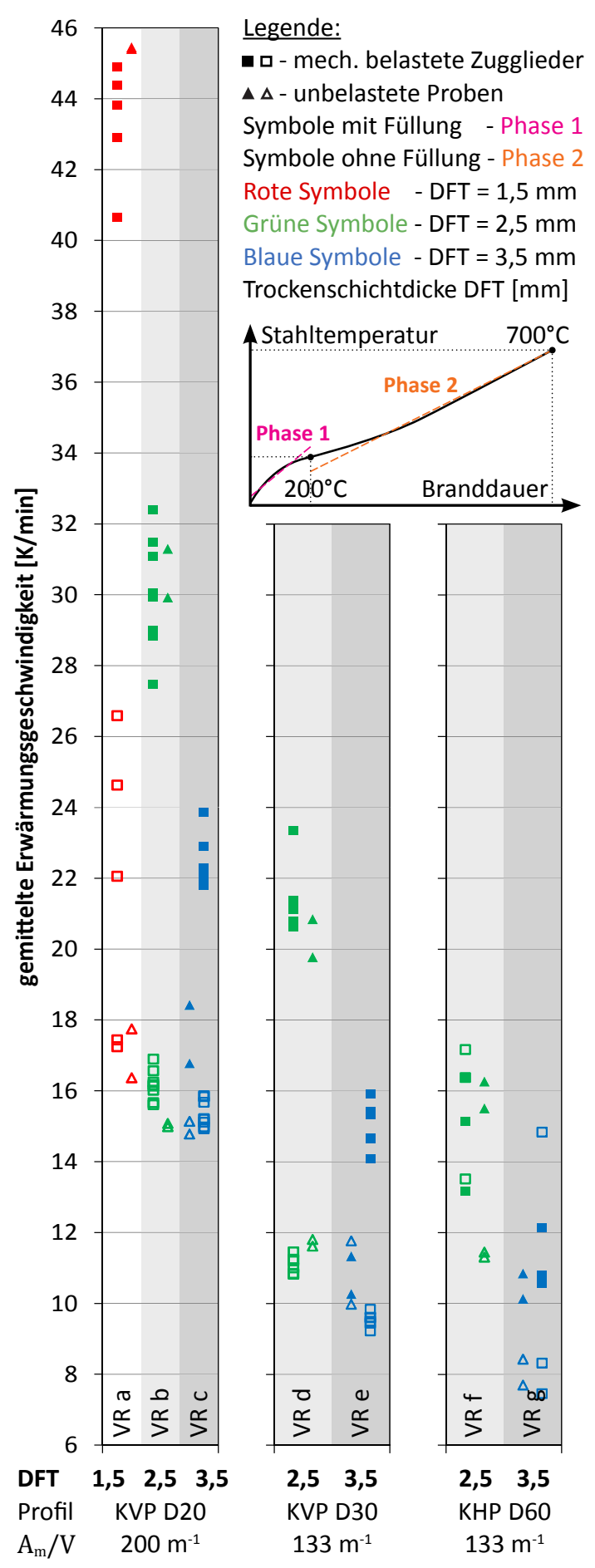

Abb. 3.21: Erwärmungsgeschwindigkeit der horizontal getesteten Versuchskörper 
wird eine vollständig aufgeschäumte Brandschutzbeschichtung angenommen. Da für die im Zugstabprüfofen getesteten Zugglieder ein anderes reaktives Brandschutzsystem verwendet wird, als für die in [37] an Trägern und Stützen ausgewerteten Brandprüfungen, ist ein Vergleichsgrundlage für die Erwärmungsgeschwindigkeit nicht gegeben.

Die in Abb. 3.21 dargestellten Ergebnisse der ermittelten Erwärmungsgeschwindigkeiten zeigen, dass sich durch die in Phase 1 noch nicht oder nur unvollständig ausgebildete thermische Schutzwirkung des reaktiven Brandschutzsystems wesentlich höhere Werte ergeben, als in Phase 2. Die Erwärmungsgeschwindigkeit lässt sich durch eine Erhöhung der Trockenschichtdicke reduzieren. Dies gilt insbesondere für den Bereich der Phase 1. Eine Vergrößerung des Stabdurchmessers führt ebenfalls zu einer signifikanten Reduzierung der Erwärmungsgeschwindigkeit. Im Allgemeinen weisen die mechanisch belasteten Zugglieder höhere Erwärmungsgeschwindigkeiten als die unbelasteten Vergleichsprüfkörper und damit in der Regel höhere Stahltemperaturen auf.

Die Kreishohlprofile besitzen gegenüber den Kreisvollprofilen bei gleicher Trockenschichtdicke und identischem Profilfaktor meist eine bessere thermische Schutzwirkung. Allerdings tritt bei den Kreishohlprofilen eine erhöhte Streuung der Werte auf. Ursächlich ist hierfür die stark ausgeprägte Längsrissbildung. Generell treten Längs- und Querrisse in der Brandschutzbeschichtung bei allen untersuchten Kreisvollprofilen auf. Die Längsrissbildung ist auf die gekrümmte Bauteiloberfläche der Zugglieder und die sich beim Aufschäumen des Brandschutzsystems infolge der Umfangsvergrößerung bildenden radialen Spannungen zurückzuführen. Des Weiteren treten bei mechanisch belasteten Zuggliedern zusätzliche Spannungen in Stablängsrichtung auf, wodurch ein dreidimensionaler Spannungszustand und damit besonders hohe Anforderungen an das reaktive Brandschutzsystem entstehen (siehe Abb. 2.06). Meist können die in der Aufschäumphase entstandenen Risse durch noch nicht vollständig reagiertes Material des Brandschutzsystems wieder geschlossen werden. Das Rissheilungsvermögen erhöht sich mit zunehmender Trockenschichtdicke.

\subsection{Einflussfaktoren auf das Verhalten des reaktiven Brand- schutzsystems und der Stahlzugglieder}

\subsubsection{Einfluss der Rissbildung auf die Branddauer bis zum Probenbruch und auf die Stahltemperatur}

Die anhand der Vorbetrachtungen in Kapitel 2.6.2 beschriebenen Versagensmechanismen der aufschäumenden Brandschutzbeschichtung, wie Rissbildung, unterschiedliche Aufschäumhöhen sowie Abgleiten, werden durch die Brandversuche bestätigt. Die durchgeführten Brandprüfungen haben gezeigt, dass das Aufschäumen der Brandschutzbeschich- 
tung überwiegend eindimensional stattfindet, d.h. senkrecht zur Bauteiloberfläche. In Querrichtung ist nur ein begrenztes Aufschäumvermögen vorhanden. Beim Aufschäumen ändern sich die Struktur und die Materialeigenschaften der Brandschutzbeschichtung von einem festen und dichten Anstrich zu einer weichen und porösen Schaumschicht. Durch das Aufschäumen des Brandschutzsystems kommt es zu einer starken Umfangsvergrößerung zwischen unaufgeschäumtem und aufgeschäumtem Zustand des reaktiven Brandschutzsystems. Das System kann die Umfangsänderung meist nicht ausgleichen und es entstehen radiale Zugspannungen in der aufschäumenden Brandschutzbeschichtung. Übersteigen diese Spannungen die Zugfestigkeit des Materials, bilden sich Längsrisse im Brandschutzsystem aus. Eine hohe Oberflächenkrümmung des Stahlbauteils, kleine Trockenschichtdicken der Brandschutzbeschichtung sowie eine mechanische Zugbeanspruchung der Stahlbauteile verstärken die Längsrissbildung. Ein ähnliches Rissverhalten des reaktiven Brandschutzsystems wurde bereits von Raveglia [49] bei Untersuchungen an Kreishohlprofilen beobachtet. An den im Zugstabprüfofen getesteten Versuchskörpern befinden sich die Längsrisse mehrheitlich auf der Stabunterseite. Längsrisse auf der Staboberseite sind nur an den mechanisch belasteten Zuggliedern aufgetreten. Ein Einfluss der Position der Thermoelemente auf die Längsrisse konnte nicht festgestellt werden.

Da die Aufschäumung des reaktiven Brandschutzsystems vorwiegend senkrecht zur Bauteiloberfläche stattfindet, entstehen durch die Längenausdehnung der Zugglieder im reaktiven Brandschutzsystem Zugspannungen in Stablängsrichtung. Sind diese Spannungen größer als die vorhandene Zugfestigkeit der aufschäumenden oder aufgeschäumten Brandschutzbeschichtung, bilden sich Querrisse. Neben dem thermischen Verformungsanteil aus der Erwärmung des Stahls wird die Längsausdehnung bei mechanisch belasteten Zuggliedern durch den Verformungsanteil infolge der Zugbeanspruchung erhöht. Da Querrisse vornehmlich an mechanisch belasteten Zuggliedern auftreten, ist ein Einfluss der Zugbeanspruchung vorhanden.

Für mechanisch belastete Zugglieder ergeben sich aufgrund des dreidimensionalen Spannungszustandes, der aus den Stablängs- und Umfangsspannungen resultiert, besonders hohe Anforderungen für das reaktive Brandschutzsystem. Die Rissbildung findet vornehmlich in der Aufschäumphase statt, erfolgt unkontrolliert und lässt sich kaum vorhersagen. Als Folge der Rissbildung verringert sich meist die thermische Schutzwirkung des reaktiven Brandschutzsystems und es kommt zu einem schnellen Anstieg der Stahltemperaturen. Die Streuungen in den gemessenen Temperatur-Zeit-Kurven der untersuchten Zugglieder sind auf die Rissbildung zurückzuführen.

Bei Vorhandensein einer ausreichend großen Trockenschichtdicke können Risse durch Nachschäumen von noch nicht vollständig reagiertem Beschichtungsmaterial teilweise oder sogar komplett wieder geschlossen werden. Die thermische Schutzwirkung des reaktiven Brandschutzsystems kann dadurch aufrecht erhalten werden. Durch das Rissheilungs- 
vermögen führt ein Riss in der Aufschäumung des reaktiven Brandschutzsystems nicht zwangsläufig zu einer Temperaturerhöhung des Zuggliedes. Die Fähigkeit, Risse wieder zu schließen, beschränkt sich jedoch auf die Aufschäumphase der Brandschutzbeschichtung.

Die Auswertung der Brandversuche hat gezeigt, dass das Rissverhalten und das Rissheilungsvermögen des reaktiven Brandschutzsystems maßgeblich durch die Profilgeometrie des Zuggliedes, die Trockenschichtdicke der Brandschutzbeschichtung, der aufgebrachten Zugbeanspruchung sowie der Bauteilorientierung des Versuchskörpers beeinflusst wird. Mögliche weitere Einflüsse, welche jedoch im Rahmen der Untersuchungen nicht systematisch untersucht werden, ergeben sich aus der Geometrie des Brandraumes, der Beflammungsrichtung, der Position der Thermoelemente sowie durch eventuell am Versuchskörper vorhandene Rand- und Abschattungseffekte. Trotz der zahlreich durchgeführten Brandversuche konnte keine Gesetzmäßigkeit zur Rissbildung und dem Rissheilungsvermögen festgestellt werden. Eine Prognose zur Anzahl, Art, Form und Position der Risse ist bisher ebenfalls nicht möglich und bedarf der weiteren Untersuchung.

Neben dem Verlauf des Risses, d.h. in Stablängs- oder Stabquerrichtung, sowie dem Rissheilungsvermögen wird die Erwärmungsgeschwindigkeit des Stahls vor allem durch die Breite und Position des Risses beeinflusst. Relativ schmale Risse führen meist zu keiner Beeinträchtigung der thermischen Schutzwirkung für das Zugglied. Längsrisse auf der Staboberseite sind als besonders kritisch anzusehen. Diese können sich aufweiten und ein teilweises Abgleiten der Brandschutzbeschichtung initiieren. Eine signifikante Verschlechterung der ther-

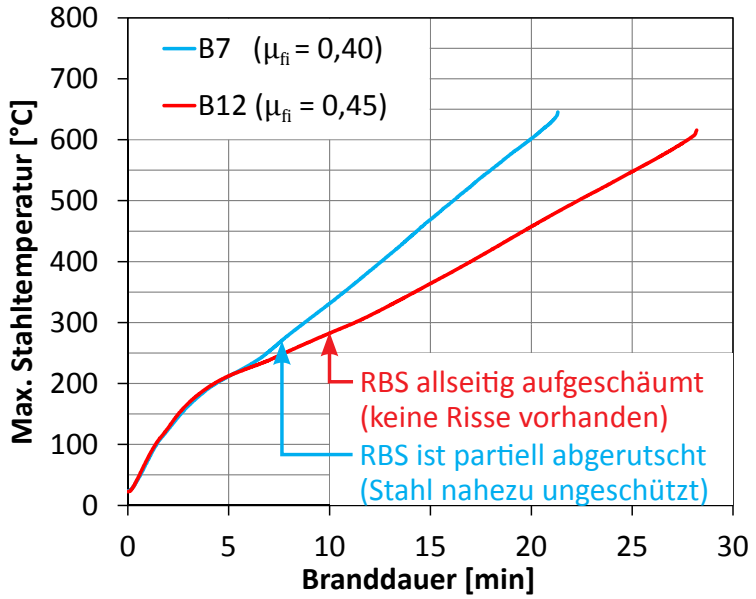

Abb. 3.22: Vergleich von Versuchskörpern mit und ohne Abgleiten des RBS (KVP D20; VR a) mischen Schutzwirkung des reaktiven Brandschutzsystems ist die Folge. Dadurch kann es bei mechanisch belasteten Zuggliedern zu einem wesentlich früheren Probenbruch kommen. Ein Vergleich des Temperaturverlaufes zwischen mechanisch belasteten Versuchskörpern mit und ohne Abgleiten des reaktiven Brandschutzsystems ist in Abb. 3.22 dargestellt. In dem Diagramm markiert das Ende der Temperaturmessung den Probenbruch. Beide Versuchskörper werden mit einer nahezu vergleichbaren Zugbeanspruchung getestet. Vorschäden sind an beiden Zuggliedern nicht vorhanden. Anhand des Diagramms ist am Versuchskörper B7 ein deutlicher Temperaturanstieg nach etwa sechs Minuten zu erkennen. Durch die Videoaufzeichnung des Brandversuches ist am Versuchskörper B7 ein Abgleiten der reaktiven Brandschutzbeschichtung nach etwa fünf Minuten zu beobachten. Die Ursache der Längsrissbildung in Verbindung mit 
dem Abgleiten des Brandschutzsystems konnte nicht festgestellt werden. Ein Zusammenhang mit dem Rissheilungsvermögen des Brandschutzsystems ist wahrscheinlich. Durch das Abgleiten der Brandschutzbeschichtung erhöht sich die Erwärmungsgeschwindigkeit des Versuchskörpers B7 auf etwa $27 \mathrm{~K} / \mathrm{min}$. Am Versuchskörper B12, der kein Abgleiten des reaktiven Brandschutzsystems und kaum Risse in der Aufschäumung aufweist, beträgt die Erwärmungsgeschwindigkeit des Stahls lediglich ca. $17 \mathrm{~K} / \mathrm{min}$. Durch eine hohe Trockenschichtdicke und damit verbundenem großen Rissheilungsvermögen ist das Brandschutzsystem in der Lage, einen auf der Staboberseite auftretenden Längsriss wieder teilweise oder sogar komplett zu schließen. Ein Abgleiten der Brandschutzbeschichtung kann dadurch verhindert werden.

\subsubsection{Einfluss der Profilgeometrie auf die Branddauer bis zum Probenbruch und auf die Stahltemperatur}

Die in den Brandversuchen getesteten Zugglieder besitzen alle einen kreisförmigen Querschnitt und unterscheiden sich hinsichtlich der Profilgeometrie jeweils in zwei der folgenden Parameter: Stabdurchmesser, Profilfaktor ( $A_{m} /$ V-Verhältnis) bzw. Profilart. Anhand der beiden Kreisvollprofile KVP D20 und KVP D30 wird der Einfluss des Stabdurchmessers und des Profilfaktors untersucht. Die Wirkung des Stabdurchmessers und der Profilart erfolgt durch Vergleich der KVP D30 mit den KHP D60. In beiden Vergleichen werden nur Versuchskörper mit mechanischer Zugbeanspruchung betrachtet.

\section{Einfluss des Stabdurchmessers und des Profilfaktors}

Bei Kreisvollprofilen hängt der Profilfaktor lediglich vom Stabdurchmesser ab und beträgt für das KVP D20 $A_{m} / V=200 m^{-1}$ und für das KVP D30 $A_{m} / V=133 m^{-1}$. Durch eine Vergrößerung des Stabdurchmessers nimmt der Profilfaktor ab. Aufgrund des direkten Zusammenhangs zwischen Stabdurchmesser und Profilfaktor wird vereinfachend nur der Einfluss des Stabdurchmessers betrachtet.

Die Wirkung des Stabdurchmessers lässt sich anhand der Branddauer bis zum Probenbruch, der Erwärmunsgeschwindigkeit des Stahls sowie des Aufschäumungs- und Rissverhaltens des reaktiven Brandschutzsystems bewerten. Allerdings werden diese Faktoren auch durch die Trockenschichtdicke der reaktiven Brandschutzbeschichtung sowie vom Lastausnutzungsgrad beeinflusst. Die Auswirkungen dieser beiden Einflussfaktoren sind im Kapitel 3.7.3 und 3.7.4 beschrieben.

Eine Vergrößerung des Stabdurchmessers von 20 auf $30 \mathrm{~mm}$ führt an den mechanisch belasteten Zuggliedern zu einer Verlängerung der Branddauer bis zum Probenbruch um etwa 13 bis 26 Minuten (siehe Tabelle 3.4) und zu einer Reduzierung der Erwärmungsgeschwindigkeit (siehe Tabelle 3.5). Durch den größeren Stabdurchmesser des KVP D30 ver- 
ringert sich die Erwärmungsgeschwindigkeit gegenüber den KVP D20 um etwa 5,5 bis 9,6 K/ min. Die Wirkung des Stabdurchmessers lässt sich durch eine Reduzierung des Lastausnutzungsgrades oder eine Erhöhung der Trockenschichtdicke weiter verstärken. Inwieweit auch der Lastausnutzungsgrad im Brandfall die Wirkung des Stabdurchmessers beeinflusst, ist anhand der Ergebnisse aus den Brandversuchen nicht eindeutig feststellbar.

Tabelle 3.4: Differenz in Minuten zwischen dem Zeitpunkt des Probenbruchs der KVP D20 und KVP D30

\begin{tabular}{|l|l|l|l|l|l|l|l|c|}
\hline \multicolumn{2}{|l|}{ Lastausnutzungsgrad [-] } & $\mathbf{0 , 1 5}$ & $\mathbf{0 , 2 5}$ & $\mathbf{0 , 3 5}$ & $\mathbf{0 , 4 5}$ & $\mathbf{0 , 5 5}$ & $\mathbf{0 , 6 5}$ & Mittelwert \\
\hline $\begin{array}{l}\text { Trockenschicht- } \\
\text { dicke (DFT) }\end{array}$ & $\mathbf{2 , 5} \mathbf{~ m m}$ & 19,3 & 15,8 & 16,2 & $15,3^{*}$ & 14,8 & 13,1 & 15,7 \\
\cline { 2 - 9 } & $\mathbf{3 , 5} \mathbf{~ m m}$ & 26,4 & 22,0 & 21,1 & 19,8 & 18,2 & 18,1 & 20,9 \\
\hline
\end{tabular}

* Dieses KVP D30 wird mit einem reduzierten Lastausnutzungsgrad von $\mu_{\mathrm{fi}}=0,42$ beansprucht. Die Differenz zwischen dem Zeitpunkt des Probenbruchs ist in Minuten angegeben und berechnet sich aus dem Zeitpunkt des Probenbruchs des KVP D30 abzüglich des Zeitpunktes für das KVP D20.

Tabelle 3.5: Differenz in K/min zwischen der Erwärmungsgeschwindigkeit der KVP D20 und KVP D30

\begin{tabular}{|l|c|c|c|c|c|c|c|c|}
\hline \multicolumn{2}{|l|}{ Lastausnutzungsgrad [-] } & $\mathbf{0 , 1 5}$ & $\mathbf{0 , 2 5}$ & $\mathbf{0 , 3 5}$ & $\mathbf{0 , 4 5}$ & $\mathbf{0 , 5 5}$ & $\mathbf{0 , 6 5}$ & Mittelwert \\
\hline $\begin{array}{l}\text { Trockenschicht- } \\
\text { dicke (DFT) }\end{array}$ & $\mathbf{2 , 5} \mathbf{~ m m}$ & 5,5 & 5,8 & 6,9 & $6,2 *$ & 6,1 & 7,0 & 6,3 \\
\cline { 2 - 9 } & $\mathbf{3 , 5} \mathbf{~ m m}$ & 7,4 & 6,0 & 7,3 & 7,4 & 9,6 & 7,6 & 7,5 \\
\hline
\end{tabular}

* Dieses KVP D30 wird mit einem reduzierten Lastausnutzungsgrad von $\mu_{\mathrm{fi}}=0,42$ beansprucht.

Die Differenz zwischen der Erwärmungsgeschwindigkeit ist in $K /$ min angegeben und berechnet sich aus dem Wert des KVP D20 abzüglich des Wertes für das KVP D30.

Die Werte für die Erwärmungsgeschwindigkeit werden anhand des Verlaufs der im Brandversuch gemessenen maximalen Stahltemperatur für einen Temperaturbereich von 350 bis $550{ }^{\circ} \mathrm{C}$ berechnet, d.h. die Aufschäumungsphase des RBS ist bereits beendet.

Ein Einfluss des Stabdurchmessers auf das Aufschäumungs- und Rissverhalten des reaktiven Brandschutzsystems ist anhand der Brandversuche ebenfalls feststellbar. An den mechanisch belasteten Zuggliedern mit KVP D20 sind in den Brandversuchen zum Teil große Längsrisse aufgetreten, die nur teilweise durch das Rissheilungsvermögen der Brandschutzbeschichtung geschlossen werden konnten. Durch die Rissbildung kommt es zu einer Reduzierung der Aufschäumhöhe des reaktiven Brandschutzsystems in diesem Bereich, der in der Regel eine Verringerung der thermischen Schutzwirkung zur Folge hat. Bei einer Vergrößerung des Stabdurchmessers der Kreisvollprofile von 20 auf $30 \mathrm{~mm}$ verbessert sich das Rissheilungsvermögen der Brandschutzbeschichtung. Die Aufschäumhöhe in den Rissbereichen erhöht sich ebenfalls. Im Allgemeinen weisen die Zugglieder mit KVP D30 gegenüber den KVP D20 sowohl in Stablängsrichtung als auch in Umfangsrichtung eine gleichmäßigere Aufschäumung des reaktiven Brandschutzsystems auf (siehe Abb. 3.23). Die Höhe der Aufschäumung wird im Wesentlichen von der Trockenschichtdicke und nicht vom Stabdurchmesser beeinflusst (siehe Tabelle A.3). 

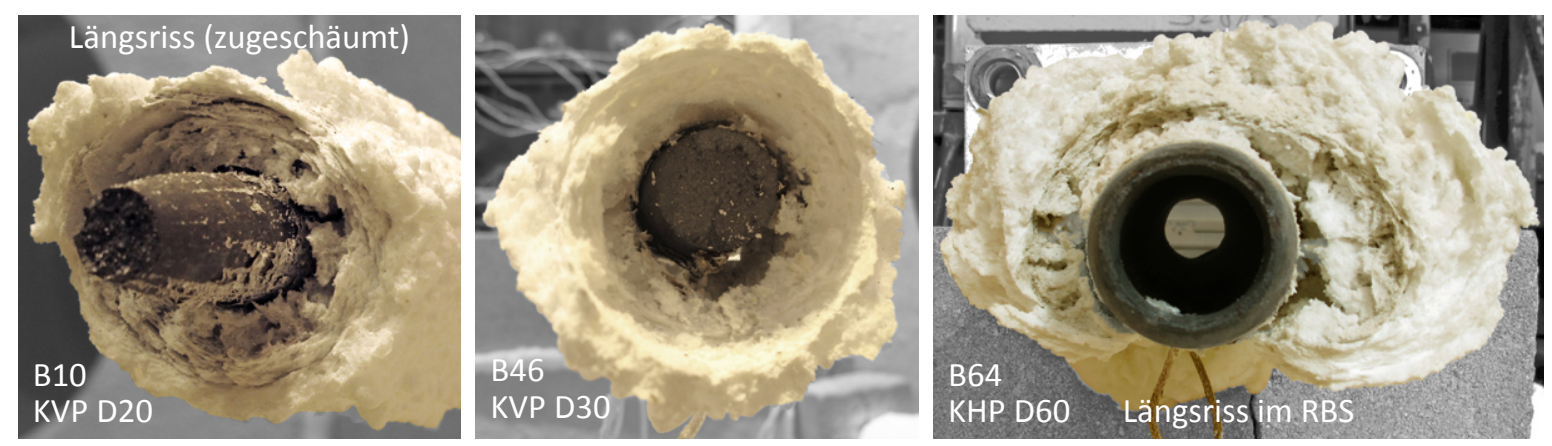

Abb. 3.23: Mechanisch belastete Zugglieder (DFT =2,5 mm) mit aufgeschäumten RBS nach dem Brandversuch (Abbildungen nicht maßstabsgerecht)

\section{Einfluss des Stabdurchmessers und der Profilart}

Die KVP D30 und die KHP D60 weisen einen kreisförmigen Querschnitt und einen Profilfaktor von $A_{m} / V=133 m^{-1}$ auf. Die Profile unterscheiden sich hinsichtlich der Profilart, d.h. Vollprofil bzw. Hohlprofil sowie der Größe des äußeren Stabdurchmessers. Außerdem wird für das KVP D30 gezogener kaltverformter Blankstahl und für das KHP D60 warmgewalzter Baustahl verwendet, wodurch sich Unterschiede in den Materialeigeschaften des Stahls ergeben. Durch die Verwendung des Lastausnutzungsgrades im Brandfall wird ein identisches Spannungsniveau für die mechanisch belasteten Zugglieder mit KVP D30 und KHP D60 erreicht. Allerdings können die Unterschiede zwischen den temperaturbedingten Abminderungsfaktoren der Festigkeitskennwerte zu einer Beeinflussung der Branddauer bis zum Probenbruch führen. Da die Materialeigenschaften des KHP D60 im Hochtemperaturbereich nicht untersucht wurden, lässt sich dieser Einfluss jedoch nicht näher quantifizieren.

Um die Auswirkung des Stabdurchmessersund der Profilart zu beurteilen, wird zunächst die Branddauer bis zum Probenbruch untersucht. Der Einfluss der unterschiedlichen Materialeigenschaften zwischen warmgewalztem und kaltverformtem Stahl wird dabei vernachlässigt. Die aus der Vergrößerung des Stabdurchmessers und der Änderung der Profilart resultierende Verlängerung der Branddauer bis zum Probenbruch ist in Tabelle 3.4 in Abhängigkeit der Trockenschichtdicke und des Lastausnutzungsgrades im Brandfall angegeben.
Tabelle 3.6: Differenz in Minuten zwischen dem Zeitpunkt des Probenbruchs der KVP D30 und KHP D60

\begin{tabular}{|l|c|c|c|c|}
\hline \multicolumn{2}{|l|}{ Lastausnutzungsgrad [-] } & $\mathbf{0 , 2 5}$ & $\mathbf{0 , 4 5}$ & $\mathbf{0 , 6 0}$ \\
\hline $\begin{array}{l}\text { Trockenschicht- } \\
\text { dicke (DFT) }\end{array}$ & $\mathbf{2 , 5} \mathbf{~ m m}$ & 0,4 & $-5,5^{*}$ & $-6,4^{* *}$ \\
\cline { 2 - 5 } & $\mathbf{3 , 5} \mathbf{~ m m}$ & $\mathbf{2 5 , 2}$ & 15,7 & $-8,8$ \\
\hline
\end{tabular}

* Das KVP D30 wird mit einem reduzierten Lastausnutzungsgrad von $\mu_{\mathrm{fi}}=0,42$ beansprucht.

** Das KVP D30 wird mit einem höheren Lastausnutzungsgrad von $\mu_{\mathrm{fi}}=0,65$ beansprucht.

Die Differenz zwischen dem Zeitpunkt des Probenbruchs ist in Minuten angegeben und berechnet sich aus dem Zeitpunkt des Probenbruchs des KHP D60 abzüglich des Zeitpunktes für das KVP D30. 
Die ermittelten Werte weisen relativ große Unterschiede auf. Trotz des wesentlich größeren Stabdurchmessers besitzen die KHP D60 bei hohen Lastausnutzungsgraden eine geringere Branddauer bis zum Probenbruch als die KVP D30. Auf Grundlage des Vergleichs der Vollprofile KVP D20 und KVP D30 hätte der größere Stabdurchmesser des KHP D60 zu längeren Branddauern bis zum Probenbruch führen müssen. Dass dies nicht eingetreten ist, lässt sich mit hoher Wahrscheinlichkeit auf die Änderung der Profilart zurückführen. Dadurch kommt es nicht nur zu einer Änderung der Materialeigenschaften des Stahls, sondern auch zu einer ausgeprägten Längsrissbildung, welche die thermische Schutzwirkung des reaktiven Brandschutzsystems verschlechtert. Erst bei vergleichsweise hohen Trockenschichtdicken von $\mathrm{DFT}=3,5 \mathrm{~mm}$ und relativ kleinen Lastausnutzungsgraden von $\mu_{\mathrm{fi}}=0,25$ und 0,45 lässt sich ein Zeitgewinn bis zum Probenbruch der KHP D60 gegenüber den KVP D30 erreichen. Dies ist wahrscheinlich auf das verbesserte Rissheilungsvermögen des reaktiven Brandschutzsystems und die daraus folgende Aufrechterhaltung der thermischen Schutzwirkung zurückzuführen. Längsrisse in der aufgeschäumten Brandschutzbeschichtung sind an den KHP D60 auch bei einer Trockenschichtdicke von 3,5 mm immer noch vorhanden. Um die thermische Schutzwirkung des reaktiven Brandschutzsystems zu prüfen, ist neben dem Stabdurchmesser und dem Profilfaktor auch die Profilart des Zuggliedes zu berücksichtigen.
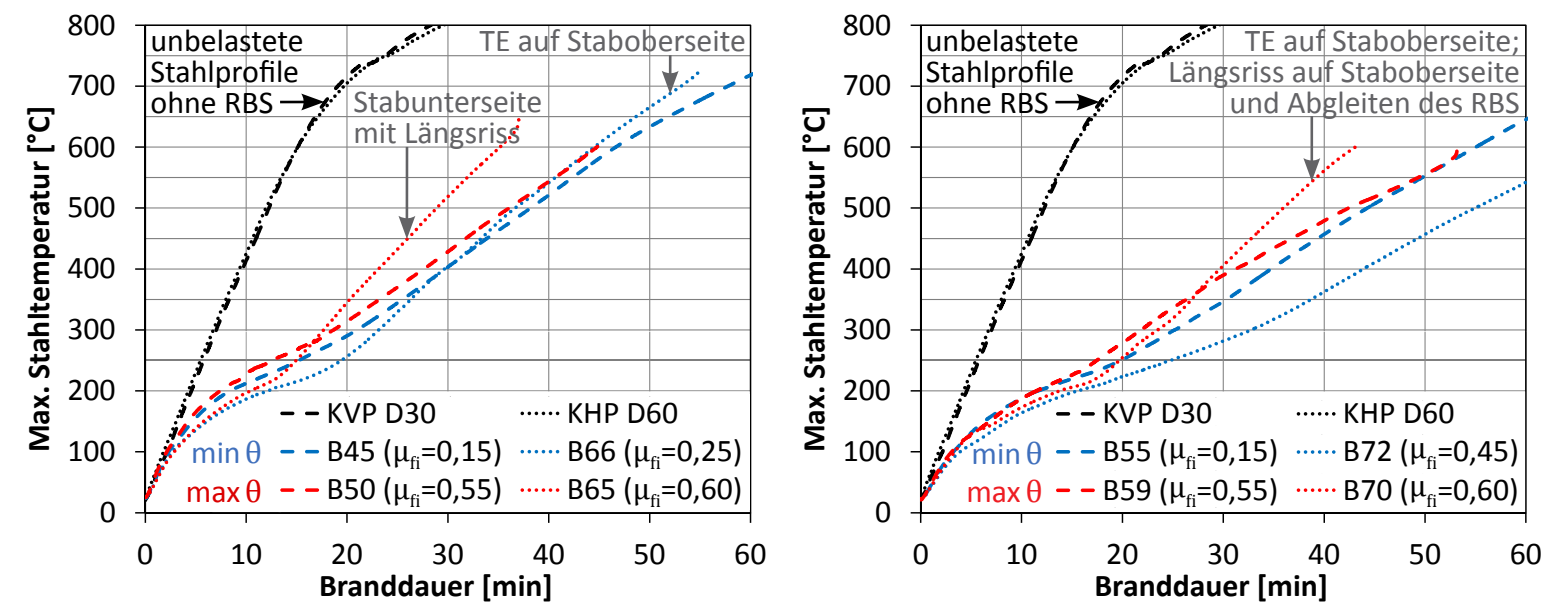

Abb. 3.24: Vergleich der kältesten ( $\min \theta)$ und heißesten (max $\theta$ ) Stahltemperaturverläufe der unter Zugbeanspruchung getesteten KVP D30 und KHP D60 (links: DFT =2,5 mm; rechts: DFT = 3,5 mm)

Um materialbedingte Einflüsse aus den unterschiedlichen Festigkeiten zwischen kaltverformten Blankstahl und warmgewalzten Baustahl auszuschließen, werden die Temperatur-Branddauer-Kurven der unter Zugbeanspruchung getesteten KVP D30 und KHP D60 miteinander verglichen (siehe Abb. 3.24). Zusätzlich zu den mechanisch belasteten Zuggliedern mit reaktiver Brandschutzbeschichtung ist in den Diagrammen für jedes Profil eine Temperatur-Branddauer-Kurve eines unbeschichteten Versuchskörpers ohne mechanische Zugbeanspruchung angegeben. Anhand der Diagramme zeigt sich, dass die Stahltemperaturen zwischen dem ungeschützten KVP D30 und KHP D60 nahezu identisch sind. Ein 
Einfluss der Profilart, des Stabdurchmessers und des Profilfaktors ist an den ungeschützten Profilen nicht vorhanden. Für den Vergleich der Zugglieder mit reaktiver Brandschutzbeschichtung werden aus einer Versuchsreihe jeweils die Versuchskörper mit dem kältesten (rot) und dem heißesten Temperaturverlauf (blau) ausgewählt. Die Stahltemperaturen beziehen sich dabei immer auf die Messstelle mit der maximal gemessenen Probekörpertemperatur. Anhand der Diagramme in Abb. 3.24 zeigt sich, dass sich die KHP D60 in der Aufschäumphase, d.h. die ersten 10 bis 20 Minuten des Brandversuches, etwas langsamer erwärmen als die KVP D30. Ein Einfluss der Profilart in Verbindung mit dem Stabdurchmesser ist daher vorhanden. Unabhängig von der untersuchten Trockenschichtdicke zeigen die KVP D30 nur geringe Unterschiede zwischen den kältesten und heißesten Temperaturverläufen. Dies ist auf das relativ homogene Aufschäumverhalten und das gute Rissheilungsvermögen des reaktiven Brandschutzsystems zurückzuführen. Im Gegensatz dazu sind zwischen den untersuchten KHP D60 große Temperaturunterschiede vorhanden. Bei einer Trockenschichtdicke von 2,5 mm treten diese nach etwa 12 Minuten und bei einer Trockenschichtdicke von 3,5 mm nach ca. 18 Minuten auf. Ursächlich für die Unterschiede zwischen den kältesten und heißesten Temperaturverläufen sind die bei einigen KHP D60 aufgetretenen großen Längsrisse im reaktiven Brandschutzsystem, welche teilweise auch zu einem Abgleiten der Brandschutzbeschichtung geführt haben. Dadurch wird die thermische Schutzwirkung erheblich reduziert und ein schneller Anstieg der Stahltemperaturen und ein frühzeitiger Probenbruch der mechanisch belasteten Zugglieder ist die Folge. Das Rissheilungsvermögen der Brandschutzbeschichtung reicht bei einigen KHP D60 nicht aus, um die entstandenen Risse wieder zu schließen. Für die Beurteilung des Einflusses der Profilgeometrie auf die Branddauer bis zum Probenbruch und die Stahltemperatur ist es daher nicht ausreichend, nur den Profilfaktor und den Stabdurchmesser eines Zuggliedes zu betrachten, sondern auch die Profilart muss berücksichtigt werden. Die Auswertung der Brandversuche hat gezeigt, dass zwischen den untersuchten Kreisvollprofilen und Kreishohlprofilen trotz des gleichen Profilfaktors Unterschiede bestehen. Eine Übertragung der Ergebnisse zum Aufschäumungs- und Rissverhalten sowie der thermischen Schutzwirkung reaktiver Brandschutzsysteme ist daher zwischen verschiedenen Profilarten nicht möglich.

\subsubsection{Einfluss der Trockenschichtdicke auf die Branddauer bis zum Proben- bruch und auf die Stahltemperatur}

Für die Untersuchung des Einflusses der Trockenschichtdicke des reaktiven Brandschutzsystems auf die Branddauer bis zum Probenbruch und die Stahltemperatur werden exemplarisch die mechanisch belasteten Zugglieder mit KVP D20 und einem Lastausnutzungsgrad von $\mu_{\mathrm{fi}}=0,45$ ausgewertet. Die Trockenschichtdicke der reaktiven Brandschutzbeschichtung variiert von 1,5 bis 4,5 mm. Der Zeitpunkt des Probenbruchs der Zugglieder lässt sich im Verformungs-Branddauer-Diagramm (Abb. 3.25) durch die fast senkrechte Verformungszunahme oder das Ende der Temperaturaufzeichnung im Stahltemperatur-Branddauer-Dia- 
gramm (Abb. 3.25) erkennen. Anhand beider Diagramme zeigt sich, dass eine Erhöhung der Trockenschichtdicke des reaktiven Brandschutzsystems zu einer Verlängerung der Branddauer bis zum Probenbruch der Zugglieder führt. Dies ist darauf zurückzuführen, dass sich mit zunehmender Trockenschichtdicke die thermische Schutzwirkung des reaktiven Brandschutzsystems verbessert. Durch die langsamere Erwärmung des Stahls bleibt dessen Festigkeit länger erhalten. Dieses Verhalten ist bereits von Brandprüfungen an beschichteten Stahlträgern und -stützen bekannt. Im Hinblick auf die maximale Stahltemperatur zum Zeitpunkt des Probenbruchs hat die Trockenschichtdicke keinen Einfluss, da alle Zugglieder mit dem gleichen Lastausnutzungsgrad belastet werden. Mit Ausnahme des Versuchskörpers B4, beträgt die zum Zeitpunkt des Probenbruchs gemessene maximale Stahltemperatur etwa $615^{\circ} \mathrm{C}$. Als mögliche Ursache für die Abweichung ist die Messung der Probekörpertemperatur zu nennen, welche nicht immer genau mit der Bruchstelle übereinstimmt.
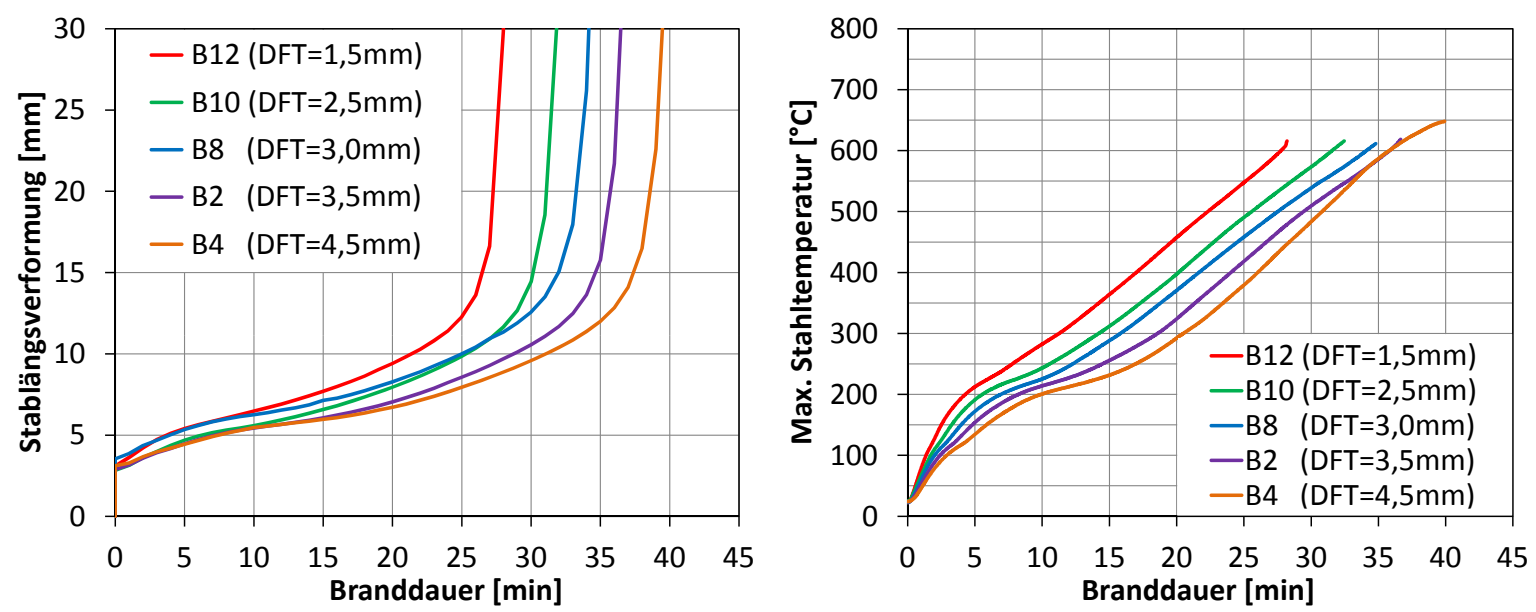

Abb. 3.25: Einfluss der Trockenschichtdicke auf Branddauer bis zum Probenbruch (links) und auf die Stahltemperatur (rechts) anhand ausgewählter Zugglieder mit KVP D20 und einem Lastausnutzungsgrad im Brandfall von $\mu_{\mathrm{fi}}=0,45$

Tabelle 3.7: Einfluss des Trockenschichtdicke auf den Zeitpunkt bis zum Erreichen einer Stahltemperatur von $550^{\circ} \mathrm{C}$

\begin{tabular}{|l|c|c|c|c|c|c|c|c|}
\hline Profilart & \multicolumn{3}{|c|}{ KVP D20 } & \multicolumn{2}{c|}{ KVP D30 } & \multicolumn{2}{c|}{ KHP D60 } \\
\hline \multicolumn{2}{|l|}{ Versuchsreihe (VR) } & a & b & c & d & e & f & g \\
\hline \multicolumn{2}{|l|}{ Trockenschichtdicke (DFT) } & $\mathbf{1 , 5} \mathbf{~ m m}$ & $\mathbf{2 , 5} \mathbf{~ m m}$ & $\mathbf{3 , 5} \mathbf{~ m m}$ & $\mathbf{2 , 5} \mathbf{~ m m}$ & $\mathbf{3 , 5} \mathbf{~ m m}$ & $\mathbf{2 , 5} \mathbf{~ m m}$ & $\mathbf{3 , 5} \mathbf{~ m m}$ \\
\hline Frühester Zeitpunkt & {$[\mathrm{min}]$} & 18,0 & 28,0 & 32,2 & 40,6 & 49,3 & 31,9 & 39,2 \\
\hline Spätester Zeitpunkt & {$[\mathrm{min}]$} & 25,1 & 30,0 & 33,0 & 42,4 & 50,1 & 40,7 & 64,6 \\
\hline Zeitdifferenz* & {$[\mathrm{min}]$} & 7,1 & 2,0 & 0,8 & 1,8 & 0,8 & 8,8 & 25,4 \\
\hline Zeitpunkt (Mittelwert) & {$[\mathrm{min}]$} & 21,6 & 29,0 & 32,5 & 41,3 & 48,8 & 35,2 & 54,9 \\
\hline Zeitgewinn** & {$[\mathrm{min}]$} & - & 7,4 & 3,5 & - & 7,5 & - & 19,7 \\
\hline
\end{tabular}

* Die Zeitdifferenz bezieht sich auf den Unterschied zwischen frühesten und spätesten Zeitpunkt.

** Der Zeitgewinn berechnet sich aus der Differenz der Mittelwerte zweier benachbarter VR gleicher Profilart. 
Um den Einfluss der Trockenschichtdicke besser bewerten zu können, werden für alle in den Brandversuchen getesteten mechanisch belasteten Zugglieder die Zeitpunkte bis zum Erreichen einer Stahltemperatur von $550{ }^{\circ} \mathrm{C}$ bestimmt. Die als Vergleichskriterium zugrunde gelegte Temperatur entspricht in etwa dem Zeitpunkt des Probenbruchs eines voll ausgelasteten Zuggliedes $\left(\mu_{\mathrm{fi}}=0,65\right)$. Darüber hinaus sind bei höheren Stahltemperaturen aufgrund des Probenbruchs nicht mehr bei allen mechanisch belasteten Zuggliedern entsprechende

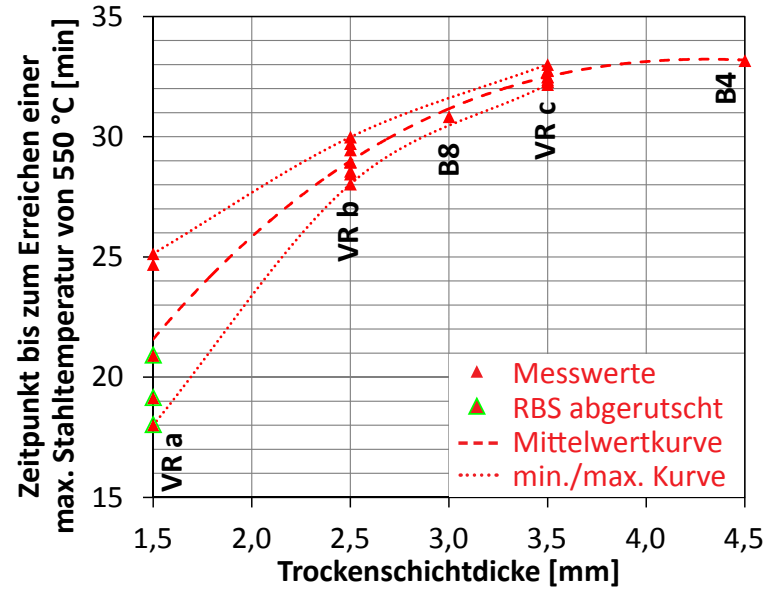

Abb. 3.26: Zeitpunkt bis zum Erreichen einer Stahltemperatur von $550^{\circ} \mathrm{C}(\mathrm{VR} a-\mathrm{c})$ Temperaturdaten vorhanden. Für den Vergleich werden auch Versuchskörper, an denen im Vorfeld des Brandversuchs Beschädigungen im reaktiven Brandschutzsystem vorgenommen wurden, berücksichtigt. In Tabelle 3.7 sind die minimalen, mittleren und maximalen Zeitpunkte für das Erreichen der Vergleichstemperatur von $550^{\circ} \mathrm{C}$ in Abhängigkeit von dem verwendeten Stahlprofil und der Trockenschichtdicke angegeben. Generell zeigt sich, dass die Zeitpunkte bis zum Erreichen der Vergleichstemperatur aufgrund der besseren thermischen Schutzwirkung des reaktiven Brandschutzsystems mit zunehmender Trockenschichtdicke ansteigen. Bei Betrachtung der in Abb. 3.26 dargestellten Werte für die einzelnen Zugglieder mit KVP D20 fällt auf, dass der Zeitgewinn bis zum Erreichen der Vergleichstemperatur von $550{ }^{\circ} \mathrm{C}$ mit steigender Trockenschichtdicke immer geringer ausfällt. Folglich lässt sich durch eine Erhöhung der Trockenschichtdicke die thermische Schutzwirkung der Brandschutzbeschichtung nicht beliebig weit steigern. Der Zeitgewinn entwickelt sich im Verhältnis zur Trockenschichtdicke unterproportional.

Durch das unterschiedliche Aufschäumungs- und Rissverhalten des reaktiven Brandschutzsystems sind auch innerhalb einer Versuchsreihe Zeitunterschiede beim Erreichen der gewählten Vergleichstemperatur vorhanden. Die großen Unterschiede in der VR a sind darauf zurückzuführen, dass an einigen Zuggliedern infolge von Längsrissen auf der Staboberseite ein teilweises Abgleiten der Brandschutzbeschichtung aufgetreten ist. Eine Vergrößerung der Trockenschichtdicke verbessert das Rissheilungsvermögen und führt zu einer gleichmäßigeren Aufschäumung des reaktiven Brandschutzsystems, wodurch die Streuungen in den Versuchsreihen reduziert werden. Beispielsweise verringert sich der Zeitunterschied zwischen minimalem und maximalem Zeitpunkt bis zum Erreichen der gewählten Vergleichstemperatur von ca. 7 Minuten in VR a auf 2 Minuten in VR b und etwa 1 Minute in VR c. Die für eine Vergleichstemperatur von $550^{\circ} \mathrm{C}$ beschriebenen Aussagen zum Einfluss der Trockenschichtdicke lassen sich auch auf andere Stahltemperaturen übertragen. 


\subsubsection{Einfluss des Lastausnutzungsgrades auf die Branddauer bis zum Pro- benbruch und auf die Stahltemperatur}

Im Laufe des Brandversuchs nimmt die Stahltemperatur des Zuggliedes aufgrund der Brandbeanspruchung zu. Die Erwärmung des Stahls wird maßgeblich von der thermischen Schutzwirkung des reaktiven Brandschutzsystems beeinflusst. Mit zunehmender Temperatur verliert der Stahl an Festigkeit. Der Einfluss der Temperatur auf die Stahlfestigkeit wird in der Regel mittels Abminderungsfaktoren, welche sich aus dem Verhältnis zwischen Festigkeit bei erhöhter Temperatur und Raumtemperatur berechnen, beschrieben. Sind die infolge einer äußeren Zugbeanspruchung im Zugglied vorhandenen Spannungen größer als die temperaturbedingte Stahlfestigkeit, kommt es zum Bruch des Bauteils. Durch eine Reduzierung des Lastausnutzungsgrades im Brandfall und die damit verbundene Verringerung der Zugbeanspruchung können die im Zugglied vorhandenen Stahlspannungen vermindert werden. Allerdings hat dies zur Folge, dass auch die Beanspruchung des Zuggliedes im Kaltfall reduziert werden muss. In Abb. 3.27 und Abb. 3.28 sind für die mechanisch belasteten Zugglieder mit Kreisvollprofil die Stablängsverformungen über die Branddauer bis zu einem Wert von $30 \mathrm{~mm}$ dargestellt. Für die Zugglieder mit KHP D60 sind entsprechende Diagramme in Abb. 3.29 angegeben. Der Zeitpunkt des Probenbruchs der Versuchskörper ist in den Diagrammen nicht enthalten, lässt sich aber anhand der großen Zunahme der Stablängsverformungen gut abschätzen.
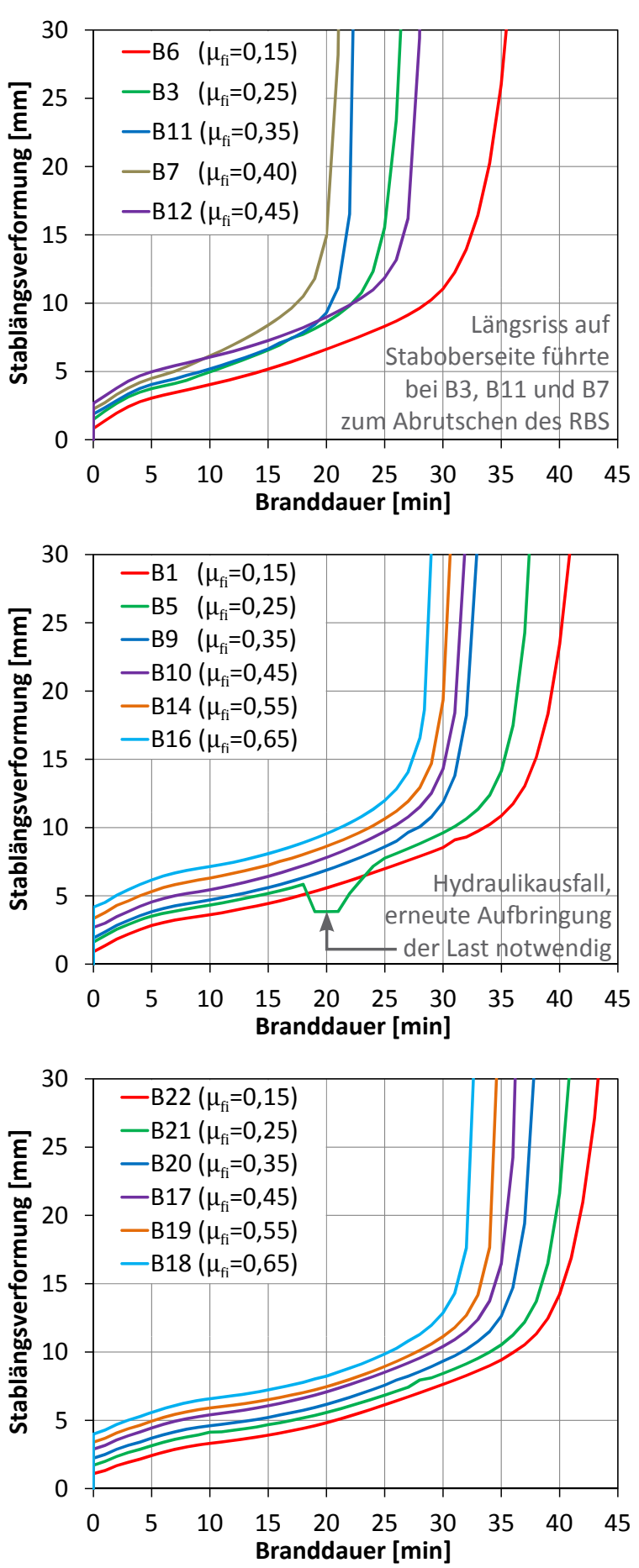

Abb. 3.27: Einfluss des Lastausnutzungsgrades im Brandfall auf die Stablängsverformung (oben: VR a; mitte: VR $b$; unten: VR c) 

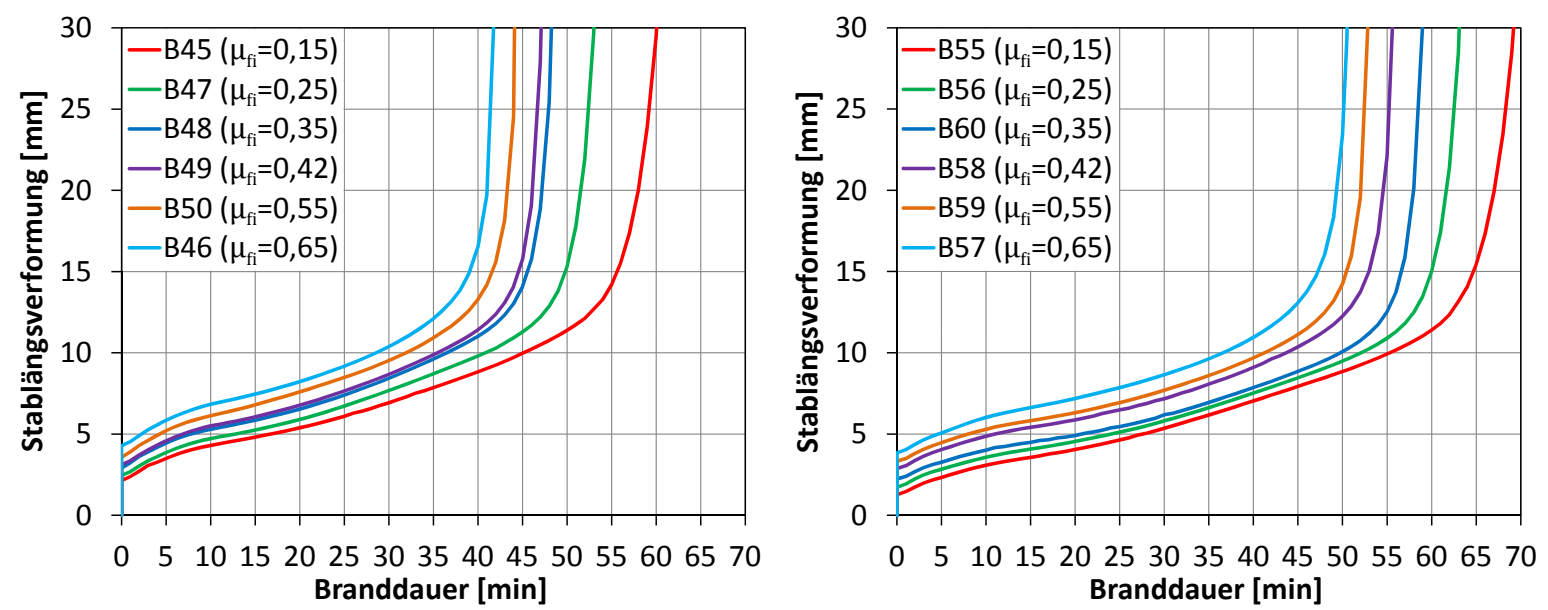

Abb. 3.28: Einfluss des Lastausnutzungsgrades im Brandfall auf die Stablängsverformung im Brandverusch (links: VR d; rechts: VR e)
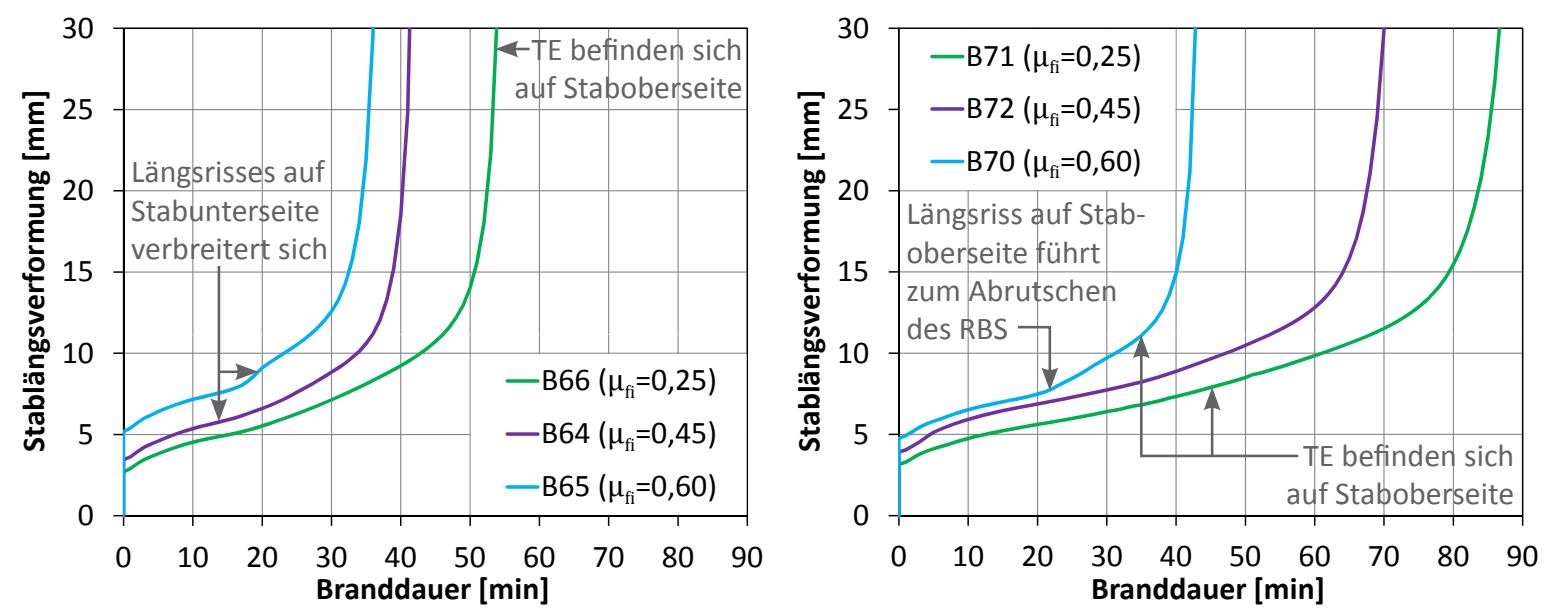

Abb. 3.29: Einfluss des Lastausnutzungsgrades im Brandfall auf die Stablängsverformung im Brandversuch (links: VR f; rechts: VR g)

In Abb. 3.30 ist die Branddauer bis zum Probenbruch der mechanisch belasteten Zugglieder mit Kreisvollprofil in Abhängigkeit vom Lastausnutzungsgrad im Brandfall dargestellt. Um den Einfluss des Lastausnutzungsgrades im Brandfall bewerten zu können, werden nur Zugglieder der selben Versuchsreihe miteinander verglichen. Wie erwartet, führt eine Reduzierung des Lastausnutzungsgrades in der Regel zu einer Erhöhung der Zeitdauer bis zum Probenbruch. Es zeigt sich, dass auch die Profilgeometrie des Zuggliedes (siehe Kapitel 3.7.2) und die Höhe der Trockenschichtdicke des reaktiven Brandschutzsystems (siehe Kapitel 3.7.3) die Branddauer bis zum Probenbruch beeinflussen. Die großen Zeitunterschiede in der VR a lassen sich auf das unterschiedliche Aufschäumungs- und Rissverhalten des reaktiven Brandschutzsystems zurückführen. An den Versuchskörpern mit einem Lastausnutzungsgrad im Brandfall von $\mu_{\mathrm{fi}}=0,25$ bis 0,42 ist ein teilweises Abgleiten des 
reaktiven Brandschutzsystems zu beobachten, wodurch die Stahltemperaturen schnell ansteigen und ein frühzeitiges Versagen der Zugglieder eintritt. Die Ergebnisse der VR b und c zeigen, dass sich eine signifikante Erhöhung der Branddauer bis zum Probenbruch erst in Bereichen geringer Lastausnutzungsgrade $\left(\mu_{\mathrm{fi}}<0,35\right)$ einstellt. Für höhere Werte des Lastausnutzungsgrades fällt der Zeitgewinn infolge einer zehnprozentigen Reduzierung des Lastniveaus vergleichsweise gering aus, d.h. etwa 1 bis 3 Minuten. Für Lastausnutzungsgrade im Brandfall zwischen $0,35<\mu_{\mathrm{fi}} \leq 0,65$ ist ein nahezu linearer Zusammenhang zwischen Branddauer bis zum Probenbruch und Lastausnutzungsgrad im Brandfall vorhanden. Eine zehnprozentige Reduzierung des Lastausnutzungsgrades im Brandfall erhöht die Branddauer bis zum Probenbruch um die in Tabelle 3.8 angegeben Werte. Für den umgekehrten Fall, d.h. eine Erhöhung des Lastausnutzungsgrades, ist die Branddauer um die in der Tabelle angegebenen Werte zu reduzie-

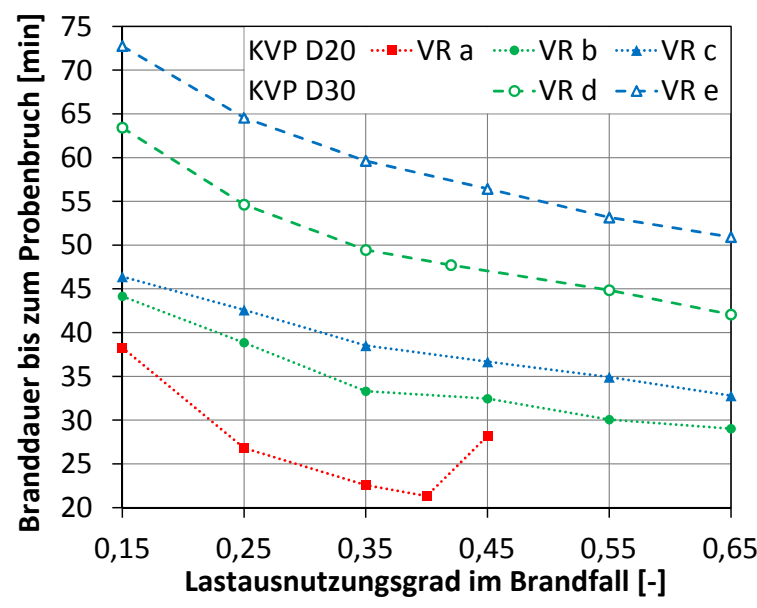

Abb. 3.30: Einfluss des Lastausnutzungsgrades auf die Branddauer bis zum Probenbruch

Tabelle 3.8: Erhöhung der Branddauer in Minuten bis zum Probenbruch durch eine zehnprozentige Reduzierung des Lastausnutzungsgrades im Brandfall

\begin{tabular}{|l|c|c|c|c|c|}
\hline Profil & \multicolumn{3}{|c|}{ KVP D20 } & \multicolumn{2}{c|}{ KVP D30 } \\
\hline VR & a & b & c & d & e \\
\hline DFT & $\mathbf{1 , 5} \mathbf{m m}$ & $\mathbf{2 , 5} \mathbf{m m}$ & $\mathbf{3 , 5} \mathbf{m m}$ & $\mathbf{2 , 5} \mathbf{m m}$ & $\mathbf{3 , 5} \mathbf{m m}$ \\
\hline$\Delta \mathrm{T}^{*}$ & - & 1,4 & 1,9 & 2,5 & 2,9 \\
\hline
\end{tabular}

Die Streuungen in VR a erlauben keine Angabe für $\Delta \mathrm{T}$. * Zeitgewinn in Minuten für mechanisch belastete Stahlzugglieder mit einem Lastausnutzungsgrad im Brandfall zwischen 0,35 $<\mu_{\mathrm{fi}} \leq 0,65$. ren. Die Wirkung des Lastausnutzungs-

grades im Brandfall auf die Branddauer bis zum Probenbruch lässt sich durch eine Erhöhung der Trockenschichtdicke oder Vergrößerung des Stabdurchmessers ebenso verstärken. Für die KHP D60 können aufgrund der relativ geringen Anzahl an getesteten Zuggliedern und der aufgetretenen großen Streuungen zwischen den einzelnen Versuchskörpern, welche auf die unterschiedliche Rissbildung und das teilweise Abgleiten der reaktiven Brandschutzbeschichtung zurückzuführen sind, keine eindeutigen Werte für die Erhöhung der Branddauer bis zum Probenbruch infolge einer zehnprozentigen Reduzierung des Lastausnutzungsgrades ermittelt werden. Die Brandversuche an mechanisch belasteten Zuggliedern mit KHP D60 bestätigen, dass eine Reduzierung des Lastausnutzungsgrades im Brandfall die Zeitdauer bis zum Probenbruch verlängert.

Neben der Branddauer bis zum Probenbruch wird auch der Zusammenhang zwischen der im Brandversuch gemessenen maximalen Stahltemperatur zum Zeitpunkt des Probenbruchs 
und dem Lastausnutzungsgrad im Brandfall untersucht. Die maximale Stahltemperatur tritt immer an der Bruchstelle des Zuggliedes auf. Die Auswertung der mechanisch belasteten Zugglieder mit KVP D20 in Abb. 3.31 zeigt, dass mit steigendem Lastausnutzungsgrad im Brandfall die gemessene maximale Stahltemperatur zum Zeitpunkt des Probenbruchs abnimmt. Grund hierfür ist die aus der Erhöhung des Lastausnutzungsgrades im Brandfall resultierende Zunahme der Zugbeanspruchung, d.h. Prüflast. Überschreitet die Zugbeanspruchung den Bauteilwiderstand kommt es zum Probenbruch des Zuggliedes. Folglich ist bei Erhöhung des Lastausnutzungsgrades im Brandfall ein höherer Bauteilwiderstand erforderlich. Da der Bauteilwiderstand infolge der Temperaturabhängigkeit der Stahlfestigkeit mit steigender Temperatur abnimmt, tritt der Probenbruch bei zunehmenden Lastausnutzungsgrad im Brandfall bereits bei geringeren Stahltemperaturen ein. In Abhängigkeit vom gewählten Lastausnutzungsgrad im Brandfall tritt der Probenbruch der mechanisch belasteten Zugglieder mit KVP D20 zwischen einer maximalen Stahltemperatur von etwa 550 bis $750{ }^{\circ} \mathrm{C}$ ein. Die Höhe der Trockenschichtdicke des reaktiven Brandschutzsystems wirkt sich aufgrund der unterschiedlichen Erwärmungsgeschwindigkeit des Stahls auf den Zeitpunkt des Probenbruchs aus, hat jedoch keinen Einfluss auf die maximale Stahltemperatur beim Probenbruchs des Zuggliedes. Diese hängt allein von der aufgebrachten Zugbeanspruchung, d.h. Lastausnutzungsgrad im Brandfall und der temperaturbedingten Festigkeit des Stahls ab. Dass zwischen den einzelnen Versuchsreihen mit KVP D20 die maximalen Stahltemperaturen zum Zeitpunkt des Probenbruchs geringfügig voneinander abweichen, ist auf die nicht immer an der Bruchstelle erfolgte Messung der Stahltemperaturen zurückzuführen. Zudem können auch kleine Imperfektionen im Materialgefüge oder dem Stabquerschnitt einen verfrühten Probenbruch verursachen, wodurch sich für das Zugglied geringere Stahltemperaturen ergeben.

Das Verhältnis zwischen maximaler Stahltemperatur zum Zeitpunkt des Probenbruchs und Lastausnutzungsgrad im Brandfall kann für die mechanisch belasteten Zugglieder mit KVP D20 vereinfacht durch eine lineare Funktion oder präziser mittels einer quadratischen Funktion approximiert werden (siehe Abb. 3.31). Trotz der voneinander abweichenden Stahlfestigkeit und der unterschiedlichen Stabdurchmesser zeigen die maximalen Stahltemperaturen zum Zeitpunkt des Probenbruchs der KVP D30 eine gute Übereinstimmung mit den Werten der KVP D20. Dies ist darauf zurückzuführen, dass Stahlfestigkeit und Stabdurchmesser bei der Bestimmung der Zugbeanspruchung berücksichtigt werden und sich dadurch gleiche Lastausnutzungsgrade im Brandfall ergeben. Darüber hinaus bestehen beide Profile aus gezogenem kaltverformten Blankstahl der Festigkeitsklasse S355, wodurch eine Übertragung der temperaturbedingten Abminderungsfaktoren für die Stahlfestigkeit gegeben ist. In Abb. 3.31 sind auch für die getesteten KHP D60 die maximalen Stahltemperaturen zum Zeitpunkt des Probenbruchs in Abhängigkeit von dem Lastausnutzungsgrad im Brandfall angegeben. Die Stahltemperaturen der KHP D60 liegen deutlich über den Werten der Kreisvollprofile. Dies ist darauf zurückzuführen, dass die Kreishohlprofile aus warmgewalztem Baustahl bestehen und dieser gegenüber dem kaltverformten Blankstahl der Kreisvollprofile andere tem- 
peraturbedingte Abminderungsfaktoren für die Stahlfestigkeit aufweist. Die relativ großen Unterschiede zwischen den maximalen Stahltemperaturen der VR $\mathrm{f}$ und $\mathrm{g}$ sind auf die Temperaturmessstellen zurückzuführen, welche sich nicht immer mit der Bruchstelle decken. Im Bereich des Probenbruchs sind voraussichtlich höhere Stahltemperaturen vorhanden. Außerdem erfolgt die Temperaturmessung lediglich auf einer Stabseite, d.h. Stabober- oder Stabunterseite. Temperaturunterschiede über den Stabquerschnitt, die sich aus dem größeren Stabdurchmesser in Zusammenhang mit der Profilart und dem Aufschäumungs- und Rissverhalten des reaktiven Brandschutzsystems ergeben können, bleiben unberücksichtigt.
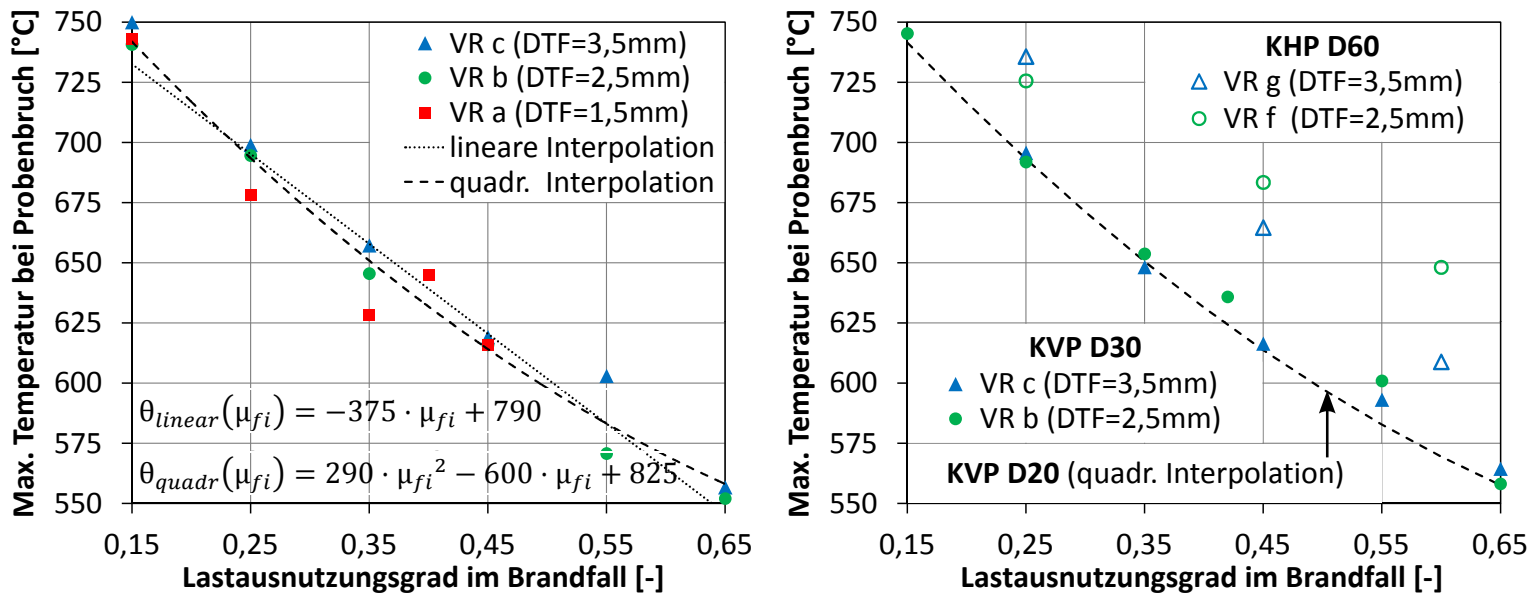

Abb. 3.31: Einfluss des Lastausnutzungsgrades auf die maximale Stahltemperatur zum Zeitpunkt des Probenbruchs (links: Vergleich der KVP D20; rechts: Vergleich der KVP D30 und KHP D60)

\subsubsection{Einfluss der axialen Zugbeanspruchung auf die Stahltemperatur}

Laut den Zulassungsgrundsätzen des DIBt [26] sind reaktive Brandschutzsysteme auf Stahlbauteilen entsprechend ihrem vorgesehenen Anwendungsbereich zu prüfen. Die sich aus dem späteren Anwendungsbereich ergebenden Beanspruchungen sind dabei zu berücksichtigen. Für Stahlbauteile, die als Träger oder Stütze verwendet werden, sind neben unbelasteten Brandprüfungen auch Versuche an belasteten Bauteilen durchzuführen. Entsprechend den Zulassunggrundsätzen werden die mit reaktiver Brandschutzbeschichtung versehenen Zugglieder mit und ohne axialer Zugbeanspruchung getestet. Im Gegensatz zu den mechanisch belasteten Versuchskörpern treten zwischen den unbelasteten Vergleichsprüfkörpern in der Regel nur relativ kleine Unterschiede der gemessenen maximalen Stahltemperatur auf.

Um den Einfluss der Zugbeanspruchung auf die Temperaturentwicklung zu untersuchen, werden in jeder Versuchsreihe zwei Brandprüfungen durchgeführt, bei der ein belastetes und ein unbelastetes Zugglied simultan und in horizontaler Bauteilorientierung geprüft werden. Für den Vergleich der maximalen Stahltemperatur wird aus jeder Versuchsreihe 
das Probenpaar mit der größten Temperaturdifferenz zwischen belastetem und unbelastetem Versuchskörper ausgewählt. Mit Ausnahme der Bauteillänge, der Anzahl der Probekörperthermoelemente und der Höhe der Zugbeanspruchung sind beide Versuchskörper identisch. Um eine Beeinflussung durch Abschattungseffekte oder herabfallende Teile der Aufschäumung des Brandschutzsystems zu vermeiden, werden die unbelasteten Vergleichsprüfkörper im Abstand von etwa $250 \mathrm{~mm}$ in vertikaler und horizontaler Richtung vom belasteten Zugglied angeordnet. Dies entspricht den Vorgaben zur Abstandregelung aus DIN EN 1363-1 [07]. Der Vergleich der maximalen Stahltemperaturen über die Branddauer ist für die ausgewählten mechanisch belasteten und unbelasteten Versuchskörper in Abb. 3.32 dargestellt. Der Verlauf der StahltemperaturZeitkurven zwischen Zuggliedern mit und ohne mechanischer Zugbeanspruchung ist prinzipiell ähnlich. Dies ist auf die identische Profilgeometrie der Probenpaare zurückzuführen. Die Erhöhung der Trockenschichtdicke des reaktiven Brandschutzsystems führt zu einer Verbesserung der thermischen Schutzwirkung der Brandschutzbeschichtung, wodurch sich die Erwärmung des Stahls verlangsamt. Zugglieder mit mechanischer Zugbeanspruchung zeigen in der Regel höhere maximale Stahltemperaturen als die unbelasteten Vergleichsprüfkörper. Ursache hierfür ist die infolge der Zugbeanspruchung erhöhte Rissneigung des aufschäumenden reaktiven Brandschutzsystems. Dadurch verschlechtert sich die thermische Schutzwirkung der
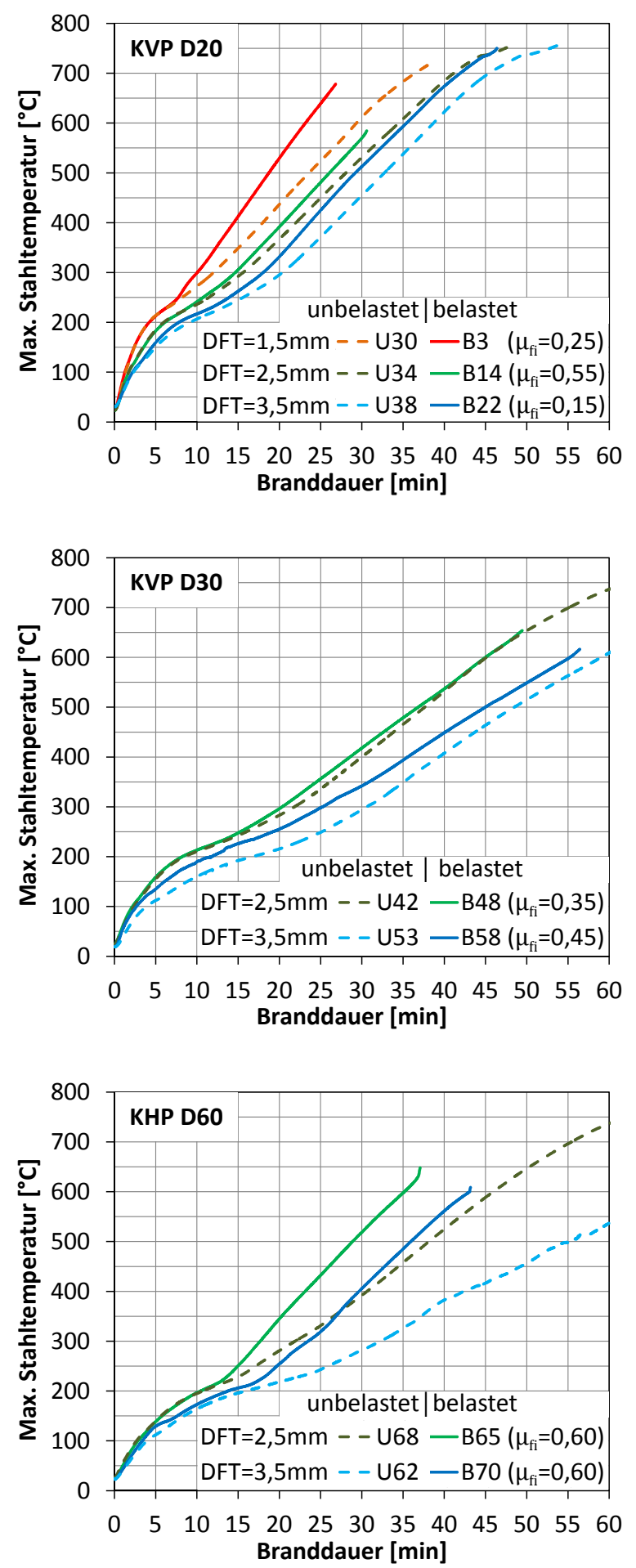

Abb. 3.32: Vergleich der maximalen Stahltemperaturen zwischen Zuggliedern mit und ohne mechanischer Zugbeanspruchung (oben: KVP D20; mitte: KVP D30; unten: KHP D60) 
Brandschutzbeschichtung und die Erwärmungsgeschwindigkeit des Stahls nimmt zu. Des Weiteren besteht bei Zuggliedern mit mechanischer Zugbeanspruchung die Gefahr eines Abgleitens des aufschäumenden reaktiven Brandschutzsystems, wodurch die Stahltemperaturen ebenfalls rapide ansteigen, z.B. Versuchskörper B3, B65 und B70.

In Abb. 3.33 ist die gemessene maximale Stahltemperatur der mechanisch belasteten und unbelasteten Zugglieder mit Kreisvollprofil nach einer Branddauer von 20 bzw. 30 Minuten in Abhängigkeit des Lastausnutzungsgrades im Brandfall dargestellt. Die Anstiege in den Trendlinien der einzelnen Versuchsreihen zeigen, dass die Zugbeanspruchung mit zunehmender Branddauer zu einer Erhöhung der maximalen Stahltemperatur führt. Versuchskörper ohne Zugbeanspruchung weisen in den Brandversuchen in der Regel eine bessere thermische Schutzwirkung des reaktiven Brandschutzsystems auf. Bei ausschließlicher Prüfung unbelasteter Versuchskörper kann dies zu einer Überschätzung der Feuerwiderstandsdauer führen. Die Festlegung eines Korrekturfaktors zwischen den Temperaturdaten mechanisch belasteter und unbelasteter Zuggliedern gestaltet sich aufgrund der kaum vorhersagbaren Rissbildung und des Rissheilungsvermögens der reaktiven Brandschutzbeschichtung schwierig. Insbesondere das Risiko eines Abgleitens des aufschäumenden Brandschutzsystems lässt sich in der Regel nur mit Hilfe von Zuggliedern mit mechanischer Zugbeanspruchung überprüfen.
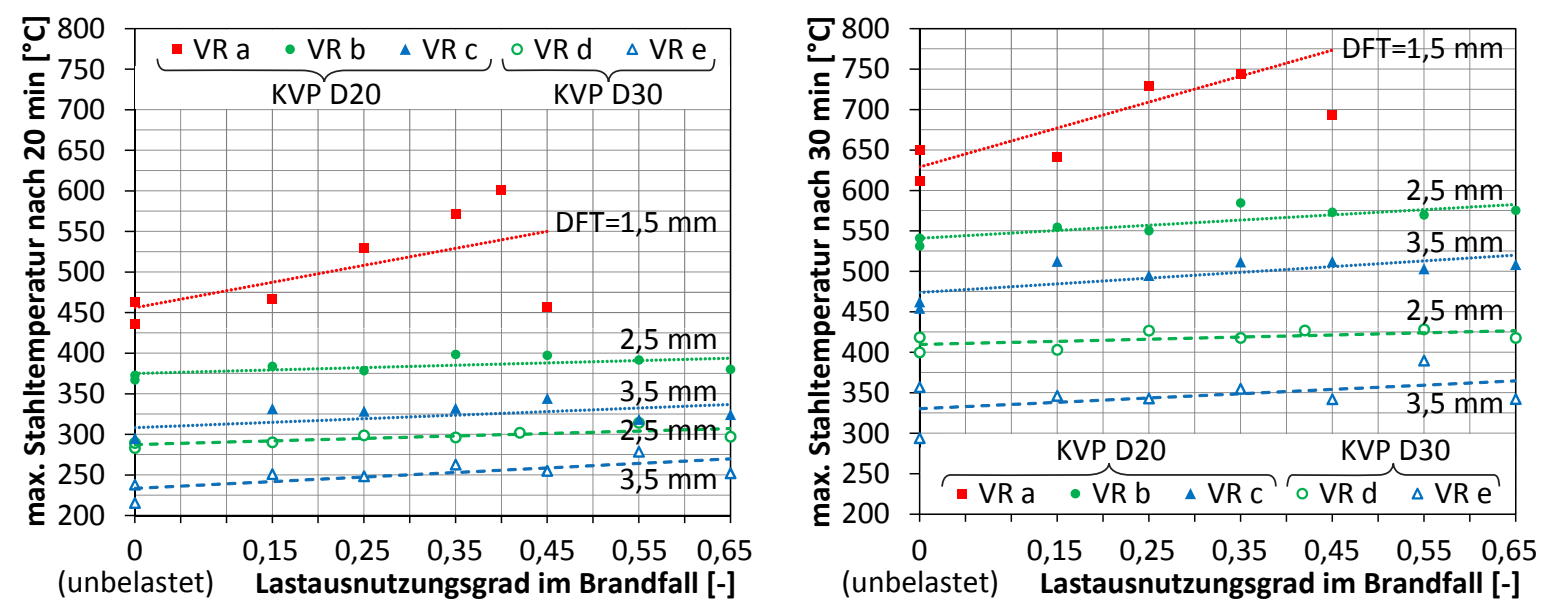

Abb. 3.33: Vergleich der maximalen Stahltemperatur in Abhängigkeit vom Lastausnutzungsgrad im Brandfall (links: nach einer Branddauer von 20 min; rechts: nach einer Branddauer von 30 min)

\subsubsection{Einfluss der Bauteilorientierung auf die Stahltemperatur}

Anhand von Zuggliedern ohne mechanischer Zugbeanspruchung, welche in horizontaler und vertikaler Prüfposition angeordnet sind, wird der Einfluss der Bauteilorientierung auf das Verhalten des reaktiven Brandschutzsystems untersucht. Die Versuchskörper mit KVP D20 werden dafür beispielhaft ausgewertet. In Abb. 3.34 ist die Temperaturentwicklung 
des Stahls über die Branddauer dargestellt. Die Brandversuche zeigen, dass sich Versuchskörper in vertikaler Einbaulage (gepunktete Linie) in der Regel schneller erwärmen als eine vergleichbare horizontal ausgerichtete Probe (gestrichelte Linie). Infolge der Bauteilorientierung ergeben sich bereits nach etwa 5 bis 10 Minuten Unterschiede in den Stahltemperatur-Branddauer-Kurven. Ursächlich für die schnellere Erwärmung der vertikalen Versuchskörper ist die ausgeprägte Längsrissbildung im Brandschutzsystem und die ungleichmäßige Verteilung der Aufschäumung des reaktiven Brandschutzsystems (siehe Abb. 3.35). Die Längsrisse verlaufen meist über die gesamte Stablänge und reichen teilweise bis auf die Stahloberfläche. Durch die Risse wird die thermische Schutzwirkung der reaktiven Brandschutzbeschichtung erheblich reduziert, wodurch die Stahltemperaturen schneller ansteigen. An den in horizontaler Prüfposition getesteten Versuchskörpern treten ebenfalls Längsrisse auf. Diese befinden sich jedoch meist auf der Stabunterseite und können in der Regel durch das Rissheilungsvermögen der Brandschutzbeschichtung wieder geschlossen werden.

Neben der Längsrissbildung kommt es an den in vertikaler Position geprüften Versuchskörpern in Stablängsrichtung vereinzelt zu einem teilweisen Abgleiten des aufschäumenden reaktiven Brandschutzsystems. Besonders am oberen Stabende ist dies zu beobachten (siehe Abb. 3.35). Für diese Bereiche ist mit einer erhöhten Stahltemperatur zu rechnen. Da der Abstand zwischen Stabende und den an
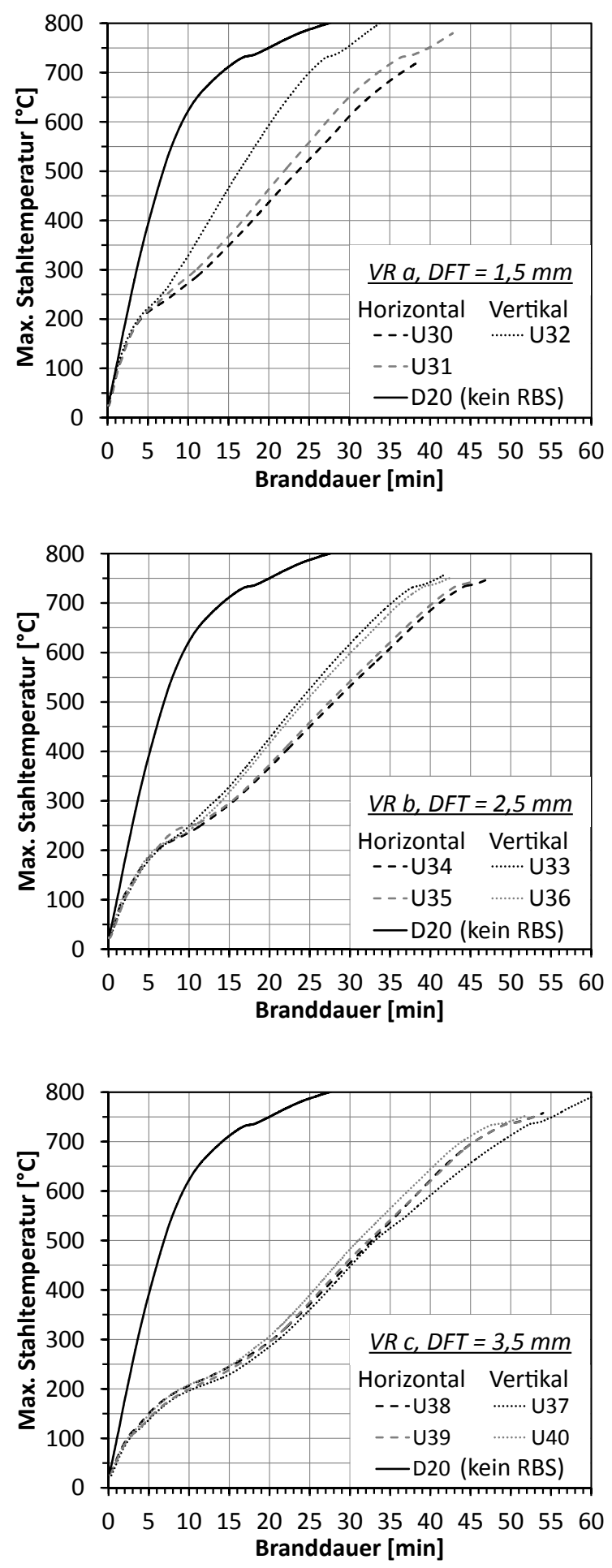

Abb. 3.34: Einfluss der Probenausrichtung auf die Temperaturentwicklung des Stahls am Beispiel der VR a bis c 
den Versuchskörper angebrachten Thermoelementen ca. $225 \mathrm{~mm}$ beträgt, kann ein Einfluss auf die Temperaturmessung ausgeschlossen werden. Ursächlich für das Abgleiten ist das Eigenwicht des aufgeschäumten Brandschutzsystems. Ein ähnliches Verhalten ist bereits aus Brandprüfungen an Stützen bekannt.

Ein weiterer Grund für den Temperaturunterschied zwischen horizontal und vertikal geprüften Versuchskörpern ist die Änderung der Beflammungsrichtung. Die horizontalen Versuchskörper werden tangential beflammt. Diese Art der Beflammung ist für Brandversuche allgemein üblich. Obwohl die in vertikaler Prüfposition untersuchten Versuchskörper seitlich versetzt von der Öffnung des Ölbrenners angeordnet sind, kommt es aufgrund der geänderten Probenausrichtung zu einer teilweisen Radialbeflammung. Als Folge dessen ergeben sich in der Umgebung des vertikalen Versuchskörpers höhere Brandgastemperaturen. Aufgrund der in den Brandversuchen aufgetretenen Längsrisse ist es wahrscheinlich, dass die Radialbeflammung die Rissbildung verstärkt hat. Generell sollte bei zukünftigen Prüfungen eine Radialbeflammung vermieden werden, da die dadurch auftretenden höheren Brandgastemperaturen eine größere Beanspruchung für das reaktive Brandschutzsystem darstellen.

Tabelle 3.9: Vergleich der Erwärmungsgeschwindigkeit zwischen horizontaler und vertikaler Prüfpostion

\begin{tabular}{|c|c|c|c|c|c|c|}
\hline \multirow{2}{*}{\multicolumn{2}{|c|}{$\begin{array}{l}\text { Profil } \\
\text { Versuchsreihe (VR) }\end{array}$}} & \multicolumn{3}{|c|}{ KVP D20 } & \multicolumn{2}{|c|}{ KVP D30 } \\
\hline & & a & b & c & d & $\mathrm{e}^{*}$ \\
\hline \multicolumn{2}{|c|}{ Trockenschichtdicke [mm] } & 1,5 & 2,5 & 3,5 & 2,5 & 3,5 \\
\hline \multirow{3}{*}{$\begin{array}{l}\text { Erwärmungs- } \\
\text { geschwindigkeit } \\
\text { [K/min] }\end{array}$} & horizontal & 18,7 & 16,5 & 16,3 & 12,5 & 12,3 \\
\hline & vertikal & 25,5 & 19,1 & 17,1 & 13,0 & - \\
\hline & Differenz & 6,8 & 2,6 & 0,8 & 0,5 & - \\
\hline
\end{tabular}

Die Anmerkungen aus Tabelle 3.10 sind zu beachten.

Die Erwärmungsgeschwindigkeit wurde für den aufgeschäumten

Zustand des reaktiven Brandschutzsystems bestimmt.

Der Temperaturunterschied zwischen horizontal und vertikal ausgerichteten Versuchskörpern mit Kreisvollprofil ist nach Versuchsreihen geordnet für ausgewählte Zeitpunkte

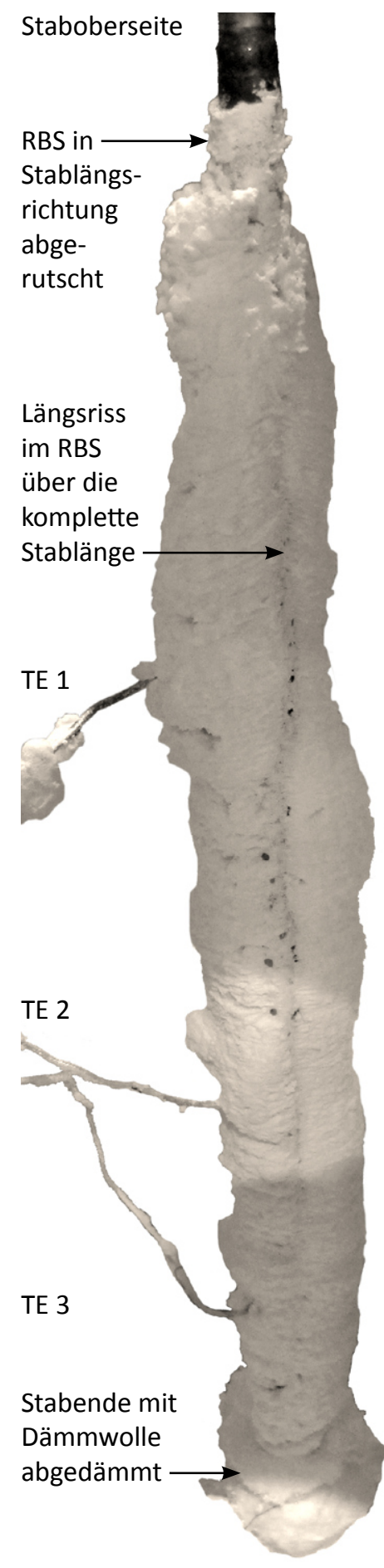

Abb. 3.35: Versuchskörper U32 nach dem Brandversuch (Nordostseite) 
in Tabelle 3.10 angegeben. Die Werte zeigen, dass innerhalb einer Versuchsreihe die Temperaturunterschiede zwischen horizontal und vertikal getesteten Versuchskörpern im Verlauf des Brandversuches ansteigen. Dies ist auf die Unterschiede in den Erwärmungsgeschwindigkeiten, welche ein Maß für die thermische Schutzwirkung des Brandschutzsystems sind, zurückzuführen. In Tabelle 3.9 ist die aus den Stahltemperatur-Branddauer-Kurven der jeweiligen, unbelasteten Versuchskörper bestimmte Erwärmungsgeschwindigkeit angegeben. Die Werte beziehen sich auf den aufgeschäumten Zustand des reaktiven Brandschutzsystems. Dieser Bereich ist durch einen annähernd konstanten zeitlichen Anstieg der Stahltemperaturen gekennzeichnet und beginnt ab einer Stahltemperatur von etwa 250 bis $300^{\circ} \mathrm{C}$. Die Erwärmungsgeschwindigkeit eines horizontal geprüften Versuchskörpers ist kleiner als die einer vertikal getesteten Probe. Durch eine Erhöhung der Trockenschichtdicke oder Vergrößerung des Stabdurchmessers lassen sich die Unterschiede in den Stahltemperaturen und Erwärmungsgeschwindigkeiten zwischen horizontal und vertikal getesteten Versuchskörpern reduzieren. Dies ist auf die Steigerung des Rissheilungsvermögens bzw. die abnehmende Rissneigung des reaktiven Brandschutzsystems zurückzuführen.

Die Untersuchungen zeigen, dass die Bauteilorientierung bei den Brandversuchen und dem späteren Anwendungsbereich des reaktiven Brandschutzsystems zu berücksichtigen ist. Eine Übertragung der Stahltemperaturen eines in horizontaler Position getesteten Zuggliedes auf die eines vertikalen Bauteils und umgedreht ist nicht möglich.

Tabelle 3.10: Vergleich der Stahltemperaturen zwischen den in horizontaler und vertikaler Prüfposition eingebauten Versuchskörpern zu verschiedenen Zeitpunkten des Brandversuches

\begin{tabular}{|c|c|c|c|c|c|c|c|c|c|c|c|c|c|c|c|}
\hline \multirow{3}{*}{$\begin{array}{l}\text { Zeitpunkt } \\
\text { Profil } \\
\text { VR }\end{array}$} & \multicolumn{5}{|c|}{15 Minuten } & \multicolumn{5}{|c|}{30 Minuten } & \multicolumn{5}{|c|}{45 Minuten } \\
\hline & \multicolumn{3}{|c|}{ KVP D20 } & \multicolumn{2}{|c|}{ KVP D30 } & \multicolumn{3}{|c|}{ KVP D20 } & \multicolumn{2}{|c|}{ KVP D30 } & \multicolumn{3}{|c|}{ KVP D20 } & \multicolumn{2}{|c|}{ KVP D30 } \\
\hline & a & b & c & d & $\mathrm{e}^{*}$ & a & b & c & d & $\mathrm{e}^{*}$ & $\mathbf{a}$ & b & c & d & $\mathrm{e}^{*}$ \\
\hline DFT [mm] & 1,5 & 2,5 & 3,5 & 2,5 & 3,5 & 1,5 & 2,5 & 3,5 & 2,5 & 3,5 & 1,5 & 2,5 & 3,5 & 2,5 & 3,5 \\
\hline$\theta_{\text {horizonta }}$ & 368 & 295 & 238 & 241 & 203 & 651 & 541 & 463 & 418 & 357 & - & 742 & 694 & 601 & 549 \\
\hline$\theta_{\text {vertikal }}$ & 465 & 329 & 246 & 275 & - & 754 & 617 & 482 & 470 & - & - & - & 712 & 652 & - \\
\hline$\Delta \theta_{\text {vertikal-horizontal }}$ & 97 & 34 & 8 & 34 & - & 103 & 76 & 19 & 52 & - & - & - & 18 & 51 & - \\
\hline
\end{tabular}

Die Werte sind in ${ }^{\circ} \mathrm{C}$ angegeben und beziehen sich auf die gemessene maximale Stahltemperatur.

* In dieser Versuchsreihe wird kein Versuchskörper in vertikaler Einbaulage untersucht.

Basiswert für den Temperaturunterschied bildet in jeder Versuchsreihe der Versuchskörper in vertikaler Bauteilorientierung.

\subsubsection{Einfluss der Stablänge auf die Stahltemperatur}

\section{Randeffekte und Abstände der Messstellen}

Um den Einfluss der beflammten Stablänge auf den Temperaturverlauf zu beurteilen, werden an den Versuchskörpern mit und ohne Zugbeanspruchung im Abstand von $150 \mathrm{~mm}$ 
Thermoelemente angebracht. Die mechanisch belasteten Zugglieder besitzen zusätzlich vier weitere Messstellen außerhalb des Brandraumes. Für die Versuchskörper mit Zugbeanspruchung zeigt sich über die Stablänge ein parabelförmiger Temperaturverlauf (siehe Abb. 3.36). Im Bereich der Brandraumwände kommt es aufgrund von Abschattungseffekten und Randeinflüssen zu einer Absenkung der Stahltemperatur des Zuggliedes. Aufgrund der Auflagerungsbedingung tritt dies auch an den unbelasteten Versuchskörpern auf. Durch die geringeren Stahltemperaturen fällt die Aufschäumung des reaktiven Brandschutzsystems in diesen Bereichen kleiner aus. Die Brandversuche zeigen, dass die Randeffekte nach etwa $150 \mathrm{~mm}$ abgeklungen sind. Die Stahltemperaturen und die Höhe der Aufschäumung des reaktiven Brandschutzsystems entsprechen dann den ungestörten Stabbereichen (Normalbereich).

Um zuverlässige und repräsentative Aussagen über die Erwärmung des Stahlbauteils im Brandversuch treffen zu können, sind mindestens drei Messquerschnitte entlang der Stablängsachse vorzusehen. Für den Abstand der Messstationen untereinander wird ein Abstand von $150 \mathrm{~mm}$ empfohlen. Darüber hinaus sind die Messquerschnitte mindestens $150 \mathrm{~mm}$ vom Stabende oder der Brandraumwand anzuordnen, so dass die Temperaturmessung in einem für die Aufschäumung des reaktiven Brandschutzsystems ungestörten Stabbereich erfolgt. Unter Berücksichtigung der genannten Empfehlungen ergibt sich für die beflammte Stablänge ein minimaler Wert von $600 \mathrm{~mm}$. Ferner ist bei der Festsetzung der Bauteillänge das Verhältbis der beflammten Stablänge $\left(L_{b}\right)$ zum Stabdurchmesser (d) zu beachten. In den Brandprüfungen hat sich ein Verhältniswert von $\mathrm{L}_{\mathrm{b}} / \mathrm{d} \geq 10$ bewährt.

\section{Einfluss der beflammten Stablänge}

Die beflammte Länge beträgt bei den mechanisch belasteten Zuggliedern etwa $900 \mathrm{~mm}$ und bei den unbelasteten Vergleichsprüfkörpern ca. $650 \mathrm{~mm}$. In Abb. 3.36 sind exemplarisch die Stahltemperatur-Branddauer-Kurven zweier Probenpaare (B14 \& U34 sowie B48 \& U42), bestehend aus einem unbelasteten und einem mechanisch belasteten Versuchskörper, dargestellt. Anhand der Diagramme zeigt sich, dass die jeweils simultan getesteten Versuchskörper bis zu einer Branddauer von etwa 12 Minuten annähernd gleiche Stahltemperaturen aufweisen. Infolge der mechanischen Beanspruchung und der damit verbundenen höheren Rissbildung im Brandschutzsystems kommt es an den mechanisch belasteten Zuggliedern noch während oder am Ende der Aufschäumphase, d.h. 10 bis 20 Minuten nach Beginn des Brandversuches, häufig zu einer Zunahme der Stahltemperaturen (siehe B14 und U34 in Abb. 3.36). Da eine Rissbildung auch an den unbelasteten Versuchskörpern auftritt, kann ein Einfluss der beflammten Stablänge ausgeschlossen werden. Zwischen dem mechanisch belasteten Versuchskörper B48 und dem unbelasteten Vergleichskörper U42 ist auch nach der Aufschäumphase kein nennenswerter Unterschied der Stahltemperaturen vorhanden. Dies ist auf den größeren Stabdurchmesser und das damit verbundene bessere Rissheilungsvermögen der reaktiven Brandschutzbeschichtung zurückzuführen. Ein aus der unterschiedlichen Stablänge hervorgerufener Temperaturunterschied ist nicht vorhanden. 
Unter der Voraussetzung, dass sich im Brandversuch ein ungestörter Stabbereich ausbilden kann, d.h. die Aufschäumung des reaktiven Brandschutzsystems wird nicht durch Abschattungseffekte gestört, existiert zwischen der Prüfung eines kurzen und eines langen Stabes kein Unterschied. Da über das gesamte mechanisch belastete Zugglied die gleiche Zugbeanspruchung vorliegt und keine Tragfähigkeitsumlagerungen möglich sind, tritt der Probenbruch des Bauteils an der Stelle mit der maximalen Stahltemperatur ein. Eine Vergrößerung der beflammten Stablänge ist daher nur sinnvoll, um einen ungestörten Aufschäumbereich für das Brandschutzsystem zu erhalten. Ist dieser bereits bei der gewählten Stablänge vorhanden, ist eine Vergrößerung der Stablänge nicht mehr sinnvoll. Um das Prüfergebnis nicht zu beeinflussen, sind Einflüsse aus der Temperaturmessung auf das Aufschäumverhalten der Brandschutzbeschichtung zu vermeiden.

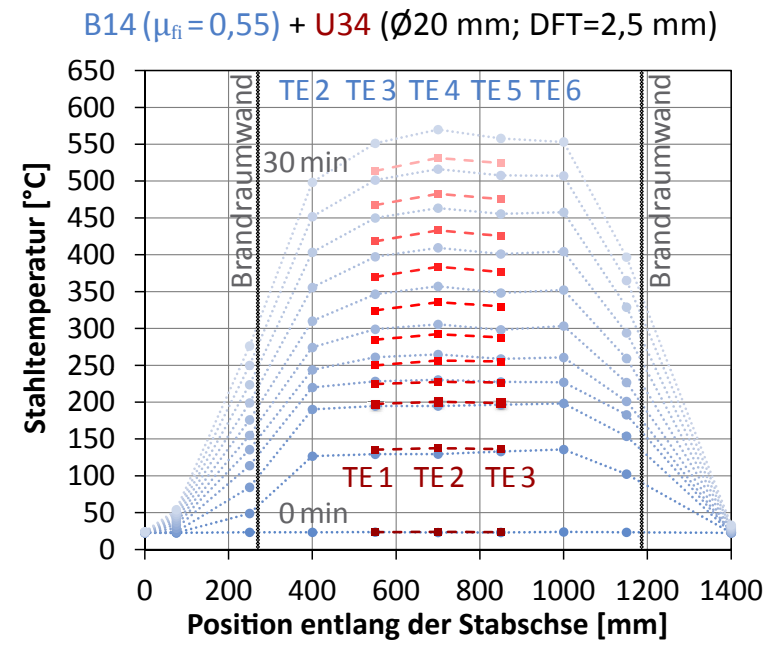

- Max. Stahltemperatur in Stabmitte (TE 2 bzw. TE 4)

- Längsrissbildung führt zu höherem Temperaturanstieg am belasteten Zugglied

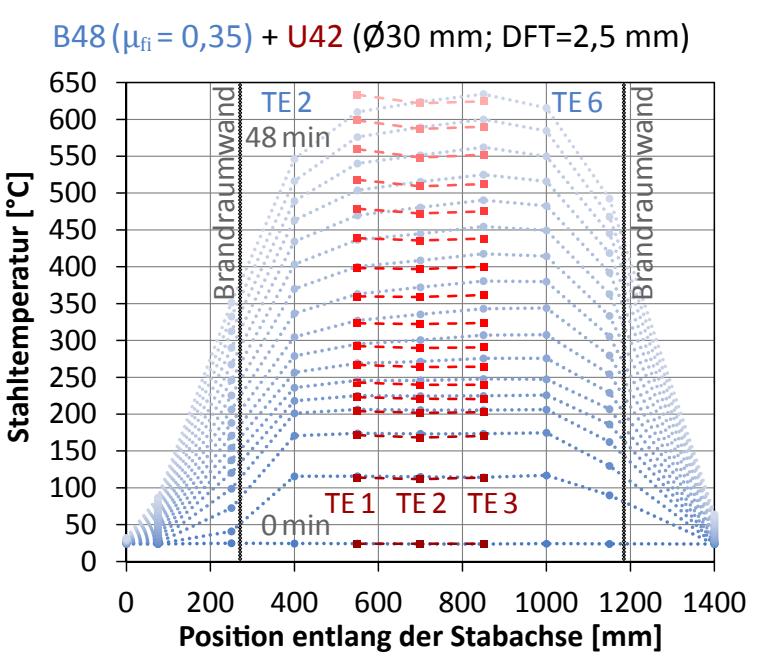

- Max. Stahltemperatur am TE 1 bzw. TE 5

- Annähernd gleiche Stahltemperatur zwischen belastetem und unbelastetem Versuchskörper

Abb. 3.36: Vergleich des Temperaturverlaufes in Stablängsrichtung über die Branddauer (zeitlicher Abstand der dargestellten Temperaturverläufe beträgt jeweils 3 min)

\subsubsection{Einfluss von Beschädigungen des reaktiven Brandschutzsystems}

Bei einigen Versuchskörpern mit mechanischer Zugbeanspruchung sind im Vorfeld des Brandversuches mittels eines Stechbeitels definierte Beschädigungen am reaktiven Brandschutzsystem vorgenommen worden (siehe Tabelle A.5), um damit die Robustheit und Empfindlichkeit der Brandschutzbeschichtung zu untersuchen. Zwei Arten von Beschädigungen, d.h. ein Querriss über den gesamten Stabumfang oder ein Längsriss auf der Staboberseite des Zuggliedes, werden getestet. Die Breite der Querrisse variierte zwischen 0,8 und $3 \mathrm{~mm}$. Die Längsrisse sind etwa $100 \mathrm{~mm}$ lang und ca. 1,0 bis $1,5 \mathrm{~mm}$ breit. Bei beiden Rissarten reicht die Beschädigung bis auf die Stahloberfläche. 
Die Brandversuche zeigen, dass Querrisse zu einer Störstelle in der Aufschäumung des reaktiven Brandschutzsystems führen (siehe Abb. 3.37). Die Temperaturmessung im Bereich der Querrisse zeigt jedoch gegenüber dem Stabbereich ohne Beschädigung meist keine erhöhten Temperaturwerte. Ein Einfluss des Querrisses auf die Stelle des späteren Probenbruchs des Zuggliedes konnte ebenfalls nicht festgestellt werden. Eine Beschädigung des Brandschutzsystems durch Querrisse ist daher als relativ unproblematisch anzusehen.

Eine Beschädigung des reaktiven Brandschutzsystems durch einen Längsriss führt zu einer ausgeprägten Rissbildung in Stablängsrichtung der aufgeschäumten Brandschutzbeschichtung (siehe Abb. 3.37). Durch das Rissheilungsvermögen des reaktiven Systems kann sich der Längsriss meist wieder vollständig schließen. In der Höhe der Aufschäumung des reaktiven Brandschutzsystems und der gemessenen Stahltemperaturen konnte zwischen geschädigtem und ungeschädigtem Bereich kein signifikanter Unterschied festgestellt werden. Ein Einfluss des Längsrisses auf die Position der Bruchstelle des mechanisch belasteten Zuggliedes ist ebenfalls nicht vorhanden. Dies ist im Wesentlichen auf das ausreichende Rissheilungsvermögen der Brandschutzbeschichtung zurückzuführen. Da das Rissheilungsvermögen des reaktiven Brandschutzsystems in hohem Maße von der Trockenschichtdicke und vom Stabdurchmesser abhängt, stellen Beschädigungen in Stablängsrichtung jedoch ein vergleichsweise hohes Risiko dar. Eine ausreichende Pflege und Wartung der Brandschutzsysteme ist daher in der Praxis sicherzustellen. In den vom DIBt ausgestellten Zulassungen wird unter anderem eine Kennzeichnung der mit reaktiven Brandschutzsystemen versehenen Oberflächen gefordert.

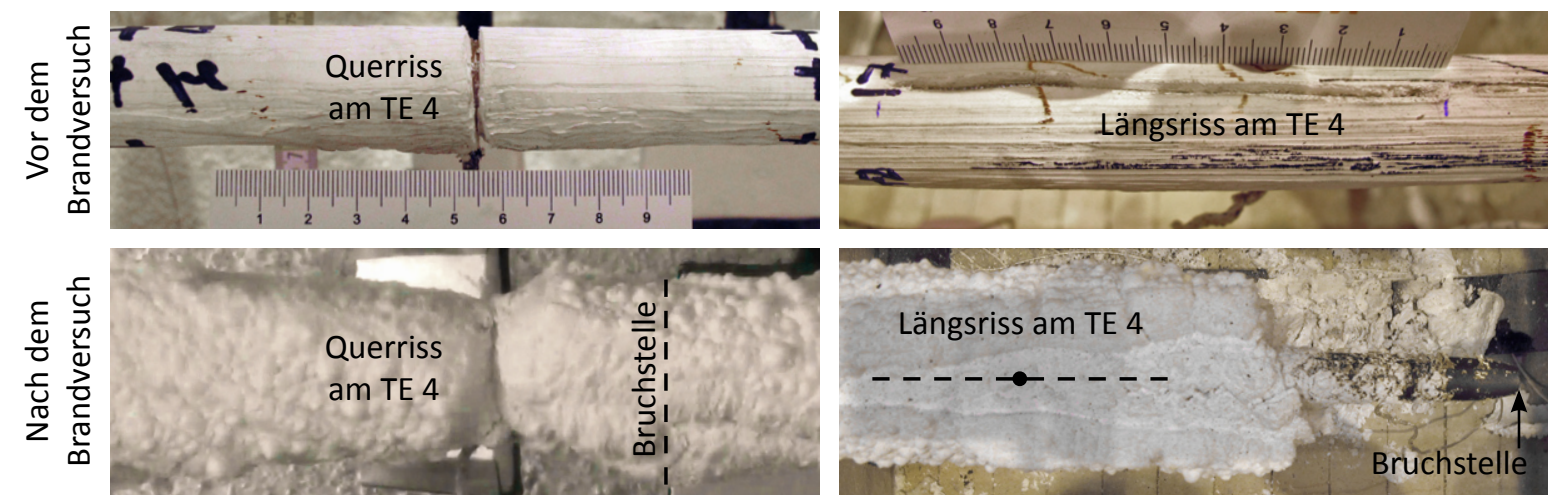

Abb. 3.37: Beschädigung des reaktiven Brandschutzsystems vor und nach dem Brandversuch (links: durch Querriss (B24); rechts: durch Längsriss (B23))

\subsubsection{Zusammenfassung der untersuchten Einflussfaktoren}

Mittels Brandprüfungen an mechanisch belasteten und unbelasteten Zuggliedern mit reaktiver Brandschutzbeschichtung wird die Wirkung verschiedener Einflussfaktoren auf die Feuerwiderstandsdauer untersucht. Als Beurteilungskriterien werden das Aufschäumungs- 
und Rissverhalten des Brandschutzsystems, die Branddauer bis zum Probenbruch der Versuchskörper mit Zugbeanspruchung sowie die Stahltemperatur und die Erwärmungsgeschwindigkeit betrachtet. Die Feststellungen beziehen sich auf das in den Untersuchungen der BAM verwendete reaktive Brandschutzsystem sowie die damit geschützten Stahlprofile. Da sich die einzelnen Produkte reaktiver Brandschutzbeschichtungen unterscheiden können, ist eine produktbezogene Prüfung in Form von Brandversuchen unverzichtbar. Es ist zu unterstellen, dass die untersuchten Phänomene und Abhängigkeiten der betrachteten Einflussfaktoren auch bei anderen reaktiven Brandschutzsystemen auftreten können.

Die wichtigsten Einflussfaktoren auf die Feuerwiderstandsdauer von Stahlzuggliedern mit reaktiver Brandschutzbeschichtung sind die Höhe der Trockenschichtdicke des Brandschutzsystems, die Profilgeometrie des Zuggliedes (Profilart, Stabdurchmesser bzw. Profilfaktor) und die Höhe der Zugbeanspruchung (Lastausnutzungsgrad). Die Wirkung der einzelnen Faktoren auf die Feuerwiderstandsdauer ist in Abb. 3.38 schematisch dargestellt. Die Auswertung der Brandversuche hat zudem gezeigt, dass eine gegenseitige Beeinflussung der genannten Faktoren vorhanden ist. Zum Beispiel führt an den Kreisvollprofilen mit einem Durchmesser von 20 und $30 \mathrm{~mm}$ die Vergrößerung der Trockenschichtdicke zu einer Verstärkung des Einflusses des Lastausnutzungsgrades und des Stabdurchmessers. Für die Auslegung des beschichteten Stahlzuggliedes entsprechend der Feuerwiderstandsklasse ergeben sich durch die Variation der drei Parameter eine Vielzahl an Möglichkeiten.

Die Untersuchungen haben gezeigt, dass sich nur innerhalb eines bestimmten Bereiches jedes einzelnen Parameters ein signifikanter Beitrag zur Erhöhung der Feuerwiderstandsdauer von Stahlzuggliedern erreichen lässt. Beispielsweise verhält sich die thermische Schutzwirkung des reaktiven Brandschutzsystems unterproportional zur Trockenschichtdicke. Um einen möglichst effizienten Einsatz der Brandschutzbeschichtung zu gewährleisten, empfiehlt es sich, die drei untersuchten Parameter aufeinander abzustimmen. Meist lässt sich durch eine kleine Variation aller Parameter eine größere Wirkung für die Erhöhung der Feuerwiderstandsdauer erreichen, als durch die übermäßige Veränderung eines einzelnen Parameters.

Neben den bereits beschriebenen Einflussfaktoren wirken sich auch die Einbaulage der Zugglieder sowie die Beflammungsrichtung auf die Feuerwiderstandsdauer des beschichteten Stahlzuggliedes aus. Brandprüfungen an Versuchskörpern ohne mechanischer Zugbeanspruchung zeigen, dass reaktive Brandschutzsysteme auf horizontalen Zuggliedern in der Regel eine bessere thermische Schutzwirkung entwickeln als auf vertikal getesteten Proben. Eine Übertragung der Ergebnisse aus Brandversuchen von horizontal getesteten auf vertikale Zugglieder und umgedreht ist nicht möglich. Die Verwendung der Brandschutzbeschichtung ist bei der Prüfung und Anwendung zu berücksichtigen. Bei der Prüfung von Stahlzuggliedern mit reaktiver Brandschutzbeschichtung ist zudem darauf zu achten, dass durch die Applikation der Thermoelemente das Verhalten des Brandschutzsystems nicht beeinflusst wird. Um das Prüfergebnis nicht zu verfälschen, ist an den Thermoelementen 
anhaftendes Beschichtungsmaterial vor dem Brandversuch zu entfernen. Des Weiteren ist eine ausreichende Anzahl an Thermoelementen am Zugglied anzubringen, um eventuell vorhandene lokale Störungen in der thermischen Schutzwirkung des Brandschutzsystem erfassen zu können. Beschädigungen und Abschattungseffekte der zu testenden Zugglieder sind zu vermeiden.

Eine Vorhersage der Feuerwiderstandsdauer von Stahlzuggliedern mit reaktiver Brandschutzbeschichtung gestaltet sich aufgrund der Vielzahl an Einflussfaktoren schwierig. Um die Zuverlässigkeit des Brandschutzsystems zu gewährleisten, sind Brandprüfungen in ausreichender Anzahl notwendig. Eine Empfehlung für ein Prüfprogramm ist im Forschungsbericht [35] angegeben. Die Anwendung reaktiver Brandschutzsysteme auf Stahlzuggliedern ist aufgrund der dabei auftretenden höheren Anforderungen zwingend durch mechanisch belastete Brandprüfungen nachzuweisen. Diese Art der Prüfung ist am besten mit der realen Beanspruchung der am Bauwerk eingesetzten Zugglieder vergleichbar. Eine alleinige Prüfung von Versuchskörpern ohne Zugbeanspruchung kann zu einer Überschätzung der Feuerwiderstandsdauer führen. Die Festlegung eines Korrekturfaktors zwischen den Temperaturdaten an mechanisch belasteten und unbelasteten Zuggliedern gestaltet sich aufgrund des zufälligen und kaum vorhersagbaren Aufschäumungs- und Rissverhaltens schwierig und bedarf weiterer Untersuchungen.

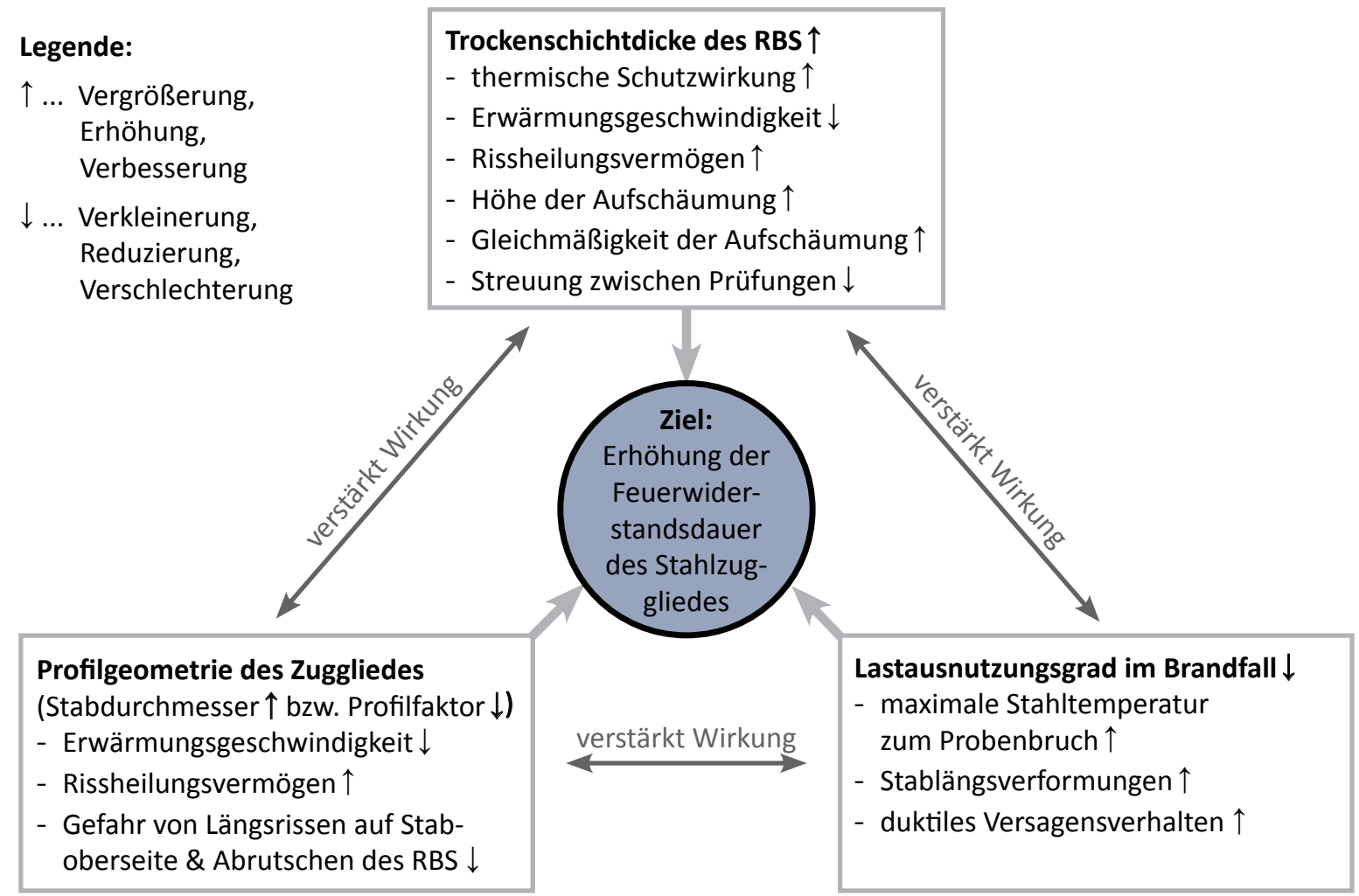

Abb. 3.38: Schematische Darstellung der drei untersuchten Parameter auf die Feuerwiderstandsdauer eines Zuggliedes mit reaktiver Brandschutzbeschichtung 


\subsection{Porenstruktur und Schichtenaufbau des aufgeschäumten reaktiven Brandschutzsystems}

Nach den Brandversuchen im Realmaßstab werden visuelle und mikroskopische Untersuchungen des aufgeschäumten reaktiven Brandschutzsystems durchgeführt, um eine genaue Kenntnis des Aufbaus und der Struktur der Brandschutzbeschichtung zu erhalten und die Wirkungsweise besser verstehen zu können. Ein Teilstück der Aufschäumung des Brandschutzsystems wird mittels Computertomographie (CT) sowie am Rasterelektronenmikroskop (REM) untersucht. Die CT- und REM-Analysen werden analog zu den Untersuchungen von Morys [42] durchgeführt.

\subsubsection{Geometrie der Aufschäumung über den Stabquerschnitt}

Die genaue Betrachtung der Geometrie des aufgeschäumten reaktiven Brandschutzsystems soll Aufschluss geben, inwieweit Trockenschichtdicke, Profilgeometrie und mechanische Zugbeanspruchung das Aufschäumverhalten beeinflussen. Zu diesem Zweck wurde nach dem Brandversuch eine Fotodokumentation der getesteten Zugglieder durchgeführt. Die Höhe der Aufschäumung der Brandschutzbeschichtung wird vier Mal um den Stabquerschnitt im Winkel von $90^{\circ}$ gemessen. Bezogen auf die Stablängsachse beträgt der Abstand der Messpunkte in jeder Messreihe ca. $50 \mathrm{~mm}$. Die Ergebnisse dieser Messungen sowie Auszüge aus der Fotodokumentation sind in den Anhängen des Forschungsberichtes [35] sowie der vorliegenden Arbeit dokumentiert. Anhand der gewonnenen Informationen wird eine Typologie der Geometrie des aufgeschäumten reaktiven Brandschutzsystems vorgenommen. Da nur eine vergleichsweise geringe Anzahl an Kreishohlprofilen untersucht wird und die Ergebnisse aus diesen Brandversuchen einer relativ großen Streuung unterliegen, werden für die Auswertung ausschließlich die Kreisvollprofile herangezogen. Insgesamt ergeben sich sechs verschiedene Aufschäumtypen (siehe Tabelle 3.11).

Im Wesentlichen ist die Aufschäumung der Brandschutzbeschichtung kreisförmig, elliptisch oder oval. Dies ist auf die kreisförmige Geometrie der Stahlprofile zurückzuführen. Bei elliptischer und ovaler Aufschäumung (Typ 2 bis 6) treten teilweise große Längsrisse in der Aufschäumung auf. Infolge lokaler Effekte, wie z.B. Längsrisse oder Bereiche mit teilweise abgerutschter Brandschutzbeschichtung, kommt es an einigen Versuchskörpern entlang der Stabachse zu einer Variation des Aufschäumtypes. Anhand der durchgeführten Brandversuche lässt sich keine Systematik zur Entstehung und Verteilung der verschiedenen Aufschäumtypen feststellen. Allerdings zeigt sich, dass eine Erhöhung der Trockenschichtdicke oder die Erhöhung des Stabdurchmessers im Allgemeinen zu einer gleichmäßigeren Aufschäumung über den Stabquerschnitt (Typ 1) führt. Der Schaumtyp 6 tritt vermehrt bei den Versuchskörpern ohne mechanische Zugbeanspruchung auf. Dies ist wahrscheinlich auf die Positionierung des Stabes unterhalb der Brennerachse und die dadurch auf der Staboberseite etwas höheren Brandgastemperaturen zurückzuführen. 
Tabelle 3.11: Typologie des aufgeschäumten reaktiven Brandschutzsystems (grau) an von horizontal getesteten Stahlzuggliedern mit Kreisvollquerschnitt (blau)

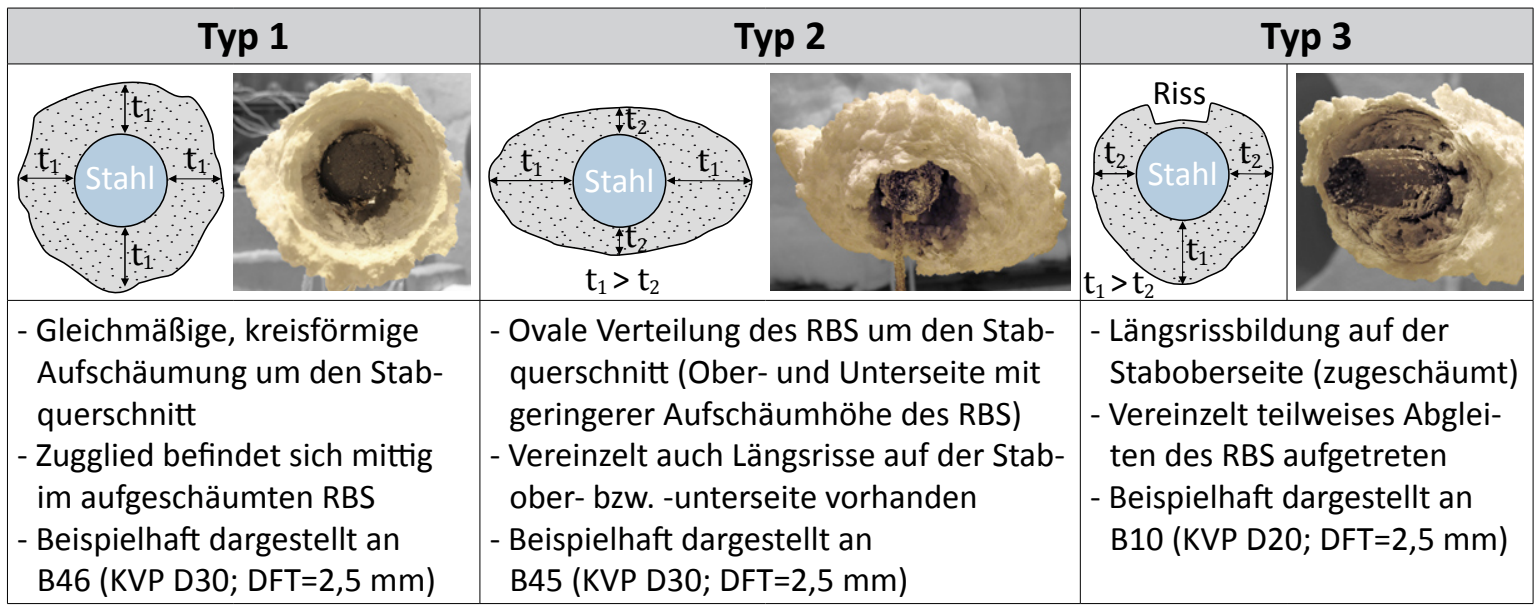

\begin{tabular}{|c|c|c|}
\hline Typ 4 & Typ 5 & Typ 6 \\
\hline & $\begin{array}{l}\text { Zugglied } \\
\text { seitlicher Längsri }\end{array}$ & $t_{1}>t_{2}>t_{3}$ \\
\hline $\begin{array}{l}\text { - Stabunterseite mit erhöhter } \\
\text { Aufschäumdicke des RBS } \\
\text { - Im Vergleich zu Typ } 3 \text { kein Riss } \\
\text { auf Staboberseite vorhanden } \\
\text { - Beispielhaft dargestellt an } \\
\text { B9 (KVP D20; DFT=2,5 mm) }\end{array}$ & $\begin{array}{l}\text { - Stabober- und -unterseite mit höherer } \\
\text { Aufschäumung des RBS } \\
\text { - Vereinzelt Längsrissbildung an den } \\
\text { seitlichen Stabseiten } \\
\text { - Beispielhaft dargestellt an } \\
\text { B3 (KVP D20; DFT=1,5 mm) }\end{array}$ & $\begin{array}{l}\text { - Staboberseite mit erhöhter } \\
\text { Aufschäumung des RBS } \\
\text { - Vereinzelt Längsriss auf der } \\
\text { Stabunterseite vorhanden } \\
\text { - Beispielhaft dargestellt an } \\
\text { U31 (KVP D20; DFT=1,5 mm) }\end{array}$ \\
\hline
\end{tabular}

\subsubsection{Untersuchung der Aufschäumung mittels CT und REM}

Das Verfahren der Computertomographie arbeitet zerstörungsfrei und berührungslos. Mit Hilfe dieser Methode können auch komplexe und nicht zugängliche Geometrien dargestellt werden. Anhand der CT-Darstellung lassen sich sowohl qualitative als auch quantitative Aussagen zur Geometrie treffen. Die Genauigkeit der CT-Untersuchung hängt unter anderem von der Probengröße und dem Abstand zwischen dem Röntgengerät und dem Versuchskörper ab. Dabei gilt, je kleiner die Probe bzw. der Abstand zur Probe, desto besser lassen sich auch kleinere Strukturen abbilden.

Für die Untersuchungen wird ein Teilstück des aufgeschäumten reaktiven Brandschutzsystems vom Zugglied B10 mittels Computertomographie (CT) und Rasterelektronenmikroskop (REM) untersucht. Die Probe ist etwa $70 \mathrm{~mm}$ lang und zeigt keine sichtbaren Beschädigungen (siehe Abb. 3.39). Auffälligstes Merkmal der Schaumprobe ist ein teilweise 
zugeschäumter Längsriss im reaktiven Brandschutzsystem. Der Riss befindet sich auf der Staboberseite und erstreckt sich etwa über die halbe Aufschäumhöhe (Aufschäumtyp 3). Die verbleibende Höhe der Aufschäumung des Brandschutzsystems beträgt im Rissbereich etwa $8 \mathrm{~mm}$. In den ungestörten Bereichen ist eine Aufschäumung von maximal $20 \mathrm{~mm}$ vorhanden. An der Stelle des Kreisvollprofils (KVP D20) sind noch Reste der Oxidschicht des Stahls vorhanden.

Bereits die Sichtprüfung des aufgeschäumten reaktiven Brandschutzsystems zeigt, dass sich im Querschnitt zwei unterschiedliche Bereiche der Aufschäumung ausgebildet haben (siehe Abb. 3.41). Der äußere Bereich der Aufschäumung hat eine blasenartige Oberfläche und eine feinporige Struktur. Der innere Schaumbereich, welcher sich rund um das Stahlbauteil erstreckt, besitzt einen lamellenartigen Schichtenaufbau. In ungestörten Bereichen ist die Dicke der beiden Schichten annähernd gleich groß.
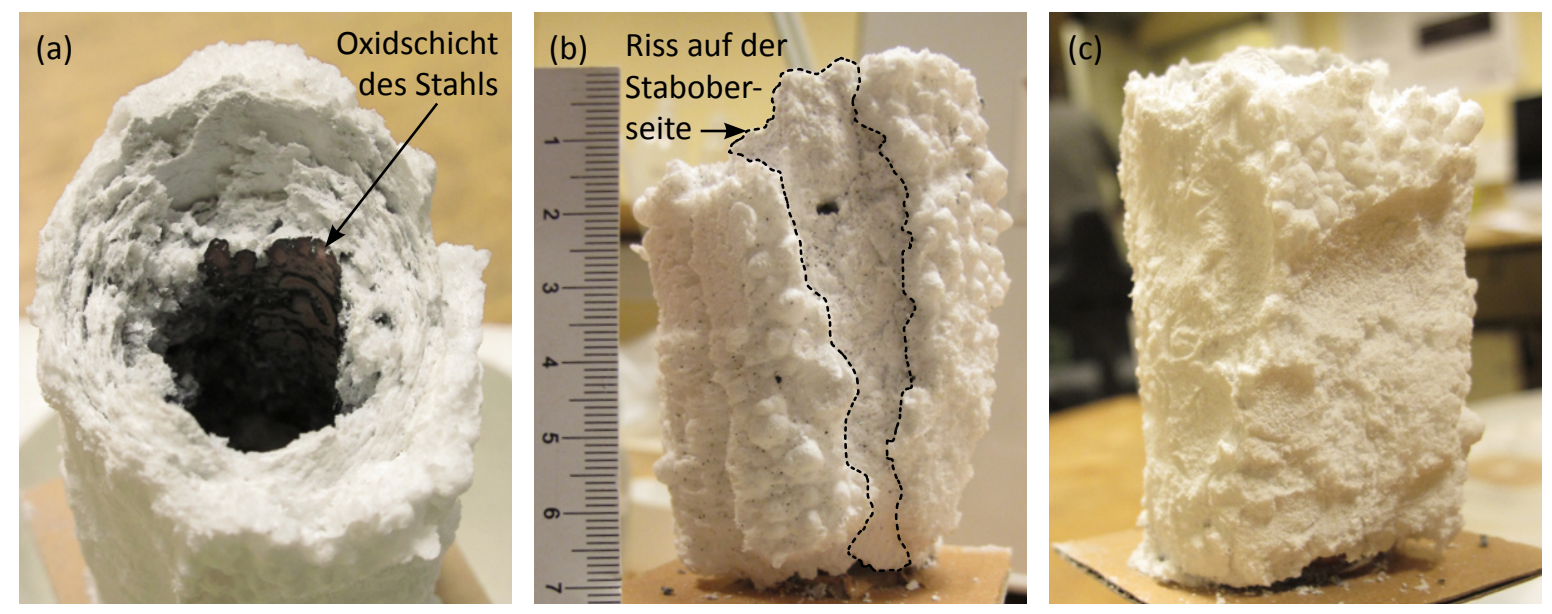

Abb. 3.39: Ansichten der mittels Computertomographie untersuchten Schaumprobe B10 a) Isometrische Ansicht b) Vorderansicht c) Rückansicht

Mit Hilfe der Computertomographie werden detaillierte Querschnittsbilder der zu untersuchenden Schaumprobe erzeugt Abb. 3.40. Die Probe wird stufenweise im Strahl des Röntgenapparates gedreht und für jede Winkelstellung ein Schnittbild erzeugt. Auf den einzelnen Röntgenbildern ist das Projektionsbild des Bauteils abgebildet. Die unterschiedliche Schwarzfärbung entsteht aufgrund abweichender Materialdicken oder -dichten. Je dicker oder dichter ein Bauteil ist, desto weniger dringt die Strahlung hindurch. Die Reste der Oxidschicht des Stahls, welche teilweise noch an der Innenseite der Schaumprobe anhaften, strahlen aufgrund der hohen Dichte besonders hell. Aus den Schnittbildern wird mit Hilfe eines Auswertungsprogramms ein 2D bzw. 3D-Modell erstellt.

Für den verwendeten Versuchsaufbau der CT-Untersuchung liegt die Bildauflösung bei 667 x 667 Pixel. Die Kantenlänge eines Pixels entspricht einer Länge von 0,034 mm. Die Bild- 
auflösung reicht jedoch nicht aus, um die feinen Poren des reaktiven Brandschutzsystems gut und in ausreichender Bildschärfe darzustellen. Ein Schnittbild der CT-Untersuchung ist in Abb. 3.42 dargestellt. Durch die Schwarz-Weiß-Darstellung der Abbildung ist die Struktur der Probe gut zu erkennen. Die bereits in der visuellen Prüfung festgestellten unterschiedlichen Aufschäumbereiche sind auch in der CT-Darstellung deutlich zu erkennen. Der äußere Bereich ist über den Querschnitt relativ unförmig. Die vergleichsweise dunkle Farbe lässt auf eine poröse Schicht schließen. Der innere Schaumbereich ist wesentlich heller und somit dichter. Die innere, lamellenartige Struktur ist ringförmig um das Zugglied angeordnet. Die Position des entfernten Stahlzuggliedes sowie dessen Größe sind nachträglich in die Abbildung der Computertomographie eingearbeitet worden. Die ursprüngliche Lage des Stahlbauteils lässt sich aus der Abbildung nicht exakt feststellen, da sich beim Ausbau der Schaumprobe kleinere Beschädigungen auf der Innenseite der Aufschäumung des reaktiven Brandschutzsystems nicht vermeiden lassen.

Um die Porengröße sowie die Oberfläche des aufgeschäumten reaktiven Brandschutzsystems noch näher zu analysieren, wird die Aufschäumung zusätzlich am Rasterelektronenmikroskop untersucht. Neben der Dicke der aufgeschäumten Brandschutzbeschichtung wird die thermische Schutzwirkung des Brandschutzsystems durch die Oberflächenbeschaffenheit der Aufschäumung beeinflusst. Untersuchungen zu diesem Thema sind in den Veröffentlichungen [62] und [42] beschrieben. Kleine Risse oder Löcher in der Oberfläche der aufgeschäumten Brandschutzbeschichtung können die thermische Schutzwirkung des Systems reduzieren. Bei der untersuchten Schaumprobe des Versuchskörpers B10 sind kleinere Löcher in der Oberfläche vorhanden (siehe Abb. 3.43). Es lässt sich jedoch nicht generell ausschließen, dass diese bei der Vorbereitung der Probe entstanden sind. Im inneren Bereich der Aufschäumung sind die Wände der Poren deutlich sichtbar. Allerdings ist eine klare Abtrennung der einzelnen Poren nicht

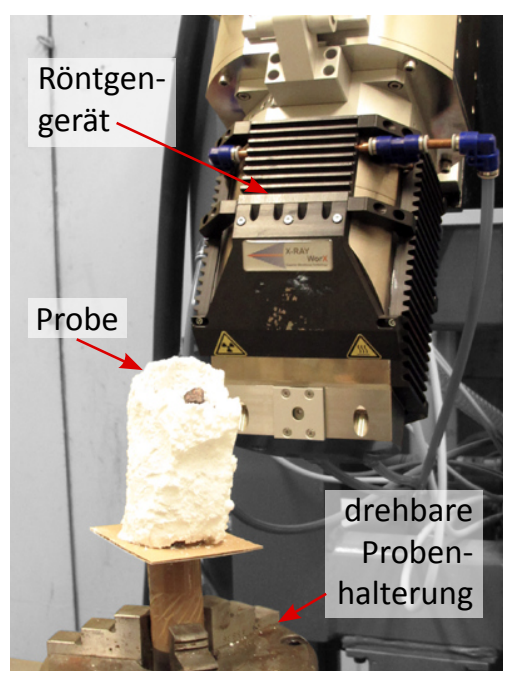

Abb. 3.40: Versuchsaufbau der Computertomographieuntersuchung

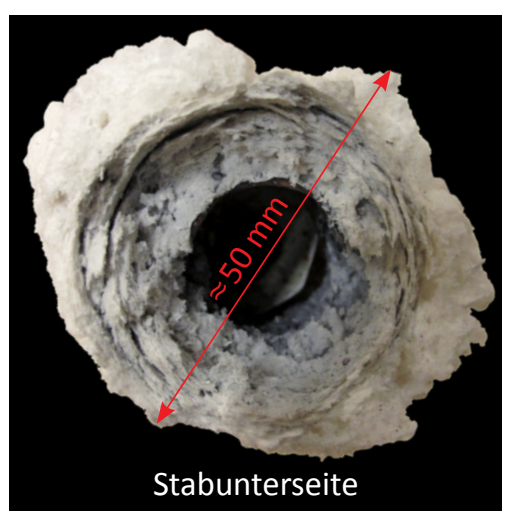

Abb. 3.41: Ansicht der Probe (nicht maßstäblich)

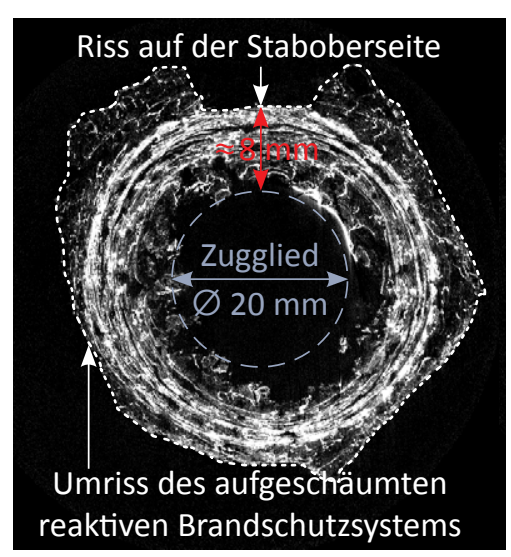

Abb. 3.42: Querschnitt der Probe aus der CT-Messung (nicht maßstäblich) 
vorhanden, sondern die einzelnen Hohlräume sind miteinander verbunden. Diese offenporige Porenstruktur resultiert wahrscheinlich aus der Pyrolyse der organischen Bestandteile des reaktiven Brandschutzsystems. Eine Systematik zur Hohlraumbildung ist auch bei einer weiteren Bildvergrößerung nicht zu erkennen. Darüber hinaus geben die REM-Untersuchungen lediglich die Struktur der Aufschäumung nach dem Brandversuch wieder.
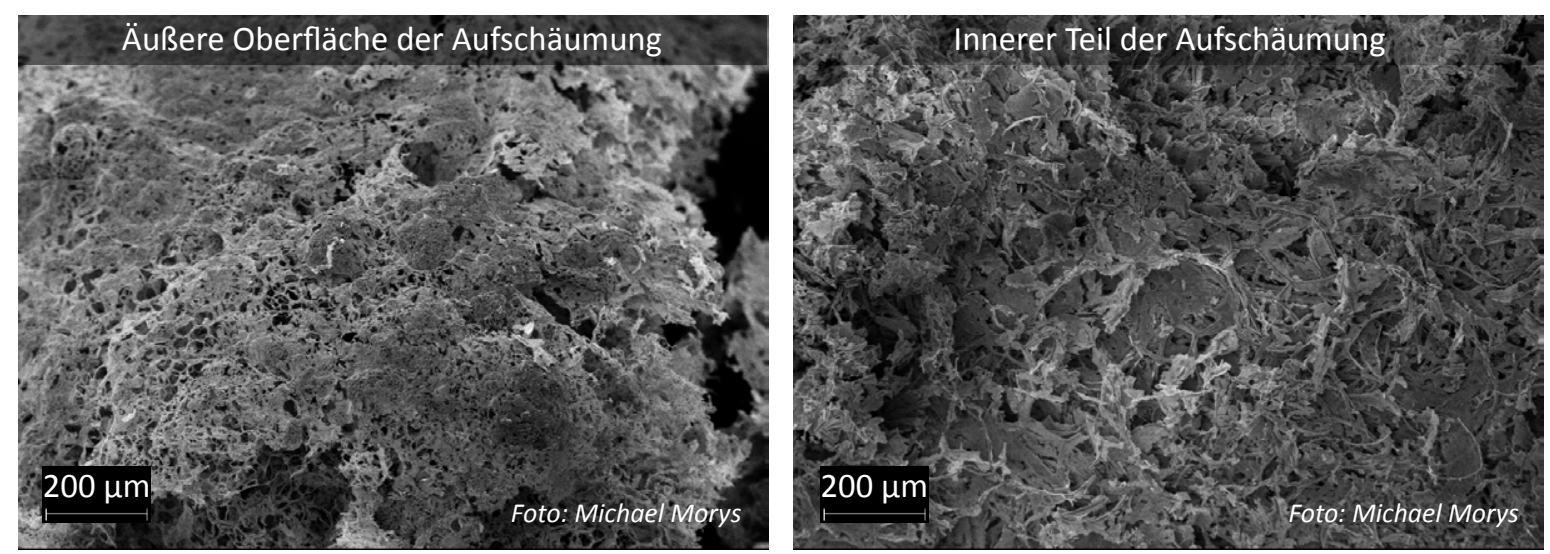

Abb. 3.43: REM-Untersuchung der Aufschäumung nach dem Brandversuch (200-fache Vergrößerung)

\section{Untersuchungen zur Porengestalt und -größenverteilung aus der Literatur}

Mikroskopische Untersuchungen an wasserbasierten und lösemittelhaltigen sowie epoxidharzbasierten Brandschutzbeschichtungen wurden bereits von Tabeling [60] durchgeführt. Dabei wurden auch unterschiedliche Reaktionsphasen des reaktiven Brandschutzsystems untersucht, d.h. unmittelbar nach der Initiierung des Aufschäumvorgangs, bei Erreichen der maximalen Aufschäumhöhe und nach der Pyrolyse der organischen Bestandteile. Aus den Untersuchungen geht hervor, dass sich zu Beginn des Aufschäumvorgangs zunächst eine kugelförmige Gestalt der Poren einstellt. Im vollständig aufgeschäumten Zustand liegt eine wabenartige Form der Poren vor. Nach der Pyrolyse der organischen Bestandteile ergibt sich eine offenporige Porenstruktur. Tabeling beschreibt, dass die Aufschäumung suksessive von der temperaturbeanspruchten Oberfläche des reaktiven Brandschutzsystems hin zum Stahlbauteil in Teilschichten erfolgt. Eine ähnliche Schaumstruktur und ein vergleichbares Aufschäumverhalten des reaktiven Brandschutzsystems zeigt sich auch an den eigenen untersuchten Proben. Durch das suksessive Aufschäumen der Brandschutzbeschichtung lassen sich auch die Rissheilungseffekte des reaktiven Brandschutzsystems erklären.

Die mittlere Porengröße in der Aufschäumung werden von Tabeling mit ca. $7 \mu \mathrm{m}$ in der Aufschäumphase bis hin zu etwa 20 - $30 \mu \mathrm{m}$ für den maximal aufgeschäumten Zustand des Brandschutzsystems angegeben. Zudem sind in der aufgeschäumten Brandschutzbeschichtung große Fehlstellen bzw. Hohlräume vorhanden. In den visuellen Untersuchungen der Proben des in der BAM getesteten reaktiven Brandschutzsystems sind Hohlräume vorhanden (siehe Abb. 3.41). Ferner weist die in der BAM untersuchte Brandschutzbeschichtung 
im aufgeschäumten Zustand einen deutlichen Unterschied der Struktur der Aufschäumung über den Querschnitt auf. Der äußere Querschnittsbereich weist eine feinporige Struktur auf, während der innere Bereich einen lamellenartigen Aufbau hat (siehe Abb. 3.41). In den Untersuchungen von Tabeling [60] ist dieses Verhalten ebenfalls zu beobachten, jedoch wesentlich schwächer ausgeprägt. Mögliche Ursachen für die unterschiedliche Beschaffenheit der Aufschäumung könnten die Geometrie der Versuchskörper, die Art der Wärmequelle sowie das verwendete reaktive Brandschutzsystem sein.

\subsection{Leistungskriterien der Tragfähigkeit}

Der Probenbruch der mechanisch belasteten Zugglieder markiert das Ende der Brandversuche. Für die Einordnung in eine Feuerwiderstandsklasse $\left(t_{F}\right)$ ist nicht der Zeitpunkt des Probenbruchs $\left(t_{\text {Bruch }}\right)$, sondern der Zeitpunkt für das Erreichen der Tragfähigkeit $\left(t_{\text {Trag }}\right)$ maßgebend. Der Verlust der Tragfähigkeit ist gleichbedeutend mit dem Versagen des Bauteils. Laut DIN EN 1363-1 [07] ist die Tragfähigkeit des Bauteils erreicht, wenn der Grenzwert der Leistungskriterien überschritten ist. Als Leistungskriterien gelten in der Norm die Größe sowie die Geschwindigkeit der Verformungen. Allerdings beinhaltet die Norm lediglich Regelungen für Brandprüfungen an mechanisch belasteten Trägern und Stützen. Für die Bewertung von Brandversuchen an Zuggliedern existieren derzeit keine normativen Vorgaben. Auf Grundlage der im Kapitel 3.5 vorgenommenen Brandversuche werden im folgenden Vorschläge für Leistungskriterien der Tragfähigkeit zur Bestimmung der Feuerwiderstandsdauer von Zuggliedern erarbeitet.

\subsubsection{Verformungskriterium}

\section{Regelungen für Träger und Stützen}

Für Träger ist in DIN EN 1363-1[07] eine Grenzdurchbiegung und für Stützen ein Grenzwert der vertikalen Stauchung angegeben (siehe Gleichung (3.2) und (3.3)). In den Gleichungen wird die Geometrie des Bauteils mit Hilfe der Spannweite und Bauteilhöhe oder der Anfangshöhe des Versuchskörpers berücksichtigt. Bei einer Überschreitung des Grenzwertes ist die Tragfähigkeit des Bauteils erreicht. Es werden jeweils nur vollendete Minuten betrachtet. Die Leistungskriterien werden zudem unabhängig von der Bauteiltemperatur berechnet. Auch der verwendete Baustoff oder eventuell eingesetzte Brandschutzmaßnahmen, z.B. reaktive Brandschutzbeschichtungen, gehen in die Bestimmung der Leistungskriterien nicht ein.

Für biegebeanspruchte Bauteile werden von Ryan \& Robertson [53] Kriterien für die Tragfähigkeit vorgeschlagen. In der Veröffentlichung wird zwischen einer Grenzdurchbiegung und einer Grenzdurchbiegungsgeschwindigkeit unterschieden. Das in der Veröffentlichung angegebene Verformungskriterium ist halb so groß, wie der in der Norm [07] angegebene 
Wert. Das von Ryan und Robertson empfohlene Verformungsgeschwindigkeitskriterium ist mit der Norm identisch. Durch die Vielzahl an Konstruktionsmöglichkeiten und den damit verbundenen Variablen, d.h. Auflagersituation, mechanische Beanspruchung, Bauteilgeometrie und Materialien, gestaltet sich die Ausarbeitung von Leistungskriterien für die Tragfähigkeit relativ schwierig. Um dennoch auf praktikable Art und Weise die Feuerwiderstandsdauer bestimmen zu können, werden zahlreiche Vereinfachungen hinsichtlich des Verformungskriteriums getroffen. Die Anwendbarkeit der von Ryan \& Robertson entwickelten Kriterien wurde anhand von 50 Brandversuchen untersucht. Die Tragfähigkeit des Bauteils gilt als erreicht, sobald beide Leistungskriterien, d.h. Verformungkriterium und Verformungsgeschwindigkeitskriterium, überschritten werden. Diese Regelung ist mit den Vorgaben in DIN EN 1363-1 [07] bis zur Fassung von 1999 identisch. Seit der Fassung der Norm von 2012 endet die Tragfähigkeit sobald eines der Leistungskriterien überschritten wird.

$$
\begin{array}{lll}
D=\frac{\mathrm{L}^{2}}{400 \cdot d} & D & \begin{array}{l}
\text { Grenzwert der Verformung (Träger) } \\
\text { Lichte Spannweite des Probekörpers }
\end{array} \\
& \begin{array}{l}
\text { Abstand zw. dem äußersten Rand der } \\
\text { Druck- und Zugzone }
\end{array} \\
C=\frac{\mathrm{h}}{100} & C & \text { Grenzwert der Verformung (Stütze) } \\
& h & \text { Anfangshöhe des Probekörpers }
\end{array}
$$

\section{Vorschlag für Zugglieder}

Die in der Prüfung gemessenen Verformungen setzen sich aus einem mechanischen und einem thermischen Verformungsanteil zusammen (siehe Gleichung (3.4)). Um die Berechnung eines Verformungskriteriums zu vereinfachen, wird zunächst unterstellt, dass sich während des Brandversuches nur die im Brandraum befindlichen Stabbereiche erwärmen. Die Temperaturmessungen aus den Brandversuchen bestätigen, dass die Stahltemperatur außerhalb des Brandraumes relativ schnell wieder Raumtemperatur annimmt (siehe Abb. 5.03.

$$
\begin{array}{lll}
\Delta L_{\text {Grenz }}=\Delta L_{\text {mech }}+\Delta L_{\text {therm }} & \Delta L_{\text {Grenz }} & \text { Grenzwert der Verformung } \\
& \Delta L_{\text {mech }} & \text { mechanischer Verformungsanteil } \\
& \Delta L_{\text {therm }} & \text { thermischer Verformungsanteil }
\end{array}
$$

Der mechanische Verformungsanteil $\left(\Delta \mathrm{L}_{\text {mech }}\right)$ setzt sich aus der Stablängsverformung der innerhalb $\left(\Delta \mathrm{L}_{\text {warm }}\right)$ und außerhalb des Brandraumes $\left(\Delta \mathrm{L}_{\text {kalt }}\right)$ befindlichen Stabbereiche zusammen (siehe Gleichung (3.5)). Für die Stabbereiche außerhalb des Brandraumes $\left(\Delta \mathrm{L}_{\text {kalt }}\right)$ wird ein anteiliger Wert der im Brandversuch gemessenen Anfangsverformung $\left(\Delta \mathrm{L}_{\mathrm{A}}\right)$ infolge der mechanischen Zugbeanspruchung nach Gleichung (3.6) ermittelt. Ist dieser nicht bekannt, kann vereinfachend die elastische Verformung des Zuggliedes nach Gleichung (3.7) angenommen werden. Die Stablängsverformung des innerhalb des Brandraumes befindlichen 
beflammten Stabbereiches des Zuggliedes $\left(\Delta \mathrm{L}_{\text {warm }}\right)$ wird nach Gleichung (3.8) berechnet. Für die Berechnung wird angenommen, dass dieser Stabbereich gleichmäßig bis zur Streckgrenze bzw. der 0,2\% Dehngrenze gedehnt wird. Nach DIN EN 1993-1-2 [13] wird bei warmgewalzten Profilen die Streckgrenze des Stahls unabhängig von der Stahltemperatur bei einer Dehnung von $2 \%$ erreicht (siehe Gleichung (3.10)). Für Zugglieder aus kaltverformtem Blankstahl wird auf Grundlage der in Kapitel 4.6 durchgeführten Materialuntersuchungen ein Dehnungswert der 0,2\%-Dehngrenze entsprechend Gleichung (3.9) vorgeschlagen. Für die Dehnungsberechnung wird eine Bauteiltemperatur von $550^{\circ} \mathrm{C}$ zugrunde gelegt, welche der maximalen Stahltemperatur zum Zeitpunkt des Probenbruchs eines im Brandfall voll ausgelasteten Zuggliedes $\left(\mu_{\mathrm{fi}}=0,65\right)$ entspricht.

$$
\Delta L_{\text {mech }}=\Delta L_{\text {kalt }}+\Delta L_{\text {warm }}
$$

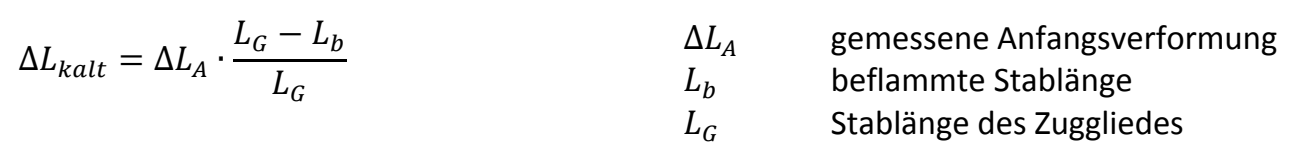

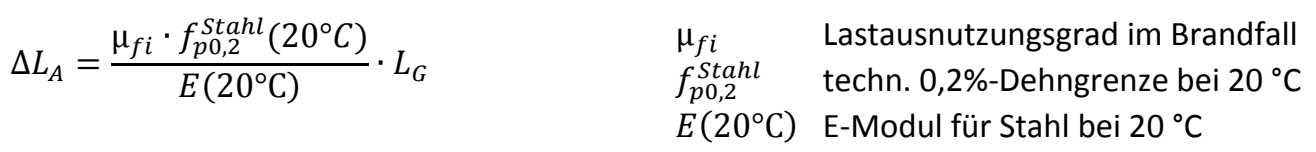

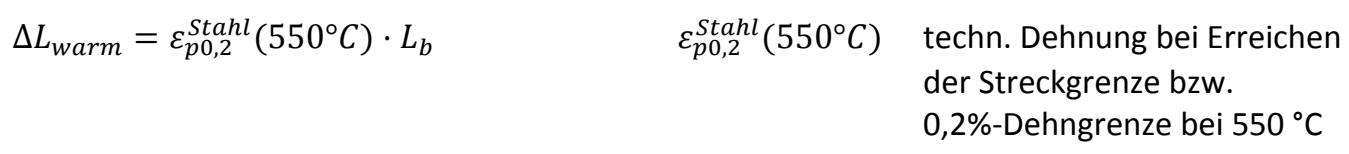

$$
\begin{aligned}
& \varepsilon_{p 0,2}^{\text {Stahl }}\left(550^{\circ} \mathrm{C}\right)=\frac{f_{00,2}^{\text {Stahl }}\left(20^{\circ} \mathrm{C}\right) \cdot f_{p 0,2}^{\text {Ref }}\left(550^{\circ} \mathrm{C}\right)}{f_{p 0,2}^{\text {Ref }}\left(20^{\circ} \mathrm{C}\right) \cdot E^{\text {Ref }}\left(550^{\circ} \mathrm{C}\right)}+0,002 \quad \begin{array}{l}
f_{p 0,2}^{\text {Stahl }} \text { techn. 0,2\%-Dehngrenze bei } 20^{\circ} \mathrm{C} \\
\text { (verwendeter Blankstahl) } \\
f_{p 0,2}^{\text {Ref }}
\end{array} \\
& \left(585 \mathrm{~N} / \mathrm{mm}^{2}\right) \text { und } 550^{\circ} \mathrm{C}\left(250 \mathrm{~N} / \mathrm{mm}^{2}\right) \\
& E^{\text {Ref }} \quad \text { E-Modul für Stahl bei } 550^{\circ} \mathrm{C} \\
& \left(159500 \mathrm{~N} / \mathrm{mm}^{2}\right)
\end{aligned}
$$

$$
\begin{array}{lr}
\varepsilon_{p 0,2}^{\text {Stahl }}\left(550^{\circ} \mathrm{C}\right)=2,6793 \cdot 10^{-6} \cdot f_{p 0,2}^{\text {Stahl }}\left(20^{\circ} \mathrm{C}\right)+0,002 & \text { für kaltverformten Blankstahl } \\
\varepsilon_{p 0,2}^{\text {Stahl }}\left(550^{\circ} \mathrm{C}\right)=0,02 & \text { für warmgewalzten Baustahl }
\end{array}
$$

Für den thermischen Verformungsanteil $\left(\Delta \mathrm{L}_{\text {therm }}\right)$ wird angenommen, dass die gesamte beflammte Stablänge eine konstante Stahltemperatur besitzt. Vereinfachend wird angenommen, dass diese Temperatur der maximalen Stahltemperatur zum Zeitpunkt des Probenbruchs entspricht. In einer ersten Näherung kann auch die Bemessungstemperatur verwendet werden. Die Berechnung der Bemessungstemperatur ist in Gleichung (3.11) angegeben. Für den Wärmeausdehnungskoeffizienten des Stahls kann für warmgewalzte 
Profile entsprechend DIN EN 1993-1-2 [2] ein Wert von 1,50 $\cdot 10^{-5} \mathrm{~K}^{-1}$ angenommen werden. Für kaltverformte Bauteile wird der aus Kapitel 4.6 ermittelte Wert von $1,75 \cdot 10^{-5} \mathrm{~K}^{-1}$ empfohlen. Beide Werte beziehen sich jeweils auf eine Bauteiltemperatur von $700{ }^{\circ} \mathrm{C}$. Der thermische Verformungsanteil berechnet sich nach Gleichung (3.12). Durch die Vereinfachungen bezüglich des Wärmeausdehnungskoeffizienten und der über die Stablänge als konstant angenommenen Stahltemperatur kann es zu einer leichten Überschätzung des thermischen Verformungsanteils kommen.

$$
\begin{aligned}
& \frac{E_{d, f i}}{R_{d, f i}}=\frac{\frac{\eta_{f i} \cdot \mu_{0}}{\gamma_{M, 0}}}{\frac{k_{y, \theta}}{\gamma_{M, f i}}} \leq 1,0 \\
& \eta_{f i} \quad \text { Abminderungsfaktor der Last im Brandfall } \\
& \mu_{0} \quad \text { Lastausnutzungsgrad im Kaltfall } \\
& \gamma_{M, 0} \quad \text { Teilsicherheitsbeiwert im Kaltfall } \\
& \gamma_{M, f i} \text { Teilsicherheitsbeiwert im Brandfall } \\
& k_{y, \Theta} \quad \text { Abminderungsfaktor der Fließspannung } \\
& \theta \quad \text { Bemessungstemperatur } \\
& \Delta L_{\text {therm }}=\alpha \cdot \theta_{s} \cdot L_{b} \\
& \alpha \quad \text { Wärmeausdehnungskoeffizient bei } 700^{\circ} \mathrm{C} \\
& \text { - warmgewalzter Stahl }=1,50 \cdot 10^{-5} K^{-1} \\
& \text { - kaltverformter Stahl }=1,75 \cdot 10^{-5} K^{-1} \\
& \theta_{s} \quad \text { max. Stahltemperatur für die Tragfähigkeit }
\end{aligned}
$$

\subsubsection{Verformungsgeschwindigkeitskriterium}

\section{Regelungen für Träger und Stützen}

Neben der Betrachtung von Grenzverformungen ist laut Norm [07] für die Bestimmung der Feuerwiderstandsdauer die Verformungsgeschwindigkeit zu berücksichtigen. Dabei wird zwischen biegebeanspruchten und vertikal belasteten Bauteilen unterschieden. Die Berechnung der Verformungsgeschwindigkeit ist in Gleichung (3.13) und (3.14) angegeben.

$\frac{d D}{d t}=\frac{\mathrm{L}^{2}}{9000 \cdot d}[\mathrm{~mm} / \mathrm{min}] \quad$ (Träger) $\begin{aligned} & \theta_{s} \\ & \frac{d D}{d t} \\ & L\end{aligned}$

$\frac{d C}{d t}=\frac{3 \cdot h}{1000} \quad[\mathrm{~mm} / \mathrm{min}] \quad$ (Stütze)

$\theta_{s} \quad$ max. Stahltemperatur für die Tragfähigkeit

$\frac{d D}{d t} \quad$ Verformungsgeschwindigkeitskriterium

Abstand zw. dem äußersten Rand der Druck- und Zugzone

\section{Vorschlag für Zugglieder}

Wie bereits bei der Berechnung des Leistungskriteriums für die Verformung setzt sich auch die Verformungsgeschwindigkeit aus einem mechanischen und einem thermischen Anteil zusammen. Die Geschwindigkeit wird in der Regel mit Hilfe der Stablängsverformung in Millimeter pro Minute Branddauer angegeben. Analog zum Vorgehen beim Verformungskriterium wird zunächst unterstellt, dass sich nur die im Brandraum befindlichen Stabberei- 
che erwärmen. Stabbereiche mit Raumtemperatur leisten daher keinen Beitrag zur Verformungsgeschwindigkeit. Der thermische Anteil an der Verformungsgeschwindigkeit ist von der beflammten Stablänge und der Erwärmungsgeschwindigkeit des Bauteils abhängig. Die Erwärmungsgeschwindigkeit wird durch die Profilgeometrie des Zuggliedes sowie der thermischen Schutzwirkung des reaktiven Brandschutzsystems beeinflusst. Da sich die thermische Schutzwirkung der Brandschutzbeschichtung im Laufe des Brandversuches ändert, lässt sich der thermische Anteil an der Verformungsgeschwindigkeit nur schwer erfassen.

Der mechanische Anteil der Verformungsgeschwindigkeit ist von der beflammten Stablänge sowie der Stahltemperatur des Zuggliedes abhängig. Je nach Temperatur ergeben sich aufgrund der temperaturabhängigen Spannungs-Dehnungs-Kennlinien des Stahls unterschiedliche Verformungen, welche die Höhe der Verformungsgeschwindigkeit beeinflussen. Die Zunahme der Stahltemperatur, welche von der Erwärmungsgeschwindigkeit abhängt, steht in direktem Zusammenhang mit der thermischen Schutzwirkung des Brandschutzsystems. Aufgrund der zeitlich veränderlichen Schutzwirkung der Brandschutzbeschichtung ist die Berechnung des mechanischen Verformungsgeschwindigkeitsanteils ebenfalls schwierig.

In den durchgeführten Brandversuchen an Zuggliedern stellte sich nach dem Aufschäumen des reaktiven Brandschutzsystems eine relativ gleichmäßige Verformungsgeschwindigkeit von etwa 0,10 bis $0,25 \mathrm{~mm} / \mathrm{min}$ ein. Bei der Annäherung an den Zeitpunkt des Probenbruchs des Zuggliedes kommt es zu einer deutlichen Zunahme der Verformungsgeschwindigkeit. Um die Sensitivität des Verformungsgeschwindigkeitskriteriums bis zum Erreichen der Tragfähigkeit besser beurteilen zu können, werden zwei Grenzverformungsgeschwindigkeiten $(0,75$ und $1,0 \mathrm{~mm} / \mathrm{min})$ untersucht. Die Auswertung dieser Untersuchung zeigt, dass sich die Zeit bis zum Erreichen der Tragfähigkeit zwischen den beiden gewählten Grenzwerten für die Verformungsgeschwindigkeit teilweise gar nicht oder nur um wenige Minuten unterscheidet. Für den untersuchten Verformungsgeschwindigkeitsbereich ist daher nur eine geringe Sensibilität vorhanden (siehe Tabelle A.4). Da die Verformungsgeschwindigkeit auch von der beflammten Stablänge abhängig ist, muss diese bei der Berechnung des Verformungsgeschwindigkeitskriteriums berücksichtigt werden. Als Grenzwert wird daher eine Dehngeschwindigkeit von $\dot{\mathrm{e}}=0,001 \mathrm{~min}^{-1}$ vorgeschlagen. Das Verformungsgeschwindigkeitskriterium ergibt sich nach Gleichung (3.15) durch Multiplikation der Dehngeschwindigkeit mit der beflammten Stablänge des Zuggliedes. Für die in der BAM untersuchten mechanisch belasteten Zugglieder $\left(\mathrm{L}_{\mathrm{b}} \approx 900 \mathrm{~mm}\right.$ ) ergibt sich ein Verformungsgeschwindigkeitskriterium von $0,9 \mathrm{~mm} / \mathrm{min}$. Dieser Wert überschreitet die Verformungsgeschwindigkeit zum Ende des Aufschäumprozesses des Brandschutzsystems um ein Mehrfaches und ist somit ein eindeutiges Zeichen dafür, dass die Tragfähigkeit des Bauteils erreicht ist.

$$
\begin{array}{llll}
\Delta V_{\text {Grenz }}=\dot{e} \cdot L_{b}[\mathrm{~mm} / \mathrm{min}] & \text { (Zugglied) } & h & \text { Anfangshöhe des Probekörpers } \\
& \Delta V_{\text {Grenz }} & \text { Verformungsgeschwindigkeitskriterium } \\
\dot{e} & \text { Dehngeschwindigkeit }=0,001 \mathrm{~min}^{-1}
\end{array}
$$




\subsubsection{Anwendung der Leistungskriterien bei Stahlzuggliedern}

Die Anwendung der entwickelten Leistungskriterien der Tragfähigkeit zur Bestimmung des Feuerwiderstandes wird nachfolgend exemplarisch an zwei Zuggliedern der VR c gezeigt. Für beide Versuchskörper wird ein Kreisvollprofil mit einem Durchmesser von $20 \mathrm{~mm}$ sowie eine Trockenschichtdicke des reaktiven Brandschutzsystems von 3,5 $\mathrm{mm}$ verwendet. Die Zugglieder unterscheiden sich lediglich in der Höhe der aufgebrachten Zugbeanspruchung. Die Lastausnutzungsgrade im Brandfall betragen $\mu_{\mathrm{fi}}=0,15$ (B22) und $\mu_{\mathrm{fi}}=0,65$ (B18). Da die beflammte Bau-

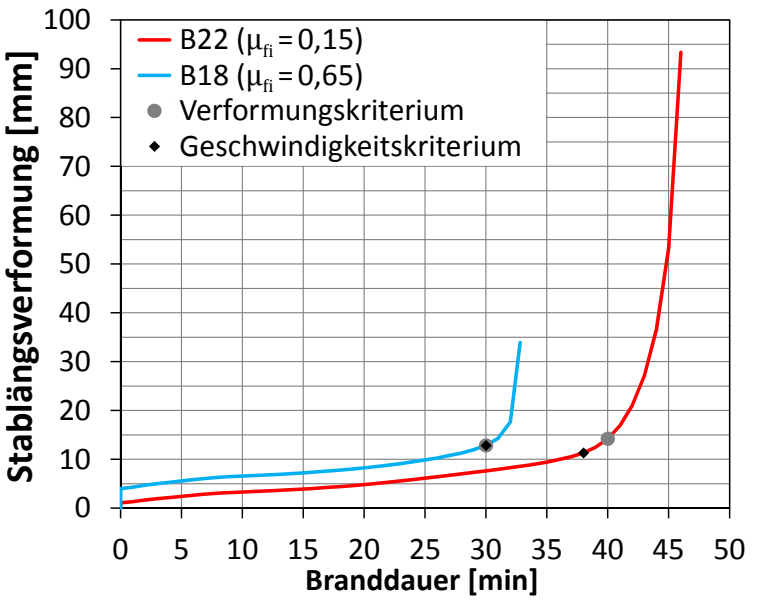

Abb. 3.44: Anwendung der entwickelten Leistungskriterien auf die Zugglieder $B 22$ und $B 18$ (KVP D20, DFT $=3,5 \mathrm{~mm})$ teillänge der Versuchskörper gleich ist, beträgt der Grenzwert des Verformungsgeschwindigkeitskriteriums für beide Versuchskörper $0,9 \mathrm{~mm} / \mathrm{min}$. Durch die unterschiedliche Stahltemperatur beim Probenbruch ergibt sich für die Verformung ein Grenzwert von 15 mm (B22) bzw. 13 mm (B18). Die beiden Leistungskriterien der Tragfähigkeit werden mit der im Brandversuch gemessenen Stablängsverformung verglichen. Es werden jeweils nur vollendete Minuten betrachtet. Die Auswertung in Abb. 3.44 zeigt, dass das Geschwindigkeitskriterium bis zu einer Branddauer von 38 min (B22) bzw. 30 min (B18) eingehalten wird. Für das Verformungskriterium ergibt sich eine Branddauer von 40 min (B22) bzw. 30 min (B18). Die jeweils an einem Zugglied bestimmte kleinste Branddauer markiert das Erreichen der Tragfähigkeit. Der Zeitunterschied zwischen den beiden Leistungskriterien liegt bei den getesteten mechanisch belasteten Zuggliedern bei 0 bis 2 Minuten. In der Regel ist für das Erreichen der Tragfähigkeit das Verformungsgeschwindigkeitskriterium maßgebend.

Da die Tragfähigkeit bis zu einem Zeitpunkt von 30 Minuten gewährleistet ist, erfüllen beide Zugglieder die Anforderung der Feuerwiderstandsklasse R30. Neben den im Brandversuch einzuhaltenden Tragfähigkeitskriterien und der Zuordnung in die einzelnen Feuerwiderstandsklassen sind in der Heißbemessung der Gesamtstatik der Konstruktion die Stablängsverformungen des Zuggliedes zu berücksichtigen. Vom Tragwerksplaner ist durch eine statische Berechnung nachzuweisen, dass die Standsicherheit infolge der auftretenden Verformungen des Zuggliedes für die gesamte Feuerwiderstandsdauer gewährleistet ist. 


\section{Bestimmung der mechanischen Hochtemperatureigen- schaften von Blankstahl}

Zusätzlich zu den Brandversuchen im Realmaßstab wird das Bauteilverhalten von Stahlzuggliedern mit reaktiver Brandschutzbeschichtung anhand von numerischen Simulationen untersucht (siehe Kapitel 5). Für diese Untersuchung sind neben den in den Brandversuchen gemessenen Temperatur-Zeit-Kurven auch die mechanischen Hochtemperatureigenschaften des verwendeten Stahls erforderlich. Dazu werden die nachfolgende Untersuchungen durchgeführt. Die Ergebnisse aus den Materialuntersuchungen sind im Forschungsbericht [29] sowie zum Teil in einer vom Verfasser betreuten Bachelorarbeit [64] enthalten.

Für Stahlbauteile mit kleinen Profilabmessungen wird häufig kaltverformter Blankstahl eingesetzt. Dieser wird aus warmgewalztem Stahl durch „Ziehen“ oder „Schälen“ hergestellt. Aufgrund der Kaltverformung beim Herstellungsverfahren kommt es zu einer Veränderung der Materialeigenschaften. Ein typischer Verlauf der Spannungs-Dehnungs-Kurven von warmgewalzten Baustahl und kaltverformten Blankstahl bei Raumtemperatur ist in Abb. 4.01 dargestellt. Die in den Brandversuchen verwendeten Stahlzugglieder mit Kreisvollprofil bestehen aus gezogenem, kaltverformtem Blankstahl der Festigkeitsklasse S355. In der Norm [13] sind Materialkennwerte lediglich für warmgewalzten Baustahl und kaltgeformte dünnwandige Stahlbauteile angegeben. Informationen zu den Hochtemperatureigenschaften von kaltverformtem Blankstahl sind in der Norm nicht enthalten. Mittels Materialuntersuchungen wird festgestellt, welche Besonderheiten der in den Brandversuchen verwendete kaltverformte Blankstahl besitzt und inwieweit sich die in der Norm für warmgewalzten Baustahl angegebenen Werte auf Blankstahl übertragen lassen. Ferner finden die Ergebnisse aus den Materialuntersuchungen Eingang in die numerische Simulation (siehe Kapitel 5).
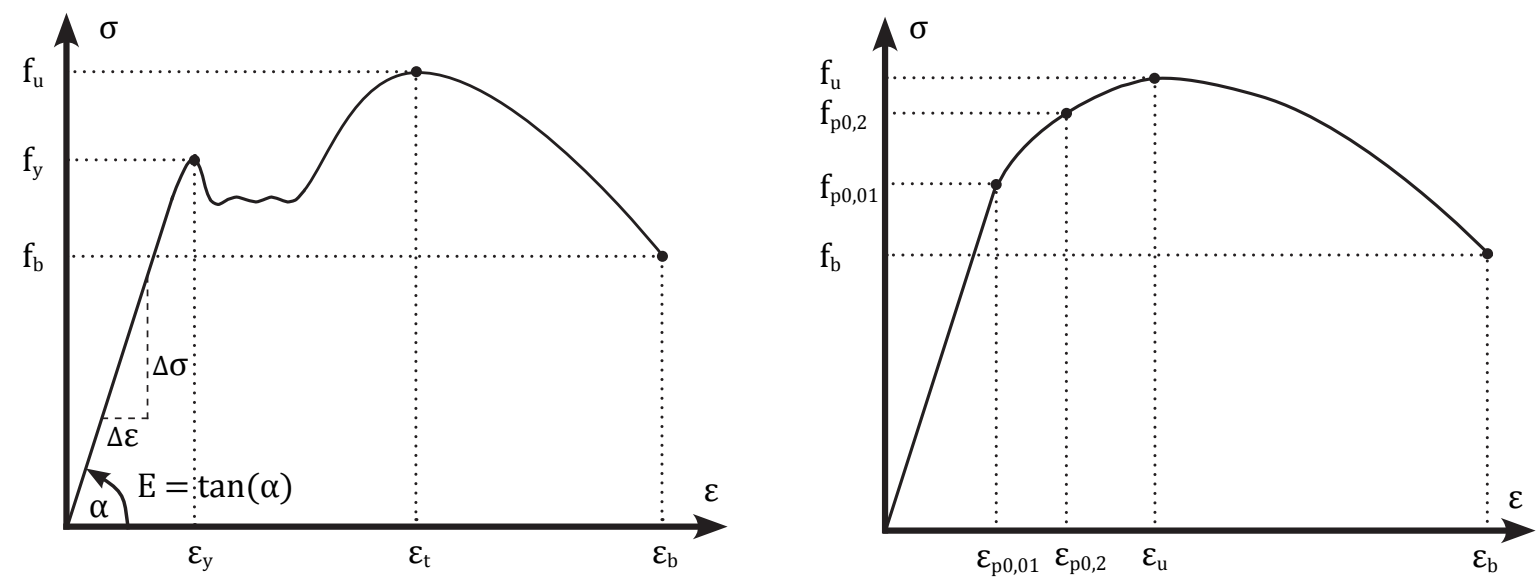

Abb. 4.01: Typische Spannungs-Dehnungs-Kurve bei Raumtemperatur ( $\varepsilon$ nicht maßstablich, links: warmgewalzter, rechts: kaltverformter Blankstahl) 


\subsection{Herstellung von Blankstahl}

Bei der Bestellung von Vollprofilen mit rundem oder rechteckigem Querschnitt wird von den Stahllieferanten überwiegend Blankstahlangeboten. DieseStähle zeichnen sich durch eine hochwertige blanke Oberfläche und Maßgenauigkeit aus. Nach DIN EN 10079 [06] wird Blankstahl je nach Fertigungsverfahren in gezogenen, geschälten und geschliffenen bzw. polierten Stahl unterteilt. Die Art des Fertigungsverfahrens wird durch Zusätze in der Bezeichnung des Stahls angegeben. Für Ziehen wird die Abkürzung,$+C$, für geschält ${ }+\mathrm{SH}^{\prime}$, für geschliffen " $+\mathrm{SL}^{\prime}$ und für poliert " $+\mathrm{PL}^{\text {" }}$ verwendet.

Die nachfolgenden Angaben entstammen dem Merkblatt des StahlInformations-Zentrums [41]. Das Ausgangmaterial für Blankstahl bildet, unabhängig vom späteren Fertigungsverfahren, warmgewalzter Stahl. Runde Blankstähle mit einem Durchmesser von weniger als $15 \mathrm{~mm}$ werden in der Regel durch Ziehen hergestellt. Stäbe mit Durchmessern von 15 bis $100 \mathrm{~mm}$ können sowohl gezogen als auch geschält werden. Bei Durchmessern von mehr als $100 \mathrm{~mm}$ wird üblicherweise nur geschält. Blankstahl, welcher durch eine spanlose Kaltverformung („Ziehen“) hergestellt wird, ist in der Regel durch eine Kaltverfestigung des Stahls gekennzeichnet. Durch eine unterhalb der Rekristallisationstemperatur stattfindende Verformung wird die Festigkeit des Stahls gesteigert. Der kaltgezogene Blankstahl besitzt unter

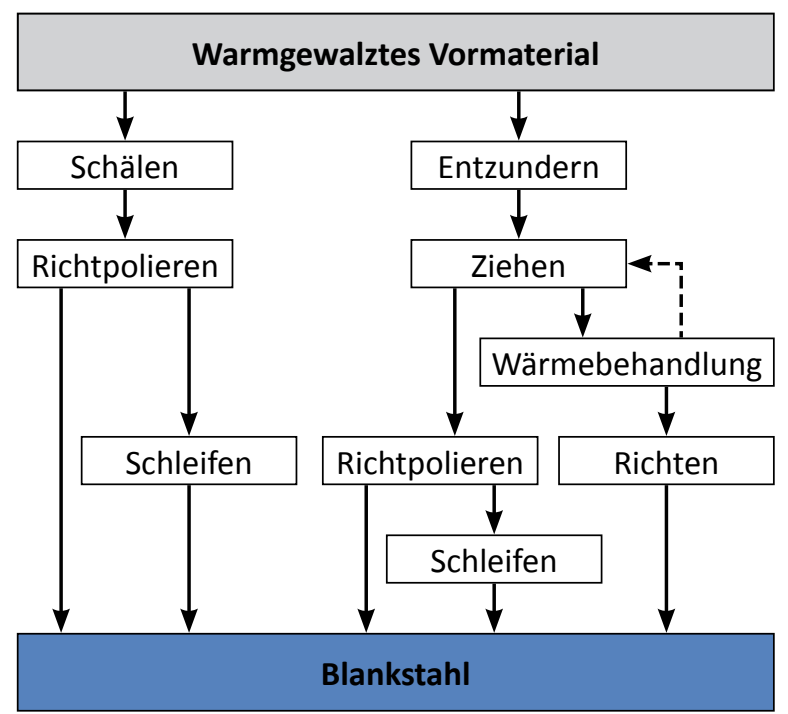

Abb. 4.02: Standardprozesse bei der Herstellung von Blankstah/[41]

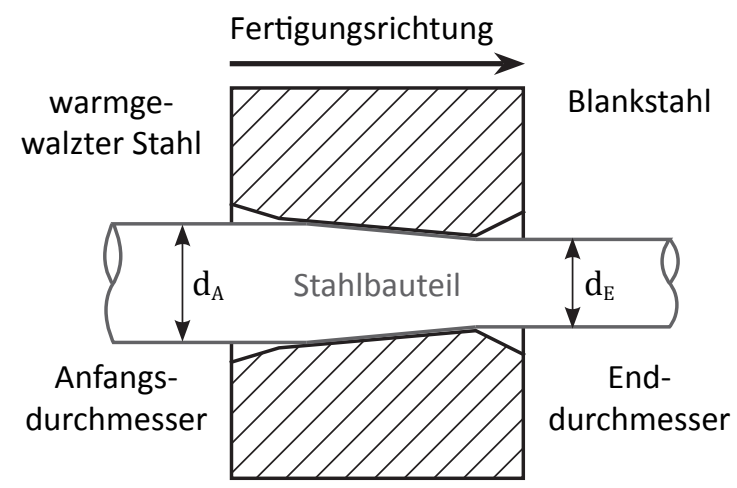

Abb. 4.03: Prinzipskizze des Ziehhofes bei der Herstellung von gezogenem Blankstahl

Tabelle 4.1: Produktgruppen für Blankstahl [41]

\begin{tabular}{|l|c|c|}
\hline Form & Prozess & Übliche Abmessungen [mm] \\
\hline Rund & Ziehen & $\varnothing 5-100$ \\
\hline Rund & Schälen & $\varnothing 15-100$ \\
\hline Flach & Ziehen & $\mathrm{b} \leq 5-500, \mathrm{~d}=3-100$ \\
\hline Vierkant & Ziehen & $5-150$ \\
\hline Sechskant & Ziehen & $5-120$ \\
\hline
\end{tabular}


anderem keine ausgeprägte Streckgrenze und kein Fließplateau. Mit Hilfe einer nachträglichen Wärmebehandlung kann die Kaltverfestigung teilweise rückgängig gemacht werden [06]. Blankstahl, der auf Schälmaschinen hergestellt wird, muss anschließend noch gerichtet werden. Unabhängig von der Art der Fertigungsmethode kann die Beschaffenheit der Oberfläche durch Schleifen oder Polieren weiter verbessert werden, um eine noch höhere Maßgenauigkeit zu erhalten.

\subsection{Herstellung der Probekörper}

Da Materialanalysen generell zeitaufwendig und kostenintensiv sind, beschränken sich die Untersuchungen vorwiegend auf den Stahl des KVP D20. Für das KVP D30 und KHP D60 werden lediglich die Spannungs-Dehnungs-Kennlinien bei Raumtemperatur bestimmt. Im Rahmen der Materialuntersuchungen werden die chemische Zusammensetzung des Blankstahls, der Elastizitätsmodul, die Spannungs-Dehnungs-Kennlinien bei Raumtemperatur und erhöhter Temperatur sowie der Wärmeausdehnungskoeffizient ermittelt. Die für die Materialuntersuchung des KVP D20 verwendeten Probekörper entstammen der gleichen Charge von Stahlbauteilen wie die in den Brandversuchen getesten Zugglieder. Eine Übersicht der Probekörper für die einzelnen Materialuntersuchungen ist in Tabelle 4.2 angegeben.

Tabelle 4.2: Übersicht der Probekörper für die Materialuntersuchungen

\begin{tabular}{|l|l|}
\hline Art der Materialuntersuchung & Spezifikation der Probekörper \\
\hline Chemische Analyse & $\begin{array}{l}\text { - zylindrische Probe mit einer Länge von } 40 \mathrm{~mm} \text { und einem } \\
\text { Durchmesser von } 20 \mathrm{~mm}\end{array}$ \\
& - polierte, glatte Oberfläche an den Stirnseiten der Probe \\
\hline Bestimmung des & - quaderförmige Probe mit den Maßen $3 \times 9 \times 100 \mathrm{~mm}$ \\
Wärmeausdehnungskoeffizienten & - planparallele Stirnflächen \\
\hline Bestimmung des & - kubische Probe mit einer Länge von $25 \mathrm{~mm}$ und einem Durch- \\
Elastizitätsmoduls & messer von $4 \mathrm{~mm}$ \\
& - planparallele Stirnflächen \\
\hline Bestimmung der & - zylindrische Probe mit einer Länge von ca. $97 \mathrm{~mm}$ und einem \\
Spannungs-Dehnungs-Kennlinien & Durchmesser von $10 \mathrm{~mm}$ (Proportionalstab siehe Tabelle B.5) \\
\hline
\end{tabular}

\subsection{Ermittlung der chemischen Zusammensetzung}

Die Materialeigenschaften von Stahl verändern sich je nach chemischer Zusammensetzung. Um den verwendeten Stahl hinsichtlich seiner Materialeigenschaften mit anderen Stählen einordnen und vergleichen zu können, wird eine Bestimmung der einzelnen chemischen Bestandteile durchgeführt. Die chemische Zusammensetzung wird mittels Funken-Emissions-Spektrometer (FES) bestimmt (siehe Abb. 4.04). Werte für die thermischen 
und mechanischen Materialeigenschaften verschiedener Stähle sind unter anderem in den Veröffentlichungen von Richter [48], [51] angegeben. Die Ergebnisse der durchgeführten chemischen Analyse sind in Tabelle 4.3 angegeben. Zum Vergleich sind in der Tabelle ebenfalls die Werte aus dem Abnahmeprüfzeugnis 3.1 des Stahlherstellers sowie ein Vergleichswerkstoff aus der Stahldatenbank [43] aufgeführt. Die gemessenen Werte stimmen mit denen des Abnahmeprüfzeugnisses gut überein. Mit dem FES können mehr chemische Elemente analysiert werden als im Abnahmeprüfzeugnis angegeben. Die für den kaltverformten Blankstahl ermittelten Kenn-

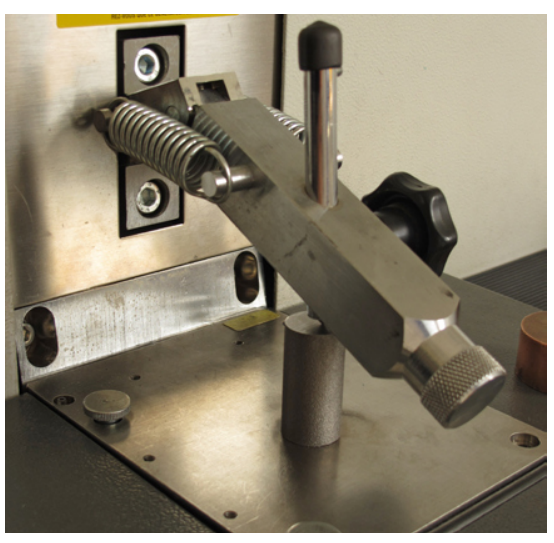

Abb. 4.04: Untersuchte Rundprobe am Funken-EmissionsSpektrometer (FES) werte der chemischen Elemente liegen innerhalb der Grenzen, die für einen vergleichbaren Werkstoff in der Stahldatenbank angegeben sind. Anhand der ermittelten chemischen Zusammensetzung und den in DIN EN 10020[05] angegebenen Grenzwerten handelt es sich bei dem untersuchten Blankstahl um einen unlegierten Qualitätsstahl. Aufgrund des Mangangehaltes von mehr als einem Prozent ergibt sich für den Stahl die Bezeichnung: 17MnCrMo5.

Tabelle 4.3: Chemische Zusammensetzung des untersuchten Blankstahls

\begin{tabular}{|l|c|c|c|}
\hline $\begin{array}{l}\text { Chemisches } \\
\text { Element [\%] }\end{array}$ & $\begin{array}{c}\text { Abnahmeprüfzeugnis 3.1 } \\
\text { (nach EN 10204) }\end{array}$ & $\begin{array}{c}\text { Chemische Analyse } \\
\text { BAM - Fachbereich 6.2 }\end{array}$ & $\begin{array}{c}\text { Vergleichswerkstoff } \\
\text { aus Stahldatenbank }\end{array}$ \\
\hline Kohlenstoff (C) & 0,16 & 0,17 & $\leq 0,20$ \\
\hline Silizium (Si) & 0,26 & 0,28 & $\leq 0,50$ \\
\hline Mangan (Mn) & 1,34 & 1,34 & $0,90-1,65$ \\
\hline Phosphor (P) & 0,023 & 0,022 & $\leq 0,035$ \\
\hline Schwefel (S) & 0,019 & 0,019 & $\leq 0,030$ \\
\hline Aluminium (Al) & 0,016 & 0,013 & $\leq 0,0200$ \\
\hline Chrom (Cr) & - & 0,16 & $\leq 0,30$ \\
\hline Kupfer (Cu) & 0,25 & 0,24 & $\leq 0,35$ \\
\hline Molybdän (Mo) & - & 0,03 & $\leq 0,10$ \\
\hline Stickstoff (N) & - & 0,008 & $\leq 0,015$ \\
\hline Niob (Nb) & - & $\leq 0,005$ & $\leq 0,050$ \\
\hline Nickel (Ni) & - & 0,10 & $\leq 0,50$ \\
\hline Titan (Ti) & - & 0,02 & $\leq 0,03$ \\
\hline Vanadium (V) & - & 0,00 & $\leq 0,12$ \\
\hline
\end{tabular}

Anmerkungen: ${ }^{1)}$ Nachschlagewerk Stahlschlüssel 2010 für MS Windows 7. Vergleichswerkstoff 1.8814 [43] 


\subsection{Ermittlung des Elastizitätsmoduls}

Der Elastizitätsmodul, auch als E-Modul bezeichnet, ist eine mechanische Werkstoffgröße und beschreibt das proportionale Verhältnis von Spannung und Dehnung im linear-elastischen Bereich. Der Anstieg im elastischen Bereich der Spannungs-Dehnungs-Kurve ist ein Maß für die Höhe des E-Moduls und gibt den nach dem Hooke'schen Gesetz geltenden linearen Zusammenhang zwischen Spannung und Dehnung wieder (siehe Gleichung (4.1)). Der nach Norm [13] für warmgewalzten Baustahl angenommene Spannungs-Dehnungs-Verlauf ist in Abb. 4.05 dargestellt. Durch den E-Modul lässt sich der elastische Bereich bis zur Proportionalitäts-

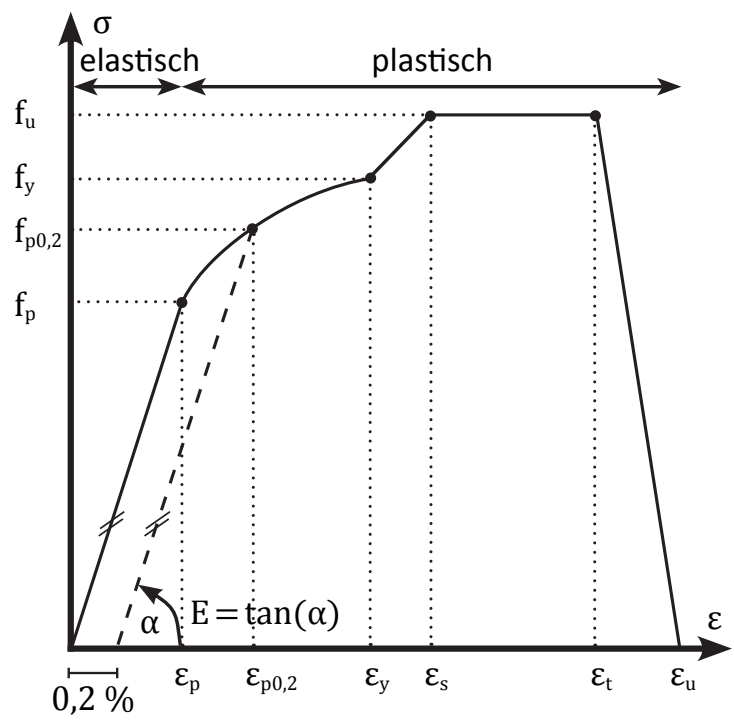

Abb. 4.05: Spannung-Dehnungs-Verlauf nach DIN EN 1993-1-2 [13] grenze $\left(f_{p}\right)$, der den Übergang zum nichtlinearen Bereich darstellt, beschreiben. Des Weiteren wird der E-Modul für die Ermittlung der $0,2 \%$-Dehngrenze $\left(\mathrm{f}_{\mathrm{p} 0,2}\right)$ benötigt. Diese ist besonders für Stähle ohne ausgeprägte Streckgrenze von Bedeutung.

$E=\frac{\sigma}{\varepsilon}$

$\begin{array}{ll}E & \text { Elastizitätsmodul } \\ \sigma & \text { Spannung } \\ \varepsilon & \text { Dehnung }\end{array}$

Da sich bei experimentell bestimmten Spannungs-Dehnungs-Kurven meist keine ideale Gerade im elastischen Bereich ausbildet, wird vereinfachend eine technische Proportionalitätsgrenze $\left(\mathrm{f}_{\mathrm{p} 0,01}\right)$ ermittelt [22]. Die technische Proportionalitätsgrenze wird durch den Schnittpunkt der Spannungs-Dehnungs-Kennlinie mit einer um 0,01 \% verschobenen Ursprungsgeraden, welche den Anstieg des E-Moduls besitzt, beschrieben.

Aufgrund des Ziehvorgangs beim Herstellen des untersuchten Blankstahls ist im SpannungsDehnungs-Verlauf keine ausgeprägte Streckgrenze vorhanden. Statt der Streckgrenze wird daher die 0,2\%-Dehngrenze $\left(\mathrm{f}_{\mathrm{p} 0,2}\right)$ bestimmt. Unabhängig von der Art des Stahls tritt unter erhöhten Temperaturen keine ausgeprägte Streckgrenze mehr auf. Die Bestimmung der Proportionalitätsgrenze und der 0,2\%-Dehngrenze erfolgt mit Hilfe des E-Moduls. Eine genaue Ermittlung des Elastizitätsmoduls ist daher wichtig. Der E-Modul kann direkt aus den Spannungs-Dehnungs-Kurven von Kleinzugversuchen (statisch) oder mit Hilfe eines Resonanzverfahrens (dynamisch) bestimmt werden. Beide Verfahren werden für den untersuchten Stahl unter Raumtemperatur und bei erhöhter Temperatur angewendet. 


\subsubsection{Bestimmung des Elastizitätsmoduls mittels Resonanzverfahren}

Beim Resonanzverfahren werden Stahlproben durch ein Sender-Empfänger-System mit sinusförmigen Signalen in Schwingung versetzt und anhand der gemessenen Frequenzen der dynamische E-Modul $\left(\mathrm{E}_{\text {dyn }}\right)$ bestimmt. Die Untersuchungen werden unter Raumtemperatur und bei erhöhter Temperatur $\left(100\right.$ bis $\left.800^{\circ} \mathrm{C}\right)$ durchgeführt. Die Messabweichung beträgt bei diesem Verfahren $\pm 1 \%$ (Raumtemperatur) bzw. $\pm 3 \%$ (Hochtemperaturbereich).

Der dynamische E-Modul wird unter erhöhter Temperatur mit Hilfe einer speziellen Prüfapparatur, dem Elastotron 2000, ermittelt (siehe Abb. 4.07). Bevor eine Messung des dynamischen E-Moduls erfolgen kann, muss eine Kalibrierung von Ofentemperatur und Stahltemperatur anhand einer Kalibrierprobe durchgeführt werden. Die Kalibrierprobe, welche vom Material und den Abmessungen identisch mit der späteren Probe zur Bestimmung des E-Moduls ist, wird zunächst freischwingend an zwei Kohlefaserschlaufen aufgehängt. An die Kalibrierprobe sind Ther-

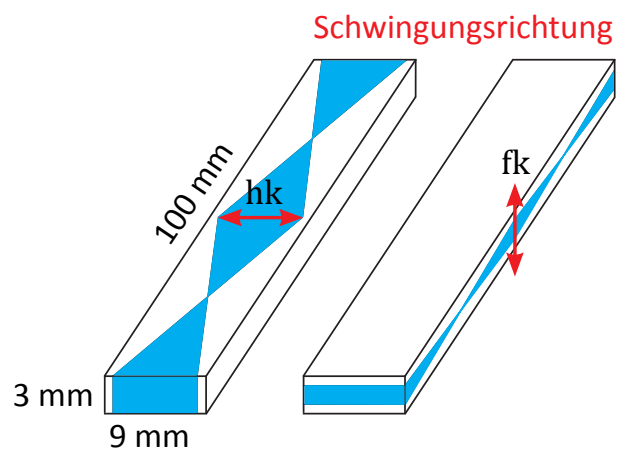

Abb. 4.06: Probekörpergeometrie und Verlauf der Schwingungen aus der Anregung des Senders moelemente zur Messung der Stahltemperatur befestigt. Rings um den Probekörper wird eine Heizspirale zur Erwärmung des Probekörpers angeordnet. Um den Wärmeverlust des Ofens an die Umgebung möglichst gering zu halten, ist der komplette Ofen mit Kohlestofffilzen isoliert. Im Ofen selbst befindet sich ein Referenzthermoelement, mit dessen Hilfe die Ofentemperatur geregelt wird. Nach dem Schließen des Ofens wird die Luft abgesaugt und ein Vakuum innerhalb des Ofens erzeugt. Nachdem sich ein konstantes Vakuum eingestellt hat, beginnt die Erwärmung des Probekörpers mittels der Heizspirale. Für die im Vorfeld festgelegten Temperaturschritte, bei denen der E-Modul bestimmt werden soll, wird zunächst mit Hilfe der Kalibrierprobe die entsprechende Ofentemperatur ermittelt. Anschließend wird der Kalibrierprobekörper ausgebaut und gegen die eigentliche E-Modul-Probe ersetzt. Allerdings wird auf die zusätzlichen Thermoelemente am Probekörper verzichtet. An einer der Kohlefaserschlaufen befindet sich ein Sender, welcher die Probe durch ein sinus-förmiges Signal in Schwingung versetzt. Über einen an der zweiten Schlaufe befestigten Empfänger erfolgt die Messung der Probenschwingung. Durch das piezoelektrische Sender-Empfänger-System kann der Probekörper in Schwingung versetzt werden. Der Frequenzbereich des Senders wird dabei kontinuierlich von 1 bis 10 $\mathrm{kHz}$ erhöht. Aus den gemessenen Resonanzpeaks wird der E-Modul für die jeweilige Stahltemperatur bestimmt (siehe Abb. 4.09). Da die Probe sowohl in Flachkantrichtung (fk) als auch in Hochkantrichtung (hk) angeregt wird, ergeben sich jeweils zwei Resonanzpeaks. Bei anisotropen Werkstoffen kann dadurch der E-Modul in zwei Richtungen bestimmt werden. 


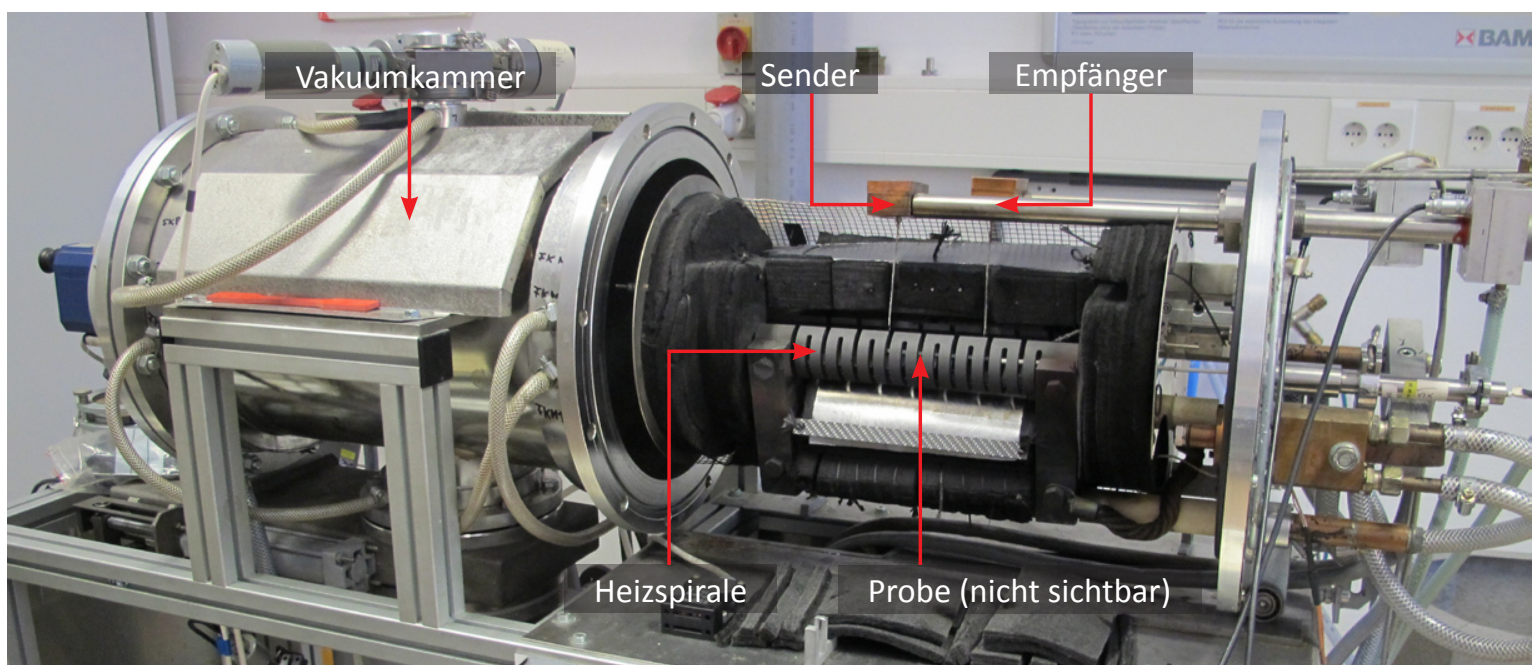

Abb. 4.07: Messvorrichtung zur Bestimmung des dynamischen E-Moduls unter erhöhter Temperatur

Die Bestimmung des Elastizitätsmoduls unter Raumtemperatur erfolgt mit einer ähnlichen Messapparatur wie bei erhöhter Temperatur (siehe Abb. 4.08). Allerdings ist bei dieser Messung kein Vakuum erforderlich. Die Ergebnisse der Messungen mittels Resonanzverfahren sind in Tabelle 4.5 angegeben.

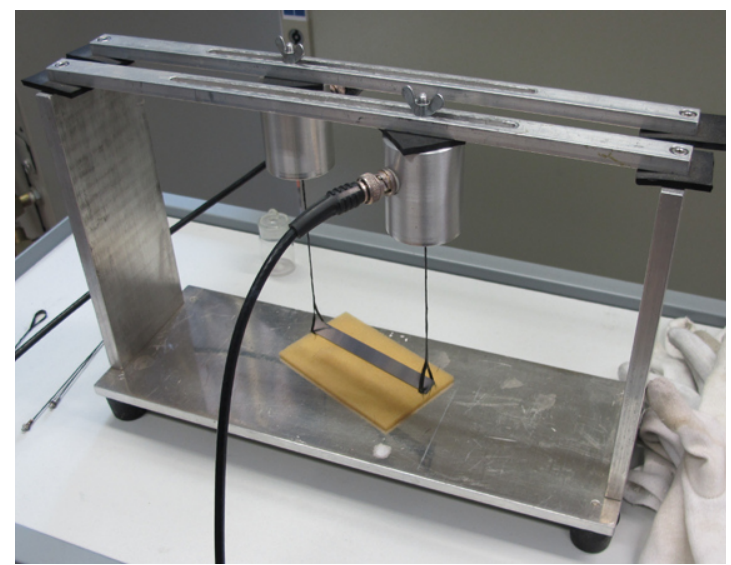

Abb. 4.08: Messvorrichtung zur Bestimmung des dynamischen E-Moduls unter Raumtemperatur

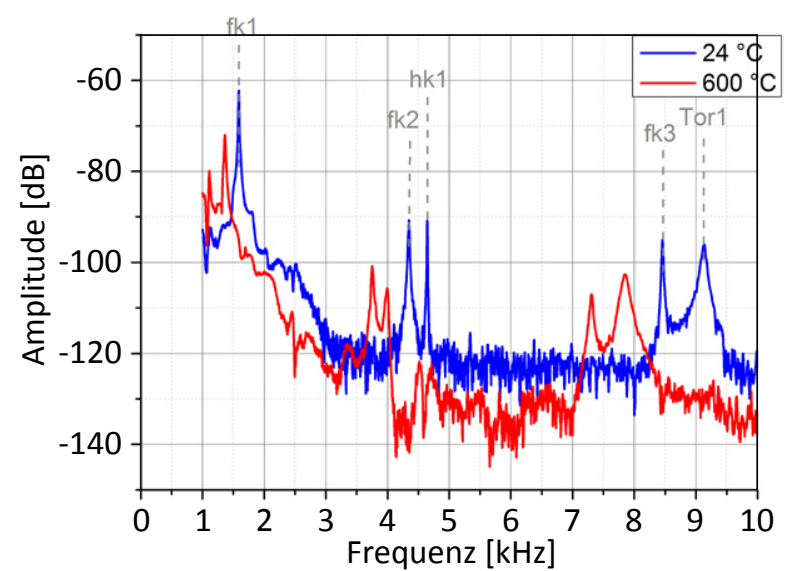

Abb. 4.09: Resonanzpeaks im Resonanzspektrum bei Raumtemperatur und $600{ }^{\circ} \mathrm{C}$

\subsubsection{Vergleich des dynamischen E-Moduls mit Werten aus der Literatur}

Im Zuge der "Stahleisen Sonderberichte" wurden in den 70er und 80er Jahren die physikalischen Eigenschaften von Stahl untersucht. In den Veröffentlichungen von Richter [48], [51] sind einige der Ergebnisse dieser Untersuchungen angegeben. Unter anderem wurde auch die Temperaturabhängigkeit des dynamischen E-Moduls für 38 unlegierte und legierte 
Stähle analysiert. Ein Hinweis auf das verwendete Messverfahren ist in den Veröffentlichungen nicht enthalten. Ferner sind in dem vom Verein deutscher Eisenhüttenleute herausgegebenen Stahl-EisenWerkstoffblatt (SEW310) [58] Werte für den dynamischen E-Modul angegeben. Der E-Modul wurde hierbei über die Messung der Schallgeschwindigkeit ermittelt. In Abb. 4.10 sind die Werte für den dynamischen E-Modul aus der Literatur den in eigenen Messungen ermittelten Werten, d.h. BAM, gegenübergestellt. Der Vergleich der Werte zeigt, dass für den untersuch-

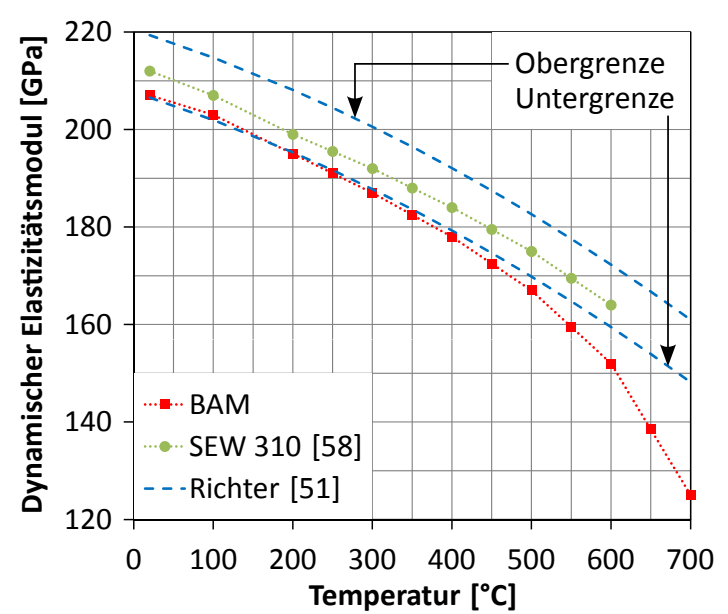

Abb. 4.10: Vergleich der Ergebnisse für den dynamischen Elastizitätsmodul ten Blankstahl die Werte des dynamischen E-Moduls bis etwa $400^{\circ} \mathrm{C}$ gut mit der Untergrenze der von Richter [51] untersuchten Stähle übereinstimmen. Für Temperaturen von mehr als $400^{\circ} \mathrm{C}$ ergeben sich für den untersuchten Stahl zunehmend geringere Werte. Die Werte aus dem SEW 310 liegen innerhalb des von Richter [51] angegebenen Bereiches, jedoch über den ermittelten Werten des Blankstahls.

\subsubsection{Bestimmung des Elastizitätsmoduls anhand von Kleinzugversuchen}

Grundlage für die Bestimmung des statischen Elastizitätsmoduls $\left(\mathrm{E}_{\mathrm{stat}}\right)$ bilden stationäre Kleinzugversuche (siehe Kapitel 4.5). Aus den aufgezeichneten Spannungs-DehnungsKurven der Kleinzugversuche wird der statische Elastizitätsmodul bestimmt. Zunächst wird dafür in der Spannungs-Dehnungs-Kurve ein Interpolationsbereich selektiert. Der Bereich wird so gewählt, dass dieser sich im elastischen Verformungsbereich befindet und somit gut durch eine Interpolationsgerade approximiert werden kann. Der Anstieg der Interpolationsgerade entspricht dem statischen Elastizitätsmodul. Die Auswahl der Messpunkte wird solange variiert bis das Bestimmtheitsmaß $\left(R^{2}\right)$, welches ein Maß für die Qualität der linearen Approximation ist, möglichst einem Wert von 1,0 entspricht. Für ein Bestimmtheitsmaß von 1,0 liegen alle selektierten Punkte auf einer Geraden. Das beschriebene Auswertungsverfahren ist in Abb. 4.11 graphisch dargestellt. Die Ergebnisse des statischen Elastizitätsmoduls sind in Tabelle 4.4 angegeben.

Insgesamt werden bei Raumtemperatur sechs Kleinzugversuche an den Proben des KVP D20 durchgeführt. Bei erhöhter Temperatur werden drei Versuchsreihen mit jeweils 12 Proben untersucht. Die Versuchsreihen unterscheiden sich lediglich in der gewählten Prüfgeschwindigkeit (siehe Tabelle 4.7 in Kapitel 4.5.2). Die Wahl der Dehngeschwindigkeit erfolgt für Raumtemperatur nach DIN EN ISO 6892-1 [14] und für erhöhte Temperatur nach DIN EN ISO 6892-2 [15]. Die Kleinzugversuche werden mit Hilfe eines am Probekörper 
angebrachten Extensometers bis zu einer Dehnung von ca. $2 \%$ dehnungsgeregelt (Bereich I) gesteuert. Anschließend wechselt die Prüfmaschine in Wegregelung (Bereich II) und der Dehnungsaufnehmer wird kurz darauf bei einer Dehnung von 3 bis $4 \%$ von der Zugprobe entfernt. Dadurch soll eine mögliche Beschädigung des Extensometers beim Probenbruch der Zugprobe verhindert werden. Die gemessenen Spannungs-Dehnungs-Kurven sind in Kapitel 4.5.1 und Kapitel 4.5.2 angegeben.

\section{Ergebnisse für den statischen E-Modul} Neben den Werten für den statischen E-Modul aus den einzelnen Versuchsreihen ist in Tabelle 4.4 auch der Mittelwert für die jeweilige Stahl-

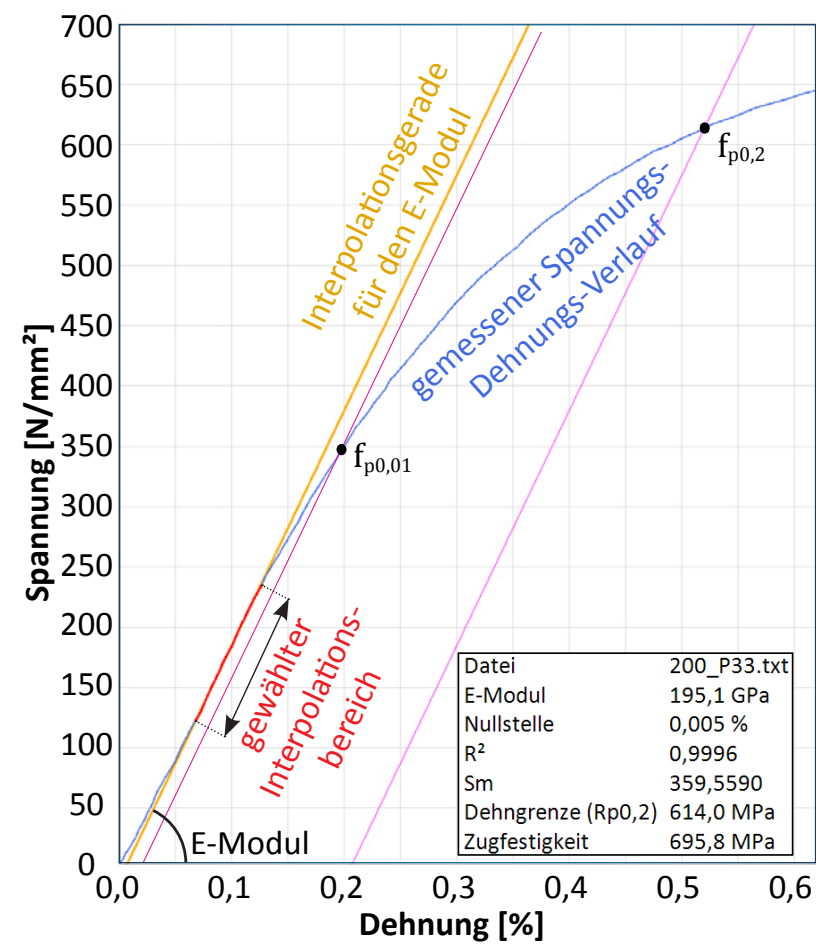

Abb. 4.11: Graphische Bestimmung des statischen E-Moduls für eine Temperatur von $200{ }^{\circ} \mathrm{C}$ temperatur $(\theta)$ sowie ein temperaturabhängiger Abminderungsfaktor $\left(\mathrm{k}_{\mathrm{E}}\right)$ angegeben. Für Raumtemperatur beträgt der statische E-Modul etwa $206 \mathrm{GPa}$. Die maximale Abweichung beträgt ca. 2,7 GPa. Für die Berechnung des temperaturabhängigen Abminderungsfaktors wird als Basiswert der statische Elastizitätsmodul bei Raumtemperatur verwendet. Anhand des Abminderungsfaktors ist zu erkennen, dass mit steigender Stahltemperatur der statische Elastizitätsmodul abnimmt. Für den untersuchten kaltverformten Blankstahl tritt bis etwa $400^{\circ} \mathrm{C}$ nur eine geringe Abminderung des Elastizitätsmoduls auf. Für Stahltemperaturen von mehr als $400^{\circ} \mathrm{C}$ ist mit einem starken Rückgang des statischen Elastizitätsmoduls zu rechnen. Bis zu einer Temperatur von etwa $200{ }^{\circ} \mathrm{C}$ sind die Werte zwischen den verschiedenen Versuchsreihen annähernd gleich groß. Für Stahltemperaturen von mehr als $200^{\circ} \mathrm{C}$ ist eine Zunahme der Abweichungen des Elastizitätsmoduls zwischen den Versuchsreihen erkennbar. Mit hoher Wahrscheinlichkeit führt eine Anhebung der Dehngeschwindigkeit im Zugversuch zu höheren Werten für den statischen Elastizitätsmodul. Eine Gesetzmäßigkeit zum Einfluss der Versuchsgeschwindigkeit auf die Höhe des statischen Elastizitätsmoduls lässt sich jedoch nicht feststellen. Dies ist unter anderem darauf zurückzuführen, dass besonders bei hohen Stahltemperaturen und dem damit verbundenen relativ kleinen linear-elastischen Bereich des Stahls eine genaue Bestimmung des Elastizitätsmoduls nicht immer eindeutig möglich ist. Auf die Schwierigkeiten bei der Ermittlung des statischen Elastizitätsmoduls wird in Kapitel 4.4.4 ausführlich eingegangen. 
Tabelle 4.4: Vergleich des statischen E-Moduls aus den Kleinzugversuchen

\begin{tabular}{|c|c|c|c|c|c|c|c|c|}
\hline Temperatur & \multicolumn{3}{|c|}{ E-Modul $\left(\mathrm{E}_{\mathrm{stat}}\right)\left[\mathrm{N} / \mathrm{mm}^{\mathbf{2}}\right]$} & \multicolumn{4}{c|}{ Abminderungsfaktor $\left(\mathrm{k}_{\mathrm{E}}\right)[-]$} \\
\hline$\theta\left[{ }^{\circ} \mathrm{C}\right]$ & $\mathrm{E}_{\mathrm{VR} 1}$ & $\mathrm{E}_{\mathrm{VR} 2}$ & $\mathrm{E}_{\mathrm{VR} 3}$ & $\mathrm{E}_{\text {Mittelwert }}$ & $\mathrm{k}_{\mathrm{E}, \mathrm{VR} 1}$ & $\mathrm{k}_{\mathrm{E}, \mathrm{VR} 2}$ & $\mathrm{k}_{\mathrm{E}, \mathrm{VR} 3}$ & $\mathrm{k}_{\mathrm{E}, \mathrm{MW}}$ \\
\hline 20 & - & - & - & 206050 & 1,000 & 1,000 & 1,000 & 1,000 \\
\hline 100 & 199200 & 191100 & 196700 & 195667 & 0,967 & 0,927 & 0,955 & 0,950 \\
\hline 200 & 188500 & 181800 & 182200 & 184167 & 0,915 & 0,882 & 0,884 & 0,894 \\
\hline 250 & 199100 & 207700 & 188800 & 198533 & 0,966 & 1,008 & 0,916 & 0,964 \\
\hline 300 & 186000 & 182600 & 170800 & 179800 & 0,903 & 0,886 & 0,829 & 0,873 \\
\hline 350 & 201000 & 184600 & 178600 & 188067 & 0,975 & 0,896 & 0,867 & 0,913 \\
\hline 400 & 175500 & 194400 & 193200 & 187700 & 0,852 & 0,943 & 0,938 & 0,911 \\
\hline 450 & 164000 & 164900 & 170000 & 166300 & 0,796 & 0,800 & 0,825 & 0,807 \\
\hline 500 & 181300 & 154000 & 176300 & 170533 & 0,880 & 0,747 & 0,856 & 0,828 \\
\hline 550 & 138100 & 137000 & 105800 & 126967 & 0,670 & 0,665 & 0,513 & 0,616 \\
\hline 600 & 95500 & 75300 & 103600 & 91467 & 0,463 & 0,365 & 0,503 & 0,444 \\
\hline 650 & 76900 & 53000 & 49900 & 59933 & 0,373 & 0,257 & 0,242 & 0,291 \\
\hline 700 & 84900 & 31200 & 24200 & 46767 & 0,412 & 0,151 & 0,117 & 0,227 \\
\hline
\end{tabular}

\subsubsection{Unsicherheiten bei der Bestimmung des statischen Elastizitätsmoduls}

Aus der Norm [14] ist bekannt, dass eine genaue Bestimmung des Elastizitätsmoduls mit Hilfe des Anstiegs im linear-elastischen Bereich der Spannungs-Dehnungs-Kennlinie nicht immer eindeutig möglich ist. Im Folgenden werden einige Einflüsse und deren Auswirkungen näher beschrieben.

\section{Ungewollte Exzentrizität in der Zugprobe}

Trotz der in der Prüfmaschine verwendeten gelenkigen Anschlüsse zur Reduzierung von Biegemomenten, lässt sich nicht ausschließen, dass sich neben der gewünschten rein axialen Zugbeanspruchung der Probekörper auch eine kleine Biegebeanspruchung eingestellt (siehe Abb. 4.12). Durch die beim Zugversuch auftretenden großen Normalkräfte kann bereits eine kleine Exzentrizität an den Probeneinspannungen eine nicht zu vernachlässigende Biegebeanspruchung hervorrufen.

Die Kleinzugversuche unter Raumtemperatur werden mittels eines Feindehnungsaufnehmers vom Typ HBM DD1 mit einer Messlänge von 50 mm durchgeführt. Dieser besitzt zwei gegenüberliegende Dehnungsaufnehmer, so dass ein eventuell vorhandener Biegeanteil in der Zugprobe durch Bildung des Mittelwertes beider Dehnungsaufnehmer nach Gleichung (4.2) herausgerechnet werden kann. Die Zugversuche unter erhöhter Temperatur werden aufgrund des gewählten Prüfaufbaus mit einem einseitigen Dehnungsaufnehmer vom Typ MTS mit Wasserkühlung durchgeführt. Der eingesetzte Hochtemperaturdeh- 
nungsaufnehmer besitzt eine Messlänge von $21 \mathrm{~mm}$. Ein vorhandener Dehnungsanteil aus einer ungewollten Biegebeanspruchung kann durch den Hochtemperaturdehnungsaufnehmer nicht ausgeglichen werden (siehe Gleichung (4.3)). Folglich ist es möglich, dass je nach Beanspruchungsrichtung und Lage des Dehnungsaufnehmers eine zu große bzw. zu kleine axiale Dehnung gemessen wird (siehe Abb. 4.13). Da die Bestimmung des statischen Elastizitätsmoduls in einem Bereich kleiner Dehnungen erfolgt, können bereits geringe Abweichungen in der Dehnungsmessung zu einer Über- bzw. Unterschätzung des Elastizitätsmoduls führen.

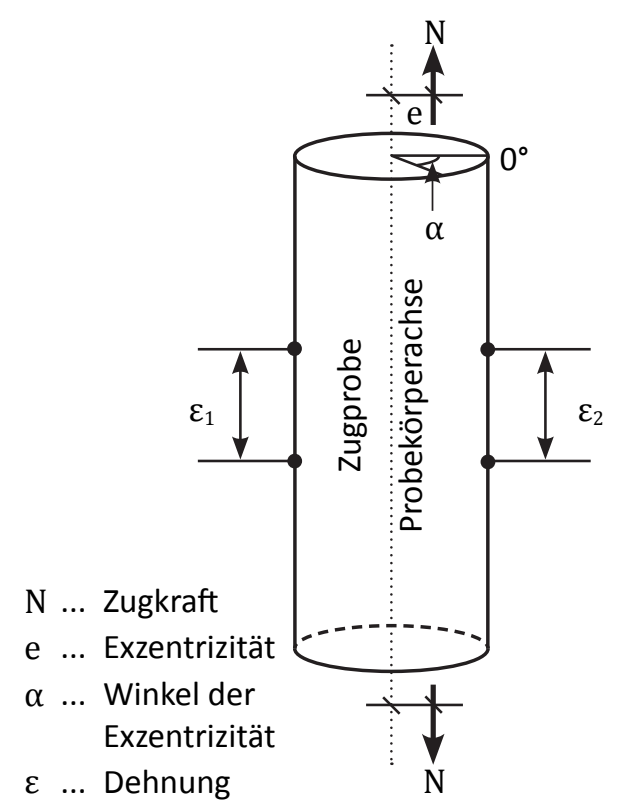

Abb. 4.12: Mögliche exzentrische Beanspruchung beim Kleinzugversuch

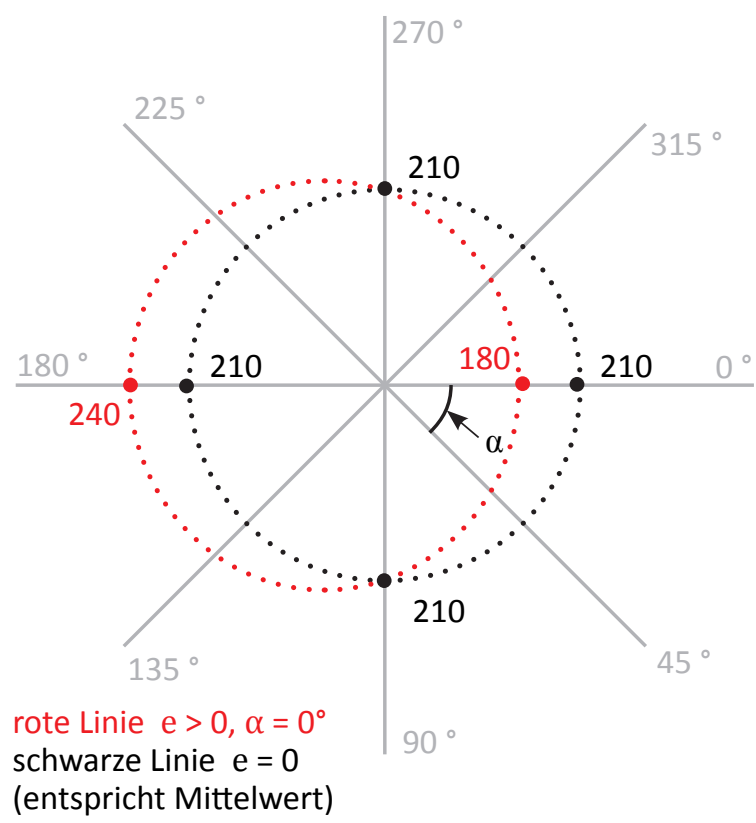

Abb. 4.13: Beispielhafte Verteilung des E-Moduls [GPa] in Abhängigkeit vom Messwinkel zur Lage der Exzentrizität (Draufsicht auf die Probe)

$\varepsilon=\frac{\varepsilon_{1}+\varepsilon_{2}}{2}$

$\varepsilon=\varepsilon_{1}$

Reduzierung des elastischen Bereiches bei erhöhter Stahltemperatur

Mit steigender Stahltemperatur nimmt die Festigkeit des Stahls ab. Ferner wird der Elastizitätsmodul kleiner und das Materialverhalten folglich "weicher". Der linear-elastische Bereich in der Spannungs-Dehnungs-Kurve wird dadurch mit zunehmender Stahltemperatur kleiner. Lässt sich der statische Elastizitätsmodul unter Raumtemperatur aufgrund des relativ langen linear-elastischen Bereiches noch gut durch eine Gerade approximieren, ist bei höheren Temperaturen nur ein vergleichsweise kurzer elastischer Bereich vorhanden. Des Weiteren geht der linear-elastische Bereich bei hohen Stahltemperaturen relativ 
schnell in einen Ausrundungsbereich über. Folglich stehen wesentlich weniger Messpunkte für eine lineare Approximation zur Verfügung. Bereits eine kleine Änderung des Interpolationsbereiches führt zu einer relativ großen Veränderung des statischen Elastizitätsmoduls. Eine eindeutige Bestimmung des Elastizitätsmoduls ist daher bei hohen Stahltemperaturen nur schwer möglich und mit einer relativ großen Unsicherheit verbunden. Beispielhaft für die genannte Problematik ist in Abb. 4.14 die Bestimmung des statischen Elastizitätsmoduls bei 100 und $700{ }^{\circ} \mathrm{C}$ dargestellt.
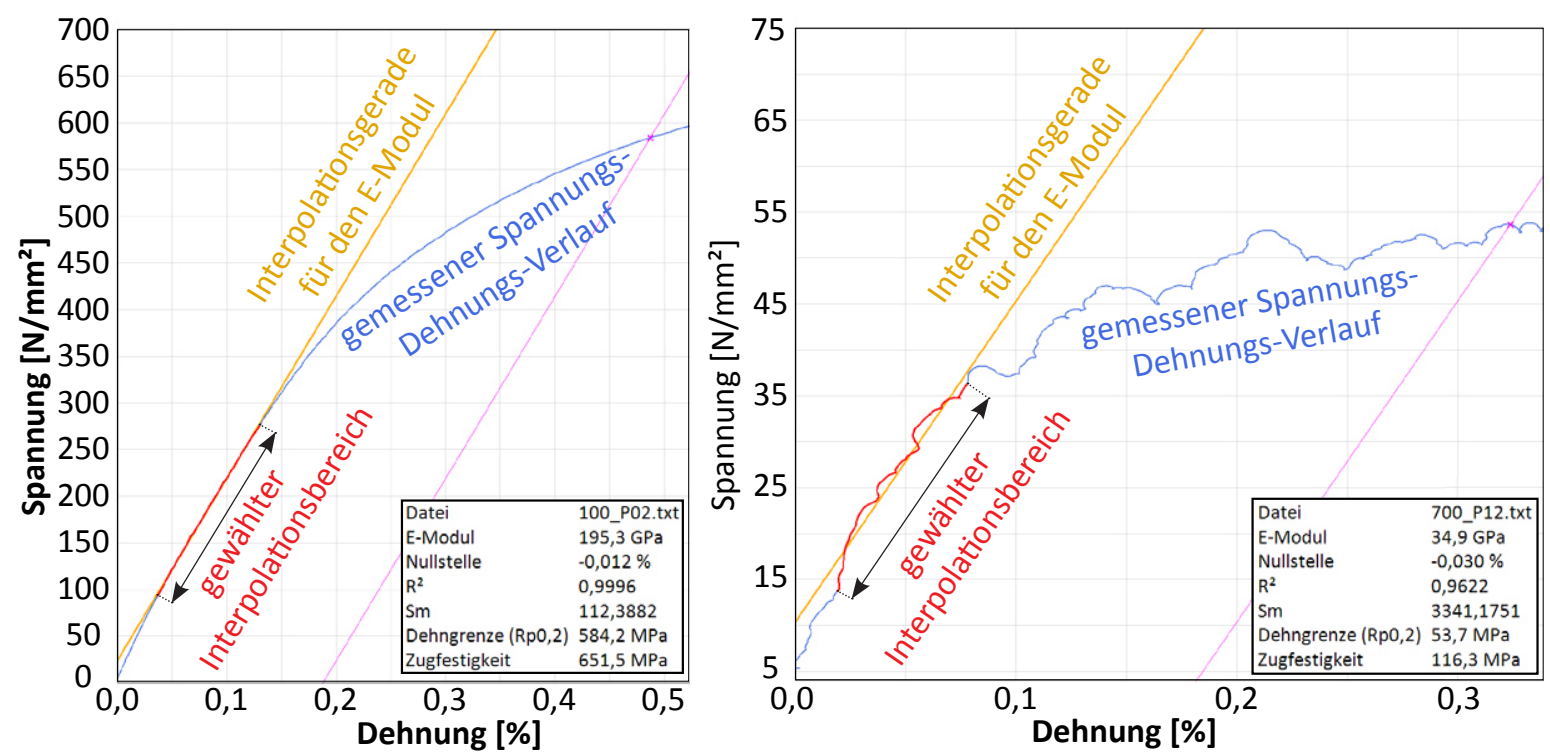

Abb. 4.14: Bestimmung des E-Moduls aus einem stationären Kleinzugversuch (links: $100{ }^{\circ} \mathrm{C}$; rechts: $700{ }^{\circ} \mathrm{C}$ )

\section{Sensibilität der Prüfmaschine}

Neben den bereits genannten Einflüssen bei der genauen und eindeutigen Bestimmung des statischen Elastizitätsmoduls besitzt auch die verwendete Messapparatur selbst ein gewisses Fehlerpotential. Die aufgezeichnete Spannungs-Dehnungs-Kurve ist nicht glatt, sondern entspricht eher einem gezackten Verlauf. Während sich bei dem mit einer Temperatur von $100^{\circ} \mathrm{C}$ durchgeführten Zugversuch eine relativ glatte Spannungs-Dehnungs-Kurve mit vielen Messpunkten ergibt, sind bei $700{ }^{\circ} \mathrm{C}$ starke Schwankungen in der Messkurve zu erkennen (siehe Abb. 4.14). Ursache für den gezackten Verlauf ist unter anderem die Sensibilität der Prüfmaschine, die eingestellte Messrate sowie die gewählte Dehnungsgeschwindigkeit. Eine eindeutige Bestimmung des statischen Elastizitätsmoduls wird dadurch bei hohen Temperaturen zusätzlich erschwert. Bereits eine kleine Variation des gewählten Interpolationsbereiches führt zu einer relativ starken Veränderung des Elastizitätsmoduls. Die Festlegung eines eindeutigen Wertes für den statischen Elastizitätsmoduls ist daher schwierig und mit großen Unwägbarkeiten verbunden. 


\subsubsection{Vergleich des statischen E-Moduls mit Werten aus der Literatur}

\section{Werte für den statischen Elastizitätsmodul aus den Normen}

In DIN EN 1993-1-2[13] (EC3 Teil 1-2) sind Abminderungsfaktoren für den statischen E-Modul bis zu einer Temperatur von $1200{ }^{\circ} \mathrm{C}$ angegeben. Die in EC3 Teil 1-2 angeführten Werte beziehen sich auf warmgewalzten Baustahl. Weitere Angaben zum statischen E-Modul sind in DIN EN 1992-1-2 [12] (EC2 Teil 1-2) angegeben. Der Elastizitätsmodul für warmgewalzten Betonstahl der Klasse N entspricht den Werten aus dem EC3 Teil 1-2. Für kaltverformten Betonstahl der Klasse $\mathrm{N}$ und $\mathrm{X}$ weichen die Werte gegenüber dem EC3 Teil 1-2 ab. In Tabelle 4.5 sind die Werte aus der Norm sowie die Werte des E-Moduls aus den Untersuchungen des kaltverformten Blankstahls angegeben.

Tabelle 4.5: Vergleich des E-Moduls zwischen untersuchtem Blankstahl und den Werten in den Normen

\begin{tabular}{|c|c|c|c|c|c|c|c|c|}
\hline Temperatur & \multicolumn{4}{|c|}{ Elastizitätsmodul [N/mm m $^{2}$} & \multicolumn{4}{c|}{ Abminderungsfaktoren } \\
\hline$\theta\left[{ }^{\circ} \mathrm{C}\right]$ & $\begin{array}{c}\mathrm{E}_{\text {Resonanz }} \\
\text { (dynamisch) }\end{array}$ & $\begin{array}{c}\mathrm{E}_{\text {Zugversuch }} \\
\text { (statisch) }\end{array}$ & $\begin{array}{c}\mathrm{E}_{\mathrm{EC} 3} \\
\text { (statisch) }\end{array}$ & $\begin{array}{c}\mathrm{E}_{\mathrm{EC} 2(\mathrm{Kl} . \mathrm{X})} \\
\text { (statisch) }\end{array}$ & $\mathrm{k}_{\mathrm{E}, \theta, \mathrm{Res}}$ & $\mathrm{k}_{\mathrm{E}, \theta, \mathrm{Zug}}$ & $\mathrm{k}_{\mathrm{E}, \theta, \mathrm{EC} 3}$ & $\begin{array}{c}\mathrm{k}_{\mathrm{E}, \theta, \mathrm{EC} 2} \\
(\mathrm{Kl} . \mathrm{X})\end{array}$ \\
\hline 20 & 207000 & 206050 & 210000 & 200000 & 1,000 & 1,000 & 1,000 & 1,000 \\
\hline 100 & 203000 & 195667 & 210000 & 200000 & 0,981 & 0,950 & 1,000 & 1,000 \\
\hline 200 & 195000 & 184167 & 189000 & 190000 & 0,942 & 0,894 & 0,900 & 0,950 \\
\hline 250 & 191000 & 198533 & 178500 & 186000 & 0,923 & 0,964 & 0,850 & 0,930 \\
\hline 300 & 187000 & 179800 & 168000 & 180000 & 0,903 & 0,873 & 0,800 & 0,900 \\
\hline 350 & 182500 & 188067 & 157500 & 166000 & 0,882 & 0,913 & 0,750 & 0,830 \\
\hline 400 & 178000 & 187700 & 147000 & 150000 & 0,860 & 0,911 & 0,700 & 0,750 \\
\hline 450 & 172500 & 166300 & 136500 & 136000 & 0,833 & 0,807 & 0,650 & 0,680 \\
\hline 500 & 167000 & 170533 & 126000 & 120000 & 0,807 & 0,828 & 0,600 & 0,600 \\
\hline 550 & 159500 & 126967 & 95550 & 92000 & 0,771 & 0,616 & 0,455 & 0,460 \\
\hline 600 & 152000 & 91467 & 65100 & 62000 & 0,734 & 0,444 & 0,310 & 0,310 \\
\hline 650 & 138500 & 59933 & 46200 & 44000 & 0,669 & 0,291 & 0,220 & 0,220 \\
\hline 700 & 125000 & 46767 & 27300 & 26000 & 0,604 & 0,227 & 0,130 & 0,130 \\
\hline 800 & 98000 & - & 18900 & 18000 & 0,473 & - & 0,090 & 0,090 \\
\hline
\end{tabular}

Anmerkung: Die hellgrau hinterlegten Werte wurden linear interpoliert.

Die für den kaltverformten Blankstahl ermittelten Werte für den statischen Elastizitätsmodul zeigen eine große Übereinstimmung mit dem kaltgeformten Betonstahl der Klasse X. Dies ist darauf zurückzuführen, dass beide Stähle durch „Ziehen“ hergestellt werden. Allerdings ist eine gute Übereinstimmung der Werte des statischen Elastizitätsmoduls lediglich bis zu einer Stahltemperatur von etwa $300^{\circ} \mathrm{C}$ gegeben. Für höhere Temperaturen weist der untersuchte Blankstahl höhere Werte auf. Generell lässt sich aus dem Vergleich erkennen, dass der untersuchte kaltverformte Blankstahl meist über ein größeres Elastizitätsmodul verfügt, als der typischerweise im Bauwesen eingesetzte warmgewalzte Baustahl. 
Werte für den statischen Elastizitätsmodul aus anderen Veröffentlichungen

In Finnland wurden von Outinen et al. [44] sowie Outinen \& Mäkeläinen [45] Untersuchungen zu den physikalischen Eigenschaften von Stahl bei erhöhter Temperatur durchgeführt. Die Festigkeiten verschiedener Stähle wurden experimentell anhand von Kleinzugversuchen untersucht. In [44] sind Werte für den statischen Elastizitätsmodul für einen Stahl der Festigkeitsklasse S355 in Form einer Funktionsgleichung angegeben (siehe Gleichung (4.4) und (4.5)). Die im EC3 Teil 1-2 angegebenen Werte für den Elastizitätsmodul bzw. dem entsprechenden Abminderungsfaktor beziehen sich auf die in [45] publizierten Kleinzugversuche.

$$
\begin{array}{ll}
E(\theta)=20600-44,44 \cdot(\theta-20) & 20^{\circ} \mathrm{C} \leq \theta<200^{\circ} \mathrm{C} \\
E(\theta)=26300-325 \cdot \theta & 200^{\circ} \mathrm{C} \leq \theta \leq 200^{\circ} \mathrm{C}
\end{array}
$$

Stationäre Kleinzugversuche an kaltgeformten Stählen wurden außerdem von Qiang [47], Wei \& Jihong [63] sowie von Ranawaka \& Mahendran [48] durchgeführt. Die Ergebnisse aus der Arbeit von Qiang bleiben jedoch aufgrund der deutlich höheren Stahlfestigkeit, d.h. S460, S690 und S960, für den Vergleich mit dem in der BAM untersuchten kaltverformten Blankstahl unberücksichtigt. In beiden Veröffentlichungen wurde Stahl der Festigkeitsklasse G550 untersucht. Die Versuche von Wei \& Jihong wurden an $1 \mathrm{~mm}$ dicken Flachproben durchgeführt. Die dabei verwendete Versuchsgeschwindigkeit ist mit der VR 1 des kaltverformten Blankstahls aus dem untersuchten Zugglied mit KVP D20 identisch. Ranawaka \& Mahendran haben für ihre Untersuchungen ebenfalls Flachproben, jedoch mit einer Dicke von 0,95 mm Dicke, verwendet. Die Prüfgeschwindigkeit betrug 0,000048 s-1. Die Versuche sind in etwa mit dem dehnungsgeregelten Bereich (Bereich I) der VR 2 und 3 des kaltverformten Blankstahls aus dem untersuchten Zugglied mit KVP D20 vergleichbar.

Der Vergleich der Werte für den statischen Elastizitätsmodul in Abb. 4.15 zeigt, dass der untersuchte Blankstahl bei Temperaturen zwischen 300 bis $600^{\circ} \mathrm{C}$ wesentlich höhere Werte aufweist, als in [13], [45], [48] oder [63] angegeben. Außerhalb des genannten Temperaturbereichs liegt eine relativ gute Übereinstimmung vor. Generell sind die Abminderungsfaktoren des warmgewalzten Stahls [13] meist deutlich geringer, als die ermittelten Werte für den untersuchten Blankstahl. Eine Übertragung der Abminderungsfaktoren des Elastizitätsmoduls $\left(E_{\theta} / E_{20}\right)$ für warmgewalzten Stahl auf kaltgezogenen Blankstahl kann somit nicht empfohlen werden.

Auch die Kleinzugversuche von Ranawaka \& Mahendran [48] an kaltgeformten Blechen sowie von Outinen \& Mäkeläinen an kaltgezogenen Hohlprofilen zeigen wesentlich geringere Abminderungsfaktoren, als die Werte des untersuchten kaltverformten Blankstahls. Die beste Übereinstimmung zeigen die Ergebnisse mit denen von Wei \& Jihong [63]. Allerdings sind im Temperaturbereich von 500 bis $600{ }^{\circ} \mathrm{C}$ ebenfalls große Unterschiede vorhanden. 
Ursächlich für die Abweichungen zwischen den stationären Kleinzugversuchen an kaltgeformten Stählen ist mit hoher Wahrscheinlichkeit das verwendete Ausgangsmaterial bzw. die Profilgeometrie. In den für den Vergleich herangezogenen Veröffentlichungen ([44], [48] und [63]) werden relativ dünne Bleche oder Profile als Zugproben verwendet. Für die Untersuchung des kaltverformten Blankstahls kommen wesentlich dickere Profile, d.h. KVP D20, zum Einsatz. Ebenso kann die gewählte Probekörpergeometrie für

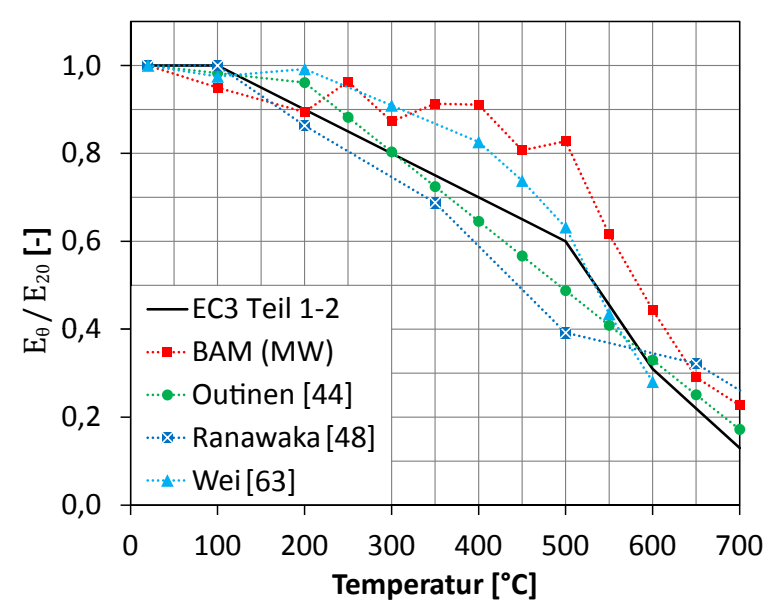

Abb. 4.15: Vergleich des Abminderungsfaktor für den statischen Elastizitätsmodul die Abweichungen mitverantwortlich sein. Im Gegensatz zu den in den Veröffentlichungen getesteten Flachproben werden für den kaltverformten Blankstahl Rundproben mit einem Durchmesser von 10 mm untersucht. Des Weiteren wird für den kaltverformten Blankstahl die Festigkeitsklasse S355 verwendet. In den Untersuchungen von Wei \& Jihong [63] sowie Ranawaka \& Mahendran [48] kommt Stahl mit einer höheren Festigkeit (G550) zum Einsatz.

\subsubsection{Vergleich des statischen und dynamischen Elastizitätsmoduls}

Aus der Literatur ist bekannt, dass sich die Werte für den statischen und dynamischen Elastizitätsmodul unterscheiden können [58]. Die durchgeführten Untersuchungen an kaltverformtem Blankstahl bestätigen dies. Eine analytische Formel, welche den Zusammenhang zwischen statischem und dynamischem Elastizitätsmodul beschreibt, ist in der Veröffentlichung von Richter [48] enthalten. Die Abhängigkeit des Elastizitätsmoduls wird dabei mit Hilfe der spezifischen Wärmekapazität (c), dem differentiellen linearen Wärmeausdehnungskoeffizienten $(\alpha)$ und der Dichte des Stahls $(\rho)$ beschrieben. Angewendet auf den untersuchten Blankstahl ergeben sich auch für hohe Stahltemperaturen nur geringe Unterschiede zwischen dem statischen und dynamischen Elastizitätsmodul. Die Verwendung der analytischen Formel von Richter [48] wird daher nicht weiter verfolgt.

Die Ergebnisse des Elastizitätsmoduls für den untersuchten kaltverformten Blankstahl sind in Tabelle 4.5 angegeben. Zur besseren Übersichtlichkeit sind die Abminderungsfaktoren in Abb. 4.16 auch graphisch dargestellt. Generell lässt sich feststellen, dass der Elastizitätsmodul zwischen Resonanzverfahren und der Auswertung der Kleinzugversuche bis etwa $500{ }^{\circ} \mathrm{C}$ gut übereinstimmt. Für Temperaturen von mehr als $500{ }^{\circ} \mathrm{C}$ nehmen die Abminderungsfaktoren aus den Kleinzugversuchen gegenüber denen aus dem Resonanzverfahren stark ab. Eine mögliche Ursache dafür ist mit hoher Wahrscheinlichkeit die mechanische Bean- 
spruchung, die die Probekörper in den Kleinzugversuchen erfahren. Dadurch können z.B. Versetzungen oder Gitterspannungen im Gefüge entstehen, die dann Auswirkung auf die SpannungsDehnungs-Kurve haben. Beim akustischen Resonanzverfahren wird das Gefüge lediglich durch die Temperatur beeinflusst. Eine weitere mögliche Ursache liegt in der schwierigen Bestimmung des statischen Elastizitätsmoduls bei hohen Temperaturen. Diese Problematik wurde bereits in Kapitel 4.4.4 beschrieben. Der rapide Abfall des Abminderungsfaktors tritt in ähnlicher Weise auch bei den in den Normen [12], [13]

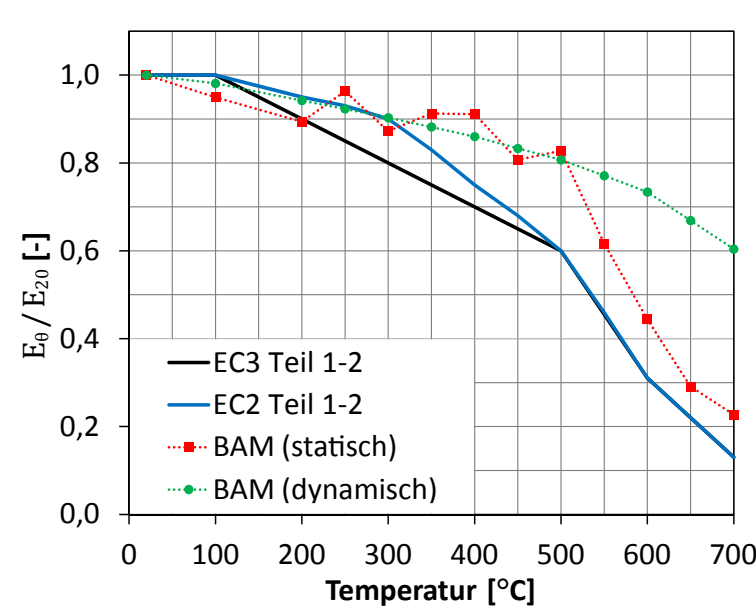

Abb. 4.16: Vergleich der Abminderungsfaktoren des Elastizitätsmoduls zwischen den untersuchten Blankstahl und den Empfehlungen aus den Normen angegebenen Kurven für den E-Modul auf. Die Werte der Abminderungsfaktoren aus den Normen sind allerdings bereits $a b 300^{\circ} \mathrm{C}$ (EC3 Teil 1-2, warmgewalzt) bzw. $400{ }^{\circ} \mathrm{C}$ (EC2 Teil 1-2, kaltverformt Klasse X) wesentlich geringer als die des untersuchten Blankstahls. Da der dynamische Elastizitätsmodul auch bei hohen Temperaturen relativ genau ermittelt werden kann, erfolgt die Bestimmung der Proportionalitätsgrenze und der 0,2\%-Dehngrenze sowie deren Abminderungsfaktoren unter Raumtemperatur und erhöhter Temperatur mittels des dynamischen E-Moduls.

\subsection{Ermittlung der Spannungs-Dehnungs-Kennlinien}

Mittels stationärer Kleinzugversuche wird das temperaturabhängige Materialverhalten des kaltverformten Blankstahls untersucht. Die Kleinzugproben werden zunächst mittels eines Elektroofens auf eine definierte Stahltemperatur erwärmt und dann bis zum Bruch gezogen. Dabei wird die Spannungs-Dehnungs-Kurve aufgezeichnet. Da die gemessene Kurve von der jeweiligen Belastungsgeschwindigkeit abhängt, werden verschiedene Versuchsreihen (VR) mit unterschiedlichen Prüfgeschwindigkeiten untersucht. Die Geschwindigkeiten werden nach DIN EN 6892-1 [14] und DIN EN 6892-2 [15] gewählt. Neben einer Versuchsreihe bei Raumtemperatur, bestehend aus sechs Kleinzugproben, werden drei Versuchsreihen unter erhöhter Temperatur mit jeweils 12 Probekörpern geprüft. Die Zuordnung der einzelnen Kleinzugproben zu den Versuchsreihen ist im Forschungsbericht [35] angegeben. Um einen Vergleich mit den Materialeigenschaften von warmgewalztem Stahl zu ermöglichen, werden aus den gemessenen Spannungs-Dehnungs-Kurven die wichtigsten Kennwerte ermittelt. Für die Bestimmung der technischen Proportionalitätsgrenze und der 0,2\%-Dehngrenze werden die Werte des in Kapitel 4.4 vorgestellten dynamischen Elastizitätsmoduls verwendet. 


\subsubsection{Kleinzugversuche bei Raumtemperatur}

\section{Versuchsaufbau und -durchführung}

Die Durchführung der Kleinzugversuche bei Raumtemperatur erfolgt an einer Universalprüfmaschine mit einer maximalen Zugraft des Hydraulikzylinders von $100 \mathrm{kN}$ (siehe Abb. 4.17). Für die Messung der Dehnungen wird ein Feindehnungsaufnehmer vom Typ HBM DD1 mit einer Messlänge von $50 \mathrm{~mm}$ verwendet. Die Zugversuche werden stationär, d.h. bei konstanter Temperatur, durchgeführt.

Vor dem Einbau werden die Maße der jeweiligen Probe ermittelt und Markierungsstriche im Abstand von $5 \mathrm{~mm}$ Markierungsstriche auf dem Probekörper aufgebracht (siehe Abb. 4.17). Um den Schlupf zu Beginn des Zugversuches zu reduzieren, wird die Probe mit einer geringen Zugkraft von etwa 0,4 kN belastet, bevor das Extensometer an die zu prüfende Probe angebracht wird (siehe Abb. 4.17). Der Zugversuch erfolgt bis zu einer Dehnung von $2 \%$ dehnungsgeregelt mit einer Dehngeschwindigkeit von 0,00025 s-1. Danach erfolgt eine Umschaltung in Wegregelung. Die Geschwindigkeit für die Wegregelung wird so gewählt, dass diese gut mit der Geschwindigkeit aus der Dehnregelung übereinstimmt. Für die Umrechnung zwischen Dehn- und Weggeschwindigkeit wird die Dehngeschwindigkeit mit der Versuchslänge der Zugprobe $\left(\mathrm{L}_{\mathrm{c}}=60 \mathrm{~mm}\right)$ multipliziert. Dadurch ergibt sich für die Wegregelung eine Geschwindigkeit von 0,015 mm/s. Das Extensometer wird bei einer Dehnung von etwa 2 bis $4 \%$ von der Zugprobe entfernt, um mögliche Beschädigungen beim Bruch des Probekörpers zu verhindern. Als Folge endet die Aufzeichnung der Dehnung. Anhand der im Vorfeld aufgebrachten Markierungsstriche wird nach dem Zugversuch die Bruchdehnung an der ausgebauten Zugprobe bestimmt.
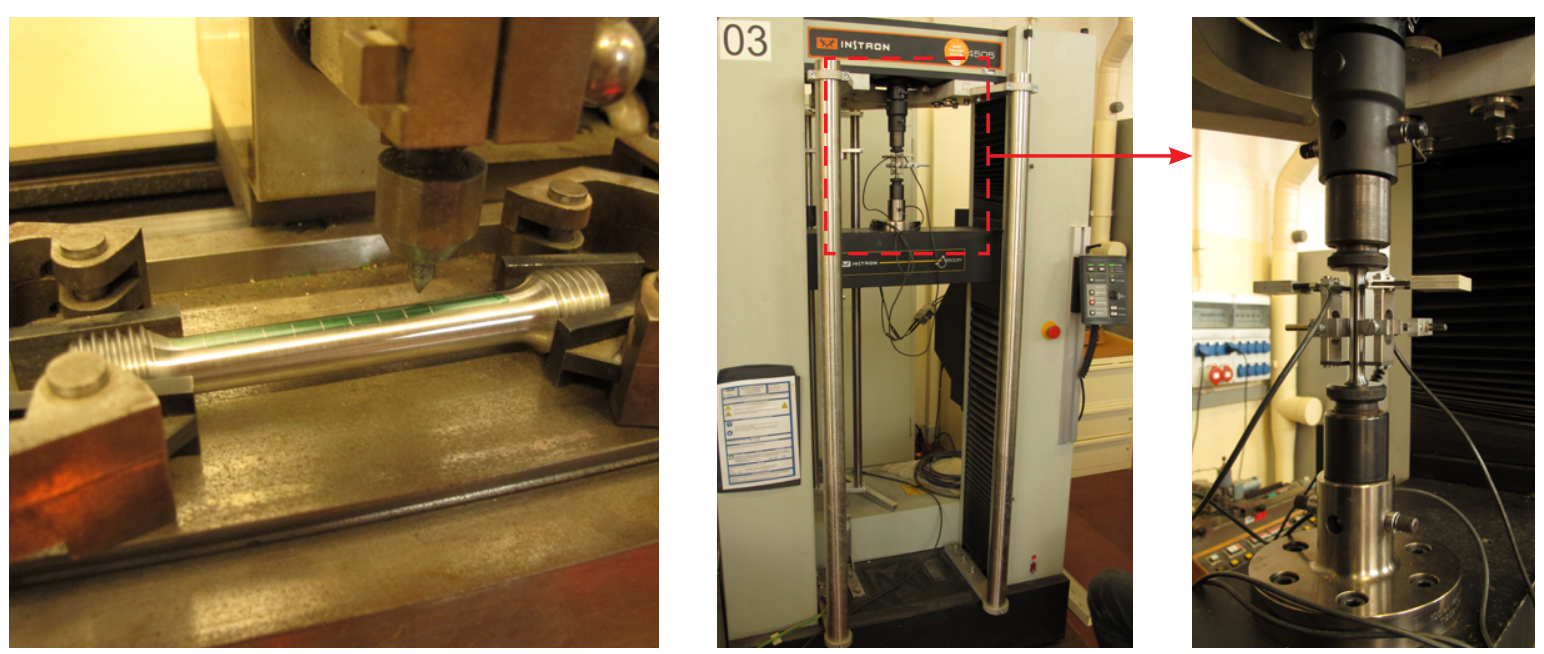

Abb. 4.17: Kleinzugversuche bei Raumtemperatur (links: Aufbringen der Markierungsstriche auf die Zugprobe; Mitte: Prüfmaschine; rechts: eingebauter Probekörper) 


\section{Auswertung der Zugversuche}

In Abb. 4.19 sind die an den Zugproben bei Raumtemperatur gemessenen Spannungs-Dehnungs-Kurven dargestellt. Die Kurven entsprechen weitgehend der schematischen Kennlinie aus Abb. 4.18. Mit der Abnahme des Extensometers endet auch die Aufzeichnung der Dehnung. Anhand der gemessenen Spannungs-Dehnungs-Kurven zeigt sich, dass der untersuchte Stahl aufgrund seines Herstellungsverfahrens über keine ausgeprägte Streckgrenze verfügt. Alternativ wird daher die 0,2\%-Dehngrenze bestimmt. Die Ermittlung der technischen Proportionalitätsgrenze $\left(\mathrm{f}_{\mathrm{p} 0,01}\right)$ und der 0,2\%-Dehngrenze $\left(\mathrm{f}_{\mathrm{p} 0,2}\right)$ erfolgt mit Hilfe des in Kapitel 4.4.1 ermittelten dynamischen Elastizitätsmoduls. Eine Zusammenfassung der für den kaltverformten Blankstahl gemessenen Kenngrößen ist in Tabelle 4.6 angegeben.

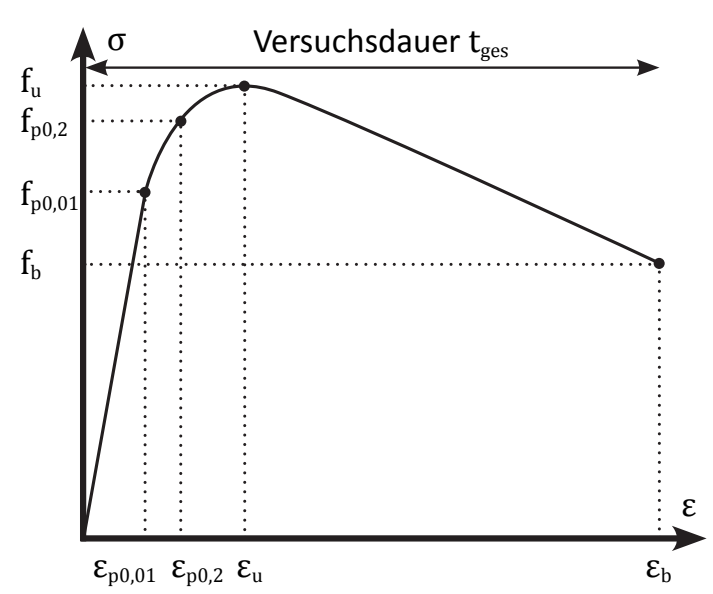

Abb. 4.18: Schematische Spannungs-DehnungsKennlinie bei Raumtemperatur

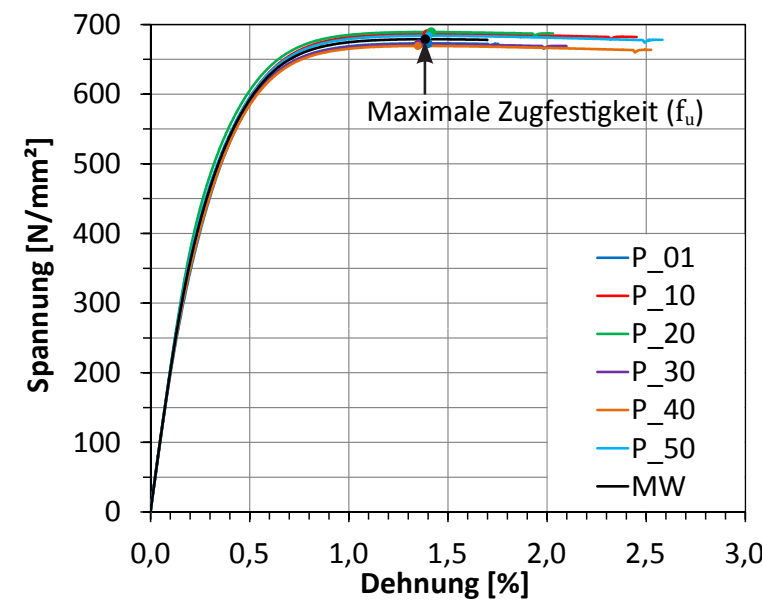

Abb. 4.19: Gemessene Spannungs-DehnungsKurven bei Raumtemperatur

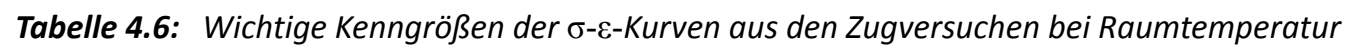

\begin{tabular}{|c|c|c|c|c|c|c|c|c|c|c|}
\hline \multirow{2}{*}{$\begin{array}{l}\text { Proben- } \\
\text { bezeich- } \\
\text { nung }\end{array}$} & \multirow{2}{*}{$\begin{array}{c}\begin{array}{c}\text { E-Modul } \\
{\left[\mathrm{N} / \mathrm{mm}^{2}\right]}\end{array} \\
E_{\mathrm{dyn}}\end{array}$} & \multicolumn{4}{|c|}{$\begin{array}{c}\text { Spannung } \\
{\left[\mathrm{N} / \mathrm{mm}^{2}\right]}\end{array}$} & \multicolumn{4}{|c|}{$\begin{array}{c}\text { Dehnung } \\
{[\%]}\end{array}$} & \multirow{2}{*}{$\begin{array}{c}\begin{array}{c}\text { Dauer } \\
\text { [s] }\end{array} \\
t_{\text {ges }}\end{array}$} \\
\hline & & $\mathrm{f}_{\mathrm{p} 0,01}$ & $\mathrm{f}_{\mathrm{p} 0,2}$ & $\mathrm{f}_{\mathrm{u}}$ & $f_{b}$ & $\varepsilon_{\mathrm{p} 0,01}$ & $\varepsilon_{\mathrm{p} 0,2}$ & $\varepsilon_{\mathrm{u}}$ & $\varepsilon_{\mathrm{b}}$ & \\
\hline P 01 & \multirow{6}{*}{207000} & 238 & 574 & 673 & 246 & 0,12 & 0,48 & 1,40 & 15,90 & 495 \\
\hline P 10 & & 337 & 597 & 687 & 245 & 0,18 & 0,51 & 1,40 & 14,88 & 489 \\
\hline P 20 & & 326 & 600 & 689 & 235 & 0,17 & 0,49 & 1,42 & 15,88 & 506 \\
\hline P 30 & & 293 & 579 & 672 & 230 & 0,15 & 0,48 & 1,35 & 14,94 & 475 \\
\hline P 40 & & 255 & 572 & 669 & 248 & 0,13 & 0,47 & 1,35 & 15,64 & 481 \\
\hline P 50 & & 296 & 587 & 684 & 247 & 0,15 & 0,48 & 1,40 & 14,38 & 459 \\
\hline Mittelwert & 207000 & 291 & 585 & 679 & 242 & 0,15 & 0,48 & 1,39 & 15,27 & 484 \\
\hline
\end{tabular}

Anhand der gemessenen Kurven in Abb. 4.19 ist erkennbar, dass die Spannung bis zum Erreichen der technischen Proportionalitätsgrenze, welche sich bei etwa 0,15\% Dehnung $\left(\varepsilon_{\mathrm{p} 0,01}\right)$ befindet, linear ansteigt. Dieser Bereich kann als elastischer Bereich bezeichnet wer- 
den. An den elastischen Bereich schließt sich ein nichtelastischer Verformungsbereich an. Für den nichtelastischen Bereich besteht kein linearer Zusammenhang zwischen Spannung und Dehnung. Die Gesamtdehnung setzt sich aus elastischen und plastischen Dehnungen zusammen. Die maximale Zugfestigkeit $\left(f_{u}\right)$ wird bei einer Dehnung von etwa 1,4 \% $\left(\varepsilon_{u}\right)$ erreicht. Danach nimmt die Spannung ab. Der Probenbruch des untersuchten Blankstahls tritt bei einer Bruchspannung $\left(\mathrm{f}_{\mathrm{b}}\right)$ von ca. $242 \mathrm{~N} / \mathrm{mm}^{2}$ und einer Bruchdehnung $\left(\varepsilon_{\mathrm{b}}\right)$ von etwa $15 \%$ auf.

\section{Fragmente der getesteten Probekörper}

Die Fragmente der getesteten Zugproben sind in Abb. 4.20 dargestellt. Bei allen untersuchten Proben bildet sich eine schwanenhalsförmige Einschnürung im Bereich der Bruchstelle aus. Beim Vergleich der Bruchstücke der Zugproben ist festzustellen, dass der Probenbruch nicht immer mittig auftritt, sondern überwiegend im oberen Bereich des Probekörpers. Die Messlänge des Extensometers ist jedoch groß genug, so dass sich dies nicht nachteilig auf die Dehnungsmessung auswirkt. Die Bruchformen der Zugproben entsprechen einem Trichter-Kegel, wodurch bestätigt wird, dass es sich um ein duktiles Material handelt.
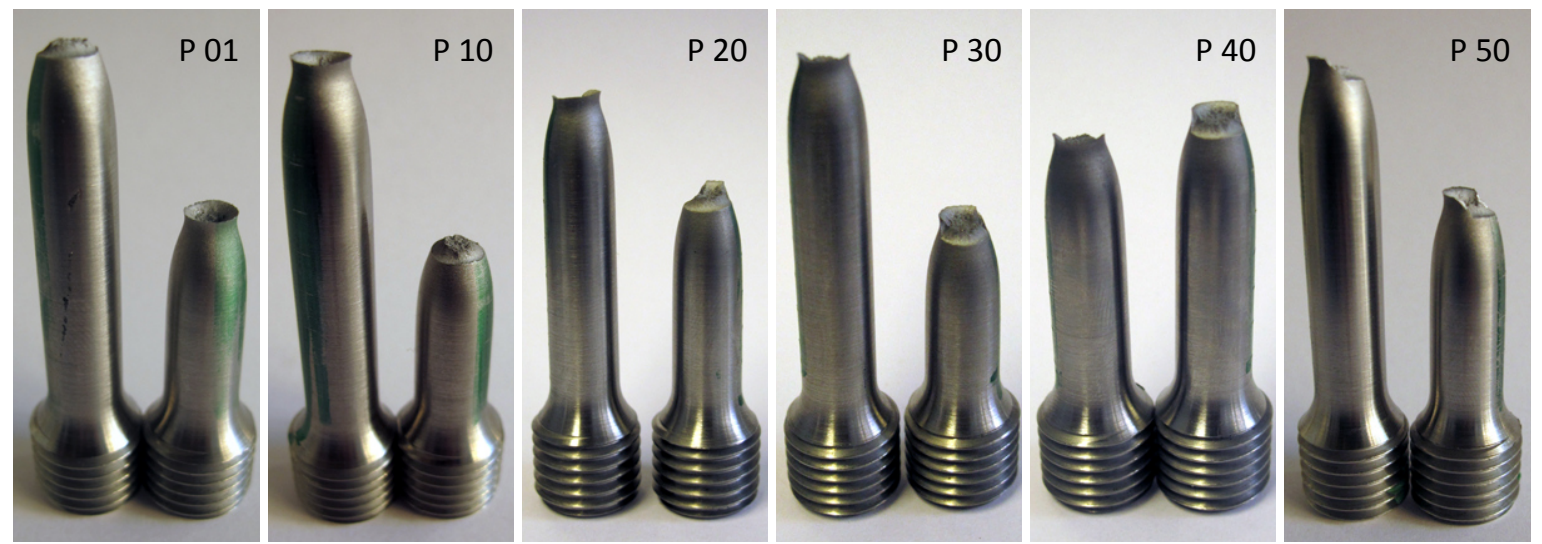

Abb. 4.20: Fragmente der Zugproben unter Raumtemperatur (Abbildungen sind nicht maßstäblich)

\subsubsection{Kleinzugversuche unter erhöhter Temperatur}

\section{Versuchsaufbau und -durchführung}

Die Kleinzugversuche unter erhöhter Temperatur werden an der gleichen Prüfmaschine, wie die Versuche bei Raumtemperatur durchgeführt. Allerdings erfolgen die Versuche in einem Hochtemperaturofen. Statt des Feindehnungsextensometers wird ein hitzeresistentes Hochtemperaturextensometer vom Typ MTS mit Wasserkühlung und einer Messlänge von $21 \mathrm{~mm}$ verwendet. Eine detaillierte Darstellung des Versuchsaufbaus mit der Bezeichnung der einzelnen Komponenten ist in Abb. 4.21 dargestellt. Mit Ausnahme des Erwärmungsvorganges werden die Kleinzugversuche unter erhöhter Temperatur analog zu den Versuchen unter Raumtemperatur durchgeführt. 

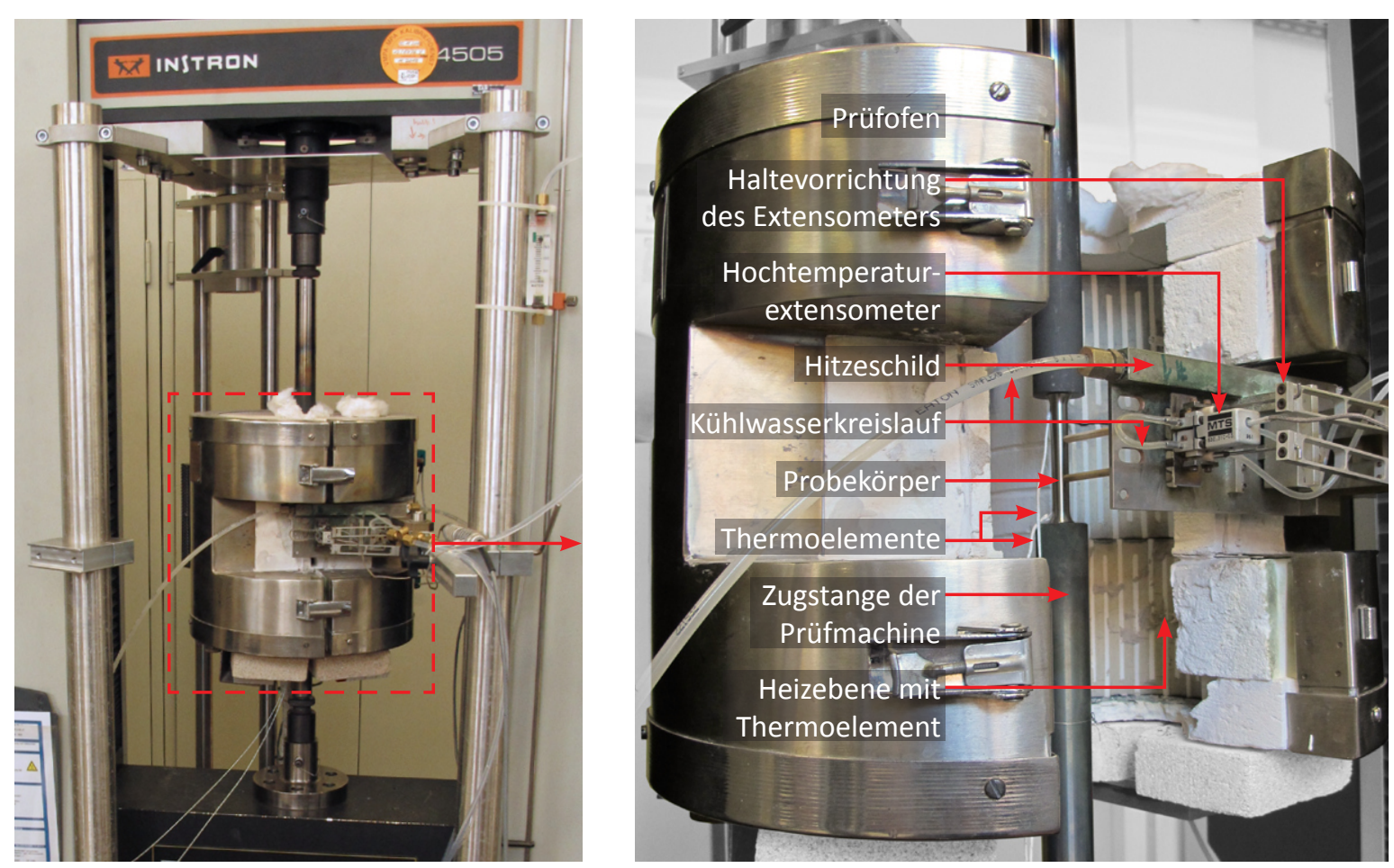

Abb. 4.21: Prüfmaschine für die Kleinzugversuche unter erhöhter Temperatur (links: geschlossener Ofen mit eingebauter Zugprobe; rechts: Probe mit angebrachten Thermoelementen sowie Extensometer

Insgesamt werden drei Versuchsreihen (VR) mit je 12 Zugproben unter erhöhter Temperatur getestet. Die einzelnen Versuchsreihen unterscheiden sich lediglich in der Versuchsgeschwindigkeit (siehe Tabelle 4.7). Durch die Variation wird der Einfluss der Prüfgeschwindigkeit auf den Verlauf der Spannungs-Dehnungs-Kurven untersucht. Wie bereits in Kapitel 4.4.3 beschrieben, erfolgen die Kleinzugversuche bis zu einer Dehnung von $2 \%$ dehnungsgeregelt (Bereich I) und danach bis zum Probenbruch der Zugprobe in Wegregelung (Bereich II). Die Dehnungsaufzeichnung endet mit der Abnahme des Extensometers bei einer Dehnung von etwa 3 bis $4 \%$. Der Beginn der Wegregelung (Bereich II) wird in den aufgezeichneten Spannungs-Dehnungs-Kurven mit einem farbigen Punkt markiert. Die gewählte Geschwindigkeit für die Wegregelung wird ebenfalls zwischen den einzelnen Versuchsreihen verändert. 


\section{Auswertung der Kleinzugversuche unter erhöhter Temperatur}

Für den untersuchten Blankstahl ist ein schematischer Verlauf der Spannungs-DehnungsKennlinie mit Angabe der wichtigsten Kenngrößen in Abb. 4.22 dargestellt. Die gemessenen Spannungs-Dehnungs-Kurven des untersuchten Blankstahls sind, nach Versuchsreihen geordnet, in Abb. 4.23 bis Abb. 4.25 für einen Dehnungsbereich bis $3 \%$ dargestellt. Zum Vergleich ist in den Abbildungen auch die Mittelwertkurve aus den Zugversuchen unter Raumtemperatur enthalten. Generell lässt sich feststellen, dass die unter Raumtemperatur und erhöhter Temperatur gemessenen Spannungs-Dehnungs-Kurven ähnlich verlaufen. Der Beginn der Kleinzugversuche ist jeweils durch einen linear-elastischen Bereich gekennzeichnet. Daran schließt sich ein nichtelastischer Verformungsbereich an. Auch bei erhöhter Temperatur weist der untersuchte Blankstahl keine ausgeprägte Streckgrenze auf. Alternativ wird die $0,2 \%$-Dehngrenze $\left(\mathrm{f}_{\mathrm{p} 0,2}\right)$ bestimmt. Sowohl die technische Proportionalitätsgrenze $\left(\mathrm{f}_{\mathrm{p} 0,01}\right)$ als auch die 0,2\%-Dehngrenze werden mit Hilfe des dynamischen Elastizitätsmoduls ermittelt (siehe Kapitel 4.4.1). Die Materialfestigkeit nimmt bei hohen Temperaturen, d.h. ab etwa $400^{\circ} \mathrm{C}$, gegenüber der Festigkeit bei Raumtemperatur deutlich ab. Der Festigkeitsverlust infolge hoher Temperaturen ist ebenfalls von warmgewalzten Baustählen bekannt. Grund hierfür ist, dass sich bei hohen Stahltemperaturen die Beweglichkeit der Atome vergrößert und die Anziehungskräfte abnehmen, welche eine leichtere Verformbarkeit und Verschiebbarkeit der Metallkristalle bewirkt. Für die untersuchten Zugproben unter erhöhter Temperatur hat sich bereits im dehnungsgeregelten Bereich oftmals ein lokales Spannungsmaximum ausgebildet. Die höhere Prüfgeschwindigkeit im Bereich der Wegregelung führt zu einem weiteren Spannungsmaximum. Die maximale Zugfestigkeit des untersuchten Blankstahls wird daher für beide Verformungsbereiche ermittelt. Nach dem Erreichen der maximalen Zugfestigkeit zeigt sich eine Abnahme der Spannung bis zum Bruch der Zugprobe. Die Anhand der Kleinzugversuche bestimmten Kenngrößen der gemessenen Spannungs-Dehnungs-Kurven sind im Anhang B angegeben.

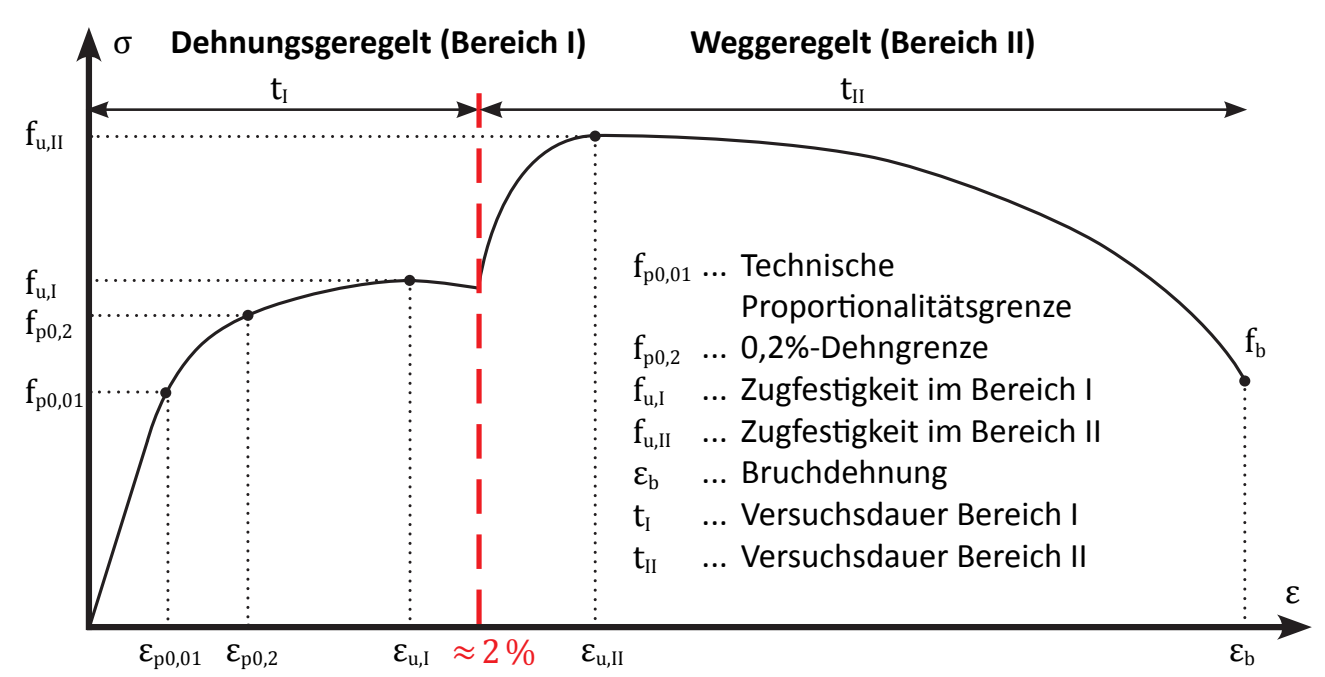

Abb. 4.22: Schematischer Spannungs-Dehnungs-Verlauf der Zugversuche unter erhöhter Temperatur 


\section{Auswertung der Zugversuche in VR 1}

Die untersuchten Zugproben werden mit einer nahezu gleichen Prüfgeschwindigkeit im Bereich der Dehn- bzw. Wegregelung geprüft. Beim Umschaltpunkt zwischen Dehn- und Wegregelung, welcher im Diagramm mit einem farbigen Punkt markiert ist, kommt es zu keinem nennenswerten Spannungsanstieg. Die gewählte Geschwindigkeit ist identisch mit den Zugproben, welche unter Raumtemperatur untersucht werden. Anhand der gemessenen Spannungs-Dehnungs-Kurven aus VR 1 (siehe Abb. 4.23) ist erkennbar, dass bei Temperaturen zwischen 200 und $300^{\circ} \mathrm{C}$ höhere Spannungen erreicht werden als bei Raumtemperatur. Ursache dafür sind mit hoher Wahrscheinlichkeit Gitterbaufehler im Stahl, welche infolge der gesteigerten Temperatur zunehmen. Diese Störungen stellen einen Widerstand gegen Verformungen dar und wirken festigkeitssteigernd ${ }^{1}$. Die gesteigerte Festigkeit hat jedoch oftmals eine Verringerung der Duktilität zur Folge, d.h. der Stahl wird spröder und besitzt eine geringere plastische Verformbarkeit. Dies ist auch bei dem untersuchten kaltverformten Blankstahl aufgrund der gemessenen Bruchdehnung bzw. der Länge des Einschnürungsbereiches der Fall.

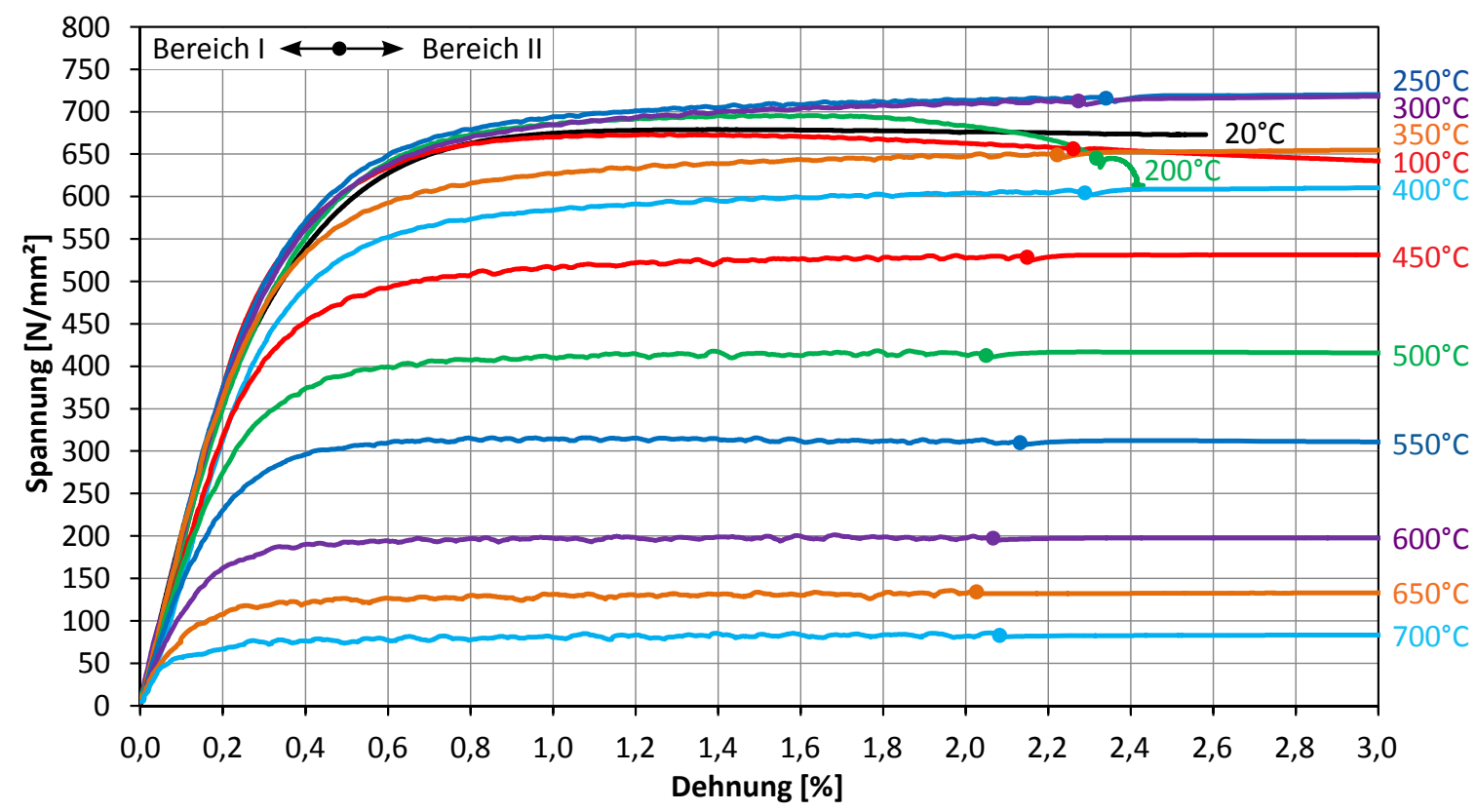

Abb. 4.23: Gemessene Spannungs-Dehnungs-Kurven der Zugversuche aus VR 1

Für Temperaturen von mehr als $350^{\circ} \mathrm{C}$ kommt es zu einer Reduzierung der maximalen Spannungen. Ab ca. $400^{\circ} \mathrm{C}$ ist zudem eine signifikante Abnahme des elastischen Bereiches sowie des statischen E-Moduls feststellbar. Besonders deutlich wird dies anhand der berechneten

1 Festigkeit charakterisiert den Widerstand gegen Verformung. Träger der plastischen Verformung bei Metallen sind Gitterfehler. Durch das gleichzeitige Auftreten von Verformungen und Temperaturerhöhungen können Lehrstellen ("nulldimensionale Gitterfehler") im Metallgitter entstehen. [27] 
Abminderungsfaktoren der Stahlfestigkeit (siehe Abb. 4.28 in Kapitel 4.5.3). Ferner nimmt die Duktilität des untersuchten Blankstahls bei Temperaturen von mehr als $400{ }^{\circ} \mathrm{C}$ merklich zu. Für eine Stahltemperatur von $700{ }^{\circ} \mathrm{C}$ betragen die Werte für die $0,2 \%$-Dehngrenze und die Zugfestigkeit lediglich etwa $10 \%$ des gemessenen Wertes bei Raumtemperatur. Die technische Proportionalitätsgrenze entspricht nur etwa $8 \%$ des bei Normaltemperatur gemessenen Wertes. Ursache für die signifikante Festigkeitsabnahme ist die bei hohen Temperaturen stattfindende Rekristallisation des Stahls. Bei diesem Vorgang kommt es durch Neubildung des Gefüges zum Abbau von Gitterfehlern, z.B. Versetzungen. Für Stahl findet dieser Vorgang überlicherweise bei Temperaturen oberhalb der Rekristallisationstemperatur $\left(\mathrm{T}_{\mathrm{R}}\right)$ statt. Im Allgemeinen beträgt die Rekristallisationstemperatur etwa $40 \%$ der Schmelztemperatur $\left(\mathrm{T}_{\mathrm{m}}\right)$. Bei einer Schmelztemperatur für Stahl von ca. $1500^{\circ} \mathrm{C}$ entspricht die Rekristallisationstemperatur ungefähr $600^{\circ} \mathrm{C}$. Für Temperaturen ab etwa $600^{\circ} \mathrm{C}$ können zudem zusätzlich Kriechverformungen auftreten [52]. Kriechen ist eine zeitabhängige, plastische Verformung des Materials unter Last. Die Kriechverformung wird von der Höhe der mechanischen Beanspruchung, der Temperatur sowie der Dauer des Zugversuches beeinflusst. Aufgrund der relativ kurzen Versuchsdauer der Kleinzugversuche, d.h. weniger als $1 \mathrm{~h}$ bei $700^{\circ} \mathrm{C}$, kann der Anteil der Kriechverformungen vernachlässigt werden.

\section{Auswertung der Zugversuche in VR 2 und 3}

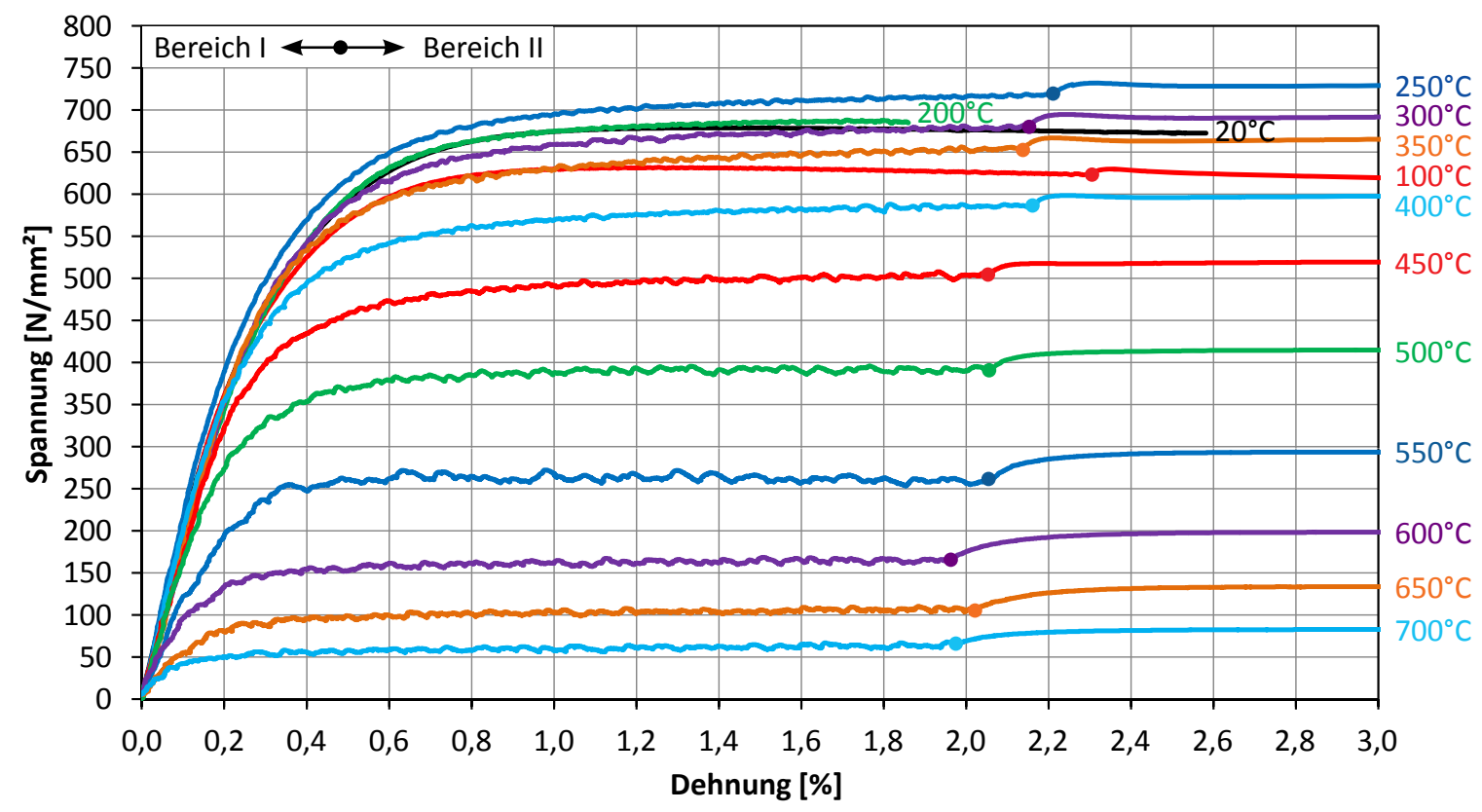

Abb. 4.24: Gemessene Spannungs-Dehnungs-Kurven der Zugversuche aus VR 2

Um den Einfluss der Versuchsgeschwindigkeit auf die Spannungs-Dehnungs-Kurven zu untersuchen, werden die VR 2 und 3 mit einer anderen Versuchsgeschwindigkeit durchgeführt als die Zugversuche in VR 1. Für den dehnungsgeregelten Bereich wird in den beiden VR 2 und 3 eine Dehngeschwindigkeit von 0,00007 s $\mathrm{s}^{-1}$ verwendet. Diese Geschwindigkeit ist 
um den Faktor 3,57 geringer als in VR 1. Im Bereich der Wegregelung wird lediglich in VR 3 eine Veränderung der Geschwindigkeit, d.h. gegenüber der VR 1 eine Erhöhung um den Faktor 5,6, vorgenommen. Anhand der in Abb. 4.24 und Abb. 4.25 dargestellten SpannungsDehnungs-Kurven der VR 2 und 3 zeigt sich, dass eine Zunahme der Versuchsgeschwindigkeit zu einer Erhöhung des Spannungsniveaus führt. Da die Spannungen von der Versuchsgeschwindigkeit abhängen, wird die maximale Zugfestigkeit sowohl für den Bereich der Dehnungsregelung (Bereich I) als auch für die Wegregelung (Bereich II) bestimmt.

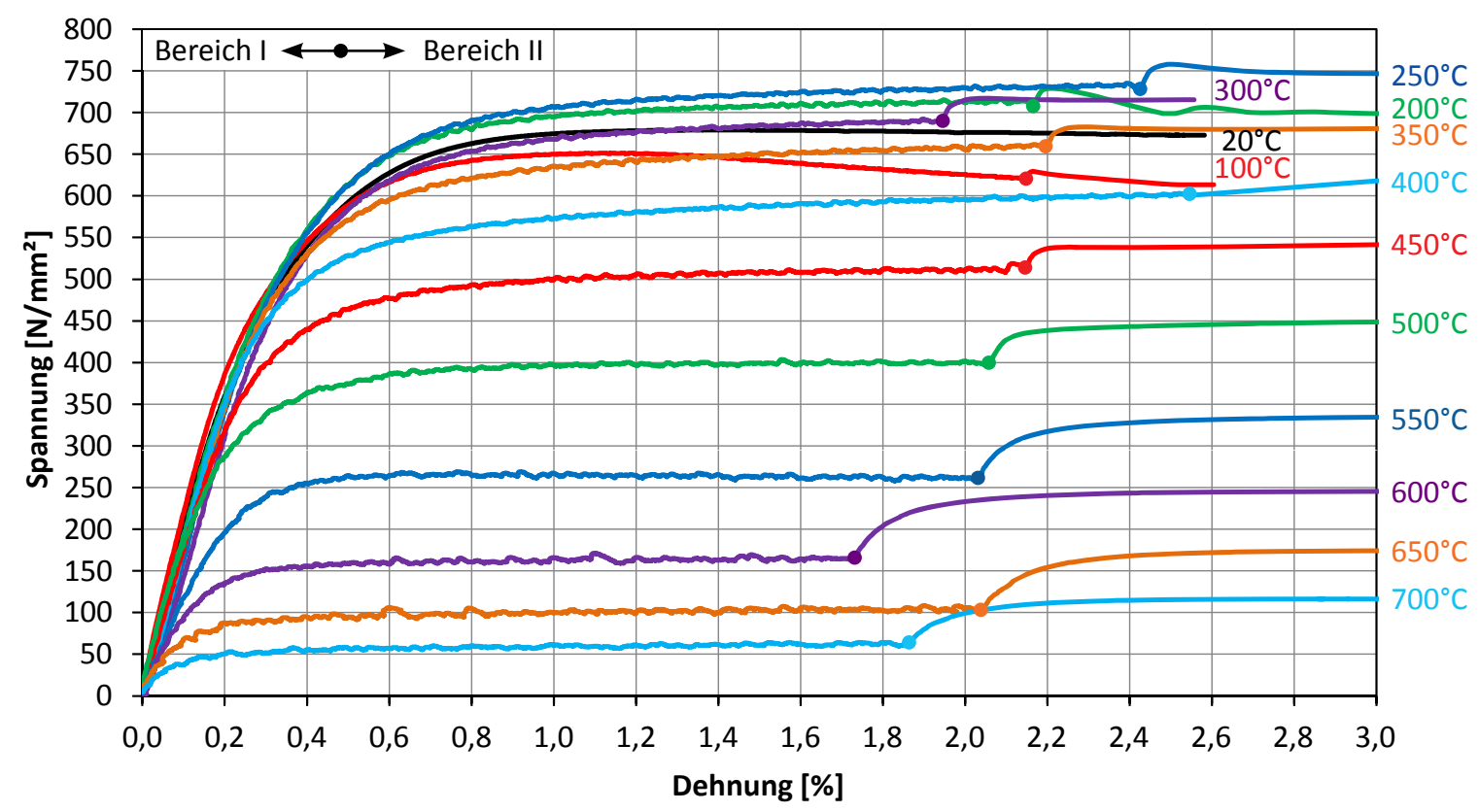

Abb. 4.25: Gemessene Spannungs-Dehnungs-Kurven der Zugversuche aus VR 3

\section{Einfluss der Versuchsgeschwindigkeit auf das Spannungsniveau}

Es ist bekannt, dass die Höhe der Versuchsgeschwindigkeit den Verlauf der Spannungs-Dehnungs-Kurven beim Kleinzugversuch beeinflusst. Um diesen Einfluss genauer zu quantifizieren, werden die Kleinzugversuche unter erhöhter Temperatur mit verschiedenen Prüfgeschwindigkeiten durchgeführt (siehe Tabelle 4.7). Exemplarisch ist der Einfluss der Versuchsgeschwindigkeit auf die Spannungs-Dehnungs-Kurven in Abb. 4.26 für die Stahltemperatur $500{ }^{\circ} \mathrm{C}$ und $600{ }^{\circ} \mathrm{C}$ dargestellt. Anhand der ausgewählten Kurven zeigt sich, dass zu

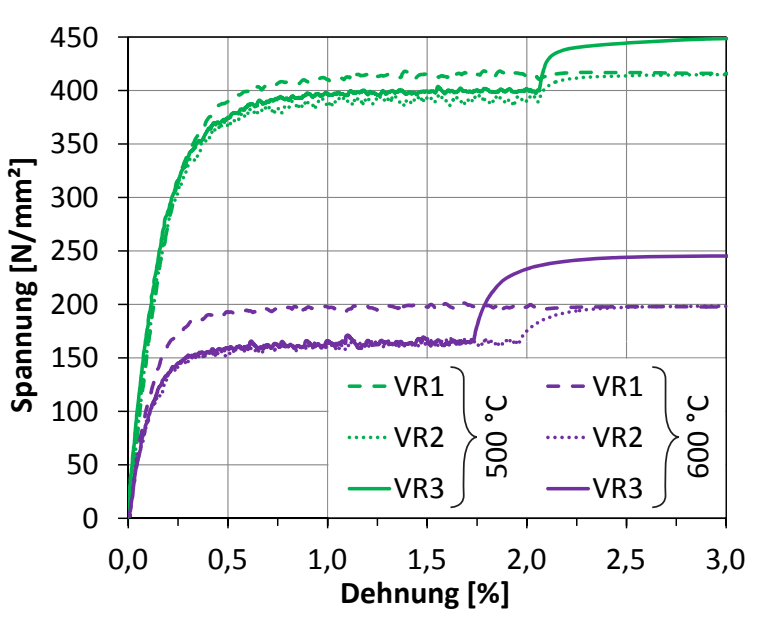

Abb. 4.26: Einfluss der Prüfgeschwindigkeit auf die $\sigma$ - $\varepsilon$-Kurven bei 500 und $600{ }^{\circ} \mathrm{C}$ 
Beginn der Kleinzugversuche, d.h. im linear-elastischen Bereich, der Anstieg der Spannungs-Dehnungs-Kurven zunächst nahezu identisch ist. Erst im anschließenden nichtelastischen Bereich ergeben sich Unterschiede. Die Zugproben aus der VR 2 und 3 besitzen im dehnungsgeregelten Bereich die gleiche Versuchsgeschwindigkeit und daher zunächst einen fast identischen Verlauf. Für den Probekörper der VR 1 ergibt sich im dehnungsgeregelten Bereich ein wesentlich höheres Spannungsniveau, welches aus der gesteigerten Versuchsgeschwindigkeit resultiert. Bei einer Dehnung von etwa $2 \%$ erfolgt der Wechsel in Wegregelung. In den VR 2 und 3 wird dabei auch die Prüfgeschwindigkeit erhöht. Infolge der gesteigerten Prüfgeschwindigkeit ergibt sich ein Anstieg der gemessenen Spannungen. Je höher die Geschwindigkeitszunahme, desto größer ist auch der Spannungszuwachs. Das Spannungsniveau der VR 2 gleicht sich im Bereich der Wegregelung an das der VR 1 an. Dies ist darauf zurückzuführen, dass die Prüfgeschwindigkeit der VR 2 auf die der VR 1 angehoben wird. Die geringere Versuchsgeschwindigkeit im dehnungsgeregelten Bereich der VR 2 hat somit keinen Einfluss auf das spätere Spannungsniveau im weggeregelten Bereich.

Eine ausführliche Beschreibung des quantitativen Einflusses der Versuchsgeschwindigkeit auf die Höhe des Spannungsniveaus ist in der Bachelorarbeit von Schramm [64] zu finden. Anhand der Ergebnisse zeigt sich, dass bis zu einer Temperatur von etwa $450{ }^{\circ} \mathrm{C}$ die Versuchsgeschwindigkeit nur im geringen Maße den Spannungsverlauf beeinflusst. Eine deutliche Auswirkung der Prüfgeschwindigkeit ist ab etwa $500{ }^{\circ} \mathrm{C}$ feststellbar. Die 0,2\%-Dehngrenze und die Zugfestigkeit im Bereich der Dehnungsregelung sind bei einer Temperatur von $500{ }^{\circ} \mathrm{C}$ in VR 1 gegenüber den VR 2 und 3 um etwa $5 \%$ höher. Für $600{ }^{\circ} \mathrm{C}$ beträgt der Unterschied bereits fast $25 \%$. Generell ist festzustellen, dass sich besonders bei hohen Stahltemperaturen eine Veränderung der Prüfgeschwindigkeit auf die Höhe der Spannungen auswirkt. Bei den im Bauwesen verwendeten Bauteilen treten in der Regel ruhende, d.h. statische Beanspruchungen auf. Die Versuchsgeschwindigkeit sollte daher möglichst klein gewählt werden. Wird beabsichtigt mit Hilfe der Spannungs-Dehnungs-Beziehungen auch das Bauteilverhalten bis in den Bereich der Zugfestigkeit und des Probenbruchs abzubilden, so ist die Prüfgeschwindigkeit zu erhöhen. Nur so lassen sich die relativ großen und schnell einsetzenden Verformungen im Einschnürungsbereich der Bruchstelle abbilden (siehe Kapitel 5.3.3).

\section{Fragmente der getesteten Zugproben}

Eine Auswahl der Fragmente der getesteten Zugproben ist in Abb. 4.27 dargestellt. Die Veränderung der Probekörper in Abhängigkeit von der Stahltemperatur ist deutlich erkennbar. Mit zunehmender Stahltemperatur kommt es zu einer Veränderung des Bruchbildes, der Einschnürungslänge sowie der Farbe des Stahls. Bis etwa $300{ }^{\circ} \mathrm{C}$ tritt der Bruch meist im oberen Bereich des Probekörpers auf. Für höhere Stahltemperaturen ereignet sich der Probenbruch gewöhnlich im Bereich der halben Probenlänge. Bei Stahltemperaturen von mehr als $500^{\circ} \mathrm{C}$ kommt es zu einer signifikanten Vergrößerung des Einschnürungsbereiches, wel- 
ches auf ein duktileres Verhalten hindeutet. Die Bruchdehnungen der einzelnen Zugproben sind im Anhang des Forschungsberichtes [35] angegeben. Ab etwa $600{ }^{\circ} \mathrm{C}$ ändert sich die sonst kraterförmig ausgebildete Bruchfläche in eine zackenförmige Fläche. Die Farbänderung des untersuchten Blankstahls entspricht im Wesentlichen den Anlassfarben von Stahl. Für Temperaturen von mehr als $350^{\circ} \mathrm{C}$ ist eine deutliche Schwarzfärbung der Zugproben und die Bildung einer Oxidschicht auf der Oberfläche zu erkennen. Die Intensität nimmt mit steigender Stahltemperatur zu. Ab ca. $500^{\circ} \mathrm{C}$ bilden sich Blasen auf der Stahloberfläche und es kommt zum Ablösen der Oxidschicht. Der Verschleiß durch Oxidation ist eine Korrosionserscheinung und wird durch die Reaktion des Stahls mit Sauerstoff hervorgerufen.
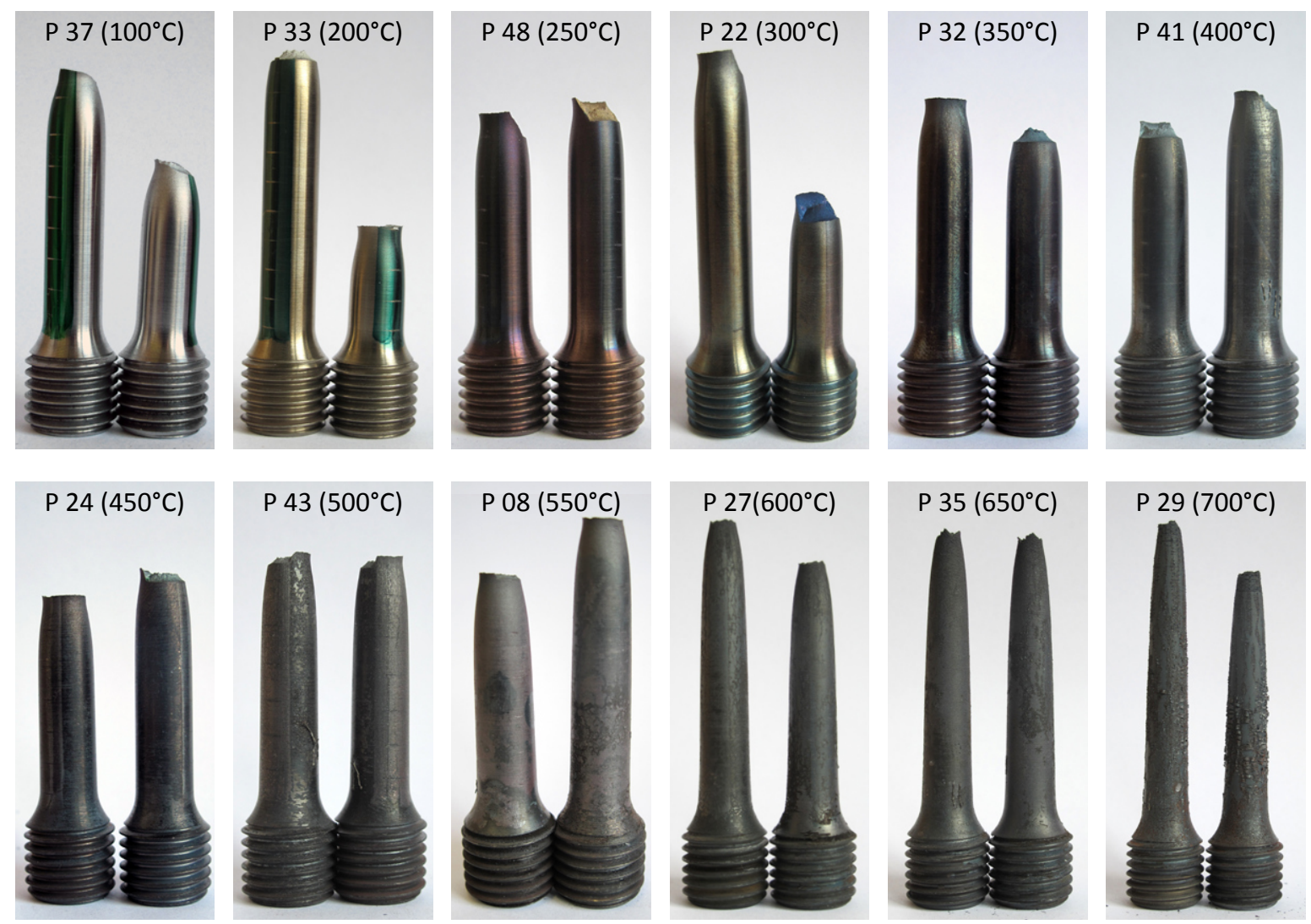

Abb. 4.27: Auswahl von Fragmenten der unter erhöhter Temperatur getesteten Zugproben (Abbildungen sind nicht maßstäblich)

\subsubsection{Vergleich der Abminderungsfaktoren der Stahlfestigkeiten}

Um das temperaturabhängige Verhalten des untersuchten Blankstahls besser bewerten zu können, werden für die gemessenen Spannungs-Dehnungs-Kurven die technische Proportionalitätsgrenze, die 0,2\%-Dehngrenze und die maximale Zugfestigkeit in den dehnungs- und weggeregelten Bereichen ermittelt. Für diese Kenngrößen werden dann Abminderungsfaktoren berechnet. Als Basiswerte dienen die Werte der Mittelwertkurve aus den Zugver- 
suchen unter Raumtemperatur. Die Ergebnisse der Abminderungsfaktoren für die Kenngrößen der Spannungs-Dehnungs-Kurven sind in Abhängigkeit von der Stahltemperatur in Abb. 4.28 sowie der Tabelle 5.2 angegeben. Es handelt sich dabei um Mittelwerte, die aus allen drei Versuchsreihen berechnet werden. Anhand der ermittelten Abminderungsfaktoren ist erkennbar, dass sich der Temperatureinfluss bei fast allen betrachteten Festigkeitskennwerten annähernd gleich auswirkt. Lediglich der Abminderungsfaktor für die technische Proportionalitätsgrenze ist meist etwas höher, als die der restlichen Kennwerte. Für einen Bereich zwischen 200 und $300{ }^{\circ} \mathrm{C}$ nehmen die Abminderungsfaktoren aller betrachteten Spannungskennwerte zu. Die Ursachen dieser Festigkeitszunahme wurden bereits bei der Auswertung der VR 1 erläutert (siehe Kapitel 4.5.2). Für Stahltemperaturen von mehr als $350^{\circ} \mathrm{C}$ kommt es mit Ausnahme der Proportionalitätsgrenze zu einer merklichen Reduzierung der Abminderungsfaktoren. Die Abminderung der Proportionalitätsgrenze setzt für den untersuchten kaltverformten Blankstahl erst bei einer Temperatur von etwa $450{ }^{\circ} \mathrm{C}$ ein. Bei einer Stahltemperatur von $700^{\circ} \mathrm{C}$ betragen alle untersuchten Spannungskennwerte nur noch etwa $10 \%$ des Wertes bei Raumtemperatur. Eine mögliche Erklärung dafür wird bereits bei der Auswertung der VR 1 angeführt (siehe Kapitel 4.5.2).

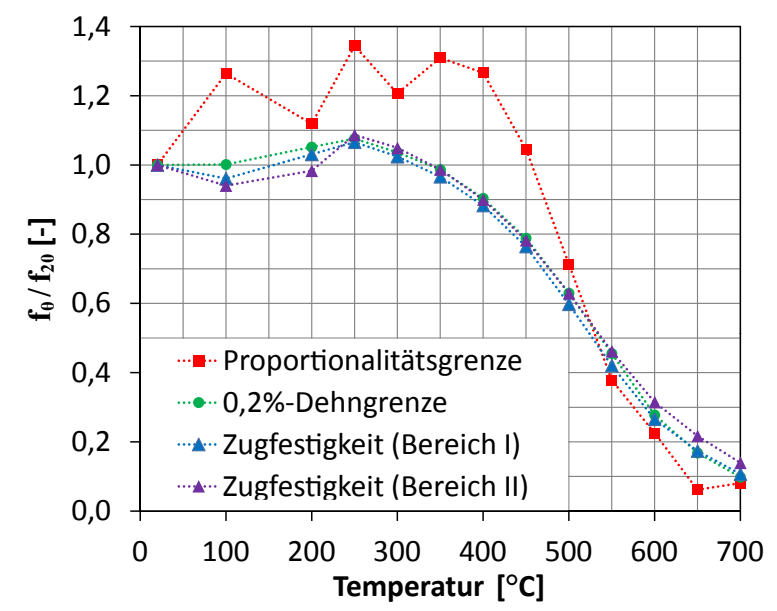

Abb. 4.28: Vergleich der Abminderungsfaktoren für die Festigkeitskennwerte des Blankstahls

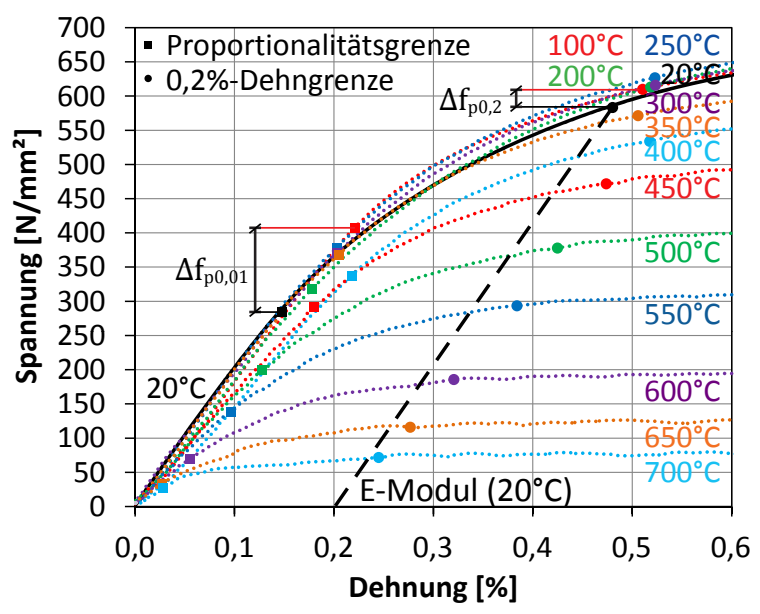

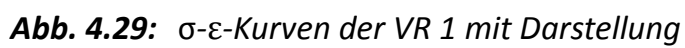
der Proportionalitätsgrenze und 0,2\%-Dehngrenze

Da die berechneten Abminderungsfaktoren der Proportionalitätsgrenze wesentlich höhere Werte aufweisen als die der anderen Spannungskennwerte, wird das temperaturabhängige Verhalten der Spannungs-Dehnungs-Kurven noch einmal speziell für den Anfangsbereich der Kleinzugversuche analysiert (siehe Abb. 4.29). Es zeigt sich, dass die technische Proportionalitätsgrenze $\left(\mathrm{f}_{\mathrm{p} 0,01}\right)$ bei Temperaturen zwischen 100 und $400{ }^{\circ} \mathrm{C}$ gegenüber dem Wert bei $20^{\circ} \mathrm{C}$ merklich ansteigt. Das Ende des elastischen Bereiches, welches durch die Proportionalitätsgrenze gekennzeichnet ist, nimmt folglich zu. Der untersuchte kaltverformte Blankstahl besitzt somit bei leicht erhöhter Temperatur einen größeren linear-elastischen Bereich 
als bei Raumtemperatur. Auch für die 0,2\%-Dehngrenze ist eine Spannungszunahme bei leicht erhöhten Stahltemperaturen zu beobachten. Allerdings fällt der Spannungszuwachs wesentlich geringer aus als bei der Proportionalitätsgrenze. Zudem setzt ein signifikanter Festigkeitsverlust der 0,2\%-Dehngrenze bereits ab einer Temperatur von $350{ }^{\circ} \mathrm{C}$ ein.

Vergleich der Abminderungsfaktoren zwischen dem untersuchten Blankstahl und den Werten aus der Literatur sowie den Normen

Mit Hilfe eines Vergleiches wird untersucht, inwieweit sich das temperaturabhängige Verhalten des untersuchten kaltverformten Blankstahls von dem eines warmgewalzten Baustahls nach EC3 Teil 1-2 [13] unterscheidet und welche spezifischen Besonderheiten auftreten. Die in [13] für kaltgeformte dünnwandige Stahlbauteile der Querschnittsklasse 4 enthaltenen Werte des Abminderungsfaktors der 0,2\%-Dehngrenze werden ebenso, wie Versuchsergebnisse aus der Literatur mit in den Vergleich einbezogen. Als Vergleichskriterium werden die Abminderungsfaktoren der Proportionalitätsgrenze, der

Tabelle 4.8: Berechnung der Abminderungsfaktoren

\begin{tabular}{|l|l|}
\hline \multicolumn{1}{|c|}{ Abminderungsfaktor } & Berechnung \\
\hline $\mathrm{k}_{\mathrm{p} 0,01}$ (BAM) & $\mathrm{f}_{\mathrm{p} 0,01, \theta} / \mathrm{f}_{\mathrm{p} 0,01}$ \\
\hline $\mathrm{k}_{\mathrm{p}} \quad$ (Outinen \& Mäkeläinen) & $\mathrm{f}_{\mathrm{p}, \theta} / \mathrm{f}_{\mathrm{p}}$ \\
\hline $\mathrm{k}_{\mathrm{p}} \quad$ (EC3 Teil 1-2 - warmgewalzt) & $\mathrm{f}_{\mathrm{p}, \theta} / \mathrm{f}_{\mathrm{y}}$ \\
\hline $\mathrm{k}_{\mathrm{p} 0,2}$ (BAM) & $\mathrm{f}_{\mathrm{p} 0,2, \theta} / \mathrm{f}_{\mathrm{p} 0,2}$ \\
\hline $\mathrm{k}_{\mathrm{p} 0,2}$ (Outinen \& Mäkeläinen) & $\mathrm{f}_{\mathrm{p} 0,2, \theta} / \mathrm{f}_{\mathrm{p} 0,2}$ \\
\hline $\mathrm{k}_{\mathrm{p} 0,2}$ (Ranawaka \& Mahendran) & $\mathrm{f}_{\mathrm{p} 0,2, \theta} / \mathrm{f}_{\mathrm{p} 0,2}$ \\
\hline $\mathrm{k}_{\mathrm{p} 0,2}$ (Wei \& Jihong) & $\mathrm{f}_{\mathrm{p} 0,2, \theta} / \mathrm{f}_{\mathrm{p} 0,2}$ \\
\hline $\mathrm{k}_{\mathrm{y}}$ (EC3 Teil 1-2 - warmgewalzt) & $\mathrm{f}_{\mathrm{y}, \theta} / \mathrm{f}_{\mathrm{y}}$ \\
\hline $\mathrm{k}_{\mathrm{p} 0,2}$ (EC3 Teil 1-2 - kaltgeformt) & $\mathrm{f}_{\mathrm{p} 0,2, \theta} / \mathrm{f}_{\mathrm{yb}}$ \\
\hline $\mathrm{k}_{\mathrm{u}}$ (BAM) & $\mathrm{f}_{\mathrm{u}, \theta} / \mathrm{f}_{\mathrm{u}}$ \\
\hline $\mathrm{k}_{\mathrm{u}}$ (Wei \& Jihong) & $\mathrm{f}_{\mathrm{u}, \theta} / \mathrm{f}_{\mathrm{u}}$ \\
\hline $\mathrm{k}_{\mathrm{u}}$ (EC3 Teil 1-2 - warmgewalzt) & $\mathrm{f}_{\mathrm{u}, \theta} / \mathrm{f}_{\mathrm{u}}$ \\
\hline
\end{tabular}
0,2\%-Dehngrenze sowie der Zugfestig-

keit betrachtet. Die Werte aus der Norm [13] entsprechen einem warmgewalzten Baustahl der Festigkeitsklasse S355. Aus der Literatur werden für den Vergleich unter anderem die von Outinen \& Mäkeläinen [45] durchgeführten Zugversuche an kaltverformtem Stahl der Festigkeitsklasse S355 J2H herangezogen. Diese Versuche wurden an verschiedenen Hohlprofilen mit quadratischem Querschnitt durchgeführt. Für die Bestimmung der Abminderungsfaktoren wird als Basiswert jeweils die bei Raumtemperatur ermittelte Proportionalitätsgrenze bzw. die 0,2\%-Dehngrenze verwendet. Für den untersuchten kaltverformten Blankstahl wird aufgrund der fehlenden Streckgrenze eine analoge Vorgehensweise gewählt. In Tabelle 4.8 ist die Grundlage für die Berechnung der Abminderungsfaktoren angegeben. Der Vergleich der Abminderungsfaktoren für die Proportionalitätsgrenze $\left(f_{p, \theta} / f_{p, 20}\right)$ ist in Abb. 4.30 dargestellt. Bei den Werten für den untersuchten kaltverformten Blankstahl und den Untersuchungen von Outinen \& Mäkeläinen [45] handelt es sich um Mittelwerte. Anhand des Diagramms zeigt sich, dass die Abminderungsfaktoren für den kaltverformten Blankstahl wesentlich größere Werte annehmen als für warmgewalzten Stahl nach EC3 Teil 1-2 [13]. Dies bedeutet, dass die Proportionalitätsgrenze des Blankstahls infolge einer erhöhten Temperatur weniger stark reduziert werden muss als bei warmgewalzten 
Stählen. Ursache dafür ist höchstwahrscheinlich die aus dem Herstellungsprozess resultierende Kaltverfestigung des Blankstahls. Die Ergebnisse des von Outinen \& Mäkeläinen getesteten kaltverformten Stahls sind ebenfalls wesentlich niedriger als die des untersuchten kaltverformten Blankstahls. Dies könnte auf das unterschiedliche Ausgangsmaterial und die Probengeometrie der Zugproben zurückzuführen sein. In den Untersuchungen von Outinen \& Mäkeläinen werden Hohlprofile mit einer Wandstärke von $4 \mathrm{~mm}$ verwendet, aus denen Flachproben gefertigt wurden. Für den kaltverformten Blankstahl wird ein Kreisvollprofil mit einem Durchmesser von $20 \mathrm{~mm}$ verwendet, aus dem anschließend Rundproben mit einem Durchmesser von $10 \mathrm{~mm}$ hergestellt werden. Erst ab Temperaturen von mehr als $400{ }^{\circ} \mathrm{C}$ kommt es zu einer langsamen Annäherung der Abminderungsfaktoren für die Proportionalitätsgrenze. Für Temperaturen von etwa $600{ }^{\circ} \mathrm{C}$ liegt nur noch eine geringe Abweichung zwischen den ausgewerteten warmgewalzten und kaltverformten Stählen vor.

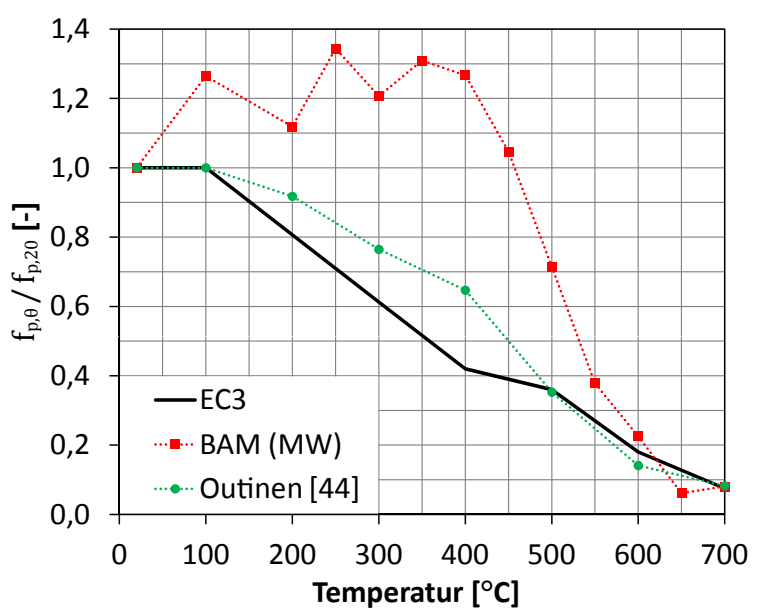

Abb. 4.30: Vergleich des Abminderungsfaktors für die Proportionalitätsgrenze

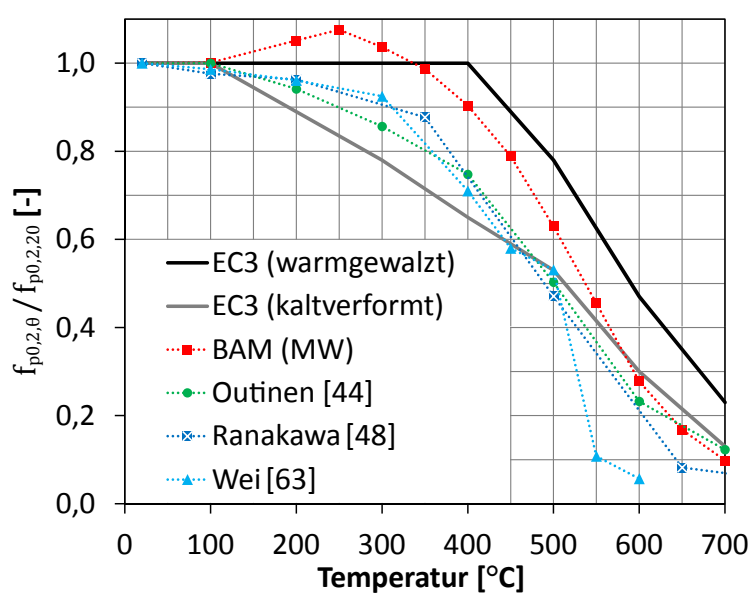

Abb. 4.31: Vergleich des Abminderungsfaktors für die 0,2\%-Dehngrenze

Wie bereits beim Vergleich des statischen Elastizitätsmoduls (siehe Kapitel 4.4.5) werden für den Vergleich der Abminderungsfaktoren der 0,2\%-Dehngrenze und der Zugfestigkeit auch die Ergebnisse anderer stationärer Kleinzugversuche ([45], [48], [63]) herangezogen. Es handelt sich dabei ebenfalls um kaltverformte Stähle. Der Vergleich der Abminderungsfaktoren für die 0,2\%-Dehngrenze $\left(f_{p 0,2, \theta} / f_{p 0,2,20}\right)$ bzw. Fließgrenze ist in Abb. 4.31 dargestellt. Auch hier handelt es sich zum Teil um gemittelte Werte. Anhand des Diagramms zeigt sich, dass die Abminderungsfaktoren für die 0,2\%-Dehngrenze für warmgewalzten Stahl nach EC3 Teil 1-2 [13] mit am höchsten ausfallen. Bis zu einer Temperatur von etwa $350^{\circ} \mathrm{C}$ stimmen die Werte des Abminderungsfaktors für den untersuchten kaltverformten Blankstahl gut mit denen des EC3 Teil 1-2 überein. Für höhere Stahltemperaturen liegen die Werte des EC3 Teil 1-2 um ca. 0,1 bis 0,2 höher als die ermittelten Werte des untersuchten Blankstahls. Dies bedeutet, dass der in den Brandversuchen verwendete kaltverformte Blankstahl wesentlich früher seine Festigkeit verliert. Im Vergleich zu den im EC3 Teil 1-2 enthaltenen 
Angaben für kaltgeformte dünnwandige Stahlbauteile der Querschnittsklasse 4 besitzt der kaltverformte Blankstahl bis etwa $550{ }^{\circ} \mathrm{C}$ eine höhere Festigkeit. Die aus anderen Veröffentlichungen herangezogenen Werte für den Abminderungsfaktor der 0,2\%-Dehngrenze zeigen eine ähnliche Tendenz wie der untersuchte kaltverformte Blankstahl. Der Festigkeitsverlust tritt jedoch bei den in der Literatur angegeben Stählen bereits bei Temperaturen von mehr als $100{ }^{\circ} \mathrm{C}$ auf. Folglich weisen diese Stähle einen noch schnelleren Rückgang der Festigkeit auf, als dies bereits bei dem untersuchten kaltverformen Blankstahl der Fall ist. Der Unterschied zwischen den von Outinen \& Mäkeläinen [44], Ranawaka \& Mahendran [48] und Wei \& Jihong [63] ermittelten Abminderungsfaktoren ist trotz der verwendeten unterschiedlichen Stahlbauteile (Festigkeitsklasse und Probengeometrie) eher gering. Die in allen drei Veröffentlichungen als Ausgangsmaterial verwendeten relativ dünnen Bleche weisen zwar ebenfalls eine Kaltverfestigung auf, wurden jedoch nicht durch Ziehen, sondern durch Walzen hergestellt. Ferner wurden in den Kleinzugversuchen der drei Veröffentlichungen Flachproben und keine Rundproben verwendet. Mit Ausnahme der Kleinzugversuche von Wei kommt es erst ab einer Temperatur von etwa $600{ }^{\circ} \mathrm{C}$ zu einer guten Übereinstimmung mit den für den kaltverformten Blankstahl gemessenen Abminderungsfaktoren für die 0,2\%-Dehngrenze.

Bei der Gegenüberstellung der Zugfestigkeit $\left(\mathrm{f}_{\mathrm{u}, \theta} / \mathrm{f}_{\mathrm{u}, 20}\right)$ der für kaltverformten Blankstahl ermittelten Werte mit denen aus der Norm [13] sowie aus der Veröffentlichung von Wei [63] zeigt sich, dass bis etwa $300{ }^{\circ} \mathrm{C}$ eine gute Übereinstimmung vorliegt (siehe Abb. 4.32). Im Bereich zwischen 300 und $500{ }^{\circ} \mathrm{C}$ fallen die Abminderungsfaktoren der Zugfestigkeit für den kaltverformten Blankstahl um etwa 0,1 höher aus, als die in der Norm angegebenen und von Wei ermittelten Werte. Für eine Stahltemperatur von $500{ }^{\circ} \mathrm{C}$ ergibt sich erneut eine gute

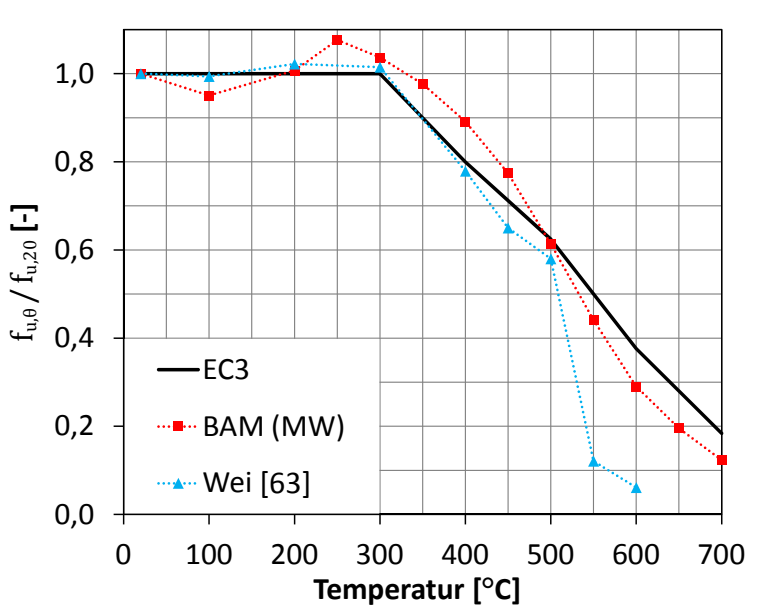

Abb. 4.32: Vergleich des Abminderungsfaktors für die Zugfestigkeit Übereinstimmung zwischen allen drei untersuchten Stählen. Bei Temperaturen von mehr als $500{ }^{\circ} \mathrm{C}$ kommt es jedoch in den Versuchen von Wei zu einer wesentlich stärkeren Reduzierung des Abminderungsfaktors als in den Kleinzugversuchen des kaltverformten Blankstahls. Ursache dafür ist mit hoher Wahrscheinlichkeit das unterschiedliche Ausgangsmaterial, die verwendete Probengeometrie sowie die unterschiedlichen Festigkeitsklassen. Die in der Norm angegebenen Werte liegen für Temperaturen von mehr als $500{ }^{\circ} \mathrm{C}$ etwa um 0,1 über den Werten des untersuchten kaltverformten Blankstahls. Die in der Norm angegebenen Werte beziehen sich auf warmgewalzten Baustahl und nicht auf kaltverformten Blankstahl. 
Fazit

Mittels stationärer Kleinzugversuche werden die Festigkeitseigenschaften von kaltgezogenem Blankstahl der Festigkeitsklasse S355 unter Raumtemperatur und erhöhter Temperatur (bis $700^{\circ} \mathrm{C}$ ) untersucht. Neben dem temperaturabhängigen Verhalten des Stahls wird auch der Einfluss der Versuchsgeschwindigkeit auf die Festigkeitsentwicklung des Stahls analysiert. Die allgemein bekannte Annahme, dass eine Erhöhung der Prüfgeschwindigkeit zu einer Zunahme der Spannungen führt, konnte anhand der durchgeführten Untersuchungen bestätigt werden. Die Festigkeitskenngrößen des untersuchten gezogenen kaltverformten Blankstahls weichen teilweise erheblich von den Werten für warmgewalzten Stahl nach Norm [13] ab. Ursache hierfür ist die aus dem Herstellungsprozess resultierende Kaltverfestigung des Blankstahls. Auch für kaltverformten Stahl weichen die für den Blankstahl ermittelten Werte der einzelnen Abminderungsfaktoren gegenüber den Angaben aus der Literatur ab. Mögliche Ursachen sind das unterschiedliche Ausgangsmaterial, die verwendete Geometrie der Zugproben sowie die abweichenden Festigkeitsklassen. Inwieweit sich diese spezifischen Besonderheiten im Festigkeitsverhalten des kaltverformten Blankstahls auf das Verhalten des reaktiven Brandschutzsystems auswirken, wurde bereits mit Hilfe der Brandversuche im Realmaßstab (siehe Kapitel 3) untersucht.

\subsection{Ermittlung des Wärmeausdehnungskoeffizienten}

Der Wärmeausdehnungskoeffizient $(\alpha)$ wird für die Validierung der numerischen Vergleichsberechnung benötigt. Er beschreibt das Ausdehnungsverhalten in Abhängigkeit von der Temperaturänderung. Mit Hilfe dieses Koeffizienten und der Temperaturdifferenz kann die thermische Dehnung des Stahls berechnet werden. Die Bestimmung des Wärmeausdehnungskoeffizienten wird mit Hilfe eines Präzisionsdilatometers durchgeführt. Zusätzlich wird der Wärmeausdehnungskoeffizient anhand der in den Kleinzugversuchen gemessenen Dehnungen während der Erwärmungsphase bestimmt.

\subsubsection{Untersuchung mittels Schubstangen-Dilatometer}

Für die Untersuchungen mittels Präzisions-Dilatometers unter Normalatmosphäre (siehe Abb. 4.33) werden aus dem kaltverformten Blankstahl des KVP D20 drei Probekörper hergestellt (siehe Tabelle 4.2). Der Wärmeausdehnungskoeffizient des Blankstahls wird für unterschiedliche Aufheizraten (D1 $=4 \mathrm{~K} / \mathrm{min} ; \mathrm{D} 2=10 \mathrm{~K} / \mathrm{min} ; \mathrm{D} 3=20 \mathrm{~K} / \mathrm{min}$ ) untersucht. Zusätzlich zum Dehnungsverhalten bei der Erwärmung des Stahls wird auch das Abkühlungsverhalten mit aufgezeichnet. Anhand des gemessenen Dehnungsverhaltens erfolgt die Berechnung des Wärmeausdehnungskoeffizienten. Nach erfolgter Kalibrierung des Dilatometers wird für die drei Blankstahlproben die Längenänderung in Abhängigkeit von der Probentemperatur bestimmt. Um eine präzise Übertragung der Längenänderung der Proben auf das Messsystem zu gewährleisten, wird der Messfühler mit einer konstanten Anpresskraft von 
0,3 N gegen die Probe gedrückt. Für den Blankstahl wird ein Temperaturbereich von Raumtemperatur bis etwa $1000{ }^{\circ} \mathrm{C}$ untersucht. Während der Untersuchung wird die Längenänderung der Probe (dL) als Funktion der Temperatur $(\theta)$ aufgezeichnet. Anhand der Messergebnisse wird die relative Längenänderung $(\mathrm{dL} /$ $\mathrm{d} \theta$ ) berechnet. Dabei ist $\mathrm{L}_{0}$ die Anfangslänge der Probe unter Raumtemperatur. Anhand der relativen Längenänderung wird der Wärmeausdehnungskoeffizient nach Gleichung (4.7) bestimmt. Die Ergebnisse der Berechnung sind in Abb. 4.34 dargestellt.

$d L=L_{0}-L(\theta)$

$L_{0} \quad$ Bauteilanfangslänge

$L(\theta)$ Bauteillänge infolge Temperatur

$\alpha(\theta)=\frac{1}{L_{0}} \cdot \frac{d L(\theta)}{d \theta}$

$\alpha \quad$ Wärmeausdehnungskoeffizient

$\theta \quad$ Bauteiltemperatur

\subsubsection{Untersuchung mittels Kleinzugversuchen}

Zu Beginn der in Kapitel 4.5.2 vorgestellten Kleinzugversuche werden die Proben zunächst auf die entsprechende Stahltemperatur erwärmt. Danach wird der stationäre Zugversuch durchgeführt. Während dieser Erwärmungsphase werden die Dehnungen der Proben mittels eines Hochtemperaturextensometers aufgezeichnet, um anschließend anhand des gemessenen Dehnungsunterschiedes den Wärmeausdehnungskoeffizienten zu berechnen. Die in den Kleinzugversuchen gemessenen Aufheizgeschwindigkeiten liegen je nach Stahltemperatur zwischen etwa 5 bis $35 \mathrm{~K} / \mathrm{min}$.

\subsubsection{Vergleich der Ergebnisse}

In Abb. 4.34 sind die Ergebnisse zum Wärmeausdehnungskoeffizienten aus den Untersuchungen mittels Präzisions-Dilatometer und den Kleinzugversuchen dargestellt. Zum Vergleich sind in dem Diagramm die Werte für warmgewalzten Baustahl nach EC3 Teil 1-2 [13] enthalten. Anhand der für den kaltverformten Blankstahl ermittelten Werte zeigt sich, dass der Wärmeausdehnungskoeffizient mit steigender Temperatur zunimmt. Für die mit dem Dilatometer gemessenen Werte lässt sich feststellen, dass sich bis zu einer Temperatur von 
etwa $300^{\circ} \mathrm{C}$ der Einfluss der Erwärmungsgeschwindigkeit kaum auf die Höhe des Wärmeausdehnungskoeffizienten auswirkt. Erst ab einer Stahltemperatur von ca. $400{ }^{\circ} \mathrm{C}$ ist ein Einfluss der Erwärmungsgeschwindigkeit erkennbar. Für eine Erwärmungsgeschwindigkeit von $10 \mathrm{~K} / \mathrm{min}$ werden die größten Werte für den Wärmeausdehnungskoeffizienten ermittelt. Ein konkreter Zusammenhang zwischen Erwärmungsgeschwindigkeit und der Höhe des Wärmeausdehnungskoeffizienten konnte jedoch nicht hergestellt werden.

Die Werte des Wärmeausdehnungskoeffizienten aus den Kleinzugversuchen fallen in der Regel geringer aus, als die aus den Untersuchungen mittels Dilatometer. Dies ist wahrscheinlich darauf zurückzuführen, dass bei der Erwärmung der Zugproben auch höhere Aufheizraten als $20 \mathrm{~K} / \mathrm{min}$ aufgetreten sind. Darüber hinaus sind die thermischen Dehnungen aus der Temperaturbeanspruchung relativ klein, so dass die Messgenauigkeit des bei den Kleinzugversuchen verwendeten Hochtemperaturextensometers möglicherweise ebenfalls Anteil an der Abweichung hat. Die Dilatometermessung mit einer Erwärmungsgeschwindigkeit von $20 \mathrm{~K} / \mathrm{min}$ zeigt die beste

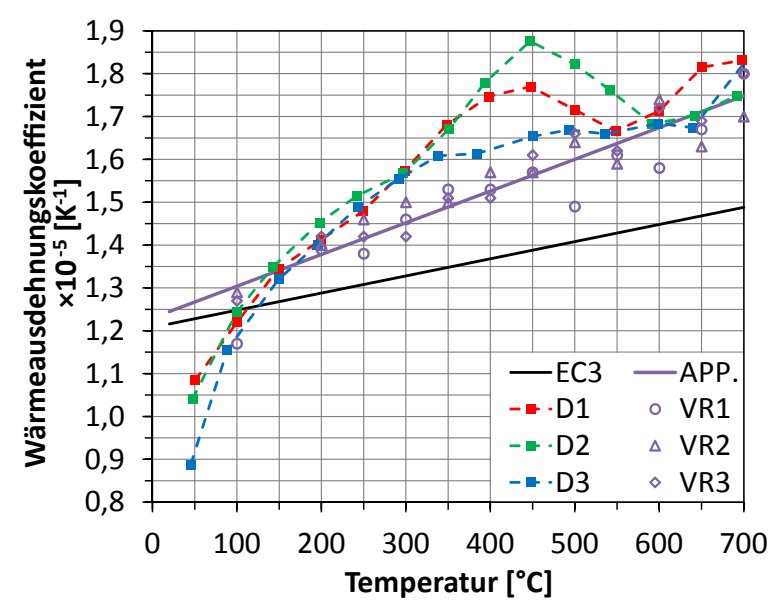

Abb. 4.34: Vergleich des Wärmeausdehnungskoeffizienten aus der Messung mittels Schubstangen-Dilatometer (D) mit den Ergebnissen aus den Kleinzugversuchen (VR) und dem EC3 Teil 1-2 [13] Übereinstimmung mit den Werten aus den Kleinzugversuchen. Die Ergebnisse aus den Kleinzugversuchen können als hinreichend genau angesehen werden. Eine aufwendige Untersuchung mittels Präzisionsdilatometer ist somit nicht zwingend erforderlich.

Der in der Norm [13] angegebene Verlauf des Wärmeausdehnungskoeffizienten, welcher sich jedoch auf warmgewalzten Baustahl bezieht, liegt wesentlich unterhalb der für kaltverformten Blankstahl experimentell ermittelten Werte. Dies ist im Herstellungsverfahren des Blankstahls, welches zu einer Kaltverfestigung des Stahls und zur Veränderung des Metallgitteraufbaus führt, begründet. Für die in Kapitel 5 durchgeführte numerische Simulation wird eine lineare Approximation (APP.) der Mittelwertkurve des in den Kleinzugversuchen bestimmten Wärmeausdehnungskoeffizienten verwendet. Die dazu erforderliche Interpolationsgerade ist in Gleichung (4.8) angegeben.

$\alpha(\theta)=7,4 \cdot 10^{-9} \cdot \theta+1,23 \cdot 10^{-5}$ 



\section{Numerische Analyse von Zuggliedern mit reaktiver Brandschutzbeschichtung}

Die numerische Untersuchung dient zur Entwicklung eines Bemessungsmodells für Zugglieder mit reaktiver Brandschutzbeschichtung. Durch die realitätsnahe Abbildung des Tragund Verformungsverhalten soll eine Bemessungsgrundlage für beschichtete Stahlzugglieder geschaffen werden. Mit Hilfe von Annahmen zur Temperaturentwicklung des Stahls kann mit dem entwickelten numerischen Modell die Feuerwiderstandsdauer von Stahlzuggliedern mit reaktiver Brandschutzbeschichtung berechnet werden. Das Modell bietet die Möglichkeit, Parameterstudien an Stahlzuggliedern mit reaktiver Brandschutzbeschichtung durchzuführen. Die Erstellung des Bemessungsmodells erfolgt mit Hilfe des Finite-Elemente-Programms ABAQUS ${ }^{\circledR}[20]$. Als Eingangsgrößen für die numerische Simulation werden die Geometrie und die mechanische Zugbeanspruchung des Zuggliedes sowie die temperaturabhängigen Materialkennwerte des verwendeten Stahls sowie die im Brandversuch gemessenen Stahltemperaturen des beschichteten Zuggliedes benötigt. Diese Kennwerte wurden im Rahmen von Materialuntersuchungen (siehe Kapitel 4) und Brandversuchen im Realmaßstab (siehe Kapitel 3) bestimmt.

Grundlage für das numerische Bemessungsmodell bildet ein an der BAM durchgeführtes Forschungsvorhaben [35], in dem bereits erste Modelle entwickelt wurden. Aufbauend auf diesen bereits vorhandenen numerischen Modellen werden verschiedene Modifikationen vorgenommen, um eine Verbesserung der Übereinstimmung von Simulation und Experiment zu erreichen. Mit Hilfe der durchgeführten Brandversuche an Zuggliedern mit reaktiver Brandschutzbeschichtung wird eine umfangreiche Verifikation des neuen numerischen Modells vorgenommen. Des Weiteren werden numerische Vergleichsrechnungen zwischen der Oberflächen- und der Kerntemperatur verschiedener Stahlzugglieder mit Kreisvollquerschnitt angestellt.

Die theoretischen Grundlagen der Temperaturfeldberechnung, einschließlich der unterschiedlichen Arten der Wärmeübertragung und der Berechnung eines transienten Temperaturfeldes sind in den Büchern zur Wärme- und Stoffübertragung von Baehr \& Stephan [21] sowie von Holman [31] beschrieben.

\subsection{Grundlagen der Temperaturfeldberechnung}

Die Grundlage für die Berechnung von Wärmetransporten bildet der erste Hauptsatz der Thermodynamik (Energieerhaltungsatz). Dieser beschreibt den quantitativen Zusammenhang zwischen Wärme, Arbeit und innerer Energie. Grundsätzlich ist eine Übertragung von Wärme durch Wärmeleitung, Konvektion und Wärmestrahlung möglich. Voraussetzung für 
einen Wärmetransport ist ein Temperaturunterschied zwischen einem wärmedurchlässigen System und seiner Umgebung. Der Wärmestrom (Energietransport) verläuft dabei immer in Richtung der abnehmenden Temperatur. Der Wärmetransport in Festkörpern findet allein durch Wärmeleitung statt. In Gasen und Flüssigkeiten überlagert sich der Wärmeleitungsvorgang mit Konvektion und Wärmestrahlung. Ursache für den Energietransport durch Wärmeleitung sind Temperaturgradienten im Material. Im Allgemeinen ändert sich die Temperatur örtlich und zeitlich. Es handelt sich dabei um transiente Temperaturfelder (instationärer Wärmeleitungsvorgang). Ist die Triebkraft des Wärmetransports ein zeitlich und örtlich unverändertes Temperaturgefälle, so liegt ein stationäres Temperaturfeld (stationärer Wärmeleitungsvorgang) vor. Mit Hilfe des Fourier'schen Gesetzes lässt sich eine Proportionalität zwischen Temperaturgradient (Ursache) und Wärmestrom (Wirkung) aufstellen. Eine ausführliche Beschreibung der theoretischen Grundlagen zur Temperaturfeldberechnung findet sich in [21] und [31].

\section{Transiente Temperaturfeldberechnung}

Diese Art der Temperaturfeldberechnung findet dann Anwendung, wenn Temperaturen und andere thermische Kennwerte in einem Bauteil durch zeitabhängige thermische Lasten bestimmt werden. Transiente Effekte, wie zum Beispiel die Wärmespeicherung des Bauteils, werden dabei berücksichtigt. Für die Berechnung eines transienten Temperaturfeldes bildet der erste Hauptsatz der Thermodynamik (Energieerhaltungssatz) und das Fourier'sche Gesetz die Grundlage. Die Lösung dieser Differentialgleichung kann analytisch oder numerisch erfolgen. Allerdings ist die analytische Lösung nur für einfache Geometrien möglich und an verschiedene Randbedingungen geknüpft (z.B. Temperaturunabhängigkeit der Werkstoffeigenschaften). Daher wird für komplexere Geometrien und bei temperaturabhängiger Änderung der Materialeigenschaften häufig die Finite-Elemente-Methode (FEM) verwendet. Die Berechnung erfolgt zunächst ähnlich einer stationären thermischen Analyse. Allerdings ist eine Zeitintegration erforderlich. Das Verhalten des Bauteils und die Auswirkungen der Lasten werden, aufbauend auf dem jeweils vorher ausgeführten Schritt, für die Bedingungen des neuen Zeitschrittes berechnet. Die Temperaturen der thermischen, transienten Temperaturfeldberechnung werden als Eingangsdaten in der Strukturanalyse verwendet.

\subsection{Untersuchung zum Erwärmungsverhalten von Stahlbauteilen}

\subsubsection{Erwärmung ungeschützter Stahlprofile}

Die Erwärmung eines ungeschützten Stahlbauteils unter Brandbeanspruchung nach Einheits-Temperaturzeitkurve ist im hohen Maße von der beflammten Bauteiloberfläche $\left(A_{m}\right)$ sowie vom zu erwärmenden Volumen des Bauteils (V) abhängig. Große und massive Stahlbauteile erwärmen sich langsamer und erreichen damit einen höheren Feuerwiderstand 
als filigrane. Im Brandingenieurwesen wird die Profilgeometrie des Bauteils mit Hilfe des

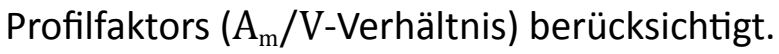

In DIN EN 1993-1-2 [13] ist eine vereinfachte Berechnung der Bauteilerwärmung ungeschützter Stahlbauteile angegeben. Die Bauteilerwärmung innerhalb eines Zeitschrittes wird in der Norm nach Gleichung (5.8) berechnet. Diese Formel wird bereits in der Veröffentlichung von Pettersson [46] verwendet. Die Methode ist im englischsprachigem Raum als "lumped capacitance method" bekannt. Dabei wird eine Energiebilanz des Netto-Wärmestroms in einem Bauteil der Änderung der inneren Energie des Körpers gegenübergestellt. Zur Verdeutlichung wird die Vorgehensweise und die Ableitung nachfolgend noch einmal näher erläutert. Die Änderung der Wärmemenge lässt sich für einen definierten Zeitschritt, welcher ausreichend klein gewählt werden muss ( $\leq 5$ Sekunden), nach Gleichung (5.1) berechnen. In Gleichung (5.2) ist die erforderliche Wärmemenge zur Temperaturerhöhung des Stahlprofils um $\Delta \mathrm{T}_{\mathrm{s}}$ angegeben.

$$
\Delta Q=\alpha \cdot A_{s} \cdot\left(T_{g}-T_{s}\right) \cdot \Delta t
$$

$\Delta Q=c_{s} \cdot \Delta T_{s} \cdot V_{s} \cdot \rho_{s}$
$\Delta Q$ Änderung der Wärmemenge

$\alpha$ gesamter Wärmeübergangskoeffizient

$A_{s} \quad$ Brandbeanspruchte Stahloberfläche $\left(A_{m}\right)$

$T_{g} \quad$ Gastemperatur

$T_{s} \quad$ Stahltemperatur auf der Oberfläche

$\Delta t \quad$ Zeitinterfall

$c_{s} \quad$ Spezifische Wärmekapazität des Stahls

$\Delta T_{S}$ Temperaturerhöhung des Stahls

$V_{s} \quad$ Volumen des Stahlprofils

$\rho_{s} \quad$ Dichte des Stahls

Durch Gleichsetzen der Gleichung (5.1) und (5.2) ergibt sich für $\Delta \mathrm{T}_{\mathrm{s}}$ :

$\Delta T_{s}=\frac{\alpha}{c_{s} \cdot \rho_{s}} \cdot \frac{A_{s}}{V_{s}} \cdot\left(T_{g}-T_{s}\right) \cdot \Delta t$

Der Wärmeübergangskoeffizient setzt sich aus einem konvektiven und einem radialen Anteil zusammen, siehe Gleichung (5.4). Gleiches gilt für den Wärmestrom, siehe Gleichung (5.5).

$$
\begin{array}{ll}
\alpha=\alpha_{c}+\alpha_{r} & \alpha_{c} \text { Wärmeübergangskoeffizient aus Konvektion } \\
& \alpha_{r} \text { Spezifische Wärmekapazität aus Radiation } \\
& \\
\dot{h}_{n e t, d}=\dot{h}_{n e t, c}+\dot{h}_{n e t, r} \quad \dot{h}_{n e t, d} \text { Gesamter Wärmestrom } \\
& \dot{h}_{n e t, r} \text { Konvektiver Anteil des Wärmestroms } \\
& \dot{h}_{n e t, c} \text { Radiativer Anteil des Wärmestroms }
\end{array}
$$


Die Berechnung des konvektiven und des radialen Anteils des Wärmestroms ist in Gleichung (5.6) und (5.7) angegeben.

$$
\begin{array}{lll}
\dot{h}_{n e t, c}=\alpha_{c} \cdot\left(T_{g}-T_{S}\right) & \alpha_{c} & \text { Wärmeübergangskoeffizient für Konvektion } \\
& T_{G} & \text { Gastemperatur } \\
& T_{S} & \text { Stahltemperatur auf der Oberfläche }
\end{array}
$$

$$
\begin{aligned}
& \dot{h}_{n e t, r}=\alpha_{r} \cdot\left(T_{g}-T_{s}\right)=\frac{\Phi \cdot \varepsilon_{m} \cdot \varepsilon_{f} \cdot \sigma}{\left(T_{g}-T_{S}\right)} \cdot\left[\left(T_{r}+273\right)^{4}-\left(T_{s}+273\right)^{4}\right] \cdot\left(T_{g}-T_{s}\right) \\
& \Phi \text { Konfigurationsfaktor } \\
& \varepsilon_{m} \text { Emissivität der Bauteiloberfläche } \\
& \varepsilon_{f} \text { Emissivität des Feuers } \\
& T_{r} \text { Strahlungstemperatur der Umgebung } \\
& \sigma \text { Stefan-Boltzmann-Konstante }
\end{aligned}
$$

Durch Einsetzen der Gleichung (5.6) und (5.7) sowie weiterem Vereinfachen ergibt sich Gleichung (5.3) zu:

$$
\begin{aligned}
& \Delta T_{s}=\frac{\alpha_{c}}{c_{s} \cdot \rho_{s}} \cdot \frac{A_{s}}{V_{s}} \cdot\left(T_{g}-T_{s}\right) \cdot \Delta t+\frac{\alpha_{r}}{c_{s} \cdot \rho_{s}} \cdot \frac{A_{s}}{V_{s}} \cdot\left(T_{g}-T_{s}\right) \cdot \Delta t \\
& \Delta T_{s}=\frac{\dot{h}_{n e t, c}}{c_{s} \cdot \rho_{s}} \cdot \frac{A_{s}}{V_{s}} \cdot \Delta t+\frac{\dot{h}_{n e t, r}}{c_{s} \cdot \rho_{s}} \cdot \frac{A_{s}}{V_{s}} \cdot \Delta t \\
& \Delta T_{s}=\frac{\dot{h}_{n e t, d}}{c_{s} \cdot \rho_{s}} \cdot \frac{A_{s}}{V_{s}} \cdot \Delta t \\
& T_{s, t+\Delta t}=T_{s, t}+\Delta T_{s, t}
\end{aligned}
$$

Mit Ausnahme des Korrekturfaktors für den Abschattungseffekt $\left(\mathrm{k}_{\mathrm{sh}}\right)$, welcher durch Multiplikation auf der rechten Seite der Gleichung ergänzt werden kann, ist Gleichung (5.8) identisch mit den Angaben in DIN EN 1993-1-2 [13]. Durch die Verwendung der in Gleichung (5.9) beschriebenen Beziehung lässt sich die zeitliche Erwärmung eines ungeschützten Stahlprofils relativ leicht mit Hilfe eines Tabellenkalkulationsprogramms berechnen. Für Träger und Stützen findet sich in der Veröffentlichung von Mensinger \& Stadler [40] eine graphische Umsetzung des in der Norm [13] angegebenen vereinfachten Nachweisverfahrens. Das vereinfachte Berechnungsverfahren der Norm geht von einer konstanten Stahltemperatur über den Bauteilquerschnitt aus. Diese Annahme begründet sich aus der hohen Wärmeleitfähigkeit des Stahls. Ferner wird eine konstante Dichte und Wärmekapazität unterstellt. Um das genannte Berechnungsverfahren anwenden zu können, wird vorausgesetzt, dass die Biot-Zahl, welche das Verhältnis des Wärmeleit- zum Wärmeübergangswiderstand beschreibt, einen entsprechend kleinen Wert annimmt (siehe Gleichung (5.10)). 
$B i=\frac{\alpha_{s} \cdot L_{c}}{\lambda_{s}}<0,1$

$\alpha_{s} \quad$ Wärmeübergangskoeffizient Stahl $\left(25 \frac{W \cdot m^{2}}{K}\right)$

$\lambda_{s} \quad$ Wärmeleitfähigkeit Stahl $\left(54 \frac{\mathrm{W}}{\mathrm{m} \cdot \mathrm{K}}\right)$

$L_{c} \quad$ Charakteristische Länge (Zylinder $=\frac{r}{2}$ )

Neben dem vereinfachten Berechnungsverfahren zur Bauteilerwärmung ist auch eine Berechnung mit Hilfe der Finite-Elemente-Methode (FEM) möglich. Mit Hilfe dieses erweiterten Berechnungsverfahrens kann die tatsächliche Temperaturverteilung im Bauteilquerschnitt zu jedem Berechnungsschritt in guter Näherung zur Realität bestimmt werden.

In den am Zugstabprüfofen durchgeführten Brandversuchen werden neben Zuggliedern mit reaktiver Brandschutzbeschichtung auch ungeschützte Profile getestet. Ein Vergleich der gemessenen und der nach DIN EN 1993-1-2 [13] analytisch berechneten Temperaturverläufe ist in Abb. 5.01 dargestellt. Die getesteten ungeschützten Stahlprofile erfüllen die Vorgaben zur Biot-Zahl. Da das KVP D30 und das KHP D60 einen identischen Profilfaktor $\left(A_{m} / V\right.$-Verhältnis) aufweisen, gibt es keinen Unterschied für den nach Norm berechneten Temperaturverlauf der bei-

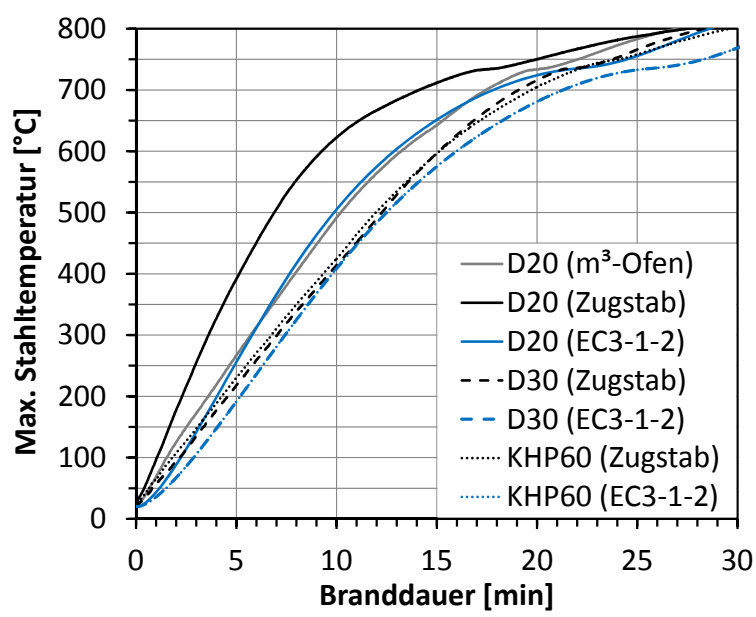

Abb. 5.01: Vergleich von Temperatur-Zeit-Verläufen für ungeschütze Stahlprofile den Profile. Wie erwartet, zeigen beide

Kreisprofile sowohl untereinander als auch gegenüber der analytischen Berechnung nur einen geringen Unterschied in den Stahltemperaturen. Dies bestätigt, dass das Erwärmungsverhalten bei ungeschützten Stahlprofilen lediglich vom Profilfaktor und von eventuell vorhandenen Abschattungseffekten beeinflusst wird.

Für das KVP D20 werden Brandversuche sowohl im Zugstabprüfofen als auch im Einkubikmeterofen durchgeführt ${ }^{2}$. Die gemessenen Temperaturverläufe variieren sehr deutlich voneinander. Dies ist zum einen auf die unterschiedliche Instrumentierung zur Messung der Brandgastemperaturen sowie die Größe des Brandraumes zurückzuführen. Der analytisch berechnete Temperaturverlauf weist eine gute Übereinstimmung mit dem Brandversuch im Einkubikmeterofen auf.

2 Im Einkubikmeterofen werden Stabthermoelemente verwendet. Diese erwärmen sich schneller als die im Zugstabprüfofen eingesetzten Plattenthermoelemente, wodurch die Brandgastemperaturen nach EinheitsTemperaturzeitkurve in der Regel mit einer geringeren Brennerleistung erreicht werden. 


\subsubsection{Erwärmung geschützter Stahlprofile}

Für geschützte Stahlbauteile gestaltet sich eine analytische oder numerische Berechnung der Erwärmung des Stahls insofern schwierig, als die thermische Schutzwirkung der Bekleidung oder des reaktiven Brandschutzsystems mit berücksichtigt werden muss. Die Norm [13] bietet zwar für geschützte Stahlbauteile die Möglichkeit einer Temperaturberechnung, diese ist jedoch aufgrund der vereinfachten Berechnungsansätze nicht für intumeszierende oder ablative Beschichtungen geeignet. Bei der Berechnungsmethode nach Norm wird beispielsweise ein linearer Temperaturgradient in der Brandschutzbekleidung unterstellt. Des Weiteren ist mit diesem Verfahren eine Berücksichtigung eines Temperaturfeldes in Stablängsrichtung nicht möglich. Hinzu kommt, dass die Anwendung der Berechnungsmethode nach Norm für Stahlkonstruktionen mit reaktiven Brandschutzsystemen dadurch erschwert wird, dass die thermische Schutzwirkung sowie entsprechende Materialkennwerte dieser Systeme im Allgemeinen unbekannt sind und sich zudem im Laufe des Brandversuches verändern.

Von Tabeling [60] wurde, basierend auf der Finite-Elemente-Methode (FEM) ein numerisches Modell entwickelt, welches den Aufschäumprozess sowie das Erwärmungsverhalten des mit einem reaktiven Brandschutzsystem geschützten Stahlbauteils prognostiziert. Allerdings wird in dem entwickelten Modell die Rissbildung sowie eventuell vorhandene Rissheilungseffekte der Brandschutzbeschichtung, welche beide sehr komplex sind, nicht berücksichtigt. Eine Erwärmungsberechnung von Stahlquerschnitten mit reaktiver Brandschutzbeschichtung ist nicht Bestandteil der vorliegenden Dissertation. Für die Validierung und Überprüfung der Ergebnisse aus der numerischen Simulation sind experimentelle Daten notwendig.

Im Allgemeinen werden die Stahltemperaturen von Stahlbauteilen mit reaktivem Brandschutzsystem mit Hilfe von Brandversuchen ermittelt. Die Messung der Stahltemperaturen erfolgt in der Regel auf der Bauteiloberfläche.

\subsubsection{Vergleich zwischen Oberflächen- und Kerntemperatur}

Aufgrund der besonderen Geometrie von Vollprofilen ist zu klären, ob im Kernbereich des Stabes geringere Stahltemperaturen auftreten als auf der Staboberfläche und inwieweit sich dadurch eventuelle Tragfähigkeitsreserven erschließen lassen. Dies wird im Folgenden durch numerische Simulationen untersucht.

Der Wärmetransport innerhalb eines Bauteils erfolgt über Wärmeleitung. Wärme fließt dabei immer in Richtung der geringeren Temperatur. Die Wärmeübertragung ist vom Temperaturgradienten und den Stoffeigenschaften, d.h. Dichte, Wärmekapazität und Wärmeleitfähigkeit, abhängig. Das Temperaturfeld eines homogenen Festkörpers mit isotropen 
Stoffeigenschaften kann mit Hilfe der Fourierschen Differentialgleichung beschrieben werden. Für ein dreidimensionales Temperaturfeld eines isotropen Körpers ergibt sich die Fouriersche Differentialgleichung. Für die numerische Untersuchung wird vereinfachend angenommen, dass in Stablängsrichtung des Zuggliedes kein Temperaturunterschied auftritt. Dadurch muss mit Hilfe des numerischen Modells lediglich eine zweidimensionale Wärmeleitung untersucht werden. In den durchgeführten Brandversuchen ändert sich die Stahltemperatur des Zuggliedes über die Branddauer. Ein instationäres Temperaturfeld liegt vor. Die im Modell aufgebrachte Temperatur des Stahls, welche der im Brandversuch gemessenen maximalen Stahltemperatur entspricht, wird als zeitabhängige Temperaturfunktion auf den äußeren Umfang des Kreisquerschnittes aufgebracht. Mit Beginn des Brandversuches bildet sich ein Temperaturgefälle zwischen der warmen Staboberfläche und dem kaltem Stabkern aus. Der Abbau dieses Temperaturunterschiedes erfolgt durch Wärmeleitung. Die Erwärmung des Stabkernes hängt vom Wärmestrom und dem Abstand zur Oberfläche, d.h. dem Radius, ab. Der Wärmestrom wird von der Höhe des Temperaturgradienten und der Wärmeleitfähigkeit des Stahls beeinflusst.

Tabelle 5.1: Vergleich der Oberflächen- und Kerntemperatur verschiedener Kreisvollprofile

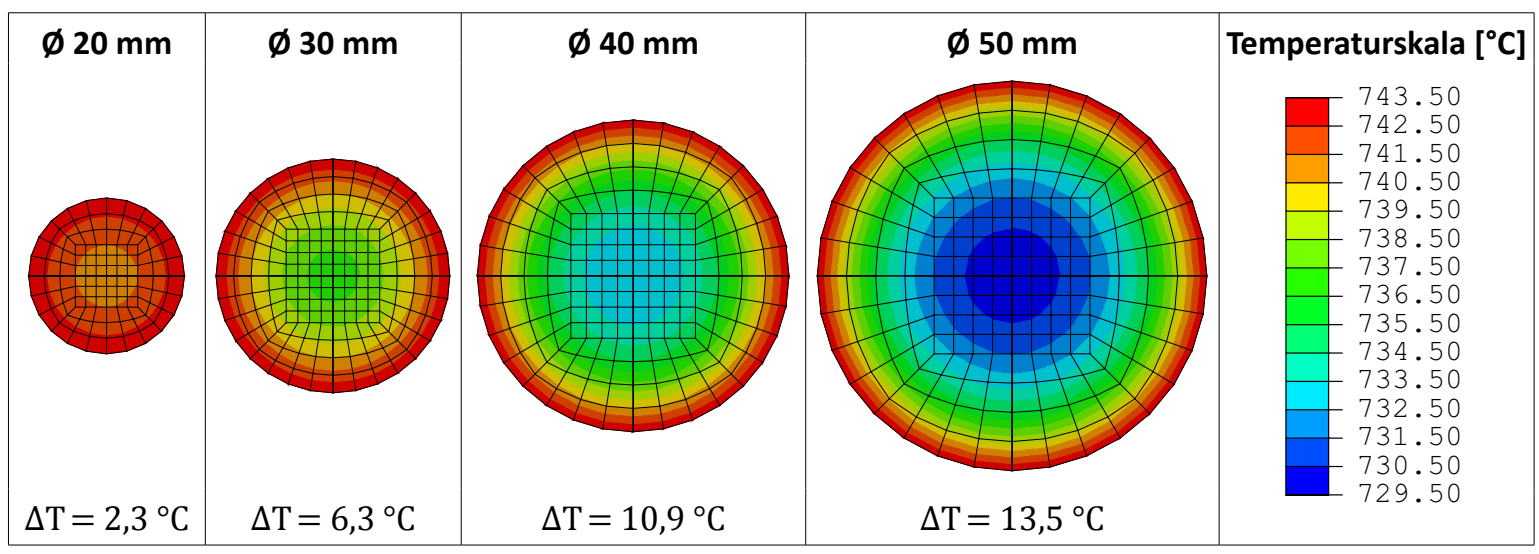

Hinweis: Auf die Oberfläche der Kreisvollprofile wird die im Brandversuch gemessene maximale Stahltemperatur des Versuchskörpers B22 ( $\varnothing 20 \mathrm{~mm}$; DFT = 3,5 mm; TE 5) aufgebracht. Die Abbildungen zeigen das Temperaturprofil nach einer Branddauer von 46 Minuten.

Insgesamt werden vier verschiedene Kreisvollprofile mit einem Durchmesser von 20 bis $50 \mathrm{~mm}$ sowie zwei Erwärmungsfunktionen untersucht. Mit Hilfe der beiden Erwärmungsfunktionen wird die unterschiedliche thermische Schutzwirkung des reaktiven Brandschutzsystems berücksichtigt. In Tabelle 5.1 sind einige der Ergebnisse aus der numerischen Simulation dargestellt. Wie erwartet, nimmt mit zunehmendem Stabdurchmesser der Temperaturunterschied zwischen der Staboberfläche und dem Stabkern zu. Zu beachten ist jedoch, dass dabei unterstellt wird, dass sich alle Profile mit der gleichen Geschwindigkeit erwärmen. Im Verhältnis zur Temperatur auf der Staboberfläche ist der Temperaturunterschied zum Stabkern äußerst gering. Die infolge einer geringeren Stahltemperatur im Stabkern vorhandenen Tragfähigkeitsreserven sind daher als vernachlässigbar anzusehen. 
In Abb. 5.02 ist der zeitliche Verlauf der Temperaturdifferenz zwischen Staboberfläche und -kern dargestellt. Der qualitative Verlauf der Temperaturdifferenz ist dabei zwischen den untersuchten Kreisvollprofilen und den beiden Erwärmungsfunktionen annähernd gleich. Zu Beginn des Brandversuches kommt es zunächst zu einem Anstieg der Temperturdifferenz. Dies ist darauf zurückzuführen, dass sich das Stahlbauteil aufgrund des noch nicht aufgeschäumten Zustandes des reaktiven Brandschutzsystems relativ schnell an der Oberfläche erwärmt. Durch das Aufschäumen des reaktiven Brandschutzsystems kommt es zu einer Reduzierung der Erwärmungsgeschwindigkeit des Stahls. Folglich nimmt auch der Temperaturunterschied zwischen Staboberfläche und Kern ab. Nachdem die Aufschäumung des Brandschutzsystems abgeschlossen ist, steigt die Erwärmungsgeschwindigkeit des Stahls auf der Staboberfläche wieder an. Folglich erhöht sich auch die Temperaturdifferenz. Kurz vor dem Probenbruch des Zuggliedes, d.h. Ende der Temperaturaufzeichnung, kommt es zu einem erneuten Anstieg des Temperaturunterschiedes. Die Ursache für diesen großen Anstieg ist die ab ca. $650^{\circ} \mathrm{C}$ einsetzende Erhöhung der spezifischen Wärmekapazität $\left(c_{p}\right)$ des Stahls. Infolgedessen verringert sich die Temperaturleitfähigkeit (a) nach Gleichung (5.11) und der Temperaturunterschied zwischen Staboberfläche und Kern erhöht sich.

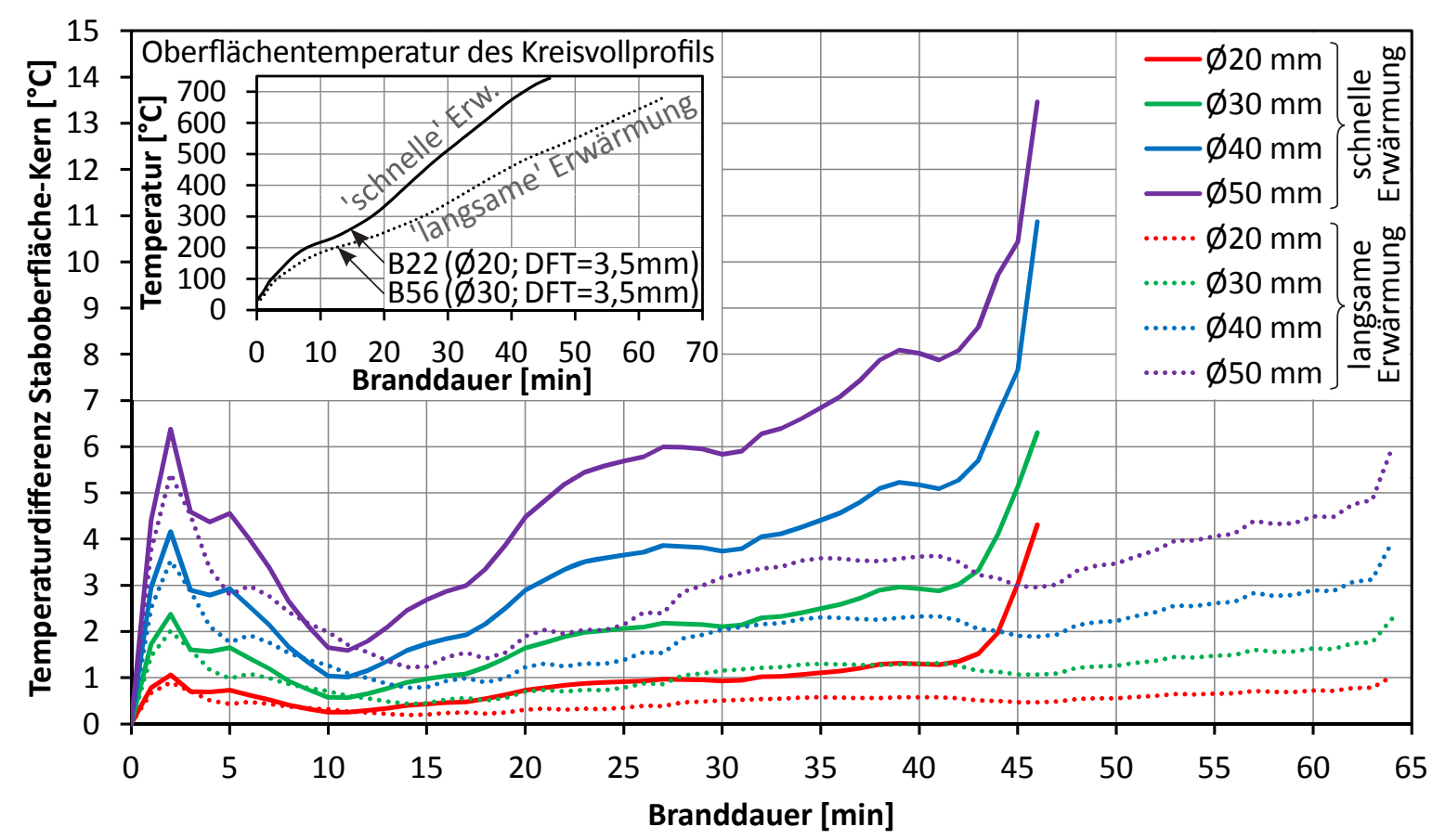

Abb. 5.02: Vergleich der zeitlichen Entwicklung der Temperaturdifferenz zwischen Staboberfläche und Kernbereich für verschiedene Kreisvollprofile bei 'schneller' und 'langsamer' Erwärmung

Die in Abb. 5.02 dargestellten Temperaturverläufe zeigen ebenfalls, dass die Vergrößerung des Stabdurchmessers oder eine Erhöhung der Erwärmungsgeschwindigkeit zu einem Anstieg der Temperaturdifferenz zwischen Staboberfläche und Kern führt. Es ist jedoch zu 
beachten, dass in der numerischen Simulation für alle vier Profile der gleiche Temperaturverlauf und somit die gleiche Erwärmungsgeschwindigkeit des Stahls verwendet wird. In der Realität wird die Erwärmungsgeschwindigkeit von der Massigkeit des Profils und der thermischen Schutzwirkung des reaktiven Brandschutzsystems beeinflusst. Kreisvollprofile mit einem großem Stabdurchmesser müssen daher nicht zwangsläufig eine höhere Temperaturdifferenz aufweisen als ein Profil mit einem kleinen Stabquerschnitt.

$a=\frac{\lambda}{\rho \cdot c_{p}}$

$\lambda \quad$ Wärmeleitfähigkeit

$\rho \quad$ Dichte

$c_{p} \quad$ spezifische Wärmekapazität

\subsection{Entwicklung eines Bemessungsmodells für Zugglieder mit reaktiver Brandschutzbeschichtung}

Mit Hilfe eines numerischen Modells soll das Trag- und Verformungsverhalten von Stahlzuggliedern mit reaktiver Brandschutzbeschichtung realitätsnah abgebildet werden. Die Erstellung des Bemessungsmodells erfolgt unter Verwendung des Finite-Elemente-Programms ABAQUS $^{\circledR}$ [20]. Grundlage für die numerische Simulation bilden die in Kapitel 4 bestimmten Materialkennwerte des verwendeten Blankstahls sowie die in den Brandversuchen im Realmaßstab gemessenen Stahltemperaturen der Zugglieder. Die Temperaturdaten sind im Forschungsbericht [35] sowie dem Anhang A enthalten. Das Verhalten des reaktiven Brandschutzsystems wird in dem numerischen Modell nicht direkt abgebildet, sondern mittels eines zeit- und ortsabhängigen Temperaturverlaufes des Stahlzuggliedes berücksichtigt. Im Forschungsbericht [35] wurde bereits ein numerisches Bemessungsmodell für Zugglieder vorgestellt. Im Rahmen der Dissertation erfolgt eine Weiterentwicklung und Verbesserung dieses bereits vorhandenen numerischen Modells. Der grundlegende Aufbau des Modells wird beibehalten.

\subsubsection{Aufbau des Bemessungsmodells aus dem Forschungsvorhaben [35]}

Für die Entwicklung eines numerischen Bemessungsmodells wird zunächst mit Hilfe des Programms MATLAB eine parametrisierte Eingabedatei für das Finite-Elemente-Programm ABAQUS $^{\circledR}$ erstellt. Um auch veränderliche Temperaturprofile über den Querschnitt zu berücksichtigen, wird ein dreidimensionales Modell gewählt. Ferner lässt sich dadurch der Einschnürungsbereich des Zuggliedes bis zum Zeitpunkt des Probenbruchs besser abbilden. Als wählbare Eingangsparameter werden in MATLAB der Stabdurchmesser, die Stablänge, die Anzahl der Knotenpunkte auf dem äußeren Kreisring sowie der Elementtyp gewählt. Das Ergebnis der MATLAB-Berechnung ist eine fertige Geometrie des Zuggliedes, welche direkt in ABAQUS ${ }^{\circledR}$ eingelesen werden kann (siehe Abb. 5.04). Die Erstellung einer Geometrie für das Zugglied ist auch direkt in ABAQUS ${ }^{\circledR}$ möglich. Allerdings ist eine Netzgenerierung 
mit rechteckigen Elementen für eine kreisförmige Geometrie relativ schwierig. Durch die Verwendung der Eingabedatei aus MATLAB ergibt sich ein regelmäßiges Netz mit relativ gleich großen Elementen. Das erstellte Elementnetz ist eine Kombination aus einem H-Netz und einem O-Netz. Mit Hilfe der parametrisierten Eingabedatei kann die Geometrie schnell und zielgerichtet verändert werden.

In dem entwickelten numerischen Modell erfolgt die Berechnung der Stahltemperaturen infolge Brandeinwirkung (Temperatur-Modell) und das mechanische Bauteilverhalten (Struktur-Modell) getrennt voneinander. Die Geometrie der beiden Modelle ist bis auf den verwendeten Elementtyp identisch. Die Ergebnisse aus dem Temperatur-Modell werden als thermische Beanspruchung im Struktur-Modell verwendet. Eine kurze Erläuterung zu den beiden Modellen findet sich nachfolgend. Ausführlichere Informationen sind im Forschungsbericht [35] enthalten.

\section{Temperatur-Modell}

Dieses numerische Modell dient dazu, aus den punktuell im Brandversuch gemessenen Stahltemperaturen ein zeitabhängiges Temperaturfeld zu erstellen, welches die Erwärmung des Zuggliedes abbildet. Das Temperaturfeld wird später als thermische Beanspruchung im Struktur-Modell verwendet. Da die Messung der Stahltemperaturen in den Brandversuchen direkt auf der Oberfläche der Zugglieder erfolgt, ist eine Modellierung des reaktiven Brandschutzsystems nicht erforderlich. In dem Temperatur-Modell werden für die thermischen Materialeigenschaften des Stahls die Werte nach DIN EN 1993-1-2 [13] verwendet. Die in den Brandversuchen punktuell gemessenen Stahltemperaturen werden so aufbereitet, dass sich in Abhängigkeit von der Branddauer ein kontinuierlicher Temperaturverlauf in Stablängsrichtung des Zuggliedes ergibt. Um Knicke im Temperaturverlauf zu vermeiden, erfolgt eine Glättung der Temperaturkurven mittels eines kubisch-hermiteschen Spline. Die Werte an den Temperaturmessstellen TE 1 bis 11 werden dabei nicht verändert. Beispielhaft ist in Abb. 5.03 der geglättete Temperaturverlauf für den Versuchskörper B9 dargestellt. Der zeitlich und örtlich veränderliche Temperaturverlauf aus den Brandversuchen wird als thermische Randbedingung auf die Knotenpunkte der Mantelfläche des Temperaturmodells des Zuggliedes aufgebracht. Die in Kapitel 5.2.3 beschriebenen Untersuchungen haben bereits gezeigt, dass sich bei den verwendeten relativ kleinen Stabdurchmessern nur ein unbedeutender Unterschied zwischen Oberflächen- und Kerntemperatur einstellt. Das Ergebnis der Berechnung des Temperatur-Modells ist ein Temperaturfeld, welches jedem Knotenpunkt des Modells einen über die Branddauer veränderlichen Temperaturverlauf zuweist. Dieses Temperaturfeld wird als thermische Beanspruchung im Struktur-Modell verwendet. 


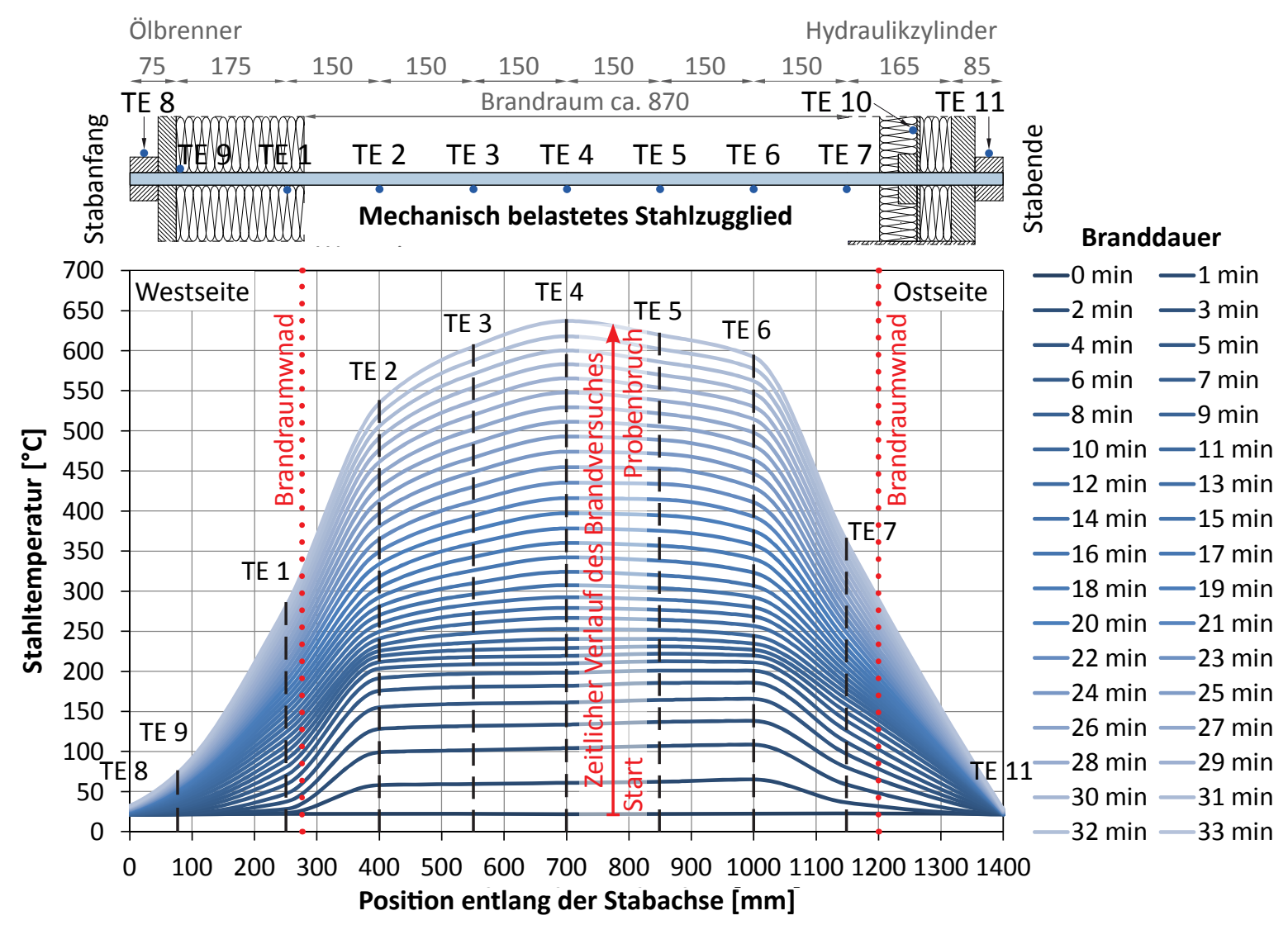

Abb. 5.03: Zeichnung der Einbausituation des mechanisch belasteten Zuggliedes mit dem nachträglich geglätteten Temperaturverlauf des Versuchskörpers $B 9$

\section{Struktur-Modell}

Mit Hilfe dieses Modells können die Verformungen des Zuggliedes sowie die im Stabquerschnitt auftretenden Spannungen und Dehnungen berechnet werden. Die Validierung des numerischen Modells erfolgt durch die Nachrechnung einzelner Brandversuche im Realmaßstab. Für die Berechnung werden unter anderem die mechanischen Hochtemperatureigenschaften des verwendeten Stahls benötigt. Die Werte für die Dichte und die Poissonzahl werden temperaturunabhängig nach DIN EN 1993-1-2 [13] angenommen. Der Elastizitätsmodul, die Spannungs-Dehnungs-Kennlinien sowie der Wärmeausdehnungskoeffizient basieren auf den in Kapitel 4 durchgeführten Materialuntersuchungen. Da die Prüfgeschwindigkeit besonders bei erhöhten Stahltemperaturen einen Einfluss auf die Höhe der gemessenen Spannungs-Dehnungs-Kurven hat, werden zunächst zwei Materialmodelle (Mat 1 und Mat 3) betrachtet. Die Materialmodelle beziehen sich auf die in den Kleinzugversuchen untersuchten VR 1 und 3. Um eine Anwendung in ABAQUS ${ }^{\circledR} z$ u ermöglichen, werden verschiedene Modifikationen an den experimentell bestimmten Spannungs-Dehnungs-Kennlinien vorgenommen. Beispielsweise erfolgt eine Umrechnung der technischen in die wahren Spannungen und Dehnungen. Eine ausführliche Beschreibung der Modifikati- 
onen ist in Kapitel 5.3.2 beschrieben. Neben den Materialeigenschaften wird auch das Temperaturfeld, welches die Erwärmung des Zuggliedes im Brandversuch beschreibt, benötigt. Entsprechende Daten werden der Temperaturfeldberechnung des Temperatur-Modells entnommen. Das Aufbringen der mechanischen Zugbeanspruchung erfolgt am Stabende des Zugstabmodells. Die Zugkraft entspricht der im Brandversuch aufgebrachten Zugbeanspruchung. Die Auflagerung des Zuggliedes wird so gestaltet, dass sich am Stabanfang ein gelenkiges, allseitig unverschiebliches Auflager und am Stabende ein gelenkiges und in Stablängsrichtung (Z-Richtung) verschiebliches Auflager befindet. Eine isometrische Ansicht des numerischen Modells ist in Abb. 5.04 dargestellt.

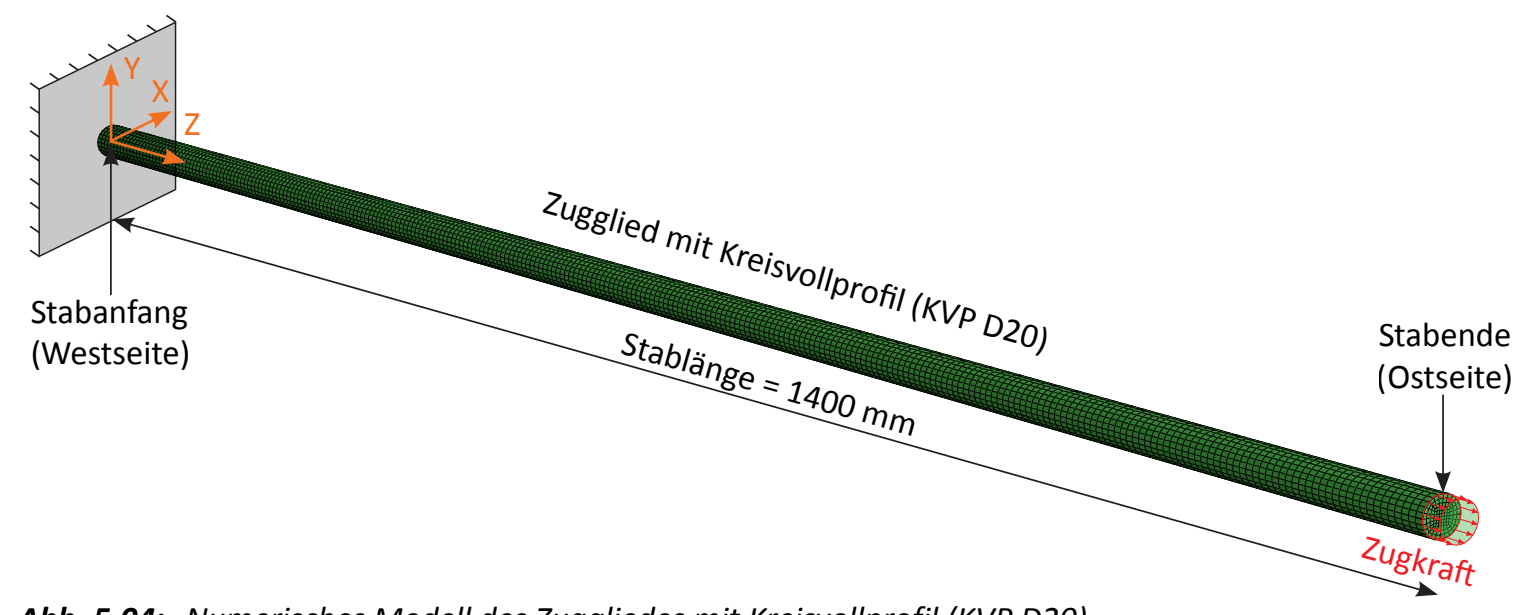

Abb. 5.04: Numerisches Modell des Zuggliedes mit Kreisvollprofil (KVP D20)

\section{Ergebnisse des Bemessungsmodells}

Die Validierung des numerischen Modells erfolgt zunächst anhand des Brandversuches des Versuchskörpers B9. Dabei werden die beiden Materialmodelle (Mat 1 und Mat 3) untersucht. Das entwickelte numerische Modell ist in der Lage das Verformungsverhalten des Zuggliedes bis in die Nähe des Zeitpunktes des Probenbruchs abzubilden. Der sich durch eine Einschnürung des Stabes ankündigende Bruch des Zuggliedes tritt auch im Bemessungsmodell auf. Hinsichtlich der Stabanfangsverformung, welche aus der aufgebrachten Zugbeanspruchung resultiert, liefert das numerische Modell meist etwas geringere Werte, als die im Brandversuch gemessenen. Trotz einer nachträglichen Korrektur der im Brandversuch gemessenen Anfangsverformung, welche die Nachgiebigkeit des Belastungsrahmens sowie den auftretenden Schlupf der Lastaufbringung berücksichtigt, bleibt ein geringer Unterschied in der Anfangsverformung zwischen Experiment und Simulation bestehen. Die im Experiment aufgetretenen leicht höheren Anfangsverformungen sind wahrscheinlich auf die Nachgiebigkeit des Belastungsrahmens und der Einspannvorrichtung für die Zugglieder sowie den bei der Lastaufbringung vorhandenen Schlupf zurückzuführen. Da es sich teilweise um Abweichungen von weniger als einen Millimeter handelt, kann auch ein Anteil der Messeinrichtung nicht ausgeschlossen werden. Zur besseren Vergleichbarkeit der über die Branddauer auftretenden Stablängsverformungen wird daher die Anfangsverformung aus 
der numerischen Simulation an die gemessenen Werte aus dem Brandversuch angepasst. Die Ergebnisse zwischen dem Brandversuch des Versuchskörpers B9 und dem numerischen Modell (Mat 1 und 3) zeigen hinsichtlich der Stablängsverformungen eine gute Übereinstimmung (siehe Abb. 5.09). Erst in der Nähe des Zeitpunktes des Probenbruchs des Zuggliedes nehmen die Unterschiede der Stablängsverformungen zu. Das im Rahmen des Forschungsprojektes [35] entwickelte numerische Bemessungsmodell ist in der Lage, den Probenbruch des Zuggliedes B9 bis auf etwa eine Minute genau zu berechnen. Die numerische Nachrechnung aller weiteren getesteten Zugglieder erfolgt im Rahmen der Dissertation.

\subsubsection{Entwicklung temperaturabhängiger Spannungs-Dehnungs-Kennlinien}

Grundlage für die in ABAQUS ${ }^{\circledR}$ verwendeten temperaturabhängigen Spannungs-DehnungsKennlinien bilden die an Kleinproben durchgeführten Zugversuche (siehe Kapitel 4). Die einzelnen Modifikationen, die erforderlich sind, um die gemessenen Spannungs-DehnungsKurven in $A B A Q U S^{\circledR}{ }^{~ z u}$ implementieren, werden nachfolgend beschrieben.

\section{Schritt 1 - Vervollständigung und Bereinigung der technischen $\sigma$ - $\varepsilon$-Kennlinien}

Um einen vollständigen Verlauf der Spannungs-Dehnungs-Kurven zu erhalten, werden die in den Kleinzugversuchen gemessenen Kurven um den Wert der Bruchspannung und -dehnung ergänzt. Vereinfachend wird zunächst ein linearer Verlauf zwischen dem Ende der Dehnungsaufzeichnung und dem Probenbruch angenommen. Anschließend wird der Schlupf zu Beginn des Kleinzugversuches entfernt (siehe Abb. 5.05, Schritt a). Mit Hilfe des dynamischen Elastizitätsmoduls aus den Materialuntersuchungen erfolgt dann die Bestimmung der technischen Proportionalitätsgrenze (siehe Abb. 5.05, Schritt b).
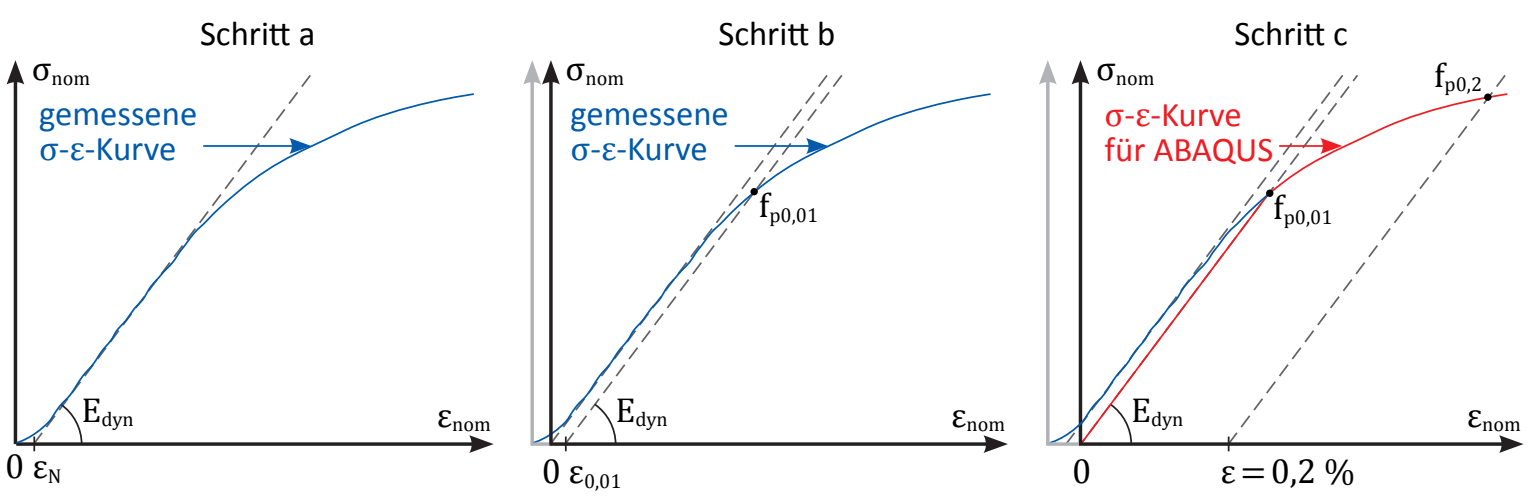

Abb. 5.05: Schematische Darstellung verschiedener Modifikationen an der Spannungs-Dehnungs-Kurve [35]

Schritt 2 - Umrechnung der technischen in die wahren $\sigma$-E-Kennlinien

Generell werden bei Kleinzugversuchen die Spannungen auf den Ausgangsquerschnitt $\left(\mathrm{A}_{0}\right)$ der Zugproben bezogen. Es handelt sich um technische Spannungs-Dehnungs-Kurven ( $\sigma_{\text {nom }}$ und $\varepsilon_{\text {nom }}$ ). Infolge der Zugspannungen treten sowohl in Stablängsrichtung, als auch in 
Querrichtung Verformungen an der Zugprobe auf. Im Einschnürungsbereich lässt sich dies besonders gut beobachten. Da in der numerischen Simulation ein dreidimensionales Modell verwendet wird, dessen Elemente sich ebenfalls in Längs- und Querrichtung verformen können, sind im Materialmodell die wahren Spannungen und Dehnungen zu berücksichtigen. Die Umrechnung der technischen in die wahren Spannungen und Dehnungen ist in Gleichung (5.12) und (5.13) angegeben.

$\varepsilon=\ln \left(1+\varepsilon_{\text {nom }}\right)$

$\sigma=\sigma_{\text {nom }} \cdot\left(1+\varepsilon_{\text {nom }}\right)$

\section{Schritt 3 - Glätten der wahren $\sigma$-ع-Kennlinien}

Durch die hohe Messrate in den Kleinzugversuchen unterliegen die aufgezeichneten Kurven einem gewissen Messrauschen. Als Folge ergibt sich ein leicht gezackter Verlauf der Spannungs-Dehnungs-Kennlinien. Dieser wirkt sich jedoch nachteilig auf die Rechenstabilität des numerischen Modells aus. Die Spannungs-Dehnungs-Kurven werden nachträglich geglättet. Eine Anpassung des zunächst angenommenen linearen Verlaufes zwischen dem Ende der Dehnungsaufzeichnung und dem Probenbruch wird vorgenommen. Knicke im Verlauf der Spannungs-Dehnungs-Kennlinien werden durch das Glätten der Kurven beseitigt. Ein Vergleich zwischen den ursprünglichen und den modifizierten Spannungs-DehnungsKurven ist in Abb. 5.06 dargestellt.

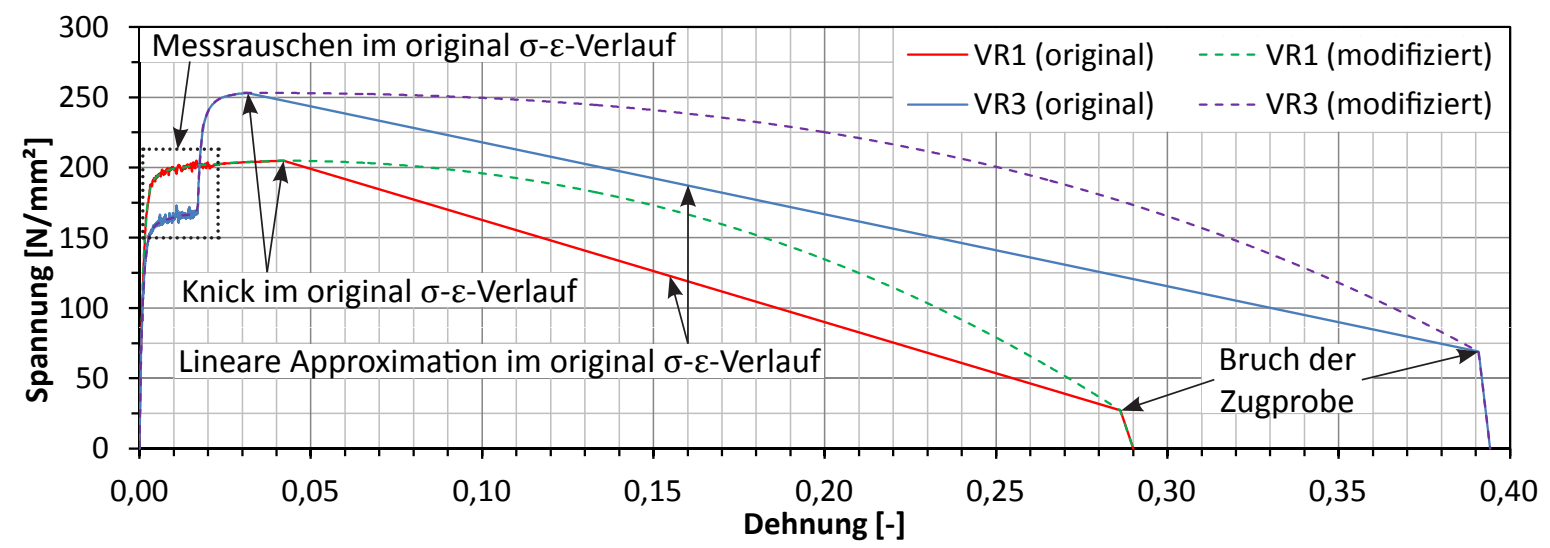

Abb. 5.06: Vergleich der wahren $\sigma-\varepsilon-K u r v e n$ bei $600^{\circ} \mathrm{C}$ vor und nach dem Glätten am Beispiel der VR 1 und $V R 3$ [35]

Schritt 4 - Eingabe der wahren $\sigma$ - $\varepsilon$-Kurven in ABAQUS ${ }^{\circledast}$

Die Eingabe der Spannungs-Dehnungs-Kennlinien in das numerische Modell erfolgt in ABAQUS $^{\circledR}$ für den elastischen und den nichtelastischen Bereich getrennt. Der elastische 
Bereich der Spannungs-Dehnungs-Kennlinie, der von einem linearen Zusammenhang zwischen Spannung und Dehnung ausgeht und sich bis zur Proportionalitätsgrenze $\left(\mathrm{f}_{\mathrm{p}}\right)$ erstreckt, wird mit Hilfe des Elastizitätsmoduls beschrieben. Der sich daran anschließende nichtelastische Verlauf der Spannungs-Dehnungs-Kurven wird im Materialmodell durch einen plastischen Dehnungsanteil $\left(\varepsilon^{\mathrm{PE}}\right)$ berücksichtigt. Bei der Berechnung der Gesamtdehnung $\left(\varepsilon^{\mathrm{E}}\right)$ wird der elastische Dehnungsanteil $\left(\varepsilon^{\mathrm{EE}}\right)$, der von der Höhe der im Bauteil vorhandenen Stahlspannung abhängt, von ABAQUS ${ }^{\circledR}$ automatisch ergänzt. Die Zusammensetzung der

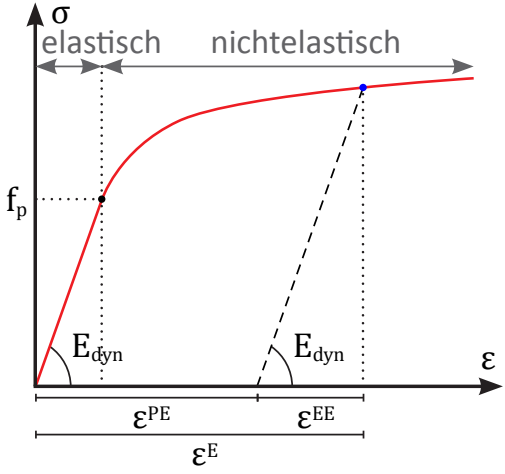

Abb. 5.07: Zusammensetzung der Dehnungsanteile in den $\sigma$ - $\varepsilon$-Kurven Gesamtdehnung ist in Abb. 5.07 schematisch dargestellt dargestellt. Im Materialmodell kann die Temperaturabhängigkeit der Stahlfestigkeit durch die Eingabe mehrerer Spannungs-Dehnungs-Kennlinien berücksichtigt werden. Die eingegebenen Spannungs-Dehnungs-Kurven besitzen jeweils nur für die dazugehörige Stahltemperatur Gültigkeit. Bei Stahltemperaturen, die sich zwischen zwei temperaturabhängigen Spannungs-Dehnungs-Kennlinien befinden, erfolgt durch ABAQUS ${ }^{\circledR}$ eine lineare Interpolation.

Vergleich der Abminderungsfaktoren der Festigkeitskennwerte aus den Materialuntersuchungen und den daraus entwickelten Materialmodellen

In Tabelle 5.2 sind die Abminderungsfaktoren der Festigkeitskennwerte aus den Materialuntersuchungen denen der entwickelten Materialmodelle gegenübergestellt. Es ist zu beachten, dass die Auswertung der Materialuntersuchungen auf zwei verschiedene Arten erfolgt ist. Einerseits berechnen sich die Abminderungsfaktoren aus dem Mittelwert der bei Raumtemperatur und erhöhter Temperatur untersuchten Kleinzugproben, d.h. VR 1 bis 3. Zum anderen wird nur die VR 3 betrachtet.

Da die entwickelten Materialmodelle (Mat 3 und 4) auf den technischen Spannungs-Dehnungs-Kennlinien der VR 3 basieren, ergeben sich zu den Abminderungsfaktoren der VR 3 nur geringfügige Unterschiede. Die Abweichungen sind vor allem auf die Umrechnung von technischer in wahre Spannung und Dehnung zurückzuführen. Vergleichsweise große Unterschiede in den Abminderungsfaktoren treten zwischen den Mittelwerten der Kleinzugversuche und der VR 3 auf. Besonders auffällig ist der Unterschied für die Abminderungsfaktoren der Zugfestigkeit bei erhöhter Stahltemperatur. Dies ist auf die in VR 3 wesentlich höhere Prüfgeschwindigkeit im weggeregelten Bereich zurückzuführen. Die Abweichungen verdeutlichen, dass trotz der sorgfältigen Durchführung und der großen Anzahl an Kleinzugversuchen die Festigkeitskennwerte und deren Abminderungsfaktoren einer gewissen Streuung unterliegen und eine eindeutige Bestimmung besonders bei erhöhter Temperatur nicht immer möglich ist. 
Tabelle 5.2: Vergleich der Abminderungsfaktoren in Bezug auf die Verwendung in dem numerischen Modell

\begin{tabular}{|c|c|c|c|c|c|c|c|c|c|c|}
\hline \multirow{4}{*}{$\begin{array}{c}\text { Stahltem- } \\
\text { peratur } \\
{\left[{ }^{\circ} \mathrm{C}\right]}\end{array}$} & \multicolumn{10}{|c|}{ Abminderungsfaktoren für die Festigkeitskennwerte des untersuchten Blankstahls } \\
\hline & \multirow{3}{*}{$\begin{array}{c}\text { Resonanz } \\
\text { dynamisch } \\
\mathrm{k}_{\mathrm{E}, \mathrm{dyn}} \\
\end{array}$} & \multirow{2}{*}{\multicolumn{3}{|c|}{$\begin{array}{l}\text { Zugversuch } \mathbf{M W} \mathbf{W}^{*} \\
\text { hnische } \sigma-\varepsilon \text {-Kennlinien }\end{array}$}} & \multirow{2}{*}{\multicolumn{3}{|c|}{$\begin{array}{c}\text { Zugversuch VR } \mathbf{3}^{* *} \\
\text { echnische } \sigma-\varepsilon \text {-Kennlinien }\end{array}$}} & \multirow{2}{*}{\multicolumn{3}{|c|}{$\begin{array}{l}\text { Mat } 3 \text { und Mat } 4 * * * \\
\text { wahre } \sigma-\varepsilon-K e n n l i n i e n\end{array}$}} \\
\hline & & & & & & & & & & \\
\hline & & $\mathrm{k}_{\mathrm{p}}$ & $\mathrm{k}_{\mathrm{p} 0,2}$ & $\mathrm{k}_{\mathrm{u}, \mathrm{I}+\mathrm{II}}$ & $\mathrm{k}_{\mathrm{p}}$ & $\mathrm{k}_{\mathrm{p} 0,2}$ & $\mathrm{k}_{\mathrm{u}, \text { II }}$ & $\mathrm{k}_{\mathrm{p}}$ & $\mathrm{k}_{\mathrm{p} 0,2}$ & $\mathrm{k}_{\mathrm{u}, \mathrm{II}}$ \\
\hline Basiswert & 207000 & 285 & 583 & 679 & 296 & 587 & 684 & 296 & 597 & 695 \\
\hline 20 & 1,000 & 1,000 & 1,000 & 1,000 & 1,000 & 1,000 & 1,000 & 1,000 & 1,000 & 1,000 \\
\hline 100 & 0,981 & 1,264 & 1,001 & 0,950 & 1,129 & 0,988 & 0,920 & 1,129 & 0,984 & ),948 \\
\hline 200 & 0,342 & 1,119 & $1,0>2$ & 1,001 & $1,0>1$ & $1,0>0$ & 1,000 & $1,0 J 1$ & דינט, & $1, \cup 4 J$ \\
\hline 250 & 3 & 1,344 & 76 & 1 & 1,251 & 1,075 & 1,1 & 1,252 & 1,072 & ,108 \\
\hline 300 & 0,903 & 1,206 & 1,036 & 1,037 & 0,910 & 1,022 & 1,051 & 0,910 & 1,020 & 1,056 \\
\hline 350 & 0,882 & 1,309 & 0,987 & 0,977 & 1,245 & 0,983 & 1,000 & 1,246 & 0,974 & 1,027 \\
\hline 400 & 0,860 & 1,266 & 0,904 & 0,892 & 1,315 & 0,900 & 0,910 & 1,316 & 0,890 & 0,929 \\
\hline 450 & 0,833 & 1,046 & 0,789 & 0,774 & 0,969 & 0,780 & 0,795 & 0,969 & 0,770 & 0,815 \\
\hline 500 & 07 & 0,714 & 30 & 0,614 & 0,7 & 0,6 & 0,6 & 0,709 & 0,613 & (8, \\
\hline 550 & 0,771 & 0,379 & 0,455 & 0,441 & 0,213 & 0,426 & 0,490 & 0,213 & 22 & 0,501 \\
\hline 600 & 0,734 & 0,225 & 0,278 & 0,290 & 0,205 & 0,258 & 0,359 & 0,204 & 0,255 & 0,364 \\
\hline 650 & 0,669 & 0,062 & 0,168 & 0,196 & 0,046 & 0,152 & 0,255 & 0,046 & 0,149 & 0,259 \\
\hline 700 & 0,604 & 0,081 & 0,098 & 0,123 & 0,077 & 0,085 & 0,170 & 0,077 & 0,086 & 0,172 \\
\hline
\end{tabular}

Hinweis: Der Basiswert bei $20^{\circ} \mathrm{C}$ ist in $\mathrm{N} / \mathrm{mm}^{2}$ angegeben. Index I bezieht sich auf den dehnungsgeregelten Bereich und Index II auf den weggeregelten Bereich des Zugversuches.

* Es handelt sich um Mittelwerte aus allen drei VR der Kleinzugversuche. Die Abminderungsfaktoren basieren auf technischen Spannungs-Dehnungs-Kennlinien. Die Basiswerte der Abminderungsfaktoren werden aus der Mittelwertkurve der bei Raumtemperatur untersuchten Proben bestimmt.

** Es handelt sich um die Ergebnisse aus der VR 3 der Kleinzugversuche. Die Abminderungsfaktoren basieren auf technischen Spannungs-Dehnungskennlinien. Die Basiswerte der Abminderungsfaktoren werden anhand der bei Raumtemperatur getesteten Zugprobe P_50 bestimmt.

*** Die Werte basieren auf den Ergebnissen der VR 3. Die Auswertung erfolgt analog zur VR 3. Allerdings handelt es sich bei den für die Auswertung zugrunde gelegten Kurven um wahre Spannungs-Dehnungs-Kennlinien. Aufgrund dessen kommt es im nichtelastischen Bereich zu einem Anstieg der Stahlfestigkeit. Die Kurvenverläufe werden entsprechend der Beschreibungen in Kapitel 5.3.2 modifiziert.

\subsubsection{Weiterentwicklungen des Bemessungsmodells}

Durch eine Weiterentwicklung des Bemessungsmodells wird eine noch bessere Übereinstimmung mit den Ergebnissen der Brandversuche angestrebt. Die Anwendbarkeit des Bemessungsmodells wird durch die Nachrechnung aller im Zugstabprüfofen getesteten Kreisvollprofile verifiziert. Die am numerischen Modell vorgenommenen Modifikationen sind nachfolgend aufgelistet:

- Verwendung einer temperaturunabhängigen Dichte des Stahls von $\rho=7850 \mathrm{~kg} / \mathrm{m}^{3}$.

Dies entspricht den Vorgaben nach DIN EN 1993-1-2 [13] 
- exakte Eingabewerte des Wärmeausdehnungskoeffizienten für kaltverformten Blankstahl nach Gleichung (4.8)

- Verbesserung der Verarbeitung des Materialmodells in ABAQUS ${ }^{\circledR}$ durch Einhaltung gleicher Dehnungsabstände in den Spannungs-Dehnungs-Kennlinien

- Entwicklung und Anwendung eines neuen Materialmodells (Mat 4) für die KVP D20. Der Einfluss hoher Dehngeschwindigkeiten auf das Spannungsniveau wird berücksichtigt

- Erstellung eines neuen Materialmodells (Mat 5) für den kaltverformten Blankstahl der Versuchskörper mit KVP D30

Erstellung eines neuen Materialmodells für hohe Dehngeschwindigkeiten (Mat 4)

Die Ergebnisse der numerischen Untersuchung aus dem Forschungsvorhaben [35] haben gezeigt, dass die Verformungen des Zuggliedes aufgrund relativ hoher Verformungsgeschwindigkeiten zum Zeitpunkt des Probenbruchs von den experimentell gemessenen Werten leicht abweichen. In den Materialuntersuchungen, welche die Grundlage für die in ABAQUS ${ }^{\circledR}$ hinterlegten Materialmodelle bilden, finden hohe Dehngeschwindigkeiten in den Kleinzugversuchen der VR 3 (Mat 3) Berücksichtigung. Allerdings setzt die Erhöhung der

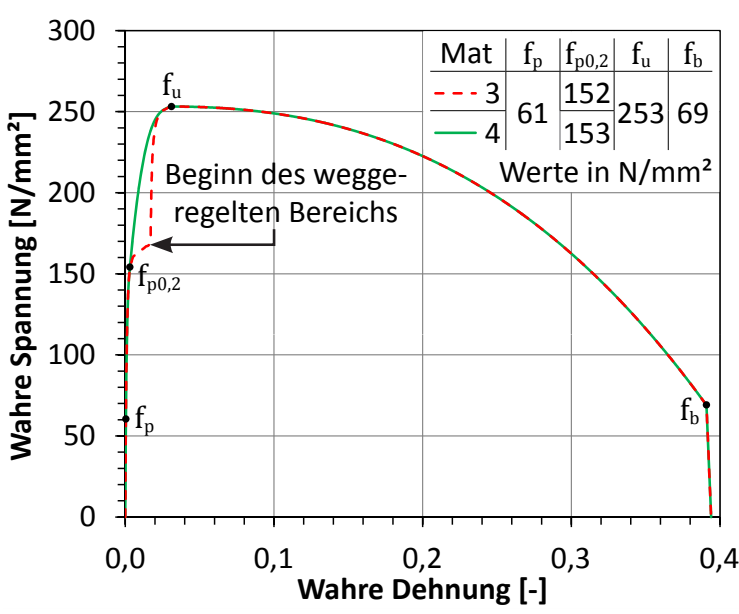

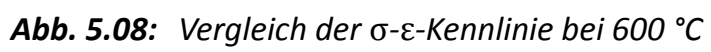
zwischen Mat 3 und 4 Dehngeschwindigkeit erst nach dem Erreichen einer Dehnung von etwa $2 \%$, d.h. nach dem Umschalten in die Wegregelung, ein. Basierend auf den Erkenntnissen zum Einfluss der Prüfgeschwindigkeit aus Kapitel 4.5.2 wird der dehnungsgeregelte Bereich der Zugversuche der VR 3 (Mat 3) modifiziert (siehe Abb. 5.08) und ein neues Materialmodell (Mat 4) entwickelt. Zwischen Mat 3 und 4 bleibt der Elastizitätsmodul unverändert. Durch die Anpassung des dehnungsgeregelten Bereiches kann für das Mat 4 über den kompletten Verlauf der Spannungs-Dehnungs-Kennlinie eine erhöhte Dehngeschwindigkeit von $\dot{\mathrm{e}}=0,0014 \mathrm{~s}^{-1}$ angenommen werden. Mit dem neuen Materialmodell (Mat 4) ist es daher möglich, die Stablängsverformungen des Zuggliedes bis zum Erreichen des in Kapitel 3.9 vom Autor vorgeschlagenen Verformungsgeschwindigkeitskriterium ( $\dot{e}=0,001 \mathrm{~s}^{-1}$ ) abzubilden. Dadurch wird eine noch bessere Übereinstimmung mit den Brandversuchen im Realmaßstab erreicht. Der komplette Verlauf der temperaturabhängigen Spannungs-Dehnungs-Kennlinien für Mat 3 und Mat 4 ist in Abb. C.01 und Abb. C.02 im Anhang dargestellt. Eine tabellarische Übersicht zu den Festigkeitsparametern aus den wahren Spannungs-Dehnungs-Kurven des Mat 3 und Mat 4 ist in Tabelle C.1 und Tabelle C.2 im Anhang zusammengestellt. 
Vergleich der entwickelten Bemessungsmodelle für das KVP D20

Anhand des weiterentwickelten Materialmodells Mat 4 sowie der zwei Materialmodelle Mat 1 und 3 aus dem Forschungsprojekt [35] erfolgt eine numerische Nachrechnung des Versuchskörpers B9. Ein Vergleich der Stablängsverformungen ist in Abb. 5.09 dargestellt. Neben den numerisch berechneten Verformungen ist im Diagramm auch die im Brandversuch gemessene Stablängsverformung abgebildet. Wie bereits in Kapitel 5.3.1 beschrieben, ist die numerisch berechnete Anfangsverformung geringfügig kleiner als im Brandversuch. Zur besseren Vergleichbarkeit der Ergebnisse wird

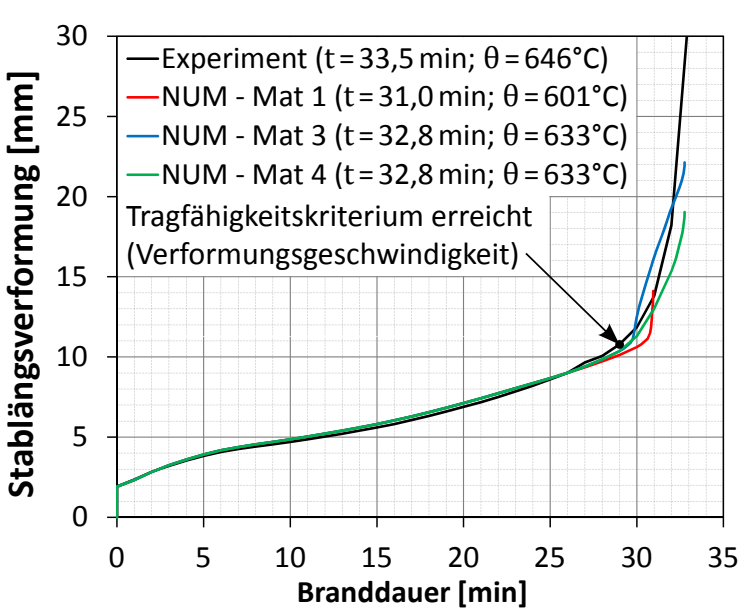

Abb. 5.09: Vergleich der gemessenen und numerisch berechneten Verformungen des Versuchskörpers $B 9$ daher die Anfangsverformung aus der numerischen Simulation an die des Brandversuches angepasst. Anhand der Abb. 5.09 zeigt sich, dass die Stablängsverformungen aller drei untersuchten Materialmodelle zunächst identisch verlaufen und sehr gut mit dem Brandversuch übereinstimmen. Neben dem Zeitpunkt des Probenbruchs bzw. dem Simulationsende $(t)$ ist in dem Diagramm auch die dazugehörige maximale Stahltemperatur $(\theta)$ angegeben. Ab der 26. Minute nehmen die Abweichungen zwischen numerischer Simulation und Experiment zu. Auch zwischen den einzelnen numerischen Modellen zeigen sich nunmehr Unterschiede. Durch die geringere Prüfgeschwindigkeit der in VR 1 durchgeführten Kleinzugversuche und dem daraus folgenden geringeren Festigkeitsniveau endet die numerische Simulation des Mat 1 nach der 31. Minute. Der Abbruch der Berechnung ist auf das Erreichen der maximalen Zugfestigkeit an der Stelle mit der höchsten Stahltemperatur zurückzuführen. Ähnlich wie im Brandversuch bildet sich um die spätere Bruchstelle eine Einschnürung aus. Der große Anstieg der Stablängsverformungen kurz vor dem Ende der Simulation ist darauf zurückzuführen, dass sich bis zum Erreichen der maximalen Zugfestigkeit ein relativ langgezogenes Festigkeitsplateau ausbildet. Für das Mat 1 beträgt die Dehnung beim Erreichen der maximalen Zugfestigkeit im Bereich der erhöhten Temperatur etwa $4 \%$.

Mit Hilfe der Materialmodelle Mat 3 und Mat 4 lässt sich eine noch bessere Übereinstimmung des Zeitpunktes für den Probenbruch sowie des Verformungsverlaufes zum Brandversuch erreichen. Die Berechnung der beiden numerischen Modelle endet jeweils nach 32,8 Minuten mit dem Erreichen der maximalen Zugfestigkeit an der Stelle mit der höchsten Stahltemperatur. Da sich die Spannungs-Dehnungs-Kennlinien der beiden Materialmodelle in diesem Bereich nicht unterscheiden, ergibt sich ein identischer Zeitpunkt für das Simula- 
tionsende. Der Unterschied im Verformungsverlauf und in der am Ende erreichten maximalen Stablängsverformung ist auf den unterschiedlichen Verlauf der Spannungs-DehnungsKurven zurückzuführen (siehe Abb. 5.08).

Der Vergleich der Stablängsverformungen hat gezeigt, dass sich prinzipiell alle drei entwickelten Materialmodelle dazu eignen, die durchgeführten Brandversuche an beschichteteten Stahlzuggliedern mit KVP D20 numerisch nachzurechnen. Bis zum Erreichen des neu entwickelten Tragfähigkeitskriteriums, welches in Kapitel 3.9 näher beschrieben ist, stimmen die numerisch berechneten Stablängsverformungen gut mit dem Brandversuch überein. Erst in der Nähe des späteren Zeitpunktes des Probenbruchs nehmen die Unterschiede zu. Ursache hierfür ist die im Brandversuch schnell anwachsende Verformungsgeschwindigkeit. Diese hat besonders bei erhöhter Stahltemperatur einen erheblichen Einfluss auf das Festigkeitsniveau des Stahls. Die beste Übereinstimmung zwischen Brandversuch und numerischer Simulation lässt sich mit Hilfe des Mat 4 erreichen. Für die Nachrechnung weiterer Zugglieder mit KVP D20 wird dieses Materialmodell verwendet.

\section{Entwicklung eines Materialmodells (Mat 5) für KVP D30}

Für die KVP D30 wurden nur Kleinzugversuche bei Raumtemperatur durchgeführt. Um die Brandversuche mit KVP D30 numerisch nachrechnen zu können, werden jedoch auch für den Hochtemperaturbereich entsprechende Spannungs-Dehnungs-Kennlinien benötigt. Auf Grundlage des für das KVP D20 erstellten Materialmodells (Mat 4) wird durch Modifikation der temperaturabhängigen Spannungs-Dehnungs-Kurven des KVP D30 ein neues Materialmodell (Mat 5) erstellt. Diese Vorgehensweise ist möglich, da beide Stähle aus gezogenem, kaltverformtem Blankstahl bestehen und bei Raumtemperatur ein ähnliches Festigkeitsniveau aufweisen. Vergleichend sind in Abb. 5.10 die technischen und wahren Spannungs-Dehnungs-Kennlinien beider Stähle bei Raumtemperatur dargestellt.

Für die Erstellung des Mat 5 wird zunächst die bei Raumtemperatur experimentell bestimmte technische Spannungs-Dehnungs-Kurve des KVP D30 nach der in Kapitel 5.3.2 beschriebenen Vorgehensweise angepasst. Anschließend werden anhand der technischen Spannungs-Dehnungs-Kurve die Festigkeitskennwerte bestimmt. Die ermittelten Werte des KVP D30 werden denen des KVP D20 gegenübergestellt und entsprechende Korrekturfaktoren (k-Faktor) berechnet (siehe Tabelle 5.3). Im Anschluss werden die Korrekturfaktoren auf die wahren Spannungs-Dehnungs-Kennlinien des Mat 4 angewendet.

Für die Zug- und Bruchfestigkeit erfolgt lediglich eine Anpassung der Spannungswerte. Die dazugehörigen Dehnungen bleiben unverändert. Da der Elastizitätsmodul des Stahls vom KVP D20 übernommen wird, ist für den neuen Wert der Proportionalitätsgrenze sowie der 0,2\%-Dehngrenze eine Anpassung der Dehnungen erforderlich. Eine schematische Darstellung der dafür erforderlichen Modifikationen ist in Abb. 5.11 dargestellt. Die für die Erstellung des Mat 5 erforderlichen Anpassungen des Mat 4 erfolgen sowohl für die Spannungs- 
Dehnungs-Kennlinien bei Raumtemperatur als auch bei erhöhter Temperatur. Die für die KVP D30 erstellten temperaturabhängigen Kennlinien und die dazugehörigen Kennwerte sind im Anhang Cangegeben.

Tabelle 5.3: Festigkeitskennwerte und Korrekurfaktoren für die KVP D20 und KVP D30 bei Raumtemperatur

\begin{tabular}{|c|c|c|c|c|c|c|c|c|c|}
\hline \multirow{2}{*}{ Material } & $\mathrm{E}_{\mathrm{dyn}}$ & $\mathrm{f}_{\mathrm{p} 0,01}$ & $\varepsilon_{\mathrm{p} 0,01}$ & $\mathrm{f}_{\mathrm{p} 0,2}$ & $\varepsilon_{\mathrm{p} 0,2}$ & $\mathrm{f}_{\mathrm{u}}$ & $\varepsilon_{\mathrm{u}}$ & $\mathrm{f}_{\mathrm{b}}$ & $\varepsilon_{\mathrm{b}}$ \\
\cline { 2 - 11 } & {$\left[\mathrm{N} / \mathrm{mm}^{2}\right]$} & {$\left[\mathrm{N} / \mathrm{mm}^{2}\right]$} & {$[-]$} & {$\left[\mathrm{N} / \mathrm{mm}^{2}\right]$} & {$[-]$} & {$\left[\mathrm{N} / \mathrm{mm}^{2}\right]$} & {$[-]$} & {$\left[\mathrm{N} / \mathrm{mm}^{2}\right]$} & {$[-]$} \\
\hline KVP D20 & 207000 & 291 & 0,00141 & 585 & 0,00483 & 679 & 0,01385 & 242 & 0,15270 \\
\hline KVP D30 & 207000 & 373 & 0,00180 & 571 & 0,00476 & 661 & 0,01810 & 236 & 0,08680 \\
\hline k-Faktor & - & $\mathrm{k}_{\sigma}=1,283$ & - & $\mathrm{k}_{\sigma}=0,976$ & - & $\mathrm{k}_{\sigma}=0,974$ & - & $\mathrm{k}_{\sigma}=0,976$ & - \\
\hline
\end{tabular}

Bei den angegebenen Werten handelt es sich um Mittelwerte aus den bei Raumtemperatur experimentell bestimmten technischen $\sigma-\varepsilon-K e n n l i n i e n$. Der Korrekturfaktor (k-Faktor) berechnet sich aus dem Quotient aus KVP D30 und KVP D20.

\begin{tabular}{|c|c|}
\hline KVP D20 & KVP D30 \\
\hline - techn. Werte & - techn. Werte \\
\hline--- wahre Werte & --- wahre Werte \\
\hline
\end{tabular}

Die Spannungs-Dehnungs-Kurven beziehen sich auf Raumtemperatur.

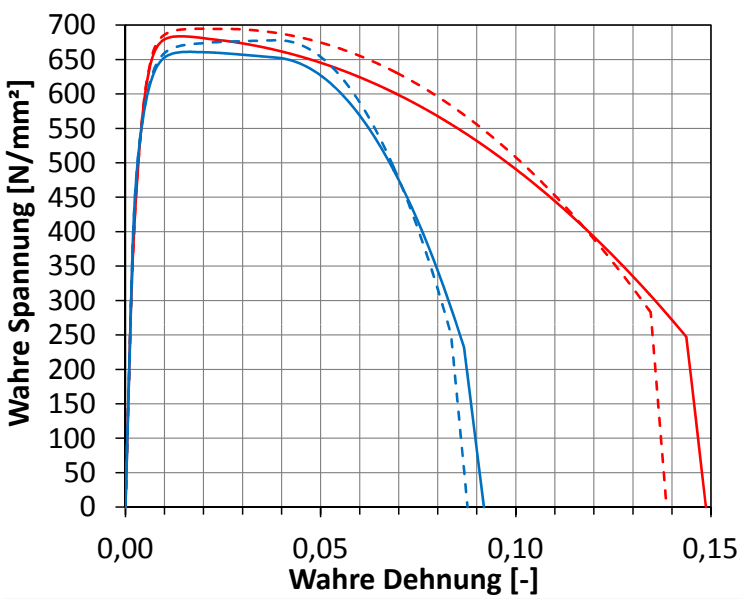

Abb. 5.10: Vergleich der technischen und wahren $\sigma$ - $\varepsilon$-Kennlinien des KVP D20 und KVP D30

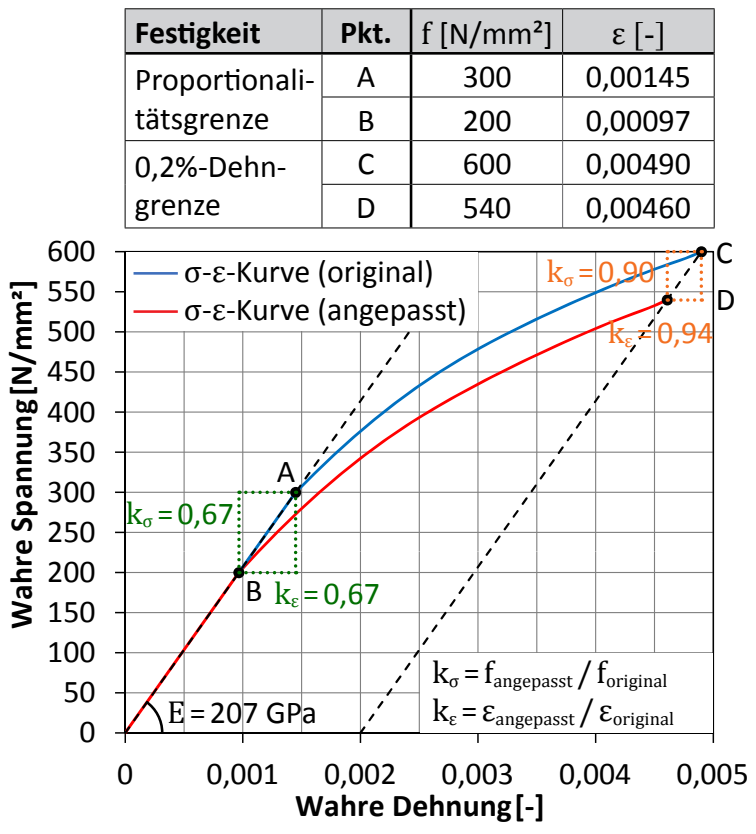

Abb. 5.11: Schematische Vorgehensweise bei der Anpassung der $\sigma-\varepsilon-K u r v e n$ von Mat 4 (KVP D20) auf Mat 5 (KVP D30)

\subsubsection{Vergleich zwischen Bemessungsmodell und Brandversuch}

Mit Hilfe der neu erstellten Materialmodelle werden die Brandversuche an den KVP D20 und KVP D30 numerisch nachgerechnet. Der Vergleich dient der Validierung des numerischen Bemessungsmodells und der erstellten Materialmodelle (Mat 4 und 5). Als weitere Eingangsdaten werden für die numerische Berechnung die Geometrie des Zuggliedes, die aufgebrachte axiale Zugbeanspruchung sowie die im Brandversuch gemessenen Stahltem- 
peraturen des Versuchskörpers benötigt. Als Vergleichsgröße zwischen Bemessungsmodell und Brandversuch wird die Stablängsverformung des Stahlzuggliedes herangezogen. Die Nachgiebigkeit des Belastungsrahmens ist in den gemessenen Verformungen aus den Brandversuchen bereits berücksichtigt. Darüber hinaus erfolgt eine Anpassung der numerisch berechneten Anfangsverformung an die des Brandversuches. Die Diagramme mit der Gegenüberstellung der Stablängsverformung aus den Brandversuchen und der numerischen Simulation sind im Abb. D.01 im Anhang enthalten.

Die numerisch berechneten Stablängsverformungen zeigen eine gute Übereinstimmung mit den experimentell gemessenen Werten. Das Ende der numerischen Simulation, welches dem Erreichen der Zugfestigkeit an der Stelle mit der maximalen Stahltemperatur entspricht, ist nur geringfügig kleiner als der Zeitpunkt des Probenbruchs der Zugglieder in den Brandversuchen. Dies zeigt, dass die entwickelten Bemessungsmodelle in der Lage sind, das Verformungsverhalten mechanisch belasteter Stahlzugglieder mit reaktiver Brandschutzbeschichtung unter Brandeinwirkung realitätsnah abzubilden.

Um eine hohe Genauigkeit der numerischen Berechnung sicherzustellen, sind genaue Kenntnisse des zeitlich und örtlich veränderlichen Temperaturfeldes des Zuggliedes sowie der mechanischen Hochtemperatureigenschaften des verwendeten Stahls erforderlich. Bestehen die Zugglieder aus gezogenem, kaltverformtem Blankstahl und liegt bei Raumtemperatur eine ähnliche Festigkeit wie bei dem untersuchten Stahl des KVP D20 vor, so können die temperaturabhängigen Spannungs-Dehnungs-Kennlinien durch Anpassung des Materialmodells (Mat 4) erstellt werden. Eine entsprechende Vorgehensweise ist in Kapitel 5.3.3 beschrieben. Liegen keine anderen Werte vor, so kann für den temperaturabhängigen Verlauf des Wärmeausdehnungskoeffizienten die Gleichung (4.8) verwendet werden.

Eine Verwendung von Temperaturdaten aus Brandversuchen an unbelasteten Versuchskörpern ist nur dann möglich, wenn die thermische Schutzwirkung des reaktiven Brandschutzsystems gleich oder geringer ist als die eines vergleichbaren, mechanisch belasteten Zuggliedes. Die in Kapitel 3.5 durchgeführten Brandversuche haben gezeigt, dass diese Annahme in der Regel nicht zutrifft. Die mechanisch belasteten Zugglieder weisen meist höhere Stahltemperaturen auf als die simultan geprüften, unbelasteten Vergleichsprüfkörper. Um die an unbelasteten Versuchskörpern gemessenen Temperaturen in der numerischen Simulation dennoch verwenden zu können, ist eine Korrektur der Temperaturdaten erforderlich. Dieser Ansatz wird bereits bei der Auswertung von beschichteten Stahlträgern und Stahlstützen angewendet. Allerdings existiert für Zugglieder zum gegenwärtigen Zeitpunkt noch kein normativ geregeltes Verfahren, wie dieser Korrekturfaktor zu bestimmen ist. Entsprechende Normen [19] und [18] zur Prüfung und Auswertung reaktiver Brandschutzsysteme auf Stahlzuggliedern mit Vollprofil sind derzeit in Arbeit. 
Unterschiede in der Anfangsverformung der mechanisch belasteten Stahlzugglieder

Es hat sich gezeigt, dass die mit Hilfe des Bemessungsmodells berechneten Anfangsverformungen teilweise geringer sind, als die im Brandversuch gemessenen Werte (siehe Abb. 5.12). Die Abweichungen sind im Wesentlichen auf die Nachgiebigkeit des Belastungsrahmens und dem Schlupf beim Aufbringen der Zugbeanspruchung zurückzuführen. Zudem kann ein geringfügiger Einfluss der Messeinrichtung nicht ausgeschlossen werden. Die Nachgiebigkeit des Stahlrahmens, der Schlupf bei der Lastaufbringung und der Einfluss

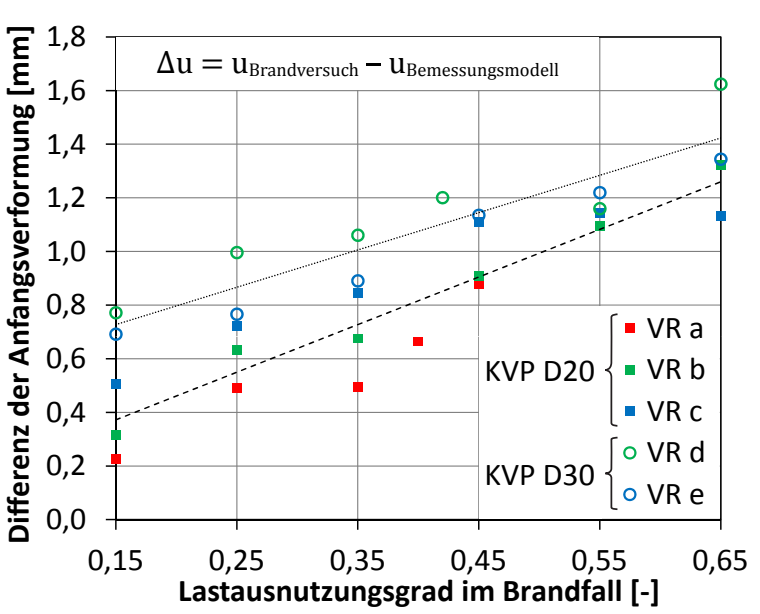

Abb. 5.12: Differenz der gemessenen und numerisch berechneten Anfangsverformung der Messeinrichtung treten lediglich im Brandversuch auf. Eine Korrektur der gemessenen Stablängsverformungen ist zwar erfolgt, jedoch ist davon auszugehen, dass die Auswirkungen dadurch nicht restlos beseitigt werden können. Im Bemessungsmodell sind ideale Randbedingungen vorhanden, wodurch kein Schlupf infolge der Lastaufbringung auftritt. Die Auflagerungsbedingungen befinden sich direkt an den Stabenden des Zuggliedes, so dass keine Nachgiebigkeit des Belastungsrahmens vorhanden ist. Um eine bessere Vergleichbarkeit der Stablängsverformungen zwischen Brandversuch und Bemessungsmodell zu erhalten, werden die Verformungswerte aus dem numerischen Modell immer an die des Brandversuches angepasst.

\subsubsection{Spannungs- und Dehnungszustand der mechanisch belasteten Stahl- zugglieder bei Erreichen der Tragfähigkeit bzw. des Probenbruchs}

Anhand des entwickelten numerischen Modells können für den Zeitpunkt des Erreichens der Tragfähigkeit (siehe Kapitel 3.9) und des Probenbruchs (Simulationsende) die Spannungs- und Dehnungszustände der mechanisch belasteten Stahlzugglieder mit Kreisvollprofil in Abhängigkeit von der Stahltemperatur berechnet werden. Dabei wird jeweils die Einschnürungs- bzw. Versagensstelle des Zuggliedes, welche sich an der Position der maximalen Stahltemperatur befindet, betrachtet. In Abb. 5.13 sind die anhand des Bemessungsmodells zum Zeitpunkt des Erreichens der Tragfähigkeit und des Probenbruchs berechneten wahren Spannungen (Mises) dargestellt (VR a bis e). Zugglieder mit gleichem Lastausnutzungsgrad im Brandfall besitzen aufgrund der identischen Zugbeanspruchung das gleiche Spannungsniveau. Die Zuordnung zwischen den wahren Spannungen und dem Lastausnutzungsgrad im Brandfall sind in Tabelle 5.4 angegeben. Dass sich für Zugglieder mit gleichem Spannungs- 
niveau und Profilquerschnitt, jedoch unterschiedlicher Trockenschichtdicke des reaktiven Brandschutzsystems bei Erreichen der Tragfähigkeit geringfügige Unterschiede für die berechnete Stahltemperatur ergeben, ist darauf zurückzuführen, dass nur vollendete Minuten für die Auswertung des Tragfähigkeitszeitpunktes betrachtet werden. Die für den Zeitpunkt des Erreichens der Tragfähigkeit und des Probenbruchs für die mechanisch belasteten Zugglieder mit KVP D20 und KVP D30 numerisch ermittelten Werte zeigen eine gute Übereinstimmung mit den temperaturabhängigen Spannungskurven der 0,2\%-Dehngrenze $\left(\mathrm{f}_{\mathrm{p} 0,2}\right)$ und der Zugfestigkeit $\left(f_{u}\right)$, welche im Materialmodell Mat 4
Tabelle 5.4: Zuordnung Lastausnutzungsgrad im Brandfall und wahre Spannung

\begin{tabular}{|c|c|c|}
\hline $\begin{array}{c}\text { Lastausnutz- } \\
\text { ungsgrad im } \\
\text { Brandfall [-] }\end{array}$ & \multicolumn{2}{|c|}{$\begin{array}{c}\text { Wahre Spannung } \\
\text { [N/mm }{ }^{2} \text { ] }\end{array}$} \\
\hline 0,15 & 88 & 86 \\
\hline 0,25 & 147 & 144 \\
\hline 0,35 & 206 & 201 \\
\hline 0,40 & 235 & - \\
\hline 0,42 & - & 241 \\
\hline 0,45 & 264 & 258 \\
\hline 0,55 & 323 & 315 \\
\hline 0,65 & 381 & 372 \\
\hline
\end{tabular}

* Basiswert $\mathrm{f}_{\mathrm{p} 0,2}=585 \mathrm{~N} / \mathrm{mm}^{2}$ [bei $20^{\circ} \mathrm{C}$ ]

** Basiswert $\mathrm{f}_{\mathrm{p} 0,2}=570 \mathrm{~N} / \mathrm{mm}^{2}$ [bei $20^{\circ} \mathrm{C}$ ] (KVP D20) sowie Mat 5 (KVP D30) der numerischen Simulation hinterlegt sind. Im Hinblick auf die Tragfähigkeit der Zugglieder bestätigt dies, dass die Leistungskriterien für die Bestimmung der Tragfähigkeit (siehe Kapitel 3.9), d.h. insbesondere das Verformungsgeschwindigkeitskriterium, sehr gut auf das Erreichen der Bemessungsfestigkeit des Stahls, d.h. 0,2\%-Dehngrenze, abgestimmt sind. Dass die anhand des Bemessungsmodells für die KVP D30 beim Erreichen der Tragfähigkeit berechneten Stahltemperaturen etwas höher ausfallen als die temperaturabhängige Kurve der 0,2\%-Dehngrenze, ist mit hoher Wahrscheinlichkeit darauf zurückzuführen, dass die temperaturabhängigen Werte der 0,2\%-Dehngrenze für die KVP D30 nicht experimentell untersucht, sondern, basierend auf den Materialuntersuchungen des KVP D20, erstellt wurden. Besonders die Festigkeitswerte der Proportionalitätsgrenze $\left(\mathrm{f}_{\mathrm{p}}\right)$ und der 0,2\%-Dehngrenze sind daher mit Unsicherheiten behaftet. Ferner zeigt sich, dass die numerische Simulation dann endet, wenn an der Stelle des Zuggliedes mit der maximalen Stahltemperatur die Zugfestigkeit erreicht wird.

Neben den Spannungen lassen sich auch die maximalen mechanischen Dehnungen der Zugglieder für den Zeitpunkt des Erreichens der Tragfähigkeit und des Probenbruchs auswerten. Auch hierbei zeigt sich eine gute Übereinstimmung der anhand des Bemessungsmodells berechneten Werte mit den temperaturabhängigen Dehnungskurven der 0,2\%-Dehngrenze $\left(\varepsilon_{\mathrm{p} 0,2}\right)$ und der Dehnung für die Zugfestigkeit $\left(\varepsilon_{\mathrm{u}}\right)$. Die bereits bei den Spannungen aufgetretenen geringfügigen Unterschiede für die numerisch berechnete Stahltemperatur bei Erreichen der Tragfähigkeit zeigen sich auch bei den Dehnungen. Zusätzlich dazu treten auch für den Zeitpunkt des Probenbruchs kleine Abweichungen zwischen den einzelnen Zuggliedern mit gleichem Lastausnutzungsgrad auf. Ferner sind kleinere Unterschiede der numerisch berechneten Dehnungen zur temperaturabhängigen Kurve der Dehnungen für die Zugfestigkeit vorhanden. Ursächlich hierfür ist der Umstand, dass im Bereich der Zugfestigkeit ein 
plateauähnlicher Verlauf der Spannungs-Dehnungs-Kurve vorhanden ist, wodurch sich ein exakter Wert für die Dehnung in der numerischen Simulation nur schwer bestimmen lässt.

\section{Fazit}

Der Vergleich hat gezeigt, dass die in Kapitel 3.9 entwickelten Leistungskriterien für die Bestimmung der Tragfähigkeit sehr gut auf das Erreichen der Bemessungsfestigkeit des Stahls, d.h. 0,2\%-Dehngrenze, abgestimmt sind. Sind die 0,2\%-Dehngrenze bei Raumtemperatur und die dazugehörigen temperaturabhängigen Abminderungsfaktoren im Hochtemperaturbereich des für die mechanisch belasteten Zugglieder eingesetzten Stahls bekannt, lässt sich der Zeitpunktes für das Erreichen der Tragfähigkeit auch auf Ebene der Stahlfestigkeiten berechnen (siehe Kapitel 5.4.3)
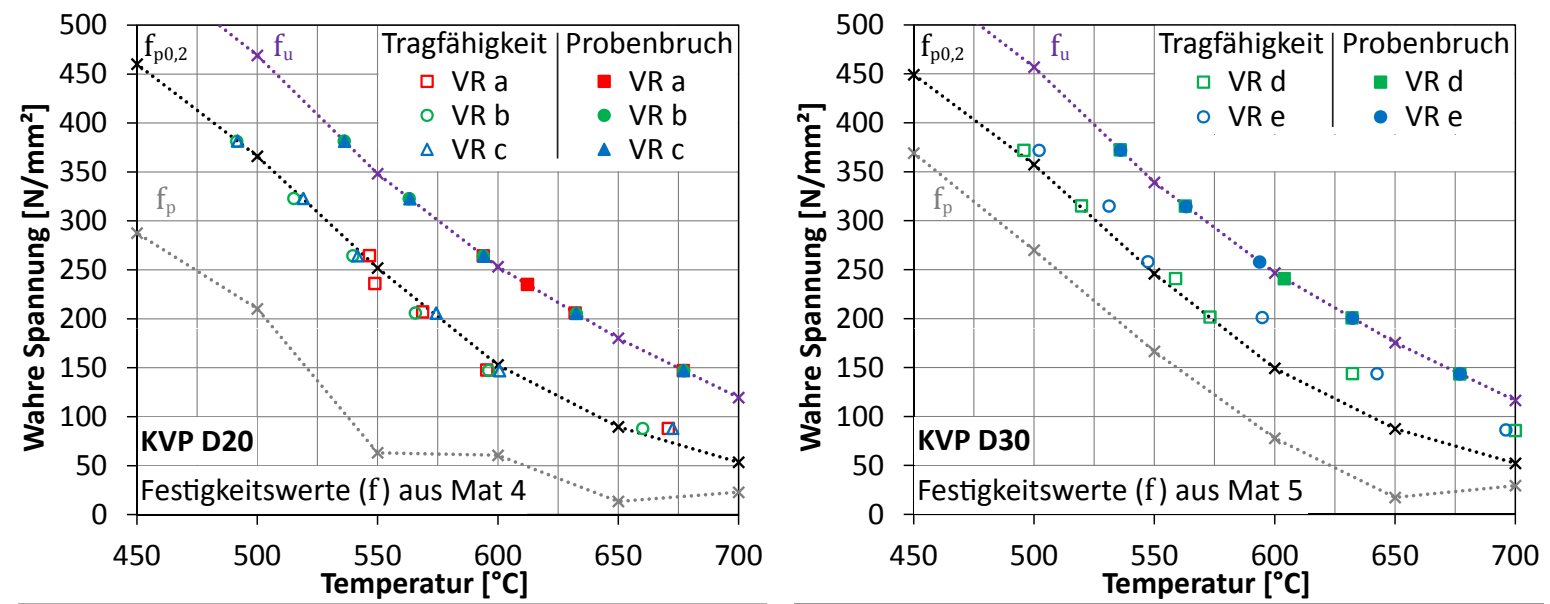

Abb. 5.13: Vergleich der an der Versagensstelle berechneten Spannungen mit den in Stahlfestigkeiten aus den Materialuntersuchungen (Zuordnung Spannung und Lastausnutzungsgrad siehe Tabelle 5.4)
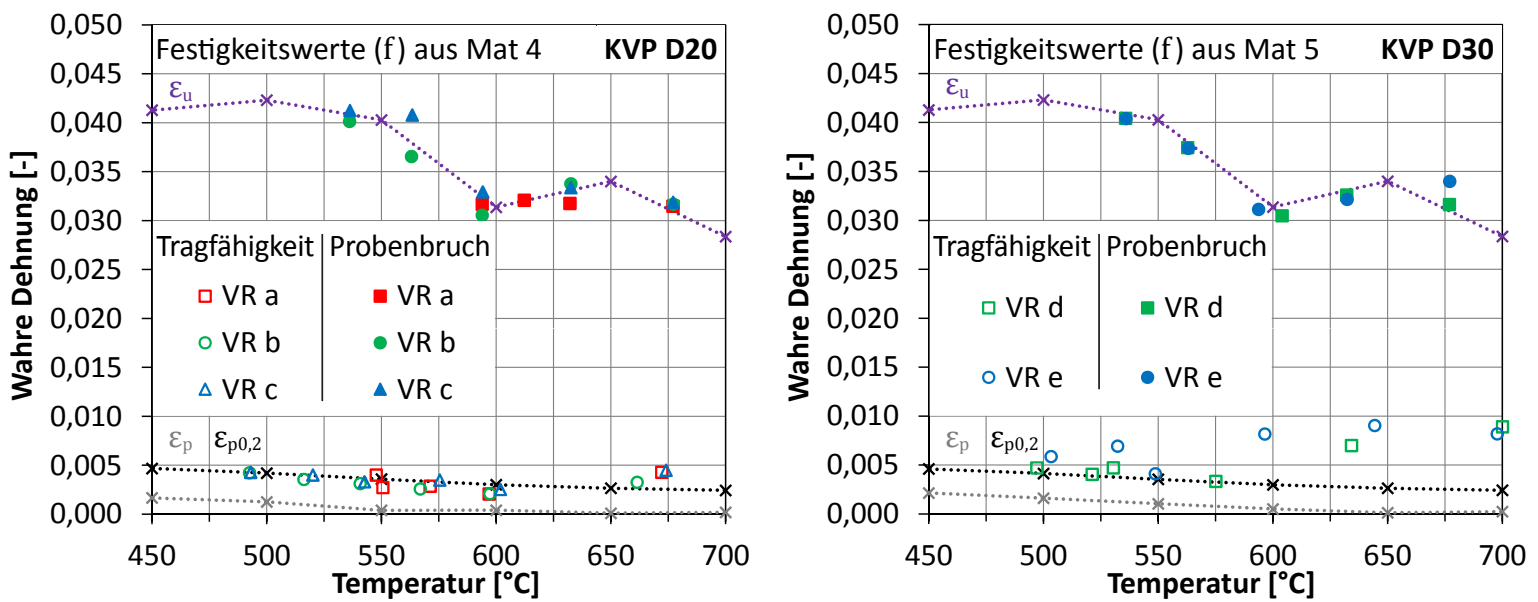

Abb. 5.14: Vergleich der an der Versagensstelle berechneten Dehnungen mit den Stahlfestigkeiten aus den Materialuntersuchungen 


\subsection{Möglichkeiten zur Bestimmung der Feuerwiderstandsdauer für Stahlzugglieder mit reaktiver Brandschutzbeschichtung}

\subsubsection{Variante 1 - Brandversuch an mechanisch belasteten Zuggliedern}

Die Grundlage für die Bestimmung der Feuerwiderstandsdauer bilden Brandversuche an mechanisch belasteten Zuggliedern mit reaktiver Brandschutzbeschichtung (siehe Kapitel 3.5). Für die Berechnung der Zugbeanspruchung des Zuggliedes, d.h. Lastausnutzungsgrad im Brandfall, wird die bei Raumtemperatur vorhandene Streckgrenze bzw. 0,2\%-Dehngrenze des verwendeten Stahls benötigt. Diese kann dem Abnahmeprüfzeugnis des Stahlherstellers entnommen oder anhand eines Kleinzugversuches nach Kapitel 4.5.1 bestimmt werden. Aus dem Brandversuch werden der zeitliche Verlauf der Stablängsverformung des Zuggliedes sowie der maximalen Stahltemperatur bestimmt. Für das getestete Zugglied werden die Leistungskriterien der Tragfähigkeit nach Gleichung (3.4) und (3.15) berechnet. Anschließend wird unter Verwendung der gemessenen Stablängsverformung und der berechneten Leistungskriterien der Zeitpunkt für das Erreichen der Tragfähigkeit $\left(t_{\text {Trag }}\right)$ bestimmt. Mittels des Zeitpunktes des Erreichens der Tragfähigkeit kann dann die Bestimmung der Feuerwiderstandsdauer $\left(t_{F}\right)$ erfolgen. Der Ablauf des beschriebenen Verfahrens ist schematisch in Abb. 5.15 dargestellt. Durch eine weiterführende Auswertung können unter Verwendung der gemessenen maximalen Stahltemperaturen Tabellen für die erforderliche Trockenschichtdicke des reaktiven Brandschutzsystems erstellt werden [01].

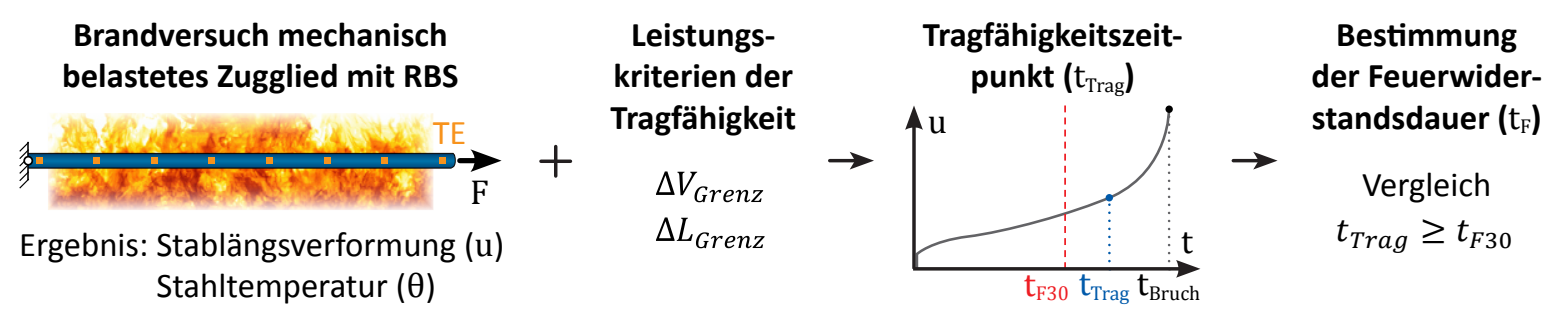

Abb. 5.15: Schematische Darstellung für die Bestimmung der Feuerwiderstandsdauer nach Variante 1

Der Vorteil dieser Variante ist, dass mit Ausnahme der Kennwerte der Stahlfestigkeit bei Raumtemperatur keine weiteren Materialeigenschaften des Stahls erforderlich sind. Materialbedingte Unsicherheiten werden dadurch auf ein Minimum reduziert. Das beschriebene Verfahren besitzt somit die größtmögliche Zuverlässigkeit und Sicherheit bei der Bestimmung der Feuerwiderstandsdauer für Stahlzugglieder mit reaktiver Brandschutzbeschichtung. Die Brandprüfungen an mechanisch belasteten Zuggliedern entsprechen am besten der tatsächlichen Situation der Bauteile in der Baupraxis. Nachteilig ist der hohe Prüfaufwand bei der Durchführung von Brandversuchen an mechanisch belasteten Zuggliedern. Des Weiteren kann mit einer Brandprüfung lediglich ein einzelner Lastausnutzungsgrad im Brandfall untersucht werden. 


\subsubsection{Variante $\mathbf{2}$ - Brandversuch an mechanisch unbelasteten Zuggliedern}

Die Grundlage dieser Variante ist ein Brandversuch an einem mit reaktivem Brandschutzsystem beschichteten Zugglied ohne mechanische Zugbeanspruchung (siehe Kapitel 3.5). Anhand des Brandversuchs wird der zeitliche Verlauf der maximalen Stahltemperatur ermittelt und ein numerisches Modell des Zuggliedes erstellt. Dabei kann auf das in Kapitel 5.3 beschriebene Bemessungsmodell zurückgegriffen werden. Für die numerische Simulation werden die Materialeigenschaften des für das Zugglied verwendeten Stahls bei Raumtemperatur und erhöhter Temperatur benötigt. Vereinfachend können bei kleinen Stabdurchmessern, d.h. $d \leq 50 \mathrm{~mm}$, die im Brandversuch gemessenen Stahltemperaturen über den Querschnitt als konstant angenommen werden. Eine numerische Temperaturfeldberechnung entfällt dadurch. Wird eine Temperaturfeldberechnung durchgeführt, so können die thermischen Materialeigenschaften aus DIN EN 1993-1-2 [13] verwendet werden. Für die Temperturfeldberechnung sind die im Brandversuch gemessenen Stahltemperaturen lediglich auf die Mantelfläche des Zuggliedes aufzubringen.

Für die Berechnung der Zugbeanspruchung des Zuggliedes, d.h. Lastausnutzungsgrad im Brandfall, wird die bei Raumtemperatur vorhandene Streckgrenze bzw. 0,2\%-Dehngrenze des verwendeten Stahls benötigt. Diese kann dem Abnahmeprüfzeugnis des Stahlherstellers oder anhand eines Kleinzugversuches nach Kapitel 4.5.1 bestimmt werden. Die mechanischen Materialeigenschaften des verwendeten Stahls sind für den Hochtemperaturbereich zu ermitteln. Wird für das Zugglied gezogener, kaltverformter Blankstahl der Festigkeitsklasse S355 verwendet, können alternativ die in Kapitel 5.3.3 vorgestellten Werte des Materialmodells (Mat 4) verwendet werden. Sind bei Raumtemperatur Unterschiede in den Festigkeitskennwerten zwischen dem Materialmodell Mat 4 und dem verwendeten Stahl vorhanden, so ist eine Anpassung der Spannungs-Dehnungs-Kurven nach Kapitel 5.3.3 vorzunehmen.

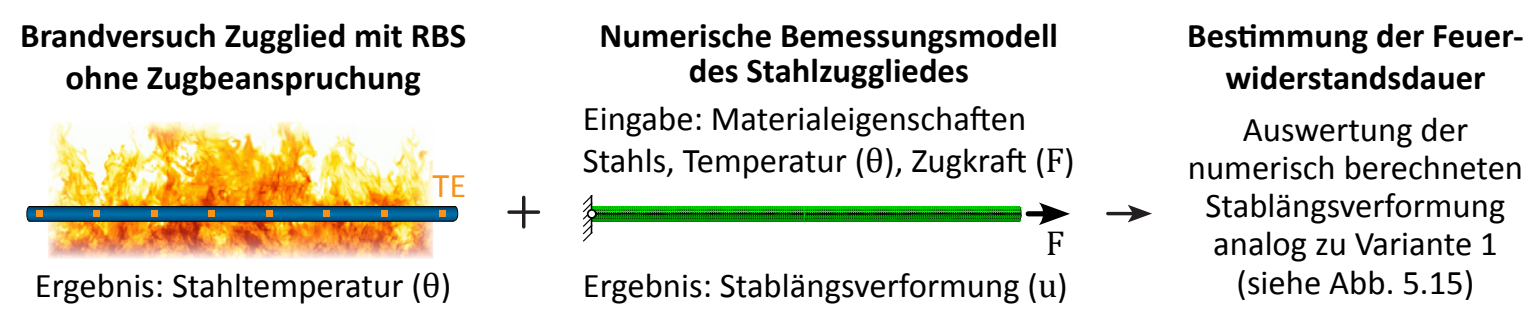

Abb. 5.16: Schematische Darstellung für die Bestimmung der Feuerwiderstandsdauer nach Variante 2

Mit Hilfe des erstellten numerischen Modells kann für verschiedene Zugbeanspruchungen der zeitliche Verlauf der Stablängsverformungen berechnet werden. Für das getestete Zugglied sind die Leistungskriterien der Tragfähigkeit nach Gleichung (3.4) und (3.15) zu berechnen. Anschließend wird unter Verwendung der numerisch ermittelten Stablängsverformung und berechneten Leistungskriterien der Tragfähigkeit der Zeitpunkt für das Errei- 
chen der Tragfähigkeit ( $t_{\text {Trag }}$ ) bestimmt. Eine Einordnung in die entsprechende Feuerwiderstandsklasse ist mittels des Vergleichs mit der erforderlichen Feuerwiderstandsdauer $\left(t_{F}\right)$ möglich. Mittels einer weiterführenden Auswertung können unter Verwendung der gemessenen maximalen Stahltemperaturen Tabellen für die erforderliche Trockenschichtdicke des reaktiven Brandschutzsystems erstellt werden [01].

Vorteil dieses Verfahrens ist, dass nur Brandversuche an Zuggliedern ohne mechanische Beanspruchung erforderlich sind, die vom Prüfaufwand wesentlich einfacher zu realisieren sind als mit mechanischer Zugbeanspruchung. Das numerische Bemessungsmodell erlaubt zudem die Untersuchung beliebiger Zugbeanspruchungen, wodurch eine Optimierung des Zuggliedes hinsichtlich des Lastausnutzungsgrades vorgenommen werden kann. Um das Verfahren der Variante 2 anwenden zu können, müssen die mechanischen Materialeigenschaften des verwendeten Stahls bei Raumtemperatur und erhöhter Temperatur bekannt sein. Da sich die genaue und eindeutige Bestimmung der mechanischen Materialeigenschaften des Stahls schwierig gestaltet, ist das Auswertungsverfahren immer mit einem gewissen Unsicherheitsgrad behaftet. Zudem wird unterstellt, dass die mechanische Zugbeanspruchung keinen Einfluss auf die Höhe der gemessenen maximalen Stahltemperatur hat. Die Untersuchungen in Kapitel 3.7.5 haben jedoch gezeigt, dass dies nur unter bestimmten Randbedingungen, d.h. für das hier untersuchte reaktive Brandschutzsystem bei einer hohen Trockenschichtdicke und einem Stabdurchmesser von mindestens $30 \mathrm{~mm}$, zutrifft. Im Allgemeinen treten an mechanisch belasteten Zuggliedern höhere Stahltemperaturen auf als an unbelasteten Versuchskörpern. Brandprüfungen an Zuggliedern ohne mechanische Beanspruchung entsprechen zudem nicht der tatsächlichen Situation der Bauteile in der Baupraxis. Die aus Brandversuchen an Zuggliedern ohne mechanische Beanspruchung gemessenen Temperaturen sind deshalb mit einem Korrekturfaktor zu erhöhen. Die Berechnung dieses Korrekturfaktors setzt jedoch Brandprüfungen an mechanisch belasteten und unbelasteten Zuggliedern voraus. Die Bestimmung eines Korrekturfaktors ist derzeit normativ nicht geregelt und gestaltet sich aufgrund der teilweise in den Brandversuchen aufgetretenen relativ großen Streuungen schwierig (siehe Kapitel 3.5). Die Anwendung dieses Verfahrens ist aufgrund der genannten Nachteile und Unsicherheiten derzeit nur theoretisch möglich.

\subsubsection{Variante $\mathbf{3}$ - Auswertung mittels Bemessungsfestigkeit}

Wie in Variante 1 bereits beschrieben, bilden Brandversuche an mechanisch belasteten oder unbelasteten Zuggliedern mit reaktiver Brandschutzbeschichtung die Grundlage für die Bestimmung des Feuerwiderstandes (siehe Kapitel 3.5). Aus dem Brandversuch wird der zeitliche Verlauf der maximalen Stahltemperatur bestimmt. Für die Berechnung der Zugbeanspruchung des Zuggliedes $\left(\sigma_{\mathrm{F}}\right)$ wird die bei Raumtemperatur vorhandene 0,2\%-Dehngrenze des verwendeten Stahls sowie der Lastausnutzungsgrad im Brandfall benötigt. Die 0,2\%-Dehngrenze kann dem Abnahmeprüfzeugnis des Stahlherstellers entnommen oder 
anhand eines Kleinzugversuches nach Kapitel 4.5.1 bestimmt werden. Des Weiteren sind die Festigkeitskennwerte der 0,2\%-Dehngrenze unter erhöhter Temperatur erforderlich. Wird für das Zugglied gezogener, kaltverformter Blankstahl der Festigkeitsklasse S355 verwendet, können die Werte der 0,2\%-Dehngrenze unter erhöhter Temperatur auch anhand der temperaturabhängigen Abminderungsfaktoren des Materialmodells Mat 4 berechnet werden (siehe Tabelle 5.5).

Für die Auswertung wird ein Spannungsniveau des Zuggliedes definiert. Dieses kann mit Hilfe des Lastausnutzungsgrades im Brandfall und der bei Raumtemperatur vorhandenen 0,2\%-Dehngrenze des verwendeten Stahls berechnet werden. Durch den Vergleich des Spannungsniveaus mit der temperaturabhängigen Festigkeit der 0,2\%-Dehngrenze kann eine Grenztemperatur $\left(\theta_{\text {grenz }}\right)$ ermittelt werden. Anhand der Untersuchungen aus Kapitel 5.3.5 wird angenommen, dass die Tragfähigkeit des Zuggliedes mit Erreichen der 0,2\%-Dehngrenze endet. Mittels des Vergleichs der Grenztemperatur mit der im Brandversuch gemessenen maximalen Stahltemperatur wird der Zeitpunkt des Erreichens der Tragfähigkeit $\left(t_{\text {Trag }}\right)$ bestimmt. Die Feuerwiderstandsklasse ergibt sich aus dem Vergleich des Zeitpunktes der Tragfähigkeit mit der Feuerwiderstandsdauer $\left(t_{\mathrm{F}}\right)$. Mittels einer weiterführenden Auswertung können unter Verwendung der gemessenen maximalen Stahltemperaturen Tabellen für die erforderliche Trockenschichtdicke des reaktiven Brandschutzsystems erstellt werden [01].
Tabelle 5.5: Bemessungsfestigkeit in Abhängigkeit von der Stahltemperatur

\begin{tabular}{|c|c|}
\hline $\begin{array}{c}\text { Stahl- } \\
\text { temperatur } \\
{\left[{ }^{\circ} \mathrm{C}\right]}\end{array}$ & $\begin{array}{c}\text { Abminderungs- } \\
\text { faktor für die } \\
\mathbf{0 , 2 \%} \begin{array}{c}\text {-Dehngrenze } \\
\left(\mathrm{k}_{\mathrm{p} 0,2}\right)\end{array}\end{array}$ \\
\hline 20 & 1,000 \\
\hline 100 & 0,984 \\
\hline 200 & 1,054 \\
\hline 250 & 1,072 \\
\hline 300 & 1,020 \\
\hline 350 & 0,974 \\
\hline 400 & 0,890 \\
\hline 450 & 0,770 \\
\hline 500 & 0,613 \\
\hline 550 & 0,422 \\
\hline 600 & 0,256 \\
\hline 650 & 0,150 \\
\hline 700 & 0,090 \\
\hline
\end{tabular}

* Werte beziehen sich auf gezogenen kaltverformten Blankstahl der Festigkeitsklasse S355.

Die Bemessungsfestigkeit berechnet sich aus der 0,2\%-Dehngrenze des verwendeten Stahls bei $20{ }^{\circ} \mathrm{C}$ multipliziert mit dem Abminderungsfaktor $\mathrm{k}_{\mathrm{p} 0,2}$.

Da bei dieser Variante der Auswertung kein zeitabhängiger Verlauf der Stablängsverformungen des Zuggliedes erforderlich ist, eignet sich das Verfahren besonders für Brandversuche an Zuggliedern ohne mechanische Beanspruchung. Für die Bestimmung der Feuerwiderstandsdauer wird lediglich der gemessene Verlauf der maximalen Stahltemperatur sowie die temperaturabhängigen Werte der 0,2\%-Dehngrenze des verwendeten Stahls benötigt. Für Zugglieder aus gezogenem, kaltverformten Blankstahl der Festigkeitsklasse S355 können die Werte der 0,2\%-Dehngrenze anhand der temperaturabhängigen Abminderungsfaktoren des Materialmodells (Mat 4) relativ einfach berechnet werden (siehe Tabelle 5.5). Dieses Verfahren bietet zudem die Möglichkeit, den Tragfähigkeitszeitpunkt des Zuggliedes 
für verschiedene Lastausnutzungsgrade im Brandfall relativ einfach zu bestimmen. Dabei wird das Zugglied bis zur Bemessungsfestigkeit des Stahls, d.h. 0,2\%-Dehngrenze, ausgenutzt. Eine genaue Bestimmung der 0,2\%-Dehngrenze sowie der dazugehörigen temperaturabhängigen Abminderungsfaktoren ist dafür erforderlich. Ferner wird bei Verwendung von Temperaturdaten aus Brandversuchen an unbelasteten Zuggliedern unterstellt, dass die mechanische Zugbeanspruchung keinen Einfluss auf die Höhe der gemessenen maximalen Stahltemperatur hat. Untersuchungen (siehe Kapitel 3.7.5) haben jedoch gezeigt, dass dies meist nicht gegeben ist. Im Allgemeinen treten an mechanisch belasteten Zuggliedern höhere Stahltemperaturen auf als an unbelasteten Versuchskörpern. Brandprüfungen an Zuggliedern ohne mechanische Beanspruchung entsprechen zudem nicht der tatsächlichen Situation der Bauteile in der Baupraxis. Die in Brandversuchen an Zuggliedern ohne mechanische Beanspruchung gemessenen Temperaturen sind daher mit einem Korrekturfaktor zu erhöhen. Die Berechnung dieses Korrekturfaktors setzt wiederum Brandprüfungen an mechanisch belasteten und unbelasteten Zuggliedern voraus. Die Bestimmung eines Korrekturfaktors ist derzeit normativ nicht geregelt und gestaltet sich aufgrund der teilweise in den Brandversuchen aufgetretenen relativ großen Streuungen schwierig (siehe Kapitel 3.5). Die Anwendung dieses Verfahrens ist aufgrund der genannten Nachteile und Unsicherheiten derzeit nur theoretisch möglich.

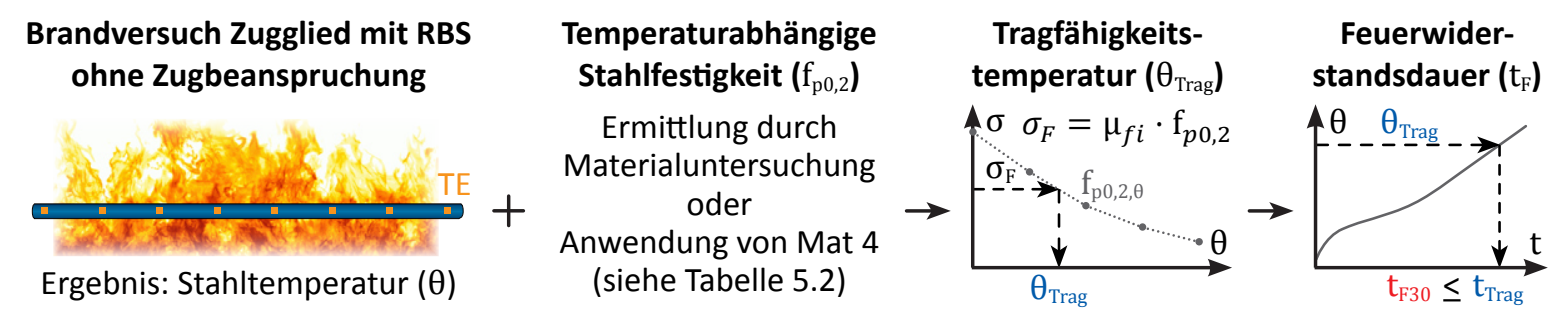

Abb. 5.17: Schematische Darstellung für die Bestimmung der Feuerwiderstandsdauer nach Variante 3 



\section{Zusammenfassung, Schlussfolgerung und Ausblick}

\subsection{Zusammenfassung und Schlussfolgerung}

Unter Brandeinwirkung verlieren ungeschützte Stahlkonstruktionen aufgrund der schnellen Erwärmung frühzeitig ihre Tragfähigkeit. Durch den Einsatz reaktiver Brandschutzsysteme kann die Erwärmung verlangsamt und der Feuerwiderstand verbessert werden. Die Optik der filigranen Zugglieder bleibt im Wesentlichen unverändert.

Für die Anwendung reaktiver Brandschutzsysteme auf Stahlzuggliedern mit Vollquerschnitt gibt es derzeit in den allgemeinen bauaufsichtlichen Zulassungen keine Regelungen. Die durchgeführten experimentellen und numerischen Untersuchungen [35], sowie die darauf aufbauenden Erkenntnisse hinsichtlich des Verhaltens reaktiver Brandschutzsysteme auf Stahlzuggliedern mit Vollprofil, bilden die Grundlage für die Erweiterung des Anwendungsbereiches dieser Produkte. Grundsätzlich ist eine Anwendung möglich. Aufgrund der meist filigranen Vollquerschnitte sowie der in Bezug auf die Tragfähigkeit fehlenden Umlagerungsmöglichkeit werden besonders hohe Anforderungen an die Wirksamkeit von reaktiven Brandschutzsystemen gestellt. Die Brandversuche sowie theoretischen Betrachtungen haben gezeigt, dass das auf kreisförmigen, zugbeanspruchten Stahlprofilen aufgebrachte reaktive Brandschutzsystem aufgrund der notwendigen dreidimensionalen Ausdehnungsrichtung der höchstmöglichen Beanspruchung ausgesetzt ist. Ein Vergleich zwischen der Biegezugzone eines mechanisch belasteten Stahlträgers mit I-Profil und einem belasteten Zugglied mit Kreisvollquerschnitt ist aufgrund fehlender wissenschaftlich fundierter Grundlagen nicht möglich. Zuverlässige Aussagen zur Feuerwiderstandsdauer von Stahlzuggliedern mit reaktiver Brandschutzbeschichtung können einzig durch Brandprüfungen mit mechanischer Zugbeanspruchung getroffen werden.

Als Kriterium für die Beurteilung der thermischen Schutzwirkung des reaktiven Brandschutzsystems sind die Erwärmungsgeschwindigkeit, die maximale Stahltemperatur sowie die Rissbildung und das Rissheilungsvermögen der Beschichtung maßgeblich. Die Brandversuche haben gezeigt, dass die Profilgeometrie, die Höhe der Trockenschichtdicke der Beschichtung, die Höhe der aufgebrachten Zugbeanspruchung sowie die Orientierungsrichtung der Zugglieder einen wesentlichen Einfluss auf die thermische Schutzwirkung des reaktiven Brandschutzsystems ausüben und in gegenseitiger Wechselwirkung stehen. Durch Vergrößerung des Stabdurchmessers, Erhöhung der Trockenschichtdicke der Brandschutzbeschichtung, Verringerung des Profilfaktors oder Reduzierung der Zugbeanspruchung lässt sich im Allgemeinen die Feuerwiderstandsdauer des Zuggliedes verbessern. Die thermische Schutzwirkung des reaktiven Brandschutzsystems auf Zuggliedern mit horizontaler Ausrichtung ist in der Regel besser als bei vertikaler Probenausrichtung. Durch Abstimmung der genannten Einflussfaktoren lässt sich die Wirkung der Brandschutzbeschichtung bis zu bestimmten Anwendungsgrenzen optimieren. Bei Brandschutzbeschichtungen mit einer 
relativ hohen Trockenschichtdicke führt eine weitere Erhöhung der Schichtdicke kaum noch zu einer signifikanten Zunahme der Feuerwiderstandsdauer. Die angestellten theoretischen Überlegungen zum Aufschäum- und Rissverhalten des reaktiven Brandschutzsystems konnten durch die Brandversuche bestätigt werden. Mechanisch belastete Zugglieder neigen aufgrund der erforderlichen dreidimensionalen Ausdehnungsrichtung der Brandschutzbeschichtung in der Regel zu einer ausgeprägteren Rissbildung. Folglich ergeben sich höhere Stahltemperaturen als bei Zuggliedern ohne mechanische Beanspruchung. Um die thermische Schutzwirkung von reaktiven Brandschutzsystemen zu beurteilen, sind mechanisch belastete Brandversuche unerlässlich. Die Festlegung eines Korrekturfaktors zwischen den Temperaturdaten an mechanisch belasteten und unbelasteten Zuggliedern gestaltet sich aufgrund des zufälligen und kaum vorhersehbaren Aufschäumungs- und Rissverhaltens des reaktiven Brandschutzsystems schwierig und ist zum gegenwärtigen Zeitpunkt offen.

Durch die Vielzahl der Produkte reaktiver Brandschutzsysteme mit variierenden chemischen Zusammensetzungen ist deren Aufschäumverhalten und thermische Schutzwirkung sehr unterschiedlich und schwer einschätzbar. Die Wirksamkeit eines reaktiven Brandschutzsystems ist deshalb für Grenzfälle des vom Hersteller definierten Anwendungsbereiches durch eine ausreichende Anzahl an Brandversuchen zu überprüfen. Dies gilt besonders für Profile mit filigranem Querschnitt, vergleichsweise dünnen Trockenschichtdicken des reaktiven Brandschutzsystems sowie für Bauteile mit hoher Zugbeanspruchung. Empfehlungen zur Durchführung dieser Versuche sind im Forschungsbericht [35] beschrieben.

Die Bestimmung der Feuerwiderstandsdauer für Stahlzugglieder mit reaktiver Brandschutzbeschichtung kann mit Hilfe entsprechender Auswertungsverfahren erfolgen. Grundlage dieser Verfahren bilden Brandversuche an mechanisch belasteten und unbelasteten Zuggliedern. Die für die Auswertung verwendeten Leistungskriterien der Tragfähigkeit für Zugglieder orientieren sich an den bereits vorhandenen Kriterien aus den etablierten Brandprüfungen an Stahlträgern und -stützen. Die Anwendung der Auswertungsverfahren auf Brandversuche an unbelasteten Zuggliedern ist derzeit nur theoretisch möglich, da ein Korrekturfaktor für die Temperaturdaten, welcher die fehlende Zugbeanspruchung im Brandversuch berücksichtigt, aus den zuvor genannten Gründen nicht vorliegt und zudem genaue Kenntnisse der Materialeigenschaften des verwendeten Stahls erforderlich sind.

Die in den Brandversuchen verwendeten Zugglieder mit Kreisvollprofil, welche mit und ohne mechanischer Zugbeanspruchung getestet wurden, bestehen aus gezogenem, kaltverformten Blankstahl der Festigkeitsklasse S355 und haben aufgrund des Herstellungsverfahrens, d.h. Ziehen, andere Materialeigenschaften als der üblicherweise im Bauwesen für Stahlträger und -stützen eingesetzte warmgewalzte Baustahl. In der Norm [12] sind Materialkennwerte für den mechanischen Hochtemperaturbereich lediglich für warmgewalzten Baustahl und kaltgeformte dünnwandige Stahlbauteile der Querschnittsklasse 4 enthalten. Anhand von stationären Kleinzugversuchen wird das temperaturabhängige Verhalten für den Elasti- 
zitätsmodul, die Proportionalitätsgrenze, die 0,2\%-Dehngrenze, die Zugfestigkeit sowie den Wärmeausdehnungskoeffizienten des Blankstahls bestimmt. Der in der Norm [12] für warmgewalzten Baustahl angegebene temperaturabhängige Verlauf des Wärmeausdehnungskoeffizienten liegt wesentlich unterhalb der für den kaltverformten Blankstahl ermittelten Werte. Ursache hierfür ist mit hoher Wahrscheinlichkeit das Fertigungsverfahren des Blankstahls und die aus Kaltverfestigung resultierende Veränderung des Gefüges des Metallgitteraufbaus. Durch den Ziehvorgang beim Herstellungsprozess besitzt der Blankstahl keine ausgeprägte Streckgrenze und kein Fließplateau. Die Festigkeitskennwerte und der Elastizitätsmodul des untersuchten gezogenen, kaltverformten Blankstahls weichen teilweise erheblich von den Werten für warmgewalzten und kaltverformten Stahl nach Norm [13] ab. Dies ist ebenfalls auf die Kaltverfestigung zurückzuführen. Die ermittelten Abminderungsfaktoren für die Proportionalitätsgrenze, d.h. Ende des elastischen Spannungs-DehnungsBereichs, sind für kaltverformten Blankstahl in der Regel wesentlich höher, als für warmgewalzten Stahl nach Norm. Ab etwa $300^{\circ} \mathrm{C}$ gilt diese Aussage auch für den Elastizitätsmodul. Dies bedeutet, dass der Blankstahl im elastischen Bereich länger seine Festigkeit behält. Im Gegensatz dazu zeigt sich für die 0,2\%-Dehngrenze des getesteten Blankstahls ab einer Stahltemperatur von etwa $400^{\circ} \mathrm{C}$ gegenüber den Werten für warmgewalzten Baustahl eine deutliche Reduzierung. Der Blankstahl verliert demnach mit steigender Temperatur schneller an Festigkeit. Die nach Norm [13] und in der Literatur [45], [48], [63] für kaltverformte dünnwandige Stahlbauteile angegebenen temperaturabhängigen Abminderungswerte der Proportionalitätsgrenze und der 0,2\%-Dehngrenze liegen meist deutlich unter den Werten des untersuchten Blankstahls. Dies könnte auf unterschiedliches Ausgangsmaterial, d.h. dünne Bleche bzw. Vollprofil sowie die in den Zugversuchen verwendeten Probengeometrien, d.h. Flachproben und Rundprobe, zurückzuführen sein. Für die temperaturabhängigen Abminderungsfaktoren der Zugfestigkeit zeigt sich zwischen den untersuchten kaltverformten Blankstahl und den in der Norm sowie Literatur angegeben Werten nur ein vergleichsweise geringer Unterschied. Um eine hohe Sicherheit und Genauigkeit bei der Bemessung oder Nachrechnung von Bauteilen aus gezogenem, kaltverformten Blankstahl zu erhalten, wird die Anwendung der in den Materialuntersuchengen ermittelten temperaturabhängigen Abminderungsfaktoren empfohlen.

Unter Verwendung der Ergebnisse aus den an mechanisch belasteten Zuggliedern mit Kreisvollprofil durchgeführten Brandversuchen und den in Materialuntersuchungen bestimmten Hochtemperatureigenschaften des verwendeten Blankstahls wird auf Grundlage der FiniteElemente-Methode das bereits aus dem Forschungsvorhaben [35] vorhandene numerische Bemessungsmodell weiterentwickelt und spezifiziert. Die numerische Nachrechnung der durchgeführten Brandversuche zeigt eine gute Übereinstimmung zwischen der im Brandversuch gemessenen und numerisch berechneten Stablängsverformung der Zugglieder. Das Bemessungsmodell ist in der Lage, das Verformungsverhalten mechanisch belasteter Zugglieder mit reaktiver Brandschutzbeschichtung im Brandversuch abzubilden. Darüber hinaus bestätigt das numerische Modell die Zuverlässigkeit und Korrektheit der auf den 
Materialuntersuchungen basierenden Materialeigenschaften. Mittels Annahmen zur thermischen Schutzwirkung des reaktiven Brandschutzsystems und der sich daraus für das Stahlbauteil ergebenden Stahltemperaturen ist es mit Hilfe des Bemessungsmodells prinzipiell möglich, die Feuerwiderstandsdauer von Zuggliedern zu berechnen. Allerdings setzt dies die Richtigkeit der verwendeten Temperaturdaten sowie Materialeigenschaften des Stahls voraus. Da mechanisch belastete Zugglieder in der Regel höhere Stahltemperaturen aufweisen als Zugglieder ohne mechanische Beanspruchung ist eine Korrektur der Temperaturdaten aus Brandversuchen an mechanisch unbelasteten Bauteilen erforderlich. Die Bestimmung eines entsprechenden Korrekturfaktors ist derzeit normativ nicht geregelt und gestaltet sich aufgrund der teilweise in den Brandversuchen aufgetretenen relativ großen Streuungen zwischen Zuggliedern mit und ohne mechanische Beanspruchung schwierig. Die Anwendung des entwickelten Bemessungsmodells ist deshalb mit Unsicherheiten verbunden und derzeit nur theoretisch möglich. 


\subsection{Ausblick}

Die in der Dissertation durchgeführten Untersuchungen erfolgten aus Gründen der Vergleichbarkeit mit einem einzigen reaktiven Brandschutzsystem eines Herstellers. Die gewonnenen Erkenntnisse sind anhand von Produkten anderer Hersteller zu überprüfen. Ebenso ist zu untersuchen, ob die ausschließlich an Stahlzuggliedern mit relativ filigranen Maßen und kreisförmigen Querschnitt, insbesondere Vollprofile, getroffenen Aussagen sich auf andere Profile, z.B. Rechteckvollprofile, übertragen lassen.

Des Weiteren bedarf es hinsichtlich der Randbedingungen in den Brandversuchen weiterer Forschung. Vorerst wurden fast ausschließlich bauteilspezifische Einflussfaktoren untersucht. Eine mögliche Beeinflussung des reaktiven Brandschutzsystems durch Variation der Probenausrichtung, z.B. schräge Orientierung, die Position der Ölbrenner und Brandraumthermoelemente, die Art und Applikation der Thermoelemente, der Zeitpunkt der Lastaufbringung, die Trockenzeitdauer der Brandschutzbeschichtung sowie weiterer Faktoren wurden in den Untersuchungen nur im Ansatz betrachtet.

Unter Verwendung des neu entwickelten und validierten numerischen Bemessungsmodells könnte die Feuerwiderstandsdauer von Zuggliedern mit reaktiver Brandschutzbeschichtung auch anhand von Brandversuchen ohne mechanische Zugbeanspruchung berechnet werden. Die Entwicklung eines dafür erforderlichen Korrekturfaktors zwischen den Temperaturdaten aus Brandversuchen an Zuggliedern mit und ohne mechanischer Zugbeanspruchung bedarf jedoch weiterer Untersuchungen.

Außerdem wurde ausschließlich das Verhalten reaktiver Brandschutzsysteme auf den Zuggliedern selbst, jedoch nicht die Anschlussbereiche zur umgebenden Tragkonstruktion untersucht. Diese besitzt zwar gegenüber dem Zugglied in der Regel eine höhere Massigkeit, welche sich positiv auf die Feuerwiderstandsdauer auswirkt, jedoch könnte besonders bei vertikalen Zuggliedern an den Verbindungsstellen ein Abgleiten des reaktiven Brandschutzsystems auftreten. Die daraus resultierende lokale Reduzierung der thermischen Schutzwirkung des reaktiven Brandschutzsystems kann die Feuerwiderstandsdauer des gesamten Zuggliedes verschlechtern.

Die vorliegenden Ergebnisse und Erkenntnisse dienen nicht nur als Grundlage für die Erweiterung des Anwendungsbereiches reaktiver Brandschutzsysteme auf Stahlzugglieder, sondern finden auch auf europäischer und internationaler Ebene Eingang in die Normungsarbeit [18], [19]. Ziel ist die Entwicklung einer Norm für die Durchführung und Bewertung von Brandprüfungen an Stahlzuggliedern mit reaktiver Brandschutzbeschichtung. 



\section{Literaturverzeichnis}

Normen, Richtlinien und Zulassungen

[01] AbZ Nr. Z-19.11-2194: Allgemeine bauaufsichtliche Zulassung für das reaktive Brandschutzsystem "Hensotherm 420 KS" zur Anwendung auf Stahlzuggliedern. Deutsches Institut für Bautechnik, Berlin, 2015.

[02] AbZ Nr. Z-200.4-15: Allgemeine bauaufsichtliche Zulassung für das reaktive Brandschutzsystem "Hensotherm 420 KS". Deutsches Institut für Bautechnik, Berlin, 2013.

[03] DIN 4102-2: Brandverhalten von Baustoffen und Bauteilen. Teil 2: Bauteile. Begriffe, Anforderungen und Prüfungen. Beuth Verlag, Berlin, September 1977.

[04] DIN 4102-8: Brandverhalten von Baustoffen und Bauteilen. Teil 8: Kleinprüfstand. Beuth Verlag, Berlin, Oktober 2003.

[05] DIN EN 10020: Begriffsbestimmung für die Einteilung der Stähle. Beuth Verlag, Berlin, Juli 2000.

[06] DIN EN 10079: Begriffsbestimmungen für Stahlerzeugnisse. Beuth Verlag, Berlin, Juni 2007.

[07] DIN EN 1363-1: Feuerwiderstandsprüfungen. Teil 1: Allgemeine Anforderungen. Beuth Verlag, Berlin, Oktober 2012.

[08] DIN EN 1363-2:1999 Feuerwiderstandsprüfungen. Teil 2: Alternative und ergänzende Verfahren. Beuth Verlag, Berlin, Oktober 1999.

[09] DIN EN 13381-8: Prüfverfahren zur Bestimmung des Beitrages zum Feuerwiderstand von tragenden Bauteilen - Teil 8: Reaktive Ummantelung von Stahlbauteilen. Beuth Verlag, Berlin, August 2013.

[10] DIN EN 13501-2: Klassifizierung von Bauprodukten und Bauarten zu ihrem Brandverhalten - Teil 2: Klassifizierung mit den Ergebnissen aus den Feuerwiderstandsprüfungen, mit Ausnahme von Lüftungsanlagen. Beuth Verlag, Berlin, Februar 2010.

[11] DIN EN 1991-1-2: Einwirkungen auf Tragwerke. Teil 1-2 Allgemeine Einwirkungen Brandeinwirkungen auf Tragwerke. Beuth Verlag, Berlin, Dezember 2010.

[12] DIN EN 1992-1-2: Bemessung und Konstruktion von Stahlbeton- und Spannbetontragwerken. Teil 1-2: Allgemeine Regeln - Tragwerksbemessung für den Brandfall. Beuth Verlag, Berlin, Dezember 2010.

[13] DIN EN 1993-1-2: Bemessung und Konstruktion von Stahlbauten. Teil 1-2: Allgemeine Regeln-Tragwerksbemessung für den Brandfall. Beuth Verlag, Berlin, Dezember 2010.

[14] DIN EN ISO 6892-1: Metallische Werkstoffe - Zugversuch. Teil 1: Prüfverfahren bei Raumtemperatur. Beuth Verlag, Berlin, Dezember 2009.

[15] DIN EN ISO 6892-2: Metallische Werkstoffe - Zugversuch. Teil 2: Prüfverfahren bei erhöhter Temperatur. Beuth Verlag, Berlin, Mai 2011. 
[16] DIN EN ISO 8501-1: Vorbereitung von Stahloberflächen vor dem Auftragen von Beschichtungsstoffen - Visuelle Beurteilung der Oberflächenreinheit. Teil 1: Rostgrade und Oberflächenvorbereitungsgrade von unbeschichteten Stahloberflächen und Stahloberflächen nach ganzflächigem Entfernen vorhandener Beschichtungen. Beuth Verlag, Berlin, Dezember 2007.

[17] ETA-12/0050: European Technical Approval for the reactive fire protection system "Hensotherm 420 KS". Warrington Certification Limited, Warrington, 2013.

[18] pr EN 13381-10: Test methods for determining the contribution to the fire resistance of structural members - Part 10: Applied protection to solid steel rods. CEN - European Committee for Standardization: Technical Committee CEN/TC 127, 2015.

[19] pr ISO/IEC WD 834-14: Fire resistance tests - Elements of building constructions Part 14: Specific requirements for the testing and assessment of applied fire protection to solid steel bar. International Organization for Standardization: Technical Committee ISO/IEC TC92/SC 2, 2015.

Monographien, Handbücher und Aufsätze

[20] ABAQUS ${ }^{\circledast}:$ Abaqus/Standard User's Manual. Version 6.11-1. Hibbit, Karlsson \& Sorensen, Inc., Pawtucket, 2011

[21] Baehr, H. D.; Stephan, K.: Wärme und Stoffübertragung. 3. Auflage, Berlin, Springer Verlag, 1998.

[22] Breternitz, V.; Schawohl, J.: Festigkeitsprüfung - WE 6. Universität IImenau, 2008.

[23] Brux, G.: Brandschutzbeschichtungen auf Stahl - Haltbarkeit und Weiterentwicklungen. Stahlbau, H. 2, S. 142-144, Berlin, Ernst \& Sohn, 2013.

[24] Brux, G: Neue Entwicklungen bei der Beschichtung in der Vorfertigung Dämmschichtbildner für Korrosions- und Brandschutz. Stahlbau, H. 3, S. 243-244, 2012.

[25] Chen, C.; Shen, B.: A Simplified Model for Describing the Effect of Intumescent Coating to Protect Steel Under Fire Conditions. Advanced Science Letters, Vol. 4, S. 1265-1269, 2011.

[26] DIBt - Deutsches Institut für Bautechnik: Zulassungsgrundsätze für reaktive Brandschutzsysteme auf Stahlbauteilen. Fassung November 1997. DIBt Mitteilungen, H. 4, Berlin, Ernst \& Sohn, 1998.

[27] Heinrich, H.: Der Einfluss der Gitterfehler auf die mechanischen Eigenschaften der Metalle. Hochschule Regensburg, Fakultät Maschinenbau, 2001.

[28] Häßler, D.; Hothan, S.: Mechanische Hochtemperatureigenschaften von Stahlzuggliedern aus kaltverformten Blankstahl der Festigkeitsklasse S355. Stahlbau, H. 5, S. 332340, Berlin, Ernst \& Sohn Verlag, 2015. 
[29] Häßler, D.; Hothan, S.: Numerical and experimental analysis of reactive fire protection systems applied to solid steel rods in tension. Applications of structural fire engineering conference, S. 454-460, Prague, 2013.

[30] Häßler, D.; Hothan, S.: Prüfmethodik für reaktive Brandschutzsysteme auf Stahlzuggliedern mit kreisrundem Vollquerschnitt. 19. DASt-Forschungskolloquium, S. 148152, Füssen, Frehner Consulting, 2014.

[31] Holman, J.P.: Heat transfer. Sixth Edition, McGraw-Hill, Tokyo, 1986.

[32] Hothan, S.: Grundlagen zur Erweiterung des Anwendungsbereiches für reaktive Brandschutzsysteme auf Stahlbauteilen im nationalen Zulassungsverfahren. DIBt Mitteilungen, H. 42, S. 187-189, Berlin, Ernst \& Sohn, 2011.

[33] Hothan, S.: Numerische Voruntersuchungen zur Anwendung von reaktiven Brandschutzsystemen auf zugbeanspruchten Stahlbauteilen. Bundesanstalt für Materialforschung und -prüfung (BAM), Berlin, 2010.

[34] Hothan, S.; Häßler, D.: Numerical and experimental analysis of reactive fire protection systems applied to solid steel rods in tension. Präsentation zur CEN/TC127/WG1Sitzung am 17. März 2015, Brüssel, 2015.

[35] Hothan, S.; Häßler, D.: Numerische und versuchstechnische Untersuchung zur Anwendung von reaktiven Brandschutzsystemen auf Zuggliedern aus Stahl. Forschungsbericht, Vh 7539, Bundesanstalt für Materialforschung und -prüfung (BAM), Berlin, 2015.

[36] Hothan, S.; Häßler, D.: Über die Entwicklung von Anwendungsregeln für reaktive Brandschutzsysteme auf Stahlzuggliedern. Festschrift Peter Schaumann, S. 141-148, Hannover, 2014.

[37] Hothan, S.; Häßler, D.: Zur Anwendung reaktiver Brandschutzsysteme auf Stahlzuggliedern. Bauphysik, H. 6, S. 275-285, Berlin, Ernst \& Sohn, 2012.

[38] Mensinger, M.; Kraus, P.: Optimierter Einsatz reaktiver Brandschutzsysteme im Stahlund Verbundbau. Stahlbau, H. 1, S. 10-18, Berlin, Ernst \& Sohn, 2015.

[39] Mensinger, M.; Schaumann, P.; Kraus, P.; Tabeling, F.: Optimierter Einsatz intumeszierender Anstriche im Stahlbau. Forschungsbericht, IGF-Vorhaben 17200 N, Deutscher Ausschuß für Stahlbau e.V. (DASt), Düsseldorf, 2014.

[40] Mensinger, M.; Stadler, M.: Aktualisierte Diagramme zur Bemessung von Stahlkonstruktionen für den Brandfall nach Eurocode 3. Stahlbau, H. 4, S. 253-258, Berlin, Ernst \& Sohn, 2009.

[41] Merkblatt 403 "Blankstahl". Stahl-Informations-Zentrum, Düsseldorf, 2002.

[42] Morys, M., Illerhaus, B., Sturm, H., Schartel, B.: Revealing the inner secrets of intumescent chars by advanced small scale tests combined with $\mu$-CT. Fire and materials - 14th International conference and exhibition, pp. 478-483, San Francisco, 2015. 
[43] Nachschlagewerk Stahlschlüssel 2010 für MS Windows 7. Verlag Stahlschlüssel Wegst GmbH, 2010.

[44] Outinen, J.; Kesti, J.; Mäkeläinen, P.: Fire design model for structural steel S355 based upon transient state tensile test results. Journal of Constructional Steel Research, Vol. 42, pp. 161-169, 1997.

[45] Outinen, J.; Mäkeläinen, P.: Mechanical properties of structural steel at elevated temperatures and after cooling down. Fire and Materials, Vol. 28, pp. 237-251, 2004.

[46] Pettersson, O.; Magnusson, S.-E.; Thor, J.: Brandschutztechnische Bemessung von Stahlkonstruktionen. deutsche Übersetzung, 1977.

[47] Qiang, X.: Behaviour of High Strength Steel Endplate Connections in Fire and after Fire. Dissertation, Department of Structural Engineering, Delft University of Technology, 2013.

[48] Ranawaka, T.; Mahendran, M.: Experimental study of the mechanical properties of light gauge cold-formed steels at elevated temperatures. Fire Safety Journal, Vol. 44, pp. 219-229, 2009.

[49] Raveglia, E.: Grundlagen der Bemessung von intumeszierenden Brandschutzsystemen im Stahlbau. Dissertation, Institut für Baustatik und Konstruktion, Eidgenössische Technische Hochschule (ETH) Zürich, Zürich, 2008.

[50] Richter, F.: Die wichtigsten physikalischen Eigenschaften von 52 Eisenwerkstoffen. Stahleisen - Sonderberichte, Heft 8, Verlag Stahleisen mbH, Düsseldorf, 1973.

[51] Richter, F.: Physikalische Eigenschaften von Stahl und ihre Temperaturabhängigkeit. Stahleisen - Sonderberichte, Heft 10, Verlag Stahleisen mbH, Düsseldorf, 1983.

[52] Rösler J.; Harders H.; Bäker M.: Mechanisches Verhalten der Werkstoffe. 3. Auflage, Verlag Vieweg und Teubner, Wiesbaden, 2008.

[53] Ryan, J. V.; Robertson, A.F.: Proposed criteria for defining load failure of beams, floors, and roof constructions during fire test. Journal of Research of the National Bureau of Standards, p. 121-124, 1959.

[54] Schaumann, P.; Tabeling, F.: Experimentelle und numerische Untersuchungen von reaktiven Brandschutzsystemen im Stahlbau. 18. DASt-Kolloquium, Aachen, S.64-69, 2012.

[55] Schaumann, P.; Tabeling, F.; Weisheim, W.: Erwärmungsverhalten dämmschichtbildender Brandschutzsysteme im Stahlbau. Stahlbau, H. 9, S. 646-651, Berlin, Ernst \& Sohn, 2014.

[56] Stahl-Eisen-Werkstoffblätter des Vereins Deutscher Eisenhüttenleute: Physikalische Eigenschaften von Stahl. SEW 310, 7. Auflage, Verlag Stahleisen $\mathrm{mbH}$, Düsseldorf, 1992. 
[57] Sothmann, J.: Zur Modellierung geschützter und ungeschützter Verbunddeckenträgersysteme im Brandfall. Dissertation, Institut für Stahlbau, Gottfried Wilhelm Leibniz Universität Hannover, Hannover, 2013.

[58] Stahl-Eisen-Werkstoffblätter des Vereins Deutscher Eisenhüttenleute: Physikalische Eigenschaften von Stahl. SEW 310, 7. Auflage, Verlag Stahleisen mbH, Düsseldorf, 1992.

[59] Stopp, V.; Proschek, P.: Reaktive Brandschutzsysteme auf Stahlbauteilen mit reiner Zugbeanspruchung. DIBt Mitteilungen, H. 42, S. 190-191, Berlin, Ernst \& Sohn, 2011.

[60] Tabeling, F.: Zum Hochtemperaturverhalten dämmschichtbildender Brandschutzsysteme auf Stahlbauteilen. Dissertation, Institut für Stahlbau, Gottfried Wilhelm Leibniz Universität Hannover, Hannover, 2014.

[61] Tabeling, F.; Weisheim, W.; Kleibömer, I.: Experimentelle Untersuchungen zur Temperaturverteilung und -entwicklung in dämmschichtbildenden Brandschutzsystemen sowie Bewertung der thermischen Schutzwirkung. Festschrift Peter Schaumann, S. 203-211, Hannover, 2014.

[62] Yew, M. C.; Sulong, N. H. R.: Fire-resistive performance of intumescent flame-retardant coatings for steel. Materials \& Design, Vol. 34, S. 719-724, 2012.

[63] Wei, C.; Jihong, Y.: Mechanical properties of G550 cold-formed steel under transient and steady state conditions. Journal of Constructional Steel Research, Vol. 73, pp. 1-11, 2012.

\section{Abschlussarbeiten}

[64] Schramm, P.: Untersuchung der mechanischen Hochtemperatureigenschaften von kaltgezogenem Blankstahl S355. Bachelorarbeit, Brandenburgische Technische Universität Cottbus-Senftenberg: Lehrstuhl für Stahl- und Holzbau, 2013, betreut durch Dustin Häßler, M.Sc..

[65] Leyli, G.: Vorbereitende Arbeiten für die numerische Untersuchung von reaktiven Brandschutzsystemen auf Zugstabgliedern. Praktikumsbericht, Bundesanstalt für Materialforschung und -prüfung (BAM), 2011, betreut durch Dr.-Ing. Sascha Hothan.

\section{Einzelne Bildnachweise}

[66] Einsatzbereich von Zugliedern: Überdachung des Carports der Abfallwirtschaft München. Fotograf: Jens Weber [http://www.ackermannarchitekten.com/entry/ ueberdachung-des-carports-des-abfallwirtschaftsamts-muenchen/] (abgerufen am 01.06.2015).

[67] Aufschäumung des verwendeten reaktiven Brandschutzsystems: REM-Untersuchung der Aufschäumung nach dem Brandversuch (200-fache Vergrößerung). Fotograf: Michael Morys, 2015. 



\section{Abbildungsverzeichnis}

2.01: Schematischer Aufbau des auf die Versuchskörper applizierten

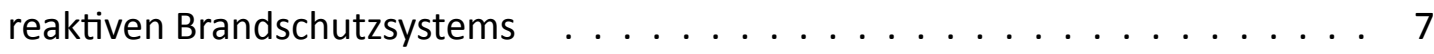

2.02: Schematische Darstellung des Aufschäumprozesses des RBS . . . . . . . . . . . 8

2.03: Wärmeleitfähigkeit und Gesamtwärmekapazität aus den Untersuchungen von Sothmann [57] . . . . . . . . . . . . . . . . . . . . . . 11

2.04: Ergebnisse der Untersuchungen des RBS von Tabeling [60] . . . . . . . . . . . . 12

2.05: Einsatzbereich von Zugliedern am Beispiel der Überdachung des Carports der Abfallwirtschaft München [66] . . . . . . . . . . . . . . . . . . . 15

2.06: Vergleich des Aufschäumverhaltens reaktiver Brandschutzsysteme auf Biegeträgern und Zuggliedern [34] . . . . . . . . . . . . . . . 18

2.07: Schematische Darstellung möglicher Versagensmechanismen des RBS auf Kreisvollprofilen (KVP) (oben: Zugglied in vertikaler

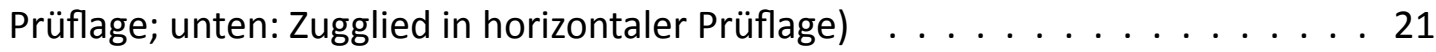

3.01: Vereinfachte Darstellung der im Zugstabprüfofen durchgeführten Brandversuche im Realmaßstab . . . . . . . . . . . . . . . . . . . 23

3.02: Detailansicht der Messstelle mit angeschweißtem Thermoelement nach dem neu entwickelten Applikationsverfahren (links: vor der Applikation des RBS, rechts: kurz vor der Brandprüfung) . . . . . . . . . . . . . 28

3.03: Versuchskörpers U91 nach der Applikation der Thermoelemente und des RBS . . . . . . . . . . . . . . . . . . . . . . 28

3.04: Applikation des reaktiven Brandschutzsystems auf die grundierten Versuchskörper V1 - V4 . . . . . . . . . . . . . . . . . . . . . . . . 29

3.05: Funktionsprinzip des magnetinduktiven Verfahrens . . . . . . . . . . . . . . 30

3.06: Detail der Versuchskörper V5 und V6 für die mikroskopische Untersuchung . . . 31

3.07: Querschliff des Versuchskörpers V5 (links) und V6 (rechts) . . . . . . . . . . . . . 32

3.08: Versuchsaufbau der Brandprüfung im Einkubikmeterofen (Versuchskörper V1 und V2; Blick aus Richtung Süden) . . . . . . . . . . . . . . 33

3.09: Detailaufnahmen der Rissbildung in den Brandversuchen . . . . . . . . . . . . . . 34

3.10: Versuchskörper V1 (oben) und V3 (unten) nach dem Brandversuch (Blick aus Richtung Norden) . . . . . . . . . . . . . . . . . . . . . . . . 35

3.11: Verlauf der gemessenen maximalen Stahltemperaturen . . . . . . . . . . 36

3.12: Detail des Versuchskörpers U91 nach dem Brandversuch (links: Bereich des Messquerschnittes 1; rechts: Bereich des Messquerschnittes 2) . . 38

3.13: Querschnitt eines Teils des Zugstabprüfofens mit Bezeichnung und Bemaßung der Thermoelemente für getesteten Versuchskörper (Blick aus Richtung Süden) . . . . . . . . . . . . . . . . . . . . . . . . 42 
3.14: 3D-Modell des Brandraumes des Zugstabprüfofens mit den eingebauten Versuchskörpern und den installierten Temperaturund Druckmessinstrumenten . . . . . . . . . . . . . . . . . . . . . . . . . 44

3.15: 3D-Modell des Zugstabprüfofens für die Durchführung der Brandversuche im Realmaßstab . . . . . . . . . . . . . . . . . . . . . . . . 45

3.16: Aufschäumungsprozess des reaktiven Brandschutzsystems am Beispiel des Versuchskörpers B3 (Branddauer $\mathrm{t}$ in min; maximale Stahltemperatur in $\theta_{\max }{ }^{\circ} \mathrm{C}$; äußere Probenabmessung $\mathrm{d}$ in $\mathrm{mm}$ ) . . . . . . . . . . 47

3.17: Beispiele mechanisch belasteter Zugglieder mit aufgeschäumten RBS (nicht maßstäblich) . . . . . . . . . . . . . . . . . . . . . . . . . . . . . 49

3.18: Beispiele unbelasteter horizontaler Vergleichsprüfkörper mit aufgeschäumten RBS (nicht maßstäblich) . . . . . . . . . . . . . . . . . . . 50

3.19: Beispiele unbelasteter vertikaler Vergleichsprüfkörper mit aufgeschäumten RBS (nicht maßstäblich) . . . . . . . . . . . . . . . . . . . . . 51

3.20: Längsrissbildung an Versuchskörpern (links: Thermoelemente auf der Stabunterseite; rechts Thermoelemente auf der Staboberseiteseite) . . . . . 52

3.21: Erwärmungsgeschwindigkeit der horizontal getesteten Versuchskörper . . . . . . 54

3.22: Vergleich von Versuchskörpern mit und ohne Abgleiten des RBS

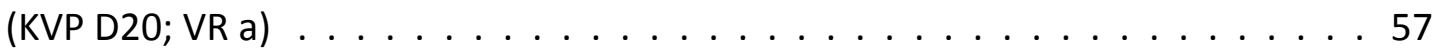

3.23: Mechanisch belastete Zugglieder (DFT $=2,5 \mathrm{~mm}$ ) mit aufgeschäumten RBS nach dem Brandversuch (Abbildungen nicht maßstabsgerecht) . . . . . . . . . . . . . . . . . 60

3.24: Vergleich der kältesten $(\min \theta)$ und heißesten $(\max \theta)$ Stahltemperaturverläufe der unter Zugbeanspruchung getesteten KVP D30 und KHP D60 (links: DFT = 2,5 mm; rechts: DFT = 3,5 mm) . . . . . . . . . . . 61

3.25: Einfluss der Trockenschichtdicke auf Branddauer bis zum Probenbruch (links) und auf die Stahltemperatur (rechts) anhand ausgewählter Zugglieder mit KVP D20 und einem Lastausnutzungsgrad im Brandfall von $\mu_{\mathrm{fi}}=0,45 \ldots \ldots \ldots$. . . . . . . . . . . . . . . . . 63

3.26: Zeitpunkt bis zum Erreichen einer Stahltemperatur von $550{ }^{\circ} \mathrm{C}(\mathrm{VR}$ a - c) . . . . 64

3.27: Einfluss des Lastausnutzungsgrades im Brandfall auf die Stablängsverformung (oben: VR a; mitte: VR b; unten: VR c) . . . . . . . . . . . . . . . 65

3.28: Einfluss des Lastausnutzungsgrades im Brandfall auf die Stablängsverformung im Brandverusch (links: VR d; rechts: VR e) . . . . . . . . . . . . . 66

3.29: Einfluss des Lastausnutzungsgrades im Brandfall auf die Stablängsverformung im Brandversuch (links: VR f; rechts: VR g) . . . . . . . . . . . . . . 66

3.30: Einfluss des Lastausnutzungsgrades auf die Branddauer bis zum Probenbruch . . 67 
3.31: Einfluss des Lastausnutzungsgrades auf die maximale Stahltemperatur zum Zeitpunkt des Probenbruchs (links: Vergleich der KVP D20; rechts: Vergleich der KVP D30 und KHP D60) . . . . . . . . . . . . . . . 69

3.32: Vergleich der maximalen Stahltemperaturen zwischen Zuggliedern mit und ohne mechanischer Zugbeanspruchung (oben: KVP D20; mitte: KVP D30; unten: KHP D60) … . . . . . . . . . . . . . . . . . . . . . 70

3.33: Vergleich der maximalen Stahltemperatur in Abhängigkeit vom Lastausnutzungsgrad im Brandfall (links: nach einer Branddauer von 20 min; rechts: nach einer Branddauer von 30 min) . . . . . . . . . . . . . . 71

3.34: Einfluss der Probenausrichtung auf die Temperaturentwicklung des Stahls am Beispiel der VR a bis c . . . . . . . . . . . . . . . . . . . . . . 72

3.35: Versuchskörper U32 nach dem Brandversuch (Nordostseite) . . . . . . . . . . . . 73

3.36: Vergleich des Temperaturverlaufes in Stablängsrichtung über die Branddauer (zeitlicher Abstand der dargestellten Temperaturverläufe beträgt jeweils $3 \mathrm{~min}) \ldots \ldots$. . . . . . . . . . . . . . 76

3.37: Beschädigung des reaktiven Brandschutzsystems vor und nach dem Brandversuch (links: durch Querriss (B24); rechts: durch Längsriss (B23)) . . 77

3.38: Schematische Darstellung der drei untersuchten Parameter auf die Feuerwiderstandsdauer eines Zuggliedes mit reaktiver Brandschutzbeschichtung . . . . . . . . . . . . . . . . . . 79

3.39: Ansichten der mittels Computertomographie untersuchten Schaumprobe B10 a) Isometrische Ansicht b) Vorderansicht c) Rückansicht . . . . 82

3.40: Versuchsaufbau der Computertomographieuntersuchung . . . . . . . . . . . 83

3.41: Ansicht der Probe (nicht maßstäblich) . . . . . . . . . . . . . . . . . . 83

3.42: Querschnitt der Probe aus der CT-Messung (nicht maßstäblich) . . . . . . . . . . 83

3.43: REM-Untersuchung der Aufschäumung nach dem Brandversuch (200-fache Vergrößerung) . . . . . . . . . . . . . . . . . . . . . . . 84

3.44: Anwendung der entwickelten Leistungskriterien auf die Zugglieder B22 und B18 (KVP D20, DFT = 3,5 mm) . . . . . . . . . . . . . . . 90

4.01: Typische Spannungs-Dehnungs-Kurve bei Raumtemperatur ( $\varepsilon$ nicht maßstablich, links: warmgewalzter, rechts: kaltverformter Blankstahl) . . . . . . 91

4.02: Standardprozesse bei der Herstellung von Blankstahl [41] . . . . . . . . . . . . . 92

4.03: Prinzipskizze des Ziehhofes bei der Herstellung von gezogenem Blankstahl . . . . 92

4.04: Untersuchte Rundprobe am Funken-Emissions-Spektrometer (FES) . . . . . . . . 94

4.05: Spannung-Dehnungs-Verlauf nach DIN EN 1993-1-2 [13] . . . . . . . . . . . . 95

4.06: Probekörpergeometrie und Verlauf der Schwingungen aus der Anregung des Senders . . . . . . . . . . . . . . . . . . . . . 96 
4.07: Messvorrichtung zur Bestimmung des dynamischen E-Moduls unter erhöhter Temperatur . . . . . . . . . . . . . . . . . . . . . . . . 97

4.08: Messvorrichtung zur Bestimmung des dynamischen E-Moduls unter Raumtemperatur . . . . . . . . . . . . . . . . . . . . . . 97

4.09: Resonanzpeaks im Resonanzspektrum bei Raumtemperatur und $600{ }^{\circ} \mathrm{C}$. . . . 97

4.10: Vergleich der Ergebnisse für den dynamischen Elastizitätsmodul . . . . . . . . . . 98

4.11: Graphische Bestimmung des statischen E-Moduls für eine Temperatur von $200{ }^{\circ} \mathrm{C} \ldots \ldots$. . . . . . . . . . . . . . . . 99

4.12: Mögliche exzentrische Beanspruchung beim Kleinzugversuch . . . . . . . . . . 101

4.13: Beispielhafte Verteilung des E-Moduls [GPa] in Abhängigkeit vom Messwinkel zur Lage der Exzentrizität (Draufsicht auf die Probe) . . . . . . . . . 101

4.14: Bestimmung des E-Moduls aus einem stationären Kleinzugversuch (links: $100{ }^{\circ} \mathrm{C}$; rechts: $700{ }^{\circ} \mathrm{C}$ ) . . . . . . . . . . . . . . . . . . . . . 102

4.15: Vergleich des Abminderungsfaktor für den statischen Elastizitätsmodul . . . . . 105

4.16: Vergleich der Abminderungsfaktoren des Elastizitätsmoduls zwischen den untersuchten Blankstahl und den Empfehlungen aus den Normen . . . . . . . . . . . . . . . . . . . . . . . 106

4.17: Kleinzugversuche bei Raumtemperatur (links: Aufbringen der Markierungsstriche auf die Zugprobe; Mitte: Prüfmaschine; rechts: eingebauter Probekörper) . . . . . . . . . . . . . . . . . . . . . . . . . 107

4.18: Schematische Spannungs-Dehnungs-Kennlinie bei Raumtemperatur . . . . . . 108

4.19: Gemessene Spannungs-Dehnungs-Kurven bei Raumtemperatur . . . . . . . . . 108

4.20: Fragmente der Zugproben unter Raumtemperatur (Abbildungen sind nicht maßstäblich) . . . . . . . . . . . . . . . . . . . . . . . . 109

4.21: Prüfmaschine für die Kleinzugversuche unter erhöhter Temperatur (links: geschlossener Ofen mit eingebauter Zugprobe; rechts: Probe mit angebrachten Thermoelementen sowie Extensometer . . . . . . . . 110

4.22: Schematischer Spannungs-Dehnungs-Verlauf der Zugversuche unter erhöhter Temperatur . . . . . . . . . . . . . . . . . . . . . . . . . 111

4.23: Gemessene Spannungs-Dehnungs-Kurven der Zugversuche aus VR 1 . . . . . . 112

4.24: Gemessene Spannungs-Dehnungs-Kurven der Zugversuche aus VR 2 . . . . . . 113

4.25: Gemessene Spannungs-Dehnungs-Kurven der Zugversuche aus VR 3 . . . . . . 114

4.26: Einfluss der Prüfgeschwindigkeit auf die $\sigma$ - $\varepsilon$-Kurven bei 500 und $600{ }^{\circ} \mathrm{C}$. . . 114

4.27: Auswahl von Fragmenten der unter erhöhter Temperatur getesteten Zugproben (Abbildungen sind nicht maßstäblich) . . . . . . . . . . . . . . 116

4.28: Vergleich der Abminderungsfaktoren für die Festigkeitskennwerte des Blankstahls 
4.29: $\sigma$ - $\varepsilon$-Kurven der VR 1 mit Darstellung der Proportionalitätsgrenze und $0,2 \%$-Dehngrenze . . . . . . . . . . . . . . . . . . . . 117

4.30: Vergleich des Abminderungsfaktors für die Proportionalitätsgrenze ..... . 119

4.31: Vergleich des Abminderungsfaktors für die 0,2\%-Dehngrenze . . . . . . . . . . 119

4.32: Vergleich des Abminderungsfaktors für die Zugfestigkeit . . . . . . . . . . . . 120

4.33: Untersuchung des Wärmeausdehnungskoeffizienten mit Hilfe eines

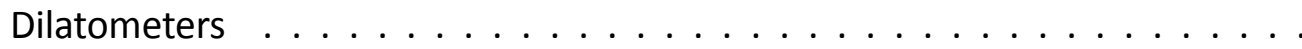

4.34: Vergleich des Wärmeausdehnungskoeffizienten aus der Messung mittels Schubstangen-Dilatometer (D) mit den Ergebnissen aus den Kleinzugversuchen (VR) und dem EC3 Teil 1-2 [13] . . . . . . . . . . . . . . . . 123

5.01: Vergleich von Temperatur-Zeit-Verläufen für ungeschütze Stahlprofile . . . . . 129

5.02: Vergleich der zeitlichen Entwicklung der Temperaturdifferenz zwischen Staboberfläche und Kernbereich für verschiedene Kreisvollprofile bei 'schneller' und 'langsamer' Erwärmung . . . . . . . . . . . . . 132

5.03: Zeichnung der Einbausituation des mechanisch belasteten Zuggliedes mit dem nachträglich geglätteten Temperaturverlauf des Versuchskörpers B9 . . . . . . . . . . . . . . . . . . . . . . 135

5.04: Numerisches Modell des Zuggliedes mit Kreisvollprofil (KVP D20) . . . . . . . . 136

5.05: Schematische Darstellung verschiedener Modifikationen an der Spannungs-Dehnungs-Kurve [35] . . . . . . . . . . . . . . . . . . . . . 137

5.06: Vergleich der wahren $\sigma-\varepsilon$-Kurven bei $600^{\circ} \mathrm{C}$ vor und nach dem Glätten am Beispiel der VR 1 und VR 3 [35] . . . . . . . . . . . . . . . . . . . . 138

5.07: Zusammensetzung der Dehnungsanteile in den $\sigma$ - $\varepsilon$-Kurven $\ldots \ldots$. . . . . . 139

5.08: Vergleich der $\sigma-\varepsilon$-Kennlinie bei $600{ }^{\circ} \mathrm{C}$ zwischen Mat 3 und $4 \ldots \ldots$. . . . . . . . 141

5.09: Vergleich der gemessenen und numerisch berechneten Verformungen des Versuchskörpers B9 . . . . . . . . . . . . . . . . . . . . . . . 142

5.10: Vergleich der technischen und wahren $\sigma$ - $\varepsilon$-Kennlinien des KVP D20 und KVP D30 . . . . . . . . . . . . . . . . . . . . . . . . . . . . . . . 144

5.11: Schematische Vorgehensweise bei der Anpassung der $\sigma$ - $\varepsilon$-Kurven von Mat 4 (KVP D20) auf Mat 5 (KVP D30) . . . . . . . . . . . . . . . . . . . . . 144

5.12: Differenz der gemessenen und numerisch berechneten Anfangsverformung 146

5.13: Vergleich der an der Versagensstelle berechneten Spannungen mit den in Stahlfestigkeiten aus den Materialuntersuchungen (Zuordnung Spannung und Lastausnutzungsgrad siehe Tabelle 5.4) . . . . . . . 148

5.14: Vergleich der an der Versagensstelle berechneten Dehnungen mit den Stahlfestigkeiten aus den Materialuntersuchungen ～. . . . . . . . . . . . . 148

5.15: Schematische Darstellung für die Bestimmung der Feuerwiderstandsdauer nach Variante 1 
5.16: Schematische Darstellung für die Bestimmung der Feuerwiderstandsdauer nach Variante $2 \ldots \ldots$. . . . . . . . . . . . . . . . . . 150

5.17: Schematische Darstellung für die Bestimmung der Feuerwiderstandsdauer nach Variante $3 \ldots \ldots$. . . . . . . . . . . . . . 153

A.01: Fertiger Aufbau des Zugstabprüfofens (es fehlen die Wandsegmente an der Ost- und Westseite sowie die Porenbetondeckenplatten und die zu prüfenden Versuchskörper) . . . . . . . . . . . . . . XXXXII

A.02: Temperatur-Zeit-Kurven der KVP D30 aus der VR e (DFT=3,5 mm) . . . . . . . XXXV

A.03: Temperatur-Branddauer-Kurven der Versuchskörper B22 und U38 sowie der Brandraumthermoelemente (BRT) . . . . . . . . . . . . XXXXV

A.04: Temperatur-Branddauer-Kurven der Versuchskörper B23 und U39 sowie der Brandraumthermoelemente (BRT) . . . . . . . . . . . . XXXVI

A.05: Temperatur-Branddauer-Kurven der Versuchskörper B58 und U53 sowie der Brandraumthermoelemente (BRT) . . . . . . . . . . . . . XXXVI

A.06: Temperatur-Branddauer-Kurven der Versuchskörper B57 und U52 sowie der Brandraumthermoelemente (BRT) . . . . . . . . . . . . XXXVII

A.07: Temperatur-Branddauer-Kurven der Versuchskörper B59 und U54

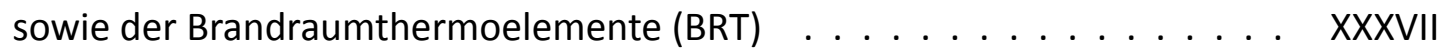

B.01: Gemessene $\sigma$ - $\varepsilon$-Kurven der in den Brandversuchen der BAM getesteten Zugglieder . . . . . . . . . . . . . . . LVI

B.02: Getestete Zugproben des KVP D30 (nicht maßstäblich) . . . . . . . . . . . . . . . LVI

B.03: Zugprüfung der Probe KHP-01 (links: vor der Prüfung; rechts: nach dem Probenbruch) . . . . . . . . . . . . . . . . . . LVI

B.04: Geteste Zugproben des KHP D60 (nicht maßstäblich) . . . . . . . . . . . . . . . LVI

C.01: Spannungs-Dehnungs-Kennlinien für das Materialmodell Mat $3 \ldots$. . . . . . . LVII

C.02: Spannungs-Dehnungs-Kennlinien für das Materialmodell Mat 4 . . . . . . . LVIII

C.03: Spannungs-Dehnungs-Kennlinien für das Materialmodell Mat 5 . . . . . . . . LIX

D.01: Vergleich der experimentell und numerisch bestimmten Stablängs-

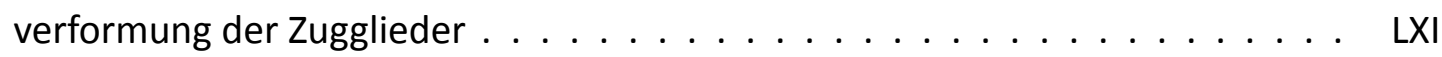




\section{Tabellenverzeichnis}

3.1: Art und Aufbau der Versuchskörper sowie deren Verwendungszeck . . . . . . 26

3.2: Versuchsprogramm für die Brandprüfungen im Realmaßstab . . . . . . . . . . . 41

3.3: Zeitpunkt bis zum Probenbruch und gemessene maximale Stahltemperaturen der im Zugstabprüfofen getesteten Versuchskörper . . . . . . . . . . . . . . 53

3.4: Differenz in Minuten zwischen dem Zeitpunkt des Probenbruchs der KVP D20 und KVP D30 . . . . . . . . . . . . . . . . . . . . . . . . . . 59

3.5: Differenz in K/min zwischen der Erwärmungsgeschwindigkeit der KVP D20

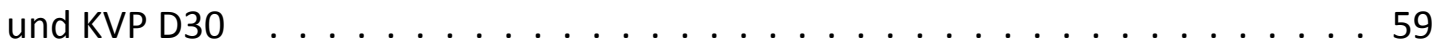

3.6: Differenz in Minuten zwischen dem Zeitpunkt des Probenbruchs der KVP D30 und KHP D60 . . . . . . . . . . . . . . . . . . . . . . . . . . . 60

3.7: Einfluss des Trockenschichtdicke auf den Zeitpunkt bis zum Erreichen einer Stahltemperatur von $550{ }^{\circ} \mathrm{C} \ldots \ldots \ldots 63$

3.8: Erhöhung der Branddauer in Minuten bis zum Probenbruch durch eine zehnprozentige Reduzierung des Lastausnutzungsgrades im Brandfall . . . . . . 67

3.9: Vergleich der Erwärmungsgeschwindigkeit zwischen horizontaler und vertikaler Prüfpostion . . . . . . . . . . . . . . . . 73

3.10: Vergleich der Stahltemperaturen zwischen den in horizontaler und vertikaler Prüfposition eingebauten Versuchskörpern zu verschiedenen Zeitpunkten des Brandversuches . . . . . . . . . . . . . . . . . . . . . . 74

3.11: Typologie des aufgeschäumten reaktiven Brandschutzsystems (grau) an von horizontal getesteten Stahlzuggliedern mit Kreisvollquerschnitt (blau) . . . . . . 81

4.1: Produktgruppen für Blankstahl [41] . . . . . . . . . . . . . . . . . . . . . 92

4.2: Übersicht der Probekörper für die Materialuntersuchungen . . . . . . . . . . 93

4.3: Chemische Zusammensetzung des untersuchten Blankstahls . . . . . . . . . 94

4.4: Vergleich des statischen E-Moduls aus den Kleinzugversuchen . . . . . . . . . 100

4.5: Vergleich des E-Moduls zwischen untersuchtem Blankstahl und den Werten in den Normen . . . . . . . . . . . . . . . . . . . . . . . . . . . 103

4.6: Wichtige Kenngrößen der $\sigma$ - $\varepsilon$-Kurven aus den Zugversuchen bei Raumtemperatur . . . . . . . . . . . . . . . . . . . . 108

4.7: Prüfgeschwindigkeiten der durchgeführten Kleinzugversuche $\ldots \ldots \ldots$. . . . 110

4.8: Berechnung der Abminderungsfaktoren . . . . . . . . . . . . . . 118

5.1: Vergleich der Oberflächen- und Kerntemperatur verschiedener Kreisvollprofile 131

5.2: Vergleich der Abminderungsfaktoren in Bezug auf die Verwendung in dem numerischen Modell . . . . . . . . . . . . . . . . . . . . . . . . 140 
5.3: Festigkeitskennwerte und Korrekurfaktoren für die KVP D20 und KVP D30 bei Raumtemperatur . . . . . . . . . . . . . . . . . . . . . . . . . . 144

5.4: Zuordnung Lastausnutzungsgrad im Brandfall und wahre Spannung . . . . . . 147

5.5: Bemessungsfestigkeit in Abhängigkeit von der Stahltemperatur . . . . . . . . . 152

A.1: Übersicht der verwendeten Stahlbauteile sowie deren Herkunft und Materialeigenschaften . . . . . . . . . . . . . XXXXI

A.2: Übersicht der Brandversuche mit KVP D30 unter Angabe des Zeitpunktes und der Position des Probenbruchs sowie der maximalen und minimalen Stahltemperatur .................... XXXII

A.3: Einfluss des Stabdurchmessers auf die Aufschäumhöhe des reaktiven Brandschutzsystems ................... XXXIII

A.4: Sensitivitätsanalyse für den Zeitpunkt des Erreichens des Verformungsgeschwindig-keitskriteriums bei Verwendung des Grenzwertes von 0,75 und $1,0 \mathrm{~mm} / \mathrm{min} \ldots \ldots \ldots \ldots \ldots$ XXXIII

A.5: Beschreibung der an ausgewählten Zuggliedern vorgenommenen Beschädigungen des reaktiven Brandschutzsystems und deren Auswirkung auf das Zugglied $\ldots \ldots \ldots \ldots \ldots \ldots \ldots$ XXXIV

B.1: Festigkeitsparameter aus den gemessenen, technischen $\sigma$ - $\varepsilon$-Kurven der VR 1 . . LIII

B.2: Festigkeitsparameter aus den gemessenen, technischen $\sigma$ - $\varepsilon$-Kurven der VR 2 . . LIII

B.3: Festigkeitsparameter aus den gemessenen, technischen $\sigma$ - $\varepsilon$-Kurven der VR 3 . LIV

B.4: Mittelwerte der Festigkeitsparameter aus allen gemessenen, technischen $\sigma$ - $\varepsilon$-Kurven (VR $1-3) \ldots \ldots \ldots \ldots$ LIV

B.5: Maße der Kleinzugproben nach DIN EN ISO 6892-2 [15] (Angaben in mm) . . . . LV

B.6: Kennwerte für das KVP D30 aus den bei Raumtemperatur durchgeführten

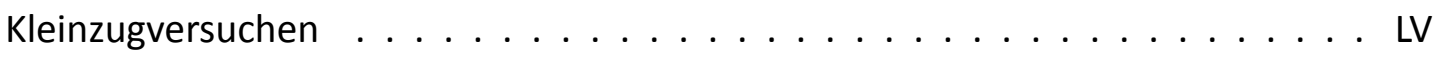

B.7: Kennwerte für das KHP D60 aus den bei Raumtemperatur durchgeführten

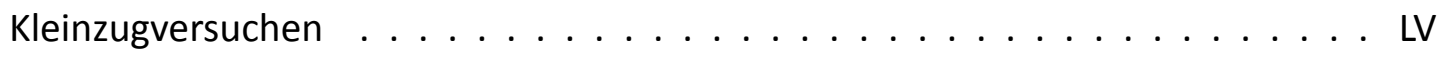

C.1: Festigkeitsparameter aus den wahren $\sigma$ - $\varepsilon$-Kurven des neu erstellten Materialmodells (Mat 3 ) . . . . . . . . . . . . . . . . . LVII

C.2: Festigkeitsparameter aus den wahren $\sigma$ - $\varepsilon$-Kurven des neu erstellten Materialmodells (Mat 4) . . . . . . . . . . . . . . . . . . LVIII

C.3: Festigkeitsparameter aus den wahren $\sigma$ - $\varepsilon$-Kurven des neu erstellten Materialmodells (Mat 5$) \ldots \ldots \ldots \ldots \ldots$. . . . . . . . . . . . . . . . . 


\section{A Ergänzende Angaben zu den in Kapitel 3 beschriebenen Brandversuchen im Realmaßstab}

Tabelle A.1: Übersicht der verwendeten Stahlbauteile sowie deren Herkunft und Materialeigenschaften

\begin{tabular}{|c|c|c|c|c|}
\hline Parameter & Einheit & $\begin{array}{c}\text { Kreisvollprofil } \\
\varnothing 20 \text { mm } \\
\text { (KVP D20) }\end{array}$ & $\begin{array}{c}\text { Kreisvollprofil } \\
\varnothing 30 \mathrm{~mm} \\
\text { (KVP D30) }\end{array}$ & $\begin{array}{l}\text { Kreishohlprofil } \\
\varnothing 60,3 \times 8,8 \mathrm{~mm} \\
\text { (KHP 60x8,8) }\end{array}$ \\
\hline Stabdurchmesser & {$[\mathrm{mm}]$} & 20 & 30 & 60,3 \\
\hline Wandstärke & {$[\mathrm{mm}]$} & - & - & 8,8 \\
\hline Stablänge & {$[\mathrm{mm}]$} & $6 \mathrm{~m}$ & $6 \mathrm{~m}$ & $6 \mathrm{~m}$ \\
\hline Profilfaktor & {$\left[\mathrm{m}^{-1}\right]$} & 200 & 133 & 133 \\
\hline Stabanzahl & {$[-]$} & 9 & 4 & 2 \\
\hline \multirow{2}{*}{ Herstellerangaben } & Name & Marcegaglia & $\begin{array}{c}\text { Riva Acciaio S.P.A. } \\
\text { Stabilimento di Malegno }\end{array}$ & $\begin{array}{l}\text { Rohrwerk Maxhütte } \\
\text { GmbH }\end{array}$ \\
\hline & Anschrift & $\begin{array}{l}\text { Gazoldo degli Ippoliti } \\
\text { Mantova (Italien) }\end{array}$ & $\begin{array}{l}\text { Malegno Brescia } \\
\text { (Italien) }\end{array}$ & $\begin{array}{l}\text { Sulzbach-Rosenberg } \\
\text { (Deutschland) }\end{array}$ \\
\hline Herstellungsdatum & {$[-]$} & 25.10 .2012 & $03.12 .2012 *$ & $24.01 .2013^{*}$ \\
\hline Material & {$[-]$} & $\begin{array}{c}\text { S355 J2C } \\
\text { (Blankstahl, } \\
\text { kaltverformt, gezogen, } \\
\text { Material-Nr. 1.0579, } \\
\text { unlegierter } \\
\text { Qualitätsstahl }{ }^{\#} \text { ) }\end{array}$ & $\begin{array}{c}\text { S355 J2C +C } \\
\text { (Blankstahl, } \\
\text { kaltverformt, gezogen, } \\
\text { Material-Nr. 1.0579, } \\
\text { unlegierter } \\
\text { Qualitätsstahl }{ }^{\#} \text { ) }\end{array}$ & $\begin{array}{c}\text { S355 J2H } \\
\text { (warmgewalzter Bau- } \\
\text { stahl, nahtlos gefertigt, } \\
\text { E355 / P355N) }\end{array}$ \\
\hline $\begin{array}{l}0,2 \% \text {-Dehngrenze } \\
\text { bzw. Streckgrenze }\end{array}$ & {$\left[\mathrm{N} / \mathrm{mm}^{2}\right]$} & $641\left(585^{* *}\right)$ & $650(570 * *)$ & $461\left(457,5^{* *}\right)$ \\
\hline Zugfestigkeit & {$\left[\mathrm{N} / \mathrm{mm}^{2}\right]$} & $701(679 * *)$ & $675\left(661^{* *}\right)$ & $615\left(600,5^{* *}\right)$ \\
\hline $\begin{array}{l}\text { Oberflächen- } \\
\text { beschaffenheit }\end{array}$ & {$[-]$} & SA 2,5 gestrahlt & SA 2,5 gestrahlt & SA 2,5 gestrahlt \\
\hline
\end{tabular}

Die Angaben sind dem Abnahmeprüfzeugnis 3.1 des jeweiligen Bauteils entnommen.

* Das angegebene Datum bezieht sich auf das Ausstelldatum des Abnahmeprüfzeugnisses 3.1.

** Anhand von Kleinzugversuchen in der BAM ermittelte Werte. Diese dienen zur Bestimmung der Prüflast.

* Unlegierte Qualitätsstähle haben im Vergleich zu unlegierten Grundstählen schärfere oder zusätzliche Anforderungen zu erfüllen, z.B. bezüglich Sprödbruchempfindlichkeit, Korngröße oder Verformbarkeit. 


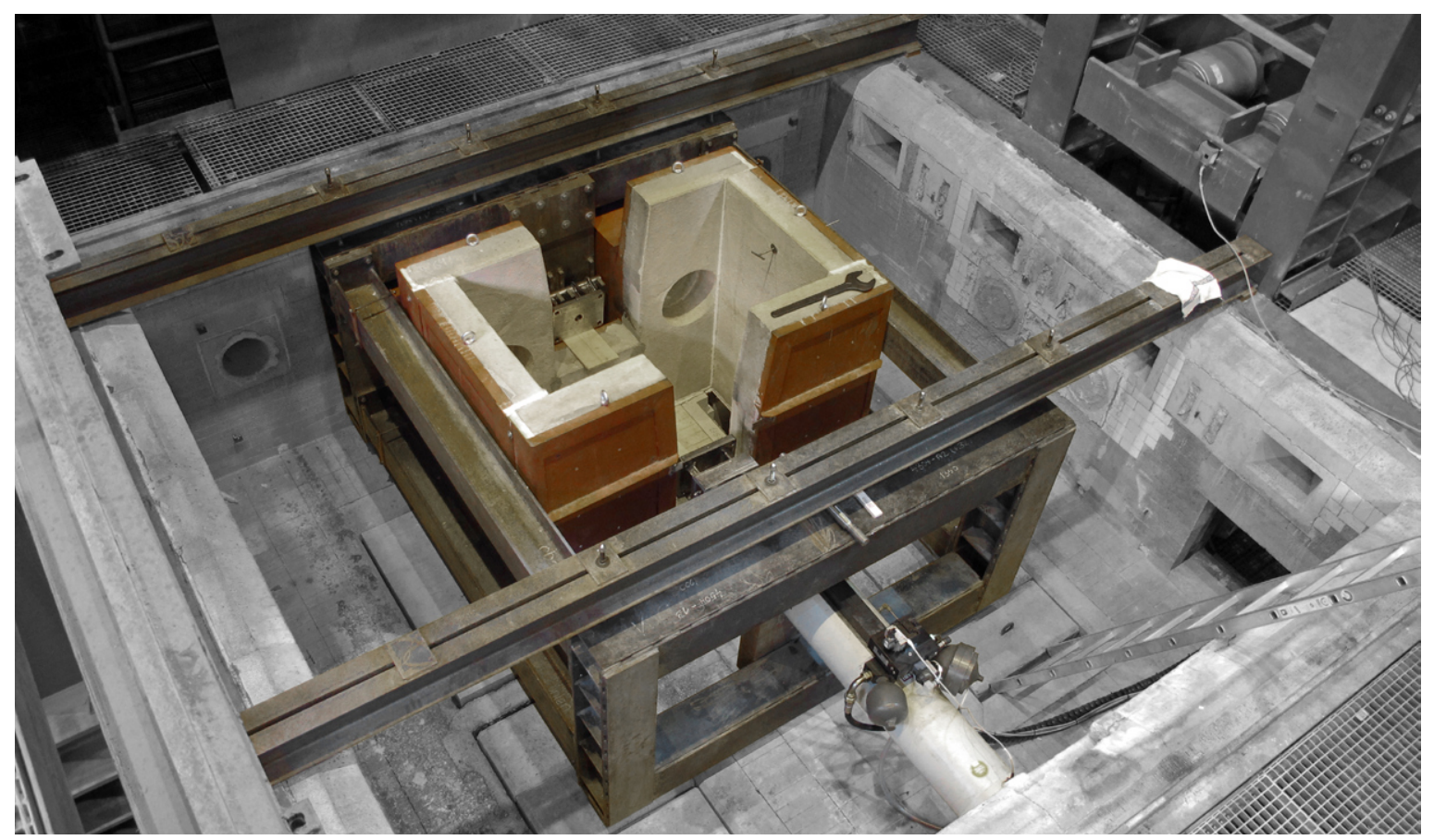

Abb. A.01: Fertiger Aufbau des Zugstabprüfofens (es fehlen die Wandsegmente an der Ost- und Westseite sowie die Porenbetondeckenplatten und die zu prüfenden Versuchskörper)

Tabelle A.2: Übersicht der Brandversuche mit KVP D30 unter Angabe des Zeitpunktes und der Position des Probenbruchs sowie der maximalen und minimalen Stahltemperatur

\begin{tabular}{|c|c|c|c|c|c|c|c|}
\hline 훙 & VR & Probe & $\begin{array}{l}\text { Lastausnutz- } \\
\text { ungsgrad im } \\
\text { Brandfall }\end{array}$ & $\begin{array}{c}\text { Zeitpunkt } \\
\text { Probenbruch } \\
\text { [min] }\end{array}$ & $\begin{array}{c}\text { Bruchstelle } \\
\text { (Position) }\end{array}$ & $\begin{array}{c}\text { Min. Temperatur } \\
\text { bei Probenbruch } \\
{\left[{ }^{\circ} \mathrm{C}\right] * *}\end{array}$ & $\begin{array}{c}\text { Max. Temperatur } \\
\text { bei Probenbruch } \\
{\left[{ }^{\circ} \mathrm{C}\right]}\end{array}$ \\
\hline \multirow{10}{*}{$\begin{array}{l}\stackrel{P}{m} \\
0 \\
\stackrel{2}{z}\end{array}$} & \multirow{10}{*}{ 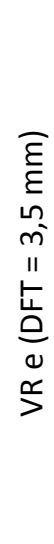 } & U51 & - & - & - & - & - \\
\hline & & U $52^{n}$ & - & 50,92 & - & TE $2=544$ & $\mathrm{TE} 1=556$ \\
\hline & & U53 & - & 56,43 & - & TE $1=560$ & TE $3=576$ \\
\hline & & U54 ${ }^{n}$ & - & 53,16 & - & TE $2=554$ & TE $3=610$ \\
\hline & & B55 & 0,15 & 72,76 & TE 5 & TE $2=626$ & TE $4=740$ \\
\hline & & B56 & 0,25 & 64,56 & zW. TE 5 - TE 6 & TE $2=556$ & TE $5=696$ \\
\hline & & B60 & 0,35 & 59,63 & zW. TE 4 - TE 5 & TE $2=579$ & TE $4=648$ \\
\hline & & B58 & 0,42 & 56,43 & zW. TE 5 - TE 6 & TE $2=536$ & TE $5=616$ \\
\hline & & B59 & 0,55 & 53,16 & TE 5 & TE $2=529$ & TE $5=593$ \\
\hline & & B57 & 0,65 & 50,92 & TE 5 & TE $2=511$ & TE $5=564$ \\
\hline
\end{tabular}

Für die unbelasteten Vergleichsprüfkörper wird jeweils der Zeitpunkt des Probenbruchs des im gleichen Brandversuchs mitgeprüften belasteten Versuchskörpers verwendet.

" Unbelaste Vergleichsprobe befindet sich ca. $150 \mathrm{~mm}$ oberhalb des belasteten Zuggliedes

** Es werden nur die Thermoelemente (TE) Nr. 2 bis 6 betrachtet. 
Tabelle A.3: Einfluss des Stabdurchmessers auf die Aufschäumhöhe des reaktiven Brandschutzsystems

\begin{tabular}{|c|c|c|c|c|c|}
\hline \multirow{2}{*}{\multicolumn{2}{|c|}{$\begin{array}{l}\text { Profil } \\
\text { Versuchsreihe (VR) }\end{array}$}} & \multicolumn{2}{|c|}{ KVP D20 } & \multicolumn{2}{|c|}{ KVP D30 } \\
\hline & & b & C & d & e \\
\hline \multicolumn{2}{|c|}{ Trockenschichtdicke (DFT) } & $2,5 \mathrm{~mm}$ & $3,5 \mathrm{~mm}$ & $2,5 \mathrm{~mm}$ & $3,5 \mathrm{~mm}$ \\
\hline \multirow{3}{*}{$\begin{array}{l}\text { Aufschäumhöhe } \\
\text { des RBS [mm] * }\end{array}$} & $\min$ & $9(5)$ & $10(8)$ & $10(7)$ & $13(11)$ \\
\hline & mittel & 19 & 20 & 19 & 21 \\
\hline & $\max$ & $27(31)$ & $29(35)$ & $26(34)$ & $27(32)$ \\
\hline
\end{tabular}

* Die Aufschäumhöhen werden entlang der Stabachse im Abstand von jeweils 50 mm gemessen. Jeder Messquerschnitt besteht aus vier Messtellen, welche im Winkel von $90^{\circ}$ zueinander angeordnet sind. Bei den in der Tabelle angegeben Werten handelt es sich um Mittelwerte aller mechanisch belasteten Versuchskörper einer Versuchsreihe. Für die Ermittlung der maximalen und minimalen Aufschäumhöhe werden nur Stabbereiche mit einem Abstand von mehr als $100 \mathrm{~mm}$ von der Brandraumwand berücksichtigt. Die mittlere Aufschäumhöhe bezieht sich auf alle Messpunkte, mit einem Abstand von mindestens 300 mm zur Brandraumwand. Werte in runden Klammern sind Extremwerte einzelner Versuchskörper aus einer Versuchsreihe.

Tabelle A.4: Sensitivitätsanalyse für den Zeitpunkt des Erreichens des Verformungsgeschwindigkeitskriteriums bei Verwendung des Grenzwertes von 0,75 und 1,0 mm/min

\begin{tabular}{|c|c|c|c|c|c|c|c|c|c|c|}
\hline Profil & VR & $\begin{array}{c}\text { DFT } \\
{[\mathrm{mm}]}\end{array}$ & Zeitu & chied f & $\begin{array}{l}\text { las Err } \\
\text { schen }\end{array}$ & $\begin{array}{l}\text { en des } \\
z=0,7\end{array}$ & $\begin{array}{l}\text { form } \\
1,0\end{array}$ & $\begin{array}{l}\text { min [r } \\
\text { minch }\end{array}$ & gkeit & eriums \\
\hline \multirow{6}{*}{$\begin{array}{l}\stackrel{ }{N} \\
0 \\
\stackrel{2}{\geq}\end{array}$} & \multirow{2}{*}{$a$} & \multirow{2}{*}{1,5} & B6 & B3 & $\mathrm{B} 11^{\#}$ & B7 & B12 & B8* & B2* & B4* \\
\hline & & & 1 & 1 & 1 & 1 & 0 & 1 & 0 & 1 \\
\hline & \multirow{2}{*}{$b$} & \multirow{2}{*}{2,5} & B1 & B5 & B9 & B13 & B10 & B15" & B14 & B16 \\
\hline & & & 1 & 1 & 0 & 1 & 1 & 1 & 1 & 1 \\
\hline & \multirow{2}{*}{ C } & \multirow{2}{*}{3,5} & B22 & B21 & B20 & B23" & B17 & B24 & B19 & B18 \\
\hline & & & 1 & 1 & 0 & 0 & 1 & 1 & 1 & 1 \\
\hline \multirow{4}{*}{$\begin{array}{l}\stackrel{一}{m} \\
0 \\
\stackrel{2}{\geq}\end{array}$} & \multirow{2}{*}{$d$} & \multirow{2}{*}{2,5} & B45 & B47 & B48 & - & B49 & - & B50 & B46 \\
\hline & & & 1 & 1 & 1 & - & 1 & - & 1 & 1 \\
\hline & \multirow{2}{*}{$\mathrm{e}$} & \multirow{2}{*}{3,5} & B55 & B56 & B60 & - & B58 & - & B59 & B57 \\
\hline & & & 2 & 1 & 1 & - & 1 & - & 0 & 1 \\
\hline \multirow{4}{*}{$\begin{array}{l}\text { 은 } \\
0 \\
\text { 몬 } \\
\text { 立 }\end{array}$} & \multirow{2}{*}{$f$} & \multirow{2}{*}{2,5} & - & $B 66^{\top}$ & - & - & B64 & - & - & B65 \\
\hline & & & - & 0 & - & - & 1 & - & - & 1 \\
\hline & \multirow{2}{*}{ g } & \multirow{2}{*}{3,5} & - & $B 71^{\top}$ & - & - & B72 & - & - & $B 70^{\top}$ \\
\hline & & & - & 1 & - & - & 1 & - & - & 1 \\
\hline
\end{tabular}

Basiswert ist das Erreichen des Verformungsgeschwindigkeitskriteriums mit 1,0 mm/min. 
Tabelle A.5: Beschreibung der an ausgewählten Zuggliedern vorgenommenen Beschädigungen des reaktiven Brandschutzsystems und deren Auswirkung auf das Zugglied

\begin{tabular}{|c|c|c|c|c|}
\hline \multirow{2}{*}{ VR } & \multirow{2}{*}{$\begin{array}{l}\text { Versuchs- } \\
\text { körper }\end{array}$} & \multirow{2}{*}{$\begin{array}{l}\text { Art und Umfang der } \\
\text { Beschädigung }\end{array}$} & \multicolumn{2}{|r|}{ Beschreibung des Brandversuches } \\
\hline & & & Zeit & Ereignis / Auswirkung \\
\hline$a$ & B11 & $\begin{array}{l}\text { Querriss am TE 4, } \\
\text { Rissbreite } \mathrm{t} \approx 3 \mathrm{~mm}\end{array}$ & $\begin{array}{r}3 \min \\
4 \min \\
6 \min \\
22 \min \end{array}$ & $\begin{array}{l}\text { - Querriss im RBS am TE } 4 \\
\text { - Längsriss auf Staboberseite zw. TE } 4 \text { und TE } 7 \\
\text { - Abrutschen des RBS im Bereich des Längsrisses } \\
\text { - Bruchstelle des Zuggliedes zw. TE } 5 \text { und TE } 6 \\
\rightarrow \text { Beschädigung hat keinen Einfluss auf Bruchstelle; } \\
\quad \text { RBS im Bereich der Beschädigung gestört }\end{array}$ \\
\hline \multirow{2}{*}{$\mathrm{b}$} & B15 & $\begin{array}{l}\text { Querriss am TE } 4, \\
\text { Rissbreite } t \approx 0,8 \mathrm{~mm}\end{array}$ & $\begin{array}{r}3 \text { min } \\
5 \text { min } \\
8 \text { min } \\
20 \text { min } \\
32 \min \end{array}$ & $\begin{array}{l}\text { - Querriss im RBS am TE } 4 \\
\text { - Längsriss auf Stabunterseite zw. TE } 1 \text { und TE } 4 \\
\text { - Längsriss auf Staboberseite zw. TE } 5 \text { und TE } 7 \\
\text { - Längsrisse nahezu komplett zugeschäumt } \\
\text { - Bruchstelle des Zuggliedes TE } 5 \text { und TE } 6 \\
\rightarrow \text { Beschädigung hat keinen Einfluss auf Bruchstelle; } \\
\text { RBS im Bereich der Beschädigung gestört }\end{array}$ \\
\hline & B13 & $\begin{array}{l}\text { Längsriss auf der } \\
\text { Staboberseite am TE 5, } \\
\mathrm{L}=100 \mathrm{~mm}, \mathrm{t} \approx 1 \mathrm{~mm}\end{array}$ & $\begin{array}{r}3 \mathrm{~min} \\
6 \mathrm{~min} \\
9 \mathrm{~min} \\
33 \mathrm{~min}\end{array}$ & $\begin{array}{l}\text { - Längsriss auf Stabunterseite zw. TE } 3 \text { und TE } 7 \\
\text { - Aufweitung des Längsrisses } \\
\text { - Rissheilung führt zum teilweisen schließen des Risses } \\
\text { - Bruchstelle des Zuggliedes am TE } 5 \\
\rightarrow \text { Beschädigung führt zu einem Längsriss auf der Sta- } \\
\text { boberseite; Rissheilung schließt den Riss komplett; kein } \\
\text { Einfluss der Beschädigung auf Aufschäumung des RBS }\end{array}$ \\
\hline \multirow[b]{2}{*}{$\mathrm{C}$} & B23 & $\begin{array}{l}\text { Längsriss auf der } \\
\text { Staboberseite am TE 4, } \\
\mathrm{L}=100 \mathrm{~mm}, \mathrm{t} \approx 1,5 \mathrm{~mm}\end{array}$ & $\begin{array}{r}4 \min \\
7 \text { min } \\
10 \min \\
11 \min \\
20 \min \\
35 \min \end{array}$ & $\begin{array}{l}\text { - Längsriss auf Staboberseite zw. TE } 3 \text { und TE } 5 \\
\text { - Querriss im RBS zw. TE } 4 \text { und TE } 5 \\
\text { - Vergrößerung des Längsrisses von TE } 3 \text { bis TE } 7 \\
\text { - Übergang des Längsrisse in einen Querriss am TE } 5 \\
\text { - Längsrisse und Querrisse fast komplett zugeschäumt } \\
\text { - Bruchstelle des Zuggliedes zw. TE } 5 \text { und TE } 6 \\
\rightarrow \text { Beschädigung hat keinen Einfluss auf Bruchstelle }\end{array}$ \\
\hline & B24 & $\begin{array}{l}\text { Querriss am TE } 4, \\
\text { Rissbreite } t \approx 1,6 \mathrm{~mm}\end{array}$ & $\begin{array}{r}3 \text { min } \\
5 \text { min } \\
5 \text { min } \\
9 \text { min } \\
20 \text { min } \\
36 \min \end{array}$ & $\begin{array}{l}\text { - Querriss im RBS am TE } 4 \\
\text { - Längsriss auf Staboberseite zw. TE } 4 \text { und TE } 7 \\
\text { - Querriss im RBS zw. TE } 3 \text { und TE } 4 \\
\text { - Längsriss auf Stabunterseite zw. TE } 1 \text { und TE } 7 \\
\text { - Längsrisse nahezu komplett zugeschäumt } \\
\text { - Bruchstelle des Zuggliedes zw. TE } 4 \text { und TE } 5 \\
\rightarrow \text { Beschädigung hat keinen Einfluss auf Bruchstelle; RBS } \\
\text { im Bereich der Beschädigung gestört }\end{array}$ \\
\hline
\end{tabular}




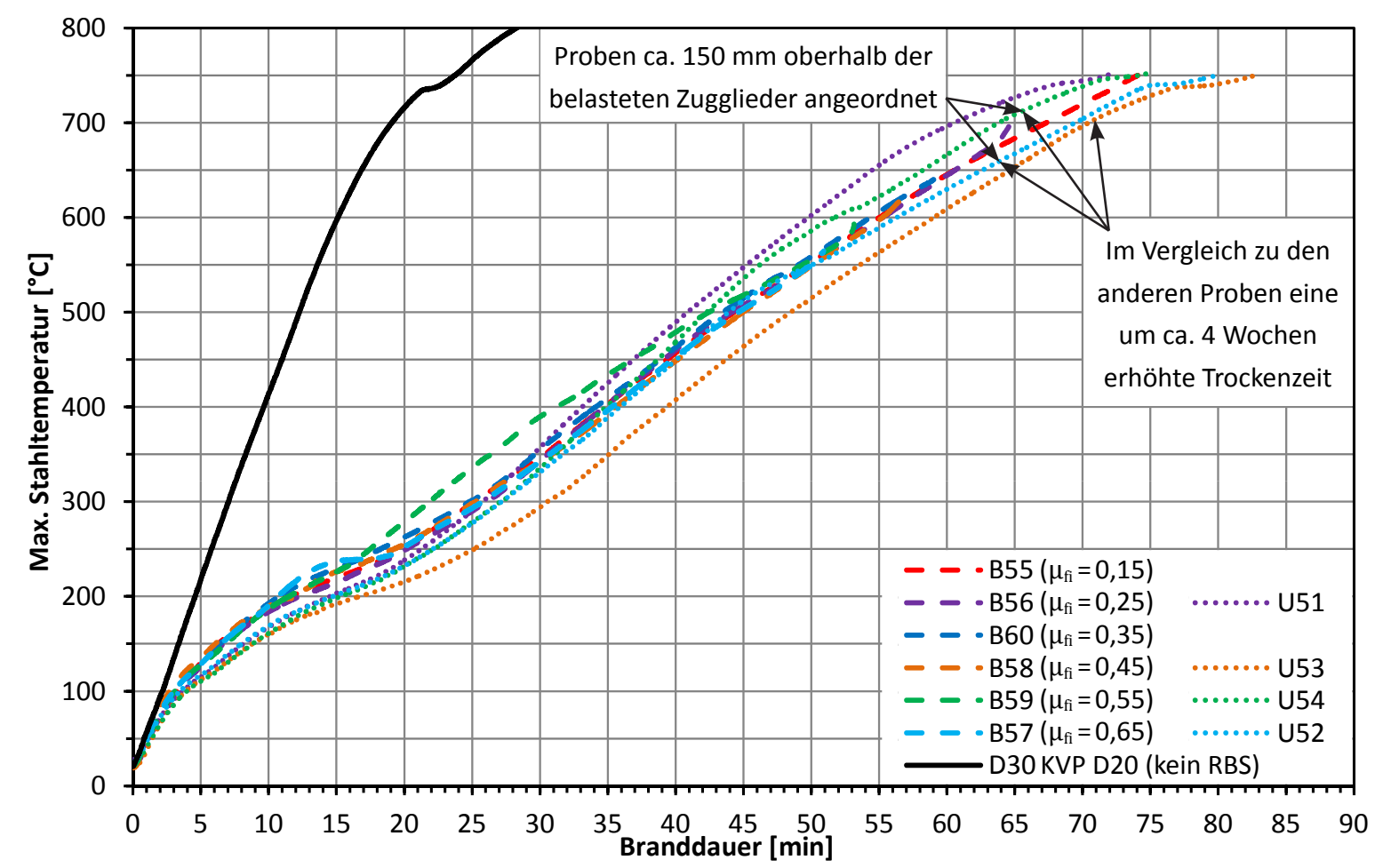

Abb. A.02: Temperatur-Zeit-Kurven der KVP D30 aus der VR e (DFT=3,5 mm)

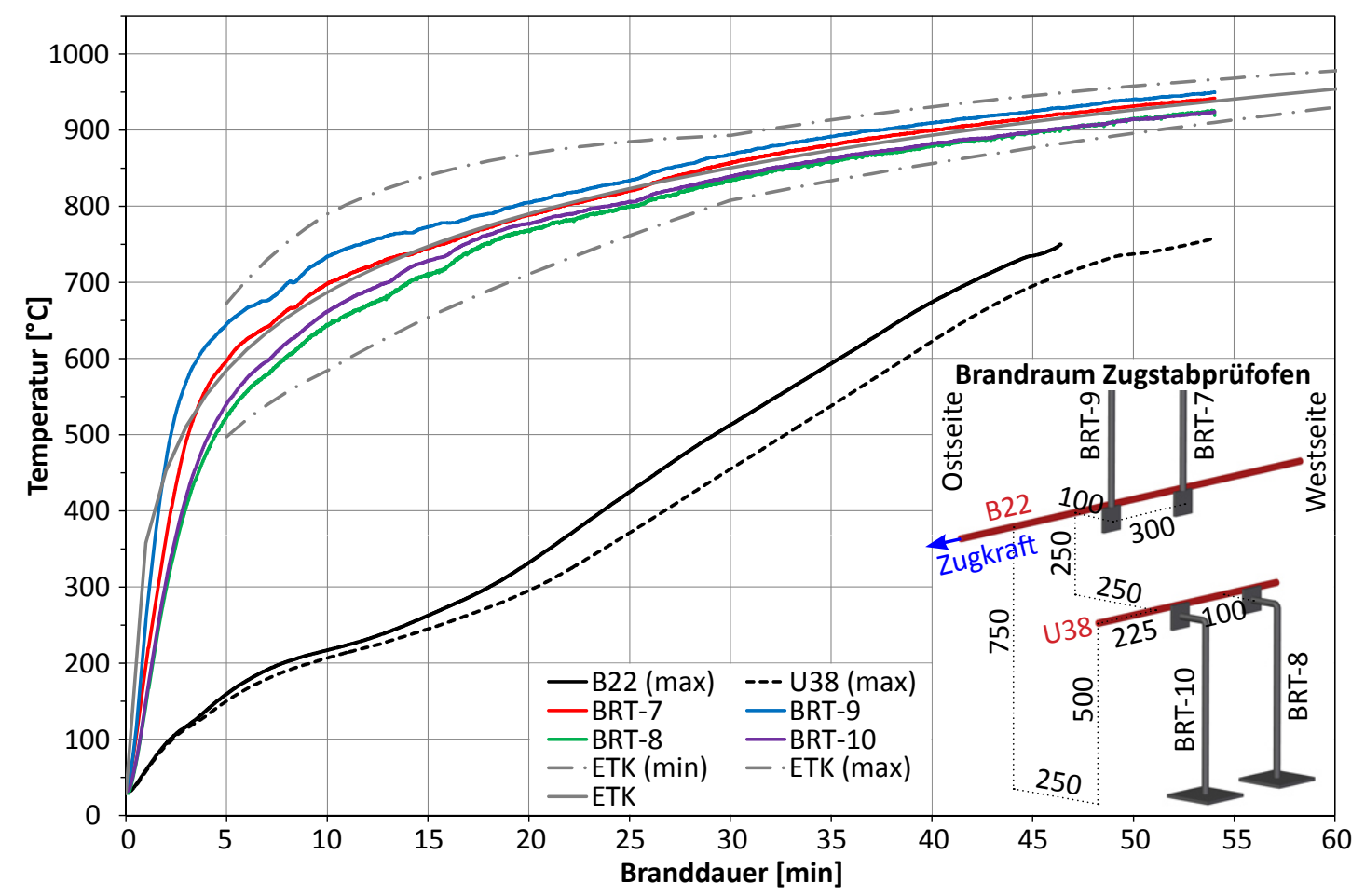

Abb. A.03: Temperatur-Branddauer-Kurven der Versuchskörper B22 und U38 sowie der Brandraumthermoelemente (BRT) 


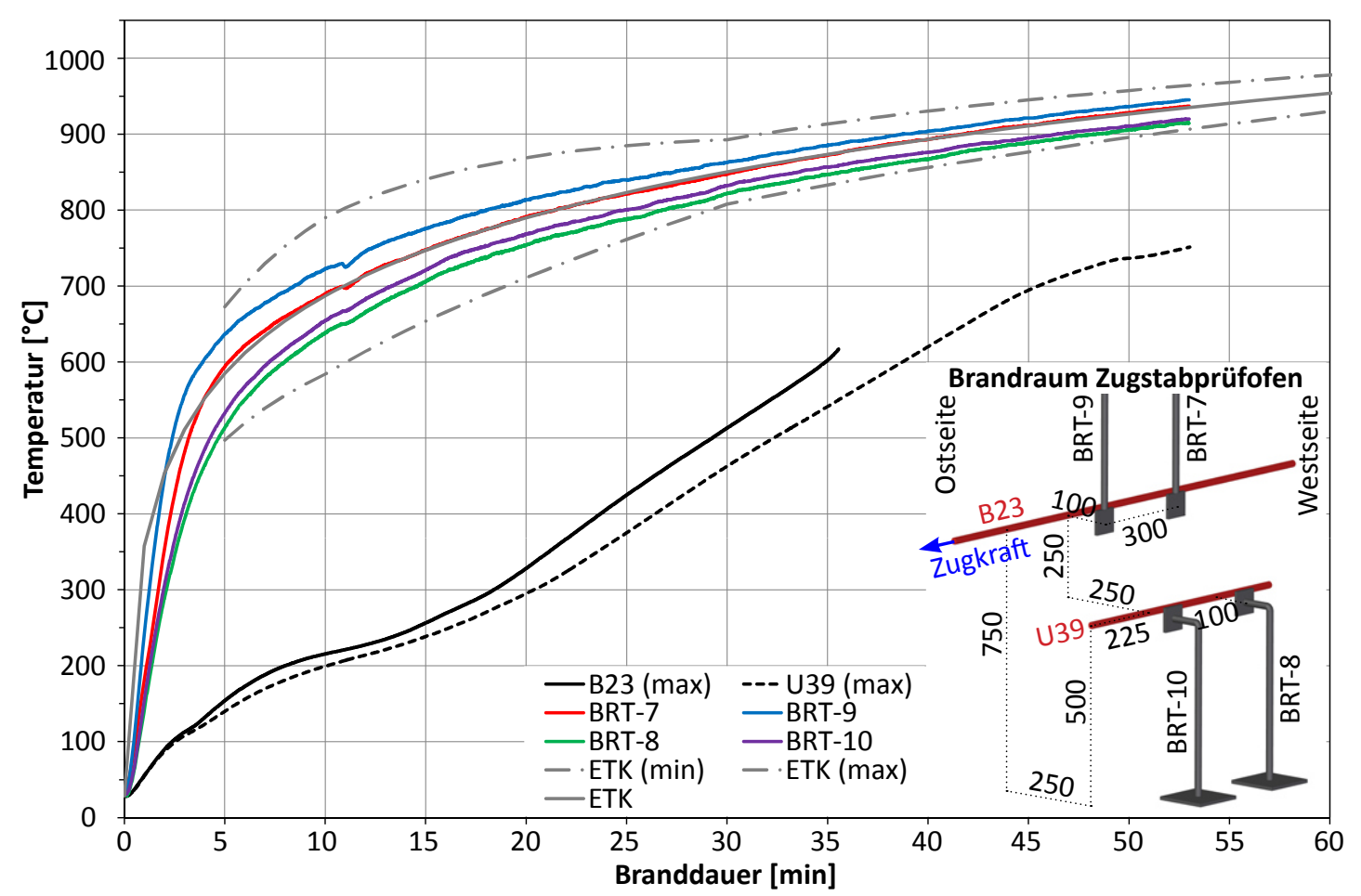

Abb. A.04: Temperatur-Branddauer-Kurven der Versuchskörper B23 und U39 sowie der Brandraumthermoelemente (BRT)

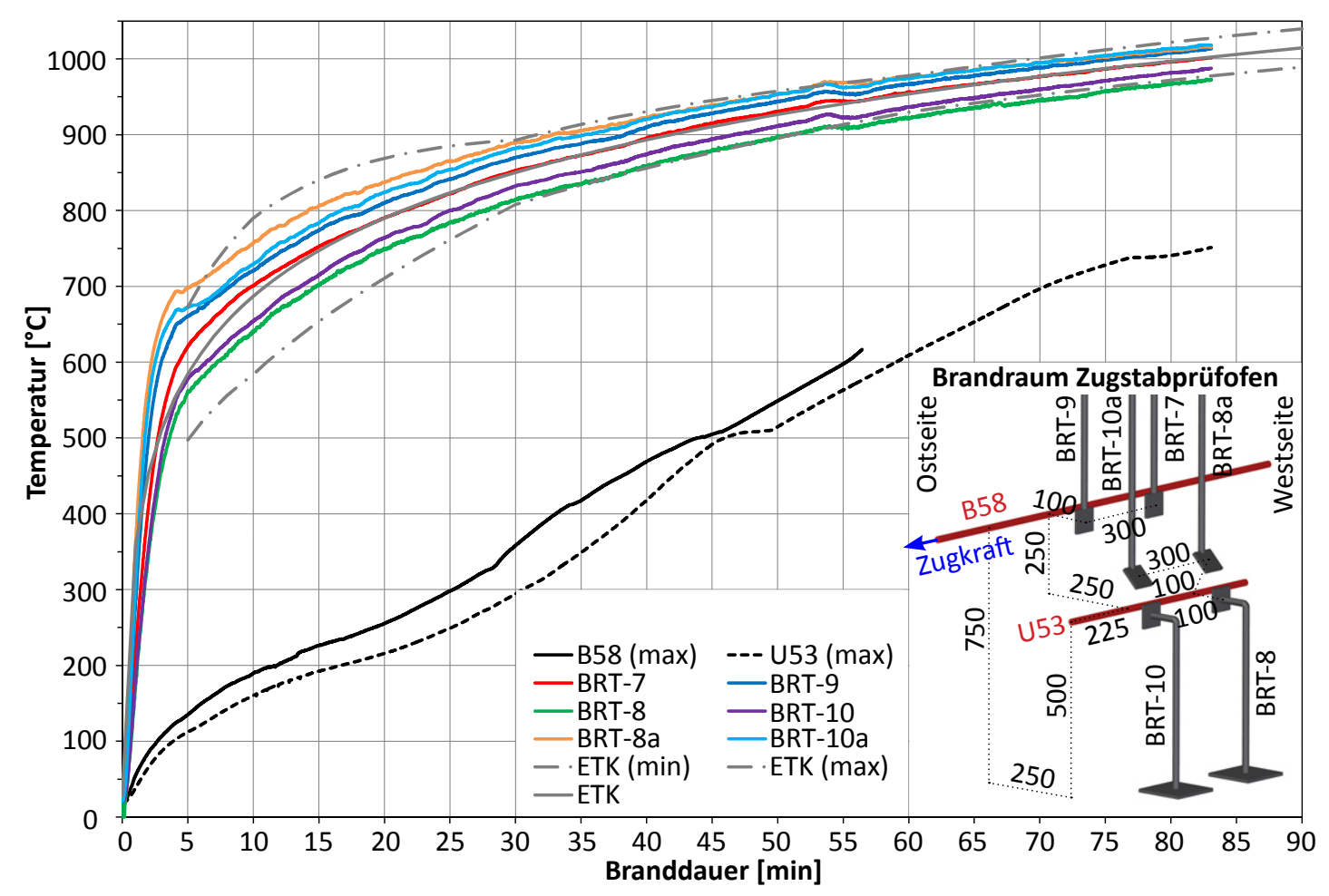

Abb. A.05: Temperatur-Branddauer-Kurven der Versuchskörper B58 und U53 sowie der Brandraumthermoelemente (BRT) 


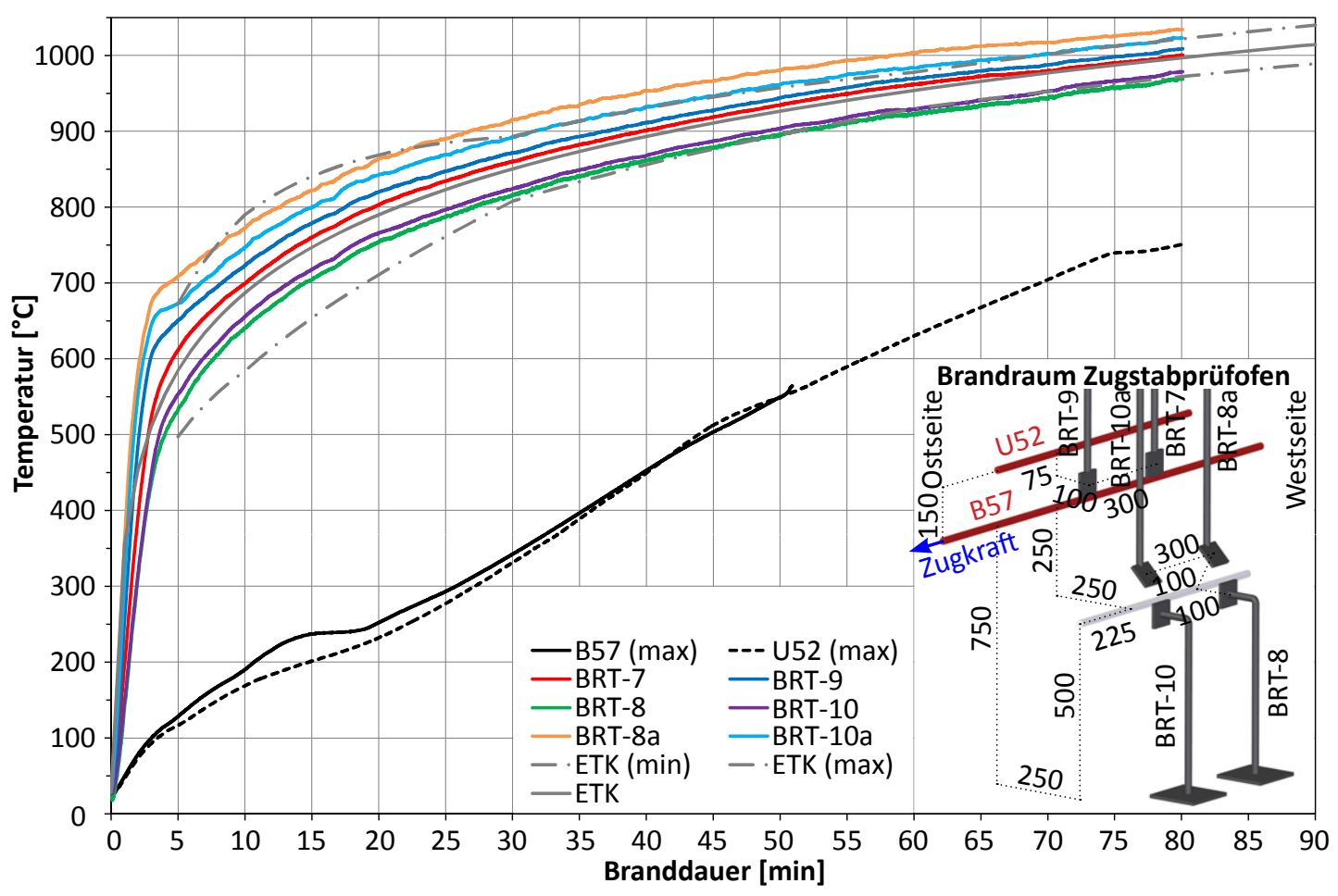

Abb. A.06: Temperatur-Branddauer-Kurven der Versuchskörper B57 und U52 sowie der Brandraumthermoelemente (BRT)

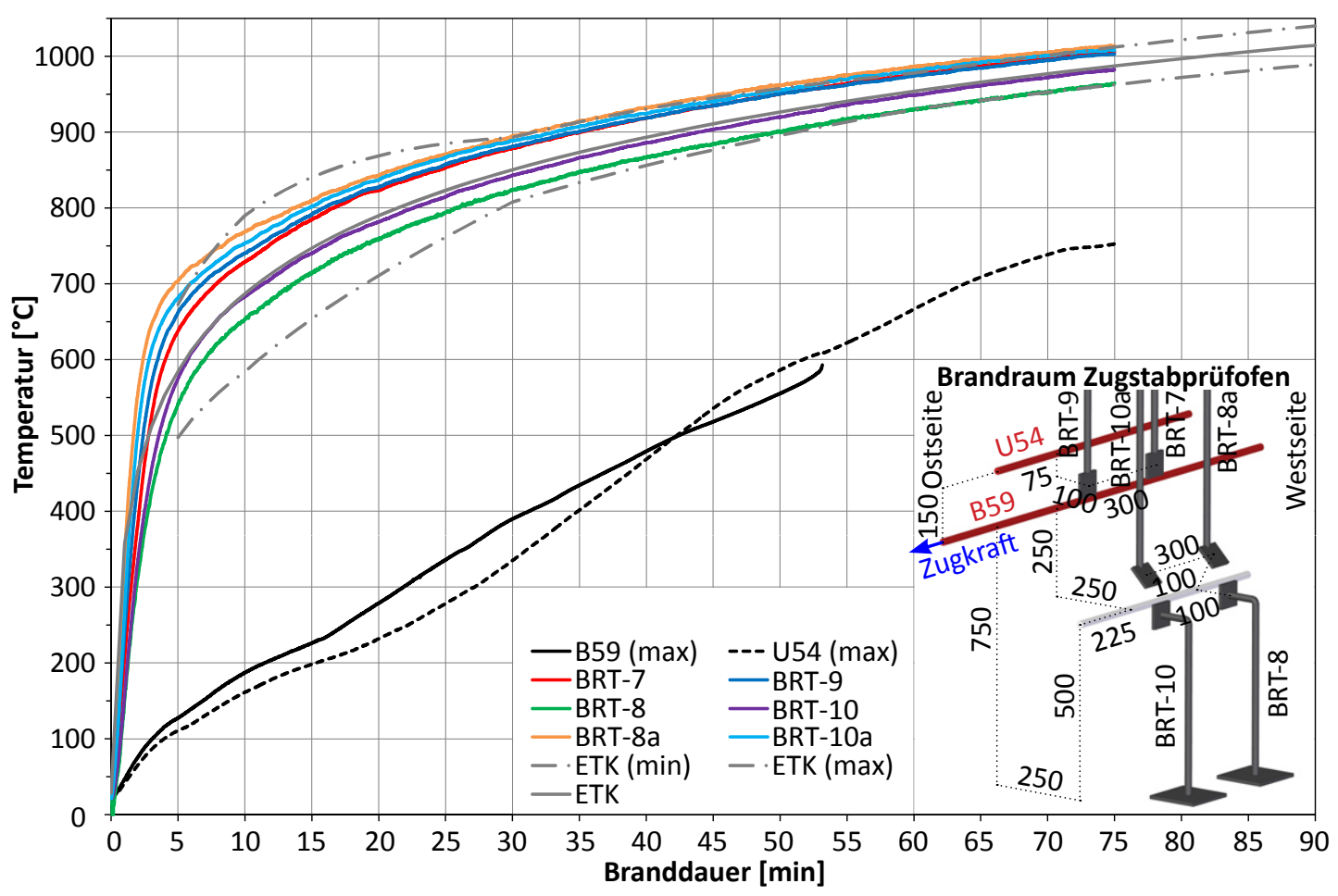

Abb. A.07: Temperatur-Branddauer-Kurven der Versuchskörper B59 und U54 sowie der Brandraumthermoelemente (BRT) 

Datenblätter zu den Brandversuchen im Realmaßstab

Mit Ausnahme der VR e sind im Forschungsbericht [35] bereits alle Datenblätter zu den in der BAM durchgeführten Brandversuchen im Realmaßstab dokumentiert. Auf den nachfolgenden Seiten sind ergänzend die Datenblätter der VR e angegeben.

Die Datenblätter dienen als Zusammenfassung der durchgeführten Brandversuche. Neben allgemeinen Angaben zum Versuchskörper ist in jedem Datenblatt der an den Thermoelementen gemessene Temperaturverlauf sowie die gemessene Gesamtverformung der belasteten Zugglieder angegeben. In einigen Diagrammen ist der Temperaturverlauf einzelner Thermoelemente nicht dargestellt. Dies ist darauf zurückzuführen, dass das entsprechende Thermoelement während des Brandversuches ausgefallen ist und keine Temperaturwerte aufgezeichnet werden konnten. Um für die spätere numerische Simulation trotzdem ein vollständiges Temperaturfeld erzeugen zu können, werden die Temperaturwerte des defekten Thermoelementes unter Zuhilfenahme der benachbarten Messtellen linear interpoliert.

Bei der im Stablängsverfomung-Branddauer-Verlauf angegebenen thermischen Verformung handelt es sich um einen rechnerisch ermittelten Verlauf. Für die Berechnung wird ein linearer Wärmeausdehnungskoeffizient sowie die im Zugglied gemessene Stahltemperatur zu Grunde gelegt. Der Wärmeausdehnungskoeffizient für die Kreisvollprofile entspricht Gleichung (4.8). Die gesamte thermische Verformung des Zuggliedes wird durch Summation der thermischen Längsverformung für die Stabbereiche jedes Thermoelementes berechnet. Der Verlauf der thermischen Verformungen wird um den Betrag der Anfangsverformung aus der mechanischen Beanspruchung verschoben. Dadurch lässt sich zeigen, ab wann der Festigkeitsverlust des Stahl einsetzt. Die in den Diagrammen dargestellt Gesamtverformung der mechanisch belasteten Zugglieder wird um den Verformungsanteil aus dem Belastungsrahmens bereinigt.

Zusätzlich zu den Diagrammen ist in jedem Datenblatt eine Skizze des Versuchskörpers, eine kurze Beschreibung wichtiger Ereignisse während des Brandversuches, ein Foto des Zuggliedes nach dem Brandversuch sowie die Ergebnisse der Messung der Schaumhöhen des reaktiven Brandschutzsystems enthalten. Stellen, an denen die Schaumhöhe nicht mehr eindeutig ermittelt werden konnte, sind mit einem Strich oder Werten in runden Klammern gekennzeichnet. Die Fotos der einzelnen Versuchskörper werden vom Autor der vorliegenden Arbeit so skaliert, dass diese möglichst gut mit der realen Stablänge übereinstimmen.

Die Auflistung der Datenblätter erfolgt mit zunehmenden Lastausnutzungsgrad. Unbelastete Versuchskörper, welche simultan mit den mechanisch belasteten Zuggliedern getestet werden, befinden sich unmittelbar nebeneinander. 


\begin{tabular}{|l|l|}
\hline \multicolumn{2}{|c|}{ Versuchskörper $\mathrm{B55}$} \\
\hline Stahlsorte & $\mathrm{S} 355 \mathrm{~J} 2 \mathrm{C}+\mathrm{C}$ \\
\hline Kreisvollprofil $\varnothing$ & $30 \mathrm{~mm}$ \\
\hline Profilfaktor & $133 \mathrm{~m}^{-1}$ \\
\hline Trockenschichtdicke & $3,5 \mathrm{~mm}$ \\
\hline Zugkraft & $60,44 \mathrm{kN}$ \\
\hline Lastausnutzung $\left(\mu_{\mathrm{f}}\right)$ & 0,15 \\
\hline Prüfdatum & 09.12 .2014 \\
\hline Zeitpunkt Probenbruch & $72,76 \mathrm{~min}$ \\
\hline Bruchposition & $\mathrm{TE} 5$ \\
\hline Max. PKT nach 20 min & $246^{\circ} \mathrm{C}(\mathrm{TE} \mathrm{5)}$ \\
\hline Max. PKT bei Bruch & $736^{\circ} \mathrm{C}(\mathrm{TE} 5)$ \\
\hline Schaumdicke (min - max) & $11-26 \mathrm{~mm} *$ \\
\hline
\end{tabular}

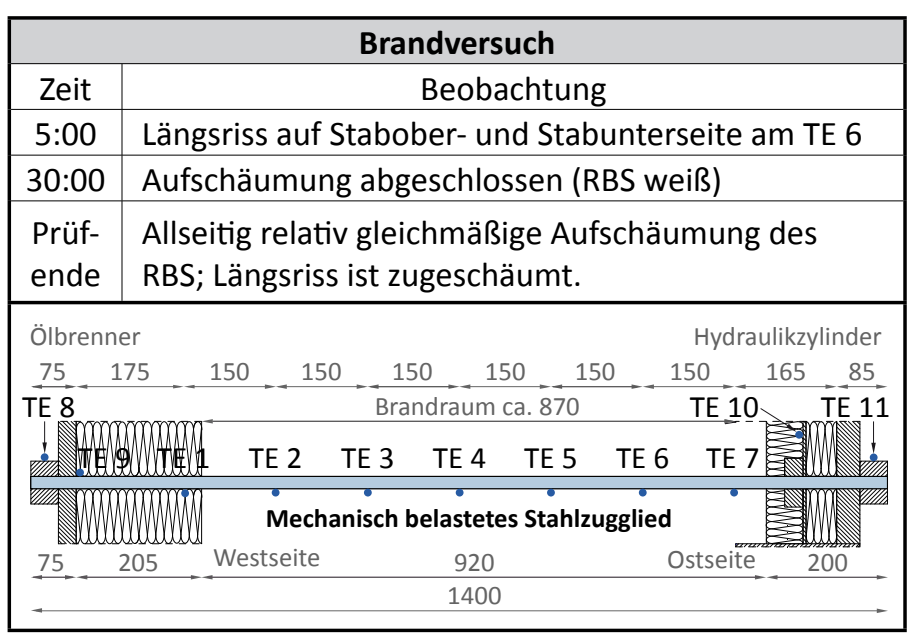

* Betrachtung zwischen $x=100-800 \mathrm{~mm}$
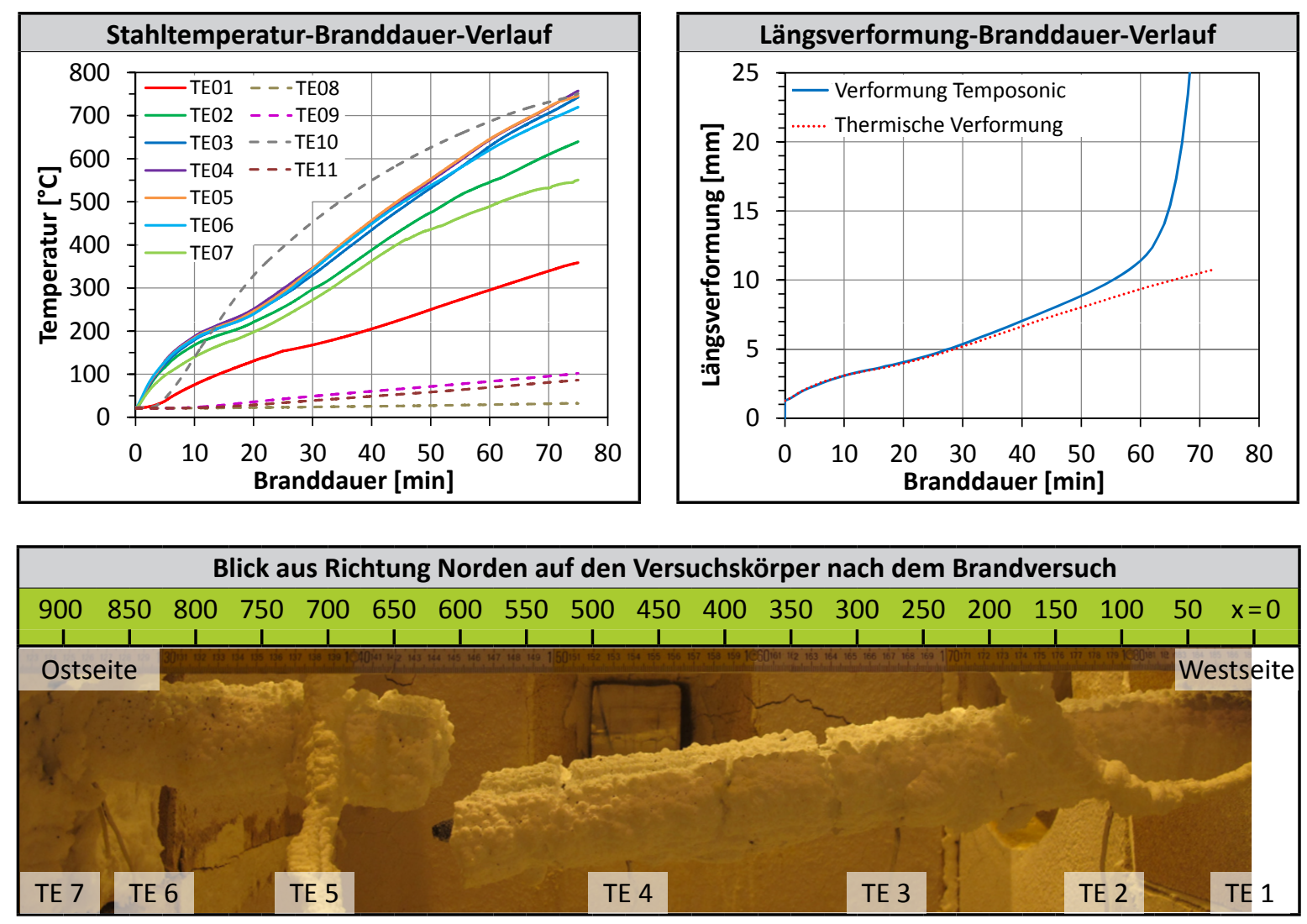

\begin{tabular}{|c|c|c|c|c|c|c|c|c|c|c|c|c|c|c|c|c|c|c|}
\hline \multicolumn{11}{|c|}{ Schaumdicke $t$ [mm], Position der Messstelle $\mathbf{~ [ m m ] ~}$} \\
\hline $\mathrm{x}=0$ & 50 & 100 & 150 & 200 & 250 & 300 & 350 & 400 & 450 & 500 & 550 & 600 & 650 & 700 & 750 & 800 & 850 & 900 \\
\hline $\mathrm{t}_{\mathrm{N}}=9$ & 22 & 26 & 26 & 24 & 23 & 20 & 23 & 25 & 24 & 22 & 24 & - & 25 & 25 & 23 & 17 & 11 & - \\
\hline $\mathrm{t}_{\mathrm{s}}=9$ & 17 & 20 & 21 & 25 & 23 & 24 & 25 & 24 & 22 & 26 & - & - & 25 & 20 & 20 & 15 & 14 & - \\
\hline $\mathrm{t}_{\mathrm{o}}=11$ & 18 & 21 & 20 & 21 & 22 & 21 & 23 & 21 & 21 & 22 & 22 & - & 18 & 20 & 19 & 20 & - & - \\
\hline $\mathrm{t}_{\mathrm{u}}=8$ & 15 & 15 & 16 & 18 & 16 & 19 & 20 & 22 & 19 & 19 & 20 & - & 20 & 17 & 13 & 11 & - & - \\
\hline
\end{tabular}




\begin{tabular}{|l|l|}
\hline \multicolumn{2}{|c|}{ Versuchskörper U91 } \\
\hline Stahlsorte & $\mathrm{S} 355 \mathrm{J2C}$ \\
\hline Kreisvollprofil $\varnothing$ & $30 \mathrm{~mm}$ \\
\hline Profilfaktor & $133 \mathrm{~m}^{-1}$ \\
\hline Trockenschichtdicke & $2,5 \mathrm{~mm}$ \\
\hline Prüfdatum & 09.12 .2014 \\
\hline Max. PKT nach 20 min & $534^{\circ} \mathrm{C}($ TE 1) \\
\hline Max. PKT bei Bruch & $848^{\circ} \mathrm{C}($ TE 1) \\
\hline Schaumdicke (min - max) & $9-27 \mathrm{~mm}^{*}$ \\
\hline
\end{tabular}

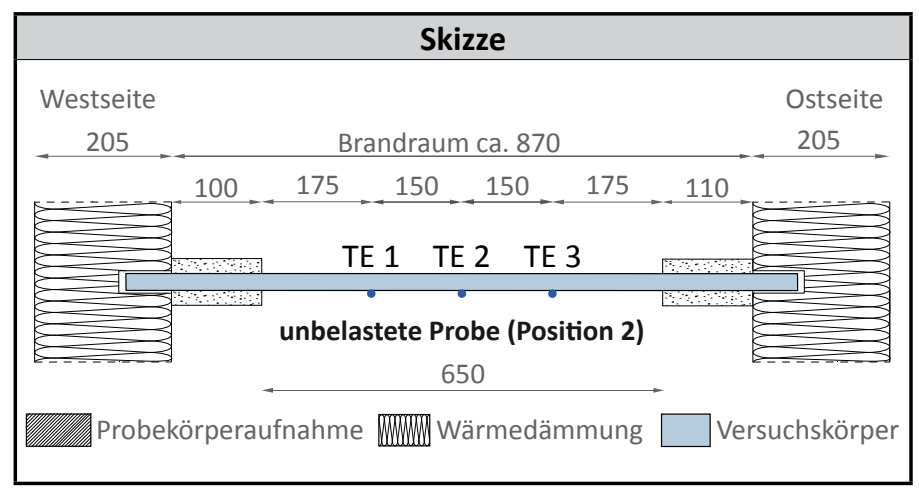

*Betrachtung zwischen $x=100-550 \mathrm{~mm}$

\begin{tabular}{|c|l|}
\hline \multicolumn{2}{|c|}{ Brandversuch } \\
\hline Zeit & \multicolumn{1}{|c|}{ Beobachtung } \\
\hline 0:00 & $\begin{array}{l}\text { Prüfung erfolgt zusammen mit B55; } \\
\text { Probe befindet sich 150 mm über B55 }\end{array}$ \\
\hline $5: 00$ & Längsriss auf Staboberseite (zugeschäumt) \\
\hline $30: 00$ & Aufschäumung abgeschlossen (RBS weiß) \\
\hline $40: 00$ & Keine sichtbare Veränderung des RBS \\
\hline $\begin{array}{l}\text { Prüf- } \\
\text { ende }\end{array}$ & $\begin{array}{l}\text { Längsriss auf der Staboberseite (zuge- } \\
\text { schäumt); relativ gleichmäßige Auf- } \\
\text { schäumung des RBS; im Bereich der } \\
\text { MTE wurde die Aufschäumung des RBS } \\
\text { beeinträchtigt; MTE 1 nach 10 Minuten } \\
\text { defekt oder nicht mehr am Stahl. }\end{array}$ \\
\hline
\end{tabular}
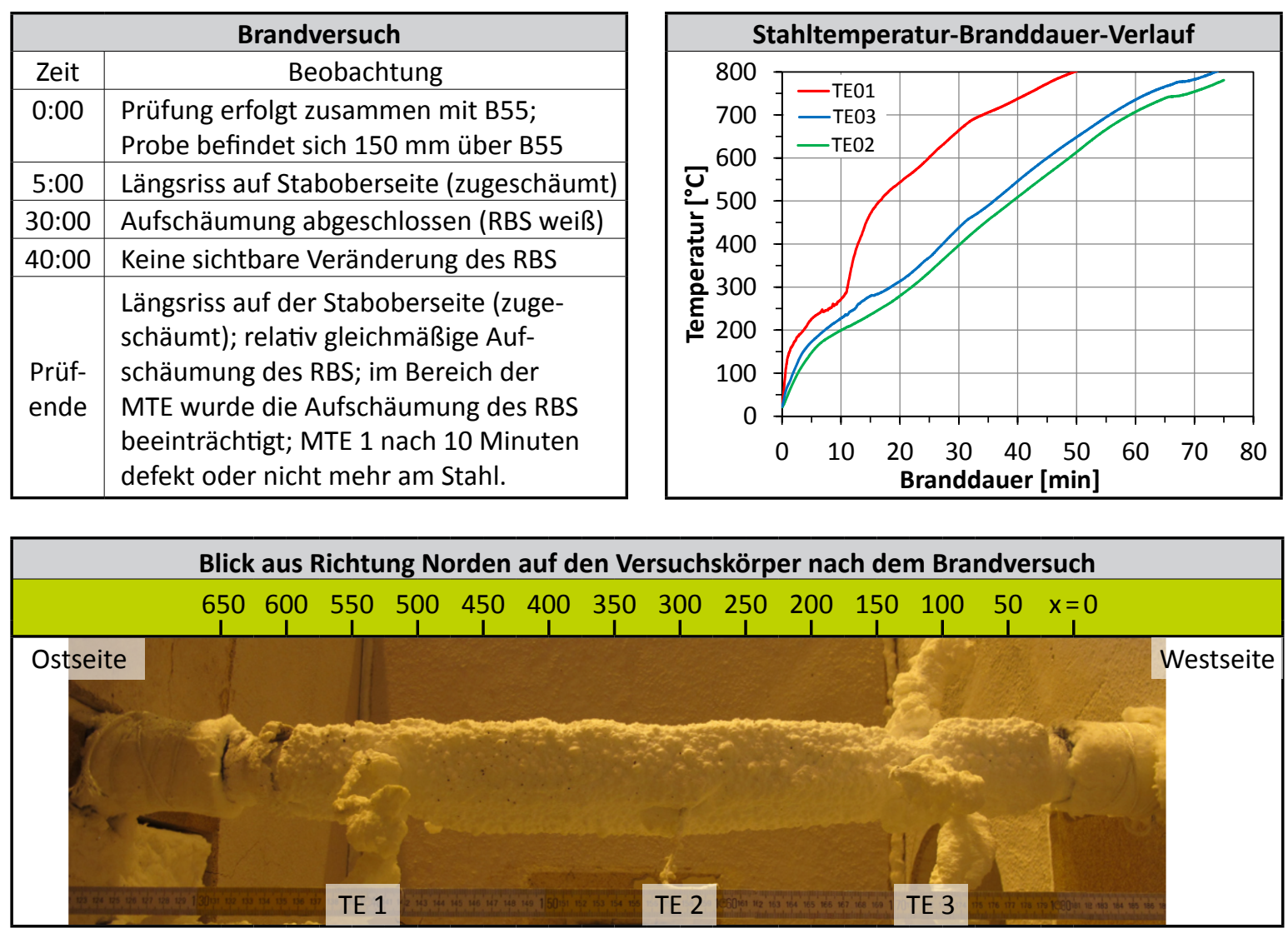

\begin{tabular}{|c|c|c|c|c|c|c|c|c|c|c|c|c|c|c|}
\hline Stabquerschnitt & \multicolumn{10}{|c|}{ Schaumdicke $\mathrm{t}[\mathrm{mm}$ ], Position der Messstelle $\mathbf{x}$ [mm } \\
\hline & $\mathrm{x}=0$ & 50 & 100 & 150 & 200 & 250 & 300 & 350 & 400 & 450 & 500 & 550 & 600 & 650 \\
\hline & $\mathrm{t}_{\mathrm{N}}=20$ & 21 & 12 & 18 & 21 & 23 & 22 & 23 & 24 & 22 & 16 & 17 & 15 & 9 \\
\hline & $\mathrm{t}_{\mathrm{s}}=18$ & 14 & 12 & 17 & 18 & 17 & 19 & 18 & 16 & 18 & 17 & 18 & 14 & 15 \\
\hline & $\mathrm{t}_{\mathrm{o}}=17$ & 17 & 9 & 14 & 16 & 17 & 15 & 17 & 17 & 18 & 18 & 17 & 14 & 15 \\
\hline & $\mathrm{t}_{\mathrm{u}}=13$ & 16 & 13 & 21 & 23 & 26 & 23 & 27 & 25 & 27 & 18 & 20 & 19 & 11 \\
\hline
\end{tabular}




\begin{tabular}{|l|l|}
\hline \multicolumn{2}{|c|}{ Versuchskörper B56 } \\
\hline Stahlsorte & S355 J2C \\
\hline Kreisvollprofil $\varnothing$ & $30 \mathrm{~mm}$ \\
\hline Profilfaktor & $133 \mathrm{~m}^{-1}$ \\
\hline Trockenschichtdicke & $3,5 \mathrm{~mm}$ \\
\hline Zugkraft & $100,73 \mathrm{kN}$ \\
\hline Lastausnutzung $\left(\mu_{\mathrm{fi}}\right)$ & 0,25 \\
\hline Prüfdatum & 12.11 .2014 \\
\hline Zeitpunkt Probenbruch & $64,56 \mathrm{~min}$ \\
\hline Bruchposition & TE 5 - TE 6 \\
\hline Max. PKT nach 20 min & $249^{\circ} \mathrm{C}(\mathrm{TE} \mathrm{5)}$ \\
\hline Max. PKT bei Bruch & $696^{\circ} \mathrm{C}(\mathrm{TE} \mathrm{5)}$ \\
\hline Schaumdicke (min - max) & $15-24 \mathrm{~mm} *$ \\
\hline
\end{tabular}

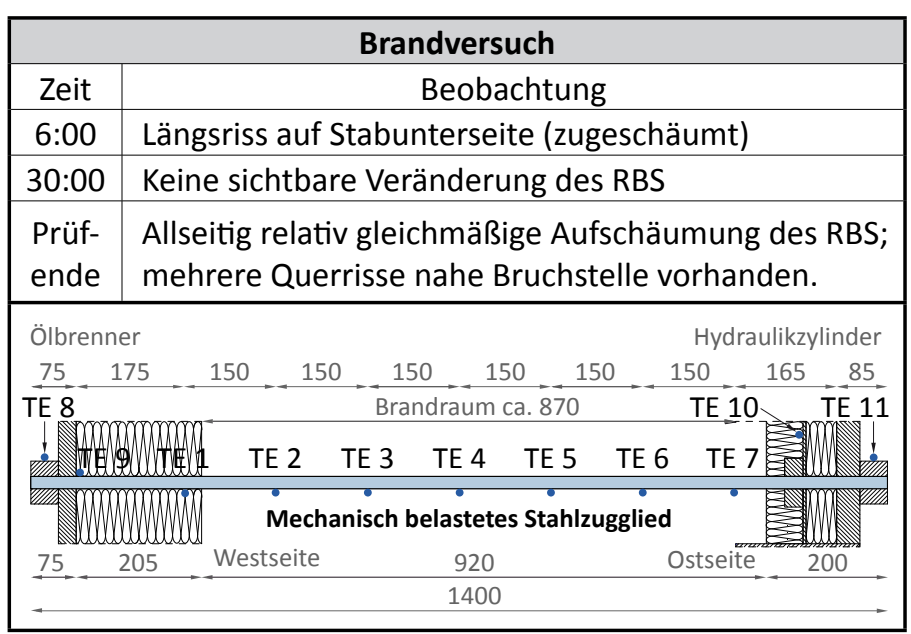

* Betrachtung zwischen $x=100-800 \mathrm{~mm}$
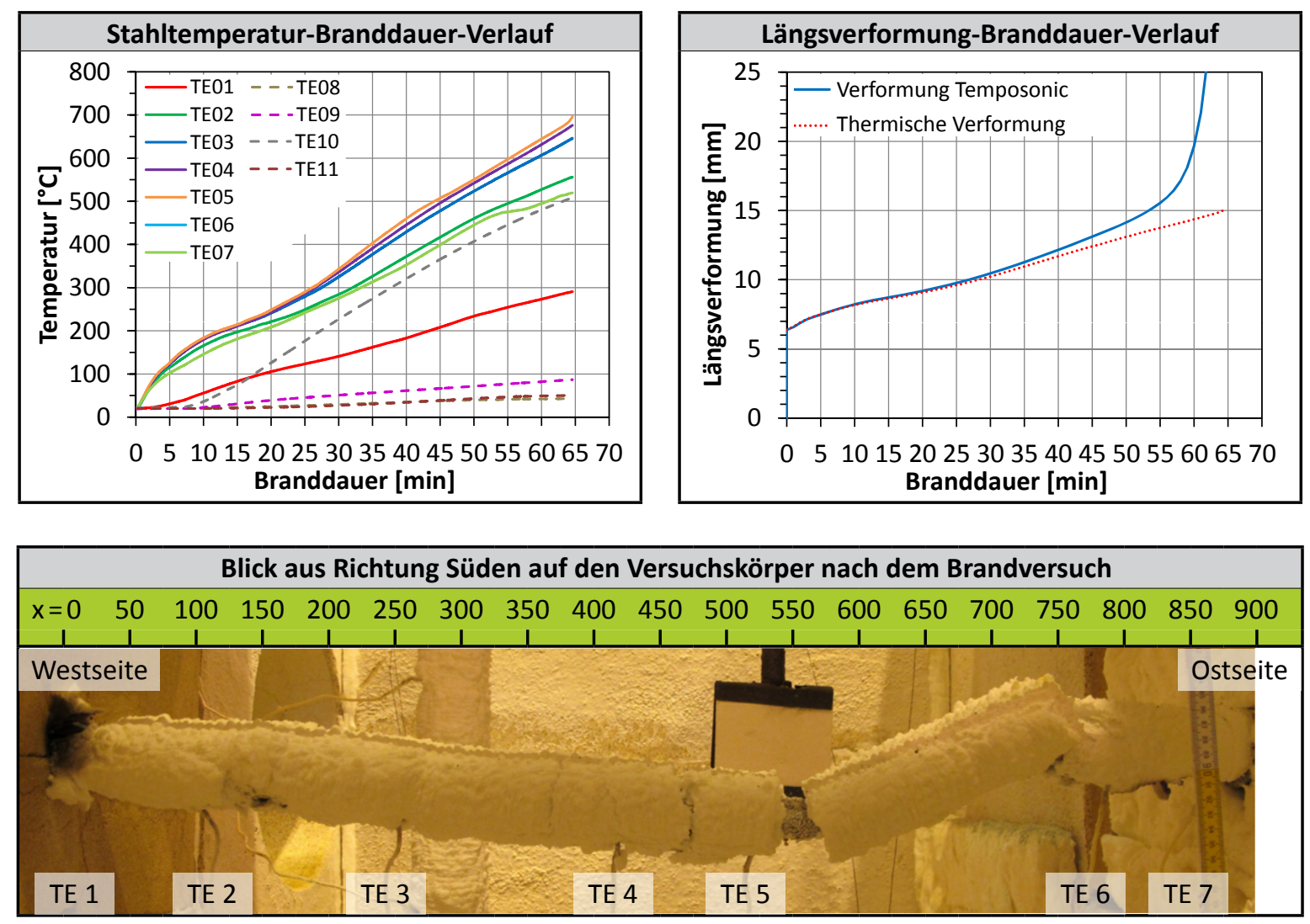

\begin{tabular}{|c|c|c|c|c|c|c|c|c|c|c|c|c|c|c|c|c|c|c|}
\hline \multicolumn{11}{|c|}{ Schaumdicke $t$ [mm], Position der Messstelle $\mathbf{~ [ m m ] ~}$} \\
\hline $\mathrm{x}=0$ & 50 & 100 & 150 & 200 & 250 & 300 & 350 & 400 & 450 & 500 & 550 & 600 & 650 & 700 & 750 & 800 & 850 & 900 \\
\hline $\mathrm{t}_{\mathrm{N}}=9$ & 17 & 24 & 21 & 21 & 21 & 21 & 23 & 22 & 23 & 22 & - & - & - & - & 22 & 19 & 11 & - \\
\hline $\mathrm{t}_{\mathrm{s}}=8$ & 17 & 21 & 22 & 22 & 22 & 15 & 19 & 19 & 20 & 21 & - & - & - & - & - & 15 & 9 & - \\
\hline $\mathrm{t}_{\mathrm{o}}=7$ & 18 & 19 & 20 & 18 & 18 & 15 & 16 & 18 & 18 & 17 & - & - & - & - & 17 & 15 & 12 & - \\
\hline $\mathrm{t}_{\mathrm{u}}=8$ & 19 & 16 & 15 & 15 & 13 & 19 & 20 & 17 & 19 & 19 & - & - & - & - & 19 & 16 & 13 & - \\
\hline
\end{tabular}




\begin{tabular}{|l|l|}
\hline \multicolumn{2}{|c|}{ Versuchskörper U51 } \\
\hline Stahlsorte & $\mathrm{S} 355 \mathrm{~J} 2 \mathrm{C}$ \\
\hline Kreisvollprofil $\varnothing$ & $30 \mathrm{~mm}$ \\
\hline Profilfaktor & $133 \mathrm{~m}^{-1}$ \\
\hline Trockenschichtdicke & $3,5 \mathrm{~mm}$ \\
\hline Prüfdatum & 21.07 .2014 \\
\hline Max. PKT nach 20 min & $238^{\circ} \mathrm{C}$ (TE 3) \\
\hline Max. PKT bei Bruch & $520^{\circ} \mathrm{C}$ (TE 3) \\
\hline Schaumdicke (min - max) & $14-26 \mathrm{~mm}$ \\
\hline
\end{tabular}

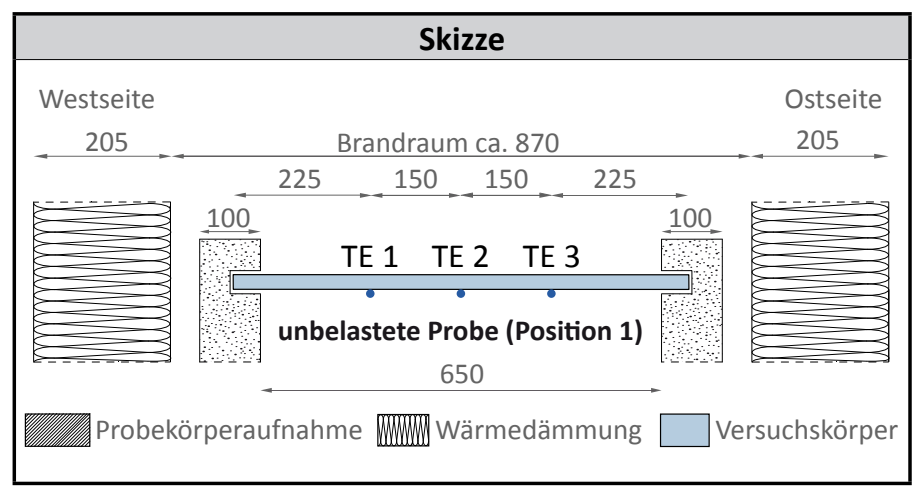

*Betrachtung zwischen $x=100-550 \mathrm{~mm}$

\begin{tabular}{|c|l|}
\hline \multicolumn{2}{|c|}{ Brandversuch } \\
\hline Zeit & \multicolumn{1}{|c|}{ Beobachtung } \\
\hline 0:00 & Prüfung erfolgt zusammen mit B21; \\
\hline 6:00 & Längsriss Stabunterseite (zugeschäumt) \\
\hline 25:00 & RBS vollständig aufgeschäumt (weiß) \\
\hline $30: 00$ & Keine sichtbare Veränderung des RBS \\
\hline $\begin{array}{l}\text { Prüf- } \\
\text { ende }\end{array}$ & $\begin{array}{l}\text { Längsriss auf der Stabunterseite (zuge- } \\
\text { schäumt); relativ gleichmäßige Aufschäu- } \\
\text { mung des RBS (Stabunterseite etwas } \\
\text { geringere Schaumdicke). }\end{array}$ \\
\hline
\end{tabular}
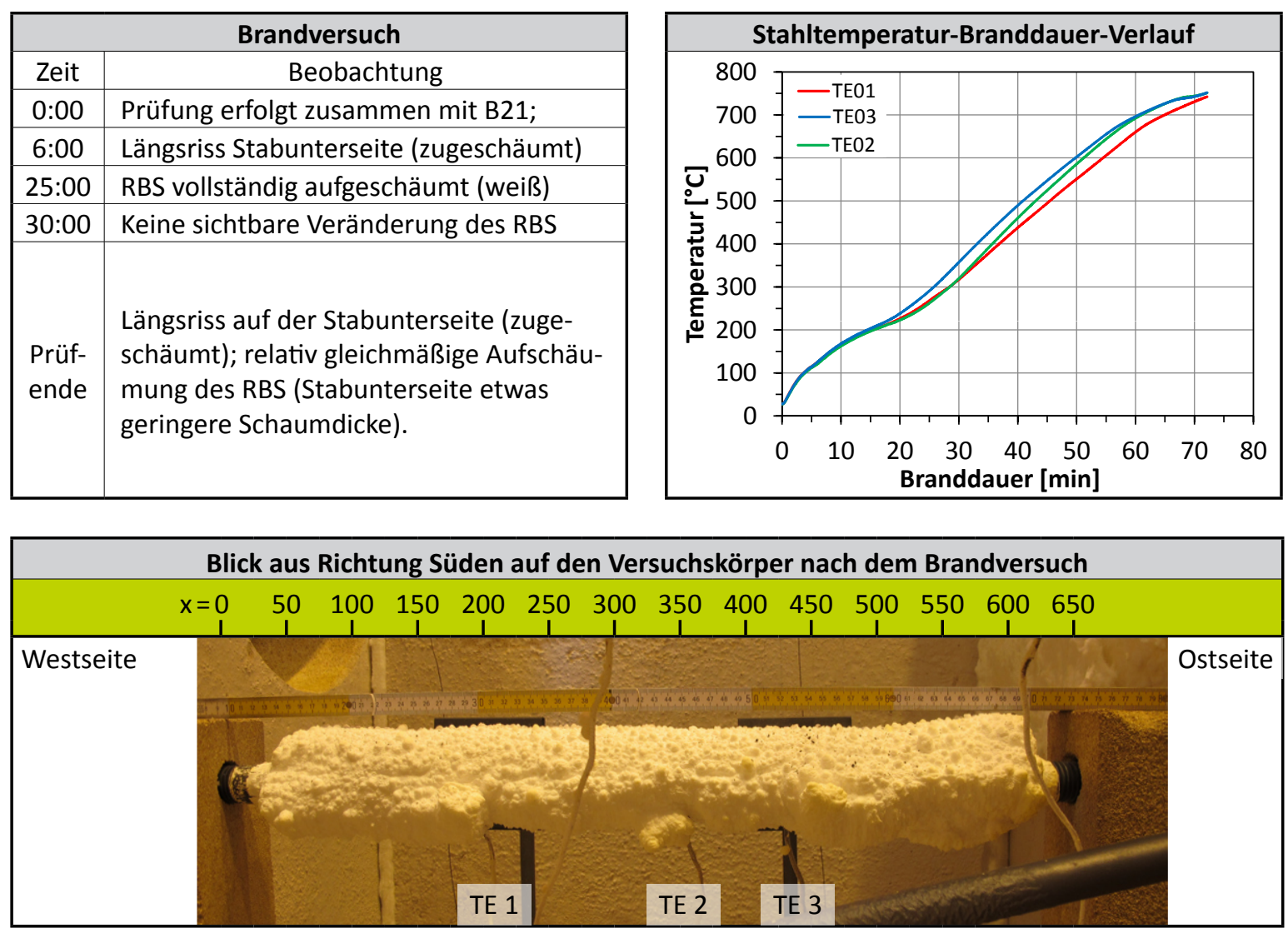

\begin{tabular}{|c|c|c|c|c|c|c|c|c|c|c|c|c|c|c|}
\hline \multirow{2}{*}{ Stabquerschnitt } & \multicolumn{14}{|c|}{ Schaumdicke $\mathrm{t}[\mathrm{mm}]$, Position der Messstelle $\mathrm{x}[\mathrm{mm}]$} \\
\hline & $x=0$ & 50 & 100 & 150 & 200 & 250 & 300 & 350 & 400 & 450 & 500 & 550 & 600 & 650 \\
\hline & $t_{N}=0$ & 30 & 30 & 35 & 38 & 27 & 30 & 22 & 23 & 28 & 26 & 27 & 28 & 0 \\
\hline & $t_{s}=0$ & 23 & 23 & 26 & 21 & 23 & 22 & 20 & 23 & 26 & 27 & 28 & 29 & 0 \\
\hline & $t_{0}=0$ & 18 & 27 & 25 & 25 & 24 & 25 & 24 & 24 & 23 & 24 & 28 & 31 & 0 \\
\hline & $t_{u}=0$ & 25 & 26 & 27 & 24 & 18 & 14 & 12 & 16 & 28 & 25 & 27 & 12 & 0 \\
\hline
\end{tabular}




\begin{tabular}{|l|l|}
\hline \multicolumn{2}{|c|}{ Versuchskörper B60 } \\
\hline Stahlsorte & S355 J2C \\
\hline Kreisvollprofil $\varnothing$ & $30 \mathrm{~mm}$ \\
\hline Profilfaktor & $133 \mathrm{~m}^{-1}$ \\
\hline Trockenschichtdicke & $3,5 \mathrm{~mm}$ \\
\hline Zugkraft & $141,02 \mathrm{kN}$ \\
\hline Lastausnutzung $\left(\mu_{\mathrm{fi}}\right)$ & 0,35 \\
\hline Prüfdatum & 11.12 .2014 \\
\hline Zeitpunkt Probenbruch & $59,63 \mathrm{~min}$ \\
\hline Bruchposition & TE 4 - TE 5 \\
\hline Max. PKT nach 20 min & $263^{\circ} \mathrm{C}(\mathrm{TE} 5)$ \\
\hline Max. PKT bei Bruch & $648^{\circ} \mathrm{C}(\mathrm{TE} 4)$ \\
\hline Schaumdicke (min - max) & $12-32 \mathrm{~mm} *$ \\
\hline
\end{tabular}

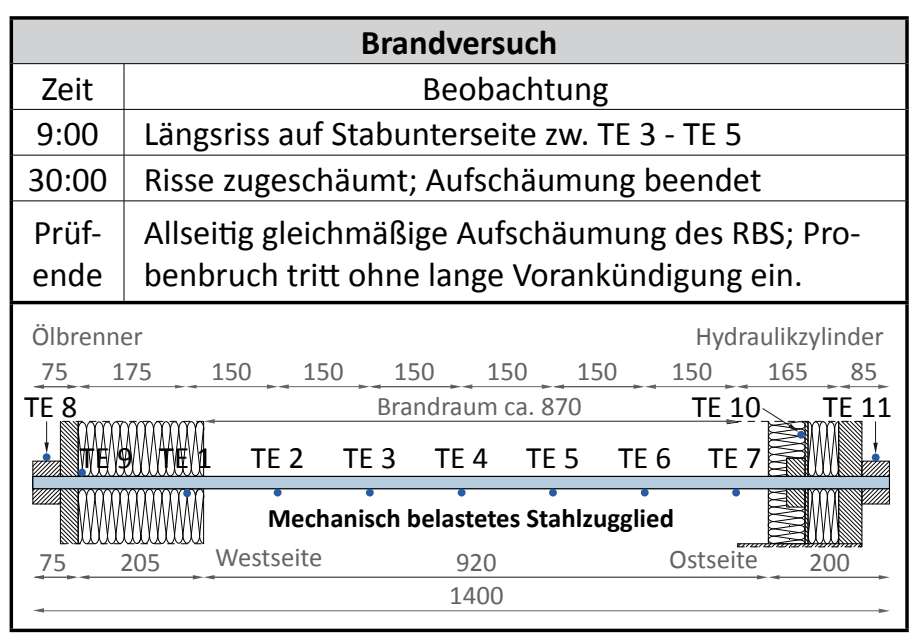

* Betrachtung zwischen $x=100-800 \mathrm{~mm}$
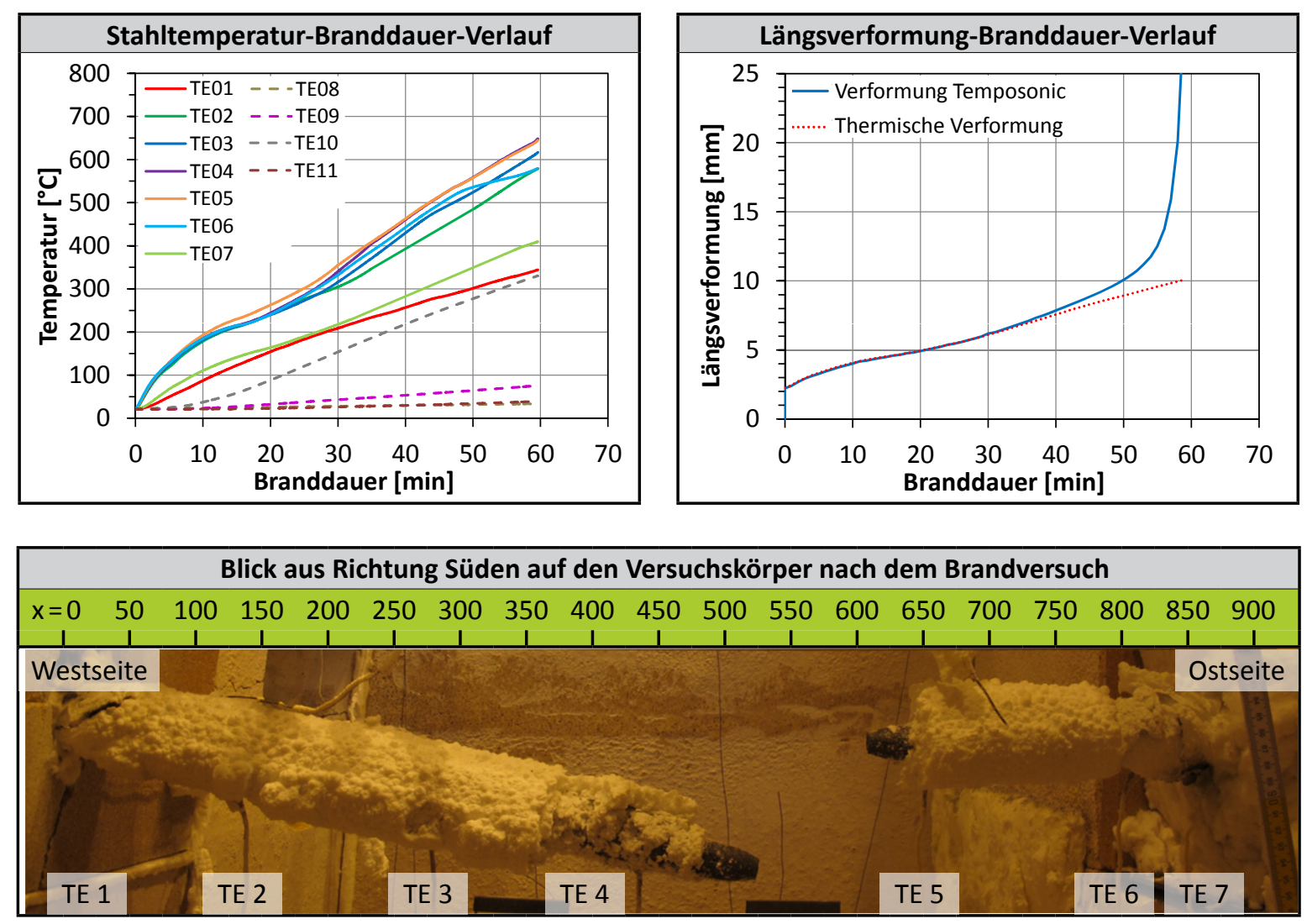

\begin{tabular}{|c|c|c|c|c|c|c|c|c|c|c|c|c|c|c|c|c|c|c|}
\hline \multicolumn{11}{|c|}{ Schaumdicke $t$ [mm], Position der Messstelle $\mathbf{~ [ m m ] ~}$} \\
\hline $\mathrm{x}=0$ & 50 & 100 & 150 & 200 & 250 & 300 & 350 & 400 & 450 & 500 & 550 & 600 & 650 & 700 & 750 & 800 & 850 & 900 \\
\hline $\mathrm{t}_{\mathrm{N}}=10$ & 15 & 21 & 20 & 22 & - & - & - & - & - & - & - & - & 32 & 31 & 21 & - & - & - \\
\hline $\mathrm{t}_{\mathrm{s}}=8$ & 15 & 18 & 19 & 19 & 21 & 22 & 26 & - & - & - & - & - & 24 & 24 & 24 & - & - & - \\
\hline $\mathrm{t}_{\mathrm{o}}=15$ & 20 & 21 & 19 & 22 & 24 & 25 & 23 & - & - & - & - & - & 25 & 25 & 24 & - & - & - \\
\hline $\mathrm{t}_{\mathrm{u}}=8$ & 15 & 13 & 14 & 19 & 16 & 16 & 18 & - & - & - & - & - & 16 & 14 & 12 & - & - & - \\
\hline
\end{tabular}




\begin{tabular}{|l|l|}
\hline \multicolumn{2}{|c|}{ Versuchskörper U92 } \\
\hline Stahlsorte & $\mathrm{S} 355 \mathrm{J2C}$ \\
\hline Kreisvollprofil $\varnothing$ & $30 \mathrm{~mm}$ \\
\hline Profilfaktor & $133 \mathrm{~m}^{-1}$ \\
\hline Trockenschichtdicke & $2,5 \mathrm{~mm}$ \\
\hline Prüfdatum & 11.12 .2014 \\
\hline Max. PKT nach 20 min & $437^{\circ} \mathrm{C}($ TE 1) \\
\hline Max. PKT bei Bruch & $769^{\circ} \mathrm{C}($ TE 1) \\
\hline Schaumdicke (min - max) & $5-27 \mathrm{~mm}^{*}$ \\
\hline
\end{tabular}

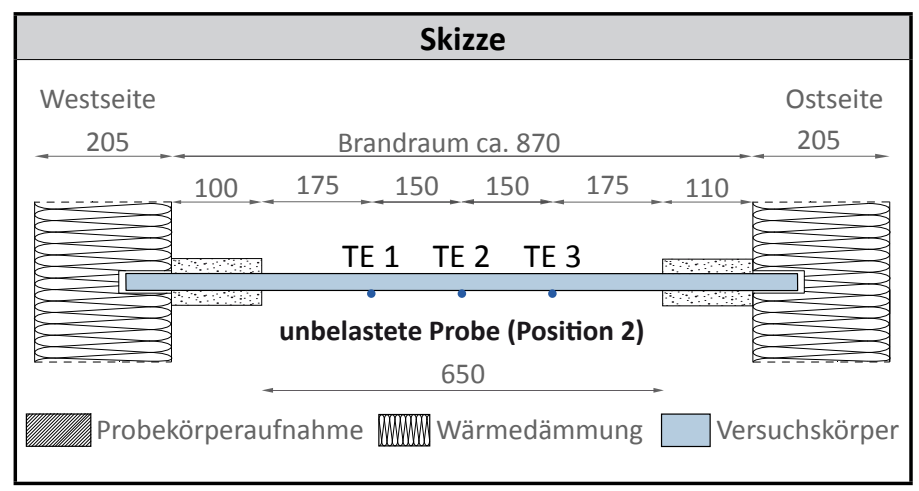

*Betrachtung zwischen $x=100-550 \mathrm{~mm}$

\begin{tabular}{|c|l|}
\hline \multicolumn{2}{|c|}{ Brandversuch } \\
\hline Zeit & \multicolumn{1}{|c|}{ Beobachtung } \\
\hline $0: 00$ & $\begin{array}{l}\text { Prüfung erfolgt zusammen mit B60; } \\
\text { Probe befindet sich 150 mm über B60 }\end{array}$ \\
\hline $5: 00$ & Längsriss auf Staboberseite (zugeschäumt) \\
\hline $30: 00$ & Risse nahezu komplett zugeschäumt \\
\hline $35: 00$ & Keine sichtbare Veränderung des RBS \\
\hline $\begin{array}{l}\text { Prüf- } \\
\text { ende }\end{array}$ & $\begin{array}{l}\text { MTE 1 wahrscheinlich defekt oder nicht } \\
\text { mehr am Probekörper; Längsriss auf der } \\
\text { Staboberseite (zugeschäumt); relativ } \\
\text { gleichmäßige Aufschäumung des RBS. }\end{array}$ \\
\hline
\end{tabular}
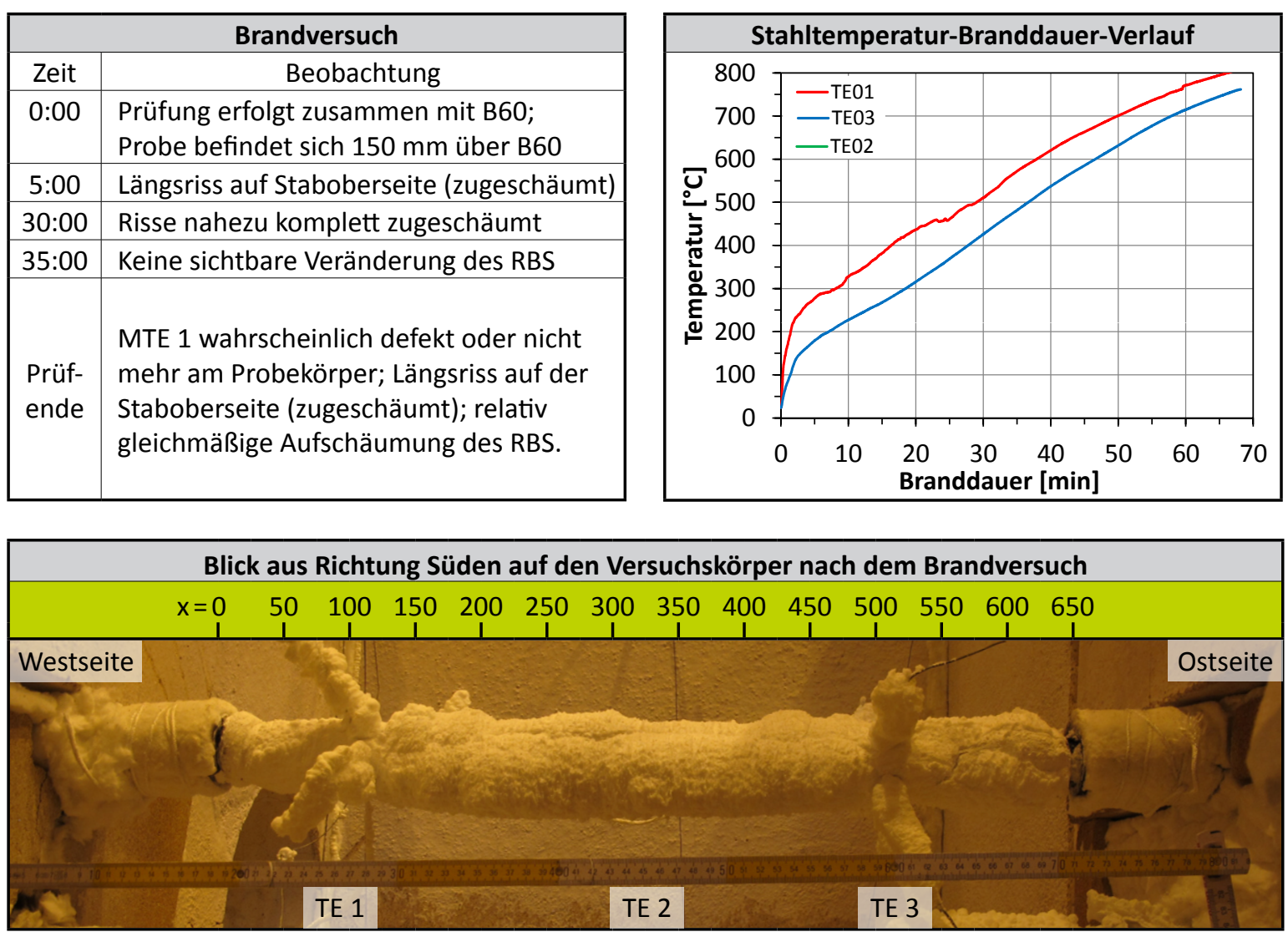

\begin{tabular}{|c|c|c|c|c|c|c|c|c|c|c|c|c|c|c|}
\hline Stabquerschnitt & \multicolumn{10}{|c|}{ Schaumdicke $\mathrm{t}$ [mm], Position der Messstelle $[\mathrm{mm}]$} \\
\hline & $\mathrm{x}=0$ & 50 & 100 & 150 & 200 & 250 & 300 & 350 & 400 & 450 & 500 & 550 & 600 & 650 \\
\hline & $\mathrm{t}_{\mathrm{N}}=19$ & 14 & 13 & 10 & 19 & 21 & 23 & 22 & 23 & 20 & 17 & 17 & 20 & 11 \\
\hline & $\mathrm{t}_{\mathrm{s}}=9$ & 10 & 5 & 8 & 7 & 11 & 11 & 12 & 9 & 7 & 7 & 5 & 10 & 10 \\
\hline & $\mathrm{t}_{\mathrm{o}}=14$ & 11 & 12 & 10 & 20 & 18 & 20 & 14 & 15 & 22 & 12 & 17 & 20 & 10 \\
\hline & $\mathrm{t}_{\mathrm{u}}=5$ & 10 & 13 & 11 & 26 & 28 & 28 & 27 & 27 & 25 & 16 & 16 & 26 & 18 \\
\hline
\end{tabular}




\begin{tabular}{|l|l|}
\hline \multicolumn{2}{|c|}{ Versuchskörper B58 } \\
\hline Stahlsorte & S355 J2C \\
\hline Kreisvollprofil $\varnothing$ & $30 \mathrm{~mm}$ \\
\hline Profilfaktor & $133 \mathrm{~m}^{-1}$ \\
\hline Trockenschichtdicke & $3,5 \mathrm{~mm}$ \\
\hline Zugkraft & $261,89 \mathrm{kN}$ \\
\hline Lastausnutzung $\left(\mu_{\mathrm{fi}}\right)$ & 0,45 \\
\hline Prüfdatum & 20.11 .2014 \\
\hline Zeitpunkt Probenbruch & $56,43 \mathrm{~min}$ \\
\hline Bruchposition & $\mathrm{TE} \mathrm{5-6}$ \\
\hline Max. PKT nach 20 min & $255^{\circ} \mathrm{C}(\mathrm{TE} \mathrm{5)}$ \\
\hline Max. PKT bei Bruch & $616^{\circ} \mathrm{C}(\mathrm{TE} \mathrm{5)}$ \\
\hline Schaumdicke (min - max) & $13-25 \mathrm{~mm} *$ \\
\hline
\end{tabular}

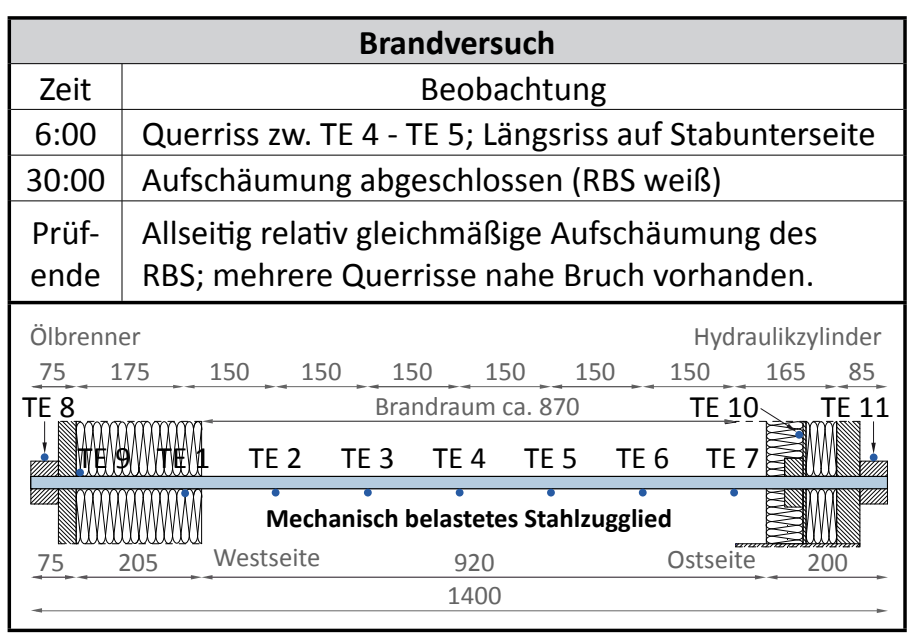

* Betrachtung zwischen $x=100-800 \mathrm{~mm}$
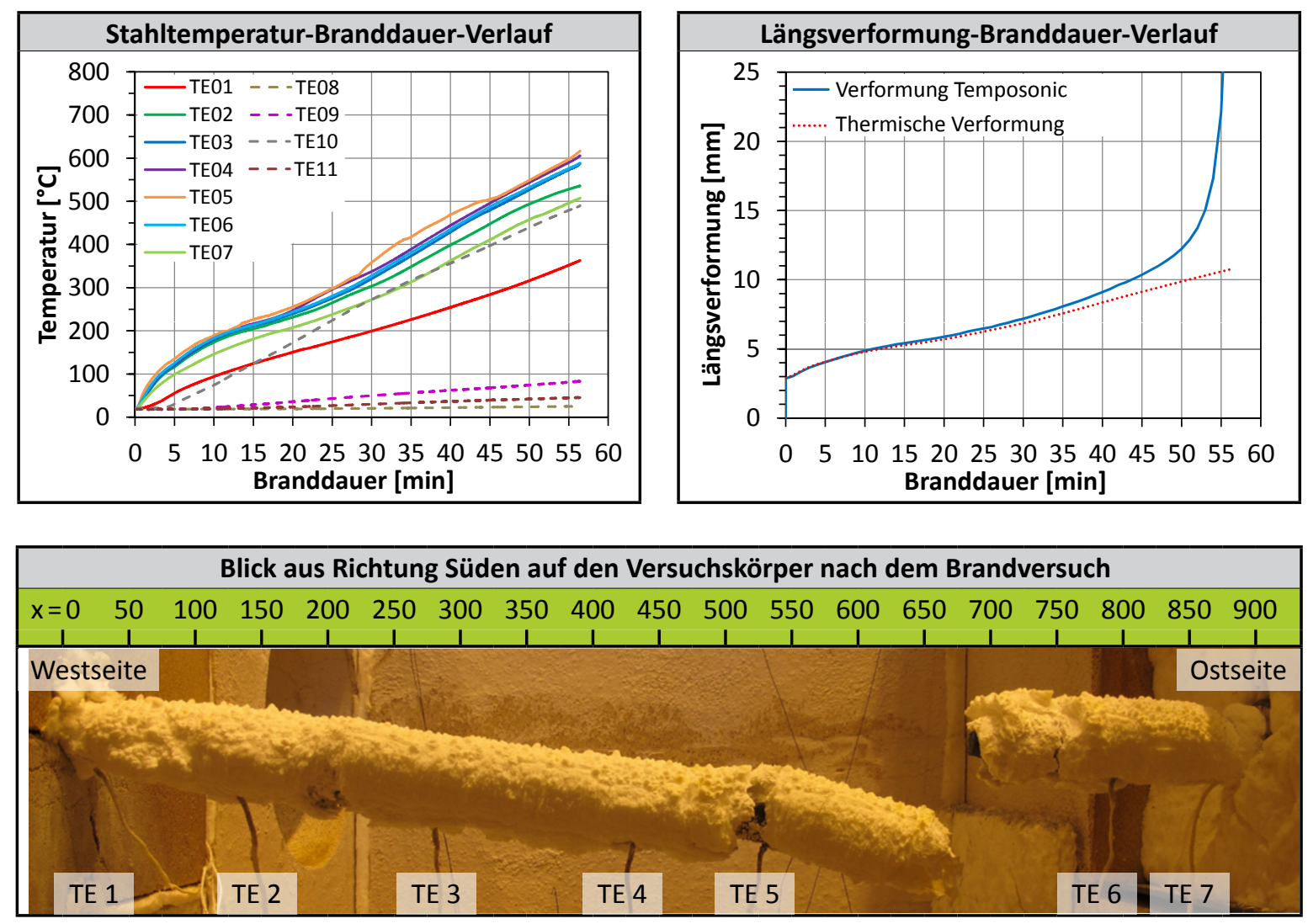

\begin{tabular}{|c|c|c|c|c|c|c|c|c|c|c|c|c|c|c|c|c|c|c|}
\hline \multicolumn{11}{|c|}{ Schaumdicke $t$ [mm], Position der Messstelle $\mathbf{~ [ m m ] ~}$} \\
\hline $\mathrm{x}=0$ & 50 & 100 & 150 & 200 & 250 & 300 & 350 & 400 & 450 & 500 & 550 & 600 & 650 & 700 & 750 & 800 & 850 & 900 \\
\hline $\mathrm{t}_{\mathrm{N}}=6$ & 19 & 18 & 23 & 23 & 23 & 22 & 23 & 25 & 21 & 23 & 22 & - & - & 23 & 23 & 21 & 18 & 15 \\
\hline $\mathrm{t}_{\mathrm{s}}=9$ & 16 & 17 & 22 & 21 & 22 & 21 & 18 & 20 & 17 & 17 & 19 & - & - & 16 & 16 & 17 & 9 & 8 \\
\hline $\mathrm{t}_{\mathrm{o}}=12$ & 16 & 18 & 20 & 19 & 20 & 18 & 17 & 18 & 19 & 20 & 19 & - & - & 22 & 20 & 19 & 16 & 9 \\
\hline $\mathrm{t}_{\mathrm{u}}=7$ & 13 & 19 & 19 & 21 & 20 & 20 & 20 & 18 & 17 & 14 & - & - & - & 13 & 13 & 13 & 12 & 11 \\
\hline
\end{tabular}




\begin{tabular}{|l|l|}
\hline \multicolumn{2}{|c|}{ Versuchskörper U53 } \\
\hline Stahlsorte & $\mathrm{S} 355 \mathrm{~J} 2 \mathrm{C}$ \\
\hline Kreisvollprofil $\varnothing$ & $30 \mathrm{~mm}$ \\
\hline Profilfaktor & $133 \mathrm{~m}^{-1}$ \\
\hline Trockenschichtdicke & $3,5 \mathrm{~mm}$ \\
\hline Prüfdatum & 20.11 .2014 \\
\hline Max. PKT nach 20 min & $216^{\circ} \mathrm{C}($ TE 2) \\
\hline Max. PKT bei Bruch & $576^{\circ} \mathrm{C}$ (TE 3) \\
\hline Schaumdicke (min - max) & $15-25 \mathrm{~mm}$ \\
\hline
\end{tabular}

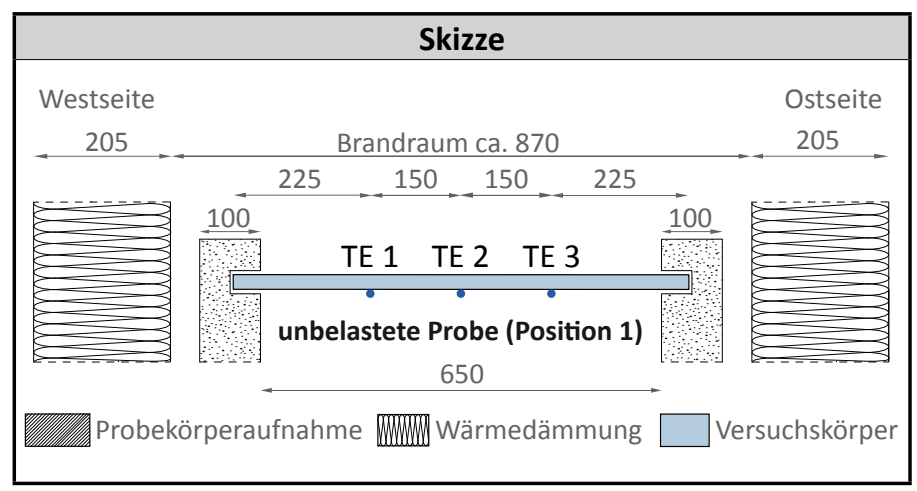

*Betrachtung zwischen $x=100-550 \mathrm{~mm}$

\begin{tabular}{|c|l|}
\hline \multicolumn{2}{|c|}{ Brandversuch } \\
\hline Zeit & \multicolumn{1}{|c|}{ Beobachtung } \\
\hline $0: 00$ & Prüfung erfolgt zusammen mit B58; \\
\hline $6: 00$ & $\begin{array}{l}\text { Längsriss auf Stabunterseite } \\
\text { (später zugeschäumt) }\end{array}$ \\
\hline $30: 00$ & Aufschäumung abgeschlossen (RBS weiß) \\
\hline $40: 00$ & Keine sichtbare Veränderung des RBS \\
\hline $\begin{array}{l}\text { Prüf- } \\
\text { ende }\end{array}$ & $\begin{array}{l}\text { Längsriss auf der Stabunterseite (zuge- } \\
\text { schäumt); relativ gleichmäßige Aufschäu- } \\
\text { mung des RBS (Stabunterseite etwas } \\
\text { geringere Schaumhöhe). }\end{array}$ \\
\hline
\end{tabular}
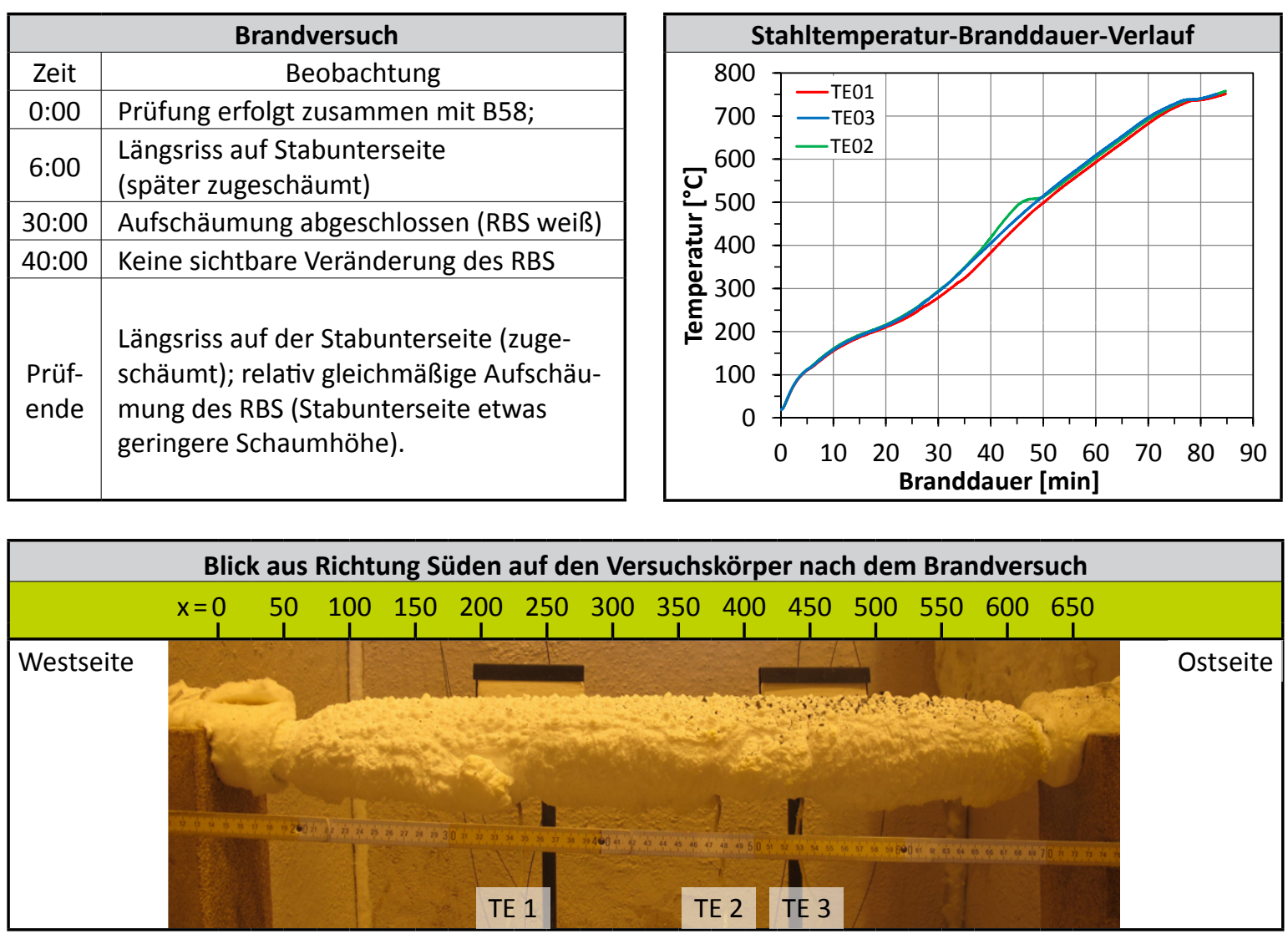

\begin{tabular}{|c|c|c|c|c|c|c|c|c|c|c|c|c|c|c|}
\hline \multirow{2}{*}{ Stabquerschnitt } & \multicolumn{14}{|c|}{ Schaumdicke $\mathrm{t}[\mathrm{mm}]$, Position der Messstelle $\mathrm{x}[\mathrm{mm}]$} \\
\hline & $x=0$ & 50 & 100 & 150 & 200 & 250 & 300 & 350 & 400 & 450 & 500 & 550 & 600 & 650 \\
\hline & $t_{N}=0$ & 11 & 21 & 25 & 27 & 21 & 23 & 25 & 22 & 22 & 22 & 21 & 20 & 18 \\
\hline & $t_{s}=0$ & 6 & 21 & 23 & 22 & 22 & 21 & 23 & 24 & 22 & 21 & 17 & 15 & 7 \\
\hline & $t_{0}=0$ & 8 & 18 & 22 & 22 & 23 & 23 & 24 & 25 & 22 & 24 & 20 & 18 & 8 \\
\hline & $t_{u}=0$ & 13 & 15 & 20 & 24 & 17 & 21 & 21 & 15 & 15 & 22 & 20 & 17 & 10 \\
\hline
\end{tabular}




\begin{tabular}{|l|l|}
\hline \multicolumn{2}{|c|}{ Versuchskörper B59 } \\
\hline Stahlsorte & S355 J2C \\
\hline Kreisvollprofil $\varnothing$ & $30 \mathrm{~mm}$ \\
\hline Profilfaktor & $133 \mathrm{~m}^{-1}$ \\
\hline Trockenschichtdicke & $3,5 \mathrm{~mm}$ \\
\hline Zugkraft & $221,60 \mathrm{kN}$ \\
\hline Lastausnutzung $\left(\mu_{\mathrm{fi}}\right)$ & 0,55 \\
\hline Prüfdatum & 04.12 .2014 \\
\hline Zeitpunkt Probenbruch & $53,16 \mathrm{~min}$ \\
\hline Bruchposition & TE 5 \\
\hline Max. PKT nach 20 min & $279^{\circ} \mathrm{C}(\mathrm{TE} \mathrm{5)}$ \\
\hline Max. PKT bei Bruch & $593^{\circ} \mathrm{C}(\mathrm{TE} \mathrm{5)}$ \\
\hline Schaumdicke (min - max) & $13-25 \mathrm{~mm} *$ \\
\hline
\end{tabular}

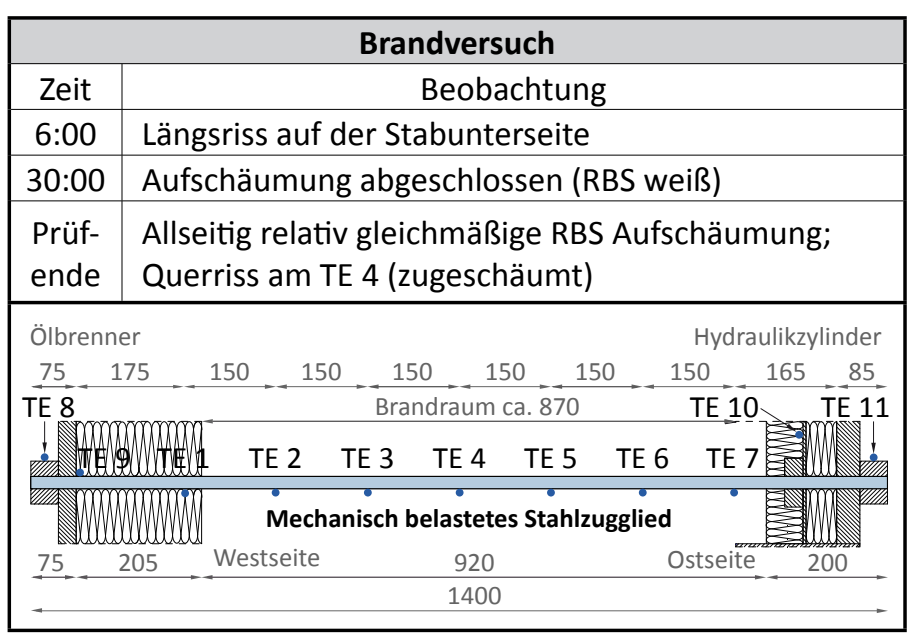

* Betrachtung zwischen $x=100-800 \mathrm{~mm}$
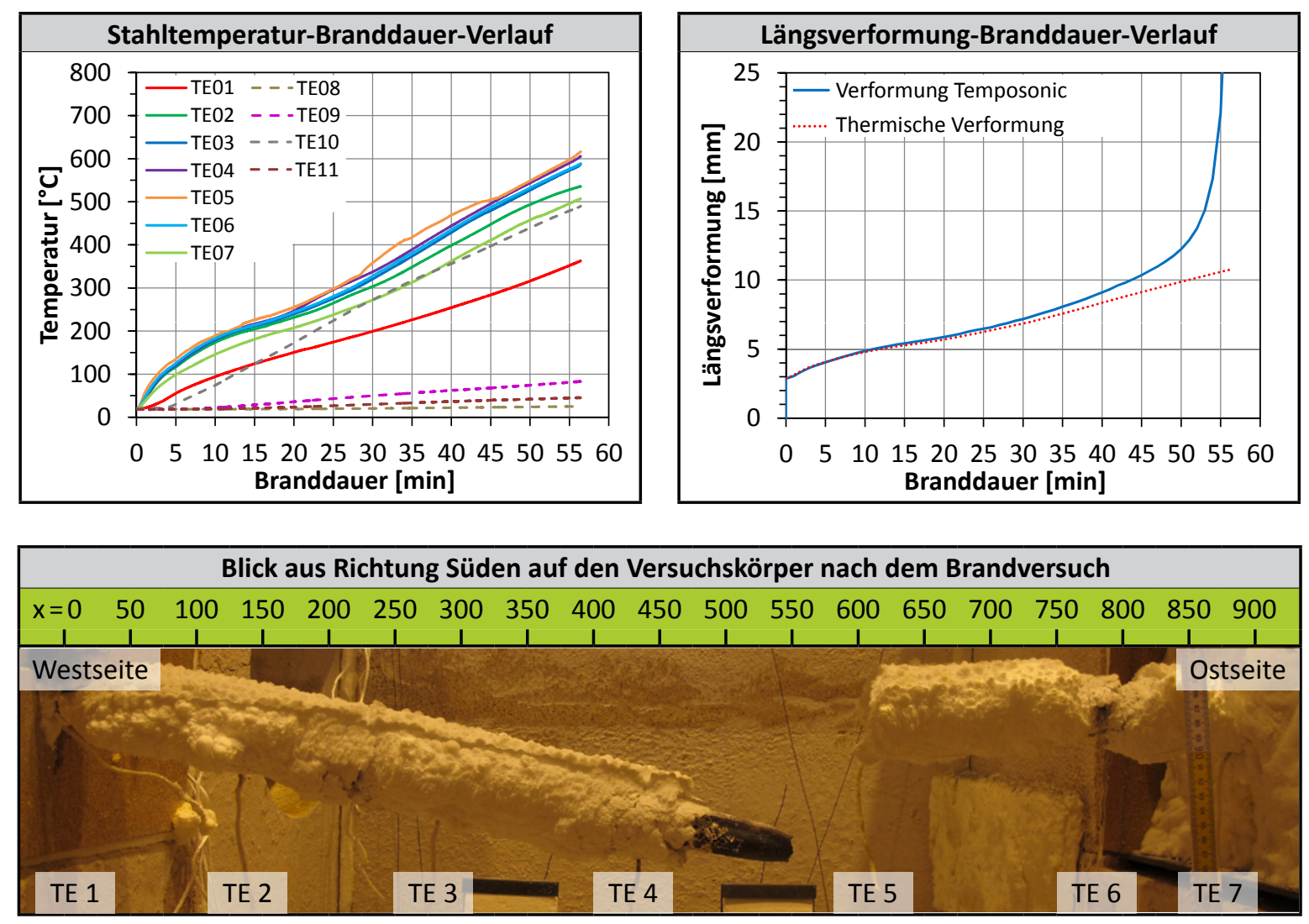

\begin{tabular}{|c|c|c|c|c|c|c|c|c|c|c|c|c|c|c|c|c|c|c|}
\hline \multicolumn{11}{|c|}{ Schaumdicke $t$ [mm], Position der Messstelle $\mathbf{~ [ m m ] ~}$} \\
\hline $\mathrm{x}=0$ & 50 & 100 & 150 & 200 & 250 & 300 & 350 & 400 & 450 & 500 & 550 & 600 & 650 & 700 & 750 & 800 & 850 & 900 \\
\hline $\mathrm{t}_{\mathrm{N}}=6$ & 22 & 23 & 23 & 22 & 24 & 24 & 19 & 23 & 23 & - & - & - & 25 & 23 & 17 & 20 & 20 & 10 \\
\hline $\mathrm{t}_{\mathrm{s}}=9$ & 12 & 18 & 21 & 18 & 18 & 21 & 19 & 13 & - & - & - & - & 21 & 17 & 21 & 22 & 19 & 10 \\
\hline $\mathrm{t}_{\mathrm{o}}=-$ & 13 & 19 & 21 & 21 & 22 & 22 & 20 & 20 & 21 & - & - & - & 19 & 20 & - & 22 & 18 & 13 \\
\hline $\mathrm{t}_{\mathrm{u}}=8$ & 18 & 18 & 16 & 24 & 20 & 21 & 20 & 22 & - & - & - & - & 19 & 15 & 13 & 14 & 11 & 8 \\
\hline
\end{tabular}




\begin{tabular}{|l|l|}
\hline \multicolumn{2}{|c|}{ Versuchskörper U54 } \\
\hline Stahlsorte & $\mathrm{S} 355 \mathrm{~J} 2 \mathrm{C}$ \\
\hline Kreisvollprofil $\varnothing$ & $30 \mathrm{~mm}$ \\
\hline Profilfaktor & $133 \mathrm{~m}^{-1}$ \\
\hline Trockenschichtdicke & $3,5 \mathrm{~mm}$ \\
\hline Prüfdatum & 04.12 .2014 \\
\hline Max. PKT nach $20 \mathrm{~min}$ & $232^{\circ} \mathrm{C}($ TE 3) \\
\hline Max. PKT bei Bruch & $610^{\circ} \mathrm{C}$ (TE 3) \\
\hline Schaumdicke (min - max) & $10-30 \mathrm{~mm}$ \\
\hline
\end{tabular}

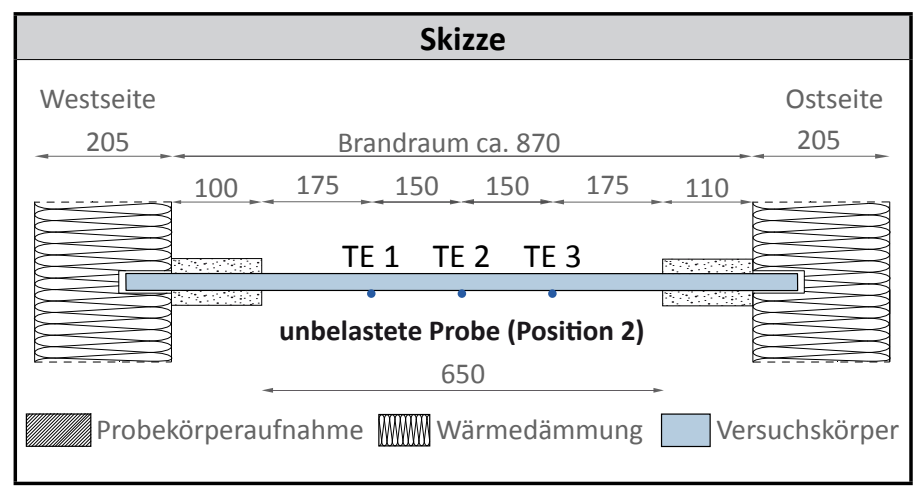

*Betrachtung zwischen $x=100-550 \mathrm{~mm}$

\begin{tabular}{|c|l|}
\hline \multicolumn{2}{|c|}{ Brandversuch } \\
\hline Zeit & \multicolumn{1}{|c|}{ Beobachtung } \\
\hline 0:00 & $\begin{array}{l}\text { Prüfung erfolgt zusammen mit B59; } \\
\text { Probe befindet sich 150 mm über B59 }\end{array}$ \\
\hline 6:00 & Längsriss auf Staboberseite (zugeschäumt) \\
\hline 14:00 & Teileweise Öffnung des Längsrisses \\
\hline $30: 00$ & Aufschäumung abgeschlossen (RBS weiß) \\
\hline $\begin{array}{l}\text { Prüf- } \\
\text { ende }\end{array}$ & $\begin{array}{l}\text { Längsriss auf der Staboberseite (zuge- } \\
\text { schäumt); relativ gleichmäßige Aufschäu- } \\
\text { mung des RBS (Staboberseite aufgrund } \\
\text { des Längsrisses etwas geringere Schaum- } \\
\text { dicke). }\end{array}$ \\
\hline
\end{tabular}
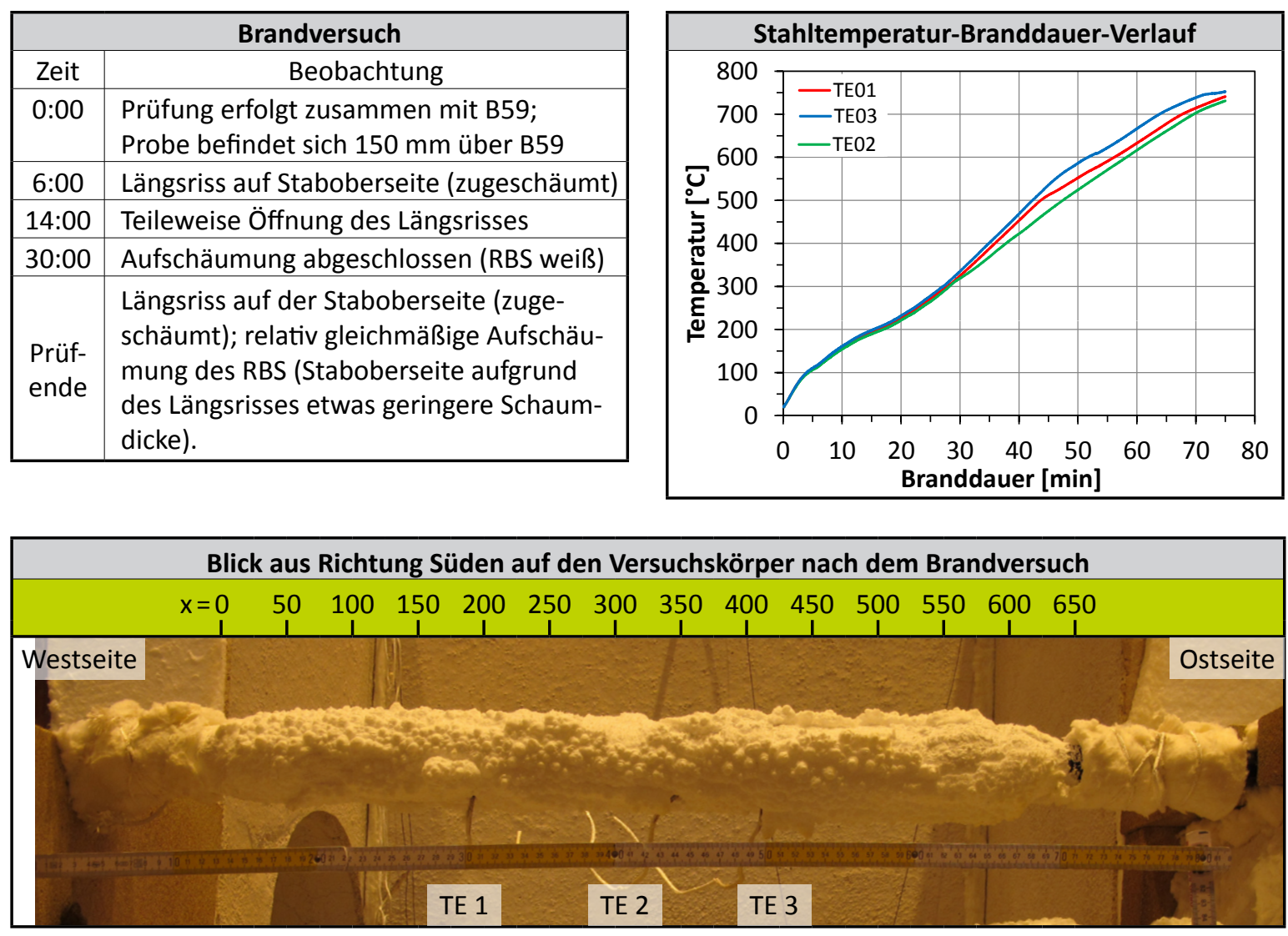

\begin{tabular}{|c|c|c|c|c|c|c|c|c|c|c|c|c|c|c|}
\hline Stabquerschnitt & \multicolumn{10}{|c|}{ Schaumdicke $\mathbf{t}[\mathrm{mm}$ ], Position der Messstelle $[\mathrm{mm}]$} \\
\hline & $\mathrm{x}=0$ & 50 & 100 & 150 & 200 & 250 & 300 & 350 & 400 & 450 & 500 & 550 & 600 & 650 \\
\hline & $\mathrm{t}_{\mathrm{N}}=12$ & 16 & 19 & 20 & 22 & 23 & 22 & 25 & 25 & 26 & 28 & 24 & 11 & 3 \\
\hline & $\mathrm{t}_{\mathrm{s}}=13$ & 17 & 20 & 18 & 18 & 19 & 19 & 20 & 22 & 20 & 18 & 17 & 15 & 0 \\
\hline & $\mathrm{t}_{\mathrm{o}}=8$ & 16 & 17 & 16 & 13 & 10 & 11 & 12 & 19 & 15 & 17 & 19 & 11 & 0 \\
\hline & $\mathrm{t}_{\mathrm{u}}=16$ & 16 & 16 & 18 & 24 & 24 & 21 & 25 & 28 & 30 & 29 & 30 & 18 & 3 \\
\hline
\end{tabular}




\begin{tabular}{|l|l|}
\hline \multicolumn{2}{|c|}{ Versuchskörper B57 } \\
\hline Stahlsorte & S355 J2C \\
\hline Kreisvollprofil $\varnothing$ & $30 \mathrm{~mm}$ \\
\hline Profilfaktor & $133 \mathrm{~m}^{-1}$ \\
\hline Trockenschichtdicke & $3,5 \mathrm{~mm}$ \\
\hline Zugkraft & $261,89 \mathrm{kN}$ \\
\hline Lastausnutzung $\left(\mu_{\mathrm{fi}}\right)$ & 0,65 \\
\hline Prüfdatum & 14.11 .2014 \\
\hline Zeitpunkt Probenbruch & $50,92 \mathrm{~min}$ \\
\hline Bruchposition & TE 5 \\
\hline Max. PKT nach 20 min & $252^{\circ} \mathrm{C}(\mathrm{TE} \mathrm{5)}$ \\
\hline Max. PKT bei Bruch & $564^{\circ} \mathrm{C}(\mathrm{TE} 5)$ \\
\hline Schaumdicke (min - max) & $14-27 \mathrm{~mm} *$ \\
\hline
\end{tabular}

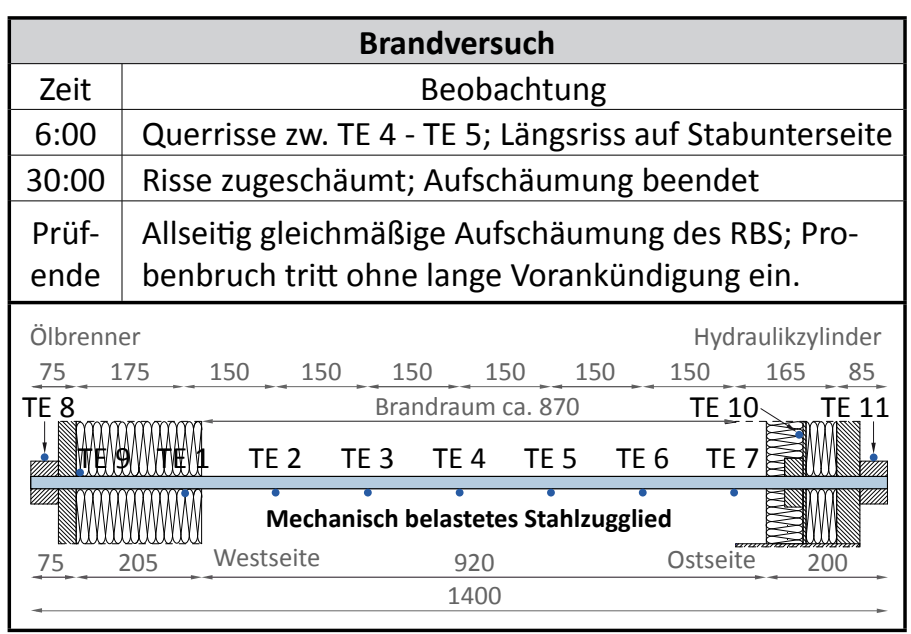

* Betrachtung zwischen $x=100-800 \mathrm{~mm}$
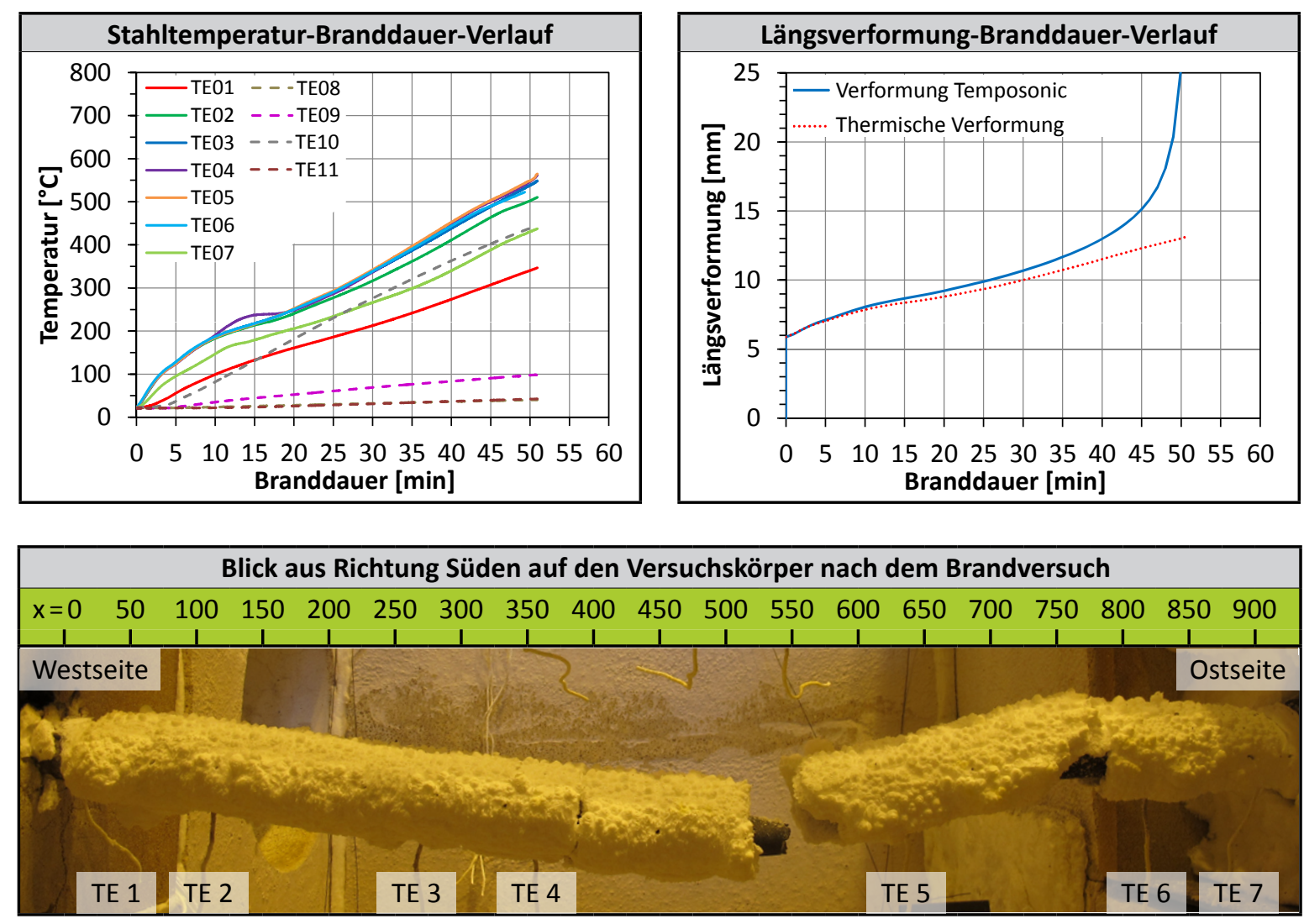

\begin{tabular}{|c|c|c|c|c|c|c|c|c|c|c|c|c|c|c|c|c|c|c|}
\hline \multicolumn{110}{|c|}{ Schaumdicke $t$ [mm], Position der Messstelle $\mathbf{~ [ m m ] ~}$} \\
\hline $\mathrm{x}=0$ & 50 & 100 & 150 & 200 & 250 & 300 & 350 & 400 & 450 & 500 & 550 & 600 & 650 & 700 & 750 & 800 & 850 & 900 \\
\hline$t_{\mathrm{N}}=11$ & 18 & 21 & 22 & 20 & 22 & 22 & 22 & 19 & 23 & 25 & - & - & 21 & 18 & 27 & 20 & 17 & 10 \\
\hline$t_{\mathrm{s}}=10$ & 21 & 14 & 23 & 25 & 24 & 26 & 26 & 27 & 20 & 21 & - & - & 21 & 22 & 20 & 21 & 14 & 10 \\
\hline$t_{\mathrm{o}}=12$ & 19 & 21 & 23 & 23 & 22 & 19 & 21 & 19 & 19 & 22 & - & - & 21 & 21 & 22 & 18 & 15 & 13 \\
\hline$t_{\mathrm{u}}=10$ & 13 & 17 & 15 & 15 & 16 & 18 & 18 & 17 & 20 & 18 & - & - & 21 & 18 & 17 & 20 & 13 & 13 \\
\hline
\end{tabular}




\begin{tabular}{|l|l|}
\hline \multicolumn{2}{|c|}{ Versuchskörper U52 } \\
\hline Stahlsorte & $\mathrm{S} 355 \mathrm{~J} 2 \mathrm{C}$ \\
\hline Kreisvollprofil $\varnothing$ & $30 \mathrm{~mm}$ \\
\hline Profilfaktor & $133 \mathrm{~m}^{-1}$ \\
\hline Trockenschichtdicke & $3,5 \mathrm{~mm}$ \\
\hline Prüfdatum & 14.11 .2014 \\
\hline Max. PKT nach 20 min & $232^{\circ} \mathrm{C}($ TE 2) \\
\hline Max. PKT bei Bruch & $556^{\circ} \mathrm{C}($ TE 1) \\
\hline Schaumdicke (min - max) & $14-26 \mathrm{~mm} *$ \\
\hline
\end{tabular}

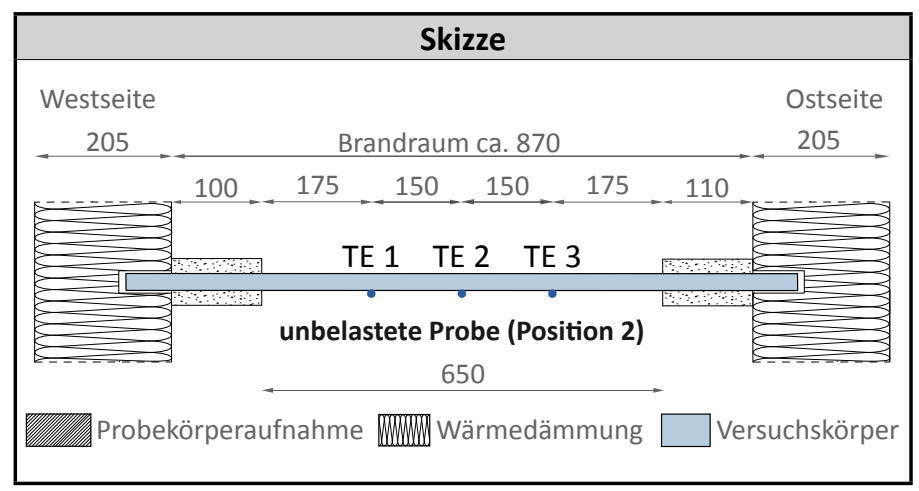

*Betrachtung zwischen $x=100-550 \mathrm{~mm}$

\begin{tabular}{|c|l|}
\hline \multicolumn{2}{|c|}{ Brandversuch } \\
\hline Zeit & \multicolumn{1}{|c|}{ Beobachtung } \\
\hline $0: 00$ & $\begin{array}{l}\text { Prüfung erfolgt zusammen mit B57; } \\
\text { Probe befindet sich 150 mm über B57 }\end{array}$ \\
\hline $6: 00$ & $\begin{array}{l}\text { Querriss zw. TE 1 - TE 2 (zugeschäumt); } \\
\text { Längsriss auf Oberseite an den Stabenden }\end{array}$ \\
\hline $30: 00$ & Risse nahezu komplett zugeschäumt \\
\hline $35: 00$ & Keine sichtbare Veränderung des RBS \\
\hline $\begin{array}{l}\text { Prüf- } \\
\text { ende }\end{array}$ & $\begin{array}{l}\text { Längsriss auf der Stabunterseite (zuge- } \\
\text { schäumt); relativ gleichmäßige Aufschäu- } \\
\text { mung des RBS. }\end{array}$ \\
\hline
\end{tabular}
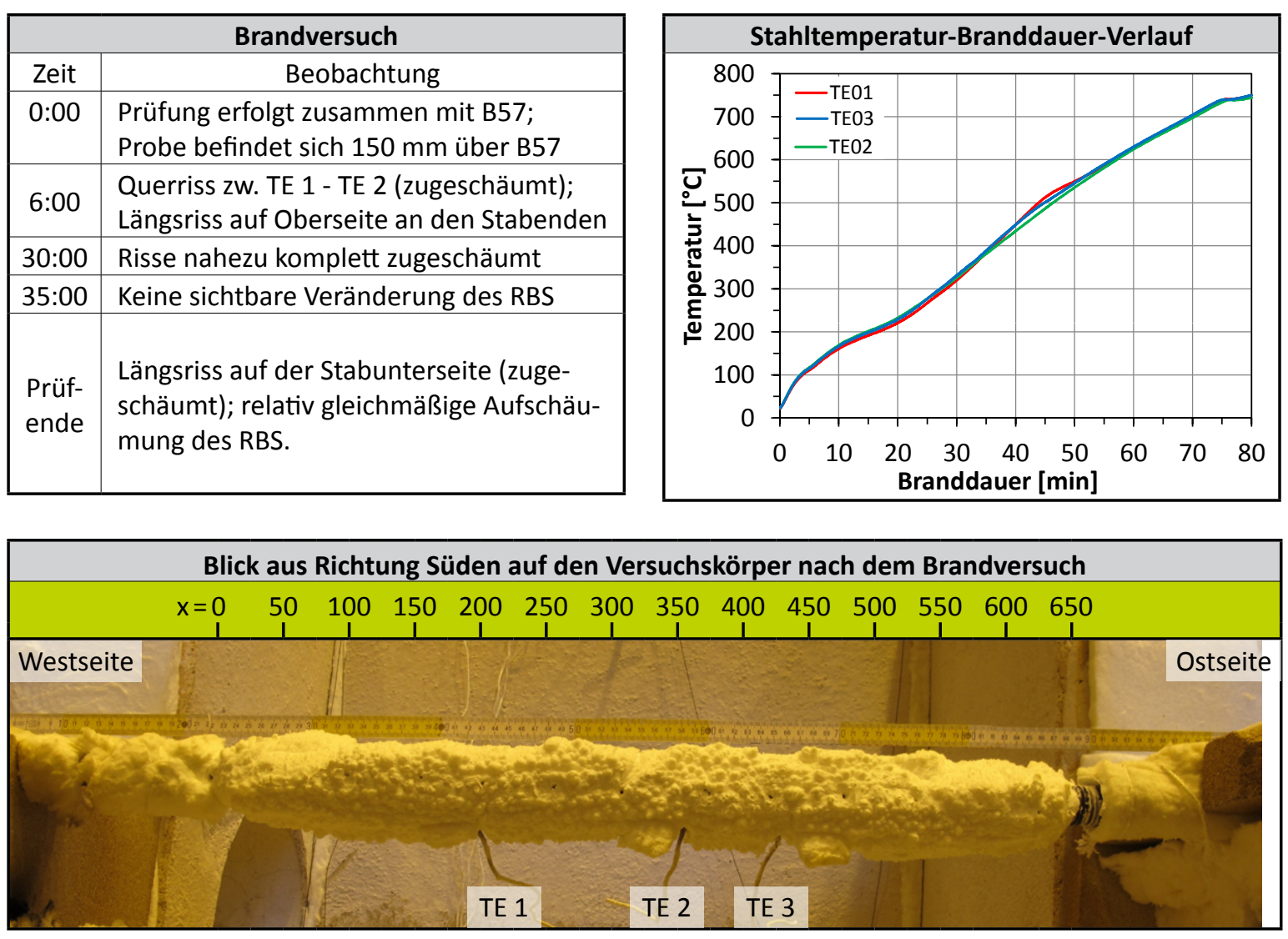

\begin{tabular}{|c|c|c|c|c|c|c|c|c|c|c|c|c|c|c|}
\hline \multirow{2}{*}{ Stabquerschnitt } & \multicolumn{14}{|c|}{ Schaumdicke $\mathrm{t}$ [mm], Position der Messstelle $\mathrm{x}$ [mm] } \\
\hline & $x=0$ & 50 & 100 & 150 & 200 & 250 & 300 & 350 & 400 & 450 & 500 & 550 & 600 & 650 \\
\hline & $t_{N}=7$ & 16 & 20 & 24 & 20 & 19 & 20 & 19 & 21 & 23 & 23 & 23 & 17 & 7 \\
\hline & $t_{S}=9$ & 19 & 22 & 23 & 21 & 22 & 20 & 20 & 20 & 22 & 21 & 26 & 21 & 9 \\
\hline & $t_{0}=8$ & 19 & 15 & 15 & 14 & 15 & 17 & 17 & 15 & 15 & 16 & 17 & 12 & 8 \\
\hline & $t_{u}=8$ & 17 & 22 & 23 & 23 & 22 & 20 & 19 & 21 & 22 & 24 & 23 & 18 & 9 \\
\hline
\end{tabular}





\section{B Zusätzliche Informationen zu den in Kapitel 4 durchge- führten Materialuntersuchungen}

Tabelle B.1: Festigkeitsparameter aus den gemessenen, technischen $\sigma-\varepsilon-K u r v e n$ der VR 1

\begin{tabular}{|c|c|c|c|c|c|c|c|c|c|c|c|}
\hline \multirow{2}{*}{$\begin{array}{c}\text { Stahltem- } \\
\text { peratur } \\
{\left[{ }^{\circ} \mathrm{C}\right]}\end{array}$} & \multirow{2}{*}{$\begin{array}{c}\text { E-Modul } \\
{\left[\mathrm{N} / \mathrm{mm}^{2}\right]}\end{array}$} & \multicolumn{5}{|c|}{$\begin{array}{c}\text { Spannungen } \\
{\left[\mathrm{N} / \mathrm{mm}^{2}\right]}\end{array}$} & \multicolumn{5}{|c|}{$\begin{array}{c}\text { Dehnungen } \\
{[\%]}\end{array}$} \\
\hline & & $f_{p}$ & $\mathrm{f}_{\mathrm{p} 0,2}$ & $\mathrm{f}_{\mathrm{u}, \mathrm{I}}$ & $\mathrm{f}_{\mathrm{u}, \mathrm{II}}$ & $f_{b}$ & $\varepsilon_{\mathrm{p}}$ & $\varepsilon_{\mathrm{p} 0,2}$ & $\varepsilon_{\mathrm{u}, \mathrm{I}}$ & $\varepsilon_{\mathrm{u}, \mathrm{II}}$ & $\varepsilon_{\mathrm{b}}$ \\
\hline 100 & 203000 & 408 & 610 & 673 & 656 & 260 & 0,221 & 0,510 & 1,280 & 2,263 & 11,040 \\
\hline 200 & 195000 & 318 & 613 & 696 & 645 & 338 & 0,178 & 0,518 & 1,459 & 2,353 & 8,960 \\
\hline 250 & 191000 & 378 & 627 & 717 & 724 & 325 & 0,203 & 0,523 & 2,311 & $-{ }^{*}$ & 12,800 \\
\hline 300 & 187000 & 371 & 616 & 713 & 724 & 324 & 0,203 & 0,523 & 2,182 & $-{ }^{*}$ & 17,620 \\
\hline 350 & 182500 & 368 & 571 & 650 & 657 & 257 & 0,204 & 0,506 & 2,033 & $-^{*}$ & 17,000 \\
\hline 400 & 178000 & 338 & 534 & 607 & 612 & 250 & 0,218 & 0,517 & 2,252 & 4,396 & 17,080 \\
\hline 450 & 172500 & 292 & 472 & 531 & 531 & 219 & 0,180 & 0,474 & 1,976 & 2,808 & 14,940 \\
\hline 500 & 167000 & 200 & 378 & 418 & 417 & 131 & 0,128 & 0,425 & 1,785 & 2,312 & 22,000 \\
\hline 550 & 159500 & 138 & 294 & 316 & 312 & 83 & 0,096 & 0,384 & 1,085 & 2,430 & 20,880 \\
\hline 600 & 152000 & 69 & 186 & 201 & 198 & 20 & 0,055 & 0,320 & 1,681 & 2,489 & 33,140 \\
\hline 650 & 138500 & 33 & 116 & 136 & 134 & 10 & 0,026 & 0,277 & 1,962 & 2,027 & 38,860 \\
\hline 700 & 125000 & 27 & 72 & 86 & 84 & 0 & 0,028 & 0,245 & 2,062 & 4,299 & 48,220 \\
\hline
\end{tabular}

* Dehnungsaufnehmer wurde zu diesem Zeitpunkt bereits entfernt.

Tabelle B.2: Festigkeitsparameter aus den gemessenen, technischen $\sigma$ - $\varepsilon$-Kurven der VR 2

\begin{tabular}{|c|c|c|c|c|c|c|c|c|c|c|c|}
\hline \multirow{2}{*}{$\begin{array}{l}\text { Stahltem- } \\
\text { peratur } \\
{\left[{ }^{\circ} \mathrm{C}\right]}\end{array}$} & \multirow{2}{*}{$\begin{array}{c}\text { E-Modul } \\
{\left[\mathrm{N} / \mathrm{mm}^{2}\right]}\end{array}$} & \multicolumn{5}{|c|}{$\begin{array}{c}\text { Spannungen } \\
{\left[\mathrm{N} / \mathrm{mm}^{2}\right]}\end{array}$} & \multicolumn{5}{|c|}{$\begin{array}{c}\text { Dehnungen } \\
{[\%]}\end{array}$} \\
\hline & & $f_{p}$ & $\mathrm{f}_{\mathrm{p} 0,2}$ & $\mathrm{f}_{\mathrm{u}, \mathrm{I}}$ & $\mathrm{f}_{\mathrm{u}, \mathrm{II}}$ & $f_{b}$ & $\varepsilon_{\mathrm{p}}$ & $\varepsilon_{\mathrm{p} 0,2}$ & $\varepsilon_{\mathrm{u}, \mathrm{I}}$ & $\varepsilon_{\mathrm{u}, \mathrm{II}}$ & $\varepsilon_{\mathrm{b}}$ \\
\hline 100 & 203000 & 337 & 563 & 632 & 630 & 237 & 0,182 & 0,483 & 1,254 & 2,347 & 12,460 \\
\hline 200 & 195000 & 326 & 606 & 688 & 629 & 307 & 0,188 & 0,521 & 1,711 & $-{ }^{*}$ & 9,700 \\
\hline 250 & 191000 & 399 & 625 & 719 & 732 & 318 & 0,206 & 0,515 & 2,146 & $-{ }^{*}$ & 15,220 \\
\hline 300 & 187000 & 390 & 597 & 681 & 695 & 293 & 0,226 & 0,527 & 2,144 & 2,240 & 17,840 \\
\hline 350 & 182500 & 381 & 578 & 656 & 669 & 257 & 0,217 & 0,516 & 1,989 & $-*$ & 18,400 \\
\hline 400 & 178000 & 354 & 519 & 588 & 599 & 239 & 0,197 & 0,480 & 1,816 & 3,915 & 16,940 \\
\hline 450 & 172500 & 313 & 450 & 508 & 520 & 190 & 0,192 & 0,462 & 1,934 & 3,511 & 17,700 \\
\hline 500 & 167000 & 200 & 360 & 396 & 415 & 131 & 0,127 & 0,413 & 1,765 & 2,956 & 25,580 \\
\hline 550 & 159500 & 122 & 253 & 272 & 293 & 73 & 0,100 & 0,371 & 0,979 & 2,820 & 43,560 \\
\hline 600 & 152000 & 62 & 148 & 169 & 198 & 25 & 0,054 & 0,306 & 1,508 & 2,937 & 34,640 \\
\hline 650 & 138500 & 6 & 89 & 110 & 134 & 10 & 0,004 & 0,255 & 1,884 & 3,470 & 53,030 \\
\hline 700 & 125000 & 19 & 50 & 68 & 84 & 0 & 0,024 & 0,236 & 1,954 & 4,112 & 55,590 \\
\hline
\end{tabular}




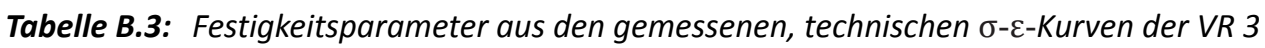

\begin{tabular}{|c|c|c|c|c|c|c|c|c|c|c|c|}
\hline \multirow{2}{*}{$\begin{array}{c}\text { Stahltem- } \\
\text { peratur } \\
{\left[{ }^{\circ} \mathrm{C}\right]}\end{array}$} & \multirow{2}{*}{$\begin{array}{c}\begin{array}{c}\text { E-Modul } \\
{\left[\mathrm{N} / \mathrm{mm}^{2}\right]}\end{array} \\
E_{\mathrm{dyn}}\end{array}$} & \multicolumn{5}{|c|}{$\begin{array}{c}\text { Spannungen } \\
{\left[\mathrm{N} / \mathrm{mm}^{2}\right]}\end{array}$} & \multicolumn{5}{|c|}{$\begin{array}{c}\text { Dehnungen } \\
{[\%]}\end{array}$} \\
\hline & & $f_{p}$ & $\mathrm{f}_{\mathrm{p} 0,2}$ & $\mathrm{f}_{\mathrm{u}, \mathrm{I}}$ & $\mathrm{f}_{\mathrm{u}, \mathrm{II}}$ & $\mathrm{f}_{\mathrm{b}}$ & $\varepsilon_{\mathrm{p}}$ & $\varepsilon_{\mathrm{p} 0,2}$ & $\varepsilon_{\mathrm{u}, \mathrm{I}}$ & $\varepsilon_{\mathrm{u}, \mathrm{II}}$ & $\varepsilon_{\mathrm{b}}$ \\
\hline 100 & 203000 & 334 & 580 & 651 & 629 & 232 & 0,164 & 0,475 & 1,120 & 2,166 & 11,300 \\
\hline 200 & 195000 & 311 & 621 & 716 & 729 & 317 & 0,168 & 0,517 & 2,085 & 2,212 & 8,640 \\
\hline 250 & 191000 & 371 & 631 & 735 & 758 & 361 & 0,219 & 0,545 & 2,393 & 2,492 & 26,620 \\
\hline 300 & 187000 & 269 & 600 & 692 & 719 & 287 & 0,175 & 0,542 & 1,898 & $-{ }^{*}$ & 27,680 \\
\hline 350 & 182500 & 369 & 577 & 662 & 684 & 262 & 0,214 & 0,518 & 2,168 & $-*$ & 18,640 \\
\hline 400 & 178000 & 390 & 528 & 603 & 622 & 227 & 0,233 & 0,500 & 2,521 & $-{ }^{*}$ & 17,500 \\
\hline 450 & 172500 & 287 & 458 & 519 & 544 & 214 & 0,173 & 0,461 & 2,114 & 3,856 & 26,620 \\
\hline 500 & 167000 & 210 & 364 & 404 & 450 & 164 & 0,121 & 0,402 & 1,555 & 3,915 & 21,560 \\
\hline 550 & 159500 & 63 & 250 & 269 & 335 & 96 & 0,052 & 0,359 & 0,918 & 3,423 & 29,720 \\
\hline 600 & 152000 & 61 & 152 & 171 & 245 & 47 & 0,057 & 0,306 & 1,100 & 3,121 & 47,820 \\
\hline 650 & 138500 & 14 & 89 & 108 & 174 & 11 & 0,004 & 0,249 & 1,899 & 3,222 & 31,700 \\
\hline 700 & 125000 & 23 & 50 & 64 & 116 & 48 & 0,031 & 0,239 & 1,812 & 2,932 & 47,680 \\
\hline
\end{tabular}

Tabelle B.4: Mittelwerte der Festigkeitsparameter aus allen gemessenen, technischen $\sigma-\varepsilon-K u r v e n ~(V R ~ 1-3)$

\begin{tabular}{|c|c|c|c|c|c|c|c|c|c|c|c|}
\hline \multirow{2}{*}{$\begin{array}{c}\text { Stahltem- } \\
\text { peratur } \\
{\left[{ }^{\circ} \mathrm{C}\right]}\end{array}$} & \multirow{2}{*}{$\begin{array}{c}\begin{array}{c}\text { E-Modul } \\
\left.\text { [N/mm } \mathbf{m m}^{2}\right]\end{array} \\
\mathrm{E}_{\mathrm{dyn}}\end{array}$} & \multicolumn{5}{|c|}{$\begin{array}{c}\text { Spannungen } \\
{\left[\mathrm{N} / \mathrm{mm}^{2}\right]}\end{array}$} & \multicolumn{5}{|c|}{$\begin{array}{c}\text { Dehnungen } \\
\text { [\%] }\end{array}$} \\
\hline & & $\mathrm{f}_{\mathrm{p}}$ & $\mathrm{f}_{\mathrm{p} 0,2}$ & $\mathrm{f}_{\mathrm{u}, \mathrm{I}}$ & $\mathrm{f}_{\mathrm{u}, \mathrm{II}}$ & $\mathrm{f}_{\mathrm{b}}$ & $\varepsilon_{\mathrm{p}}$ & $\varepsilon_{\mathrm{p} 0,2}$ & $\varepsilon_{\mathrm{u}, \mathrm{I}}$ & $\varepsilon_{\mathrm{u}, \mathrm{II}}$ & $\varepsilon_{\mathrm{b}}$ \\
\hline 100 & 203000 & 360 & 584 & 652 & 639 & 243 & 0,189 & 0,490 & 1,218 & 2,259 & 11,600 \\
\hline 200 & 195000 & 318 & 613 & 700 & 668 & 321 & 0,178 & 0,519 & 1,752 & 2,283 & 9,100 \\
\hline 250 & 191000 & 382 & 628 & 724 & 738 & 335 & 0,209 & 0,528 & 2,283 & 2,492 & 18,213 \\
\hline 300 & 187000 & 343 & 604 & 696 & 713 & 302 & 0,201 & 0,531 & 2,075 & 2,240 & 21,047 \\
\hline 350 & 182500 & 373 & 575 & 656 & 670 & 259 & 0,212 & 0,513 & 2,063 & $-*$ & 18,013 \\
\hline 400 & 178000 & 360 & 527 & 600 & 611 & 239 & 0,216 & 0,499 & 2,196 & 4,155 & 17,173 \\
\hline 450 & 172500 & 298 & 460 & 519 & 532 & 208 & 0,182 & 0,466 & 2,008 & 3,392 & 19,753 \\
\hline 500 & 167000 & 203 & 367 & 406 & 427 & 142 & 0,125 & 0,413 & 1,702 & 3,061 & 23,047 \\
\hline 550 & 159500 & 108 & 265 & 286 & 314 & 84 & 0,083 & 0,371 & 0,994 & 2,891 & 31,387 \\
\hline 600 & 152000 & 64 & 162 & 180 & 214 & 31 & 0,055 & 0,311 & 1,430 & 2,849 & 38,533 \\
\hline 650 & 138500 & 18 & 98 & 118 & 147 & 10 & 0,011 & 0,260 & 1,915 & 2,907 & 41,197 \\
\hline 700 & 125000 & 23 & 57 & 73 & 95 & 16 & 0,027 & 0,240 & 1,943 & 3,781 & 50,497 \\
\hline
\end{tabular}

* Dehnungsaufnehmer wurde zu diesem Zeitpunkt bereits entfernt. 
Zur Bestimmung der in den Brandversuchen im Realmaßstab aufzubringenden Prüflast werden Kleinzugversuche bei Raumtemperatur auch an dem KVP D30 und KHP D60 durchgeführt. Die Durchführung der Prüfungen erfolgt analog zu den Zugversuchen des KVP D20. Nachfolgend sind die Ergebnisse dieser Kleinzugversuche angegeben. Es ist zu beachten, dass das Ende der Kurven in Abb. B.01 nicht den Probenbruch darstellt, sondern lediglich die Dehnungsaufzeichnung beendet wird.

Tabelle B.5: Maße der Kleinzugproben nach DIN EN ISO 6892-2 [15] (Angaben in mm)

\begin{tabular}{|c|c|c|c|c|c|c|}
\hline $\mathrm{d}_{\mathrm{o}}$ & $\mathrm{L}_{\mathrm{o}}$ & $\mathrm{d}_{1}$ & $\mathrm{r}$ & $\mathrm{h}$ & $\mathrm{L}_{\mathrm{c}}$ & $\mathrm{L}_{\mathrm{t}}$ \\
\hline 10 & 50 & $\mathrm{M} 16$ & 8 & 12 & 60 & 97 \\
\hline
\end{tabular}

Tabelle B.6: Kennwerte für das KVP D30 aus den bei Raumtemperatur durchgeführten Kleinzugversuchen

\begin{tabular}{|c|c|c|c|c|c|c|c|c|c|c|}
\hline \multirow{2}{*}{ 풍 } & \multirow{2}{*}{$\begin{array}{c}\text { Proben- } \\
\text { name }\end{array}$} & \multirow{2}{*}{$\begin{array}{c}\text { E-Modul } \\
\text { [GPa] }\end{array}$} & \multicolumn{4}{|c|}{ Spannung $\left[\mathrm{N} / \mathrm{mm}^{2}\right]$} & \multicolumn{4}{|c|}{ Dehnung [\%] } \\
\hline & & & $\mathrm{f}_{\mathrm{p} 0,01}$ & $\mathrm{f}_{\mathrm{p} 0,2}$ & $\mathrm{f}_{\mathrm{u}}$ & $\mathrm{f}_{\mathrm{b}}$ & $\varepsilon_{\mathrm{p} 0,01}$ & $\varepsilon_{\mathrm{p} 0,2}$ & $\varepsilon_{\mathrm{u}}$ & $\varepsilon_{\mathrm{b}}$ \\
\hline \multirow{3}{*}{$\begin{array}{l}\stackrel{\rho}{m} \\
\text { D } \\
\stackrel{2}{\gtrless}\end{array}$} & P_60 & $207^{*}$ & 380,0 & 577,3 & 661,9 & 230,2 & 0,189 & 0,474 & 1,559 & 8,685 \\
\hline & P_61 & $207^{*}$ & 366,6 & 564,8 & 660,8 & 241,7 & 0,184 & 0,470 & 2,061 & 8,675 \\
\hline & MW & $207^{*}$ & 373,3 & 571,0 & 661,3 & 236,0 & 0,187 & 0,472 & 1,810 & 8,680 \\
\hline
\end{tabular}

Die Zugproben entsprechen einem Proportionalstab der Form B mit einem Durchmesser von $10 \mathrm{~mm}$.

* Der Wert bezieht sich auf den am KVP D20 bestimmten dynamischen Elastizitätsmodul.

Tabelle B.7: Kennwerte für das KHP D60 aus den bei Raumtemperatur durchgeführten Kleinzugversuchen

\begin{tabular}{|c|c|c|c|c|c|c|c|c|}
\hline \multirow{2}{*}{ 동 } & \multirow{2}{*}{$\begin{array}{c}\text { Proben- } \\
\text { name }\end{array}$} & \multirow{2}{*}{$\begin{array}{c}\text { E-Modul } \\
\text { [GPa] }\end{array}$} & \multicolumn{3}{|c|}{ Spannung $\left[\mathrm{N} / \mathrm{mm}^{2}\right]$} & \multicolumn{3}{|c|}{ Dehnung [\%] } \\
\hline & & & $\mathrm{f}_{\mathrm{p}}=\mathrm{f}_{\mathrm{y}}$ & $f_{u}$ & $\mathrm{f}_{\mathrm{b}}$ & $\varepsilon_{\mathrm{p}}=\varepsilon_{\mathrm{y}}$ & $\varepsilon_{\mathrm{u}}$ & $\varepsilon_{\mathrm{b}}$ \\
\hline \multirow{3}{*}{$\begin{array}{l}\text { 윰 } \\
\text { D } \\
\text { 号 } \\
\text { 立 }\end{array}$} & KHP-01 & $213^{\#}$ & 458,1 & 596,3 & 192,8 & 0,2188 & - & 30,500 \\
\hline & KHP-02 & $213^{\#}$ & 456,8 & 605,1 & 204,6 & 0,2197 & - & 27,670 \\
\hline & MW & $213^{\#}$ & 457,5 & 600,7 & 198,7 & 0,2193 & - & 29,085 \\
\hline
\end{tabular}

Die Zugproben entsprechen einem Proportionalstab der Form B mit einem Durchmesser von 5 mm.

Da es sich um einen warmgewalzten Stahl handelt und eine ausgeprägte Streckgrenze existiert, sind Proportionalitätsgrenze und Streckgrenze identisch.

Die fehlende Angabe zur Dehnung der Zugfestigkeit ist darauf zurückzuführen, dass das Extensometer bereits zuvor von der Probe entfernt wird und daher keine Messaufzeichung existiert.

\# Mittelwert des aus den $\sigma$-ع-Kurven bestimmten statischen Elastizitätsmoduls. 


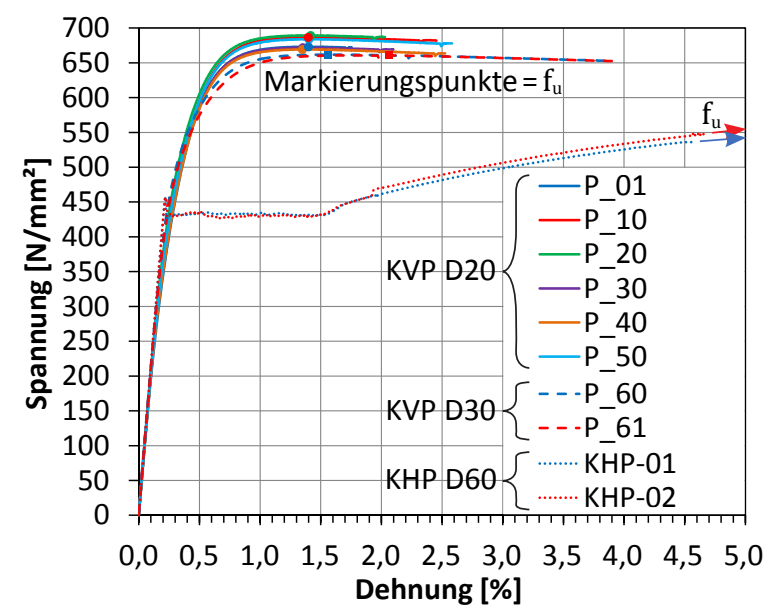

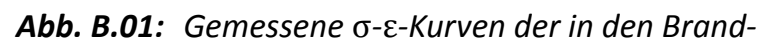
versuchen der BAM getesteten Zugglieder
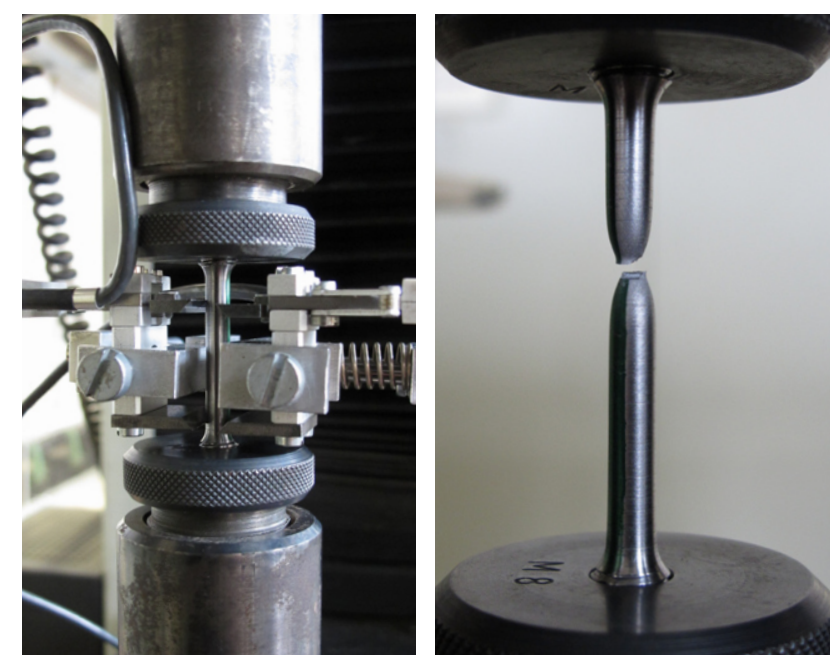

Abb. B.03: Zugprüfung der Probe KHP-01 (links: vor der Prüfung; rechts: nach dem Probenbruch)
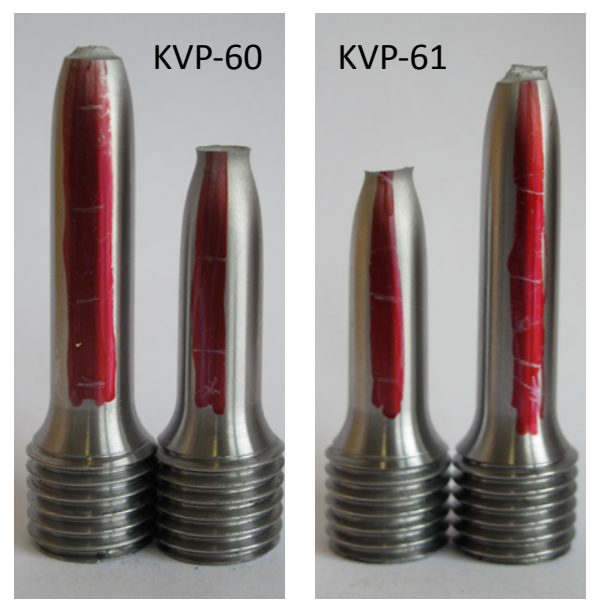

Abb. B.02: Getestete Zugproben des KVP D30 (nicht maßstäblich)
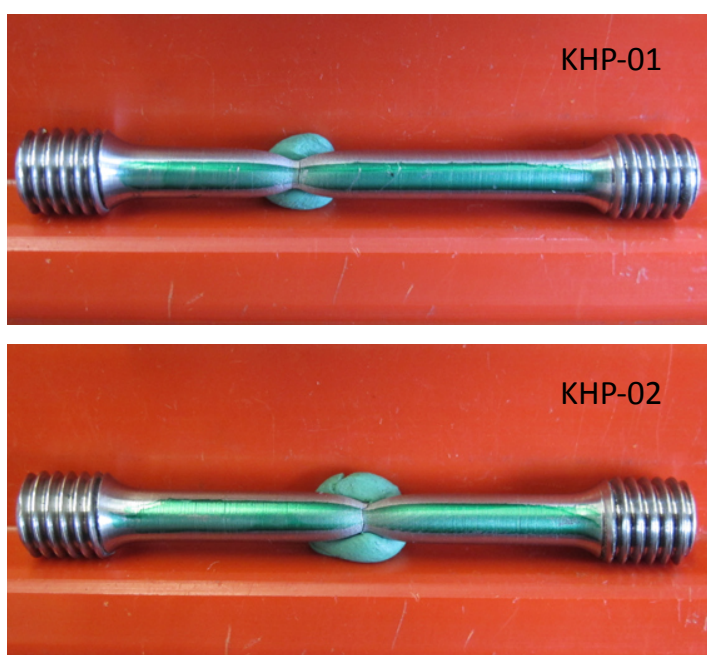

Abb. B.04: Geteste Zugproben des KHP D60 (nicht maßstäblich) 


\section{Information zu den in Kapitel 5 verwendeten Material- modellen}

Tabelle C.1: Festigkeitsparameter aus den wahren $\sigma-\varepsilon-K u r v e n$ des neu erstellten Materialmodells (Mat 3)

\begin{tabular}{|c|c|c|c|c|c|c|c|c|c|}
\hline \multirow{2}{*}{$\begin{array}{l}\text { Stahltem- } \\
\text { peratur } \\
{\left[{ }^{\circ} \mathrm{C}\right]}\end{array}$} & \multirow{2}{*}{$\begin{array}{c}\begin{array}{c}\text { E-Modul } \\
{\left[\mathrm{N} / \mathrm{mm}^{2}\right]}\end{array} \\
E_{\mathrm{dyn}} \\
\end{array}$} & \multicolumn{4}{|c|}{$\begin{array}{c}\text { Spannungen } \\
{\left[\mathrm{N} / \mathrm{mm}^{2}\right]}\end{array}$} & \multicolumn{4}{|c|}{$\begin{array}{c}\text { Dehnungen } \\
{[-]}\end{array}$} \\
\hline & & $f_{p}$ & $f_{p 0,2}$ & $f_{u}$ & $\mathrm{f}_{\mathrm{b}}$ & $\varepsilon_{\mathrm{p}}$ & $\varepsilon_{\mathrm{p} 0,2}$ & $\varepsilon_{\mathrm{u}}$ & $\varepsilon_{\mathrm{b}}$ \\
\hline 20 & 207000 & 296,6 & 597,4 & 694,8 & 282,8 & 0,00143 & 0,00489 & 0,01966 & 0,13457 \\
\hline 100 & 203000 & 334,8 & 587,9 & 658,5 & 258,7 & 0,00165 & 0,00490 & 0,01164 & 0,10727 \\
\hline 200 & 195000 & 311,7 & 629,5 & 728,6 & 346,0 & 0,00160 & 0,00523 & 0,02214 & 0,08297 \\
\hline 250 & 191000 & 371,3 & 640,2 & 770,0 & 457,0 & 0,00194 & 0,00535 & 0,03363 & 0,23669 \\
\hline 300 & 187000 & 269,8 & 609,3 & 733,5 & 366,8 & 0,00144 & 0,00526 & 0,02512 & 0,24486 \\
\hline 350 & 182500 & 369,5 & 581,9 & 713,8 & 311,5 & 0,00202 & 0,00519 & 0,04291 & 0,17131 \\
\hline 400 & 178000 & 390,4 & 531,9 & 645,6 & 267,2 & 0,00219 & 0,00499 & 0,03833 & 0,16150 \\
\hline 450 & 172500 & 287,6 & 460,1 & 566,2 & 271,3 & 0,00167 & 0,00467 & 0,04128 & 0,23647 \\
\hline 500 & 167000 & 210,2 & 366,0 & 469,0 & 199,1 & 0,00126 & 0,00419 & 0,04231 & 0,19559 \\
\hline 550 & 159500 & 63,2 & 251,9 & 348,2 & 125,2 & 0,00040 & 0,00358 & 0,04028 & 0,26039 \\
\hline 600 & 152000 & 60,6 & 152,2 & 253,2 & 69,0 & 0,00040 & 0,00300 & 0,03137 & 0,39095 \\
\hline 650 & 138500 & 13,5 & 89,0 & 180,2 & 14,0 & 0,00010 & 0,00264 & 0,03400 & 0,27540 \\
\hline 700 & 125000 & 22,9 & 51,3 & 119,6 & 70,4 & 0,00018 & 0,00241 & 0,02836 & 0,39006 \\
\hline
\end{tabular}

Anmerkung: Zwischen den Materialmodellen Mat 3 und Mat 4 treten für die Kennwerte des Blankstahls lediglich für die 0,2\%-Dehngrenze in den höheren Temperaturbereichen kleinere Abweichungen auf. Alle anderen Werte sind identisch.

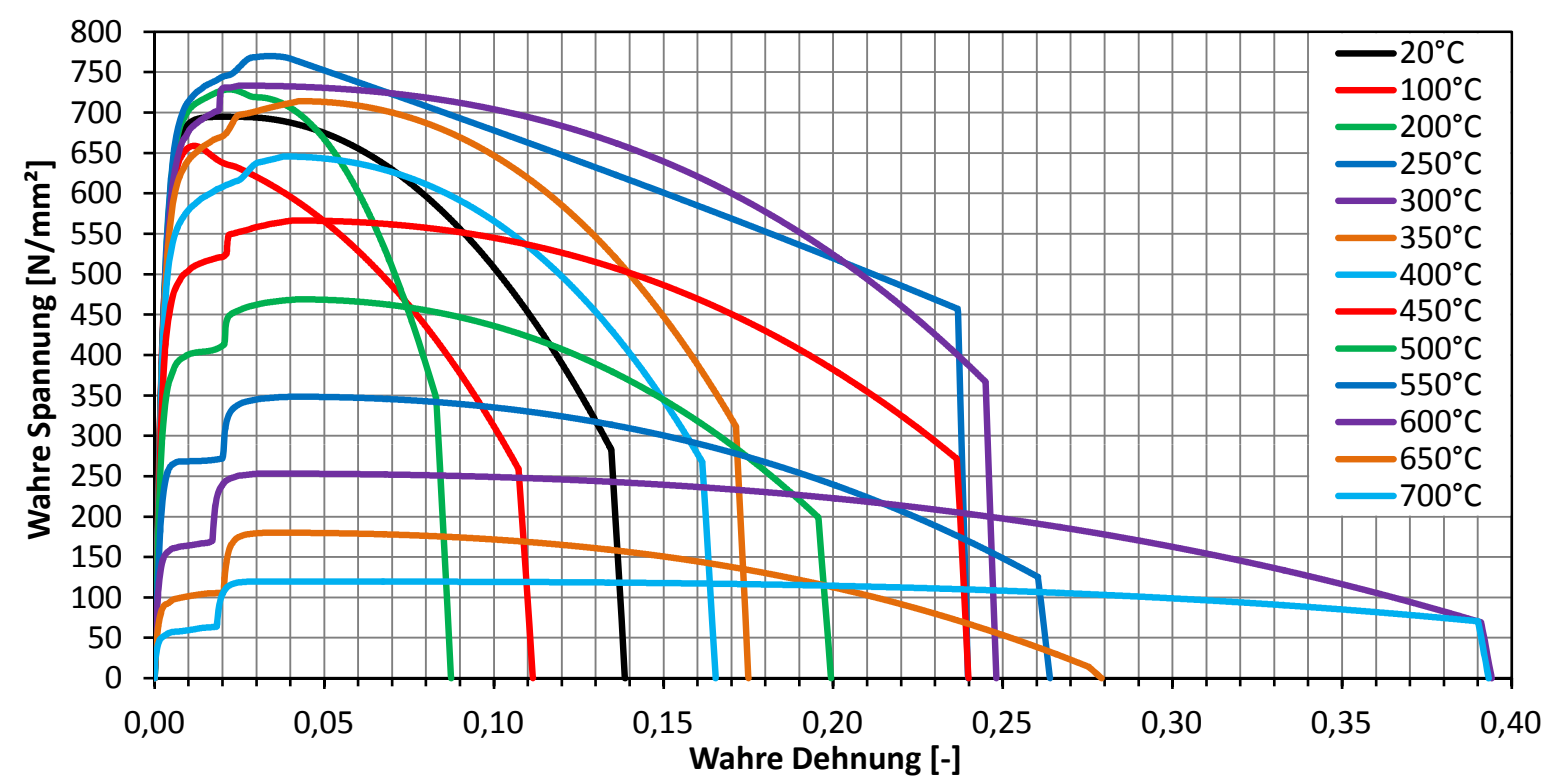

Abb. C.01: Spannungs-Dehnungs-Kennlinien für das Materialmodell Mat 3 
Tabelle C.2: Festigkeitsparameter aus den wahren $\sigma-\varepsilon-K u r v e n$ des neu erstellten Materialmodells (Mat 4)

\begin{tabular}{|c|c|c|c|c|c|c|c|c|c|}
\hline \multirow{2}{*}{$\begin{array}{c}\text { Stahltem- } \\
\text { peratur } \\
{\left[{ }^{\circ} \mathrm{C}\right]}\end{array}$} & \multirow{2}{*}{$\begin{array}{c}\text { E-Modul } \\
{\left[\mathrm{N} / \mathrm{mm}^{2}\right]}\end{array}$} & \multicolumn{4}{|c|}{$\begin{array}{c}\text { Spannungen } \\
{\left[\mathrm{N} / \mathrm{mm}^{2}\right]}\end{array}$} & \multicolumn{4}{|c|}{$\begin{array}{c}\text { Dehnungen } \\
{[-]}\end{array}$} \\
\hline & & $f_{p}$ & $\mathrm{f}_{\mathrm{p} 0,2}$ & $\mathrm{f}_{\mathrm{u}}$ & $f_{b}$ & $\varepsilon_{\mathrm{p}}$ & $\varepsilon_{\mathrm{p} 0,2}$ & $\varepsilon_{\mathrm{u}}$ & $\varepsilon_{\mathrm{b}}$ \\
\hline 20 & 207000 & 296,6 & 597,4 & 694,8 & 282,8 & 0,00143 & 0,00489 & 0,01966 & 0,13457 \\
\hline 100 & 203000 & 334,8 & 587,9 & 658,5 & 258,7 & 0,00165 & 0,00490 & 0,01164 & 0,10727 \\
\hline 200 & 195000 & 311,7 & 629,5 & 728,6 & 346,0 & 0,00160 & 0,00523 & 0,02214 & 0,08297 \\
\hline 250 & 191000 & 371,3 & 640,2 & 770,0 & 457,0 & 0,00194 & 0,00535 & 0,03363 & 0,23669 \\
\hline 300 & 187000 & 269,8 & 609,3 & 733,5 & 366,8 & 0,00144 & 0,00526 & 0,02512 & 0,24486 \\
\hline 350 & 182500 & 369,5 & 581,9 & 713,8 & 311,5 & 0,00202 & 0,00519 & 0,04291 & 0,17131 \\
\hline 400 & 178000 & 390,4 & 531,9 & 645,6 & 267,2 & 0,00219 & 0,00499 & 0,03833 & 0,16150 \\
\hline 450 & 172500 & 287,6 & 460,1 & 566,2 & 271,3 & 0,00167 & 0,00467 & 0,04128 & 0,23647 \\
\hline 500 & 167000 & 210,2 & 366,0 & 469,0 & 199,1 & 0,00126 & 0,00419 & 0,04231 & 0,19559 \\
\hline 550 & 159500 & 63,2 & 251,9 & 348,2 & 125,2 & 0,00040 & 0,00358 & 0,04028 & 0,26039 \\
\hline 600 & 152000 & 60,6 & 152,8 & 253,2 & 69,0 & 0,00040 & 0,00301 & 0,03137 & 0,39095 \\
\hline 650 & 138500 & 13,5 & 89,7 & 180,2 & 14,0 & 0,00010 & 0,00265 & 0,03400 & 0,27540 \\
\hline 700 & 125000 & 22,9 & 53,5 & 119,6 & 70,4 & 0,00018 & 0,00243 & 0,02836 & 0,39006 \\
\hline
\end{tabular}

Anmerkung: Zwischen den Materialmodellen Mat 3 und Mat 4 treten für die Kennwerte des Blankstahls lediglich für die 0,2\%-Dehngrenze in den höheren Temperaturbereichen kleinere Abweichungen auf. Alle anderen Werte sind identisch.

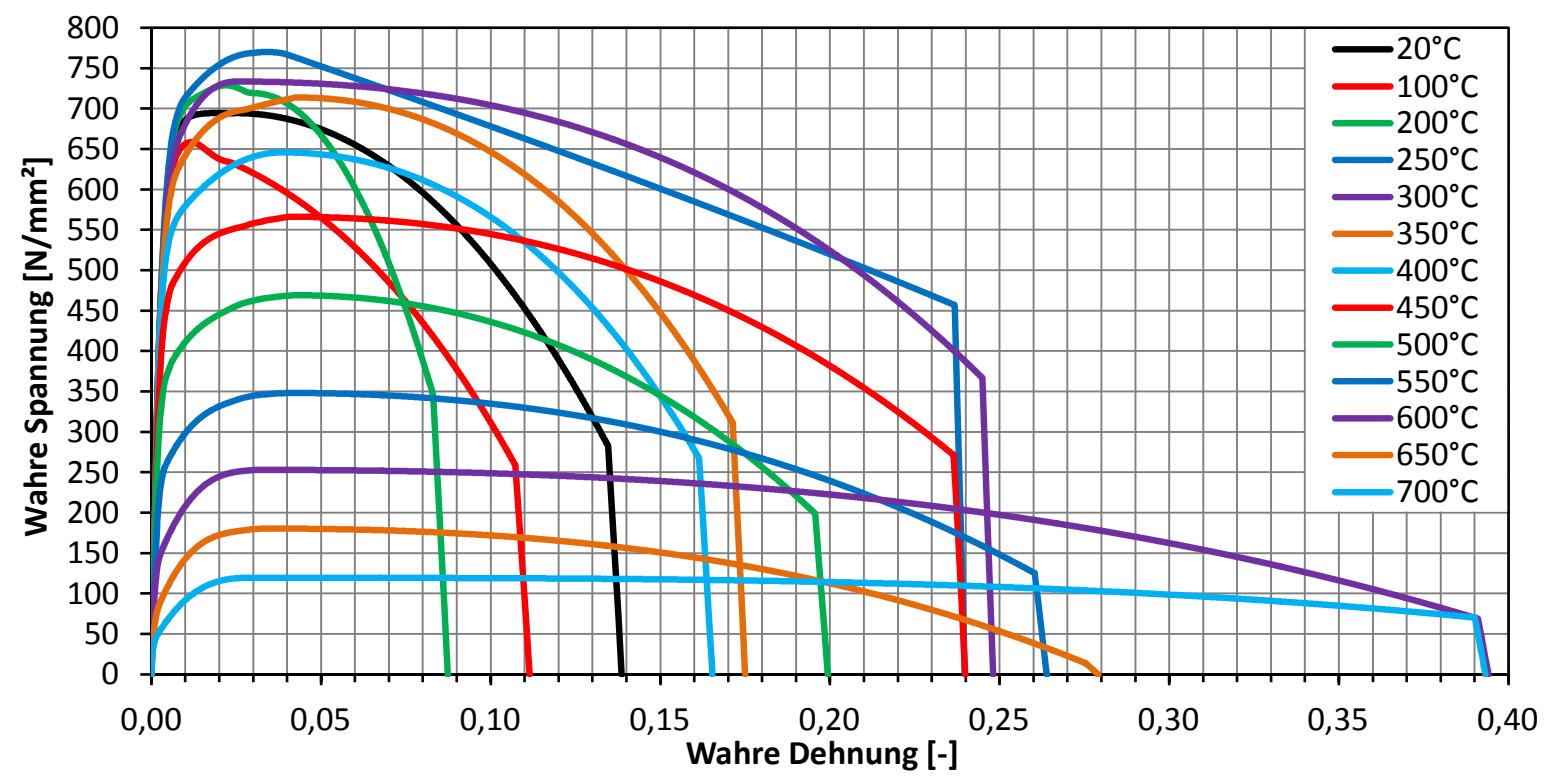

Abb. C.02: Spannungs-Dehnungs-Kennlinien für das Materialmodell Mat 4 


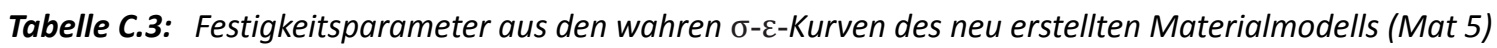

\begin{tabular}{|c|c|c|c|c|c|c|c|c|c|}
\hline \multirow{2}{*}{$\begin{array}{c}\text { Stahltem- } \\
\text { peratur } \\
{\left[{ }^{\circ} \mathrm{C}\right]}\end{array}$} & \multirow{2}{*}{$\begin{array}{c}\begin{array}{c}\text { E-Modul } \\
{\left[\mathrm{N} / \mathrm{mm}^{2}\right]}\end{array} \\
\mathrm{E}_{\mathrm{dyn}}\end{array}$} & \multicolumn{4}{|c|}{$\begin{array}{c}\text { Spannungen } \\
{\left[\mathrm{N} / \mathrm{mm}^{2}\right]}\end{array}$} & \multicolumn{4}{|c|}{$\begin{array}{c}\text { Dehnungen } \\
{[-]}\end{array}$} \\
\hline & & $f_{p}$ & $\mathrm{f}_{\mathrm{p} 0,2}$ & $\mathrm{f}_{\mathrm{u}}$ & $\mathrm{f}_{\mathrm{b}}$ & $\varepsilon_{\mathrm{p}}$ & $\varepsilon_{\mathrm{p} 0,2}$ & $\varepsilon_{\mathrm{u}}$ & $\varepsilon_{\mathrm{b}}$ \\
\hline 20 & 207000 & 380,7 & 583,2 & 676,7 & 275,9 & 0,00184 & 0,00482 & 0,01966 & 0,13457 \\
\hline 100 & 203000 & 429,8 & 573,9 & 641,4 & 252,4 & 0,00212 & 0,00483 & 0,01164 & 0,10727 \\
\hline 200 & 195000 & 400,0 & 614,5 & 709,6 & 337,6 & 0,00205 & 0,00515 & 0,02214 & 0,08297 \\
\hline 250 & 191000 & 476,5 & 625,0 & 750,0 & 446,0 & 0,00250 & 0,00527 & 0,03363 & 0,23669 \\
\hline 300 & 187000 & 346,3 & 594,8 & 714,4 & 358,0 & 0,00185 & 0,00518 & 0,02512 & 0,24486 \\
\hline 350 & 182500 & 474,3 & 568,1 & 695,3 & 303,9 & 0,00260 & 0,00511 & 0,04291 & 0,17131 \\
\hline 400 & 178000 & 432,4 & 519,3 & 628,8 & 260,8 & 0,00243 & 0,00492 & 0,03833 & 0,16150 \\
\hline 450 & 172500 & 369,1 & 449,1 & 551,4 & 264,7 & 0,00214 & 0,00460 & 0,04128 & 0,23647 \\
\hline 500 & 167000 & 269,9 & 357,3 & 456,8 & 194,3 & 0,00162 & 0,00414 & 0,04231 & 0,19559 \\
\hline 550 & 159500 & 166,5 & 245,9 & 339,1 & 122,2 & 0,00104 & 0,00354 & 0,04028 & 0,26039 \\
\hline 600 & 152000 & 77,8 & 149,2 & 246,6 & 67,3 & 0,00051 & 0,00298 & 0,03137 & 0,39095 \\
\hline 650 & 138500 & 17,4 & 87,5 & 175,5 & 13,6 & 0,00013 & 0,00263 & 0,03400 & 0,27540 \\
\hline 700 & 125000 & 29,4 & 52,2 & 116,5 & 68,7 & 0,00023 & 0,00242 & 0,02836 & 0,39006 \\
\hline
\end{tabular}

Anmerkung: Zwischen den Materialmodellen Mat 3 und Mat 4 treten für die Kennwerte des Blankstahls lediglich für die 0,2\%-Dehngrenze in den höheren Temperaturbereichen kleinere Abweichungen auf. Alle anderen Werte sind identisch.

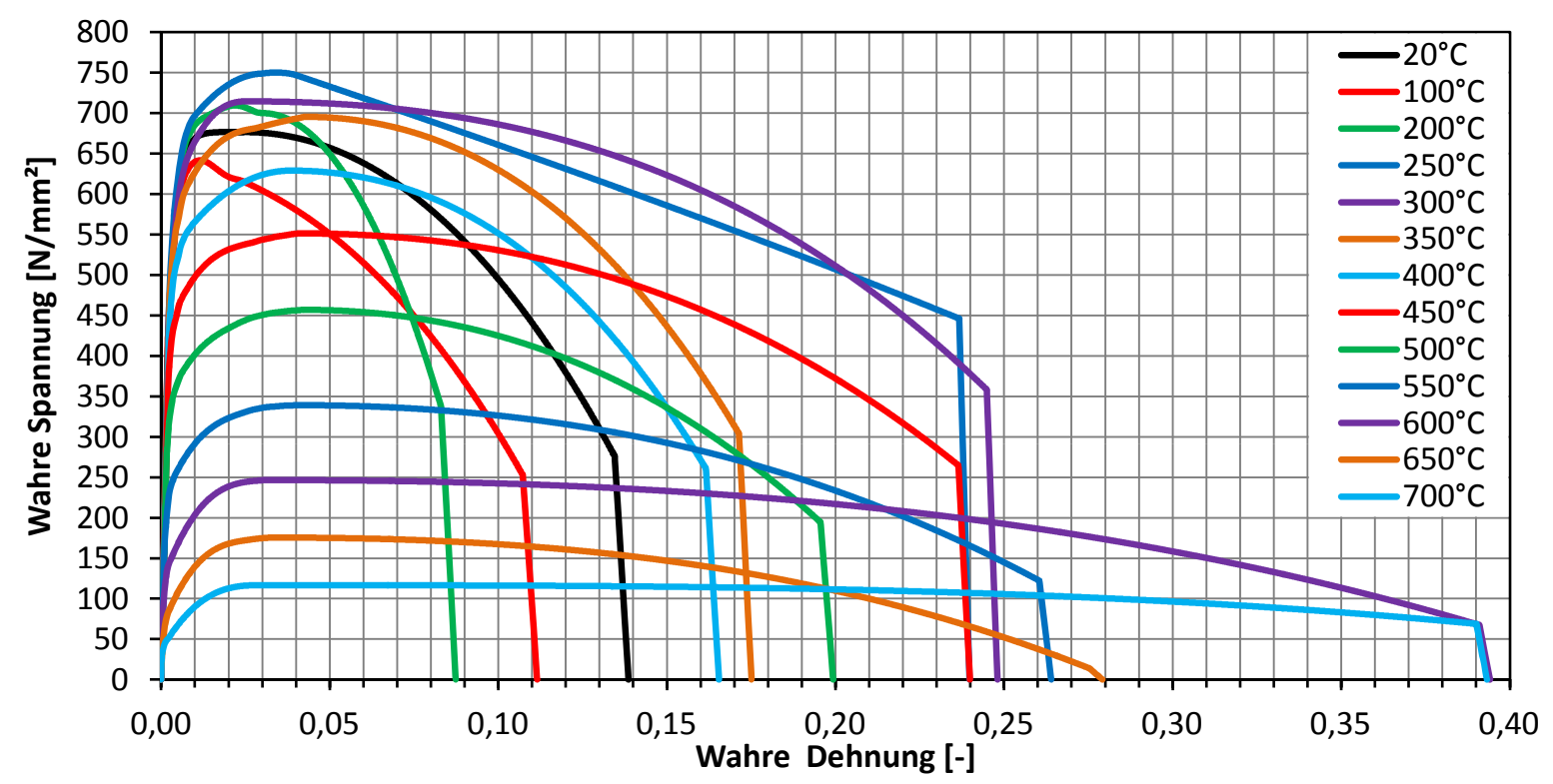

Abb. C.03: Spannungs-Dehnungs-Kennlinien für das Materialmodell Mat 5 



\section{Gegenüberstellung der in Brandversuchen gemessenen und numerisch berechneten Stablängsverformungen}
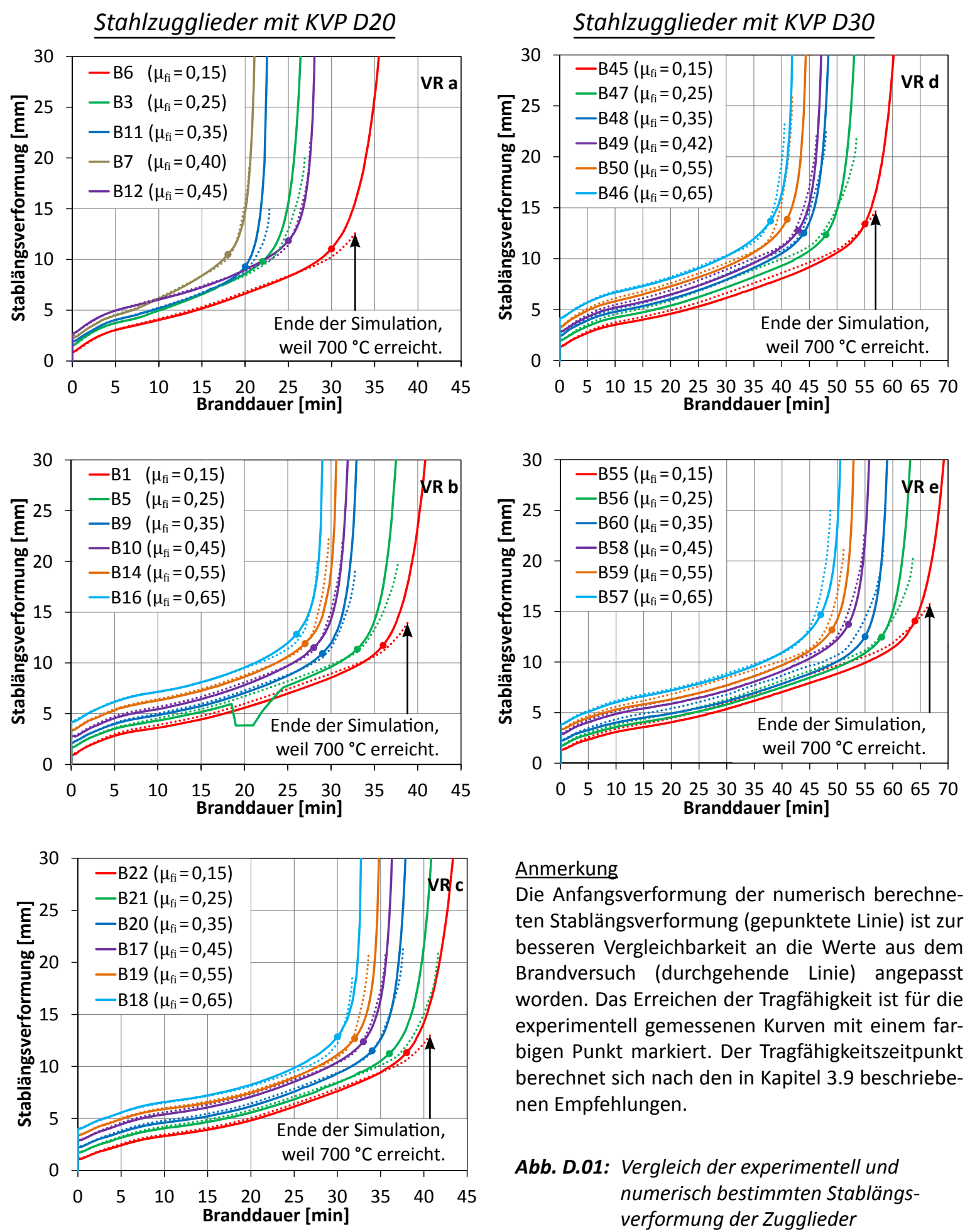

Anmerkung

Die Anfangsverformung der numerisch berechneten Stablängsverformung (gepunktete Linie) ist zur besseren Vergleichbarkeit an die Werte aus dem Brandversuch (durchgehende Linie) angepasst worden. Das Erreichen der Tragfähigkeit ist für die experimentell gemessenen Kurven mit einem farbigen Punkt markiert. Der Tragfähigkeitszeitpunkt berechnet sich nach den in Kapitel 3.9 beschriebenen Empfehlungen.

Abb. D.01: Vergleich der experimentell und numerisch bestimmten Stablängsverformung der Zugglieder 



\section{Lebenslauf}

Name:

Dustin Häßler

Geburtsdatum: 26.07.1986

Geburtsort: Karl-Marx-Stadt (jetzt Chemnitz)

Nationalität: Deutsch

Familienstatus: Ledig

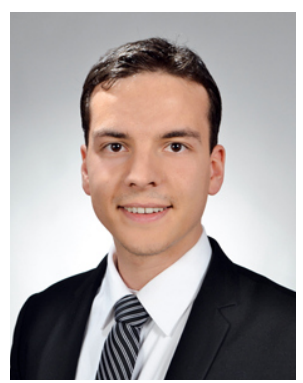

\section{Ausbildung}

2012

2011

Sachverständiger für den konstruktiven Ingenieurbau (EIPOS)

$2008-2011$ Master of Science (Konstruktiver Ingenieurbau)

mauingenieurwesens an der

2010

Brandenburgischen Technischen Universität Cottbus

$2009-2010$

Master of Science (Structural Analysis of Monuments and Historical Constructions - SAHC) Gebäude (SAHC) an der Technischen Universität Prag (CZ) und der Universität von Minho (PT)
2008 Bachelor of Science (Konstruktiver Ingenieurbau)
2005-2008 Studium des Bauingenieurwesens an der Brandenburgischen Technischen Universität Cottbus
1997-2005 Gymnasium in Olbernhau Vertiefungsstudium im Bereich der konstruktiven Analyse historischer

\section{Beruflicher Werdegang}

$2011 \rightarrow$

Wissenschaftlicher Mitarbeiter an der BAM Bundesanstalt für Materialforschung und -prüfung (Fachbereich 7.3-Brandingenieurwesen) Akademischer Mitarbeiter an der Brandenburgischen Technischen Universität Cottbus (Lehrstuhl für Statik und Dynamik) Freier Mitarbeiter im Bauplanungs- und Sachverständigenbüro Dr. Dietmar Häßler

\section{Auszeichnungen und Preise}

2015

2014

2012

2011
Auszeichnung beim BAM Posterwettbewerb, 1. Platz

Auszeichnung des beim 19. DASt-Kolloquium Stahlbau in Hannover vorgestellten Vortrages, 3. Preis

Nachwuchs-Förderpreis des Ernst \& Sohn Verlages

Nachwuchsinnovationspreis Bauwerkserhaltung des Bundesverbandes

für Feuchte und Altbausanierung e. V. (BuFAS), 2. Preis 



\section{Erklärung zur Dissertation}

gemäß der Promotionsordnung vom 05.05.2014

Ich erkläre, dass ich meine Dissertation mit dem Titel

„Verhalten reaktiver Brandschutzsysteme auf kreisförmigen Zuggliedern aus Blank- und Baustahl"

entsprechend den Vorgaben nach $\S 5$ der Promotionsordnung verfasst habe.

Diesen Vorgaben entsprechend erkläre ich im Einzelnen:

a. die Regeln der geltenden Promotionsordnung zu kennen und eingehalten zu haben und mit einer Prüfung nach den Bestimmungen der Promotionsordnung einverstanden zu sein,

b. die Dissertation selbst verfasst zu haben (Selbständigkeitserklärung), keine Textabchnitte von Dritten oder eigener Prüfungsarbeiten ohne Kennzeichnung übernommen und alle von ihr oder ihm benutzten Hilfsmittel und Quellen in seiner Arbeit angegeben zu haben,

c. Dritten weder unmittelbar noch mittelbar geldwerte Leistungen für Vermittlungstätigkeiten oder für die inhaltliche Ausarbeitung der Dissertation erbracht zu haben (d.h. die wissenschaftliche Arbeit darf weder in Teilen noch in Gänze von Dritten gegen Entgelt oder sonstige Gegenleistung erworben oder vermittelt worden sein),

d. die Dissertation noch nicht als Prüfungsarbeit für eine staatliche oder andere wissenschaftliche Prüfung eingereicht zu haben,

e. ob sie bzw. er die gleiche oder eine in wesentlichen Teilen ähnliche Arbeit bei einer anderen Hochschule als Dissertation eingereicht hat und gegebenenfalls mit welchem Ergebnis; zugleich ist mitzuteilen, ob eine andere Abhandlung als Dissertation eingereicht wurde und gegebenenfalls mit welchem Ergebnis,

f. damit einverstanden zu sein, dass die Dissertation auch zum Zwecke der Überprüfung der Einhaltung allgemein geltender wissenschaftlicher Standards genutzt wird, insbesondere auch unter Verwendung elektronischer Datenverarbeitungsprogramme.

Datum, Ort, Unterschrift

Dustin Häßler

Name (in Druckbuchstaben) 